Bond University

Research Repository

\title{
Fluphenazine decanoate (depot) and enanthate for schizophrenia
}

Maayan, Nicola; Quraishi, Seema N.; David, Anthony; Jayaswal, Aprajita; Eisenbruch, Maurice; Rathbone, John; Asher, Rosie; Adams, Clive E.

Published in:

Cochrane Database of Systematic Reviews

DOI:

10.1002/14651858.CD000307.pub2

Licence:

Other

Link to output in Bond University research repository.

Recommended citation(APA):

Maayan, N., Quraishi, S. N., David, A., Jayaswal, A., Eisenbruch, M., Rathbone, J., Asher, R., \& Adams, C. E. (2015). Fluphenazine decanoate (depot) and enanthate for schizophrenia. Cochrane Database of Systematic Reviews, 2015(2), [CD000307]. https://doi.org/10.1002/14651858.CD000307.pub2

\section{General rights}

Copyright and moral rights for the publications made accessible in the public portal are retained by the authors and/or other copyright owners and it is a condition of accessing publications that users recognise and abide by the legal requirements associated with these rights.

For more information, or if you believe that this document breaches copyright, please contact the Bond University research repository coordinator. 


\section{(E) Cochrane Library}

Cochrane Database of Systematic Reviews

\section{Fluphenazine decanoate (depot) and enanthate for schizophrenia (Review)}

Maayan N, Quraishi SN, David A, Jayaswal A, Eisenbruch M, Rathbone J, Asher R, Adams CE

Maayan N, Quraishi SN, David A, Jayaswal A, Eisenbruch M, Rathbone J, Asher R, Adams CE.

Fluphenazine decanoate (depot) and enanthate for schizophrenia.

Cochrane Database of Systematic Reviews 2015, Issue 2. Art. No.: CD000307.

DOI: 10.1002/14651858.CD000307.pub2.

www.cochranelibrary.com 
TABLE OF CONTENTS

HEADER . . . . . . . . . . . . . . . . . . . . . . . . . . . . . . . . . . . . . . . . 1

ABSTRACT . . . . . . . . . . . . . . . . . . . . . . . . . . . . . . . . . . . . . . . . . . . . . . . 1

PLAIN LANGUAGE SUMMARY . . . . . . . . . . . . . . . . . . . . . . . . . . . . . . . . . . . . . . . 2

SUMMARY OF FINDINGS FOR THE MAIN COMPARISON . . . . . . . . . . . . . . . . . . . . . . . . . . . . 4

BACKGROUND . . . . . . . . . . . . . . . . . . . . . . . . . . . . . . . . . . . . . 6

OBJECTIVES . . . . . . . . . . . . . . . . . . . . . . . . . . . . . . . . . . . . . . . . . . . . . .

METHODS . . . . . . . . . . . . . . . . . . . . . . . . . . . . . . . . . . . . . . . . .

RESULTS . . . . . . . . . . . . . . . . . . . . . . . . . . . . . . . . . . . . . . . 11

Figure 1. . . . . . . . . . . . . . . . . . . . . . . . . . . . . . . . . . . . . . 13

Figure 2. . . . . . . . . . . . . . . . . . . . . . . . . . . . . . . . . . . . . . . . . . . 16

Figure 3. . . . . . . . . . . . . . . . . . . . . . . . . . . . . . . . . . . . . . . . . . .

ADDITIONAL SUMMARY OF FINDINGS . . . . . . . . . . . . . . . . . . . . . . . . . . . . . . . . . .

DISCUSSION . . . . . . . . . . . . . . . . . . . . . . . . . . . . . . . . . . . . . . . . . . 30

AUTHORS' CONCLUSIONS . . . . . . . . . . . . . . . . . . . . . . . . . . . . . . . . . . . . . .

ACKNOWLEDGEMENTS . . . . . . . . . . . . . . . . . . . . . . . . . . . . . . . . . . . . . . . . . . .

REFERENCES . . . . . . . . . . . . . . . . . . . . . . . . . . . . . . . . . . . . . . 33

CHARACTERISTICS OF STUDIES . . . . . . . . . . . . . . . . . . . . . . . . . . . . . . . . . . 50

DATA AND ANALYSES . . . . . . . . . . . . . . . . . . . . . . . . . . . . . . . . . . . . . . . . 160

Analysis 1.1. Comparison 1 FLUPHENAZINE DECANOATE vs PLACEBO, Outcome 1 Death. . . . . . . . 170

Analysis 1.2. Comparison 1 FLUPHENAZINE DECANOATE vs PLACEBO, Outcome 2 Global state: 1 . Relapse. 171

Analysis 1.3. Comparison 1 FLUPHENAZINE DECANOATE vs PLACEBO, Outcome 3 Global state: 2. GAS (short term -6 weeks to 5 months) (high score $=$ worse)..$\quad$. . . . . . . . . . . . . . . . . . . . . . . . . $\quad$. 172

Analysis 1.4. Comparison 1 FLUPHENAZINE DECANOATE vs PLACEBO, Outcome 4 Leaving the study early. . 173

Analysis 1.5. Comparison 1 FLUPHENAZINE DECANOATE vs PLACEBO, Outcome 5 Mental state: 1. BPRS (endpoint scores - high score $=$ worse).$\quad$. . . . . . . . . . . . . . . . . . . . . . . . . . . . . . . . . 174

Analysis 1.6. Comparison 1 FLUPHENAZINE DECANOATE vs PLACEBO, Outcome 6 Mental state: 2. Depression (medium term -6 months to 1 year). . . . . . . . . . . . . . . . . . . . . . . . . . . . . . . . . .

Analysis 1.7. Comparison 1 FLUPHENAZINE DECANOATE vs PLACEBO, Outcome 7 Adverse effects: 1. Movement disorders - tardive dyskinesia (longer term - more than 1 year). . . . . . . . . . . . . . . . . . . . . 175

Analysis 1.8. Comparison 1 FLUPHENAZINE DECANOATE vs PLACEBO, Outcome 8 Adverse effects: 2. Toxicity. 175

Analysis 2.1. Comparison 2 FLUPHENAZINE DECANOATE vs ORAL NEUROLEPTICS, Outcome 1 Global state: 1.

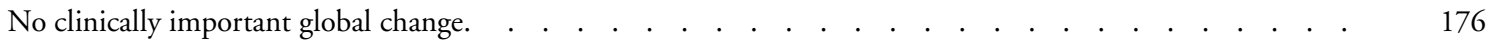

Analysis 2.2. Comparison 2 FLUPHENAZINE DECANOATE vs ORAL NEUROLEPTICS, Outcome 2 Global state: 2. Relapse. . . . . . . . . . . . . . . . . . . . . . . . . . . . . . . . . . . . . 177

Analysis 2.3. Comparison 2 FLUPHENAZINE DECANOATE vs ORAL NEUROLEPTICS, Outcome 3 Global state: 3. Clinical Global Impression (short term - 6 weeks to 5 months) (high score = worse). $\quad$. . . . . . . . . . . . . 178

Analysis 2.4. Comparison 2 FLUPHENAZINE DECANOATE vs ORAL NEUROLEPTICS, Outcome 4 Leaving the study early.

Analysis 2.5. Comparison 2 FLUPHENAZINE DECANOATE vs ORAL NEUROLEPTICS, Outcome 5 Behaviour: 1. NOSIE-30 - endpoint scores (high score = poor). $\quad . \quad$. . . . . . . . . . . . . . . . . . . . . . . . . . . . . . 180

Analysis 2.7. Comparison 2 FLUPHENAZINE DECANOATE vs ORAL NEUROLEPTICS, Outcome 7 Mental state: 1. BPRS - endpoint scores (longer term - more than 1 year) (high score = poor). . . . . . . . . . . . . . . . . . . 181

Analysis 2.8. Comparison 2 FLUPHENAZINE DECANOATE vs ORAL NEUROLEPTICS, Outcome 8 Mental state: 2. Depression

Analysis 2.9. Comparison 2 FLUPHENAZINE DECANOATE vs ORAL NEUROLEPTICS, Outcome 9 Adverse effects: 1a. Movement disorders - general. . . . . . . . . . . . . . . . . . . . . . . . . . . . . . . . . . . 183

Analysis 2.10. Comparison 2 FLUPHENAZINE DECANOATE vs ORAL NEUROLEPTICS, Outcome 10 Adverse effects: 1b. Movement disorders - akathisia.

Analysis 2.11. Comparison 2 FLUPHENAZINE DECANOATE vs ORAL NEUROLEPTICS, Outcome 11 Adverse effects: 1c. Movement disorders - needing anticholinergic drugs.

Fluphenazine decanoate (depot) and enanthate for schizophrenia (Review)

Copyright $\odot 2016$ The Cochrane Collaboration. Published by John Wiley \& Sons, Ltd. 
Analysis 2.12. Comparison 2 FLUPHENAZINE DECANOATE vs ORAL NEUROLEPTICS, Outcome 12 Adverse effects: $1 \mathrm{~d}$. Movement disorders - tardive dyskinesia. $\quad . \quad$. . . . . . . . . . . . . . . . . . . . . . . . .

Analysis 2.13. Comparison 2 FLUPHENAZINE DECANOATE vs ORAL NEUROLEPTICS, Outcome 13 Adverse effects: 1e. Movement disorders - tremor (longer term - more than 1 year) . . . . . . . . . . . . . . . . . .

Analysis 2.14. Comparison 2 FLUPHENAZINE DECANOATE vs ORAL NEUROLEPTICS, Outcome 14 Adverse effects: 1f. Movement disorders - average score (Simpson \& Angus, 0 to 5 weeks, high = poor). . . . . . . . .

Analysis 2.15. Comparison 2 FLUPHENAZINE DECANOATE vs ORAL NEUROLEPTICS, Outcome 15 Adverse effects: 2. Blurred vision - medium term (6 months to 1 year).

Analysis 2.16. Comparison 2 FLUPHENAZINE DECANOATE vs ORAL NEUROLEPTICS, Outcome 16 Adverse effects: 3. Toxicity - medium term (6 months to 1 year).

Analysis 2.17. Comparison 2 FLUPHENAZINE DECANOATE vs ORAL NEUROLEPTICS, Outcome 17 Adverse effects: 4. General adverse effects. . . . . . . . . . . . . . . . . . . . . . . . . . . . . . . . .

Analysis 2.18. Comparison 2 FLUPHENAZINE DECANOATE vs ORAL NEUROLEPTICS, Outcome 18 SENSITIVITY ANALYSIS Global state: 2 . Relapse. $\quad . \quad$. . . . . . . . . . . . . . . . . . . . . . . . .

Analysis 3.1. Comparison 3 FLUPHENAZINE DECANOATE vs OTHER DEPOT NEUROLEPTICS, Outcome 1 Death.

Analysis 3.2. Comparison 3 FLUPHENAZINE DECANOATE vs OTHER DEPOT NEUROLEPTICS, Outcome 2 Global state: 1 . No clinically important global change.

Analysis 3.3. Comparison 3 FLUPHENAZINE DECANOATE vs OTHER DEPOT NEUROLEPTICS, Outcome 3 Global state: 2. Relapse. . . . . . . . . . . . . . . . . . . . . . . . . . . . . . .

Analysis 3.4. Comparison 3 FLUPHENAZINE DECANOATE vs OTHER DEPOT NEUROLEPTICS, Outcome 4 Global state: 3. Severly ill (medium term 6 months to 1 year).

Analysis 3.5. Comparison 3 FLUPHENAZINE DECANOATE vs OTHER DEPOT NEUROLEPTICS, Outcome 5 Global state: 4. Needing additional antipsychotic treatment (6 months to 1 year). . . . . . . . . . . . . . .

Analysis 3.7. Comparison 3 FLUPHENAZINE DECANOATE vs OTHER DEPOT NEUROLEPTICS, Outcome 7 Global state: 6. Clinical Global Impression. (medium term - 6 months to 1 year).

Analysis 3.8. Comparison 3 FLUPHENAZINE DECANOATE vs OTHER DEPOT NEUROLEPTICS, Outcome 8 Global state: 7. Clinical Global Impression - not improved (high score = poor). . . . . . . . . . . . . .

Analysis 3.9. Comparison 3 FLUPHENAZINE DECANOATE vs OTHER DEPOT NEUROLEPTICS, Outcome 9 Leaving the study early.

Analysis 3.10. Comparison 3 FLUPHENAZINE DECANOATE vs OTHER DEPOT NEUROLEPTICS, Outcome 10 Behaviour: 1. NOSIE-30 - endpoint scores (high score = poor). . . . . . . . . . . . . . . . . . . . . . . .

Analysis 3.11. Comparison 3 FLUPHENAZINE DECANOATE vs OTHER DEPOT NEUROLEPTICS, Outcome 11 Mental state: 1. BPRS (endpoint scores - high score = poor). . . . . . . . . . . . . . . . . . . . . . . . . . .

Analysis 3.12. Comparison 3 FLUPHENAZINE DECANOATE vs OTHER DEPOT NEUROLEPTICS, Outcome 12 Mental state: 2. BPRS (endpoint scores 6 months to 1 year - dichotomous data). . . . . . . . . . . . . . .

Analysis 3.13. Comparison 3 FLUPHENAZINE DECANOATE vs OTHER DEPOT NEUROLEPTICS, Outcome 13

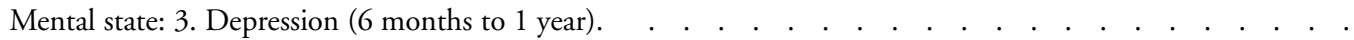

Analysis 3.15. Comparison 3 FLUPHENAZINE DECANOATE vs OTHER DEPOT NEUROLEPTICS, Outcome 15 Adverse effects: 1a. Movement disorders - general.

Analysis 3.16. Comparison 3 FLUPHENAZINE DECANOATE vs OTHER DEPOT NEUROLEPTICS, Outcome 16 Adverse effects: 1b. Movement disorders - needing anticholinergic drugs.

Analysis 3.17. Comparison 3 FLUPHENAZINE DECANOATE vs OTHER DEPOT NEUROLEPTICS, Outcome 17 Adverse effects: 1c. Movement disorders - parkinsonism. . . . . . . . . . . . . . . . . . . . . . . . . . . . .

Analysis 3.18. Comparison 3 FLUPHENAZINE DECANOATE vs OTHER DEPOT NEUROLEPTICS, Outcome 18 Adverse effects: $1 \mathrm{~d}$. Movement disorders - tardive dyskinesia: longer term (more than 1 year). . . . . . . .

Analysis 3.19. Comparison 3 FLUPHENAZINE DECANOATE vs OTHER DEPOT NEUROLEPTICS, Outcome 19 Adverse effects: 1e. Movement disorders - tremor. . . . . . . . . . . . . . . . . . . . . . . . . . . .

Analysis 3.21. Comparison 3 FLUPHENAZINE DECANOATE vs OTHER DEPOT NEUROLEPTICS, Outcome 21 Adverse effects: 2. Blurred vision. . . . . . . . . . . . . . . . . . . . . . . . . . . . . . . . . . . . . .

Analysis 3.22. Comparison 3 FLUPHENAZINE DECANOATE vs OTHER DEPOT NEUROLEPTICS, Outcome 22 Adverse effects: 3. Dry mouth: longer term (more than 1 year). . . . . . . . . . . . . . . . . . . . . . . . .

Fluphenazine decanoate (depot) and enanthate for schizophrenia (Review)

Copyright () 2016 The Cochrane Collaboration. Published by John Wiley \& Sons, Ltd. 
Analysis 3.23. Comparison 3 FLUPHENAZINE DECANOATE vs OTHER DEPOT NEUROLEPTICS, Outcome 23 Adverse effects: 4 . General adverse effects. . . . . . . . . . . . . . . . . . . . . . . . .

Analysis 3.24. Comparison 3 FLUPHENAZINE DECANOATE vs OTHER DEPOT NEUROLEPTICS, Outcome 24 SENSITIVITY ANALYSIS Global state: 2. Relapse.

Analysis 4.1. Comparison 4 FLUPHENAZINE DECANOATE - DOSAGE STUDIES - HIGH DOSE vs STANDARD, Outcome 1 Global state: 1 . Relapse (medium term -6 months to 1 year). . . . . . . . . . . . . . . . . . 210

Analysis 4.2. Comparison 4 FLUPHENAZINE DECANOATE - DOSAGE STUDIES - HIGH DOSE vs STANDARD, Outcome 2 Global state: 2. Needing additional antispsychotic treatment (medium term - 6 months to 1 year).

Analysis 4.3. Comparison 4 FLUPHENAZINE DECANOATE - DOSAGE STUDIES - HIGH DOSE vs STANDARD, Outcome 3 Global state: 3. Not improved (medium term -6 months to 1 year). . . . . . . . . . . . . . . 212

Analysis 4.4. Comparison 4 FLUPHENAZINE DECANOATE - DOSAGE STUDIES - HIGH DOSE vs STANDARD, Outcome 4 Leaving the study early (medium term - 6 months to 1 year).

Analysis 4.5. Comparison 4 FLUPHENAZINE DECANOATE - DOSAGE STUDIES - HIGH DOSE vs STANDARD, Outcome 5 Mental state: BPRS endpoint scores (medium term - 6 months to 1 year, high score = poor). $\quad . \quad$. 213

Analysis 4.6. Comparison 4 FLUPHENAZINE DECANOATE - DOSAGE STUDIES - HIGH DOSE vs STANDARD, Outcome 6 Adverse effects: Movement disorders - needing anticholinergic drugs (medium term - 6 months to 1 year). . . . . . . . . . . . . . . . . . . . . . . . . . . . . . . . . . . . . . . . . . .

Analysis 5.1. Comparison 5 FLUPHENAZINE DECANOATE - DOSAGE STUDIES - LOW DOSE vs STANDARD, Outcome 1 Global state: Relapse.

Analysis 5.2. Comparison 5 FLUPHENAZINE DECANOATE - DOSAGE STUDIES - LOW DOSE vs STANDARD, Outcome 2 Leaving the study early. . . . . . . . . . . . . . . . . . . . . . . . . . . . . . .

Analysis 5.3. Comparison 5 FLUPHENAZINE DECANOATE - DOSAGE STUDIES - LOW DOSE vs STANDARD, Outcome 3 Adverse effects: 1 . Movement disorders (medium term -6 months to 1 year). . . . . . . . . .

Analysis 6.1. Comparison 6 FLUPHENAZINE ENANTHATE vs PLACEBO, Outcome 1 Adverse effects: Movement disorders - general. . . . . . . . . . . . . . . . . . . . . . . . . . . . . . . . . . . .

Analysis 7.1. Comparison 7 FLUPHENAZINE ENANTHATE vs ORAL NEUROLEPTICS, Outcome 1 Global state: No clinically important global change (immediate -0 to 5 weeks). . . . . . . . . . . . . . . . . . . .

Analysis 7.2. Comparison 7 FLUPHENAZINE ENANTHATE vs ORAL NEUROLEPTICS, Outcome 2 Adverse effects: Movement disorders - general. . . . . . . . . . . . . . . . . . . . . . . . . . . . . . . . . . .

Analysis 8.1. Comparison 8 FLUPHENAZINE ENANTHATE vs OTHER DEPOT NEUROLEPTICS, Outcome 1 Global state: 1 . Needing additional antipsychotic treatment $(6$ months to 1 year $) . \quad$. . . . . . . . . . . .

Analysis 8.2. Comparison 8 FLUPHENAZINE ENANTHATE vs OTHER DEPOT NEUROLEPTICS, Outcome 2 Global state: 2. Relapse.

Analysis 8.3. Comparison 8 FLUPHENAZINE ENANTHATE vs OTHER DEPOT NEUROLEPTICS, Outcome 3 Leaving the study early. . . . . . . . . . . . . . . . . . . . . . . . . . . . . . . . . . . Analysis 8.4. Comparison 8 FLUPHENAZINE ENANTHATE vs OTHER DEPOT NEUROLEPTICS, Outcome 4 Mental state: 1 . BPRS - endpoint scores (medium term -6 months to 1 year) (high score = poor). . . . . . Analysis 8.5. Comparison 8 FLUPHENAZINE ENANTHATE vs OTHER DEPOT NEUROLEPTICS, Outcome 5 Mental state: 2. Depression (medium term -6 months to 1 year). . . . . . . . . . . . . . . . . . . . . . . Analysis 8.6. Comparison 8 FLUPHENAZINE ENANTHATE vs OTHER DEPOT NEUROLEPTICS, Outcome 6 Adverse effects: 1a. Movement disorders - general (medium term - 6 months to 1 year). . . . . . . . . . . . Analysis 8.7. Comparison 8 FLUPHENAZINE ENANTHATE vs OTHER DEPOT NEUROLEPTICS, Outcome 7 Adverse effects: 1 b. Movement disorders - needing additional anticholinergic drugs. . . . . . . . . . . . . Analysis 8.8. Comparison 8 FLUPHENAZINE ENANTHATE vs OTHER DEPOT NEUROLEPTICS, Outcome 8 Adverse effects: 1c. Movement disorders - tardive dyskinesia: medium term (6 months to 1 year). . . . . . Analysis 8.9. Comparison 8 FLUPHENAZINE ENANTHATE vs OTHER DEPOT NEUROLEPTICS, Outcome 9 Adverse effects: $1 \mathrm{~d}$. Movement disorders - tremor (medium term - 6 months to 1 year).

Analysis 8.10. Comparison 8 FLUPHENAZINE ENANTHATE vs OTHER DEPOT NEUROLEPTICS, Outcome 10 Adverse effects: 2 . Blurred vision (medium term -6 months to 1 year). . . . . . . . . . . . . . . . . . . . .

Analysis 8.11. Comparison 8 FLUPHENAZINE ENANTHATE vs OTHER DEPOT NEUROLEPTICS, Outcome 11 Adverse effects: 3. Dry mouth (medium term -6 months to 1 year). . . . . . . . . . . . . . . . . . . . . . 
Analysis 9.2. Comparison 9 FLUPHENAZINE ENANTHATE - DOSAGE STUDIES - LOW DOSE vs

INTERMEDIATE/HIGH DOSE, Outcome 2 Leaving the study early (short term - 6 weeks to 5 months).

Analysis 10.1. Comparison 10 FLUPHENAZINE DECANAOTE vs FLUPHENAZINE ENANTHATE, Outcome 1

Global state: 1 . Needing additional antipsychotic treatment. . . . . . . . . . . . . . . . . . . . . . . . . .

Analysis 10.2. Comparison 10 FLUPHENAZINE DECANAOTE vs FLUPHENAZINE ENANTHATE, Outcome 2

Global state: 2 . Relapse. . . . . . . . . . . . . . . . . . . . . . . . . . . . . . .

Analysis 10.3. Comparison 10 FLUPHENAZINE DECANAOTE vs FLUPHENAZINE ENANTHATE, Outcome 3 Behavioiur: Leaving the study early.

Analysis 10.4. Comparison 10 FLUPHENAZINE DECANAOTE vs FLUPHENAZINE ENANTHATE, Outcome 4

Mental State: BPRS medium term $(6$ months to 1 year - high score = poor $) . \quad$. . . . . . . . . . . . . . . . .

Analysis 10.5. Comparison 10 FLUPHENAZINE DECANAOTE vs FLUPHENAZINE ENANTHATE, Outcome 5 Adverse effects: 1a. Movement disorders - general. . . . . . . . . . . . . . . . . . . . . . . . . . . . . . . .

Analysis 10.6. Comparison 10 FLUPHENAZINE DECANAOTE vs FLUPHENAZINE ENANTHATE, Outcome 6 Adverse effects: 1 b. Movement disorders - needing anticholinergic drugs. . . . . . . . . . . . . . . . . . . . .

Analysis 10.7. Comparison 10 FLUPHENAZINE DECANAOTE vs FLUPHENAZINE ENANTHATE, Outcome 7 Adverse effects: 1c. Movement disorders - parkinsonism (short term - 6 weeks to 5 months). . . . . . . . . .

Analysis 10.8. Comparison 10 FLUPHENAZINE DECANAOTE vs FLUPHENAZINE ENANTHATE, Outcome 8 Adverse effects: 1d. Movement disorders - akathisia (Immediate - 0 to 5 weeks). . . . . . . . . . . . . .

Analysis 10.9. Comparison 10 FLUPHENAZINE DECANAOTE vs FLUPHENAZINE ENANTHATE, Outcome 9 Adverse effects: 2. General adverse effects (immediate -0 to 5 weeks). . . . . . . . . . . . . . . . . . . . .

Analysis 11.1. Comparison 11 FLUPHENAZINE DECANOATE - DOSAGE STUDIES - 2 WEEKS vs 6 WEEKS, Outcome 1 Global state: 1 . Relapse $(1$ year). $\quad . \quad$. . . . . . . . . . . . . . . . . . . . . . . . . . . . . .

Analysis 11.2. Comparison 11 FLUPHENAZINE DECANOATE - DOSAGE STUDIES - 2 WEEKS vs 6 WEEKS,

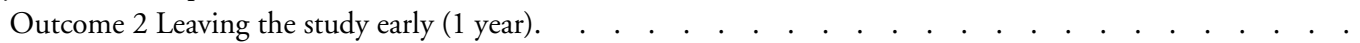

Analysis 11.3. Comparison 11 FLUPHENAZINE DECANOATE - DOSAGE STUDIES - 2 WEEKS vs 6 WEEKS,

Outcome 3 Mental state: 1 . BPRS - endpoint scores (1 year) (high score = poor).

Analysis 11.4. Comparison 11 FLUPHENAZINE DECANOATE - DOSAGE STUDIES - 2 WEEKS vs 6 WEEKS, Outcome 4 Adverse effects: 1 . Movement disorders - MPRC (1 year, high = poor). . . . . . . . . . . . . .

Analysis 11.5. Comparison 11 FLUPHENAZINE DECANOATE - DOSAGE STUDIES - 2 WEEKS vs 6 WEEKS,

Outcome 5 Quality of life: Quality of life scale (1 year) (high score = good). . . . . . . . . . . . . 238

ADDITIONAL TABLES . . . . . . . . . . . . . . . . . . . . . . . . . . . . . . . . . . . . . . . . . . . . .

APPENDICES . . . . . . . . . . . . . . . . . . . . . . . . . . . . . . . . . . . . . . . 239

WHAT'S NEW . . . . . . . . . . . . . . . . . . . . . . . . . . . . . . . . . . . . . . 241

HISTORY . . . . . . . . . . . . . . . . . . . . . . . . . . . . . . . . . . . . . . . 242

CONTRIBUTIONS OF AUTHORS . . . . . . . . . . . . . . . . . . . . . . . . . . . . . . . . . . . . 242

DECLARATIONS OF INTEREST . . . . . . . . . . . . . . . . . . . . . . . . . . . . . . . . . . . . . . . . 243

SOURCES OF SUPPORT . . . . . . . . . . . . . . . . . . . . . . . . . . . . . . . . . . . . . . . . . . . . . . .

DIFFERENCES BETWEEN PROTOCOL AND REVIEW . . . . . . . . . . . . . . . . . . . . . . . . . . 243

INDEX TERMS . . . . . . . . . . . . . . . . . . . . . . . . . . . . . . . . . . . . . . . . . . 243

Fluphenazine decanoate (depot) and enanthate for schizophrenia (Review) 


\title{
[Intervention Review]
}

\section{Fluphenazine decanoate (depot) and enanthate for schizophrenia}

\author{
Nicola Maayan $^{1}$, Seema N Quraishi ${ }^{2}$, Anthony David ${ }^{3}$, Aprajita Jayaswal ${ }^{4}$, Maurice Eisenbruch ${ }^{5}$, John Rathbone ${ }^{6}$, Rosie Asher $^{7}$, Clive $^{-}$ \\ E Adams ${ }^{8}$ \\ ${ }^{1}$ Enhance Reviews Ltd, Wantage, UK. ${ }^{2}$ SQ Therapy, London, UK. ${ }^{3}$ Institute of Psychiatry, London, UK. ${ }^{4}$ Psychiatry, Paras Hospital, \\ Gurgoan, India. ${ }^{5}$ Monash University, Melbourne, Australia. ${ }^{6}$ Faculty of Health Sciences and Medicine, Bond University, Gold Coast, \\ Australia. ${ }^{7}$ Enhance Reviews Ltd, Central Office, Cobweb Buildings, Wantage, UK. ${ }^{8}$ Cochrane Schizophrenia Group, The University \\ of Nottingham, Nottingham, UK
}

Contact address: Nicola Maayan, Enhance Reviews Ltd, Central Office, Cobweb Buildings, The Lane, Lyford, Wantage, OX12 0EE, UK. Nicola@enhance-reviews.com.

Editorial group: Cochrane Schizophrenia Group.

Publication status and date: Edited (no change to conclusions), published in Issue 2, 2016.

Citation: Maayan N, Quraishi SN, David A, Jayaswal A, Eisenbruch M, Rathbone J, Asher R, Adams CE. Fluphenazine decanoate (depot) and enanthate for schizophrenia. Cochrane Database of Systematic Reviews 2015, Issue 2. Art. No.: CD000307. DOI: 10.1002/14651858.CD000307.pub2.

Copyright (C) 2016 The Cochrane Collaboration. Published by John Wiley \& Sons, Ltd.

\begin{abstract}
A B S T R A C T
Background

Intramuscular injections (depot preparations) offer an advantage over oral medication for treating schizophrenia by reducing poor compliance. The benefits gained by long-acting preparations, however, may be offset by a higher incidence of adverse effects.
\end{abstract}

Objectives

To assess the effects of fluphenazine decanoate and enanthate versus oral anti-psychotics and other depot neuroleptic preparations for individuals with schizophrenia in terms of clinical, social and economic outcomes.

\section{Search methods}

We searched the Cochrane Schizophrenia Group's Trials Register (February 2011 and October 16, 2013), which is based on regular searches of CINAHL, BIOSIS, AMED, EMBASE, PubMed, MEDLINE, PsycINFO, and registries of clinical trials.

\section{Selection criteria}

We considered all relevant randomised controlled trials (RCTs) focusing on people with schizophrenia comparing fluphenazine decanoate or enanthate with placebo or oral anti-psychotics or other depot preparations.

\section{Data collection and analysis}

We reliably selected, assessed the quality, and extracted data of the included studies. For dichotomous data, we estimated risk ratio (RR) with $95 \%$ confidence intervals (CI). Analysis was by intention-to-treat. We used the mean difference (MD) for normal continuous data. We excluded continuous data if loss to follow-up was greater than $50 \%$. Tests of heterogeneity and for publication bias were undertaken. We used a fixed-effect model for all analyses unless there was high heterogeneity. For this update. we assessed risk of bias of included studies and used the GRADE (Grading of Recommendations Assessment, Development and Evaluation) approach to create a 'Summary of findings' table.

Fluphenazine decanoate (depot) and enanthate for schizophrenia (Review)

Copyright @ 2016 The Cochrane Collaboration. Published by John Wiley \& Sons, Ltd. 


\section{Main results}

This review now includes 73 randomised studies, with 4870 participants. Overall, the quality of the evidence is low to very low.

Compared with placebo, use of fluphenazine decanoate does not result in any significant differences in death, nor does it reduce relapse over six months to one year, but one longer-term study found that relapse was significantly reduced in the fluphenazine arm ( $\mathrm{n}=54,1$ RCT, RR 0.35 , CI 0.19 to 0.64 , very low quality evidence). A very similar number of people left the medium-term studies (six months to one year) early in the fluphenazine decanoate (24\%) and placebo (19\%) groups, however, a two-year study significantly favoured fluphenazine decanoate ( $\mathrm{n}=54,1$ RCT, RR 0.47 , CI 0.23 to 0.96 , very low quality evidence). No significant differences were found in mental state measured on the Brief Psychiatric Rating Scale (BPRS) or in extrapyramidal adverse effects, although these outcomes were only reported in one small study each. No study comparing fluphenazine decanoate with placebo reported clinically significant changes in global state or hospital admissions.

Fluphenazine decanoate does not reduce relapse more than oral neuroleptics in the medium term ( $\mathrm{n}=419,6 \mathrm{RCTs}$, RR 1.46 CI 0.75 to 2.83 , very low quality evidence). A small study found no difference in clinically significant changes in global state. No difference in the number of participants leaving the study early was found between fluphenazine decanoate (17\%) and oral neuroleptics (18\%), and no significant differences were found in mental state measured on the BPRS. Extrapyramidal adverse effects were significantly less for people receiving fluphenazine decanoate compared with oral neuroleptics ( $\mathrm{n}=259$, 3 RCTs, RR 0.47 CI 0.24 to 0.91 , very low quality evidence). No study comparing fluphenazine decanoate with oral neuroleptics reported death or hospital admissions.

No significant difference in relapse rates in the medium term between fluphenazine decanoate and fluphenazine enanthate was found ( $\mathrm{n}=49,1$ RCT, RR 2.43, CI 0.71 to 8.32 , very low quality evidence), immediate- and short-term studies were also equivocal. One small study reported the number of participants leaving the study early (29\% versus $12 \%)$ and mental state measured on the BPRS and found no significant difference for either outcome. No significant difference was found in extrapyramidal adverse effects between fluphenazine decanoate and fluphenazine enanthate. No study comparing fluphenazine decanoate with fluphenazine enanthate reported death, clinically significant changes in global state or hospital admissions.

\section{Authors' conclusions}

There are more data for fluphenazine decanoate than for the enanthate ester. Both are effective antipsychotic preparations. Fluphenazine decanoate produced fewer movement disorder effects than other oral antipsychotics but data were of low quality, and overall, adverse effect data were equivocal. In the context of trials, there is little advantage of these depots over oral medications in terms of compliance but this is unlikely to be applicable to everyday clinical practice.

\section{PLAIN LANGUAGE SUMMARY}

\section{Depot fluphenazine decanoate and enanthate for schizophrenia}

People with schizophrenia often hear voices or see things (hallucinations) and have strange beliefs (delusions). The main treatment for these symptoms of schizophrenia are antipsychotic drugs, which can be taken by mouth (tablet) or by an injection (depot). Fluphenazine was one of the first antipsychotic to be produced in depot form. The depot comes in two forms (decanoate and enanthate). Depot injections are often used for people who refuse or forget to take tablets (showing poor compliance or adherence with medication). Fluphenazine is an older antipsychotic drug that is very effective in the treatment of schizophrenia. However, when compared to newer antipsychotic drugs, fluphenazine may have serious side effects (such as involuntary shaking, tremors, muscle stiffness and the inability to sit still) and is known to lower people's mood.

This review aimed to investigate the effects of fluphenazine (decanoate and enanthate) for schizophrenia. Searches for relevant randomised controlled trials was run in February 2011 and October 16, 2013. Authors could include and extract data from 73 studies with a total of 4870 participants. There were more studies on fluphenazine decanoate than enanthate. The review authors rated the quality of the evidence in the included trials to be low or very low. A long-term result from only one trial indicated fluphenazine decanoate reduces the rate of relapse when compared with placebo ('dummy treatment'). Three studies found that fluphenazine decanoate produced fewer general movement disorders than oral antipsychotics. However, other results showed, overall, the effects and outcomes, including adverse effects for fluphenazine (decanoate and enanthate) are similar to other oral and depot antipsychotics. Important outcomes and information about use of services, going into hospital, satisfaction with care and costs were not reported in any study.

Depot injections may offer an advantage over tablets (oral medication) in terms of people taking their medication (complying and adhering to treatment). However, this needs to be balanced with the likelihood of serious side effects, such as involuntary shaking,

Fluphenazine decanoate (depot) and enanthate for schizophrenia (Review)

Copyright () 2016 The Cochrane Collaboration. Published by John Wiley \& Sons, Ltd. 
muscle stiffness, the inability to sit still and lowering in people's mood. Results did not show any strong evidence that depot fluphenazine produced more adverse effects than other antipsychotics.

This should be addressed in future large scale and high quality studies.

This plain language summary has been written by a consumer Ben Gray from Rethink Mental Illness. 


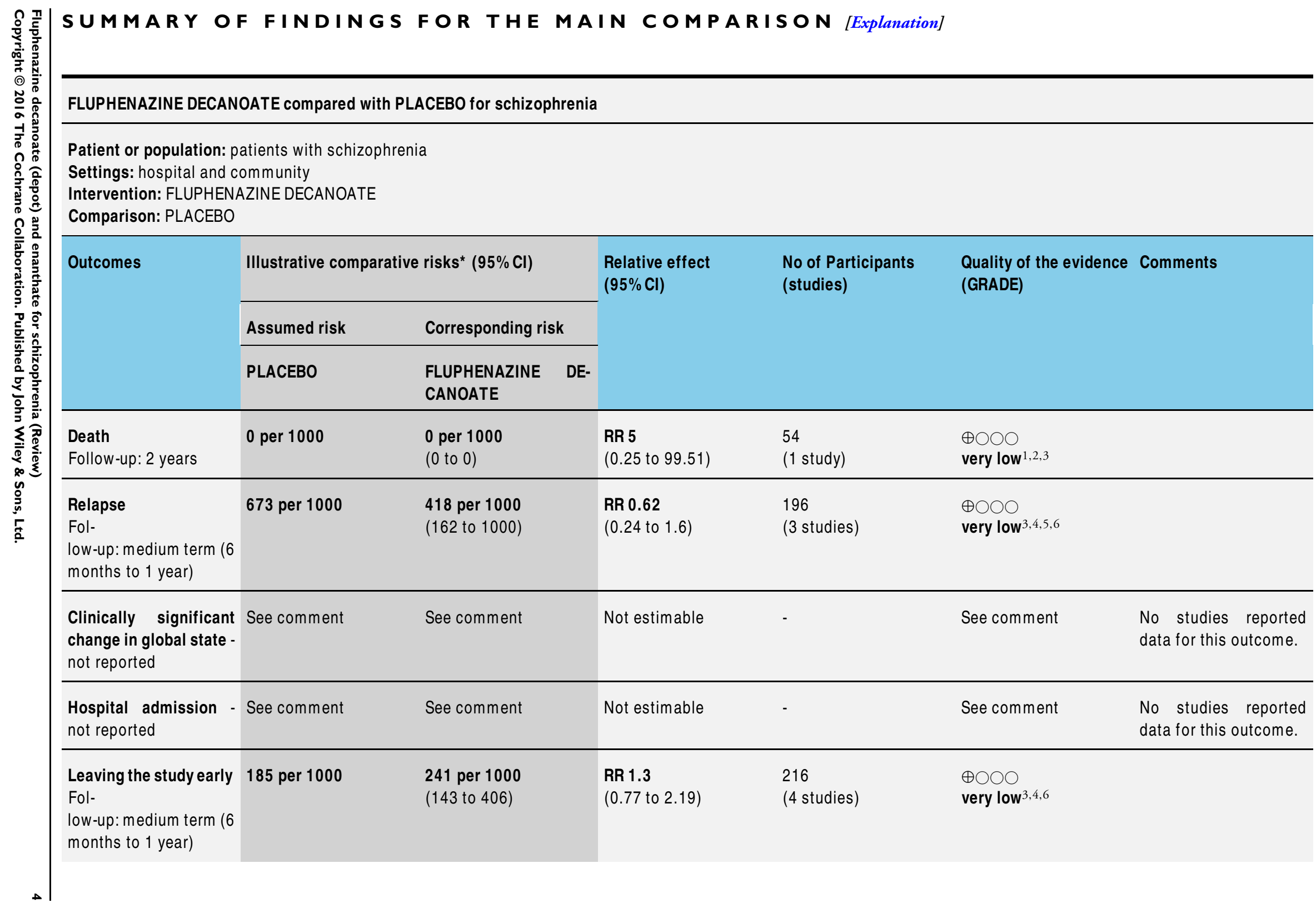




\begin{tabular}{|c|c|c|c|c|c|}
\hline $\begin{array}{l}\text { Mental state } \\
\text { BPRS } \\
\text { Follow-up: } 9 \text { months }\end{array}$ & & $\begin{array}{l}\text { The mean mental state } \\
\text { in the intervention } \\
\text { groups was } \\
2.03 \text { lower } \\
\text { ( } 4.51 \text { lower to } 0.45 \\
\text { higher) }\end{array}$ & & $\begin{array}{l}16 \\
\text { (1 study) }\end{array}$ & $\begin{array}{l}\oplus \bigcirc \bigcirc \bigcirc \\
\text { very low } 3,6,7\end{array}$ \\
\hline $\begin{array}{l}\text { Extrapyra- } \\
\text { midal adverse effects - } \\
\text { tardive dyskinesia } \\
\text { Follow-up: } 2 \text { years }\end{array}$ & 852 per 1000 & $\begin{array}{l}707 \text { per } 1000 \\
(528 \text { to } 946)\end{array}$ & $\begin{array}{l}\text { RR } 0.83 \\
(0.62 \text { to } 1.11)\end{array}$ & $\begin{array}{l}54 \\
\text { (1 study) }\end{array}$ & $\begin{array}{l}\oplus \bigcirc \bigcirc \bigcirc \\
\text { very low }\end{array}$ \\
\hline
\end{tabular}

*The basis for the assumed risk (e.g. the median control group risk across studies) is provided in footnotes. The corresponding risk (and its $95 \%$ confidence interval) is based on the assumed risk in the comparison group and the relative effect of the intervention (and its $95 \% \mathrm{Cl}$ ).

Cl: Confidence interval; RR: Risk ratio;

GRADE Working Group grades of evidence

High quality: Further research is very unlikely to change our confidence in the estimate of effect.

Moderate quality: Further research is likely to have an important impact on our confidence in the estimate of effect and may change the estimate.

Low quality: Further research is very likely to have an important impact on our confidence in the estimate of effect and is likely to change the estimate.

Very low quality: We are very uncertain about the estimate.

${ }^{1}$ Risk of bias: serious - This study had an unclear risk of bias for randomisation, allocation concealment and blinding.

${ }^{2}$ Imprecision: very serious - There were few participants and few events; the confidence intervals are wide.

3 Publication bias: strongly suspected - Four studies or fewer reported data for this outcome.

${ }^{4}$ Risk of bias: serious - The studies had an unclear risk of bias for randomisation, allocation concealment and blinding of participants.

${ }^{5}$ Inconsistency: very serious - There is very high heterogeneity.

${ }^{6}$ Imprecision: serious - There are wide confidence intervals.

${ }^{7}$ Risk of bias: very serious - This study had a high risk of bias for incomplete outcome data, and an unclear risk of bias for randomisation, allocation concealment and blinding. 


\section{B A C K G R O U N D}

\section{Description of the condition}

One in every 10,000 people per year are diagnosed with schizophrenia, with a lifetime prevalence of about $1 \%$ (Jablensky 1992). It often runs a chronic course with acute exacerbations and often partial remissions. The neuroleptic group of drugs is the mainstay treatment for this illness (Dencker 1980). These are generally regarded as highly effective, especially in controlling such symptoms as hallucinations and fixed false beliefs (delusions) (Kane 1986). They seem to reduce the risk of acute relapse.

Anti-psychotic drugs are usually given orally (Aaes-Jorgenson 1985), but compliance with medication given by this route may be difficult to quantify. Problems with treatment adherence are common throughout medicine (Haynes 1979). Those who suffer from long-term illness such as schizophrenia are less likely to take medication regularly if experiencing adverse effects (Kane 1998), or if they experience cognitive impairments (David 1994) and erosion of insight. The development of depot injections in the 1960s and initial clinical trials (Hirsch 1973b) gave rise to extensive use of depots as a means of long-term maintenance treatment.

\section{Description of the intervention}

Fluphenazine was one of the first oral antipsychotics to be produced in a depot form. Two forms of the depot, a decanoate (Modecate) and an enanthate (Moditen) are available. The decanoate is more commonly prescribed (Marder 1990) and lasts about four to six weeks in the body, while a single dose of the enanthate is shorter acting (one to three weeks). Evidence also suggests that the decanoate may produce slightly less adverse effects than its enanthate counterpart (Kurland 1970). However, in comparison with newer depot formulations fluphenazine decanoate has been reported to cause greater extrapyramidal adverse effects (Knights 1979) and to significantly lower mood (De Alarcon 1969a).

\section{How the intervention might work}

Depots mainly consist of an ester of the active drug held in an oily suspension. This is injected intramuscularly and is slowly released. Depots may be given every one to six weeks. Individuals may be maintained in the community with regular injections administered by community psychiatric nurses, sometimes in clinics set up for this purpose (Barnes 1994). The use of depots eradicates covert non-compliance.

\section{Why it is important to do this review}

A systematic review undertaken over a decade ago suggested that, for those with serious mental illness, stopping anti-psychotics resulted in $58 \%$ of people relapsing, whereas only $16 \%$ of those who were still on the drugs became acutely ill within a one-year period (Davis 1986). Evidence also points to the fact that experiencing a relapse of schizophrenia lowers a person's level of social functioning and quality of life (Curson 1985). Relapse prevention has also enormous financial implications. For example, within the UK, a Department of Health burden of disease analysis in 1996 indicated that schizophrenia accounted for $5.4 \%$ of all National Heath Service inpatient expenditure, placing it behind only learning disability and stroke in magnitude (DoH 1996).

\section{O B J E C T I V E S}

To assess the effects of fluphenazine decanoate and enanthate versus oral anti-psychotics and other depot neuroleptic preparations for individuals with schizophrenia in terms of clinical, social and economic outcomes.

\section{METHODS}

\section{Criteria for considering studies for this review}

\section{Types of studies}

All relevant randomised controlled trials. If a trial had been described as 'double blind' but implied randomisation, we would have included such trials in a sensitivity analysis (see Sensitivity analysis). If their inclusion did not result in a substantive difference, they would have remained in the analyses. If their inclusion did result in statistically significant differences, we would not have added the data from these lower quality studies to the results of the better trials, but presented such data within a subcategory.

We excluded quasi-randomised studies, such as those allocating by alternate days of the week. Where people were given additional treatments with fluphenazine decanoate or fluphenazine enanthate, we only included data if the adjunct treatment was evenly distributed between groups and it was only the fluphenazine decanoate or fluphenazine enanthate that was randomised.

\section{Types of participants}

Adults, however defined, with schizophrenia or related disorders, including schizophreniform disorder, schizoaffective disorder and delusional disorder, again, by any means of diagnosis. 
We are interested in making sure that information is as relevant to the current care of people with schizophrenia as possible so proposed to clearly highlight the current clinical state (acute, early post-acute, partial remission, remission) as well as the stage (prodromal, first episode, early illness, persistent) and as to whether the studies primarily focused on people with particular problems (for example, negative symptoms, treatment-resistant illnesses).

\section{Types of interventions}

1. Fluphenazine decanoate: any dose.

2. Fluphenazine enanthate: any dose.

3. Oral anti-psychotics (with the exception of fluphenazine hydrochloride): any dose.

4. Other depot preparations: any dose.

5. Placebo.

\section{Types of outcome measures}

Outcomes were grouped into immediate (zero to five weeks), short term (six weeks to five months), medium term (six months to one year) and longer term (over 12 months)

\section{Primary outcomes}

\section{Death and all causes of mortality}

2. Clinical global state

2.1 Relapse

2.2 Clinically significant change in global state - as defined by each of the studies

3. Leaving the study early

4. Service utilisation outcomes

4.1 Hospital admission

\section{Secondary outcomes}

1. Clinical global state

1.1 Mean score/change in global state

2. Behaviour*

\section{Mental state}

3.1 Clinically significant change in psychotic symptoms - as defined by each of the studies

3.2 Mean score/change in psychotic symptoms

3.3 Clinically significant change in positive symptoms - as defined by each of the studies

3.4 Mean score/change in positive symptoms

3.5 Clinically significant response in negative symptoms - as defined by each of the studies

3.6 Mean score/change in negative symptoms

\section{Extrapyramidal adverse effects}

4.1 Incidence of use of antiparkinson drugs

4.2 Clinically significant extrapyramidal adverse effects - as defined by each of the studies

4.3 Mean score/change in extrapyramidal adverse effects
5. Other adverse effects, general and specific

6. Service utilisation outcomes

6.1 Days in hospital

\section{Economic outcomes}

8. Quality of life/satisfaction with care for either recipients of care or carers

8.1 Significant change in quality of life/satisfaction - as defined by each of the studies

8.2 Mean score/change in quality of life/satisfaction.

9. 'Summary of findings' table

We used the GRADE approach to interpret findings (Schünemann 2008) and used GRADE profiler to import data from RevMan 5 (Review Manager) to create 'Summary of findings' tables. These tables provide outcome-specific information concerning the overall quality of evidence from each included study in the comparison, the magnitude of effect of the interventions examined, and the sum of available data on all outcomes we rated as important to patient-care and decision making. We selected the following main outcomes at medium-term follow-up for inclusion in the 'Summary of findings' table.

1. Death and all causes of mortality

2. Relapse

3. Clinically significant change in global state

4. Hospital admission

5. Leaving the study early

6. Mental state

7. Extrapyramidal adverse effects

* additional outcome

\section{Search methods for identification of studies}

\section{Electronic searches}

\section{Cochrane Schizophrenia Group's Trials Register}

The Trials Search Co-ordinator (TSC) searched the Cochrane Schizophrenia Group's Registry of Trials (February 2011 and October 16, 2013) using the following search strategies which have been developed based on literature review and consulting the authors of the review:

( $\left({ }^{*}\right.$ anaten ${ }^{*}$ or ${ }^{*}$ Cardilac $^{*}$ or ${ }^{*}$ Cenilene* or ${ }^{*}$ dapotum ${ }^{*}$ or ${ }^{*}$ Decafen* or ${ }^{*}$ decazate* or $^{*}$ Decentan* or $^{*}$ enanthate* or ${ }^{*}$ eutimox* or *Fludeca* or *flufen* or *flunanthate* or *fluphen* or *Idazoxan* or ${ }^{*}$ Lyogen ${ }^{*}$ or ${ }^{*}$ lyoridin* or ${ }^{*}$ Mirenil* or ${ }^{*} \operatorname{modec}^{*}$ or ${ }^{*}$ moditen* or ${ }^{*}$ Omca* ${ }^{*}{ }^{*}$ Oxyprothepin* ${ }^{*}{ }^{*}$ Pacinol* ${ }^{*}{ }^{*}$ Permitil ${ }^{*}$ or ${ }^{*}$ phenathiazine* or *piperazine* or *prolixin* or *Prolongatum* or *Rx $781094^{*}$ or * ${ }^{*}$ sediten* ${ }^{*}{ }^{*}$ selecten* ${ }^{*}{ }^{*}$ Sevinol* or ${ }^{*}$ sinqualone* or *siqualone* or *trancin*) and (*decanoat* or *depot* or *long? act* ${ }^{*}{ }^{*}$ delayed?act $\left.{ }^{*}\right)$ ):ti,ab,kw of REFERENCE or (*fluphenaz* and ${ }^{*}$ depot $\left.^{*}\right): \sin$ of STUDY 
The Cochrane Schizophrenia Group's Registry of Trials is compiled by systematic searches of major resources (including AMED, BIOSIS, CINAHL, EMBASE, MEDLINE, PsycINFO, PubMed, and registries of clinical trials) and their monthly updates, handsearches, grey literature, and conference proceedings (see Group Module). There is no language, date, document type, or publication status limitations for inclusion of records into the register. For previous searches, see Appendix 1.

\section{Searching other resources}

\section{Reference searching}

We also inspected the references of all identified trials for more studies. We sought each of the included studies as a citation on the SCISEARCH database. Then we inspected reports of articles that had cited these studies in order to identify further trials.

\section{Personal contact}

We tried to contact the first author of each included study for information regarding unpublished trials. We contacted companies producing depots and made requests for reports of published and unpublished trials. Where authors responded, this is noted in the Characteristics of included studies.

\section{Data collection and analysis}

This is an update of the original review (David 2004). Methods used in data collection and analysis for this update are below, for previous methods please see Appendix 2.

\section{Selection of studies}

For the update screening, two members of the Enhance Reviews team (NM and RA) independently inspected citations from the 2011 and 2013 searches and identified relevant abstracts. A random $20 \%$ sample was independently re-inspected by a senior researcher in the team to ensure reliability. Where disputes arose, the full report was acquired for more detailed scrutiny. If citations met inclusion criteria, we obtained full reports of the papers for more detailed inspection. Again, a random $20 \%$ of reports were re-inspected by a senior researcher in the team in order to ensure reliable selection. Where it was not possible to resolve disagreement by discussion, we attempted to contact the authors of the study for clarification.

\section{Data extraction and management}

\section{Extraction}

For this update, review author NM extracted data from included studies and RA checked the data. We extracted data presented only in graphs and figures whenever possible, but only included if two review authors had the same results. When further information was necessary, we contacted authors of studies in order to obtain missing data or for clarification. No studies were multi-centre; had there been, we would have extracted data relevant to each component centre separately where possible.

\section{Management}

\subsection{Forms}

We extracted data onto standard, simple forms.

\subsection{Scale-derived data}

We included continuous data from rating scales only if: a. the psychometric properties of the measuring instrument have been described in a peer-reviewed journal (Marshall 2000); and b. the measuring instrument has not been written or modified by one of the trialists for that particular trial.

\subsection{Endpoint versus change data}

There are advantages to both endpoint and change data. Change data can remove a component of between-person variability from the analysis. On the other hand, calculation of change needs two assessments (baseline and endpoint), which can be difficult in unstable and difficult to measure conditions such as schizophrenia. We decided primarily to use endpoint data, and only use change data if the former were not available. We combined endpoint and change data in the analysis as we used mean differences (MD) rather than standardised mean differences throughout (Higgins 2011, Chapter 9.4.5.2).

\subsection{Skewed data}

Continuous data on clinical and social outcomes are often not normally distributed. To avoid the pitfall of applying parametric tests to non-parametric data, we aimed to apply the following standards to all data before inclusion: a) standard deviations and means are reported in the paper or obtainable from the authors; b) when a scale starts from the finite number zero, the standard deviation, when multiplied by two, is less than the mean (as otherwise the mean is unlikely to be an appropriate measure of the centre of the distribution (Altman 1996)); c) if a scale started from a positive value (such as the Positive and Negative Syndrome Scale (PANSS), Kay 1986), which can have values from 30 to 210), we modified the calculation described above to take the scale starting point into account. In these cases skew is present if $2 \mathrm{SD}>(\mathrm{S}-\mathrm{S}$ $\min$ ), where $S$ is the mean score and $S$ min is the minimum score. Endpoint scores on scales often have a finite start and end point and these rules can be applied. We entered skewed endpoint data from studies of fewer than 200 participants in 'other tables' within the data and analyses section rather than into a statistical analysis. Skewed data pose less of a problem when looking at mean if the 
sample size is large; we entered such data from studies with over 200 participants into syntheses.

When continuous data are presented on a scale that includes a possibility of negative values (such as change data), it is difficult to tell whether data are skewed or not; we entered skewed change data into analyses.

\subsection{Common measure}

To facilitate comparison between trials, we intended to convert variables that can be reported in different metrics, such as days in hospital (mean days per year, per week or per month) to a common metric (e.g. mean days per month).

\subsection{Conversion of continuous to binary}

Where possible, we made efforts to convert outcome measures to dichotomous data. This can be done by identifying cut-off points on rating scales and dividing participants accordingly into 'clinically improved' or 'not clinically improved'. It is generally assumed that if there is a $50 \%$ reduction in a scale-derived score such as the Brief Psychiatric Rating Scale (BPRS, Overall 1962) or the Positive and Negative Syndrome Scale (PANSS, Kay 1986), this could be considered as a clinically significant response (Leucht 2005; Leucht 2005a). If data based on these thresholds were not available, we used the primary cut-off presented by the original authors.

\subsection{Direction of graphs}

We entered data in such a way that the area to the left of the line of no effect indicated a favourable outcome for fluphenazine esters.

\section{Assessment of risk of bias in included studies}

For this 2013 update, the Enhanced Reviews team used the criteria described in the Cochrane Handbook for Systematic Reviews of Interventions (Higgins 2011) to assess trial quality. This new set of criteria is based on evidence of associations between overestimate of effect and high risk of bias of the article such as sequence generation, allocation concealment, blinding, incomplete outcome data and selective reporting.

We have noted the level of risk of bias in both the text of the review and in the Summary of findings for the main comparison, Summary of findings 2 and Summary of findings 3 .

\section{Measures of treatment effect}

\section{Binary data}

For binary outcomes, we calculated a standard estimation of the risk ratio (RR) and its $95 \%$ confidence interval (CI). It has been shown that RR is more intuitive (Boissel 1999) than odds ratios and that odds ratios tend to be interpreted as RR by clinicians (Deeks 2000). The Number Needed to Treat/Harm (NNT/H) statistic with its confidence intervals is intuitively attractive to clinicians but is problematic both in its accurate calculation in meta-analyses and interpretation (Hutton 2009). Therefore, we did not present NNTs.

\section{Continuous data}

For continuous outcomes, we estimated mean difference (MD) between groups. We prefer not to calculate effect size measures (standardised mean difference SMD). However, if scales of very considerable similarity were used, we presumed there was a small difference in measurement, and we calculated effect size and transformed the effect back to the units of one or more of the specific instruments. Where trials reported mean data adjusted for baseline and standard error using ANCOVA, we entered this data using the generic inverse variance according to section 9.4.5.2 of the Cochrane Handbook (Higgins 2011).

\section{Unit of analysis issues}

\section{Cluster trials}

Studies increasingly employ 'cluster randomisation' (such as randomisation by clinician or practice), but analysis and pooling of clustered data poses problems. Authors often fail to account for intra-class correlation in clustered studies, leading to a 'unit of analysis' error (Divine 1992) whereby P values are spuriously low, confidence intervals unduly narrow and statistical significance overestimated. This causes type I errors (Bland 1997; Gulliford 1999). None of the trials we included were cluster trials. Had there been, where clustering was not accounted for in primary studies, we would have presented data in a table, with a $\left(^{*}\right)$ symbol to indicate the presence of a probable unit of analysis error. In subsequent versions of this review, we would seek to contact first authors of studies to obtain intra-class correlation coefficients (ICCs) for their clustered data and to adjust for this by using accepted methods (Gulliford 1999). Where clustering had been incorporated into the analysis of primary studies, we would have presented these data as if from a non-cluster randomised study, but adjusted for the clustering effect.

We have sought statistical advice and have been advised that the binary data as presented in a report should be divided by a 'design effect'. This is calculated using the mean number of participants per cluster $(\mathrm{m})$ and the ICC [Design effect $=1+(\mathrm{m}-1)^{*} \mathrm{ICC}$ ] (Donner 2002). If the ICC was not reported we would have assumed it to be 0.1 (Ukoumunne 1999).

If cluster studies have been appropriately analysed taking into account ICCs and relevant data documented in the report, synthesis with other studies would have been possible using the generic inverse variance technique. 


\section{Cross-over trials}

Some included studies used a cross-over design. A major concern of cross-over trials is the carry-over effect. It occurs if an effect (e.g. pharmacological, physiological or psychological) of the treatment in the first phase is carried over to the second phase. As a consequence, on entry to the second phase the participants can differ systematically from their initial state despite a wash-out phase. For the same reason cross-over trials are not appropriate if the condition of interest is unstable (Elbourne 2002). As both effects are very likely in severe mental illness, we only used data of the first phase of cross-over studies.

\section{Studies with multiple treatment groups}

For the included studies with more than two treatment arms, we presented the additional treatment arms in comparisons. Where data were binary, we simply added these and combined them within the two-by-two table. Where data were continuous we combined data following the formula in section 7.7.3.8 (Combining groups) of the Handbook (Higgins 2011). Where the additional treatment arms were not relevant, we did not reproduce these data.

\section{Dealing with missing data}

\section{Overall loss of credibility}

At some degree of loss of follow-up, data must lose credibility (Xia 2009). We chose that, for any particular outcome, where more than $30 \%$ of those randomised were lost to follow-up by six months, or $50 \%$ of data by beyond that time be unaccounted for, we did not reproduce these data or use them within analyses.

\section{Continuous}

\subsection{Attrition}

In the case where attrition for a continuous outcome was between $0 \%$ and $50 \%$ and completer-only data were reported, we reproduced these.

\subsection{Standard deviations}

If standard deviations (SDs) were not reported, we first tried to obtain the missing values from the authors. If not available, where there are missing measures of variance for continuous data, but an exact standard error (SE) and confidence intervals available for group means, and either $\mathrm{P}$ value or $\mathrm{T}$ value available for differences in mean, we can calculate them according to the rules described in the Handbook (Higgins 2011): When only the SE is reported, $\mathrm{SDs}$ are calculated by the formula $\mathrm{SD}=\mathrm{SE} *$ square root (n). Chapters 7.7.3 and 16.1.3 of the Handbook (Higgins 2011) present detailed formulae for estimating SDs from $\mathrm{P}$ values, $\mathrm{T}$ or
F values, confidence intervals, ranges or other statistics. If these formulae do not apply, we would calculate the SDs according to a validated imputation method which is based on the SDs of the other included studies (Furukawa 2006). Although some of these imputation strategies can introduce error, the alternative would be to exclude a given study's outcome and thus to lose information. We nevertheless examined the validity of the imputations in a sensitivity analysis excluding imputed values.

\section{Assessment of heterogeneity}

\section{Clinical heterogeneity}

We considered all included studies initially, without seeing comparison data, to judge clinical heterogeneity. We simply inspected all studies for clearly outlying people or situations which we had not predicted would arise. When such situations or participant groups arose, we fully discussed these.

\section{Methodological heterogeneity}

We considered all included studies initially, without seeing comparison data, to judge methodological heterogeneity. We simply inspected all studies for clearly outlying methods which we had not predicted would arise. When such methodological outliers arose, we fully discussed these.

\section{Statistical heterogeneity}

\subsection{Visual inspection}

We visually inspected graphs to investigate the possibility of statistical heterogeneity.

\subsection{Employing the $\mathrm{I}^{2}$ statistic}

We investigated heterogeneity between studies by considering the $\mathrm{I}^{2}$ method alongside the $\mathrm{Chi}^{2} \mathrm{P}$ value. The $\mathrm{I}^{2}$ provides an estimate of the percentage of inconsistency thought to be due to chance (Higgins 2003). The importance of the observed value of $I^{2}$ depends on i. magnitude and direction of effects and ii. strength of evidence for heterogeneity (e.g. P value from $\mathrm{Chi}^{2}$ test, or a confidence interval for $\mathrm{I}^{2}$ ). an $\mathrm{I}^{2}$ estimate greater than or equal to around $50 \%$ accompanied by a statistically significant $\mathrm{Chi}^{2}$ statistic was interpreted as evidence of substantial levels of heterogeneity (Higgins 2011). When substantial levels of heterogeneity were found in the primary outcome, we explored reasons for heterogeneity (see Subgroup analysis and investigation of heterogeneity). 


\section{Assessment of reporting biases}

Reporting biases arise when the dissemination of research findings is influenced by the nature and direction of results (Egger 1997). These are described in Section 10 of the Handbook (Higgins 2011). We are aware that funnel plots may be useful in investigating reporting biases but are of limited power to detect small-study effects. We did not use funnel plots for outcomes where there were 10 or fewer studies, or where all studies were of similar sizes. In other cases, where funnel plots were possible, we sought statistical advice in their interpretation.

\section{Data synthesis}

We understand that there is no closed argument for preference for use of fixed-effect or random-effects models. The random-effects method incorporates an assumption that the different studies are estimating different, yet related, intervention effects. This often seems to be true to us and the random-effects model takes into account differences between studies, even if there is no statistically significant heterogeneity. There is, however, a disadvantage to the random-effects model: it puts added weight onto small studies which often are the most biased ones. Depending on the direction of effect, these studies can either inflate or deflate the effect size. We chose the fixed-effect model for all analyses, unless there was high heterogeneity (see Assessment of heterogeneity), in which case we used the random-effects model. The reader is, however, able to choose to inspect the data using the random-effects model.

\section{Subgroup analysis and investigation of heterogeneity}

\section{Subgroup analyses - only primary outcomes}

\subsection{Clinical state, stage or problem}

We proposed to undertake this review and provide an overview of the effects of fluphenazine esters for people with schizophrenia in general. In addition, however, we tried to report data on subgroups of people in the same clinical state, stage and with similar problems. In order to do subgroup analyses, we needed to have at least six studies for an outcome.

\section{Sensitivity analysis}

We aimed to apply all sensitivity analyses to the primary outcomes of this review, again, if there were at least six studies with data for a particular outcome.

\section{Implication of randomisation}

We aimed to include trials in a sensitivity analysis if they were described in some way so as to imply randomisation. For the primary outcomes, we would have included these studies and if there was no substantive difference when the implied randomised studies were added to those with better description of randomisation, then we would have entered all data from these studies.

\section{Assumptions for lost binary data}

If assumptions had to be made regarding people lost to follow-up and missing SDs data (see Dealing with missing data), we would have compared the findings on primary outcomes when we used our assumption compared with completer data only. We would have undertaken a sensitivity analysis to test how prone results were to change when 'completer' data only were compared to the imputed data using the above assumption. If there was a substantial difference, we would have reported results and discussed them, but continued to employ our assumption.

\section{Risk of bias}

We analysed the effects of excluding trials that were judged to be at high risk of bias across one or more of the domains of randomisation (implied as randomised with no further details available): allocation concealment, blinding and outcome reporting for the meta-analysis of the primary outcome. If the exclusion of trials at high risk of bias did not substantially alter the direction of effect or the precision of the effect estimates, then we included data from these trials in the analysis.

\section{Imputed values}

If we had included cluster trials, we also would have completed a sensitivity analysis to assess the effects of including data from trials if we needed to use imputed values for ICC in calculating the design effect in cluster randomised trials.

If we had noted substantial differences in the direction or precision of effect estimates in any of the sensitivity analyses listed above, we would not have pooled data from the excluded trials with the other trials contributing to the outcome, but would have presented them separately.

\section{Fixed-effect and random-effects}

We synthesised data using a fixed-effect model.

\section{Dose}

We tested the sensitivity of the primary outcomes as to whether high (250 mg) or low (25 mg) dose of fluphenazine decanoate was used or whether the trials used an intermediate/high $(0.5 \mathrm{mg})$ or low $(0.25 \mathrm{mg})$ dose of fluphenazine enanthate. 


\section{Description of studies}

Please see Characteristics of included studies, Characteristics of excluded studies and Characteristics of studies awaiting classification.

\section{Results of the search}

The original search yielded 982 citations using the search strategy. Two hundred and forty-eight citations were related to fluphenazine decanoate or enanthate but only 62 referred to controlled clinical trials (all published in journals). The review was also updated in
May 2002, a further electronic search yielded 247 citations from which we obtained 124 articles for further inspection.

The 2013 update search identified 44 potential studies and after screening we added four new studies (Ju 2000; Kane 1978; Khazaie 2005; Shenoy 1981) to the included studies. Two previously included studies, Marder 1984 and Marder 1987, were found to include the same participants, and so were added as the same study. The total number of included studies is now 73 randomised controlled trials with a total of 90 reports.

For overall screening from the three searches see Figure 1. 
Figure I. Study flow diagram.

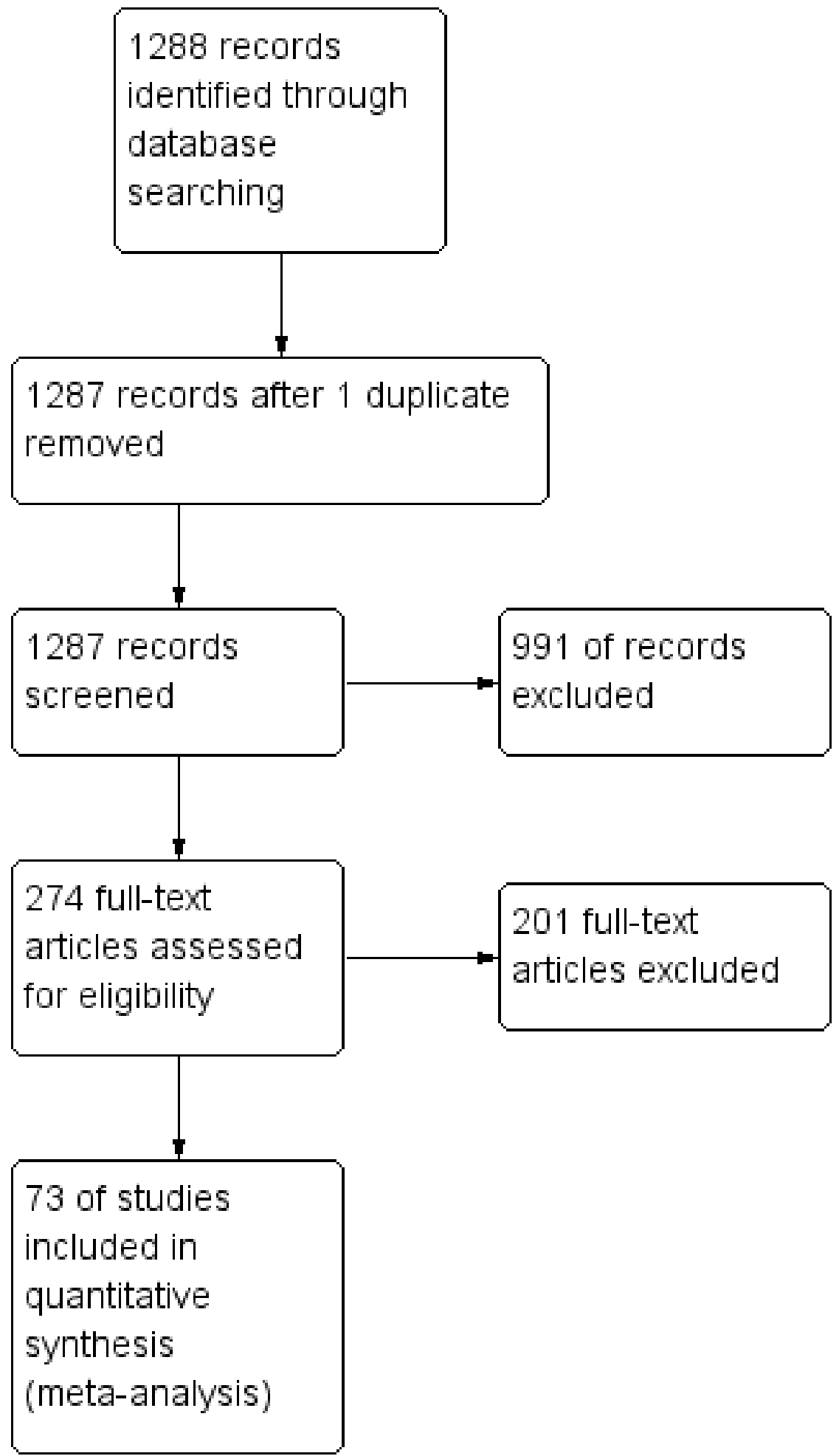




\section{Included studies}

\section{Length of trials}

The duration for all the studies ranged between two weeks (Kane 1978) to three years (Dencker 1973).

\section{Participants}

The diagnoses of all participants were schizophrenia or some other similar psychotic disorder. Most of the studies included people of both sexes, although seven studies (Albert 1980; Asarnow 1988; Kurland 1966; Marder 1987; McCreadie 1980; McCreadie 1982) included only men and 16 trials failed to mention the sex of participants. Ages ranged between 13 and 81 years, but most people were in the 18 to 65 age range. Most trial participants had long histories of schizophrenia, although many studies $(n=44)$ failed to mention the length of time people had been ill. Researchers frequently used operational criteria for diagnoses (RDC, Schneider's first rank symptoms, Hay \& Forrest 1972 criteria, PSE, Kraepelinian, ICD -9, DSM-II/III, Bleuler's criteria, Feighner 1972 criteria, Chinese Classification of Mental Disorders and Huangshan council schizophrenia standard), although 31 (43\%) trials did not specify which diagnostic criteria were used.

\section{Setting}

The trials were both community- and hospital-based. People in two studies (Schooler 1980; Wistedt 1984) were given the first two injections whilst in hospital and after which medication continued to be administered in the community. Both Dencker 1973 and Wistedt 1984 studied people initially in a hospital setting followed by a continuation in the community. Several studies involved people from both hospital and community settings (Dencker 1973; Donlon 1976; Kaneno 1991; Magnus 1979; Marder 1987; McCreadie 1980; Rifkin 1977; Schooler 1997; Simon 1978). A surprisingly large number (11) of studies did not mention the setting used (Albert 1980; Javed 1991; Kissling 1985; McKane 1987; Odejide 1982; Quitkin 1978; Russell 1982; Schneider 1981; Schlosberg 1978; Sharma 1991; Wistedt 1983). Thirty-one trials were conducted in North America and another 29 in Europe, 10 in Asia, one in Africa; and two did not report the country.

\section{Study size}

The largest study was by Schooler 1997 who randomised 313 people, whereas Altamura 1985 only included 11. The majority randomised between 30 and 60 people.

\section{Interventions}

Six of the included trials compared fluphenazine decanoate with placebo (Dotti 1979; Hirsch 1975; Jolley 1990; Odejide 1982; Rifkin 1977; Shenoy 1981) and one study compared fluphenazine enanthate with placebo (Van Praag 1970). Ten studies compared fluphenazine decanoate with enanthate (Altamura 1985; Asarnow 1988; Chouinard 1978; Chouinard 1982; Donlon 1976; Kane 1978; Keskiner 1971; Kurland 1966; MacCrimmon 1978; Van Praag 1973). Fourteen studies compared fluphenazine esters with oral antipsychotics. Thirty-five trials compared fluphenazine decanoate or enanthate with other depot formulations. There were 10 dosage studies - nine comparing fluphenazine decanoate and one comparing fluphenazine enanthate (Goldstein 1978). Of the 73 included trials, 66 used fluphenazine decanoate as an intervention.

\section{Outcomes}

\subsection{Outcome reporting}

Many of the trials presented their findings in graphs or using $\mathrm{P}$ values alone. Graphical presentation made it impossible to acquire raw data for synthesis. Requests for raw data from authors have so far failed with the exception of Pinto 1979 and Quitkin 1978. It was also common to use $\mathrm{P}$ values as a measure of association between intervention and outcomes instead of showing the strength of the association.

\subsection{Missing outcomes}

No study reported on hospital and service outcomes or commented on participants' overall satisfaction during or after the trial. Economic outcomes were not reviewed by any of the included studies.

\subsection{Outcome scales}

Scales that provided usable data are listed below. We listed data that were not usable in the Characteristics of included studies under outcomes, 'unable to use'.

\subsubsection{Global functioning}

a) Clinical Global Impression - CGI (Guy 1976)

This is a three-item rating instrument commonly used in schizophrenia studies. It enables clinicians to quantify the severity of illness and overall clinical improvement during therapy. A 
seven-point scoring system is usually used with low scores indicating decreased severity and/or greater recovery.

b) Global Assessment Scale - GAS (Endicott 1976)

This is an observer-rated scale for evaluating the overall functioning of an individual during a specified time period on a continuum from psychological or psychiatric sickness to health. Score ranges from zero to 100 , where a higher score indicates better functioning.

\subsubsection{Mental state}

a) Brief Psychiatric Rating Scale - BPRS (Overall 1962) The BPRS is an 18-item scale measuring positive symptoms, general psychopathology and affective symptoms. The original scale has 16 items, but a revised 18-item scale is commonly used. Scores can range from zero to 126 . Each item is rated on a seven-point scale, with high scores indicating more severe symptoms.

b) Comprehensive Psychopathological Rating Scale - CPRS (Asberg 1978)

The scale is designed to measure psychopathology over time via a clinical interview. It contains 67 items, including one global rating and one item documenting the reliability of the interview. The majority of the items (40) are based upon reported symptoms. Assumed reliability of the rating is scored as zero (very poor), one (fair), two (good) or three (very good).

c) Krawiecka Scale (Krawiecka 1977)

This mental state scale encompasses both positive and negative symptoms of schizophrenia. It is used to evaluate the mental state and behaviour in chronic psychotic people with higher scores indicating greater severity. It is also known as the Manchester Scale. d) Scale for the Assessment of Negative Symptoms - SANS (Andreasen 1983)

This scale allows a global rating of the following negative symptoms: alogia (impoverished thinking), affective blunting, avolition-apathy, anhedonia-asociality, and attention impairment. Assessments are made on a six-point scale from zero (not at all) to five (severe). Higher scores indicate more symptoms.

e) Scale for the Assessment of Positive Symptoms - SAPS (Andreasen 1984)

This six-point scale gives a global rating of positive symptoms such as delusions, hallucinations and disordered thinking. Higher scores indicate more symptoms.

\subsubsection{Behaviour}

a) Nurses Observational Scale of Inpatients Evaluation - NOSIE (Honigfeld 1962).

This is an 80-item scale with items rated on a five-point scale from zero (not present) to four (always present). Ratings are based on behaviour over the previous three days. The seven headings are social competence, social interest, personal neatness, co-operation, irritability, manifest psychosis and psychotic depression. The total score ranges from zero to 320 with high scores indicating a poor outcome.

\subsubsection{Adverse effects}

a) Abnormal Involuntary Movement Side Effects Scale - AIMS (Guy 1976)

This is a 12-item scale designed to record the occurrence of dyskinetic movements. Ten items of this scale have been used to assess tardive dyskinesia, a long-term drug-induced movement disorder. A five-point scoring system (from zero - none to four - severe) has been used to rate each of the 10 items. Using this scale in shortterm treatment may be helpful in assessing some short-term abnormal movement disorders. A low score indicates low levels of dyskinetic movements.

b) Dosage Record and Treatment Emergent Symptoms Scale DOTES (Guy 1976)

This adverse effect tool seems less of a scale, where the degree and severity of a symptom is recorded, and more of a checklist. The DOTES seems to record the presence or absence of a list of adverse effects.

c) Extrapyramidal Symptom Rating Scale - ESRS (Chouinard 1980)

This consists of a questionnaire relating to parkinsonian symptoms (nine items), a physician's examination for parkinsonism and dyskinetic movements (eight items), and a clinical global impression of tardive dyskinesia. High scores indicate severe levels of movement disorder.

d) Simpson and Angus Scale - SAS (Simpson 1970b)

The SAS is a 10 -item scale, used to evaluate the presence and severity of drug-induced parkinsonian symptomatology. The ten items focus on rigidity rather than bradykinesia, and do not assess subjective rigidity or slowness. Items are rated for severity on a zero to four scale, with a scoring system of zero to four for each item. This scale is referred to as the RSESE in Ju 2000. A low score indicates low levels of parkinsonism.

e) UKU Side Effects Rating Scale - UKU-SERS (Lingjaerde 1987). The UKU rates four major topics: psychological adverse effects (10 items), neurological adverse effects (eight items), autonomic adverse effects (11 items) and other adverse effects (19 items). Each item is defined by means of a four-point scale where zero means not present or doubtfully present. Scoring range zero to144.

f) Treatment Emergent Symptom Scale -TESS (Guy 1976)

This checklist assesses a variety of characteristics for each adverse event, including severity, relationship to the drug, temporal characteristics (timing after a dose, duration and pattern during the day), contributing factors, course, and action taken to counteract the effect. Symptoms can be listed a priori or can be recorded as observed by the investigator. High scores indicate worse symptoms.

g) Symptom Checklist 90 - SCL-90 (Derogatis 1977) 
This is a self-report scale of physical symptoms.

h) Maryland Psychiatric Research Center Involuntary Movement Scale (Cassady 1997)

The MPRC rates the severity of tardive dyskinesia. It gives a global rating of dyskinesia in 11 body areas and two ratings during gait. It is rated on an eight-point scale (zero to seven), with higher scores indicating worse symptoms.

\subsubsection{Quality of life}

a) Quality of life scale - QLS (Heinrich 1984)

This 21-item scale is based on a semi-structured interview providing information on symptoms and functioning during the preceding four weeks. There are seven severity steps (zero to six; six being adequately functioning and zero being deficient). Four item categories have been identified by factor analysis i) interpersonal relationships (seven items), ii) instrumental role (four items), iii) intrapsychic function (seven items) and iv) common place objects and activities. Higher scores indicate better quality of life.

\section{Excluded studies}

We excluded 201 studies, mainly because they were not randomised controlled trials (RCTs), or controlled clinical trials
(CCTs), because neither fluphenazine decanoate nor fluphenazine enanthate were included in the interventions or because trialists did not report any usable data. In the latter case, we contacted authors requesting raw data but we have, in most cases, received no reply. Other reasons for exclusion were that the two drugs were not analysed (Crawford 1974; Wistedt 1983a) or clinical outcomes were not measured (Landmark 1994; Leff 1973; Marder 1990a; Marder 1991a; Stevens 1973).

\section{Awaiting assessment}

Six studies await assessment. del Giudice 1970; Jue 1996; Kabes 1984; Ravanic 1996 are reports for which we have citations but no papers. These are currently being sought. Two papers await translation (Angst 1973; Ushakov 1990a).

\section{Ongoing studies}

We have not identified any ongoing studies.

\section{Risk of bias in included studies}

See also 'Risk of bias' tables in Characteristics of included studies, and Figure 2 and Figure 3.

Figure 2. 'Risk of bias' graph: review authors' judgements about each risk of bias item presented as percentages across all included studies.

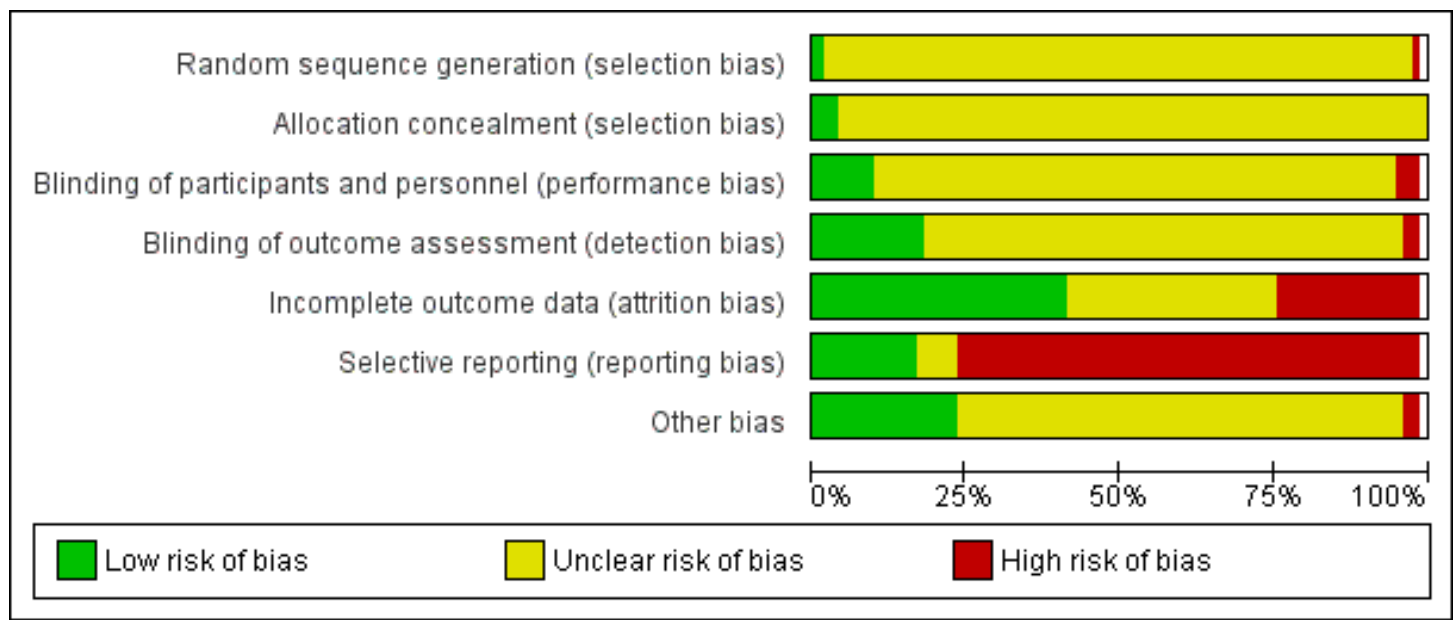

Fluphenazine decanoate (depot) and enanthate for schizophrenia (Review) 
Figure 3. 'Risk of bias' summary: review authors' judgements about each risk of bias item for each included study.

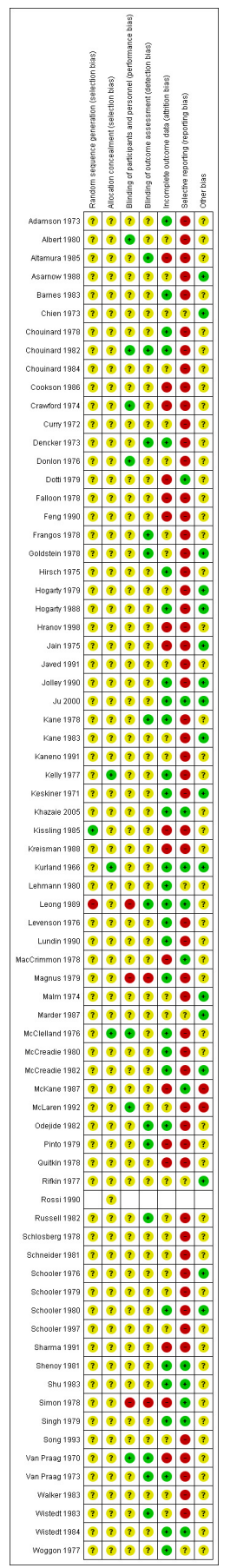

Fluphenazine decanoate (depot) and enanthate for schizophrenia (Review)

Copyright $\odot 2016$ The Cochrane Collaboration. Published by John Wiley \& Sons, Ltd. 
Since the last version of this review was published, the methods for assessing the risk of bias of studies has changed. All studies were re-assessed for risk of bias in the current version of this review. The risk of bias has not been fully assessed for two studies as they are not reported in English and we were not able to translate them (Kaneno 1991; Rossi 1990), although Kaneno 1991 had a section in English and the risk of bias has been completed based on this.

\section{Allocation}

All included studies were reported as randomised, but only five described the method of randomisation: Kissling 1985 used a cointhrowing method, Frangos 1978 a randomisation code, Magnus 1979 a pre-arranged prescribing list, and Wistedt 1984 a randomisation list. Leong 1989 described the method of randomisation as the next available study number in the numerical sequence. However only Kissling 1985 was rated as low risk, Leong 1989 was rated to be a high risk and the other studies were all rated as unclear risk of bias for sequence generation.

Three other studies did not report method of randomisation but did describe allocation concealment (Kelly 1977; Kurland 1966; McClelland 1976). In these three trials the allocation codes were known only to the hospital pharamcists or nurses involved in the trial, and were rated as low risk of bias; the remaining studies were rated as unclear risk of bias.

\section{Blinding}

Most studies reported using double-blind methodology, although the technique used was not described in the majority of these studies. Seven studies were rated low risk of bias for blinding of participants and personnel, and stated blindness was achieved through use of identical injections of medication (Albert 1980; Chouinard 1982; Crawford 1974; Donlon 1976; McClelland 1976; McLaren 1992; Van Praag 1970).

Only 14 studies described outcome assessors as being blinded to treatment and were rated low risk of bias (Altamura 1985; Chouinard 1982; Dencker 1973; Frangos 1978; Goldstein 1978; Kane 1978; Leong 1989; McClelland 1976; Odejide 1982; Pinto 1979; Russell 1982; Van Praag 1970; Van Praag 1973; Wistedt 1983). Magnus 1979 and Simon 1978 were open label trials and Leong 1989 described using a 'partially-blinded' method where only outcome assessors were blinded. The remaining studies were of unclear risk of bias as no information on blinding of outcome assessors was provided.

\section{Incomplete outcome data}

Twenty-nine studies were rated as low risk of bias for incomplete outcome data and 25 studies had an unclear risk of bias. Seventeen studies were rated as high risk of bias, for two of these it was due to more than 50\% of losses to follow-up (Jain 1975; Kissling 1985), and only data for the outcome "Leaving the study early" was used for these studies.

\section{Selective reporting}

Only 12 studies were of low risk of bias with regard to selective reporting, and five were unclear. The remaining studies were of high risk of bias, mainly due to poor data reporting. Continuous data were particularly problematic as many studies presented findings without standard deviations or any other measure of variance, in graphs, in percentiles or by inexact P values. Furthermore, many pre-planned outcomes were not reported at all.

\section{Other potential sources of bias}

McKane 1987 and McLaren 1992 were subject to other biases as they were funded by the pharmaceutical industry. Eighteen studies were rated as low risk of bias for other potential sources of bias, and the remaining had an unclear risk of bias.

\section{Effects of interventions}

See: Summary of findings for the main comparison FLUPHENAZINE DECANOATE compared with PLACEBO for schizophrenia; Summary of findings 2 FLUPHENAZINE DECANOATE compared with ORAL NEUROLEPTICS for schizophrenia; Summary of findings 3 FLUPHENAZINE DECANAOTE compared to FLUPHENAZINE ENANTHATE for schizophrenia

We calculated risk ratios (RR) for dichotomous data and estimated mean differences (MD) for continuous data, with their respective $95 \%$ confidence intervals (CIs) throughout.

\section{COMPARISON I: FLUPHENAZINE DECANOATE versus PLACEBO}

\section{I.I Death}

The only study reporting mortality was Jolley 1990 where two deaths occurred in the treatment group (fluphenazine decanoate) compared to none in the placebo group ( $n=54$, RR 5.00, CI 0.30 to 99.51). Nevertheless, the result was not statistically significant (Analysis 1.1). 


\section{I.2 Global state}

Heterogeneous data from three studies (Hirsch 1975; Odejide 1982; Rifkin 1977) found relapse rates to be equivocal over six months to one year for the fluphenazine decanoate group compared with people receiving placebo $(\mathrm{n}=196,3$ RCTs, RR 0.62 , CI 0.24 to 1.60 ). Shenoy 1981 reported no relapses in the short term at six weeks. Relapse rates for longer-term studies (Jolley 1990) at two years significantly favoured fluphenazine decanoate $(\mathrm{n}=54$, RR 0.35, CI 0.19 to 0.64 ) compared to placebo (Analysis 1.2). Furthermore, one short-term study of six weeks found no significant difference between treatment group when global state was measured on the Global Assessment Scale (GAS) (Analysis 1.3).

\section{I.3 Leaving the study early}

Four trials in which 216 people had been randomised to fluphenazine decanoate or placebo had, in total, $21 \%$ attrition (Analysis 1.4). No significant difference was found in people leaving the study early between groups (RR 1.30 , CI 0,77 to 2.19 ). Shenoy 1981 reported short-term data at six weeks and also found no significant difference between treatment groups. Jolley 1990 reported longer-term data at two years for leaving the study early that significantly favoured depot fluphenazine compared to placebo (n $=54, \mathrm{RR} 0.47$, CI 0.23 to 0.96 ).

\section{I.4 Mental state}

Only one trial (Dotti 1979) reported general mental state on the Brief Psychiatric Rating Scale (BPRS), the results were equivocal (Analysis 1.5). The single study by Odejide 1982 reporting on depression showed equivocal results between fluphenazine decanoate and placebo (Analysis 1.6).

\section{I.5 Adverse effects}

Limited data were available. Jolley 1990 reported equivocal data for incidence of tardive dyskinesia (Analysis 1.7). Rifkin 1977 reported on toxicity (no further details reported), which was significantly higher in the depot fluphenazine group $(n=45 R R, 7.65$, CI 1.04 to 56.26; Analysis 1.8).

\section{COMPARISON 2: FLUPHENAZINE DECANOATE versus ORAL NEUROLEPTICS}

\section{I Death}

There were no reports of death in any of the studies comparing depot fluphenazine versus other oral neuroleptics.

\subsection{Global state}

Using the negative outcome, 'no clinically important global change' Adamson 1973 and Curry 1972 produced results favouring fluphenazine decanoate at 0 to 5 weeks $(\mathrm{n}=74,2$ RCTs, RR 0.61 CI 0.46 to 0.81 ; Analysis 2.1). Song 1993 reported on outcomes at 6 months to one year, with equivocal findings $(\mathrm{n}=102$, RR 0.85, CI 0.56 to 1.27). Using the CGI scale, Shu 1983 also reported equivocal findings ( $\mathrm{n}=34, \mathrm{MD}$ at 6 weeks $-0.10 \mathrm{CI}$ 2.79 to 2.59; Analysis 2.3). There was no significant difference between those taking fluphenazine decanoate and people on oral neuroleptics for relapse at 6 months to one year $(n=419,6$ RCTs, RR 1.46, CI 0.75 to 2.83); relapse data recorded at more than one year were also not significant $(\mathrm{n}=216,3$ RCTs, RR 1.250 .81 to 1.95; Analysis 2.2).

\subsection{Leaving the study early}

Nine trials reported no significant difference between the number of people who left the study early over six months to one year in either the fluphenazine decanoate group or the oral antipsychotic group ( $\mathrm{n}=887$, RR 0.96, CI 0.73 to 1.25 ; Analysis 2.4 ). Studies by Curry 1972 (at 28 days), Shu 1983 (at six weeks) and Falloon 1978 and Simon 1978 (at more than one year) were also equivocal.

\subsection{Behaviour}

Simon 1978 found no difference in Nurses Observational Scale of Inpatients Evaluation (NOSIE) scale scores between groups (n $=120, \mathrm{MD}-0.56, \mathrm{CI}-6.92$ to 5.80; Analysis 2.5). Barnes 1983 reported a significant difference for change in disturbed behaviour $(\mathrm{n}=36)$; these data are skewed (Analysis 2.6).

\subsection{Mental state}

Only Simon 1978, reported on mental state (BPRS endpoint scores) and found no significant difference between groups ( $\mathrm{n}=$ 120, MD -0.75, CI -5.75 to 4.25; Analysis 2.7). Schooler 1979 and Falloon 1978 reporting on depression found no significant difference between those receiving fluphenazine decanoate and oral neuroleptics $(\mathrm{n}=214, \mathrm{RR}$ six months to one year 0.89 , CI 0.60 to $1.32 ; \mathrm{n}=44, \mathrm{RR}$ more than one year 1.53 , CI 0.91 to 2.57 ; Analysis 2.8).

\subsection{Adverse effects}

Three studies, McCreadie 1980, McCreadie 1982 and Schooler 1980 , report homogenous data for general movement disorders (six months to one year), which significantly favoured fluphenazine decanoate compared to oral neuroleptics ( $\mathrm{n}=259$, RR 0.47, CI 0.24 to 0.91; Analysis 2.9). The single longer-term study by Falloon 1978 found no significant difference for incidence of movement disorders $(\mathrm{n}=44$, RR 0.40 , CI 0.12 to 
1.28). Rifkin 1977 reported on akathisia at one year. Akathisia was significantly lower in the oral fluphenazine group ( $\mathrm{n}=51$, RR 20.54 CI 1.25 to 337.94; Analysis 2.10). Trials reported limited data for the outcome 'needing anticholinergic drugs' and all findings were equivocal (Analysis 2.11). McCreadie 1982 found tardive dyskinesia to be significantly less common for those allocated fluphenazine decanoate compared with people on pimozide ( $\mathrm{n}=28$, RR medium term 0.62, CI 0.41 to 0.93 ; Analysis 2.12). The other study that reported on tardive dyskinesia was Simon 1978. Trialists did not find any difference between fluphenazine decanoate and oral neuroleptic $(\mathrm{n}=120, \mathrm{RR}$ at 18 months 0.16 , CI 0.01 to 2.99). Shu 1983, using the Simpson and Angus Scale (SAS) reported no significant difference at six weeks between fluphenazine decanoate and penfluridol ( $\mathrm{n}=32$, MD 1.30, CI 0.01 to 2.59; Analysis 2.14). Adamson 1973 (immediate), McCreadie 1982 and Schooler 1980 (medium term) reported general adverse effects. Outcomes are equivocal (Analysis 2.17). Falloon 1978 was the only longer-term study to report on tremor, with equivocal results for depot fluphenazine and pimozide $(n=44$, RR 0.80 , CI 0.26 to 2.45; Analysis 2.13). Schooler 1976 reports equivocal data for the adverse effect of blurred vision (Analysis 2.15). Rifkin 1977 also reported on toxicity (no further details), which was more frequent for the depot fluphenazine group $(n=51$, RR 4.87, CI 1.14 to 20.72; Analysis 2.16).

\section{COMPARISON 3: FLUPHENAZINE DECANOATE versus OTHER DEPOT NEUROLEPTICS}

\section{I Death}

McKane 1987 reported one death occurring in the treatment group (fluphenazine decanoate) compared to none in the haloperidol decanoate group ( $\mathrm{n}=38$, RR 3.0, CI 0.13 to 69.31; Analysis 3.1). Nevertheless, the result was not statistically significant.

\subsection{Global state}

Eleven studies reported the outcome of 'relapse' at six months to one year. We found no statistically significant difference between the fluphenazine decanoate group and the other depot groups (n $=581$, RR 0.82, CI 0.56 to 1.18). Longer studies (more than one year) also found no difference between interventions ( $\mathrm{n}=$ 252, RR 1.22, CI 0.77 to 1.92 ). Wistedt 1984 did report relapse data at 20 weeks but, again, results were equivocal (Analysis 3.3). Outcomes for 'no clinically important global change' at six months to one year reported by Dencker 1973, Leong 1989 and Schlosberg 1978 were not significant for the fluphenazine decanoate and other depot neuroleptic groups ( $\mathrm{n}=187$, RR 1.04, CI 0.96 to 1.12 ; Analysis 3.2); Ju 2000 also reported short-term data and also found no significant difference. Leong 1989 supported this result by reporting no significant differences in the number of people who became severely ill in the comparison of fluphenazine decanoate with other depot drugs ( $\mathrm{n}=60$, RR 1.07, CI 0.94 to 1.23; Analysis 3.4).

Chouinard 1984 and Schlosberg 1978 report continuous data at six months to one year on clinical global impression. There is no clear advantage between fluphenazine decanoate and other depot neuroleptics ( $\mathrm{n}=90, \mathrm{MD}-0.10, \mathrm{CI}-0.41$ to 0.21 ; Analysis 3.7). These findings were confirmed by Chouinard 1984 and Cookson 1986 who reported no significant difference in needing additional antipsychotics at six months to one year between the depot groups $(\mathrm{n}=91$, RR 0.53, CI 0.14 to 1.96; Analysis 3.5). Frangos 1978 also reported the outcome of 'not improved' ( $n=50, R R$ at four months RR 2.50, CI 0.53 to 11.70) and Leong 1989, at seven months (n $=60$, RR 0.75, CI 0.18 to 3.07) (Analysis 3.8). Finally, Wistedt 1984 reported non-significant data for clinical global impression at zero to five weeks. These data are skewed so are not displayed graphically (Analysis 3.6).

\subsection{Leaving the study early}

Fifteen included trials found no significant difference in the number of people who left the study early in either the fluphenazine decanoate group or the other depot group ( $n=775$, RR medium term 1.13, CI 0.89 to 1.44 ). Studies found no differences across any time period from the immediate to those lasting longer than one year (Analysis 3.9).

\subsection{Behaviour}

Simon 1978 supported this outcome by reporting no difference in NOSIE-30 scores between the groups ( $\mathrm{n}=118$, MD - -5.21 10.85 to 0.43 ; Analysis 3.10).

\subsection{Mental state}

We found short- and medium-term studies assessing mental state (BPRS endpoint scores) to significantly favour 'other depot neuroleptics' for the short term ( $\mathrm{n}=203,2$ RCTs MD 1.11, CI 0.86 to $1.36)$ and medium term $(\mathrm{n}=162,3$ RCTs, MD 1.20, CI 1.10 to 1.30) (Analysis 3.11). Longer-term studies (McKane 1987, Simon 1978) did not show any differences for mental state in either intervention ( $\mathrm{n}=141$, MD 0.85 CI -2.32 to 4.03; Analysis 3.11). Dichotomised medium-term BPRS data reported by Dencker 1973 found no significant difference between depot fluphenazine and pipothiazine palmitate (Analysis 3.12). The only study reporting on the outcome of depression was Dencker 1973 who found no significant difference between fluphenazine decanoate and pipothiazine palmitate $(\mathrm{n}=67$, RR medium term 1.02 , CI 0.81 to 1.28; Analysis 3.13). Ju 2000 reported data for positive and negative symptoms on the Scale for the Assessment of Positive Symptom(SAPS) and SANS, respectively, from six weeks to five months, but these data are skewed so are not displayed graphically (Analysis 3.14). 


\subsection{Adverse effects}

The occurrence of dyskinetic movements in general was the same across short-, medium- and longer-term studies (Analysis 3.15). Feng 1990 reporting on a small, short-term study found no significant difference between fluphenazine decanoate and haloperidol decanoate ( $\mathrm{n}=30$ RR 2.00, CI 0.43 to 9.32). Dencker 1973, Leong 1989 and Schlosberg 1978 (comparing fluphenazine decanoate with pipothiazide palmitate) and McLaren 1992 (comparing with bromperidol decanoate) found no significant difference in the occurrence of dyskinestic movements $(\mathrm{n}=234, \mathrm{RR}$ at six months to one year 1.08 , CI 0.86 to 1.34 ). Longer-term studies also found no significant difference with movement disorders between fluphenazine decanoate and other depot neuroleptics. For the outcome of 'needing anticholinergic medication', eight studies, when synthesised, found in favour of other depots by one year $(\mathrm{n}=448$, RR 1.240 .93 to 1.64$)$. However, these data were heterogeneous and using the random-effects model (as per protocol), the result was not statistically significant (Analysis 3.16). For the same outcome, three longer-term studies were equivocal but significantly favoured the 'other depot neuroleptics' group when analysed with a fixed-effect model $(\mathrm{n}=220, \mathrm{RR} 1.28, \mathrm{CI}$ 1.08 to 1.51 ). Outcomes such as dry mouth, tardive dyskinesia and parkinsonism were not significantly different between depot fluphenazine and other depot neuroleptics (Analysis 3.22; Analysis 3.18; Analysis 3.17, respectively). Tremor (short term, 2 RCTs and medium term, 3 RCTs) was not more common for people given the depot flupenthixol (Analysis 3.19). Ju 2000 reported data for movement disorders from six weeks to five months on the TESS and RSESE, but these data are skewed so are not displayed graphically (Analysis 3.20). When reporting blurred vision, the results of one medium term trial were not significant, but one longerterm study, Pinto 1979, did report significant results $(\mathrm{P}=0.04)$ favouring flupenthixol decanoate $(\mathrm{n}=64, \mathrm{RR} 17.88$, CI 1.08 to 294.82; Analysis 3.21). General adverse effects (short-term data) were reported by Frangos 1978 and Javed 1991 and favoured other depot neuroleptics ( $\mathrm{n}=88$, RR 1.36, CI 1.07 to 1.74 ). However, medium-term data $(n=249$, six months to one year) were equivocal (Analysis 3.23).

\section{COMPARISON 4: FLUPHENAZINE DECANOATE - DOSAGE STUDIES (HIGH DOSE versus STANDARD DOSE)}

\section{I Global state}

McClelland 1976 and Kreisman 1988 reported no significant difference in relapse scores (medium term) between either depot group ( $\mathrm{n}=182$, RR 2.11, CI 0.30 to 14.91; Analysis 4.1). Also, McClelland 1976 reported no significant difference in needing additional antipsychotics (six months to one year) between fluphenazine decanoate (high dose) group and the standard dosage groups ( $\mathrm{n}=50,1$ RCTs, RR 1.67, CI 0.45 to 6.24; Analysis 4.2). Outcomes for global improvement 'not improved' were reported by Lehmann 1980 (nurse and psychiatrist rated) at six months to one year (Analysis 4.3). Results for nurse rated outcomes significantly favoured the standard dosage group $(n=40,1$ RCT, RR 1.58 , CI 1.09 to 2.30). However, results for psychiatrist rated were not significant for either dosage intervention at six months ( $\mathrm{n}=$ 40, 1 RCT, RR 1.15, CI 0.77 to 1.74 ).

\subsection{Leaving the study early}

Lehmann 1980 and McClelland 1976 reported no difference in the number leaving the study (six months to one year) for either intervention ( $\mathrm{n}=90,2$ RCTs, RR 0.60, CI 0.15 to 2.36; Analysis 4.4).

\subsection{Mental state}

McClelland 1976 further reported no difference in BPRS endpoint score ( $\mathrm{n}=50,1$ RCT, MD -0.03, CI -5.79 to 5.73) for either the high or standard dosage group (Analysis 4.5).

\subsection{Adverse effects}

McClelland 1976 reported no difference between the groups for those needing anticholinergic medication $(\mathrm{n}=50$, RR 1.67, CI 0.45 to 6.24 ) at six months to one year, suggesting the incidence of adverse effects is comparable between the groups, as the use of anticholinergic drugs is considered to be a direct measure of the severity of adverse effects due to medication (Analysis 4.6).

\section{COMPARISON 5: FLUPHENAZINE DECANOATE - DOSAGE STUDIES - (LOW DOSE versus STANDARD DOSE)}

\section{I Global state}

Relapse data, assessed over six months to one year were equivocal. Longer-term studies (more than one year) reported by Asarnow 1988, Hogarty 1988 and Marder 1987 were also equivocal (Analysis 5.1).

\subsection{Leaving the study early}

Asarnow 1988, Hogarty 1988, and Marder 1987 reported no difference in the number of people who left the study early in each dosage group after more than one year of medication $(n=172$, RR 0.67, CI 0.33 to 1.36; Analysis 5.2). 


\subsection{Mental state}

The data obtained for mental state (e.g. BPRS score etc.) were skewed and therefore could not be included in the analyses.

\subsection{Adverse effects}

Marder 1987 reported that there was no significant difference in the number of people requiring additional anticholinergic drugs at six months to one year ( $\mathrm{n}=50$, RR 2.55, CI 0.72 to 9.05). Kane 1983 supported this finding by reporting that the number of people with tardive dyskinesia ( $\mathrm{n}=126$, RR 0.52 , CI 0.10 to 2.72) at six months to one year, was not significantly different between the groups receiving low doses of fluphenazine decanoate and standard dosage fluphenazine. Kane 1983, however, did report a statistically significant $(\mathrm{P}=0.03)$ difference at endpoint analysis with the Simpson Dyskinesia Scale $(\mathrm{n}=126)$, which favoured low-dose fluphenazine decanoate, although data were skewed and therefore not graphically reported (Analysis 5.3).

\section{COMPARISON 6: FLUPHENAZINE ENANTHATE versus PLACEBO}

\section{I Adverse effects - at eight weeks}

Only Van Praag 1973 reported for this comparison. This small trial reported no significant difference in the number of people needing anticholinergic drugs in the fluphenazine enanthate and placebo groups ( $\mathrm{n}=25$, RR 9.69, CI 0.58 to 163.02; Analysis 6.1).

\section{COMPARISON 7. FLUPHENAZINE ENANTHATE versus ORAL NEUROLEPTICS}

\section{I Global state}

Chien 1973 reported no significant difference in global change (immediate term, zero to five weeks) between fluphenazine enanthate and chlorpromazine ( $\mathrm{n}=31$, RR 0.67 , CI 0.27 to 1.66 ; Analysis 7.1).

\subsection{Adverse effects}

Reports of adverse effects, again from the same study and for the immediate term were all not significantly different $(n=31$, RR movement disorders 2.34, CI 0.53 to 10.30 ; RR general adverse effects 2.81, CI 0.94 to 8.45 ; RR parkinsonism 6.56, CI 0.91 to 47.21; Analysis 7.2).

\section{COMPARISON 8: FLUPHENAZINE ENANTHATE versus OTHER DEPOT NEUROLEPTICS}

\section{I Global state}

Albert 1980 and Chouinard 1978 reported no significant difference in needing additional antipsychotics (at six months to one year) for fluphenazine enanthate compared with other depot groups ( $\mathrm{n}=65$, RR 0.50 , CI 0.24 to 1.05 ; Analysis 8.1 ). Both Malm 1974, at six weeks to five months ( $\mathrm{n}=57$, RR 2.38, CI 0.66 to 8.61) and Chouinard 1978, at six months to one year (n $=32$, RR 0.33 , CI 0.04 to 2.87 ) reported no statistically significant differences in relapse rates between the fluphenazine enanthate group and the other depot (pipothiazine palmitate) groups (Analysis 8.2).

\subsection{Leaving the study early}

Only Jain 1975 provided data for numbers leaving the study early (zero to five weeks). These data significantly favoured fluphenazine enanthate compared with the other depot neuroleptics - pipothiazine palmitate ( $\mathrm{n}=30$, RR 0.09 , CI 0.01 to 0.62 ). However, this outcome should be interpreted with caution given the limited number of participants. The number of people who left the study early by six weeks to five months, in the single study by Malm 1974 using fluspirilene as a control, was not significant ( $n=57, R R$ 2.38, CI 0.66 to 8.61). Similarly, Chouinard 1978 found no difference between the fluphenazine enanthate group and the other depot neuroleptic group - pipothiazine palmitate at six months to one year ( $\mathrm{n}=32$, RR 0.33, CI 0.04 to 2.87) (Analysis 8.3).

\subsection{Mental state}

Singh 1979 reported general BPRS scores and found a significant difference between the two groups favouring the other depot group ( $\mathrm{n}=30$, MD 0.40, CI 0.34 to 0.46; Analysis 8.4). Specific scores on, for example, depression found no difference between the two groups (Singh 1979, $\mathrm{n}=30, \mathrm{RR} 7.00, \mathrm{CI} 0.39$ to 124.83; Analysis 8.5).

\subsection{Adverse effects}

Findings were equivocal for outcomes of 'movement disorders' (medium term: $\mathrm{n}=63,2$ RCTs, RR 1.52, CI 0.75 to 3.07; Analysis 8.6), tardive dyskinesia (medium term: $\mathrm{n}=32,1 \mathrm{RCT}, \mathrm{RR} 0.89$, CI 0.46 to 1.71 ; Analysis 8.8), tremor (medium term: $\mathrm{n}=95,3$ RCTs, RR 1.24, CI 0.82 to 1.87; Analysis 8.9), blurred vision (medium term: $\mathrm{n}=30,1$ RCT, RR 3.00, CI 0.13 to 68.26; Analysis 8.10) and dry mouth (medium term: $\mathrm{n}=62,2$ RCTs, RR 0.80, CI 0.36 to 1.76; Analysis 8.11). Malm 1974 reported that those receiving fluspirilene required significantly less anticholinergic drugs at six weeks to five months than the fluphenazine enanthate group ( $\mathrm{n}=$ 
57, RR 2.86, CI 1.16 to 7.06). The numbers of people needing additional anticholinergic drugs at six months to one year were found (Albert 1980; Chouinard 1978) to be equivocal ( $n=65$, $R R$ 1.02 , CI 0.76 to 1.35 ) for the fluphenazine enanthate and other depot neuroleptic groups (Analysis 8.7).

\section{COMPARISON 9: FLUPHENAZINE ENANTHATE - DOSAGE STUDIES (LOW DOSE versus INTERMEDIATE/HIGH DOSE)}

\section{I Global state}

A single study by Goldstein 1978 reported the global outcome of relapse at six weeks to five months (Analysis 9.1). Trialists found statistically significant differences favouring the highdosage fluphenazine enanthate group compared with low-dosage fluphenazine enanthate ( $\mathrm{n}=104$, RR 9.35, CI 2.28 to 38.29). For every fourth person administered a low dose of fluphenazine decanoate, one would relapse. However, this result must be treated with caution as only one study is involved.

\subsection{Leaving the study early}

Goldstein 1978 found no significant difference in the number of people who left the study early (six weeks to five months) whilst receiving either high or low dosages of fluphenazine enanthate ( $\mathrm{n}$ $=104, \mathrm{RR} 3.12$, CI 0.66 to 14.74; Analysis 9.2).

\section{COMPARISON I0: FLUPHENAZINE DECANOATE versus FLUPHENAZINE ENANTHATE}

\section{I Global state}

Van Praag 1973 reported data for 'needing additional antipsychotic treatment' at zero to five weeks (Analysis 10.1). This trial found a significant difference between the fluphenazines (decanoate and enanthate) ( $\mathrm{n}=33$, RR 0.39 , CI 0.18 to 0.86 ). Chouinard 1982 was the only study to report the numbers of people requiring additional antipsychotic treatment at six months to one year and found no significant difference.

The number of people who relapsed whilst receiving medication at zero to five weeks was not significant for the two studies available ( $\mathrm{n}=44,2$ RCTs, RR 0.66, CI 0.18 to 2.43). Donlon 1976 reported no significant difference in relapse rates at six weeks to five months between the fluphenazine decanoate group and the fluphenazine enanthate group ( $\mathrm{n}=30$, RR 2.29, CI 0.70 to 7.48 ). MacCrimmon 1978 , reporting on relapse over the medium term (six months to one year) found no significant difference $(\mathrm{n}=49$, RR 2.43 , CI 0.71 to 8.32 ) (Analysis 10.2).

\subsection{Leaving the study early}

The number of people leaving the study early (Analysis 10.3) at zero to five weeks was not significantly different between the fluphenazine decanoate and enanthate groups ( $\mathrm{n}=44,2$ RCTs, RR 0.66 , CI 0.18 to 2.43 ). Short term outcomes ( 6 weeks to 5 months) were also not significantly different between the fluphenazine ester groups ( $\mathrm{n}=42,2$ RCTs, RR 2.29 CI 0.70 to 7.48 ). Medium-term data (six months to one year) were consistent with the results of the two shorter study periods, finding no difference in the number of people leaving the study early for the two fluphenazine ester groups ( $\mathrm{n}=49$, 1 RCT, RR 2.43, CI 0.71 to 8.32 ).

\subsection{Mental state}

Only one study by MacCrimmon 1978 reported on mental state, using BPRS endpoint scores at one year. They found no significant difference between the fluphenazine esters ( $\mathrm{n}=39$, MD 0.00, CI -3.93 to 3.93; (Analysis 10.4).

\subsection{Adverse effects}

The number of people in these studies reporting movement disorders for immediate (zero to five weeks) or short term (six weeks to five months) was not significantly different between the fluphenazine esters (Analysis 10.5). Reports of adverse effects (zero to five weeks), parkinsonism (six weeks to five months) and akathisia (zero to five weeks) were equivocal for fluphenazine decanoate and enanthate groups (Analysis 10.9; Analysis 10.7; Analysis 10.8, respectively).

The number of people needing anticholinergic drugs at zero to five weeks was found by Van Praag 1973 to be significantly lower for the fluphenazine decanoate group ( $\mathrm{n}=33$, RR 0.29 , CI 0.12 to 0.70 ). For longer-term studies (six weeks to five months and six months to one year) there were no significant differences in the number of people needing anticholinergic drugs (Analysis 10.6).

\section{COMPARISON I I: FLUPHENAZINE DECANOATE EVERY TWO WEEKS verSUS EVERY SIX WEEKS}

We found only one relevant trial (Khazaie 2005) that compared the use of fluphenazine decanoate every two weeks with every six weeks.

\section{I.I Global state}

There was no significant difference between fluphenazine decanoate every two weeks and every six weeks in the number of participants relapsing $(\mathrm{n}=37$, RR 0.89, CI 0.55 to 1.44 ; Analysis 11.1). 


\section{I.2 Leaving the study early}

The number of participants leaving the study early also did not differ significantly between fluphenazine decanoate every two weeks and every six weeks ( $\mathrm{n}=37$, RR 1.17, CI 0.46 to 2.98; Analysis 11.2).

\section{I.3 Mental state}

Total BPRS scores were not significantly different between fluphenazine decanoate every two weeks and every six weeks ( $\mathrm{n}=$ 37, MD 2.72, CI -1.16 to 6.60, Analysis 11.3), nor was the BPRS sub-scale for thought disorder $(\mathrm{n}=37, \mathrm{MD}-0.39$, CI -1.51 to 0.73; Analysis 11.3).

\section{I.4 Adverse effects}

There was no significant difference between fluphenazine decanoate every two weeks and every six weeks in parkinsonian symptoms ( $\mathrm{n}=37$, MD 1.30, CI -0.03 to 2.63) and dyskinesia $(\mathrm{n}=37$, MD 2.40, CI -1.77 to 6.57) measured on the Maryland Psychiatric Research Center Involuntary Movement Scale (MPRC) scale (Analysis 11.4).

\section{1.5 Quality of life}

We did not find any difference between fluphenazine decanoate every two weeks and every six weeks in the quality of life of participants ( $\mathrm{n}=37$, MD 1.42, CI -9.68 to 12.52 , Analysis 11.5$)$.

\section{Subgroup analyses}

The only primary outcome with enough studies (at least six) to warrant subgroup analysis was relapse from Comparison 2 and Comparison 3. However, studies either included chronic patients, a mix of chronic and acute, or did not report the stage of the illness, therefore, it was not possible to undertake any subgroup analyses.

\section{Sensitivity analysis}

The only primary outcome with enough studies (at least six) to warrant sensitivity analysis was relapse from Comparison 2 and Comparison 3.

\section{3.I Implication of randomisation}

All trials were described as randomised in the review.

\subsection{Assumptions for lost binary data}

We did not make any assumptions about lost binary data.

\section{3.3 Risk of bias}

For relapse at medium term in Comparison 2 (Analysis 3.3), only Leong 1989 had a high risk of bias for randomisation. The results remained non-significant with the removal of this trial. For this outcome, all trials had a high risk of bias for selective reporting except, again, for Leong 1989 and results were unchanged.

For relapse at medium term in Comparison 3 (Analysis 2.2), all studies had unclear risk of bias for randomisation and all had a high or unclear risk of bias for selective reporting.

\subsection{Imputed values}

We did not have any cluster randomised trials and did not impute any data.

\subsection{Dose}

The mean daily dose of fluphenazine decanoate at endpoint ranged from 0.3 to $300 \mathrm{mg}$ and for fluphenazine enanthate ranged from 2.35 to 387.5 mg. Two studies, Cookson 1986 and Curry 1972, did not specify the average dose. The way data were reported did not permit any more sensitivity analyses than those which have already been presented. 


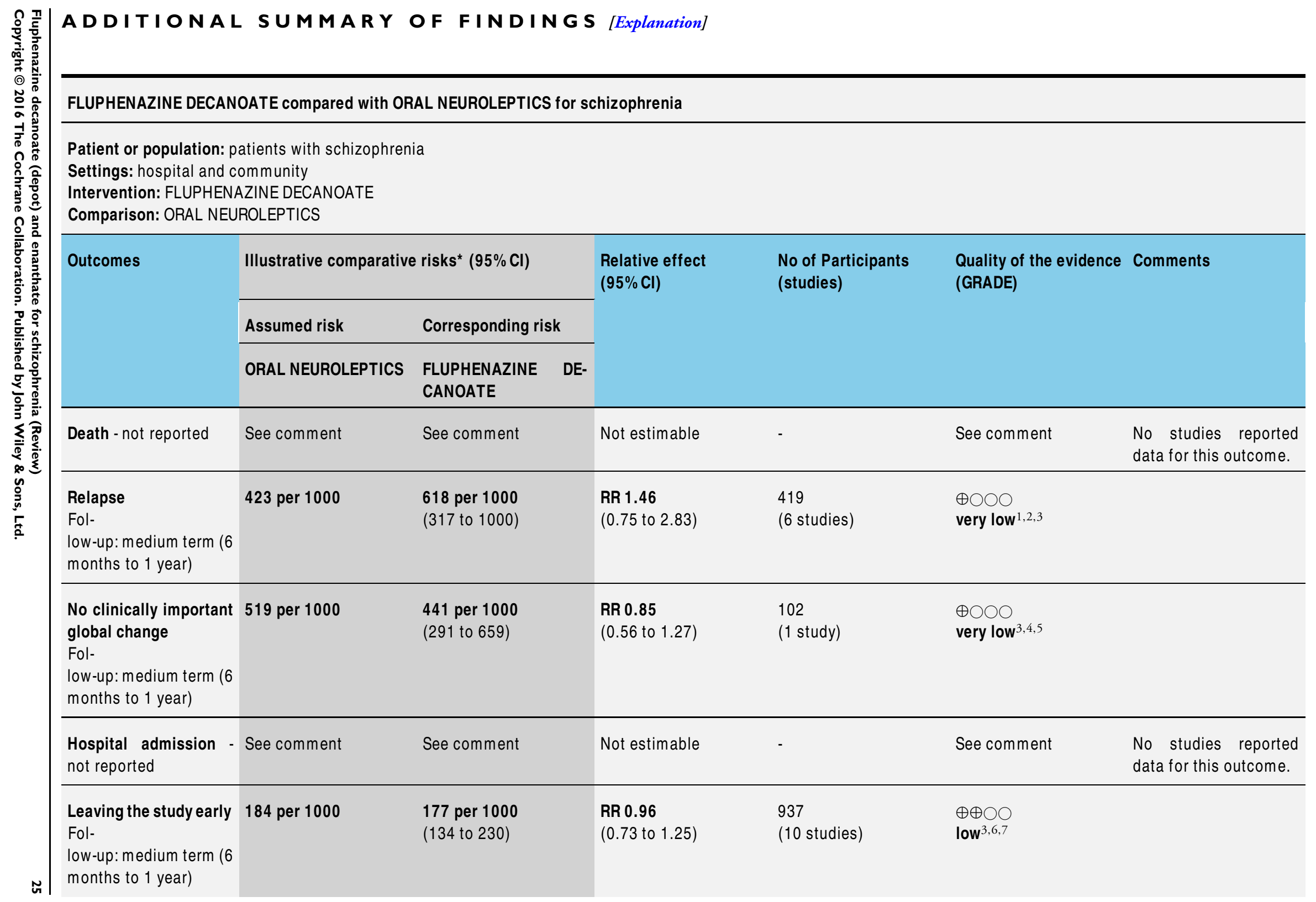




\begin{tabular}{|c|c|c|c|c|c|c|}
\hline $\begin{array}{l}\text { Mental state } \\
\text { BPRS endpoint score } \\
\text { Follow-up: (longer term } \\
\text { - more than } 1 \text { year) }\end{array}$ & & $\begin{array}{l}\text { The mean mental state } \\
\text { in the intervention } \\
\text { groups was } \\
0.75 \text { lower } \\
\text { ( } 5.75 \text { lower to } 4.25 \\
\text { higher) }\end{array}$ & & $\begin{array}{l}120 \\
\text { (1 study) }\end{array}$ & $\begin{array}{l}\oplus \bigcirc \bigcirc \bigcirc \\
\text { very low } \\
3,5,8\end{array}$ & \\
\hline $\begin{array}{l}\text { Extrapyramidal ad- } \\
\text { verse effects - general } \\
\text { Fol- } \\
\text { low-up: medium term ( } 6 \\
\text { months to } 1 \text { year) }\end{array}$ & 137 per 1000 & $\begin{array}{l}64 \text { per } 1000 \\
(33 \text { to } 125)\end{array}$ & $\begin{array}{l}\mathbf{R R} 0.47 \\
(0.24 \text { to } 0.91)\end{array}$ & $\begin{array}{l}259 \\
\text { (3 studies) }\end{array}$ & $\begin{array}{l}\oplus \bigcirc \bigcirc \bigcirc \\
\text { very low } \\
3,5,9\end{array}$ & $\begin{array}{l}\text { Studies also reported } \\
\text { equivocal results for } \\
\text { akathisia, needing an- } \\
\text { ticholinergic drugs, tar- } \\
\text { dive dyskinesia, tremor, } \\
\text { and symptoms on the } \\
\text { Simpson Angus scale }\end{array}$ \\
\hline
\end{tabular}

*The basis for the assumed risk (e.g. the median control group risk across studies) is provided in footnotes. The corresponding risk (and its $95 \%$ confidence interval) is based on the assumed risk in the comparison group and the relative effect of the intervention (and its $95 \% \mathrm{Cl}$ ).

Cl: Confidence interval; RR: Risk ratio;

GRADE Working Group grades of evidence

High quality: Further research is very unlikely to change our confidence in the estimate of effect.

Moderate quality: Further research is likely to have an important impact on our confidence in the estimate of effect and may change the estimate.

Low quality: Further research is very likely to have an important impact on our confidence in the estimate of effect and is likely to change the estimate.

Very low quality: We are very uncertain about the estimate.

${ }^{1}$ Risk of bias: serious - Studies had an unclear risk of bias for randomisation, allocation concealment and blinding. One study

had a high risk of bias for incomplete outcome data.

${ }^{2}$ Inconsistency: serious - There is high heterogeneity.

${ }^{3}$ Imprecision: serious - There are wide confidence intervals.

${ }^{4}$ Risk of bias: serious - This study had an unclear risk of bias for randomisation, allocation concealment, blinding and incomplete outcome data.

${ }_{5}^{5}$ Publication bias: strongly suspected - Three studies or fewer reported data for this outcome.

${ }^{6}$ Risk of bias: serious - Studies had an unclear risk of bias for randomisation, allocation concealment and blinding, although one study had a low risk of bias for blinding of participants. One study had a high risk of bias for incomplete outcome data.

${ }^{7}$ Publication bias: undetected - It seems that larger trials have not been performed, see Figure 4.

${ }^{8}$ Risk of bias: very serious - This study had a high risk of bias for blinding as it is an open label study. It has an unclear risk of bias for randomisation and allocation concealment. 


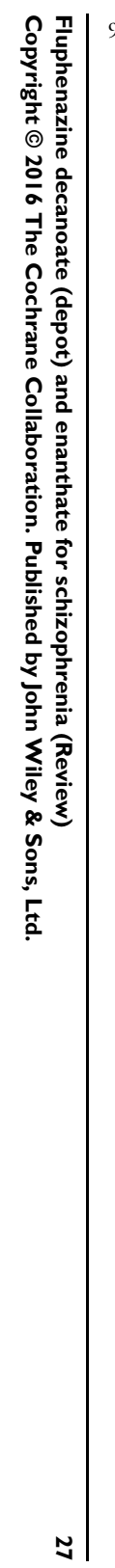




\section{FLUPHENAZINE DECANAOTE compared to FLUPHENAZINE ENANTHATE for schizophrenia}

Patient or population: patients with schizophrenia

Settings: hospital and community

Intervention: FLUPHENAZINE DECANAOTE

Comparison: FLUPHENAZINE ENANTHATE

\begin{tabular}{|c|c|c|c|c|c|c|}
\hline \multirow[t]{3}{*}{ Outcomes } & \multicolumn{2}{|c|}{ Illustrative comparative risks ${ }^{\star}(95 \% \mathrm{Cl})$} & \multirow{3}{*}{$\begin{array}{l}\text { Relative effect } \\
(95 \% \mathrm{Cl})\end{array}$} & \multirow{3}{*}{$\begin{array}{l}\text { No of Participants } \\
\text { (studies) }\end{array}$} & \multirow{3}{*}{$\begin{array}{l}\text { Quality of the evidence } \\
\text { (GRADE) }\end{array}$} & \multirow{3}{*}{ Comments } \\
\hline & Assumed risk & Corresponding risk & & & & \\
\hline & $\begin{array}{l}\text { FLUPHENAZINE ENAN- } \\
\text { THATE }\end{array}$ & $\begin{array}{l}\text { FLUPHENAZINE DE- } \\
\text { CANAOTE }\end{array}$ & & & & \\
\hline Death - not reported & See comment & See comment & Not estimable & - & See comment & $\begin{array}{l}\text { No studies reported } \\
\text { data for this outcome. }\end{array}$ \\
\hline $\begin{array}{l}\text { Relapse } \\
\text { Fol- } \\
\text { low-up: medium term ( } 6 \\
\text { months to } 1 \text { year) }\end{array}$ & 120 per 1000 & $\begin{array}{l}292 \text { per } 1000 \\
(85 \text { to } 998)\end{array}$ & $\begin{array}{l}\text { RR } 2.43 \\
(0.71 \text { to } 8.32)\end{array}$ & $\begin{array}{l}49 \\
\text { (1 study) }\end{array}$ & $\begin{array}{l}\oplus \bigcirc \bigcirc \bigcirc \\
\text { very low }\end{array}$ & \\
\hline $\begin{array}{l}\text { Clinically significant } \\
\text { change in global state - } \\
\text { not reported }\end{array}$ & See comment & See comment & Not estimable & - & See comment & $\begin{array}{l}\text { No studies reported } \\
\text { data for this outcome. }\end{array}$ \\
\hline $\begin{array}{l}\text { Hospital admission } \\
\text { not reported }\end{array}$ & See comment & See comment & Not estimable & - & See comment & $\begin{array}{l}\text { No studies reported } \\
\text { data for this outcome. }\end{array}$ \\
\hline $\begin{array}{l}\text { Leaving the study early } \\
\text { Fol- } \\
\text { low-up: medium term ( } 6 \\
\text { months to } 1 \text { year) }\end{array}$ & 120 per 1000 & $\begin{array}{l}292 \text { per } 1000 \\
(85 \text { to } 998)\end{array}$ & $\begin{array}{l}\text { RR } 2.43 \\
(0.71 \text { to } 8.32)\end{array}$ & $\begin{array}{l}49 \\
\text { (1 study) }\end{array}$ & $\begin{array}{l}\oplus \bigcirc \bigcirc \bigcirc \\
\text { very low } 1,2,3\end{array}$ & \\
\hline
\end{tabular}




\begin{tabular}{|c|c|c|c|c|c|c|}
\hline $\begin{array}{l}\text { Mental State } \\
\text { BPRS } \\
\text { Fol- } \\
\text { low-up: medium term (6 } \\
\text { months to } 1 \text { year) }\end{array}$ & & $\begin{array}{l}\text { The mean mental state } \\
\text { in the intervention } \\
\text { groups was } \\
0 \text { higher } \\
\text { ( } 3.93 \text { lower to } 3.93 \\
\text { higher) }\end{array}$ & & $\begin{array}{l}39 \\
\text { (1 study) }\end{array}$ & $\begin{array}{l}\oplus \bigcirc \bigcirc \bigcirc \\
\text { very low } \\
1,3,4\end{array}$ & \\
\hline $\begin{array}{l}\text { Extrapyra- } \\
\text { midal adverse effects - } \\
\text { general } \\
\text { Follow-up: short term (6 } \\
\text { weeks to } 5 \text { months) }\end{array}$ & 538 per 1000 & $\begin{array}{l}614 \text { per } 1000 \\
(425 \text { to } 883)\end{array}$ & $\begin{array}{l}\text { RR } 1.14 \\
\text { (0.79 to } 1.64)\end{array}$ & $\begin{array}{l}49 \\
\text { (2 studies) }\end{array}$ & $\begin{array}{l}\oplus \bigcirc \bigcirc \bigcirc \\
\text { very low } \\
3,4,5\end{array}$ & $\begin{array}{l}\text { Stud- } \\
\text { ies also reported equiv- } \\
\text { ocal results for Parkin- } \\
\text { sonism, akathisia and } \\
\text { needing anticholinergic } \\
\text { drugs }\end{array}$ \\
\hline
\end{tabular}

*The basis for the assumed risk (e.g. the median control group risk across studies) is provided in footnotes. The corresponding risk (and its $95 \%$ confidence interval) is based on the assumed risk in the comparison group and the relative effect of the intervention (and its $95 \% \mathrm{Cl}$ ).

Cl: Confidence interval; RR: Risk ratio;

GRADE Working Group grades of evidence

High quality: Further research is very unlikely to change our confidence in the estimate of effect.

Moderate quality: Further research is likely to have an important impact on our confidence in the estimate of effect and may change the estimate.

Low quality: Further research is very likely to have an important impact on our confidence in the estimate of effect and is likely to change the estimate.

Very low quality: We are very uncertain about the estimate.

${ }^{1}$ Risk of bias: serious - This study had an unclear risk of bias for randomisation, allocation concealment and blinding.

${ }^{2}$ Imprecision: very serious - There were few participants and few events; the confidence intervals are wide.

${ }^{3}$ Publication bias: strongly suspected - Fewer than three studies reported data for this outcome.

${ }^{4}$ Imprecision: serious - There are wide confidence intervals.

${ }^{5}$ Risk of bias: serious - The studies had an unclear risk of bias for randomisation and blinding of outcome assessors. One

was low risk of bias for allocation concealment and the other for blinding of participants. 


\section{ISCUSSION}

\section{Summary of main results}

Seventy-three trials including 4870 participants were included in the review. The summary below reflects the outcomes chosen for Summary of findings for the main comparison, Summary of findings 2 and Summary of findings 3, and are considered the main findings of this review. Overall, the quality of the evidence was rated as low to very low.

\section{Fluphenazine decanoate versus placebo}

\section{I.I Death}

Only one, small study (Jolley 1990) reported on death and no significant differences were observed.

\section{I.2 Global state}

\subsubsection{Clinically significant change in global state}

No studies reported data for this outcome.

\subsubsection{Relapse}

Very low quality evidence from three studies showed that mediumterm relapse rates were not significantly lower in the fluphenazine decanoate group (43\%) compared with placebo (67\%). Only longer-term data from one small trial (Jolley 1990) significantly reduced relapse.

\section{I.3 Hospital admission}

No studies reported data for this outcome.

\section{I.4 Leaving the study early}

The numbers of people leaving the study early in the fluphenazine decanoate $(24 \%)$ and placebo $(19 \%)$ groups were very similar, however, the quality of the evidence was very low. This figure could be higher in clinical practice because rigorous adherence to protocols in these randomised studies may decrease attrition, although the opposite could also be true. Although adherence to protocol improves internal validity, it can potentially decrease the external validity and applicability of results. The single twoyear study significantly favoured fluphenazine decanoate compared with placebo (Jolley 1990).

\section{I.5 Mental state}

One very small study (Dotti 1979) found no difference in mental state measured on the BPRS.

\section{I.6 Extrapyramidal adverse effects}

The occurrence of tardive dyskinesia (long term) was not significantly different, although the quality of the evidence is very low and data were again from a single small study (Jolley 1990).

\section{Fluphenazine decanoate versus oral neuroleptics}

\section{I Death}

No studies reported data for this outcome.

\subsection{Relapse}

Very low quality evidence from six studies showed that mediumterm rates of relapse were not significantly different in the fluphenazine decanoate group (49\%) compared with oral neuroleptics (42\%).

\subsection{Clinically significant change in global state}

One small study (Song 1993) found no difference in the number of participants showing a clinically significant change in global state.

\subsection{Hospital admission}

No studies reported data for this outcome.

\subsection{Leaving the study early}

Low-quality evidence showed no difference in the number of participants leaving the study early for fluphenazine decanoate (17\%) versus oral neuroleptics (18\%).

\subsection{Mental state}

Very low quality evidence from one study (Simon 1978) found no difference in mental state measured on the BPRS.

\subsection{Extrapyramidal adverse effects}

Three small studies showed that general extrapyramidal adverse effects were lower in the fluphenazine decanoate group (7\%) compared to oral neuroleptics (14\%). However, the quality of the evidence was judged to be very low, and there was no difference with longer-term data. The outcome of 'needing additional anticholinergic drug' was equivocal over short-, medium- and longer-term, suggesting oral neuroleptics and fluphenazine decanoate are similar in their ability to induce movement disorders. Also, tardive 
dyskinesia was significantly lower for the fluphenazine decanoate group during medium-term evaluation, but was not different to oral neuroleptics with longer-term data.

\section{Fluphenazine decanoate versus other depot antipsychotics}

\section{I Death}

One study did report a death in fluphenazine decanoate treatment group, however, this did produce an effect with no significant differences between groups for death.

\subsection{Global state}

Eleven studies reported equivocal data fro the outcome of 'relapse' at six months to one year. Other global state outcomes such as significant clinical improvement, clinical global impression, needing additional antipsychotics and 'not improved' were also equivocal.

\subsection{Leaving the study early}

Fifteen included trials found people were no more likely to leave the study early if they were receiving fluphenazine decanoate or other depot antipsychotics.

\subsection{Behaviour}

Only one study reported equivocal data.

\subsection{Mental state}

Short- and medium-term studies assessing mental state (BPRS endpoint scores) to significantly favour 'other depot neuroleptics' for the short term and medium term. Long-term studies did not find such difference in mental state. One study reported on the outcome of depression; Dencker 1973, found no significant difference between fluphenazine decanoate and pipothiazine palmitate.

\subsection{Adverse effects}

The occurrence of dyskinetic movements in general was the same across short-, medium- and longer-term studies. Outcomes such as dry mouth, tardive dyskinesia and parkinsonism were not significantly different between depot fluphenazine and other depot neuroleptics. When reporting blurred vision, the results of one medium-term trial were not significant, but one longer-term study, Pinto 1979, did report significant results favouring flupenthixol decanoate. General adverse effects (short-term data) were reported by Frangos 1978 and Javed 1991 and favoured other depot neuroleptics. However, medium-term data were equivocal.

\section{Fluphenazine decanoate versus fluphenazine enanthate}

\section{I Death}

No studies reported data for this outcome.

\subsection{Relapse}

Very low quality evidence from only one small study ( MacCrimmon 1978) found no significant difference in the number of participants experiencing relapse in the medium term. Results were also equivocal for immediate- and short-term studies.

\subsection{Clinically significant change in global state}

No studies reported data for this outcome.

\subsection{Hospital admission}

No studies reported data for this outcome.

\subsection{Leaving the study early}

No difference in the number of participants leaving the study early was found between fluphenazine decanoate (29\%) and fluphenazine enanthate (12\%), but this is based on one small study (MacCrimmon 1978) and considered to be very low quality evidence.

\subsection{Mental state}

BPRS data were only available from one small trial (MacCrimmon 1978). This study reported identical scores for both of the fluphenazine depots groups.

\subsection{Extrapyramidal adverse effects}

Very low evidence from two very small studies showed that two preparations caused roughly equal incidences of general movement disorders. Results were also equivocal for parkinsonism, akathisia and needing additional anticholinergics in the short and immediate term.

\section{Overall completeness and applicability of evidence}

Trials were based mainly in the community, or combined both hospital and community settings. Ages ranged between 13 and 81 years, but most people were in the 18 to 65 age range. The duration of illness was long in most participants in the studies that reported this. Included trials were conducted around the world, although the majority (56) were based in North America and Europe and none were based in Australia or South America. There is a broad 
mixture of participants, settings and clinical applicability of the interventions which should increase generalisability. The dosages of fluphenazine decanoate and enanthate reflected current clinical practice. Outcomes were, however, limited. No trials reported data on service utilisation, hospital admission and economic outcomes. It is a shame that so few outcomes were included.

\section{Quality of the evidence}

The quality of the evidence is low to very low based on GRADE (Schünemann 2008). Although all studies were reported as randomised, only five reported the method of randomisation and another three the method of allocation concealment. For the majority of studies the method of blinding was not reported despite most stating that they were double blind. In $83 \%$ of studies, the risk of bias for selective reporting was rated as high; many preplanned outcomes were not reported and continuous data were often reported as mean without standard deviation, only as P values, or graphically. Poor presentation of data meant that a lot of potentially informative data were lost.

\section{Potential biases in the review process}

A thorough search strategy was used in this review. There may still be gaps in the search strategy such as unpublished data (grey literature), which are difficult to obtain. Futhermore, when assessing the risk of bias of studies previously included in this review, we were unable to assess the risk of bias for one study in Italian. However, this study did not contribute to any outcomes in the 'Summary of findings' tables and therefore the omission of this one study did not affect the overall quality judgement.

For most outcomes we are not able to tell if there was publication bias, as no more than 10 trials reported data for these, and a funnel plot could not be performed. For leaving the study early in the comparison of fluphenazine decanoate versus oral neuroleptics, there does appear to be publication bias, as the funnel plot indicates that larger trials have not been performed.

\section{Agreements and disagreements with other studies or reviews}

We do not know of any other systematic reviews on this topic.

\section{A U THORS, CONCLUSIONS}

\section{Implications for practice}

\section{For people with schizophrenia}

When compared with either placebo or oral neuroleptics, fluphenazine decanoate does not appear to have a clinically important effect in terms of improving relapse rates based on mediumterm (six months to one year) data. Longer-term data, however, do support the use of fluphenazine decanoate to reduce relapse when compared to placebo, but not when compared to oral neuroleptics. Relapse data for fluphenazine enanthate were limited and no data comparing it with placebo or oral neuroleptics were available. Fluphenazine depot preparations, especially the decanoate, seem equivalent to oral medications and may even cause less adverse effects.

\section{For clinicians}

The data on the effects of fluphenazine decanoate are clearer than for fluphenazine enanthate. Within the highly unusual setting of a randomised trial, the decanoate may have some advantages over the oral antipsychotics. In clinical life there may be greater advantages in terms of compliance. There are no data to support the claim that depots cause more adverse effects than oral preparations. There are also no data to support the use of high doses.

\section{For managers or policy makers}

Studies did not report data relating to service utilisation and care management. Outcomes relating to use of hospitals and services, satisfaction with care and economics were not reported in any study. This deficiency remains and should be addressed in real world randomised studies.

\section{Implications for research}

\section{General}

Trialists involved in future studies should implement the CONSORT statement (Moher 2001) to ensure that outcomes are more relevant. Inclusion of hospital and services outcomes, satisfaction with care and economic outcomes would provide valuable data for people with schizophrenia, clinicians and policy makers.

\section{Specific}

A recurring failure to report the exact methodology of allocation was evident throughout the included trials. Only five studies stated the randomisation process used; Kissling 1985 used a coin-throwing method, Frangos 1978 a randomisation code, Magnus 1979 a pre-arranged prescribing list, and Wistedt 1984 a randomisation list and Leong 1989 allocated to the next available study number. Allocation concealment is essential to ensure that selection bias is kept to a minimum.

Only 14 studies described outcome assessors as being blinded to treatment and were rated low risk of bias (Altamura 1985; Chouinard 1982; Dencker 1973; Frangos 1978; Goldstein 1978; 
Kane 1978; Leong 1989; McClelland 1976; Odejide 1982; Pinto 1979; Russell 1982; Van Praag 1970; Van Praag 1973; Wistedt 1983). Magnus 1979 and Simon 1978 were open label trials and Leong 1989 described using a 'partially-blinded' method where only outcome assessors were blinded. The remaining studies were of unclear risk of bias as no information on blinding of outcome assessors was provided. Although most studies were stated to be double blind, only 14 studies described outcome assessors as being blinded to treatment and were rated low risk of bias (Altamura 1985; Chouinard 1982; Dencker 1973; Frangos 1978; Goldstein 1978; Kane 1978; Leong 1989; McClelland 1976; Odejide 1982; Pinto 1979; Russell 1982; Van Praag 1970; Van Praag 1973; Wistedt 1983). This is an important strategy for avoiding performance and detection bias. Odejide 1982 included participants who were unaccounted for after randomisation was undertaken. This study did not specify from which groups this withdrawal had occurred. In 12 trials the number of people who left the study was not reported. It is important to know how many, and from which groups, people were withdrawn in order to evaluate exclusion bias. Studies included both community-based and hospitalised people but 16 failed to report the setting (Albert 1980; Feng 1990; Hranov 1998; Javed 1991; Kissling 1985; Kreisman 1988; Lehmann 1980; McKane 1987; Odejide 1982; Quitkin 1978; Rossi 1990; Russell 1982; Schlosberg 1978; Schneider 1981; Sharma 1991; Wistedt 1983). A few studies, all using fluphenazine decanoate as an intervention, involved people in hospital at the beginning of the trial but these people were later discharged into the community (Dencker 1973; Donlon 1976; Magnus 1979; Marder 1987; McCreadie 1980; Rifkin 1977; Schooler 1980; Schooler 1997; Simon 1978; Wistedt 1984). More community-based studies would be welcome.

This review highlights the need for good controlled clinical trials to address the effects of fluphenazine decanoate and fluphenazine enanthate and to assess their clinical suitability in certain situations. Despite the many studies that are in existence, we do think that more studies are required in each category but particularly in the case of fluphenazine enanthate where data were particularly few. We realise that such design needs a great deal of planning but suggest a simple outline in Table 1 .

\section{ACKNOW LEDGEMENTS}

We would like to thank Leanne Roberts for her help and support as well as Drs Cookson, Merskey, Russell, Quitkin, Pinto and McCreadie for their assistance.

Thank you to Mingming Zhang, Huilin Liu and Jun Xie for translation of Chinese papers and to Dr. Simi Pereira for peer reviewing comments.

\section{R E F E R E N C E S}

\section{References to studies included in this review}

\section{Adamson 1973 \{published data only\}}

Adamson L, Currey SH, Bridges PK, Firestone AF, Lavin NI, Lewis DM, et al. Fluphenazine decanoate trial in chronic inpatient schizophrenics failing to absorb oral chlorpromazine. Diseases of the Nervous System 1973;34(4): 181-91.

Albert 1980 \{published data only\} Albert JM, Elie R, Cooper SF. Long term double-blind evaluation of pipotiazine palmitate and fluphenazine decanoate. Current Therapeutic Research 1980;27(6): 897-907.

Altamura 1985 \{published data only\}

Altamura AC, Curry SH, Montgomery S, Wiles DH. Early unwanted effects of fluphenazine esters related to plasma fluphenazine concentrations in schizophrenic patients. Psychopharmacology 1985;87:30-3.

Asarnow 1988 \{published data only\}

Asarnow RE, Marder SR, Mintz J, Van Putten T, Zimmerman KE. Differential effect of low and conventional doses of fluphenazine on schizophrenic outpatients with good or poor information-processing skills. Archives of General Psychiatry 1988;45:822-7.
Barnes 1983 \{published data only\} Barnes TRE, Milavic G, Curson DA, Platt SD. Use of the social behaviour assessment schedule (SBAS) in a trial of maintenance antipsychotic therapy in schizophrenic outpatients: pimozide versus fluphenazine. Social Psychiatry 1983;18:193-9.

Chien 1973 \{published data only\} Chien CP, Cole JO. Depot phenothiazine treatment in acute psychosis: A sequential comparative clinical study. American Journal of Psychiatry 1973;130(1):13-7.

Chouinard 1978 \{published data only\} Chouinard G, Annable L, Kropsky M. A doubleblind controlled study of pipothiazine palmitate in the maintenance treatment of schizophrenic outpatients. Journal of Clinical Psychopharmacology 1978; Feb-Mar: $148-54$.

Chouinard 1982 \{published data only\} Chouinard G, Annable L, Ross-Chouinard A. Fluphenazine enanthate and fluphenazine decanoate in the treatment of schizophrenic outpatients: Extrapyramidal symptoms and therapeutic effect. American Journal of Psychiatry 1982;139 (3):312-18

Chouinard 1984 \{published data only\} Chouinard G, Annable L, Campbell W. A randomized 
clinical trial of haloperidol decanoate and fluphenazine decanoate in the outpatient treatment of schizophrenia. Journal of Clinical Psychopharmacology 1989;9(4):247-53. * Chouinard G, Annable L, Campbell W, Boisvert D, Bradwejn J. A double-blind, controlled clinical trial of haloperidol decanoate and fluphenazine decanoate in the maintenance treatment of schizophrenia. Psychopharmacology Bulletin 1984;20:108-9.

\section{Cookson 1986 \{published data only\}}

Cookson JC, Kennedy NM, Gribbon D. Weight gain and prolactin levels in patients on long-term antipsychotic medication: a double-blind comparative trial of haloperidol decanoate and fluphenazine decanaote. International Clinical Psychopharmacology 1986;Suppl 1:41-51.

Crawford 1974 \{published data only\} Crawford R, Forrest A. Controlled trial of depot fluphenazine in out-patient schizophrenics. British Journal of Psychiatry 1974;124:385-91.

Curry 1972 \{published data only\} Curry SH, Adamson L. Double-blind trial of fluphenazine decanoate. Lancet 1972;2(776):543-4.

Dencker 1973 \{published data only\} Dencker SJ, Frankenberg K, Lepp M, Lindberg D, Malm $\mathrm{U}$. How schizophrenic patients change during 3 years' treatment with depot neuroleptics. Acta Psychiatria Scandanavia 1978;57:115-23. Dencker SJ, Frankenberg K, Lepp M, Lindberg D, Malm U. Three years' maintenance neuroleptic treatment in schizophrenia - before and beyond. Acta Psychiatria Scandinavica 1978;57:103-14.

* Dencker SJ, Frankenberg K, Malm U, Zell B. A controlled one-year study of pipotiazine palmitate and fluphenazine decanoate in chronic schizophrenic syndromes. Acta Psychiatria Scandinavica 1973;241(Suppl):101-18.

\section{Donlon 1976 \{published data only\}}

Donlon PT, Axelrad AD, Tupin JP, Chien C. Comparison of depot fluphenazines: Duration of action and incidence of side effects. Comprehensive Psychiatry 1976;17(2):369-76.

Dotti 1979 \{published data only\}

Dotti A, Bersani G, Rubino IA, Elliseo C. Double blind trial of fluphenazine decanoate against placebo in ambulant maintenance treatment of chronic schizophrenics [Studio in doppio cieco della flufenazina decanoato versus placebo nella terapia ambulatoriale di mantenimento di pazienti schizofrenici cronici]. Rivista di Psichiatria 1979;14(5): 374-83. MEDLINE: 75003702

Falloon 1978 \{published data only\}

* Fallon I, Watt DC, Shepherd M. The social outcome of patients in a trial of long-term continuation therapy in schizophrenia: pimozide vs fluphenazine. Psychological Medicine 1978;8:265-74.

Falloon I, Watt D. A comparative controlled trial of pimozide and fluphenazine decanoate in the continuation therapy of schizophrenia. Psychological Medicine 1978;8: 59-70.

Shepherd M. Medical-social evaluation of the long term pharmacotherapy of schizophrenia. Progress NeuroPsychopharmacology 1979;3:383-9.

Feng 1990 \{published data only\}

Feng L. Double blind controlled trial of haloperidol decanoate and fluphenazine decanoate in chronic schizophrenia. Chinese Journal of Nervous and Mental Disorders 1990;16(5):299.

Frangos 1978 \{published data only\}

Frangos H, Zississ NP, Leontopoulos I, Diamentas N, Tsitouridis S, Gavriil I, et al. Double blind therapeutic evaluation of fluspirilene compared with fluphenazine decanoate in chronic schizophrenics. Acta Psychiatria Scandinavica 1978;57:436-46.

Goldstein 1978 \{published data only\}

Goldstein MJ, Rodnick EH, Evans JR, May PRA, Steinberg MR. Drug and family therapy in the aftercare of acute schizophrenics. Archives of General Psychiatry 1978;35: 1169-77.

Hirsch 1975 \{published data only\}

Hirsch SR. The interaction between depot-phenothiazines and the social and clinical manifestations of behaviour in patients with stabilised chronic schizophrenia. In: Van Praag HM editor(s). On the Origin of Schizophrenic Psychoses. De Erven Bohn BV, 1975:209-22. MEDLINE: 76263054

Hogarty 1979 \{published data only\} Hogarty GE, Schooler NR, Ulrich R, Mussare I, Ferro P, Herron E. Fluphenazine and social therapy in the aftercare of schizophrenic patients. Archives of General Psychiatry 1979;36:1283-94.

Hogarty 1988 \{published data only\} Hogarty GE, McEvoy JP, Munetz M, DiBarry AL, Bartone P, Cather R, et al. Dose of fluphenazine, familial expressed emotion, and outcome in schizophrenia. Archives of General Psychiatry 1988;45:797-805.

Hranov 1998 \{published data only\} Hranov LG, Yanakiev N, Stefanov S, Nikolova N, Yakimova R. Haloperidol decanoate and fluphenazine decanoate for schizophrenia: a comparative naturalistic medium term study of efficacy and tolerability. 11th Congress of The European College of Neuropsychopharmacology; 1998 Oct 3 - Nov 4; Paris, France. 1998.

Jain 1975 \{published data only\}

* Jain RC, Ananth JV, Lehmann HE, Ban TA. A comparative study with pipothiazine palmitate and fluphenazine enanthate in the treatment of schizophrenic patients. Current Therapeutic Research 1975;18(4):585-9.

Javed 1991 \{published data only\} Javed MA, Chaudhry MR. Double blind comparison of flupenthixol decanoate and fluphenazine decanoate in the treatment of chronic schizophrenia. Pakistan Journal of Clinical Psychiatry 1991;1(2):69-74. 
Jolley 1990 \{published data only\}

Jolley AG, Hirsch SR, Morrison E, McRink A, Wilson L. Trial of brief intermittent neuroleptic prophylaxis for selected schizophrenic outpatients: clinical and social outcome at two years. BMJ 1990;301:837-42.

Ju 2000 \{published data only\}

Ju H, Cong Z, Deng P. A control study on using pipothiazine palmitate to treat schizophrenic patients. Journal of Clinical Psychological Medicine 2000;10(1):24-5.

Kane 1978 \{published data only\}

Kane J, Quitkin F, Rifkin A, Klein DF. Comparison of the incidence and severity of extrapyramidal side effects with fluphenazine enanthate and fluphenazine decanoate. American Journal of Psychiatry 1978;135:1539-42.

Kane 1983 \{published data only\}

Kane JM, Rifkin A, Woerner M, Reardon G. Lowdose neuroleptics in outpatient schizophrenics. Psychopharmacology Bulletin 1982;18(1):20-1.

* Kane JM, Rifkin A, Woerner M, Reardon G, Sarantakos $\mathrm{S}$, Schiebel D, et al. Low-dose neuroleptic treatment of outpatient schizophrenics. Archives of General Psychiatry 1983;40:893-6.

\section{Kaneno 1991 \{published data only\}}

Kaneno S, Ohkuma T, Yamashita I, Mori A, Yagi G, Kudo $\mathrm{Y}$, et al. A double blind comparative study on the efficacy and safety of fluphenazine decanoate (SQ10, 733) and oral haloperidol in the treatment of schizophrenic patients. Rinsho Hyoka (Clinical Evaluation) 1991;19(1):15-45. MEDLINE: 70166858

Kelly 1977 \{published data only\}

Kelly HB, Freeman HL, Banning B, Schiff AA. Clinical and social comparison of fluphenazine decanoate and flupenthixol decanoate in the community maintenance therapy of schizophrenia. International Pharmacopsychiatry 1977;12:54-64.

Keskiner 1971 \{published data only\}

Keskiner A, Itil T, Han H, Hsu W, Ulett G. EEG changes after fluphenazine enanthate and decanoate based on analog power spectra and digital computer period analysis. Psychopharmacologia 1971;20(3):230-41. MEDLINE: 71275801

Khazaie 2005 \{published data only\}

Khazaei H, Habibi F, Pourafkari N. The effect of fluphenazine decanoate every 2 weeks versus 6 weeks in the treatment of schizophrenia and schizoaffective disorders. Iranian Journal of Psychiatry and Clinical Psychology 2003;8 (4):11-5.

* Khazaie H, Shakeri J. Comparative efficacy of every 2 weeks versus every 6 weeks injections of fluphenazine decanoate. Archives of Iranian Medicine 2005;8(2):109-14.

Kissling 1985 \{published data only\} Kissling W, Moller HJ, Walter K, Wittman B, Kruegger R, Trenk D. Double-blind comparison of haloperidol and fluphenazine decanoate effectiveness, side effects, dosage and serum levels during a six months treatment for relapse prevention. Pharmacopsychiatry 1985;18:240-5.
Kreisman 1988 \{published data only\}

Kreisman D, Blumenthal R, Borenstein M, Woerner M, Kane J, Rifkin A, et al. Family attitudes and patient social adjustment in a longitudinal study of outpatient schizophrenics receiving low-dose neuroleptics: the family's view. Psychiatry 1988;51(1):3-13. MEDLINE: 88218101

\section{Kurland 1966 \{published data only\}}

Kurland AA, Richardson JH. A comparative study of two long acting phenothiazine preparations, fluphenazine enanthate and fluphenazine decanoate. Psychopharmacologia (Berl) 1966;9:320-7.

Lehmann 1980 \{published data only\}

Lehmann E, Quadbeck H, Tegeler J, Fararuni M, Heinrich K. Drug-response differences of high and standard dosage of fluphenazine-decanoate in relation to schizophrenic symptoms. Pharmakopsychiatrie und Neuropsychopharmakologie 1980;13(3):117-29.

Leong 1989 \{published data only\} Leong OK, Wong KE, Tay WK, Gill RC. A comparative study of pipothiazine palmitate and fluphenazine decanoate in the maintenance of remission of schizophrenia. Singapore Medical Journal 1989;30:536-40.

\section{Levenson 1976 \{published data only\}}

Levenson AJ, Burnett GB, Nottingham JD, Sermas CE, Thornby JI. Speed and rate of remission in acute schizophrenia: a comparison of intramuscularly administered fluphenazine $\mathrm{HC} 1$ with thiothixene and haloperidol. Current Therapeutic Research Clinical and Experimental 1976;20(5):695-700. MEDLINE: 77048014

Lundin 1990 \{published data only\}

* Lundin L, Dencker SJ, Malm U. Community-based rehabilitation of schizophrenia. Nordisk Psykiatrisk Tidsskrift 1990;44:81-7.

Lundin L, Dencker SJ, Malm U. Community-based rehabilitation of schizophrenia: corrected version. Nordisk Psykiatrisk Tidsskrift 1992;46(2):121-7.

MacCrimmon 1978 \{published data only\} MacCrimmon DJ, Saxena B, Foley P, Grof P. Fluphenazine decanoate and fluphenazine enanthate in the out-patient management of chronic schizophrenia. Neuropsychobiology 1978;4:360-5.

Magnus 1979 \{published data only\}

* Magnus RV. A comparative study of fluspirilene and fluphenazine decanoate in schizophrenic patients. Journal of Pharmacotherapy 1979;2(3):109-14.

Magnus RV. Fluspirilene and fluphenazine decanoate for schizophrenic patients. Controlled study [Fluspirilen und Fluphenazin-Dekanoat bei schizophrenen Patienten. Vergleichende Studie]. Munchener Medizinische Wochenschriftenschrift 1980;122:1758-60.

Malm 1974 \{published data only\} Malm U, Perris C, Rapp W, Wedren G. A multicenter controlled trial of fluspirilene and fluphenazine enanthate in chronic schizophrenic syndromes. Acta Psychiatrica Scandinavica Supplementum 1974;249:94-116. 
Marder 1987 \{published data only\}

* Marder SR, Van Putten T, Mintz J, Lebell M, McKenzie J, May PRA. Low and conventional dose maintenance therapy with fluphenazine decanoate. Archives of General Psychiatry 1987;44:518-21.

Marder SR, Van Putten T, Mintz J, McKenzie J, Lebell M, Faltico G, et al. Costs and benefits of two doses of fluphenazine. Archives of General Psychiatry 1984;41: 1025-9.

McClelland 1976 \{published data only\} McClelland H, Farquharson RG, Leyburn P, Furness JA, Schiff AA. Very high dose fluphenazine decanoate. Archives of General Psychiatry 1976;33:1435-9.

McCreadie 1980 \{published data only\} McCreadie RG, Dingwall JM, Wiles DH, Heykants JJP. Intermittant pimozide versus fluphenazine decanoate as maintenance therapy in chronic schizophrenia. British Journal of Psychiatry 1980;137:510-7.

McCreadie 1982 \{published data only\} McCreadie R, Mackie M, Morrison D, Kidd J. Once weekly pimozide versus fluphenazine decanoate as maintenance therapy in chronic schizophrenia. British Journal of Psychiatry 1982;140:280-6.

McKane 1987 \{published data only\} McKane JP, Robinson ADT, Wiles DH, McCreadie RG, Stirling GS. Haloperidol decanoate versus fluphenazine decanoate as maintenance therapy in chronic schizophrenic in-patients. British Journal of Psychiatry 1987;151:333-6.

McLaren 1992 \{published data only\}

McLaren S, Cookson J, Silverstone J. A comparative trial of bromperidol decanoate and fluphenazine decanoate in chronic. Annual Meeting of the British Association for Psychopharmacology; 1990 July 15, Cambridge, England. 1990.

* McLaren S, Cookson JC, Silverstone T. Postive and negative symptoms, depression and social disability in chronic schizophrenia: a comparative trial of bromoperidol and fluphenazine decanoates. International Clinical Psychopharmacology 1992;7(2):67-72.

Odejide 1982 \{published data only\}

Odejide OA, Aderounmu AF. Double-blind placebo substitution: Withdrawal of fluphenazine decanoate in schizophrenic patients. Psychopharmacology 1982;43(5): 195-6.

Pinto 1979 \{published data only\}

Pinto R, Banerjee A, Ghosh N. A double-blind comparison of flupenthixol decanoate and fluphenazine decanoate int he treatment of chronic schizophrenia. Acta Psychiatria Scandinavia 1979;60:313-22.

Quitkin 1978 \{published data only\}

Quitkin F, Rifkin A, Kane J, Ramos-Lorenzi JR, Klein DF. Long-acting oral vs injectable antipsychotic drugs in schizophrenics. Archives of General Psychiatry 1978;35: 889-92.
Rifkin 1977 \{published data only\}

* Rifkin A, Quitkin F, Kane J, Klein DF. Fluphenazine decanoate, oral fluphenazine, and placebo in the treatment of remitted schizophrenics II. Rating scale data. Psychopharmacology Bulletin 1977;13(2):40-50.

Rifkin A, Quitkin F, Kane J, Klein DF, Ross D. The effect of fluphenazine upon social and vocational functioning in remitted schizophrenics. Biological Psychiatry 1979;14(3): 499-508.

Rifkin A, Quitkin F, Klein D. Fluphenazine decanoate, oral fluphenazine, and placebo in treatment of remitted schizophrenics - Rating scale data. Archives of General Psychiatry 1977;34:15-9.

Rifkin A, Quitkin F, Rabiner C, Klein DF. Fluphenazine decanoate, fluphenazine hydrochloride given orally, and placebo in remitted schizophrenics - Relapse years after one year. Archives of General Psychiatry 1977;34:43-7.

Rossi 1990 \{published data only\}

Rossi A, Di Michele VD, Volonte MV, Casacchia M. Therapeutic evaluation of bromperidol decanoate in schizophrenia - a double-blind comparison vs. Fluphenazine decanoate [Efficacia terapeutica e tollerabilita del Bromperidolo decanoato VS Flufenazina decanoato nel Disturbo Schizofrenico]. Rivista Sperimentale di Freniatria e Medicina Legale delle Alienazioni Mentali 1990;114(6): 1379-86.

Russell 1982 \{published data only\} Russell N, Landmark J, Merskey H, Turpin T. A doubleblind comparison of fluspirilene and fluphenazine decanoate in schizophrenia. Canadian Journal of Psychiatry 1982;27: 593-6.

Schlosberg 1978 \{published data only\}

Schlosberg A, Shadmi M. A comparative controlled study of two long-acting phenothiazines: Pipothiazine palmitate and fluphenazine decanoate. Current Therapeutic Research 1978;23(5):642-54.

Schneider 1981 \{published data only\}

Schneider SJ, Kirby EJ, Itil TM. Clinical blood chemistry values and long acting phenothiazines. Pharmacopsychitria 1981;14:107-14

Schooler 1976 \{published data only\} Schooler NR, Levine J, NIMH-PRB Collaborative Fluphenazine Study Group. The initiation of longterm pharmacotherapy in schizophrenia: dosage and side effect comparisons between oral and depot fluphenazine. Pharmakopsychiatria 1976;9(4):159-69.

Schooler 1979 \{published data only\}

Schooler NR, Levine J, Severe JB. Depot fluphenazine in the prevention of relapse in schizophrenia: evaluation of a treatment regimen. Psychopharmacology Bulletin 1979;15 (2):44-7.

Schooler 1980 \{published data only\} Gelenberg AJ, Dollar JC, Schooler NR, Mieske M, Severe J, Mandel MR. Acute extrapyramidal reactions with 
fluphenazine hydrochloride and fluphenazine decanoate. Amercian Journal of Psychiatry 1979;136(2):217-9. Levine J, Schooler NR, Severe J, Escobar J, Geleberg A, Mandel M, et al. Discontinuation of oral and depot fluphenazine in schizophrenic patients ater one year of continuous medication: A controlled study. Advanced Biochemical Psychopharmacology 1980;24:483-93.

Mandel MR, Severe JB, Schooler NR, Gelenberg AJ, Mieske M. Development and prediction of postpsychotic depression in neuroleptic-treated schizophrenics. Archives of General Psychiatry 1982;39:197-203.

Schooler NR, Levine J, NIMH-PRB Collaborative Fluphenazine Study Group. The initiation of longterm pharmacotherapy in schizophrenia: dosage and side effect comparison between oral and depot fluphenazine. Pharmakopsychiatria 1976;9:159-69.

* Schooler NR, Levine J, Severe JB, Brauzer B, DiMascio A, Klerman GL, et al. Prevention of relapse in schizophrenia - An evaluation of fluphenazine decanoate. Archives of General Psychiatry 1980;37:16-24.

Schooler 1997 \{published data only\} Schooler NR, Keith SJ, Sever JB, Matthews SM, Bellack AS, Glick ID, et al. Relapse and rehospitalisation during maintenance treatment of schizophrenia: the effects of dose reduction and family treatment. Archives of General Psychiatry 1997;54(5):453-63.

Sharma 1991 \{published data only\} Sharma SK, Jaigirdar SH. A comparison of fluphenazine decanoate and haloperidol decanoate in chronic schizophrenia. British Journal of Clinical Research 1991;2: $177-86$.

Shenoy 1981 \{published data only\}

Shenoy RS, Sadler SG, Goldberg SC, Hamer RM, Ross B. Effects of a six-week drug holiday on symptom status, relapse, and tardive dyskinesia in chronic schizophrenics. Journal of Clinical Psychopharmacology 1981;1:141-5.

Shu 1983 \{published data only\}

Shu L. Double-blind study of domestic penfluridol and fluphenazine decanoate. Chung Hua Shen Ching Ching Shen Ko Tsa Chih (Chinese Journal of Neurology and Psychiatry) 1983;16(3):141-5. MEDLINE: 84083784

Simon 1978 \{published data only\} Simon P, Fermanian J, Ginestet D, Goujet MA, PeronMagnan P. Standard and long-acting depot neuroleptics in chronic schizophrenics. Archives of General Psychiatry 1978; 35:893-7.

Singh 1979 \{published data only\}

Singh AN, Saxena B. A comparative study of prolonged action (depot) neuroleptics: pipotiazine palmitate versus fluphenazine enanthate in chronic schizophrenic patients. Current Therapeutic Research 1979;25(1):121-32.

Song 1993 \{published data only\}

Song Y. A double-blind control study on the effect of pipotiazine palmitate and fluphenazine decanoate in the treatment of schizophrenia [data not available]. Chinese Journal of Neurology and Psychiatry 1993;26(3):137-40.
Van Praag 1970 \{published data only\}

Van Praag HM, Breetveld J, Van Mesdag-etty H, Westerhuis

R, Pen A, Schut T. A controlled comparative study of fluphenazine and fluphenazine enanthate in acute and chronic psychotic patients. Psychiatria, Neurologia, Neurochirurgia 1970;73:165-75.

Van Praag 1973 \{published data only\}

Van Praag HM, Dols LCW. Fluphenazine enanthate and fluphenazine decanoate: A comparison of their duration of action and motor side effects. American Journal of Psychiatry 1973;130(7):801-4.

Walker 1983 \{published data only\}

Walker CA. A double-blind comparative trial of the decanoates of clopenthixol and fluphenazine in the treatment of chronic schizophrenic out-patients. Pharmatherapeutica 1983;3(5):289-93.

Wistedt 1983 \{published data only\}

Wistedt B, Ranta J. Comparative double-blind study of flupenthixol decanoate and fluphenazine decanoate in the treatment of patients relapsing in a schizophrenic symptomatology. Acta Psychiatria Scandinavica 1983;67: 378-88.

Wistedt 1984 \{published data only\}

Wistedt B. A comparative trial of haloperidol decanoate and fluphenazine decanoate in chronic schizophrenic patients. International Clinical Psychopharmacology 1986;1(Suppl 1): $15-23$.

Wistedt B, Persson T, Helbom E. A clinical doubleblind comparison between haloperidol decanoate and fluphenazine decanoate. Current Therapeutic Research 1984; 35(5):804-14.

Woggon 1977 \{published data only\}

Woggon B, Dick P, Fleischhauer HJ, Gmur M, Gruber G, Angst J, et al. Comparison of the effects of pipothiazine palmitate and fluphenazine decanoate. International Pharmacopsychiatry 1977;12(4):193-209.

\section{References to studies excluded from this review}

Abuzzahab 1976a \{published data only\} Abuzzahab FS. Fluphenazine in chronic schizophrenia. Unidentified report 1976:72-3.

Abuzzahab 1976b \{published data only\} Abuzzahab FS Sr, Zimmermann RL. A three-year doubleblind investigation of pimozide versus fluphenazine in chronic schizophrenia. Psychopharmacology Bulletin 1976; 12(2):26-7. MEDLINE: 76152826

Abuzzahab 1977 \{published data only\} Abuzzahab FS Sr. Evaluations of social functioning in a 3-year double-blind investigation of pimozide versus fluphenazine in chronic schizophrenia. Psychopharmacology Bulletin 1977;13:71-3. MEDLINE: 77103340

Abuzzahab 1980 \{published data only\} Abuzzahab FS, Zimmermann RL. Factors determining patient tenure on a 3-year double-blind investigation of pimozide versus fluphenazine $\mathrm{HCl}$. Advances in Biochemical 
Psychopharmacology 1980;24:547-50. MEDLINE: 80262450

Ahlfors 1971 \{published data only\}

Ahlfors UG. A double-blind trial with long-acting neuroleptics. 5th World Congress of Psychiatry; 1971 Nov 28 - Dec 4; Ciudad de Mexico, Mexico. 1971. MEDLINE: 80262450

Ahlfors 1973 \{published data only\}

Ahlfors UG. Controlled clinical evaluation of depot neuroleptics. A double-blind trial with pipotiazine undecylenate and fluphenazine decanoate. Acta Psychiatria Scandinavica 1973;241:95-9.

Altamura 1987 \{published data only\}

Altamura AC, Mauri MC, Guercetti G, Cazullo CL. Fluphenazine decanoate in acute and maintenance therapy of schizophrenia. Progressive Neuro-Psychopharmacological and Biological Psychiatry 1987;11:613-23.

Angst 1975 \{published data only\} Angst VJ, Woggon B. Clinical study on five depot neuroleptics. Comparison of effective profiles of fluphenazine decanoate, fluspirilene, penfluridol, perphenazine enanthate and pipothiazine palmitate [Klinische prufung von funf depotneuroleptika]. Arzneimittel Forschung 1975;25(2):267-70.

Arato 1979 \{published data only\}

Arato M, Erdos A. Experience with depot neuroleptics [Tapasztalataink depo neuroleptikumokkal]. Orvosi Hetilap 1979;not known(17):1015-21. MEDLINE: 79157578

Astrup 1974 \{published data only\}

Astrup G, Grimsgard A, Hebnes K, Kruse-Jensen A, Lid M. A study of flupenthixol decanoate and pipothiazine undecylenate in schizophrenics. Acta Psychiatria Scandinavica 1974;50:481-91.

Balon 1982 \{published data only\}

Balon R, Kabes J, Parezova G. A comparison of the efficacy of fluphenazine decanoate and hydroxyprotepine decanoate in the treatment of schizophrenia (a double-blind crossover study). 1982;82:116-8. Zhurnal Nevropatologii i Psikhiatrii imeni S.S. Korsakova 1982;82(1):116-8.

Bankier 1968 \{published data only\}

Bankier RG, Pettit DE, Bergen B. A comparative study of fluphenazine enanthate and trifluoperazine in chronic schizophrenic patients. Diseases of the Nervous System 1968; 29(1):56-60.

Bao 1991 \{published data only\}

Bao GW. Efficacy and safety of fluphenthixol decanoate in chronic schizophrenia. Chinese Journal of Nervous and Mental Disorders 1991;17(1):49-50.

Barnes 2010 \{published data only\}

Barnes T, Jones P, Dunn G, Hayhurst K, Drake R, Lewis $S$. Large effect of baseline treatment with long acting antipsychotic drugs on randomized treatment outcomes. Schizophrenia Research 2010;117(2-3):380.
Barsa 1965 \{published data only\}

Barsa JA, Saunders JC. A double blind study of fluphenazine enanthate. Diseases of the Nervous System 1965;26:496-8. MEDLINE: 77103340

Bastie 1974 \{published data only\}

Bastie Y. Outcome in 64 chronic psychotics treated with delayed action Moditen or Modecate [Devenir de 64 psychotiques chroniques sous Moditen retard ou Modecate]. Annales Medico Psychologiques 1974;1(4):531-6. MEDLINE: 75003702

Benassi 1968 \{published data only\}

Benassi P, Bertolotti P, De Pietri L. High doses of fluphenazine: clinico-therapeutical trials [Flufenazina as alte dosi: esperienze clinico-terapeutiche]. Rivista Sperimentale di Freniatria e Medicina Legale Delle Alienazioni Mentali 1968;92(6):1803-25. [PUBMED: 4888750]

Berliner 1974 \{published data only\}

Berliner J. Trial at the guidance center of ixelles of a major neuroleptic drug: anatensol decanoate [Eperimentation au niveau du centre de guidance d'lxelles d'un neuroleptique majeur: l'Anatensol decanoate]. Acta Psychiatria Belgique 1974;74(3):305-16. [PUBMED: 4455024]

Bilone 1988 \{published data only\}

Bilone F, Carcereri G, Lamonaca D. Fluphenazine decanoate and haloperidol decanoate - 2 neuroleptics compared in nonacute psychiatric pathology. Long-term crossover trial. II. Tolerability and side effects [Flufenazina decanoato e aloperidolo decanoato: due neurolettici a confronto nella patologia psichiatrica non acuta studio cross-over a lungo termine. II. Tollerrabilita ed effetti collaterali]. Neurologia Psichiatria Scienze Umane 1988;8(Suppl 3):49-59.

Bloch 2004 \{published data only\}

Bloch Y, Levkovitz Y, Atshuler A, Dvoretzki V, Fenning $S$, Ratzoni G. Use of topical application of lidocaineprilocaine cream to reduce injection-site pain of depot antipsychotics. Psychiatric Services 2004;55(8):940-1. MEDLINE: 15292547

Boyer 1987 \{published data only\}

Boyer P, Puech AJ. Determinants for clinical activity of neuroleptic drugs: chemical substances, doses, assessment tools. Psychiatrie and Psychobiologie 1987;2(4):296-305.

Brankovic 1998 \{published data only\}

Brankovic S, Milovanovic S, Damjanovic A, Paunovic VR. No difference between the effects of clozapine and fluphenazine on probabilistic reasoning in paranoid schizophrenia. 21st Congress of the Collegium Internationale Neuro-psychopharmacologicum; 1998 Jul 12-16; Glasgow, Scotland. 1998. MEDLINE: 94212428

Breier 1987 \{published data only\}

Breier A, Wolkowitz OM, Doran AR, Roy A, Boronow J, Hommer DW, et al. Neuroleptic responsivity of negative and positive symptoms in schizophrenia. American Journal of Psychiatry 1987;144:1549-55. MEDLINE: 88074868 
Caranza 1973 \{published data only\}

Caranza J, Vargas L, Gomez J. A double-blind evaluation of sulpiride, a new antipsychotic compound. American Social Clinical Pharmacological Therapeutics 1973;14:132-2.

Carpenter 1992 \{published data only\} Carpenter WT, Buchanan RW, Breier A, Kirkpatrick B, Hanlon T, Levine J, et al. Novel neuroleptic dosage reduction strategies. Schizophrenia Research 1992;6(2):107. MEDLINE: 75116033

Carpenter 1993 \{published data only\} Carpenter WT, Buchanan RW, Breier A. The effect of dosage interval on the efficacy of fluphenazine decanoate in the prevention of relapse. Schizophrenia Research 1993;9: 258-9.

Carpenter 1999 \{published data only\}

Carpenter WT Jr, Buchanan RW, Kirkpatrick B, Lann HD, Breier AF, Summerfelt AT. Comparative effectiveness of fluphenazine decanoate injections every 2 weeks versus every 6 weeks. American Journal of Psychiatry 1999;156(3): 412-8. MEDLINE: 99178642

Casacchia 1989 \{published data only\}

Casacchia M, Michele DIV, Volonte MV, Rossi A. Bromperidol decanoate vs fluphenazine decanoate in schizophrenia. Psychiatry Today: Accomplishments and Promises. Proceedings of the 8th World Congress of Psychiatry; 1989 Oct 13-19; Athens, Greece. 1989. MEDLINE: 94212428

Castellini \{published data only\}

Castellini A, Lorini M. Flufenazina decanoato e aloperidolo decanoato: due neurolettici a confronto nella patologia psichiatrica non acuta studio crossover a lunga termine. not known. not known, not known:33-48.

Chacon 1972 \{published data only\} Chacon C, Harper P, Harvey GF. Work study in the assessment of the effects of phenothiazines in schizophrenia. Comprehensive Psychiatry 1972;13(6):549-54. MEDLINE: 73051431

Chacon 1973 \{published data only\} Chacon C, Harper P. Clinical and work performance variables in phenothiazine therapy of schizophrenia. Acta Psychiatria Scandinavica 1973;49:65-76.

Charalampous 1977 \{published data only\} Charalampous K, Thornby J, Ford BK, Freemesser GF. Penfluridol versus oral fluphenazine in the maintenance treatment of chronic schizophrenics. Current Therapeutic Research 1977;21(2):215-23.

Chien 1974 \{published data only\} Chein CP, Dimascio A, Cole JO. Antiparkinsonian agents and depot phenothiazine. American Journal of Psychiatry 1974;131(1):86-90.

Childers 1964 \{published data only\} Childers RT. Comparison of four regimens in newly admitted female schizophrenics. American Journal of Psychiatry 1964;120:1010-1. MEDLINE: 72165461
Chouinard 1970 \{published data only\}

Chouinard G, Lehmann HE, Ban TA. Pimozide in the treatment of chronic schizophrenic patients. Current Therapeutic Research Clinical and Experimental 1970;12(9): 598-603. MEDLINE: 70285984

Chowdhury 1980 \{published data only\}

Chowdhury MEH, Chacon C. Depot fluphenazine and flupenthixol in the treatment of stabilized schizophrenics. Comprehensive Psychiatry 1980;21(2):135-9.

Clark 1971 \{published data only\} Clark ML, Huber WK, Charalampous KD, Serafetinides EA, Trousdale W, Colmore JP. Drug treatment in newly admitted schizophrenic patients. Archives of General Psychiatry 1971;25(5):404-9. MEDLINE: 72081786

Cohen 1985 \{published data only\} Cohen BM, Waternaux C, Chouinard G, Sommer BR, Jones B. Plasma levels of neuroleptic in patients receiving depot fluphenazine. Journal of Clinical Psychopharmacology 1985;5(6):328-32.

\section{Cole 1967 \{published data only\}}

Cole JO, Schooler NR National Institute of Mental Health Psychopharmacology Research Branch Collaborative Study Group. Differences in clinical effects of three phenothiazines in "acute" schizophrenia. Diseases of the Nervous System 1967;446(6):369-83. MEDLINE: 67248496

Cookson 1991 \{published data only\} Cookson JC. Side effects during long-term treatment with depot antipsychotic medication. Clinical Neuropharmacology 1991;14(Suppl 2):S24-32. MEDLINE: 92089872

Coufal 1981 \{published data only\}

Coufal J, Novotny M. Fluphenazine and flupenthixol decanoate in psychiatric outpatients practice. Activa Nervosa Superior (Praha) 1981;23(4):269-71.

Curry 1979 \{published data only\} Curry SH, Altamura AC, Montgomery S. Unwanted effects of fluphenazine enanthate and decanoate (letter). Lancet 1979;1(8111):331-2. MEDLINE: 79133726

Curson 1985 \{published data only\} Curson DA, Barnes TRE, Bamber RW, Platt SD, Hirsch SR, Duffy JC. Long term depot maintenance of chronic schizophrenic out-patients: The seven year follow-up of the Medical Reseach Council fluphenazine/placebo trial. British Journal of Psychiatry 1985;146:464-80.

Curson 1986 \{published data only\} Curson DA, Hirsch SR, Platt SD, Bamber RW, Barnes TR. Does short term placebo treatment of chronic schizophrenia produce long term harm?. British Medical Journal Clinical Research Edition 1986;293(6549):726-8. MEDLINE: 87027218

De Alarcon 1969 \{published data only\} De Alarcon R, Carney MWP. Severe depressive mood changes following slow-release intramuscular fluphenazine injection. British Medical Journal 1969;3(6 September): 564-7. 
De Buck 1973 \{published data only\}

De Buck RP. Relative safety and efficacy of high and low dose administration of fluphenazine-hcl to psychotic patients. In: Ban TA editor(s). Psychopharmacology Sexual Disorders and Drug Abuse. North Holland, 1973.

Del Giudice 1975 \{published data only\} Del Giudice J, Clark WG, Gocka EF. Prevention of recidivism of schizophrenics treated with fluphenazine enanthate. Psychosomatics 1976;16:32-6.

Dencker 1978 \{published data only\}

Dencker SJ, Johansson R, Lundin L, Malm U. High doses of fluphenazine enanthate in schizophrenia. Acta Psychiatria Scandinavica 1978;57:405-14.

Dencker 1981 \{published data only\}

Dencker SJ, Johansson R, Lundin L. Pharmaco kinetics of fluphenazine depot in megadose: Comparison between the enanthate and the decanoate form. Nordic Psykiatria Tidskkia 1981;35(5-6):365-9.

Dengler 1969 \{published data only\} Dengler K. Contribution to the infusion therapy with fluphenazine in schizophrenics with regard to the control of the neuroleptic threshold [Beitrag zur Infusionstherapie mit Fluphenazin bei schizophrenen im Hinblick auf die Steuerbarkeit der neuroleptischen Schwelle]. Medizinische Welt 1969;16:977-81. MEDLINE: 69204321

DeWolfe 1971 \{published data only\}

DeWolfe AS, Barrell RP, London L, Spaner FE. Prolixin enanthate and thorazine-stelazine regimens in the treatment of schizophrenic patients: An experimental evaluation. Psychosomatics 1971;12:186-90.

Donlon 19761 \{published data only\}

Donlon PT, Rada RT, Arora KK. Depression and the reintegration phase of acute schizophrenia. American Journal of Psychiatry 1976;133:1265-8. MEDLINE: 77042351

Donlon 1977 \{published data only\} Donlon PT, Swaback DO, Osborne ML. Pimozide versus fluphenazine in ambulatory schizophrenics: a 12-month comparison study. Diseases of the Nervous System 1977;38 (2):119-23. MEDLINE: 77115433

Donlon 1978 \{published data only\} Donlon PT, Meadow A, Tupin JP, Wahba M. High vs standard dosage fluphenazine HCL in acute schizophrenia. Journal of Clinical Psychiatry 1978;39(11):800-4. MEDLINE: 79067694

Doongaji 1988 \{published data only\} Doongaji DR, Sheth AS, Apte JS, Desai AB, Gupta L. Penfluridol vs fluphenazine decanaote: A double-blind clinical study in chronic and subchronic schizophrenia. Current Therapeutic Research 1988;43(3):416-22.

Dossenbach 1997 \{published data only\}

* Dossenbach M, Friedel P, Jakovljevic M, Hotujac L, Folnegovic V, Uglesic B, et al. Olanzapine versus fluphenazine - six weeks' treatment of acute schizophrenia. 10th European College of Neuropsychopharmacology
Congress; 1997 Sep 13-17; Vienna, Austria. 1997.

MEDLINE: 21196608

Dossenbach M, Jakovljevic M, Folnegovic F, Uglesic B, Dodig G, Friedel P, et al. Olanzapine versus fluphenazine - 6 weeks treatment of anxiety symptoms during acute schizophrenia. Schizophrenia Research 1998;29(1,2):203.

Downing 1963 \{published data only\}

Downing RW, Ebert JN, Shubrooks SJ. Effect of phenothiazines on the thinking of acute schizophrenics. Perceptual and Motor Skills 1963;17(2):511-20.

Emsley 1999 \{published data only\} Emsley R, Jones AM, Bailey P, Raniwalla J. 'Seroquel' In partially responsive schizophrenics. 11th World Congress of Psychiatry; 1999 Aug 6-11; Hamburg, Germany. 1999: 287.

Engelhardt 1973 \{published data only\}

Engelhardt DM, Polizos P, Waizer J, Hoffman SP. A double blind comparison of fluphenazine and haloperidol in outpatient schizophrenic children. Journal of Autism and Childhood Schizophrenia 1973;3(2):128-37.

Engstrand 1969 \{published data only\}

Engstrand E. Fluphenazine enanthate in long term treatment of psychoses [Flufenazin-enantat i langtidsbehandling av psykoser]. Nordisk Psykiatrisk Tidsskrift 1969;23:401-3. MEDLINE: 77103340

Faltus 1974 \{published data only\}

Faltus F. The depot compounds moditen (fluphenazine) and IMAP (flusipirilene) in clinical practice. Active Nervosa Superior 1974;16(3):167-8.

Faretra 1970 \{published data only\}

Faretra G, Dooher L, Dowling J. Comparison of haloperidol and fluphenazine in disturbed children. American Journal of Psychiatry 1970;126:1670-3. MEDLINE: 70181755

Ferenc 2000 \{published data only\}

Ferenc M, Dossenbach M, Jakovljevic M, Metcalfe S. Predictive value of early anti anxiety effect on the acute antipsychotic outcome: a comparison of fluphenazine and olanzapine. 153rd Annual Meeting of the American Psychiatric Association; 2000 May 13-18; Chicago, USA. 2000.

Filip 1985 \{published data only\}

Filip V, Faltus F, Hanakova S, Janeckova E, Raboch J, Dobiasova A, et al. Oxyprothepin decanoate in maintenance therapy of schizophrenia - a double-blind, standardized controlled study [Oxyprothepin dekanoat v udrzovaci lecbe schizofrenie-dvojite slepa, standardem kontrolovana studie]. Ceskoslovenska Psychiatrie 1985;81(1):6-14. MEDLINE: 85152093

Floru 1974 \{published data only\}

Floru L, Heinrich K, Wittek F. Problem of depressions during long-time neuroleptic treatment of schizophrenia - single blind-study of fluphenazine-decanoate and flupenthixol-decanoate. Journal De Pharmacologie 1974;5: 31. 
Floru 1975 \{published data only\}

Floru L, Heinrich K, Wittek F. The problem of postpsychotic schizophrenic depressions and pharmacological induction. International Pharmacopsychiatria 1975;10(4): 230-9.

Giannelli 1990 \{published data only\}

Giannelli A, Rabboni M, Zarattini F. Clinical profiles of the effects, indications, preferential therapeutic effect and contraindication of three depot neuroleptics compared in a multicentre trial [Profili clinici di azione, indicazioni preferenziali, effetti terapeutici e controindicazioni di tre neurolettici depot in trial multicentrico di confronto]. Rivista di Psichiatria 1990;25(1):7-24.

Gillis 1981 \{published data only\}

Gillis JS, Parkison S. The effects of fluphenazine decanoate injection and chlorpromazine on symptom severity and learning in outpatient schizophrenics. Current Therapeutic Research Clinical and Experimental 1981;29:551-66.

Gitlin 1988 \{published data only\}

Gitlin MJ, Midha KK, Fogelson D, Nuechterlein K. Persistance of fluphenazine in plasma after decanoate withdrawal. Journal of Clinical Psychopharmacology 1988;8 (1):53-6.

Gitlin 2001 \{published data only\}

Gitlin M, Neuchterlein K, Subotnik KL, Ventura J, Mintz J, Fogelson DL, et al. Clinical outcome following neuroleptic discontinuation in patients with remitted recent-onset schizophrenia. American Journal of Psychiatry 2001;158: 1835-42.

Goff 2005 \{published data only\}

Goff DC, Herz L, Posever T, Shih V, Tsai G, Henderson DC, et al. A six-month, placebo-controlled trial of $\mathrm{d}$-cycloserine co-administered with conventional antipsychotics in schizophrenia patients. Psychopharmacology 2005;179(1): 144-50. MEDLINE: 15502972

Goldberg 1967 \{published data only\} Goldberg SC, Mattsson N, Cole JO, Klerman GI. Prediction of improvement in schizophrenia under four phenothiazines. Archives General Psychiatry 1967;16: $107-17$.

Goldberg 1968 \{published data only\} Goldberg SC, Mattsson NB. Schizophrenic subtypes defined by response to drugs and placebo. Diseases of the Nervous System 1968;29(5):153-8. MEDLINE: 68399894

Goldberg 1970 \{published data only\} Goldberg HL, DiMascio A, Chaudhary B. A clinical evaluation of prolixin enanthate. Psychosomatics 1970;11

(3):173-7. MEDLINE: 70229686

Goldberg 1981 \{published data only\} Goldberg SC, Shenoy RS, Sadler A, Hamer R, Ross B. The effects of a drug holiday on relapse and tardive dyskinesia in chronic schizophrenics. Psychopharmacology Bulletin 1981; 17(1):116-7. MEDLINE: 81200009

Gopalakrishnan 2006 \{published data only\} Gopalakrishnan R, Jacob KS, Kuruvilla A, Vasantharaj B, John JK. Sildenafil in the treatment of antipsychotic- induced erectile dysfunction: a randomized, doubleblind, placebo-controlled, flexible-dose, two-way crossover trial. American Journal of Psychiatry 2006;163(3):494-9. MEDLINE: 16513872

Grosser 1970 \{published data only\} Grosser HH. Experience of psychiatric management of schizophrenia with fluphenazine decanoate. Diseases of the Nervous System 1970;31(Supp):32-6.

Haider 1968 \{published data only\}

Haider I. A controlled trial of fluphenazine enanthate in hospitalized chronic schizophrennics. British Journal of Psychiatry 1968;114:837-41.

Hall 1968 \{published data only\}

Hall WB, Vestre ND, Schiele BC, Zimmermann R. A controlled comparison of haloperidol and fluphenazine in chronic treatment-resistant schizophrenics. Diseases of the Nervous System 1968;29(6):405-8. MEDLINE: 68409364

Hamilton 1979 \{published data only\}

Hamilton M, Card IR, Wallis GG, Mahmoud MR. A comparative trial of the decanoates of flupenthixol and fluphenazine. Psychopharmacology 1979;64:225-9.

Hanlon 1965 \{published data only\} Hanlon TE, Michaux MH, Ota KY, Shaffer JW, Kurland AA. The comparative effectiveness of eight phenothiazines. Psychopharmacologia 1965;7(2):89-106. MEDLINE: 66010572

Harper 1976 \{published data only\} Harper P, Chacon C. Work performance versus clinical assessment in the evaluation of phenothiazine therapy. British Journal of Clinical Pharmacology 1976;3:50-5.

Haslam 1975 \{published data only\}

Haslam MT, Bromham BM, Schiff AA. A comparative trial of fluphenazine decanoate and flupenthixol decanoate. Acta Psychiatria Scandinavica 1975;51:92-100.

Held 1970 \{published data only\} Held JM, Cromwell RL, Frank ET Jr, Fann WE. Effect of phenothiazines on reaction time in schizophrenics. Journal of Psychiatric Research 1970;7(3):209-13. MEDLINE: 70166858

Hirsch 1973 \{published data only\}

* Hirsch SR, Gaind R, Rohde PD, Stevens BC, Wing J. Outpatients maintenance of chronic schizophrenic pateints with long-acting fluphenazine: double-blind placebo trial. British Medical Journal 1973;1:633-7.

Hirsch SR, Leff JP, Wing JK. Outpatient maintenance of chronic schizophrenics with long acting fluphenazine. British Medical Journal 1973;17(5868):715-6.

Stevens B. The social value of fluphenazine decanoate. Acta Psychitria Belgique 1976;76:792-803.

Hirsch 1978 \{published data only\} Hirsch SR, Knights A, Okasha MS, Salih MA. Maintenance therapy in out-patient schizophrenics: a report of a doubleblind trial comparison of fluphenazine decanoate and flupenthixol decanoate. British Journal of Psychiatry 1978; 133:371.

Fluphenazine decanoate (depot) and enanthate for schizophrenia (Review)

Copyright @ 2016 The Cochrane Collaboration. Published by John Wiley \& Sons, Ltd. 
Hirsch 1989 \{published data only\}

Hirsch SR, Jolley AG. The dysphoric syndrome in schizophrenia and its implications for relapse. British Journal of Psychiatry 1989;155(5):46-50.

Hogarty 1995 \{published data only\} Hogarty GE, McEvoy JP, Ulrich RF, DiBarry AL, Bartone P, Cooley $S$, et al. Pharmacotherapy of impaired affect in recovering schizophrenic patients. Archives of General Psychiatry 1995;52:29-41.

Holden 1970 \{published data only\} Holden JM, Itil TM, Keskiner A. Assessment and significance of changes in laboratory values with haloperidol and fluphenazine hydrochloride therapy. Biological Psychiatry 1970;2(2):173-82. MEDLINE: 70291696

Holt 1984 \{published data only\}

Holt RJ. Neuroleptic drug-induced changes in platelet levels. Journal of Clinical Psychopharmacology 1984;4(3): 130-2. MEDLINE: 84240338

Hsu 1967 \{published data only\}

Hsu JJ, Nol E, Martinez ML, Lessien B, Paragas PG, Puhac $\mathrm{M}$, et al. One year study of fluphenazine enanthate. Diseases of the Nervous System 1967;28:807-11.

Inderbitzen 1994 \{published data only\} Inderbitzin LB, Lewine RRJ, Scheller-Gilkey G, Swofford $\mathrm{CD}$, Egan GJ, Gloersen BA, et al. A double-blind dosereduction trial of fluphenazine decanoate for chronic, unstable schizophrneic patients. American Journal of Psychiatry 1994;151:1753-9.

Inderbitzin 1993 \{published data only\} Inderbitzin LB, Lewine R, Scheller-Gilkey G. Most effective dose study (meds): a double - blind reduction trial of fluphenazine decanoate. Schizophrenia Research 1993;9: 238-9.

Ionescu 1983 \{published data only\} Ionescu R, Tiberiu C, Miklos R, Angelescu C, Persiceanu AM. Penfluridol in the maintenance therapy of schizophrenia. Neurologie et Psychiatrie 1983;21(1):33-41. MEDLINE: 83197141

Iqbal 1978 \{published data only\} Iqbal MJ, Young MA, Charles J, Elgart B, Von Greiff H, Simpson GM. A long term comparative trial of penfluridol and fluphenazine decanoate in schizophrenic outpatients. Journal of Clinical Psychiatry 1978;39(4):375-7.

Irwin 1986 \{published data only\}

Irwin M, Fuentenebro F, Marder SR Yuwiler A. L-5Hydroxytryptophan-induced delirium. Biological Psychiatry 1986;21(7):673-6. MEDLINE: 86216405

\section{Itil 1970a \{published data only\}}

Itil T, Keskiner A. Fluphenazine hydrochloride, enanthate, and decanoate in the management of chronic psychosis. Diseases of the Nervous System 1970;31(5-9):37-42.

Itil 1970b \{published data only\}

Itil T, Keskiner A, Heinemann L, Han T, Gannon P, Hsu W. Treatment of resistant schizophrenics with extreme high dosage fluphenazine hydrochloride. Psychosomatics 1970;11

(5):456-63. MEDLINE: 71007605

Itil 1971 \{published data only\}

Itil TM, Saletu B, Hsu W, Kiremitci N, Keskiner A. Clinical and quantitative EEG changes at different dosage levels of fluphenazine treatment. Acta Psychiatrica Scandinavica 1971;47(4):440-51. MEDLINE: 72165461

Itil 1978 \{published data only\}

Itil TM, Reisberg B, Patterson C, Amin A, Wadud A, Herrman WM. Pipothiazine palmitate, A long-acting neuroleptic: Clinical and computerized EEG effects. Current Therapeutic Research 1978;24(6):689-707.

Jakovljevic 1999 \{published data only\} Jakovljevic M, Dossenbach MRK. Olanzapine versus fluphenazine in the acute (six-week) treatment of schizophrenia. Psychiatria Danubina 1999;11(1-2):3-11. MEDLINE: 77103340

James 1977 \{published data only\} James NM. Penfluridol: a double blind trial in chronic schizophrenia. New Zealand Medical Journal 1977;85(580): 53-4. MEDLINE: 77147536

Johnson 1975 \{published data only\} Johnson DA, Malik NA. A double-blind comparison of fluphenazine decanoate and flupenthixol decanoate in the treatment of acute schizophrenia. Acta Psychiatria Scandinavica 1975;51:257-67.

Jones 2006 \{published data only\} Jones PB, Barnes TRE, Davies L, Dunn G, Lloyd H, Hayhurst KP, et al. Randomized controlled trial of the effect on quality of life of second- vs first-generation antipsychotic drugs in schizophrenia: cost utility of the latest antipsychotic drugs in schizophrenia Study (CUtLASS 1). Archives of General Psychiatry 2006;63(10):1079-87. MEDLINE: 17015810

Kabes 1980a \{published data only\} Kabes J, Balon R, Papezova H. Maintenance therapy with fluphenazine decanoate and oxyprothepine decanoate in schizophrenic patients - a DOUBLE blind cross-over comparative study. Activitas Nervosa Superior 1980;22(3): 160-2. MEDLINE: 90076019

Kabes 1980b \{published data only\}

Kabes J, Balon R, Hanzlicek L, Skovajsova M, Kozakova H. Clinical trial of oxyprothepin decanoate [Klinicke zkouseni oxyprothepin dekanoatu]. Ceskoslovenska Psychiatrie 1980; 76(1):16-25. MEDLINE: 80200044

Kabes 1981 \{published data only\}

Kabes J. Oxyprothepine decanoate in the maintenance treatment of schizophrenia: multiclinical controlled comparison with fluphenazine decanoate. Activitas Nervosa Superior 1981;23(3):241-3.

Kane 1979 \{published data only\} Kane JM, Rifkin A, Quitkin F, Nayak D, Saraf K, RamosLorenzi JR, et al. Low dose fluphenazine decanoate in maintenance treatment of schizophrenia. Psychiatry Research 1979;1:341-8. 
Kane 1982 \{published data only\}

Kane JM, Rifkin A, Quitkin F, Nayak D, Ramos-Lorenzi $\mathrm{J}$. Fluphenazine vs placebo in patients with remitted, acute first-episode schizophrenia. Archives of General Psychiatry 1982;39:70-3.

Kane 1983 b \{published data only\}

Kane JM. Low dose medication in the treatment of schizophrenia. Schizophrenia Bulletin 1983;9(4):528-31.

Keith 2002 \{published data only\}

Keith SJ. Research experience with a long-acting atypical antipsychotic medications. Proceedings of the 155th Annual Meeting of the American Psychiatric Association; 2002 May 18-23; Philadelphia, Pennsylvania, USA. 2002.

Kelly 1999 \{published data only\}

Kelly D, Conley RR. Sexual side effects of quetiapine and risperidone compared with fluphenazine. Stanley Foundation Research Awards - 1999 Research Award Recipients (http://www.stanleyresearch.org/ accessed February 2001) 1999. MEDLINE: 98271218

Kenway 1971 \{published data only\} Kenway AK, Masheter HC. Pimozide compared with fluphenazine in schizophrenia. British Journal of Clinical Practice 1971;25(2):69-72. MEDLINE: 71157161

Keskiner 1968a \{published data only\} Keskiner A, Simeon J, Fink M, Itil TM. Long acting phenothiazine (fluphenazine decanoate) treatment of psychosis. Archives of General Psychiatry 1968;18:477-81.

Keskiner 1968b \{published data only\}

Keskiner A, Holden JMC, Itil TM. Maintenance treatment of schizophrenic outpatients with a depot phenothiazine. Psychosomatics 1968;9:166-71.

King 1979 \{published data only\} King CE, Goldstein MJ. Therapists rating of achievement of objectives in psychotherapy with acute schizophrenics. Schizophrenia Bulletin 1979;5(1):118-29.

Kinon 1993 \{published data only\}

Kinon BJ, Kane JM, Johns C, Perovich R, Ismi M, Koreen A, et al. Treatment of neuroleptic-resistant schizophrenic relapse. Psychopharmacology Bulletin 1993;29(2):309-14. MEDLINE: 94120060

Kinross-Wright 1963 \{published data only\} Kinross-Wright J, Vogt AH, Charalampous KD. A new method of drug therapy. American Journal of Psychiatry 1963;119:779-80.

\section{Knights 1979 \{published data only\}}

Knights A, Okasha MS, Salih MA, Hirsch SR. Depressive and extrapyramidal symptoms and clinical effects: A trial of fluphenazine versus flupenthixol in maintenance of schizophrenic out-patients. British Journal of Psychiatry 1979;135:515-23.

Kong 1989 \{published data only\}

Kong DSG, Yeo SH. An open clinical trial with the longacting neuroleptics flupenthixol decanoate and fluphenazine decanoate in the maintenance treatment of schizophrenia. Pharmatherapeutica 1989;5:371-9.
Landmark 1994 \{published data only\}

Landmark J, Merskey H, Cernovsky ZZ. Fluphenazine treatment of DSM-III-R Male Schizophrenic Patients Among the Xhosa. Canadian Journal of Psychiatry 1994;39: 219-22.

Lapierre 1975 \{published data only\} Lapierre YD, Lavallee J. Pimozide and the social behavior of schizophrenics. Current Therapeutic Research 1975;18(1): 181-8.

\section{Lapierre 1976 \{published data only\}} Lapierre Y, Lavallee J. A controlled pimozide, fluphenazine and group psychotherapy study of chronic schizophrenics. Psychiatric Journal of the University of Ottawa 1976;1(1-2): 8-13.

Lapierre 1978 \{published data only\} Lapierre YD. A controlled study of penfluridol in the treatment of chronic schizophrenia. American Journal of Psychiatry 1978;135:956-9. MEDLINE: 78208642

Lapierre 1983 \{published data only\} Lapierre YD, von Frenckell R. AMDP psychopathology factors in chronic schizophrenia: a clinical trial of two longacting neuroleptics. Modern Problems of Pharmacopsychiatry 1983;2:193-203. MEDLINE: 84013802

Lasky 1962 \{published data only\} Lasky JJ, Klett CJ, Caffey EM, Bennett JL, Rosenblum MP, Hollister LE. Drug treatment of schizophrenic patients. A comparitive evaluation of chlorpromazine, chloprothixene, fluphenazine, reserpine, thioridazine and triflupromazine. Diseases of the Nervous System 1962;23(12):698-706.

Leff 1971 \{published data only\}

Leff JP, Wing JK. Trial of maintenance therapy in schizophrenia. British Medical Journal 1971;11(12): 599-604.

Leff 1973 \{published data only\}

Leff JP, Hirsch SR, Gaind R, Rohde PD, Stevens BC. Life events and maintenance therapy in schizophrenic therapy. British Journal of Psychiatry 1973;123:659-69.

Levinson 1990 \{published data only\} Levinson DF, Simpson GM, Lo ES, Cooper TB, Singh $\mathrm{H}$, Yadalam K, et al. Fluphenazine plasma levels, dosage, efficacy, and side effects. American Journal of Psychiatry 1995;152(5):765-71. MEDLINE: 95243387

* Levinson DF, Simpson GM, Singh H, Yadalam K. Fluphenazine dose, clinical response, and extrapyramidal symptoms during acute treatment. Archives of General Psychiatry 1990;47(8):761-8. MEDLINE: 90334499 Levinson DF, Singh H, Simpson GM. Timing of acute clinical response to fluphenazine. British Journal of Psychiatry 1992;160:365-71. MEDLINE: 92223990

Lewis 2003 \{published data only\}

* Lewis S, Davies L, Jones P, Barnes T, Murray R, Kerwin $\mathrm{R}$, et al. Randomised controlled trials of conventional antipsychotic versus new atypical drugs, and new atypical drugs versus clozapine, in people with schizophrenia 
responding poorly to, or intolerant of, current drug treatment. Draft final report for NCCHTA 2003. Lewis S, Davies L, Jones P, BarnesT, Murray R, Kerwin $\mathrm{R}$, et al. Randomised controlled trials of conventional antipsychotic versus new atypical drugs, and new atypical drugs versus clozapine, in people with schizophrenia responding poorly to, or intolerant of, current drug treatment. Health Technology Assessment 2006;10(17):iii-iv, ix-xi, 1-165.

Lewis SW, Davies L, Jones PB, Barnes TRE, Murray RM, Kerwin R, et al. Randomised controlled trials of conventional antipsychotic versus new atypical drugs, and new atypical drugs versus clozapine, in people with schizophrenia responding poorly to, or intolerant of, current drug treatment. Health Technology Assessment 2006;10(17): iii-xi, 1-182. MEDLINE: 16707074

Litman 1994 \{published data only\}

Litman RE, Hommer DW, Radant A, Clem T, Pickar D. Quantitative effects of typical and atypical neuroleptics on smooth pursuit eye tracking in schizophrenia. Schizophrenia research 1994;12(2):107-20.

Ljubin 2000 \{published data only\} Ljubin T, Milas DZ, Mimica N, Folnegovic Smalc V, Makaric G. A preliminary study of the comparative effects of olanzapine and fluphenazine on cognition in schizophrenic patients. Human Psychopharmacology 2000;15(7):513-9.

Mahmoud 2004 \{published data only\} Mahmoud RA, Engelhart LM, Janagap CC, Oster G, Ollendorf D. Risperidone versus conventional antipsychotics for schizophrenia and schizoaffective disorder: symptoms, quality of life and resource use under customary clinical care. Clinical Drug Investigation 2004;24 (5):275-86. MEDLINE: 17503889

Marder 1986 \{published data only\}

Marder SR, Hawes EM, van Putten T, Hubbard JW. Fluphenazine plasma levels in patients receiving low and conventional doses of fluphenazine decanoate. Psychopharmacology 1986;88(4):480-3.

Marder 1989 \{published data only\}

Marder SR, Van Putten T, Aravagiri M, Hubbard JW, Hawes EM, McKay G, et al. Plasma levels of parent drug and metabolites in patients recieving oral and depot fluphenazine. Psychopharmacology Bulletin 1989;25(3): 479-82.

Marder 1990a \{published data only\}

Marder SR, Van Putten T, Aravagiri M, Hawes EM, Hubbard JW, McKay G, et al. Fluphenazine plasma levels and clinical response. Psychopharmacology Bulletin 1990;26 (2):256-9.

Marder 1991a \{published data only\}

Marder SR, Midha KK, Van Putten T, Aravagiri M, Hawes EM, Hubbard JW, et al. Plasma levels of fluphenazine in patients receiving fluphenazine decanoate. British Journal of Psychiatry 1991;158:658-65.
Marder 1991b \{published data only\}

Marder SR, Mintz J, Van Putten T, Lebell M, Wirshing WC, Johnston-Cronk K. Early prediction of relapse in schizophrenia: An application of receiver operating characteristic (ROC) methods. Psychopharmalogical Bulletin 1991;27(1):79-82.

Marder 1996 \{published data only\}

Marder SR, Wirshing WC, Mintz J, McKenzie J, Johnston K, Eckman TA, Lebell M, Zimmerman K, Liberman RP. Two-year outcome of social skills training and group psychotherapy for outpatients with schizophrenia. American Journal of Psychiatry 1996;153:1585-92.

Marder 2002 \{published data only\}

Marder SR, Aravagiri M, Wirshing WC, Wirshing DA, Lebell M, Mintz J. Fluphenazine plasma level monitoring for patients receiving fluphenazine decanoate. Schizophrenia Research 2002;53:25-30.

\section{Martenyi 2000 \{published data only\}}

Martenyi F, Dossenbach M, Jakovljevic M, Metcalfe S. Predictive value of early anti anxiety effect on the acute antipsychotic outcome: a comparison of fluphenazine and olanzapine. Schizophrenia Research 2000;41(1):191. MEDLINE: 70166858

\section{Martin 1972 \{published data only\}}

Martin A, Masson JM, Quentin JC, Verrier JP, Jusseaume P. Comparative study of the action of fluphenazine oenanthate and decanoate in chronic psychoses $(73$ cases) [Etude comparative de l'action de l'oenanthate de fluphenazine et du decanoate dans les psychoses chroniques (73 cas]. Annales Medico Psychologiques 1972;2(5):705-8. MEDLINE: 73200093

\section{Mattes 1984 \{published data only\}}

Mattes JA, Nayak D. Lithium versus fluphenazine for prophylaxis in mainly schizophrenic schizo-affectives. Biological Psychiatry 1984;19(3):445-9. MEDLINE: 84203817

\section{McCreadie 1983 \{published data only\}}

McCreadie RG, McKane JP, Mackie M. Weekly pimozide versus fluphenazine decanoate in schizophrenic out and day patients. British Journal of PSychiatry 1983;143:97-8.

McCreadie 1986 \{published data only\} McCreadie RG, McKane JP, Robinson ADT, Wiles DH, Stirling GS. Depot neuroleptics as maintenance therapy in chronic schizophrenic in-patients. International Clinical Psychopharmacology 1986;1(Suppl 1):13-4. MEDLINE: 83284029

\section{Meco 1987 \{published data only\}}

Meco G, Aniello R, Lestingi L, Petrini P, Castellano A, Casacchia M. Haloperidol decanoate (a new depot neuroleptic drug): double-blind study versus fluphenazine decanoate in schizophreniform disorders and chronic schizophrenia. In: Cazullo CL, Invernizzi G, Bressi C, Ghedini E editor(s). Schizophrenia: an Intergrative View. Milan: John Libbey, 1987:155-70. 
Mimica 1998 \{published data only\}

Mimica N, Dossenbach M, Friedel P, Folnegovic-Smalc V, Makaric G, Jakovlijevic M, et al. Olanzapine compared to fluphenazine in the treatment of schizophrenia. Schizophrenia Research 1998;29(1,2):150. MEDLINE: 84013802

Montejo 2010 \{published data only\}

Montejo AL, Majadas S, Rico-Villademoros F, Llorca G, De La Gándara J, Franco M, et al. Frequency of sexual dysfunction in patients with a psychotic disorder receiving antipsychotics. Journal of Sexual Medicine 2010;7(10): 3404-13.

Morris 1970 \{published data only\}

Morris PA, MacKenzie DH, Masheter HC. A comparative double blind trial of pimozide and fluphenazine in chronic schizophrenia. British Journal of Psychiatry 1970;117: 683-4. MEDLINE: 71077810

National 1964 \{published data only\}

National Institute of Mental Health Psychopharmacology Service Center Collaborative Study Group. Phenothiazine treatment in acute schizophrenia. Archives of General Psychiatry 1964;10:246-61. MEDLINE: 68399894

Nestoros 1978 \{published data only\}

Nestoros JN, Lehmann HE, Ban TA. Butaclamol in the treatment of schizophrenia. A standard-controlled clinical trial. International Pharmacopsychiatry 1978;13(3):138-50. MEDLINE: 78241394

Owen 1993 \{published data only\}

Owen RR, Gutierrez ER, Hsiao J, Hadd K. Effects of clozapine and fluphenazine treatment on responses to $\mathrm{m}$ chlorophenylpiperazine infusions in schizophrenia. Archives of General Psychiatry 1993;50(8):636-44. MEDLINE: 93343730

Palma 1997 \{published data only\}

Palma Wenzel MI, Parada R, Osorio C, Dorr A, Bauer S. Flupentixol decanoate versus other neuroleptics in chronic schizophrenia [Decanoato de flupentixol versus otros neurolepticos en esquizofrenicos cronicos]. Revista Chilena de Neuropsiquiatria 1997;35(1):29-35. MEDLINE: 97353440

Pichot 1988 \{published data only\}

Pichot P, Boyer P. A double blind, controlled, multicenter trial of low dose amisulpride (Solian(R) 50) versus low dose fluphenazine in the treatment of negative symptoms in chronic schizophrenia [Essai multicentrique controle, en double insu, amisulpride (solian(r) 50) contre fluphenazine a faibles doses dans le traitement du syndrome deficitaire des schizophrenies chroniques]. Annales de Psychiatrie 1988; 3(3):312-20.

Pickar 1987 \{published data only\}

Pickar D, Breier A, Wolkowitz OM, Pato C. Profiles of the pharmacologic response of positive and negative symptoms in schizophrenia. Psychiatrie and Psychobiologie 1987;2(4): 277-87.
Pickar 1992 \{published data only\}

Pickar D, Owen RR, Litman RE, Konicki E, Gutierrez R, Rapaport $\mathrm{MH}$. Clinical and biologic response to clozapine in patients with schizophrenia. Crossover comparison with fluphenazine. Archives of General Psychiatry 1992;49(5): 345-53. MEDLINE: 92264872

Pickar 1994 \{published data only\}

Pickar D, Litman RE, Hong WW, Su TP, Weissman EM. Clinical response to clozapine in patients with schizophrenia. Archives of General Psychiatry 1994;51: 159-60.

Pollack 1964 \{published data only\} Pollack SL, Tourlentes TT, Zocchi AF. Clinical trial of fluphenazine enanthate- A long-acting injectable tranquilizer. American Jornal of Psychiatry 1964;121:73-4.

Preussler 1995 \{published data only\} Preussler B, Bohle C, Jeschke G, Volz H P, Sauer H. Psychometric performance of clozapine and fluphenazine treated schizophrenics. Pharmacopsychiatry 1995;28:204. MEDLINE: 20326685

Preussler 1997 \{published data only\} Preussler B, Hubner G, Rossger G, Jeschke G, Lorenz $S$, Volz HP, et al. Psychometric performance of chronic schizophrenics treated with a typical neuroleptic (fluphenazine) or an atypical neuroleptic drug (clozapine) a double-blind controlled clinical trial. Pharmacopsychiatry 1997;30:207. MEDLINE: 20326685

Quitkin 1975 \{published data only\}

Quitkin F, Rifkin A, Klein DF. Very high dosage vs standard dosage fluphenazine in schizophrenia. A double-blind study of nonchronic treatment-refractory patients. Archives of General Psychiatry 1975;32(10):1276-81. MEDLINE: 76038904

Quitkin 1977 \{published data only\} Quitkin F, Rifkin A, Klein DF. A one year doubleblind comparison of long-acting oral (penfluridol) versus long-acting injectable (fluphenazine decanoate) antipsychotic drugs in multiple episode schizophrenics. Psychopharmacological Bulletin 1977;12:27-9.

\section{Ravaris 1965 \{published data only\}} Ravaris CL, Weaver LA, Brooks GW. A controlled study of fluphenazine enanthate in chronic schizophrenic patients. Diseases of the Nervous System 1965;25:33-9.

Ravaris 1967 \{published data only\}

Ravaris CL, Weaver LA, Brooks GW. Further studies with fluphenazine enanthate: II. Relapse rate in patients deprived of medication. Americal Journal of Psychology 1967;124: 248-9.

Rifkin 1976 \{published data only\} Rifkin A, Quitkin F, Rabiner CJ, Klein DF. Comparison of fluphenazine decanoate, oral fluphenazine, and placebo in remitted outpatient schizophrenics. Psychopharmacology Bulletin 1976;12:24-6.

Roose 1982 \{published data only\}

Roose K. Haloperidol decanoate as a replacement therapy with intramuscular fluphenazine decanoate in schizophrenia 
and other chronic psychoses. Acta Psychiatria Belgique 1982; 82:216-23.

Rossger 1997 \{published data only\}

Rossger G, Preussler B, Rauch J, Kunze M, Lorenz S, Harting J, et al. Neuropsychological test performance of chronic schizophrenics treated with clozapine or fluphenazine - a double-blind, controlled clinical trial. Pharmacopsychiatry 1997;30:212. MEDLINE: 20326685

Saxena 1996 \{published data only\}

Saxena B. The value of depot neuroleptic injections in the treatment of chronic schizophrenia. Conference: schizophrenia 1996: Breaking down the barriers, 4th International Conference, Vancouver, B.C. Breaking down the barrires, 4th International Conference, Vancouver, B.C., Canada October 6-9, 1996. 1996.

Schausberger 1999 \{published data only\}

Schausberger B, Dossenbach M, Hotujac L, FolnegovicSmalc V, Uglesic B, Jakovljevic M. Impact of olanzapine versus fluphenazine on patient's drug acceptance during acute treatment of schizophrenia. Journal of the European College of Neuropsychopharmacology 1999;9:S292.

* Schausberger B, Folnegovic-Smalc V, Hotujac L, Uglesic

B, Jakovljevic M, Dossenbach M. Impact of olanzapine vs. Fluphenazine on patient's drug acceptance during acute treatment of schizophrenia. 11th World Congress of Psychiatry; 1999 Aug 6-11; Hamburg, Germany. 1999.

Schipper 1971 \{published data only\}

Schipper JA. Depot neuroleptics in the treatment of schizophrenia [Depot-neuroleptica bij de behandeling van schizofrenie]. Nederlands Tijdschrift Voor Geneeskunde 1971; 115(16):707-9. MEDLINE: 71156843

Schooler 1971 \{published data only\}

Schooler NR, Boothe H, Goldberg SC. Life history and symptoms in schizophrenia. Severity at hospitalization and response to phenothiazines. Archives of General Psychiatry 1971;25(2):138-47. MEDLINE: 71286095

Schooler 1977 \{published data only\}

Schooler NR, Levine J. Dosage and side effect comparisons between oral and depot fluphenazine. Psychopharmacology Bulletin 1977;13:29-31.

Schubert 1988 \{published data only\}

Schubert H, Zangerl K, Wibmer M. On minus symptoms in chronic schizophrenic patients and their reactions to neuroleptic drugs [Zur Minussymptomatik bei chronisch schizophrenen Patienten und deren Ansprechen auf Neuroleptika]. In: Bender W, Dencker SJ, Kulhanek F editor(s). Schizophrene Erkrankungen. Therapie Therapieresistenz eine Standortbestimmung. Vieweg, 1988: 50-9.

Shafti 2009 \{published data only\}

Shafti SS. Augmentation of olanzapine by fluphenazine decanoate in poorly responsive schizophrenia. Clinical Schizophrenia and Related Psychoses 2009;3(2):97-102.

Simpson 1970 \{published data only\}

Simpson GM, Krakov L, Mattke D, St Phard G. A controlled comparison of the treatment of schizophrenic patients when treated according to the neurologic threshold or by clinical judgment. Acta Psychiatrica Scandinavica Supplementum 1970;212:38-43. MEDLINE: 70291112

Siris 1990 \{published data only\}

Siris SG, Bermanzohn PC, Gonzalez A, Mason SE, White $\mathrm{CV}$, Shuwall MA. The use of antidepressants for negative symptoms in a subset of schizophrenic-patients. Proceedings of the 29th Annual Meeting of the American College of Neuropsychopharmacology. San Juan: Us Government Printing Office, 1990:331-5.

Siris 1991 \{published data only\}

Siris SG, Bermanzohn PC, Mason SE, Shuwall MA. Maintenance imipramine therapy for secondary depression in schizophrenia - a controlled trial. Proceedings of the 30th Annual Meeting of the American College of Neuropsychopharmacology; 1991 Dec 10. San Juan: American Medical Association, 1991:109-15.

Steingard 1994 \{published data only\}

Steingard S, Allen M, Schooler NR. A study of the pharmacologic treatment of medication-compliant schizophrenics who relapse. Journal of Clinical Psychiatry 1994;55(11):470-2. MEDLINE: 95081045

\section{Stevens 1973 \{published data only\}}

Stevens BC. Role of fluphenazine decanaote in lessening the burden of chronic schizophrenics on the community. Psychological Medicine 1973;3:141-58.

Tegeler 1985 \{published data only\}

Tegeler J. A comparative trial of CIS(Z)-clopenthixol decanoate and fluphenazine decanoate. Pharmacopsychiatria 1985;18:78-9.

Tetreault 1969 \{published data only\}

Tretreault L, Bordeleau JM, Albert JM, Rajotte P. Comparative study of fluphenazine ethanate, fluphenazine bichlorhydrate and placebos in chronic schizophrenics [Etude comparative de l'enanthate de fluphenazine du bichlorhydrate de fluphenazine et du placebo chez le schizophrene chronique]. Canadian Psychiatric Associative Journal 1969;14(2):191-8. [PUBMED: 4891134]

Tran 1998 \{published data only\}

Tran PV, Jakovljevic M, Tollefson GD, Crawford AM, Dossenbach M, Friedel P, et al. Olanzapine versus fluphenazine: treatment of acute schizophrenic symptomatology and anxiety. Biological Psychiatry 1998;43: 109S. MEDLINE: 77103340

Tran PV, Tollefson GD, Crawford AM, Dossenbach M, Friedel P, Folnegovic V, et al. Olanzapine versus fluphenazine in schizophrenia. 151st Annual Meeting of the American Psychiatric Association; 1998 May 30-Jun 4; Toronto, Canada. 1998.

Tsai 2004 \{published data only\} Tsai G, Lane HY, Yang P, Chong MY, Lange N. Glycine transporter I inhibitor, N-Methylglycine (sarcosine), added to antipsychotics for the treatment of schizophrenia. Biological Psychiatry 2004;55(5):452-6. MEDLINE: 15023571

Fluphenazine decanoate (depot) and enanthate for schizophrenia (Review)

Copyright $\odot 2016$ The Cochrane Collaboration. Published by John Wiley \& Sons, Ltd. 
Tsai 2006 \{published data only\}

Tsai GE, Yang P, Chang Y-C, Chong M-Y. D-alanine added to antipsychotics for the treatment of schizophrenia. Biological Psychiatry 2006;59(3):230-4. MEDLINE: 16154544

Turner 1966 \{published data only\}

Turner P. A comparison of fluphenazine and chlorpromazine on critical flicker fusion frequency. Journal of Pharmacy and Pharmacology 1966;18:836. MEDLINE: 67163670

Turner 2004 \{published data only\}

Turner M, Eerdekens E, Jacko M, Eerdekens M. Longacting injectable risperidone: safety and efficacy in stable patients switched from conventional depot antipsychotics. International Clinical Psychopharmacology 2004;19(4): 241-9.

Ushakov 1990 \{published data only\} Ushakov IuV, Kravchenko NE, Kopeiko GI, Kalugina LI, Mirzoian MG. Neurophysiological dynamics in the treatment of endogenous depression using reflexotherapy [[data not available]]. Zhurnal Nevropatologii i Psikhiatrii imeni S.S. Korsakova 1990;90(7):99-104. MEDLINE: 91075905

van Putten 1986 \{published data only\} van Putten T, Marder SR. Low-dose treatment strategies. Journal of Clinical Psychiatry 1986;47(Suppl):12-6. MEDLINE: 86195892

van Putten 1991 \{published data only\} van Putten T, Aravagiri M, Marder SR, Wirshing WC, Mintz J, Chabert N. Plasma fluphenazine levels and clinical response in newly admitted schizophrenic patients. Psychopharmacology Bulletin 1991;27(2):91-6. MEDLINE: 92021361

Verster 1998 \{published data only\} Verster GC, Joubert G, Stevens M, van-der-Merwe H. Generic substitution: comparing the clinical efficacy of a generic substitute for fluphenazine decanoate with the original product. South African Medical Journal 1998;88(3): 260-2. MEDLINE: 98271218

Vestre 1962 \{published data only\}

Vestre ND, Hall WB, Schiele BC. A comparison of fluphenazine, triflupromazine, and phenobarbital in the treatment of chronic schizophrenic patients: a doubleblind controlled study. Journal of Clinical and Experimental Psychopathology 1962;23:149-59. MEDLINE: 77103340

Viala 1988 \{published data only\}

Viala A, Durand A, Gouezo F, Hou N, Jorgensen A. Comparative study of the pharmacokinetics of zuclopenthixol decanoate and fluphenazine decanoate. Psychopharmacology 1988;94:293-7.

Villeneuve 1970 \{published data only\} Villeneuve A, Dogan K, Lachance R, Proulx C. A controlled study of fluspirilene in chronic schizophrenia. Current Therapeutic Research Clinical and Experimental 1970;12 (12):819-27. MEDLINE: 71159104
Vinar 1970 \{published data only\}

Vinar O, Taussigova D, Bastecky J, Boleloucky Z. Long acting peroral fluphenazine and its dosage in psychoses. Activitas Nervosa Superior 1970;12(3):248-9. MEDLINE: 70287097

Weiden 1993 \{published data only\} Weiden P, Schooler NR, Severe JB, Lee HJ, Schulz SC. Stabilization and depot neuroleptic dosages. Psychopharmacology Bulletin 1993;29:269-75.

Wiles 1990 \{published data only\} Wiles DM, McCreadie RG, Whitehead A. Pharmacokinetics of haloperidol and fluphenazine decanoates in chronic schizophrenia. Psychopharmacology 1990;101:274-81.

Winter 1973 \{published data only\}

Winter K, Fullerton AG, Hussain K, Tarlo L. A comparative double-blind trial of fluspirilene and fluphenazine decanoate in the treatment of chronic schizophrenia. British Journal of Clinical Practice 1973;27(10):377-80.

Wistedt 1981 \{published data only\}

* Wistedt B. A controlled study of the clinical effects of the withdrawal of depot fluphenazine decanoate and depot flupenthixol decanoate in chronic schizophrenic patients. Acta Psychiatria Scandinavica 1981;64:65-84.

Wistedt B, Jorgensen A, Wiles D. A depot withdrawal study. Palsma concentration of fluphenazine and flupenthixol and relapse frequency. Psychopharmacology 1982;78:301-4.

Wistedt 1983a \{published data only\} Wistedt B, Palmstierna T. Depressive symptoms in chronic schizophrenic patients after withdrawal of long-acting neuroleptics. Journal of Clinical Psychiatry 1983;44:369-71.

Wistedt 1983b \{published data only\} Wistedt B, Wiles D, Jorgensen A. A depot neuroleptic withdrawal study neurological effects. Psychopharmacology 1983;80(2):101-5. MEDLINE: 93126518

Zapletalek 1981 \{published data only\} Zapletalek M, Zbytovsky J, Preiningerova O, Kindernayova $\mathrm{H}$. Maintenance treatment of schizophrenia with oxyprothepine decanoate: double-blind comparison with standard. Activis Nervosa Superior (Praha) 1981;23(3): 243-4.

\section{References to studies awaiting assessment}

Angst 1973 \{published data only\}

Angst J, Frei M, Scharfetter C. The depot neuroleptic agent fluspirilene. Pharmakopsychiatrie und Neuropsychopharmakologie 1973;6:13-28.

del Giudice 1970 \{published data only\} del Giudice J, Okun R, Clark WG. Recidivism with long acting fluphenazine. VII meeting of the International College of Neuropsychopharmacology. 1970. MEDLINE: 21196608

Jue 1996 \{published data only\} Jue FY, Wang CZ, Yue XC, Fang YR, Xue HD, Chen SX. Flupentixol vs fluphenazine in schizophrenia patients: 
a randomized controlled trial. New Drugs and Clinical

Remedies 1996;15(1):19-22.

Kabes 1984 \{published data only\}

Kabes J, Filip V, Sikora J. A multiclinical study of the effectiveness of meclopin in maintenance therapy of schizophrenic patients - A DOUBLE-blind crossover comparative study with flufenazine decanoate. Farmakoterapeuticke Zpravy 1984;30(2):159-81.

Ravanic 1996 \{published data only\}

Ravanic DB, Djukic-Dejanovic SM, Stojiljkovic M, Jankovic S, Paunovic VR, Bankovic D. Antipsychotic efficacy of clozapine vs fluphenazine in positive and negative schizophrenia syndrome. Journal of Neural Transmission 1996;103:XLVI. MEDLINE: 20445745

\section{Ushakov 1990a \{published data only\}}

Ushakov IV, Kravchenko NE, Kopeiko GI, Kalugina LI, Mirzoian MG. Clinical and economic approach to evaluation of the effectiveness of moditen-depot and haloperidol decanoate in the maintenance therapy of attacklike schizophrenia [Klinicheskii ekonomicheskii podkhod $\mathrm{k}$ otsenke sravnitel'noi effektivnosti moditena-depo i galoperidola dekanoata $\mathrm{v}$ podderzhi- vaiushchei terapii u bol'nykh pristupoobraznoi shizofreniei.]. Zhurnal Nevropatologii i Psikhiatrii Imeni S. S. Korsakova 1990;90 (7):99-104. MEDLINE: 91075905

\section{Additional references}

\section{Aaes-Jorgenson 1985}

Aaes-Jorgenson A. Pharmacokinetics of oral and depot neuroleptics-Clinical relevance. Symposium Espo, 1985 Feb 1.

\section{Alderson 2004}

Alderson P, Green S, Higgins JPT. Cochrane Reviewers' Handbook 4.2.2 [updated December 2003]. The Cochrane Library. Chichester, UK: John Wiley \& Sons, Ltd, 2004, issue 1.

\section{Altman 1996}

Altman DG, Bland JM. Statistics Notes: Detecting skewness from summary information. BMJ 1996;313:1200.

\section{Andreasen 1983}

Andreasen NC. The Scale for the Assessment of Negative Symptoms (SANS). Iowa City, Iowa: Univ. of Iowa, 1983.

Andreasen 1984

Andreasen NC. The Scale for the Assessment of Positive Symptoms (SAPS). Iowa City, Iowa: Univ. of Iowa, 1984.

\section{Asberg 1978}

Asberg M, Montgomery SA, Perris C, Schalling D, Sedavll GA. A comprehensive psychopathological rating scale. Acta Psychiatria Scandinavca 1978;Supp 271:5-27.

\section{Barnes 1994}

Barnes TRE, Curson DA. Long term depot antipsychotics. A risk benefit assessment. Drug Safety 1994;10(6):464-79.

\section{Bland 1997}

Bland JM. Statistics notes. Trials randomised in clusters. BMJ 1997;315:600.

\section{Boissel 1999}

Boissel JP, Cucherat M, Li W, Chatellier G, Gueyffier F, Buyse $\mathrm{M}$, et al. The problem of therapeutic efficacy indices. 3. Comparison of the indices and their use [Apercu sur la problematique des indices d'efficacite therapeutique, 3: comparaison des indices et utilisation. Groupe d'Etude des Indices D'efficacite]. Therapie 1999;54(4):405-11. [PUBMED: 10667106]

Cassady 1997

Cassady SL, Thacker GK, Summerfelt A, Tamminga CA. The Maryland Psychiatric Research Center scale and the characterization of involuntary movements. Psychiatry Research 1997;70(1):21-37.

\section{Chouinard 1980}

Chouinard G, Ross-Chouinard A, Annable L. Extrapyramidal symptom rating scale. Canadian Journal of Neurological Science 1980;7:233.

\section{David 1994}

David AS, Cutting JC (Eds.). The Neuropsychology of Schizophrenia. Hove, E. Sussck: Lawrence Erlbaum Assoc, 1994.

\section{Davis 1986}

Davis JM, Andriukatis S. The natural course of schizophrenia and effective maintenance drug therapy. Journal of Clinical Psychopharmacology 1986;6(1Suppl): 2S-10S.

\section{De Alarcon 1969a}

De Alarcon R, Carney MW. Severe depressive mood changes following slow-release intramuscular fluphenazine injection. British Medical Journal 1969;3(670):564-7.

\section{Deeks 2000}

Deeks J. Issues in the selection for meta-analyses of binary data. Proceedings of the 8 th International Cochrane Colloquium; 2000 Oct 25-28; Cape Town. Cape Town: The Cochrane Collaboration, 2000.

\section{Dencker 1980}

Dencker SJ, Lepp M, Malm U. Do schizophrenics well adapted in the community need neuroleptics? A depot neuroleptic withdrawal study. Acta Psychiatria Scandinavica 1980;61(supp 279):64-76.

\section{Derogatis 1977}

Derogatis LR. SCL-90-R: Administration, procedures and scoring manual, for the revised version. Clinical Psychometrics Reasearch Unit, John Hopkins University, Baltimore 1977.

\section{Divine 1992}

Divine GW, Brown JT, Frazier LM. The unit of analysis error in studies about physicians' patient care behavior. Journal of General Internal Medicine 1992;7(6):623-9.

\section{DoH 1996}

Department of Health. Burdens of Disease: a Discussion Document. Department of Health. NHS Executive 1996.

\section{Donner 2002}

Donner A, Klar N. Issues in the meta-analysis of cluster randomized trials. Statistics in Medicine 2002;21:2971-80. 
Egger 1997

Egger M, Davey-Smith G, Schneider M, Minder C. Bias in meta-analysis detected by a simple, graphical test. $B M J$ 1997;315:629-34.

Elbourne 2002

Elbourne D, Altman DG, Higgins JPT, Curtina F, Worthingtond HV, Vaile A. Meta-analyses involving crossover trials: methodological issues. International Journal of Epidemiology 2002;31(1):140-9.

\section{Endicott 1976}

Endicott J, Spitzer RL, Fleiss JL, Cohen J. The Global Assessment Scale: A procedure for measuring overall severity of psychiatric disturbance. Archives of General Psychiatry 1976;33(6):766-71.

\section{Feighner 1972}

Feighner JP, Robins E, Guze S, Woodruff RA, Winokur G, Munoz R. Diagnostic criteria for use in psychiatric research. Archives of General Psychiatry 1972;26:57-62.

\section{Furukawa 2006}

Furukawa TA, Barbui C, Cipriani A, Brambilla P, Watanabe $\mathrm{N}$. Imputing missing standard deviations in meta-analyses can provide accurate results. Journal of Clinical Epidemiology 2006;59(7):7-10.

\section{Gulliford 1999}

Gulliford MC. Components of variance and intraclass correlations for the design of community-based surveys and intervention studies: data from the Health Survey for England 1994. American Journal of Epidemiology 1999;149: $876-83$.

\section{Guy 1976}

Guy W. Clinincal Global Impression (CGI). In: Early clinical drug evaluation (ECDUE) assessment manual for psychopharmacology. Washington DC: National Institute of Mental Health, 1976.

\section{Haynes 1979}

Haynes RB, Taylor WD, Sackett DL (eds). Compliance in health care. Baltimore: John Hopkins University Press, 1979.

\section{Heinrich 1984}

Heinrich DW, Hanlon TE, Carpenter WT. The quality of life scale: an instrument for rating the schizophrenic deficit syndrome. Schizophrenia Bulletin 1984;10(3):388-98.

\section{Higgins 2003}

Higgins JPT, Thompson SG, Deeks JJ, Altman DG. Measuring inconsistency in meta-analyses. BMJ 2003;327: 557-60.

\section{Higgins 2011}

Higgins JPT, Green S (editors). Cochrane Handbook for Systematic Reviews of Interventions Version 5.0.2 [updated September 2011]. The Cochrane Collaboration, 2011. Available from www.cochrane-handbook.org..

Hirsch 1973b

Hirsch SR, Gaind R, Rohde PD. Outpatient maintenance of chronic schizophrenic patients with long-acting fluphenazine: double-blind placebo controlled trial. British Medical Journal 1973;1:633-7.

\section{Honigfeld 1962}

Honigfeld G, Gillis RD, Klett CJ. NOSIE-30: A treatment sensitive ward behavior scale. Psychological Reports 1962;10: 799-812.

\section{Hutton 2009}

Hutton JL. Number needed to treat and number needed to harm are not the best way to report and assess the results of randomised clinical trials. British Journal of Haematology 2009;146(1):27-30.

\section{Jablensky 1992}

Jablensky A, Sartorius N, Ernberg G, Anker M, Korten A, Cooper J, et al.A World Health Organization ten-country study. Schizophrenia: manifestations, incidence and course indifferent cultures. Psychological Medicine Monograph Supplement 1992;20:1-97.

\section{Kane 1986}

Kane JM, Woerner M, Sarantakos S. Depot neuroleptics: A comparison review of standard, intermediate and low-dose regimens. Journal of Clinical Psychiatry 1986;47(suppl 5): $30-3$.

Kane 1998

Kane JM, Aguglia E, Carlo Altamura A, Guiterrez JLA, Brunello N, Fleischhacker WW, et al. Guidelines for depot antipsychotic treatment in schizophrenia. European Neuropschopharmacology 1998;8:55-66.

Kay 1986

Kay SR, Opler LA, Fiszbein A. Positive and Negative Syndrome Scale (PANSS) Manual. North Tonawanda, NY: Multi-Health Systems, 1986.

\section{Krawiecka 1977}

Krawiecka M, Goldberg D, Vaughan M. A standardised psychiatric assessment scale for rating psychotic patients. Acta Psychiatria Scandinavica 1977;55:299-308.

\section{Kurland 1970}

Kurland AA, Dim BM, Olssen JE. The effectiveness of parenteral administration of fluphenazine decanoate in the treatment of chronic schizophrenics. Diseases of the Nervous System 1970;31(Supp9):18-23.

\section{Leucht 2005}

Leucht S, Kane JM, Kissling W, Hamann J, Etschel E, Engel R. Clinical implications of Brief Psychiatric Rating Scale scores. British Journal of Psychiatry 2005;187:366-71. [PUBMED: 16199797]

\section{Leucht 2005a}

Leucht S, Kane JM, Kissling W, Hamann J, Etschel E, Engel RR. What does the PANSS mean?. Schizophrenia Research 2005;79(2-3):231-8. [PUBMED: 15982856]

\section{Lingjaerde 1987}

Lingjaerde O, Ahlfors UG, Bech P, Dencker SJ, Elgen K. The UKU side effect rating scale. A new comprehensive rating scale for psychotropic drugs and a cross-sectional study of side effects in neuroleptic-treated patients. Acta Psychiatrla Scandinavica Supplement 1987;334:1-100. 


\section{Marder 1984}

Marder SR, Van Putten T, Mintz J, McKenzie J, Lebell M, Faltico G, et al. Costs and benefits of two doses of fluphenazine. Archives of General Psychiatry 1984;41: 1025-9.

\section{Marder 1990}

Marder SR, Van Putten T, Aravagiri M. Fluphenazine plasma levels and clinical response. Psychopharmacology Bulletin 1990;26:256-8.

\section{Marshall 1998}

Marshall. Personal communication. Email 1998.

\section{Marshall 2000}

Marshall M, Lockwood A, Bradley C, Adams CE, Joy C, Fenton M. Unpublished rating scales: a major source of bias in randomised controlled trials of treatments for schizophrenia. British Journal of Psychiatry 2000;176: $249-52$.

\section{Moher 2001}

Moher D, Schulz KF, Altman D. The CONSORT statement: revised recommendations for improving the quality of reports of parallel-group randomized trials. JAMA 2001;285:1987-91.

\section{Overall 1962}

Overall JE, Gorham DR. The Brief Psychiatric Rating Scale. Psychological Reports 1962;10:799-812.

\section{Schünemann 2008}

Schünemann HJ, Oxman AD, Vist GE, Higgins JPT, Deeks JJ, Glasziou P, et al. Chapter 12: Interpreting results and drawing conclusions. In: Higgins JPT, Green S editor(s). Cochrane Handbook for Systematic Reviews of Interventions. The Cochrane Collaboration, 2008:359-83.

\section{Simpson 1970b}

Simpson GM, Angus JWS. A rating scale for extrapyramidal side effects. Acta Psychiatria Scandinavica 1970;212:s11-9.

Ukoumunne 1999

Ukoumunne OC, Gulliford MC, Chinn S, Sterne JAC, Burney PGJ. Methods for evaluating area-wide and organistation-based intervention in health and health care: a systematic review. Health Technology Assessment 1999;3(5): $1-75$.

\section{Xia 2009}

Xia J, Adams CE, Bhagat N, Bhagat V, Bhoopathi P, ElSayeh $\mathrm{H}$, et al. Loss to outcomes stakeholder survey: the LOSS study. Psychiatric Bulletin 2009;33(7):254-7.

\section{References to other published versions of this review}

\section{David 2004}

David A, Adams CE, Eisenbruch M, Quraishi SN, Rathbone J. Depot fluphenazine decanoate and enanthate for schizophrenia. Cochrane Database of Systematic Reviews 2004, Issue 2. [DOI: 10.1002/14651858.CD000307]

* Indicates the major publication for the study 


\section{CHARACTERISTICS OF STUDIES}

\section{Characteristics of included studies [ordered by study ID]}

\section{Adamson 1973}

$\begin{array}{ll}\text { Methods } & \text { Allocation: randomised. } \\ & \text { Blindness: double. } \\ & \text { Duration: } 28 \text { days. } \\ \text { Design: cross-over } \mathrm{x} 2 . \\ \text { Country: UK. }\end{array}$

\begin{tabular}{ll}
\hline Participants & Diagnosis: schizophrenia. \\
& N=37 (in phase II). \\
Age: $24-65$ years. \\
Sex: $22 \mathrm{M}, 15 \mathrm{~F}$. \\
History: all in hospital for $>1$ year. \\
Setting: hospital. \\
\hline
\end{tabular}

Interventions $\quad$ 1. Fluphenazine decanoate: dose $12.5 \mathrm{mg} / \mathrm{IM}$ day one, $25 \mathrm{mg} / \mathrm{IM}$ day $7 . \mathrm{N}=19$.

2. Chloropromazine: dose $50-100 \mathrm{mg} / \mathrm{bid} . \mathrm{N}=18$.

\begin{tabular}{|c|c|c|}
\hline Outcomes & \multicolumn{2}{|c|}{$\begin{array}{l}\text { Behaviour: leaving the study early. Adverse effects: various side effects } \\
\text { Unable to use - } \\
\text { Mental state: BPRS (no data). } \\
\text { Behaviour: WWBRS (no data). }\end{array}$} \\
\hline Notes & \multicolumn{2}{|l|}{ No usable continuous data. } \\
\hline \multicolumn{3}{|l|}{ Risk of bias } \\
\hline Bias & Authors' judgement & Support for judgement \\
\hline $\begin{array}{l}\text { Random sequence generation (selection } \\
\text { bias) }\end{array}$ & Unclear risk & $\begin{array}{l}\text { "Divided randomly", "matched for sex, } \\
\text { mean age, mean weight and mean plasma } \\
\text { chlorpromazine concentrations" }\end{array}$ \\
\hline Allocation concealment (selection bias) & Unclear risk & $\begin{array}{l}\text { Details of allocation concealment not re- } \\
\text { ported. }\end{array}$ \\
\hline
\end{tabular}

Blinding of participants and personnel Unclear risk (performance bias)

"Double-blind", no further details reAll outcomes ported.

Blinding of outcome assessment (detection Unclear risk bias)

"Double-blind", no further details reAll outcomes ported.

Incomplete outcome data (attrition bias) Low risk All outcomes

No losses to follow-up reported. 
Adamson 1973 (Continued)

\begin{tabular}{|c|c|c|}
\hline Selective reporting (reporting bias) & High risk & $\begin{array}{l}\text { Not all outcomes fully reported, no data } \\
\text { reported for BPRS and WWBRS }\end{array}$ \\
\hline Other bias & Unclear risk & Source of funding not reported. \\
\hline
\end{tabular}

\section{Albert 1980}

\begin{tabular}{ll}
\hline Methods & Allocation: randomised. \\
& Blindness: double. \\
& Duration: 39 weeks. \\
Design: drug stabilisation period 2 months, treatment 3 months. \\
Country: Canada.
\end{tabular}

$\begin{array}{ll}\text { Participants } & \text { Diagnosis: schizophrenia. } \\ & \text { N=33. } \\ & \text { Age: approximate age mid 40s. } \\ \text { Sex: all male. } \\ \text { History: average duration spent in hospital 16-20 years. } \\ \text { Setting: hospital. }\end{array}$

Interventions

1. Fluphenazine enanthate: dose mean $50 \mathrm{mg} / \mathrm{IM} /$ biweekly. $\mathrm{N}=11$.

2. Pipothiazine palmitate: dose mean $100 \mathrm{mg} / \mathrm{IM}$ or $150 \mathrm{mg} / \mathrm{IM}^{*} / \mathrm{monthly} . \mathrm{N}=11$

Outcomes

Global state: need for additional medication.

Behaviour: leaving the study early.

Adverse effects: Evaluation Scale.

Unable to use -

Global state: CGI (no SD).

Mental state: BPRS (no SD).

Adverse effects: NOSIE (no SD).

\begin{tabular}{l|l}
\hline Notes & $* 2$ different dosage groups for PP. \\
& Authors contacted.
\end{tabular}

Risk of bias

\begin{tabular}{l|l|l}
\hline Bias & Authors' judgement & Support for judgement \\
\hline $\begin{array}{l}\text { Random sequence generation (selection } \\
\text { bias) }\end{array}$ & Unclear risk & $\begin{array}{l}\text { "patients were randomly assigned". } \\
\text { Method not reported. }\end{array}$ \\
\hline Allocation concealment (selection bias) & Unclear risk & $\begin{array}{l}\text { Details of allocation concealment not re- } \\
\text { ported. }\end{array}$ \\
\hline
\end{tabular}

Blinding of participants and personnel Low risk (performance bias)

"The identity of the medications was All outcomes masked and the double blind character of the study preserved by inserting an identi- 
Albert 1980 (Continued)

cal placebo injection at two week intervals"

\begin{tabular}{|c|c|c|}
\hline $\begin{array}{l}\text { Blinding of outcome assessment (detection } \\
\text { bias) } \\
\text { All outcomes }\end{array}$ & Unclear risk & $\begin{array}{l}\text { "The identity of the medications was } \\
\text { masked and the double blind character of } \\
\text { the study preserved by inserting an iden- } \\
\text { tical placebo injection at two week inter- } \\
\text { vals". Blinding of outcome assessment not } \\
\text { specifically reported }\end{array}$ \\
\hline
\end{tabular}

Incomplete outcome data (attrition bias) Unclear risk

Number of patients randomised to each All outcomes group have been reported. However, it is unclear if all participants completed the study

\begin{tabular}{|l|l|l}
\hline Selective reporting (reporting bias) & High risk & $\begin{array}{l}\text { Standard deviations and information on } \\
\text { Serious Adverse Events have not been re- } \\
\text { ported }\end{array}$
\end{tabular}

Other bias

Unclear risk

Source of funding not reported.

\section{Altamura 1985}

\begin{tabular}{|c|c|}
\hline Methods & $\begin{array}{l}\text { Allocation: randomised. } \\
\text { Blindness: double. } \\
\text { Duration: } 2-3 \text { week (2 periods). } \\
\text { Design: parallel group. } \\
\text { Country: United Kingdom. }\end{array}$ \\
\hline Participants & $\begin{array}{l}\text { Diagnosis: schizophrenia (PSE- DSM III). } \\
\mathrm{N}=11 \text {. } \\
\text { Age: } 35-60 \text { years. } \\
\text { Sex: } 2 \mathrm{M} \text {, } 9 \mathrm{~F} \text {. } \\
\text { History: duration illness }<2 \text { yrs. } \\
\text { Setting: community. }\end{array}$ \\
\hline Interventions & $\begin{array}{l}\text { 1. Fluphenazine decanoate: dose } 25 \mathrm{mg} / \mathrm{IM} \text { every } 3-4 \text { weeks. } \mathrm{N}=6 \text {. } \\
\text { 2. Fluphenazine enanthate: dose } 25 \mathrm{mg} / \mathrm{IM} \text { every } 3-4 \text { weeks. } \mathrm{N}=5\end{array}$ \\
\hline Outcomes & $\begin{array}{l}\text { Behaviour: leaving the study early. } \\
\text { Adverse effects: various side effects. } \\
\text { Unable to use - } \\
\text { Mental state: CPRS (no data). } \\
\text { Physiological: (various measures, blood tests - non-clinical outcomes, data unusable). } \\
\text { Cognitive: handwriting (non-clinical outcomes, data unusable) }\end{array}$ \\
\hline Notes & $\begin{array}{l}\text { No usable continuous data. } \\
\text { Authors contacted. }\end{array}$ \\
\hline
\end{tabular}


Altamura 1985 (Continued)

Risk of bias

Risk of bias

\begin{tabular}{|c|c|c|}
\hline Bias & Authors' judgement & Support for judgement \\
\hline $\begin{array}{l}\text { Random sequence generation (selection } \\
\text { bias) }\end{array}$ & Unclear risk & $\begin{array}{l}\text { "The assignment of order of treatments was } \\
\text { randomised". Method not reported }\end{array}$ \\
\hline Allocation concealment (selection bias) & Unclear risk & $\begin{array}{l}\text { Details of allocation concealment not re- } \\
\text { ported. }\end{array}$ \\
\hline
\end{tabular}

Blinding of participants and personnel Unclear risk (performance bias)

All outcomes

An uninvolved person administered the doses and the ampules were not seen by the patients or rater. Other involved personnel might have been unblinded

Blinding of outcome assessment (detection Low risk bias)

An uninvolved person administered the All outcomes doses and the ampules were not seen by the Alloutcomes

Incomplete outcome data (attrition bias) High risk All outcomes rater

4/11 participants left early. Reasons are not reported per intervention group. Losses to follow-up/missing data imbalanced in numbers across intervention groups. $2 / 5$ vs $2 / 6$. The study was terminated due to to high incidence of side effects

Selective reporting (reporting bias) High risk

Results for "unwanted effects" not reported separately (akinesia, involuntary movement, autonomic disturbances and drowsiness) and only part of the rating scale was used. "only two scores on the four point rating scale were... used... absent and maximum...no attempt could be made to derive a figure representing a grading of effect". Although this is mainly a safety study, presence/absence of Serious Adverse Events not reported

Other bias Unclear risk Source of funding not reported. 
Methods
Allocation: randomised.

Blindness: double.

Duration: 2 years.

Design: dosage study.

Country: United States.

\begin{tabular}{ll}
\hline Participants & Diagnosis: schizophrenia. \\
& $\begin{array}{l}\mathrm{N}=36 . \\
\text { Age: } 34-41 \text { years. } \\
\text { Sex: all male. } \\
\text { History: stabilised for }<2 \text { months, informed consent given. } \\
\text { Setting: community. }\end{array}$ \\
\hline Interventions & $\begin{array}{l}\text { 1. Fluphenazine decanoate: dose } 25 \mathrm{mg} / \mathrm{IM} \text { (standard) biweekly. } \mathrm{N}=14 . \\
\text { 2. Fluphenazine decanoate: dose } 5 \mathrm{mg} / \mathrm{IM} \text { (low) biweekly. } \mathrm{N}=22\end{array}$ \\
\hline Outcomes & $\begin{array}{l}\text { Behaviour: leaving the study early. } \\
\text { Unable to use - } \\
\text { Mental state: BPRS (no SD). } \\
\text { Cognitive: information-processing skills (non-clinical outcomes, data unusable) }\end{array}$ \\
\hline Notes & Very little usable data \\
\hline
\end{tabular}

Risk of bias

\begin{tabular}{lll}
\hline Bias & Authors' judgement & Support for judgement \\
\hline $\begin{array}{l}\text { Random sequence generation (selection } \\
\text { bias) }\end{array}$ & Unclear risk & $\begin{array}{l}\text { "randomly assigned". Method not re- } \\
\text { ported. }\end{array}$ \\
\hline $\begin{array}{l}\text { Allocation concealment (selection bias) } \\
\text { Blinding of participants and personnel } \\
\text { (performance bias) }\end{array}$ & Unclear risk & $\begin{array}{l}\text { Details of allocation concealment not re- } \\
\text { ported. }\end{array}$ \\
\hline $\begin{array}{l}\text { Blinding of outcome assessment (detection } \\
\text { bias) } \\
\text { All outcomes }\end{array}$ & Unclear risk & $\begin{array}{l}\text { "double-blind". Blinding details not re- } \\
\text { ported. }\end{array}$ \\
\hline $\begin{array}{l}\text { Incomplete outcome data (attrition bias) } \\
\text { All outcomes }\end{array}$ & Unclear risk & $\begin{array}{l}\text { "double-blind". Blinding details not re- } \\
\text { ported. }\end{array}$ \\
\hline
\end{tabular}


Asarnow 1988 (Continued)

\begin{tabular}{l|l|l}
\hline Selective reporting (reporting bias) & High risk & $\begin{array}{l}\text { Although the results for stated outcomes } \\
\text { have been reported, the results are not pre- } \\
\text { sented as useful data (i.e. mean, SD). Also, } \\
\text { not all expected outcomes were reported (e. } \\
\text { g. presence/absence adverse events, serious } \\
\text { adverse events) }\end{array}$ \\
\hline Other bias & Low risk & $\begin{array}{l}\text { The study seems to be free of other sources } \\
\text { of bias. }\end{array}$ \\
\hline
\end{tabular}

Barnes 1983

Methods Allocation: assigned to two groups by independent statistician.

Blindness: double.

Duration: 1 year.

Design: parallel group.

Country: United Kingdom.

\begin{tabular}{|c|c|c|}
\hline Participants & \multicolumn{2}{|c|}{$\begin{array}{l}\text { Diagnosis: schizophrenia (PSE). } \\
\mathrm{N}=36 . \\
\text { Age: mean }-49 \text { years. } \\
\text { Sex: } 18 \mathrm{M}, 18 \mathrm{~F} \text {. } \\
\text { History: not stated. } \\
\text { Setting: community. }\end{array}$} \\
\hline Interventions & \multicolumn{2}{|c|}{$\begin{array}{l}\text { 1. Fluphenazine decanoate: dose } 25 \mathrm{mg} / \mathrm{IM} \text { biweekly. } \mathrm{N}=19 \text {. } \\
\text { 2. Pimozide: dose } 8 \mathrm{mg} \text { biweekly. } \mathrm{N}=17 \text {. }\end{array}$} \\
\hline Outcomes & \multicolumn{2}{|c|}{$\begin{array}{l}\text { Behaviour: leaving the study early. } \\
\text { Unable to use - } \\
\text { Behaviour: SBAS (non-clinical outcomes, data unusable). }\end{array}$} \\
\hline Notes & \multicolumn{2}{|c|}{$\begin{array}{l}\text { Analysis: last observation carried forward. } \\
\text { No continuous outcomes measured. }\end{array}$} \\
\hline \multicolumn{3}{|l|}{ Risk of bias } \\
\hline Bias & Authors' judgement & Support for judgement \\
\hline $\begin{array}{l}\text { Random sequence generation (selection } \\
\text { bias) }\end{array}$ & Unclear risk & $\begin{array}{l}\text { "Patients were assigned to two groups by } \\
\text { an independent statistician, matched on } \\
\text { the basis of age, sex and calculated weekly } \\
\text { fluphenazine dose" }\end{array}$ \\
\hline Allocation concealment (selection bias) & Unclear risk & $\begin{array}{l}\text { Details of allocation concealment not re- } \\
\text { ported. }\end{array}$ \\
\hline
\end{tabular}


Barnes 1983 (Continued)

\begin{tabular}{|c|c|c|}
\hline $\begin{array}{l}\text { Blinding of participants and personnel } \\
\text { (performance bias) } \\
\text { All outcomes }\end{array}$ & Unclear risk & $\begin{array}{l}\text { "double blind...double dummy" Blinding } \\
\text { details not reported. }\end{array}$ \\
\hline $\begin{array}{l}\text { Blinding of outcome assessment (detection } \\
\text { bias) } \\
\text { All outcomes }\end{array}$ & Unclear risk & Blinding details not reported. \\
\hline $\begin{array}{l}\text { Incomplete outcome data (attrition bias) } \\
\text { All outcomes }\end{array}$ & Low risk & $\begin{array}{l}\text { In "the } 1 \text {-year follow-up results of all pa- } \\
\text { tients who had begun the trial, includ- } \\
\text { ing drop-outs, withdrawals and relapsers, } \\
\text { were involved in the analysis... Unfortu- } \\
\text { nately, the SBAS data were incomplete for } \\
\text { one relapsed patient who was abroad from } \\
\text { the time of relapse to the end of the trial." }\end{array}$ \\
\hline Selective reporting (reporting bias) & High risk & $\begin{array}{l}\text { Results for SBAS have been reported as per- } \\
\text { centages, only. Other expected outcomes } \\
\text { (e.g. presence/absence of adverse events, } \\
\text { serious adverse events have not been re- } \\
\text { ported) }\end{array}$ \\
\hline Other bias & Unclear risk & Source of funding has not been reported. \\
\hline
\end{tabular}

\section{Chien 1973}

\begin{tabular}{|c|c|}
\hline Methods & $\begin{array}{l}\text { Allocation: randomised. } \\
\text { Blindness: single. } \\
\text { Duration: } 30 \text { days. } \\
\text { Design: parallel group. } \\
\text { Country: USA. }\end{array}$ \\
\hline Participants & $\begin{array}{l}\text { Diagnosis: psychosis. } \\
\mathrm{N}=31 \\
\text { Age: } 17-62 \text { years, mean }-37 \text { years. } \\
\text { Sex: } 24 \mathrm{M}, 22 \mathrm{~F} \text {. } \\
\text { History: acutely psychotic, recently admitted. } \\
\text { Setting: hospital. }\end{array}$ \\
\hline Interventions & $\begin{array}{l}\text { 1. Fluphenazine enanthate: dose } 12.5-75 \mathrm{mg} / \mathrm{IM} \text {, mean } 28.5 \mathrm{mg} / \mathrm{IM} \text { every } 12 \text { days. } \mathrm{N} \\
=16 . \\
\text { 2. Chlorpromazine: dose mean } 388 \mathrm{mg} / \text { day. } \mathrm{N}=15 \text {. }\end{array}$ \\
\hline Outcomes & $\begin{array}{l}\text { Global state: need for additional medication. } \\
\text { Behaviour: leaving the study early. } \\
\text { Adverse effects: TESF. } \\
\text { Unable to use - } \\
\text { Behaviour: NOSIE (no data). }\end{array}$ \\
\hline
\end{tabular}


Chien 1973 (Continued)

Notes

Risk of bias

\begin{tabular}{|c|c|c|}
\hline Bias & Authors' judgement & Support for judgement \\
\hline $\begin{array}{l}\text { Random sequence generation (selection } \\
\text { bias) }\end{array}$ & Unclear risk & $\begin{array}{l}\text { "randomly assigned". Method not re- } \\
\text { ported. }\end{array}$ \\
\hline Allocation concealment (selection bias) & Unclear risk & $\begin{array}{l}\text { Details of allocation concealment not re- } \\
\text { ported. }\end{array}$ \\
\hline
\end{tabular}

Blinding of participants and personnel Unclear risk (performance bias)

Blinding of participants and personnel not All outcomes

Blinding of outcome assessment (detection Unclear risk bias)

All outcomes reported.

\begin{tabular}{|c|c|c|}
\hline & & $\begin{array}{l}\text { psychiatrist who had no knowledge of the } \\
\text { patient's medication". Blinding methods of } \\
\text { nurses rating NOISE not reported }\end{array}$ \\
\hline $\begin{array}{l}\text { Incomplete outcome data (attrition bias) } \\
\text { All outcomes }\end{array}$ & Unclear risk & Losses to follow-up not reported. \\
\hline Selective reporting (reporting bias) & Unclear risk & Data for NOSIE not reported. \\
\hline Other bias & Low risk & $\begin{array}{l}\text { "supported in part by Public Health Ser- } \\
\text { vice research grant MH-16128 from the } \\
\text { National Institute of Mental Health." }\end{array}$ \\
\hline
\end{tabular}

Chouinard 1978

\begin{tabular}{ll}
\hline Methods & Allocation: randomised. \\
& Blindness: double. \\
& Duration: 9 months. \\
& Design: parallel group. \\
& Country: Canada. \\
\hline Participants & Diagnosis: schizophrenia. \\
& N = 32. \\
Age: $20-60$ years. \\
Sex: $16 \mathrm{M}, 16 \mathrm{~F}$. \\
History: informed consent given. \\
Setting: community.
\end{tabular}

"each patient was rated after 10 days of treatment on a seven-point scale of global improvement by an independent research psychiatrist who had no knowledge of the patient's medication". Blinding methods of 
Chouinard 1978 (Continued)

\begin{tabular}{ll}
\hline Interventions & $\begin{array}{l}\text { 1. Fluphenazine enanthate: dose } 6.25-100 \mathrm{mg} / \mathrm{IM} \text { biweekly. } \mathrm{N}=16 . \\
\text { 2. Pipothiazine palmitate: dose } 25-100 \mathrm{mg} / \mathrm{IM} \text { monthly. } \mathrm{N}=16 \\
\text { Dose adjusted to therapeutic response. }\end{array}$ \\
\hline Outcomes & $\begin{array}{l}\text { Global state: CGI, need for additional medication. } \\
\text { Mental state: BPRS. } \\
\text { Behaviour: leaving the study early. } \\
\text { Adverse effects: HRSD, EPS, TESF. } \\
\text { Unable to use - } \\
\text { Adverse effects: various effects (no SD). } \\
\text { Physiological: various measures (non-clinical outcomes, data unusable) }\end{array}$ \\
\hline Notes & Analysis: last observation carried forward.
\end{tabular}

Risk of bias

\begin{tabular}{l|ll}
\hline Bias & Authors' judgement & Support for judgement \\
\hline $\begin{array}{l}\text { Random sequence generation (selection } \\
\text { bias) }\end{array}$ & Unclear risk & $\begin{array}{l}\text { Random allocation. Methods of randomi- } \\
\text { sation not reported. }\end{array}$ \\
\hline Allocation concealment (selection bias) & Unclear risk & $\begin{array}{l}\text { Allocation concealment details not re- } \\
\text { ported. }\end{array}$ \\
\hline
\end{tabular}

Blinding of participants and personnel Unclear risk (performance bias)

"Except for the nurse responsible for giving All outcomes the injections, the procedure was double blind."

Blinding of outcome assessment (detection Unclear risk bias)

Outcome blinding details not reported.

All outcomes

Incomplete outcome data (attrition bias) Low risk All outcomes

4 patients did not complete the study. Reasons for dropping out have been described. Number of drop-outs was balanced between the groups. "Psychiatric evaluations of these four patients were made on the days they left the study and were used ...for the statistical analysis of the rating scale data."

Not all outcomes fully reported. The study reports most but not all expected outcomes (e.g.presence/absence serious adverse events) 


\begin{tabular}{|c|c|}
\hline Methods & $\begin{array}{l}\text { Allocation: randomised. } \\
\text { Blindness: double. } \\
\text { Duration: } 7 \text { months, preceded by } 1 \text { month stabilisation period. } \\
\text { Design: parallel group. } \\
\text { Country: Canada. }\end{array}$ \\
\hline Participants & $\begin{array}{l}\text { Diagnosis: schizophrenia (DSM II). } \\
\mathrm{N}=50^{*} \\
\text { Age: } 24-65 \text { years, median }-41 \text { years. } \\
\text { Sex: } 27 \mathrm{M}, 21 \mathrm{~F} \text {. } \\
\text { History: on FE for } 1 \text { month, able to give informed consent. } \\
\text { Setting: community. }\end{array}$ \\
\hline Interventions & $\begin{array}{l}\text { 1. Fluphenazine decanoate: dose } 2.5-250 \mathrm{mg} / \mathrm{IM} \text {, mean } 27 \mathrm{mg} / \mathrm{IM} \text { monthly. } \mathrm{N}=24 \text {. } \\
\text { 2. Fluphenazine enanthate: dose } 2.5-325 \mathrm{mg} / \mathrm{IM} \text {, mean }-35 \mathrm{mg} / \mathrm{IM} \text { biweekly. } \mathrm{N}=24 \\
\text { Dose adjusted to therapeutic response. }\end{array}$ \\
\hline Outcomes & $\begin{array}{l}\text { Global state: need for additional medication. } \\
\text { Behaviour: leaving the study early. } \\
\text { Additional medication. } \\
\text { Unable to use - } \\
\text { Mental state: BPRS (no SD). } \\
\text { Adverse effects: TESF (no data); ESRS (authors own scale**). }\end{array}$ \\
\hline Notes & $\begin{array}{l}\text { Authors contacted. } \\
\text { Results for FE \& FD pooled. } \\
\text { * } 2 \text { dropped out after randomisation/ moved \& suicide. } \\
\text { ** see Marshall et al } 1998\end{array}$ \\
\hline
\end{tabular}

Risk of bias

\begin{tabular}{lll}
\hline Bias & Authors' judgement & Support for judgement \\
\hline $\begin{array}{l}\text { Random sequence generation (selection } \\
\text { bias) }\end{array}$ & Unclear risk & $\begin{array}{l}\text { "randomly assigned”. Method not re- } \\
\text { ported. }\end{array}$ \\
\hline Allocation concealment (selection bias) & Unclear risk & $\begin{array}{l}\text { Details of allocation concealment not re- } \\
\text { ported. }\end{array}$ \\
\hline
\end{tabular}

Blinding of participants and personnel Low risk (performance bias)

All outcomes

"double-blind". "Both preparations were administered as identical suspensions in oil”

Blinding of outcome assessment (detection Low risk bias)

All outcomes

"Assessment of psychiatric symptoms was based on clinical interviews conducted by the psychiatrist...under blind conditions" 
Chouinard 1982 (Continued)

\begin{tabular}{|c|c|c|}
\hline $\begin{array}{l}\text { Incomplete outcome data (attrition bias) } \\
\text { All outcomes }\end{array}$ & Low risk & $\begin{array}{l}\text { "Two patients did not complete the } 28 \\
\text { week trial...One of these left the coun- } \\
\text { try after } 12 \text { weeks of treatment with } \\
\text { fluphenazine decanoate; the other commit- } \\
\text { ted suicide after } 22 \text { weeks of treatment with } \\
\text { fluphenazine enanthate" }\end{array}$ \\
\hline
\end{tabular}

Selective reporting (reporting bias) High risk

Results reported incompletely for BPRS scores. Adverse events,other than EPS and TD not reported

\begin{tabular}{|c|c|c|}
\hline Other bias & Unclear risk & Source of funding not reported. \\
\hline
\end{tabular}

\section{Chouinard 1984}

Methods

Allocation: randomised, stratified by sex \& past frequency of depot administration.

Blindness: double.

Duration: 8 months.

Design: parallel group.

Country: Canada.

\begin{tabular}{ll} 
Participants & Diagnosis: schizophrenia (DSM III). \\
& $\mathrm{N}=72$. \\
& Age: $18-66$ years, mean -44 years. \\
& Sex: $36 \mathrm{M}, 36 \mathrm{~F}$. \\
& History: on depot $>3$ months; duration illness 3-38 years, mean 16 years, able to give \\
& informed consent. \\
& Setting: community. \\
\hline Interventions & $\begin{array}{l}\text { 1. Fluphenazine decanoate: dose } 2.5-300 \mathrm{mg} / \mathrm{IM} \text {, mean } 75 \mathrm{mg} / \mathrm{IM} \text { every } 2-4 \text { weeks. N } \\
=36 .\end{array}$ \\
& 2. Haloperidol decanoate: dose $15-900 \mathrm{mg} / \mathrm{IM}$, mean $225 \mathrm{mg} / \mathrm{IM}$ every $2-4$ weeks. N \\
& $=36$
\end{tabular}

Outcomes

Global state: CGI, need for additional medication.

Mental state: BPRS.

Behaviour: leaving the study early.

Unable to use -

Adverse effects: ESRS (authors own scale*), TESF (no data).

Physiological: various measures (non clinical outcomes, data unusable)

Notes Statistics: last observation brought forward.

*see Marshall et al 1998.

Risk of bias 


\section{Chouinard 1984 (Continued)}

\begin{tabular}{|c|c|c|}
\hline $\begin{array}{l}\text { Random sequence generation (selection } \\
\text { bias) }\end{array}$ & Unclear risk & $\begin{array}{l}\text { "randomly assigned". Method not re- } \\
\text { ported. }\end{array}$ \\
\hline Allocation concealment (selection bias) & Unclear risk & $\begin{array}{l}\text { Details of allocation concealment not re- } \\
\text { ported. }\end{array}$ \\
\hline $\begin{array}{l}\text { Blinding of participants and personnel } \\
\text { (performance bias) } \\
\text { All outcomes }\end{array}$ & Unclear risk & $\begin{array}{l}\text { "double -blind". Blinding details not re- } \\
\text { ported. }\end{array}$ \\
\hline $\begin{array}{l}\text { Blinding of outcome assessment (detection } \\
\text { bias) } \\
\text { All outcomes }\end{array}$ & Unclear risk & Blinding details not reported. \\
\hline $\begin{array}{l}\text { Incomplete outcome data (attrition bias) } \\
\text { All outcomes }\end{array}$ & Unclear risk & Losses to follow-up have not been reported. \\
\hline Selective reporting (reporting bias) & High risk & $\begin{array}{l}\text { Most of the outcomes have been reported } \\
\text { only as } \mathrm{P}=.05 \text {. }\end{array}$ \\
\hline Other bias & Unclear risk & Source of funding has not been reported. \\
\hline
\end{tabular}

\section{Cookson 1986}

Methods

Allocation: randomised, separate randomisation sequences for males and females.

Blindness: double.

Duration: 8 months.

Design: parallel group.

Country: United Kingdom.

\begin{tabular}{ll} 
Participants & Diagnosis: schizophrenia implied. \\
& $\mathrm{N}=19$. \\
& Age: $26-60$ years. \\
& Sex: $9 \mathrm{M}, 10 \mathrm{~F}$. \\
& History: $1 \mathrm{yr}$ treatment with fluphenazine decanoate, overweight BMI \\
& fit, stable during previous year \\
& Setting: community. \\
\hline Interventions & Fluphenazine decanoate: dose $26.4 \mathrm{mg} / \mathrm{IM}$, every 2-6 weeks, average 3.6 \\
& 9. \\
& 2. Haloperidol decanoate: dose $22.2 \mathrm{mg} / \mathrm{IM}$ every 2-5 weeks, average 3.6 \\
& 10 \\
\hline \multirow{3}{*}{ Outcomes } & Behaviour: leaving the study early. \\
& Unable to use - \\
& Mental state: CPRS, KGS (no data). \\
Adverse effects: SAS, AIMS (no data). \\
Physiological: various measures (non clinical outcomes, data unusable)
\end{tabular}


Cookson 1986 (Continued)

\begin{tabular}{|c|c|c|}
\hline Notes & \multicolumn{2}{|c|}{ Analysis: last observation carried forward. } \\
\hline \multicolumn{3}{|l|}{ Risk of bias } \\
\hline Bias & Authors' judgement & Support for judgement \\
\hline $\begin{array}{l}\text { Random sequence generation (selection } \\
\text { bias) }\end{array}$ & Unclear risk & $\begin{array}{l}\text { “...randomly assigned...Separate randomi- } \\
\text { sation sequences were used for male and fe- } \\
\text { male patients." Method not reported }\end{array}$ \\
\hline Allocation concealment (selection bias) & Unclear risk & $\begin{array}{l}\text { Details of allocation concealment not re- } \\
\text { ported. }\end{array}$ \\
\hline $\begin{array}{l}\text { Blinding of participants and personnel } \\
\text { (performance bias) } \\
\text { All outcomes }\end{array}$ & Unclear risk & $\begin{array}{l}\text { "double-blind". Blinding details not re- } \\
\text { ported. }\end{array}$ \\
\hline $\begin{array}{l}\text { Blinding of outcome assessment (detection } \\
\text { bias) } \\
\text { All outcomes }\end{array}$ & Unclear risk & $\begin{array}{l}\text { "double-blind". Blinding details not re- } \\
\text { ported. }\end{array}$ \\
\hline $\begin{array}{l}\text { Incomplete outcome data (attrition bias) } \\
\text { All outcomes }\end{array}$ & High risk & $\begin{array}{l}\text { Losses to follow-up are imbalanced. "Two } \\
\text { patients- both on the haloperidol de- } \\
\text { canoate-dropped out". Also, prolactin lev- } \\
\text { els not measured for all subjects i.e. "avail- } \\
\text { able for six patients on fluphenazine de- } \\
\text { canoate ... and eight on haloperidol de- } \\
\text { canoate." }\end{array}$ \\
\hline Selective reporting (reporting bias) & High risk & $\begin{array}{l}\text { Body weight and prolactin levels have been } \\
\text { reported. However, results of clinical assess- } \\
\text { ments not fully reported }\end{array}$ \\
\hline Other bias & Unclear risk & Source of funding not reported. \\
\hline
\end{tabular}

\section{Crawford 1974}

\begin{tabular}{ll} 
Methods & Allocation: randomised. \\
& Blindness: double. \\
& Duration: 40 weeks. \\
& Design: parallel group. \\
& Country: United Kingdom (Scotland). \\
\hline Participants & Diagnosis: schizophrenia (Forest \& Hay $1971 / 72$ criteria). \\
& N = 31. \\
& Age: $20-65$ years. \\
& Sex: $9 \mathrm{M}, 22 \mathrm{~F}$. \\
& History: mean duration illness $1-27$ years, mean -14 years.
\end{tabular}




\section{Crawford 1974 (Continued)}

Setting: community.

$\begin{array}{ll}\text { Interventions } & \text { 1. Fluphenazine decanoate: (dosage not stated). } \mathrm{N}=14 . \\ \text { 2. Trifluoperazine hydrochloride (oral): (dosage not stated). } \mathrm{N}=17\end{array}$

Outcomes Behaviour: leaving the study early.

Unable to use -

Mental state: BPRS (no data).

Notes

Risk of bias

Bias

Random sequence generation (selection Unclear risk bias)

Allocation concealment (selection bias) Unclear risk

\section{Support for judgement}

"randomly allocated". Method not reported.

Details of allocation concealment not reported.

"double-blind". "The preparations employed had identical appearances"

"double-blind". Blinding details not reported.

bias)

Blinding of outcome assessment (detection Unclear risk

Blinding of participants and personnel Low risk

(performance bias)

All outcomes

All outcomes

Incomplete outcome data (attrition bias) High risk

All outcomes

2 participants, both from the trifluoperazine hydrochloride group, dropped out "within four weeks" of the study and were not included in the analysis. Withdrawal rate was $6 / 15$ in the trifluoperazine hydrochloride group and $2 / 12$ in the fluphenazine group

Selective reporting (reporting bias)

High risk

Not all outcome data was fully reported.

Other bias

Unclear risk
Source of funding not reported. 


\begin{tabular}{|c|c|c|}
\hline Methods & \multicolumn{2}{|l|}{$\begin{array}{l}\text { Allocation: randomised. } \\
\text { Blindness: double. } \\
\text { Duration: } 28 \text { days. } \\
\text { Design: parallel group. } \\
\text { Country: United Kingdom. }\end{array}$} \\
\hline Participants & \multicolumn{2}{|l|}{$\begin{array}{l}\text { Diagnosis: schizophrenia. } \\
\mathrm{N}=37 \text {. } \\
\text { Age: not stated. } \\
\text { Sex: male and female. } \\
\text { History: chronically ill. } \\
\text { Setting: hospital. }\end{array}$} \\
\hline Interventions & \multicolumn{2}{|c|}{$\begin{array}{l}\text { 1. Fluphenazine decanoate: (dosage not stated). } \mathrm{N}=19 \text {. } \\
\text { 2. Chlorpromazine (oral): (dosage not stated). } \mathrm{N}=18 \text {. }\end{array}$} \\
\hline Outcomes & \multicolumn{2}{|l|}{$\begin{array}{l}\text { Behaviour: WWBRS. } \\
\text { Leaving the study early. } \\
\text { Unable to use - } \\
\text { Mental state: BPRS (no SD). }\end{array}$} \\
\hline Notes & \multicolumn{2}{|l|}{ Authors contacted. } \\
\hline \multicolumn{3}{|l|}{ Risk of bias } \\
\hline Bias & Authors' judgement & Support for judgement \\
\hline $\begin{array}{l}\text { Random sequence generation (selection } \\
\text { bias) }\end{array}$ & Unclear risk & “randomised". Method not reported. \\
\hline Allocation concealment (selection bias) & Unclear risk & $\begin{array}{l}\text { Details of allocation concealment not re- } \\
\text { ported. }\end{array}$ \\
\hline $\begin{array}{l}\text { Blinding of participants and personnel } \\
\text { (performance bias) } \\
\text { All outcomes }\end{array}$ & Unclear risk & $\begin{array}{l}\text { "double-blind". Blinding details not re- } \\
\text { ported. }\end{array}$ \\
\hline $\begin{array}{l}\text { Blinding of outcome assessment (detection } \\
\text { bias) } \\
\text { All outcomes }\end{array}$ & Unclear risk & $\begin{array}{l}\text { "double-blind". Blinding details not re- } \\
\text { ported. }\end{array}$ \\
\hline $\begin{array}{l}\text { Incomplete outcome data (attrition bias) } \\
\text { All outcomes }\end{array}$ & Unclear risk & Losses to follow-up not reported. \\
\hline Selective reporting (reporting bias) & High risk & $\begin{array}{l}\text { Incomplete study results. Results not re- } \\
\text { ported as mean (SD). }\end{array}$ \\
\hline Other bias & Unclear risk & Source of funding not reported. \\
\hline
\end{tabular}


Methods
Allocations: randomised.

Blindness: double.

Duration: 3 years.

Design: 3 months adjustment, 1-3 months maintenance, 2-6 months maintenance, 2year follow-up.

Country: Sweden.

\begin{tabular}{|c|c|}
\hline Participants & $\begin{array}{l}\text { Diagnosis: schizophrenia. } \\
\mathrm{N}=67 . \\
\text { Age: } 18-65 \text { years, mean }-41 \text { years. } \\
\text { Sex: } 51 \mathrm{M}, 14 \mathrm{~F} \text {. } \\
\text { History: duration illness }>5 \text { years. } \\
\text { Setting: } 1 \text { year in hospital, } 2 \text { years in community. }\end{array}$ \\
\hline Interventions & $\begin{array}{l}\text { 1.Fluphenazine decanoate: dose } 3.1-50 \mathrm{mg} / \mathrm{IM} \text {, mean } 6.25 \mathrm{mg} / \mathrm{IM} \text { monthly (mean } \\
\text { monthly dose for } 2 \text {-year continuation phase } 27.8 \mathrm{mg} / \mathrm{IM} \text { ). } \mathrm{N}=35 \text {. } \\
\text { 2. Pipothiazine palmitate: dose } 25-400 \mathrm{mg} / \mathrm{IM} \text {, mean } 50 \mathrm{mg} / \mathrm{IM} \text { monthly (mean } \\
\text { monthly dose for 2-year continuation phase } 152.3 \mathrm{mg} / \mathrm{IM} \text { ). } \mathrm{N}=32\end{array}$ \\
\hline Outcomes & $\begin{array}{l}\text { Global state: need for additional medication. } \\
\text { Behaviour: leaving the study early. } \\
\text { Adverse effects: EPS, HRSD. } \\
\text { Unable to use - } \\
\text { Mental state: BPRS, S-Scale, HRSD (no SD). } \\
\text { Cognitive: Handwriting test (non-clinical outcomes, data not usable). } \\
\text { Social ability: ADL, work performance, SRE (non-clinical outcomes, data not usable). } \\
\text { Adverse effects: EPS (no data). }\end{array}$ \\
\hline Notes & Authors contacted. \\
\hline
\end{tabular}

Random sequence generation (selection Unclear risk bias)

Allocation concealment (selection bias) Unclear risk

Blinding of participants and personnel Unclear risk (performance bias)

All outcomes

Blinding of outcome assessment (detection Low risk bias)

All outcomes
Method of sequence generation not reported.

Details of allocation concealment not reported.

"double blind". " the injections were given by a nurse, who was the only person who knew the code"

"double blind". " the injections were given by a nurse, who was the only person who knew the code." "most of rating were made by one psychologist, and the rest by one of the psychiatrists" 


\section{Dencker 1973 (Continued)}

\begin{tabular}{l|l|l}
\hline $\begin{array}{l}\text { Incomplete outcome data (attrition bias) } \\
\text { All outcomes }\end{array}$ & Low risk & $\begin{array}{l}\text { Losses to follow-up balanced in numbers } \\
\text { across intervention groups } 9 \% \text { vs } 17 \%\end{array}$ \\
\hline Selective reporting (reporting bias) & High risk & $\begin{array}{l}\text { Although all outcomes have been reported, } \\
\text { data for most of the outcomes (BPRS, } \\
\text { Hamilton depression scale, side effects rat- } \\
\text { ing scale, rating scale for extra-pyramidal } \\
\text { side effect) cannot be used i.e. only mean } \\
\text { reported (SD not reported) }\end{array}$ \\
\hline Other bias & Unclear risk & Source of funding not reported \\
\hline
\end{tabular}

\section{Donlon 1976}

\begin{tabular}{|c|c|}
\hline Methods & $\begin{array}{l}\text { Allocation: randomised. } \\
\text { Blindness: double. } \\
\text { Duration: } 2 \text { months. } \\
\text { Design: parallel group. } \\
\text { Country: USA. }\end{array}$ \\
\hline Participants & $\begin{array}{l}\text { Diagnosis: schizophrenia. } \\
\mathrm{N}=40 / 41^{*} \text {. } \\
\text { Age: } 18-57 \text { years, mean }-29 \text { years. } \\
\text { Sex: } 12 \mathrm{M}, 18 \mathrm{~F} \text {. } \\
\text { History: able to give informed consent. } \\
\text { Setting: hospital \& community. }\end{array}$ \\
\hline Interventions & $\begin{array}{l}\text { 1. Fluphenazine decanoate: dose } 75-500 \mathrm{mg} / \mathrm{IM} \text {, mean } 296.4 \mathrm{mg} / \mathrm{IM} 2-3 \mathrm{x} \text { week. } \mathrm{N}= \\
\text { 14. } \\
\text { 2. Fluphenazine enanthate: dose } 50-550 \mathrm{mg} / \mathrm{IM} \text {, mean } 387.5 \mathrm{mg} / \mathrm{IM} 2-3 \mathrm{x} \text { week. } \mathrm{N}= \\
16\end{array}$ \\
\hline Outcomes & $\begin{array}{l}\text { Global state: need for additional medication. } \\
\text { Behaviour: leaving the study early. } \\
\text { Adverse effects: EPS Rating Scale. } \\
\text { Unable to use - } \\
\text { Global state: CGI (no data). } \\
\text { Mental state: BPRS (no data). }\end{array}$ \\
\hline Notes & $\begin{array}{l}\text { Data put in depot vs depot category in both FE \& FD treatment groups } \\
{ }^{*} 2 \text { different } \mathrm{N} \text { values in the paper. }\end{array}$ \\
\hline
\end{tabular}

Bias

Authors' judgement

Support for judgement 


\section{Donlon 1976 (Continued)}

\begin{tabular}{|c|c|c|}
\hline $\begin{array}{l}\text { Random sequence generation (selection } \\
\text { bias) }\end{array}$ & Unclear risk & $\begin{array}{l}\text { "assigned...on a random basis". Method not } \\
\text { reported. }\end{array}$ \\
\hline Allocation concealment (selection bias) & Unclear risk & $\begin{array}{l}\text { Details of allocation concealment not re- } \\
\text { ported. }\end{array}$ \\
\hline $\begin{array}{l}\text { Blinding of participants and personnel } \\
\text { (performance bias) } \\
\text { All outcomes }\end{array}$ & Low risk & $\begin{array}{l}\text { "double blind", "FE and FD were supplied } \\
\text { in identical bottles" }\end{array}$ \\
\hline $\begin{array}{l}\text { Blinding of outcome assessment (detection } \\
\text { bias) } \\
\text { All outcomes }\end{array}$ & Unclear risk & $\begin{array}{l}\text { "double-blind". Blinding of outcome asses- } \\
\text { sors not reported. }\end{array}$ \\
\hline $\begin{array}{l}\text { Incomplete outcome data (attrition bias) } \\
\text { All outcomes }\end{array}$ & Unclear risk & $\begin{array}{l}\text { Losses to follow-up not reported. } 41 \text { par- } \\
\text { ticipants randomised, but number in each } \\
\text { group not reported. Allocation per group } \\
\text { reported for } 30 \text { patients: "Eleven patients } \\
\text { either voluntarily dropped out or were lost } \\
\text { to follow-up prior to the end of } 1 \text { month. } \\
\text {..3 patients on FE terminated during the } \\
\text { second month", other reasons for leaving } \\
\text { early not reported }\end{array}$ \\
\hline Selective reporting (reporting bias) & High risk & $\begin{array}{l}\text { CGI and BPRS results incompletely re- } \\
\text { ported (only P values). }\end{array}$ \\
\hline Other bias & Unclear risk & Source of funding not reported. \\
\hline
\end{tabular}

Dotti 1979

Methods

Allocation: randomised.

Blindness: double.

Duration: 9 months.

Design: parallel group.

Country: Italy.

Participants

Diagnosis: schizophrenia.

$\mathrm{N}=20$.

Age: $19-32$ years.

Sex: all male.

History: previous episodes of psychosis.

Setting: community.

Interventions

1. Fluphenazine decanoate: dose $25-50 \mathrm{mg}$ (frequency not stated). $\mathrm{N}=10$.

2. Placebo: (frequency not stated). $\mathrm{N}=10$. 


\section{Dotti 1979 (Continued)}

\begin{tabular}{|c|c|c|}
\hline Outcomes & \multicolumn{2}{|l|}{$\begin{array}{l}\text { Behaviour: leaving the study early. } \\
\text { Mental state: BPRS. }\end{array}$} \\
\hline Notes & & \\
\hline \multicolumn{3}{|l|}{ Risk of bias } \\
\hline Bias & Authors' judgement & Support for judgement \\
\hline $\begin{array}{l}\text { Random sequence generation (selection } \\
\text { bias) }\end{array}$ & Unclear risk & "Randomised", no further details reported. \\
\hline Allocation concealment (selection bias) & Unclear risk & $\begin{array}{l}\text { Medication was prepared in a pharmacy, } \\
\text { but no details of allocation concealment re- } \\
\text { ported }\end{array}$ \\
\hline $\begin{array}{l}\text { Blinding of participants and personnel } \\
\text { (performance bias) } \\
\text { All outcomes }\end{array}$ & Unclear risk & "Double blind", no details reported. \\
\hline $\begin{array}{l}\text { Blinding of outcome assessment (detection } \\
\text { bias) } \\
\text { All outcomes }\end{array}$ & Unclear risk & "Double blind", no details reported. \\
\hline $\begin{array}{l}\text { Incomplete outcome data (attrition bias) } \\
\text { All outcomes }\end{array}$ & High risk & $\begin{array}{l}\text { Four participants left the study early, one } \\
\text { from the fluphenazine group and three } \\
\text { from the placebo group, reasons not re- } \\
\text { ported }\end{array}$ \\
\hline Selective reporting (reporting bias) & Low risk & All outcomes reported. \\
\hline Other bias & Unclear risk & Source of funding not reported. \\
\hline
\end{tabular}

Falloon 1978

\begin{tabular}{ll} 
Methods & Allocation: randomised. \\
& Blindness: double. \\
& Duration: 20 months. \\
& Design: 2 trials - I \& II. \\
& Country: United Kingdom. \\
\hline Participants & Diagnosis: schizophrenia (Schneider). \\
& N $=44$. \\
Age: $17-60$ years, mean -39 years. \\
Sex: $20 \mathrm{M}, 24 \mathrm{~F}$. \\
History: stabilised prior to study entry. \\
Setting: community.
\end{tabular}


Falloon 1978 (Continued)

\begin{tabular}{|c|c|c|}
\hline Interventions & \multicolumn{2}{|c|}{$\begin{array}{l}\text { 1. Fluphenazine decanoate: dose mean } 25 \mathrm{mg} / \mathrm{IM} \text { weekly, maximum } 50 \mathrm{mg} / \text { biweekly. } \\
\mathrm{N}=20 \text {. } \\
\text { 2. Pimozide: dose mean } 8 \mathrm{mg} / \text { day, maximum } 16 \mathrm{mg} / \text { day. } \mathrm{N}=24 \text {. } \\
\text { Flexible dosage. }\end{array}$} \\
\hline Outcomes & \multicolumn{2}{|c|}{$\begin{array}{l}\text { Global state: need for additional medication. } \\
\text { Behaviour: leaving the study early. } \\
\text { Adverse effects: checklist for SE's. } \\
\text { Unable to use - } \\
\text { Mental state: PSE (no data). } \\
\text { Social ability: SPS (non-clinical outcome, data unusable). }\end{array}$} \\
\hline \multicolumn{3}{|l|}{ Notes } \\
\hline \multicolumn{3}{|l|}{ Risk of bias } \\
\hline Bias & Authors' judgement & Support for judgement \\
\hline $\begin{array}{l}\text { Random sequence generation (selection } \\
\text { bias) }\end{array}$ & Unclear risk & $\begin{array}{l}\text { "randomly allocated". Method not re- } \\
\text { ported. }\end{array}$ \\
\hline Allocation concealment (selection bias) & Unclear risk & $\begin{array}{l}\text { Details of allocation concealment not re- } \\
\text { ported. }\end{array}$ \\
\hline $\begin{array}{l}\text { Blinding of participants and personnel } \\
\text { (performance bias) } \\
\text { All outcomes }\end{array}$ & Unclear risk & $\begin{array}{l}\text { "double- blind control conditions...active } \\
\text { pimozide tablets +inert fluphenazine in- } \\
\text { jections, or...active fluphenazine injections } \\
\text { +inert pimozide tablets." Blinding of per- } \\
\text { sonnel unclear }\end{array}$ \\
\hline $\begin{array}{l}\text { Blinding of outcome assessment (detection } \\
\text { bias) } \\
\text { All outcomes }\end{array}$ & Unclear risk & $\begin{array}{l}\text { "double- blind". Blinding of outcome as- } \\
\text { sessor unclear. }\end{array}$ \\
\hline $\begin{array}{l}\text { Incomplete outcome data (attrition bias) } \\
\text { All outcomes }\end{array}$ & High risk & $\begin{array}{l}\text { Three patients (all from one group) were } \\
\text { withdrawn, losses to follow-up are unbal- } \\
\text { anced }\end{array}$ \\
\hline Selective reporting (reporting bias) & High risk & Incomplete reporting of data. \\
\hline Other bias & Unclear risk & Sources of funding not reported. \\
\hline
\end{tabular}




\begin{tabular}{l|l} 
Methods & Allocation: randomised. \\
& Blindness: double. \\
& Duration: 12 weeks. \\
Design: parallel group. \\
Country: China. \\
\hline
\end{tabular}

Participants

Diagnosis: schizophrenia (Huangshan council schizophrenia standard 1984).

$\mathrm{N}=30$.

Age: $27-54$ years, mean -41 years.

Sex: 24M, 64F.

History: all chronically ill $>5$ years.

Setting: not stated.

Interventions

1. Fluphenazine decanoate: dose $25 \mathrm{mg} / \mathrm{mL}$ fortnightly injections. $\mathrm{N}=15$.

2. Haloperidol decanoate: dose $25 \mathrm{mg} / \mathrm{mL}$ monthly injections. $\mathrm{N}=15$

Outcomes

Behaviour: leaving the study early.

Adverse effects.

Unable to use -

Mental state: MIE (data unusable).

Adverse effects: SAS (data unusable).

Global state: CGI (not reported).

Notes

Risk of bias

\begin{tabular}{lll}
\hline Bias & Authors' judgement & Support for judgement \\
\hline $\begin{array}{l}\text { Random sequence generation (selection } \\
\text { bias) }\end{array}$ & Unclear risk & $\begin{array}{l}\text { "randomly assigned”. Method not re- } \\
\text { ported. }\end{array}$ \\
\hline $\begin{array}{l}\text { Allocation concealment (selection bias) } \\
\text { Unclear risk }\end{array}$ & Details of allocation concealment not re- \\
ported.
\end{tabular}

Blinding of participants and personnel Unclear risk (performance bias)

All outcomes

"double blind...patients and doctors did not know what kind of medicine to be applied till end of treatment". Blinding details not reported

Blinding of outcome assessment (detection Unclear risk bias)

"double blind...patients and doctors did

All outcomes not know what kind of medicine to be applied till end of treatment”. Blinding details not reported

Incomplete outcome data (attrition bias) High risk All outcomes

Losses to follow-up unbalanced between groups: $3(20 \%)$ in the haloperidol group and $1(7 \%)$ in the fluphenazine group 
Feng 1990 (Continued)

\begin{tabular}{|c|c|c|}
\hline Selective reporting (reporting bias) & High risk & $\begin{array}{l}\text { CGI outcome data not reported, no usable } \\
\text { data for MIE and SAS }\end{array}$ \\
\hline Other bias & Unclear risk & Source of funding not reported. \\
\hline
\end{tabular}

Frangos 1978

Methods

Allocation: randomised (randomisation code).

Blindness: double.

Duration: 16 weeks.

Design: parallel group.

Country: Greece.

Darticipants
Niagnosis: schizophrenia.
Age: $21-62$ years, mean -44 years.
Sex: $25 \mathrm{M}, 25 \mathrm{~F}$.
History: hospitalised for at least 2 years.
Setting: hospital.

Interventions

1. Fluphenazine decanoate: dose $25-150 \mathrm{mg} / \mathrm{IM}$, mean $76 \mathrm{mg} / \mathrm{IM}$ biweekly. $\mathrm{N}=25$.

2. Fluspirilene decanoate: dose 4-20 mg/IM, mean $12 \mathrm{mg} / \mathrm{IM}$ weekly. $\mathrm{N}=25$

\begin{tabular}{ll} 
Outcomes & Adverse effects: SE Rating Scale. \\
& Unable to use - \\
& Global state: CGI (no data). \\
& Mental state: BPRS (no SD). \\
& Behaviour: NOSIE (no SD). \\
\hline Notes & Authors contacted. \\
\hline
\end{tabular}

Risk of bias

\begin{tabular}{lll}
\hline Bias & Authors' judgement & Support for judgement \\
\hline $\begin{array}{l}\text { Random sequence generation (selection } \\
\text { bias) }\end{array}$ & Unclear risk & $\begin{array}{l}\text { "randomly allocated”, "according to a ran- } \\
\text { domisation code". }\end{array}$ \\
\hline Allocation concealment (selection bias) & Unclear risk & $\begin{array}{l}\text { Details of allocation concealment not re- } \\
\text { ported. }\end{array}$ \\
\hline
\end{tabular}

Blinding of participants and personnel Unclear risk (performance bias)

All outcomes

"all patients were treated with weekly injections; fluspirilene administered every week and fluphenazine decanoate every 2 weeks with placebo...in the between periods". Blinding details of personnel administering the injections not reported 


\section{Frangos 1978 (Continued)}

\begin{tabular}{|c|c|c|}
\hline $\begin{array}{l}\text { Blinding of outcome assessment (detection } \\
\text { bias) } \\
\text { All outcomes }\end{array}$ & Low risk & $\begin{array}{l}\text { "randomisation code unknown to the in- } \\
\text { vestigators involved with the patient evalu- } \\
\text { ation". "investigators concerned with drug } \\
\text { administration were not involved in the pa- } \\
\text { tient evaluations" }\end{array}$ \\
\hline $\begin{array}{l}\text { Incomplete outcome data (attrition bias) } \\
\text { All outcomes }\end{array}$ & Unclear risk & $\begin{array}{l}\text { No details reported, losses to follow-up not } \\
\text { reported. }\end{array}$ \\
\hline Selective reporting (reporting bias) & High risk & $\begin{array}{l}\text { Data reported incompletely: only P values } \\
\text { for BPRS, NOISE-30, and extrapyramidal } \\
\text { symptoms. Adverse events reported, pres- } \\
\text { ence/absence of serious adverse events not } \\
\text { reported }\end{array}$ \\
\hline Other bias & Unclear risk & Source of funding has not been reported. \\
\hline
\end{tabular}

\section{Goldstein 1978}

Allocation: randomised.
Blindness: single.
Duration: 6 weeks.
Design: 6 month follow-up (not controlled).
Country: United States.

Diagnosis: schizophrenia.
N $=104$.
Age: mean -23 years.
Sex: $45 \mathrm{M}, 37 \mathrm{~F}$.
History: acutely ill, 1st or 2nd admission, able to give informed consent.
Setting: community.

Interventions $\quad$ 1. Fluphenazine enanthate: dose (high) $1 \mathrm{~mL} / \mathrm{IM}$ biweekly. $\mathrm{N}=53$.

2. Fluphenazine enanthate: dose (low) $0.25 \mathrm{~mL} / \mathrm{IM}$ biweekly. $\mathrm{N}=51$

Outcomes

Behaviour: leaving the study early.

Unable to use -

Mental state: BPRS (no data).

Family therapy: non-clinical outcome (data unusable).

Analysis: last observation carried forward. 


\section{Goldstein 1978 (Continued)}

\begin{tabular}{|c|c|c|}
\hline $\begin{array}{l}\text { Random sequence generation (selection } \\
\text { bias) }\end{array}$ & Unclear risk & $\begin{array}{l}\text { "...patient was... assigned by a random } \\
\text { method". Details of method not reported }\end{array}$ \\
\hline Allocation concealment (selection bias) & Unclear risk & $\begin{array}{l}\text { Details of allocation concealment not re- } \\
\text { ported. }\end{array}$ \\
\hline $\begin{array}{l}\text { Blinding of participants and personnel } \\
\text { (performance bias) } \\
\text { All outcomes }\end{array}$ & Unclear risk & $\begin{array}{l}\text { " single blind...the patient was blind to the } \\
\text { dose level but the treating psychiatrist was } \\
\text { not" }\end{array}$ \\
\hline $\begin{array}{l}\text { Blinding of outcome assessment (detection } \\
\text { bias) } \\
\text { All outcomes }\end{array}$ & Low risk & $\begin{array}{l}\text { "...all ratings of clinical behavior were car- } \\
\text { ried out by raters blind to drug and family } \\
\text { therapy status." }\end{array}$ \\
\hline $\begin{array}{l}\text { Incomplete outcome data (attrition bias) } \\
\text { All outcomes }\end{array}$ & Unclear risk & $\begin{array}{l}\text { "...eight withdrew after release from the } \\
\text { hospital... treatment refusers came from } \\
\text { three groups; high dose therapy (two), low } \\
\text { dose therapy (three), low dose no ther- } \\
\text { apy (three)." None of the participants from } \\
\text { high dose no therapy dropped out }\end{array}$ \\
\hline Selective reporting (reporting bias) & High risk & $\begin{array}{l}\text { Relapse reported. Data for BPRS not re- } \\
\text { ported as mean SD, data for family therapy } \\
\text { not reported }\end{array}$ \\
\hline Other bias & Low risk & $\begin{array}{l}\text { Supported by grants from National Insti- } \\
\text { tute for Mental Health }\end{array}$ \\
\hline
\end{tabular}

Hirsch 1975

\begin{tabular}{ll}
\hline Methods & Allocation: randomised. \\
& Blindness: double. \\
& Duration: 7 months. \\
& Design: parallel group. \\
& Country: Norway. \\
\hline Participants & Diagnosis: schizophrenia. \\
& $\begin{array}{l}\text { N = 81. } \\
\text { Age: under } 67 \text { years. }\end{array}$ \\
& Sex: male \& female. \\
& History: chronically ill. \\
& Seting: community. \\
\hline Interventions & 1. Fluphenazine decanoate: dose monthly average 25 mg/IM. N = 40. \\
& 2. Placebo. $\mathrm{N}=41$. \\
\hline
\end{tabular}


Hirsch 1975 (Continued)

\begin{tabular}{|c|c|c|}
\hline Outcomes & \multicolumn{2}{|l|}{$\begin{array}{l}\text { Global state: relapse. } \\
\text { Behaviour: leaving the study early. } \\
\text { Unable to use - } \\
\text { Mental state: PSE (data unusable). } \\
\text { Behaviour: SPS (data unusable). }\end{array}$} \\
\hline Notes & \multicolumn{2}{|c|}{ Unable to complete risk of bias table - PDF missing. } \\
\hline \multicolumn{3}{|l|}{ Risk of bias } \\
\hline Bias & Authors' judgement & Support for judgement \\
\hline $\begin{array}{l}\text { Random sequence generation (selection } \\
\text { bias) }\end{array}$ & Unclear risk & $\begin{array}{l}\text { "Randomly allocated by a research assis- } \\
\text { tant", no further details reported }\end{array}$ \\
\hline Allocation concealment (selection bias) & Unclear risk & $\begin{array}{l}\text { Details of allocation concealment not re- } \\
\text { ported. }\end{array}$ \\
\hline $\begin{array}{l}\text { Blinding of participants and personnel } \\
\text { (performance bias) } \\
\text { All outcomes }\end{array}$ & Unclear risk & $\begin{array}{l}\text { "Double-blind", "A number of checks by } \\
\text { questionnaires filled out by the doctors, } \\
\text { nurses, and the patient's general practi- } \\
\text { tioner confirmed that the double-blind } \\
\text { procedure had been successful" }\end{array}$ \\
\hline $\begin{array}{l}\text { Blinding of outcome assessment (detection } \\
\text { bias) } \\
\text { All outcomes }\end{array}$ & Unclear risk & $\begin{array}{l}\text { "Double-blind", details of blinding of out- } \\
\text { come assessors not reported }\end{array}$ \\
\hline $\begin{array}{l}\text { Incomplete outcome data (attrition bias) } \\
\text { All outcomes }\end{array}$ & Low risk & $\begin{array}{l}\text { "One patient had [drugs from other } \\
\text { sources], and he was excluded from the } \\
\text { analysis" }\end{array}$ \\
\hline Selective reporting (reporting bias) & High risk & $\begin{array}{l}\text { Some outcomes were not fully reported: no } \\
\text { data for PSE and SPS }\end{array}$ \\
\hline Other bias & Unclear risk & Source of funding not reported. \\
\hline
\end{tabular}

\section{Hogarty 1979}

Methods

Allocation: randomised.

Blindness: double.

Duration: 2 years.

Design: parallel study.

Country: United States.
Diagnosis: schizophrenia.

$$
\mathrm{N}=105 \text {. }
$$


Hogarty 1979 (Continued)

Age: $18-55$ years, mean -34 years.

Sex: 46M, 54F.

History: received no other psychotropic medication, able to give informed consent.

Setting: community.

Interventions

1. Fluphenazine decanoate: dose 12.5-125 mg/IM, mean $25 \mathrm{mg} / \mathrm{IM}$ biweekly. $\mathrm{N}=27$.

2. Fluphenazine hydrochloride (oral): dose $2.5-40 \mathrm{mg} / \mathrm{IM}$, mean $2.5 \mathrm{mg} / \mathrm{IM}$ daily. $\mathrm{N}=$

25

Outcomes

Behaviour: leaving the study early.

Unable to use -

Mental state: BPRS (no SD).

Behaviour: KAS (no data).

Adverse effects: SSI, SEC, HSC, TESS (no data).

Notes

Last observation carried forward

Risk of bias

Bias

Authors' judgement

Support for judgement

Random sequence generation (selection Unclear risk bias)

"randomly assigned". Method not reported.

Allocation concealment (selection bias) Unclear risk

Details of allocation concealment not reported.

Blinding of participants and personnel Unclear risk (performance bias)

All outcomes

“double-blind”. Subjects blinded; “...providing each patient with an injection and tablets i.e. active injections of fluphenazine decanoate with inactive placebo tablets or placebo injections...with active...tablets. The active and placebo forms of the medication were identical in appearance. " Blinding of details of personnel not reported

Blinding of outcome assessment (detection Unclear risk Blinding of details not reported.

bias)

All outcomes

Incomplete outcome data (attrition bias) Unclear risk All outcomes

Insufficient information: number of subjects allocated per group unclear; "...the analyses included only patients known to be receiving their injection... and who appeared for their prescribed oral medication, independent whether they... took their tablets or not." 
Hogarty 1979 (Continued)

\begin{tabular}{|c|c|c|}
\hline Selective reporting (reporting bias) & High risk & $\begin{array}{l}\text { Outcomes not reported (SSI, HCI, KAS) } \\
\text { or incompletely reported (BPRS) }\end{array}$ \\
\hline Other bias & Low risk & $\begin{array}{l}\text { “...supported by Psychopharmacology Re- } \\
\text { search Branch, National Institute of Men- } \\
\text { tal Health" }\end{array}$ \\
\hline
\end{tabular}

\section{Hogarty 1988}

Methods
Allocation: randomised, stratification by dose $\&$ household EE.

Blindness: double.

Duration: 2 years.

Design: dosage study.

Country: United States.

Outcomes

Behaviour: leaving the study early.

Unable to use -

Mental state: BPRS, SCL-90 (no data).

Adverse effects: MRQ (no data).

Notes

Risk of bias

\begin{tabular}{l|ll}
\hline Bias & Authors' judgement & Support for judgement \\
\hline $\begin{array}{l}\text { Random sequence generation (selection } \\
\text { bias) }\end{array}$ & Unclear risk & "random". Method not reported. \\
\hline $\begin{array}{l}\text { Allocation concealment (selection bias) } \\
\text { Unclear risk }\end{array}$ & Details of allocation concealment not re- \\
\hline
\end{tabular}

Blinding of participants and personnel Unclear risk (performance bias)

All outcomes

"double-blind”. “...injections were provided by two non blinded nurses who scrupulously concealed the information on 
Hogarty 1988 (Continued)

dose assignment from patients and the treating clinical research team. Blinding details not reported

Blinding of outcome assessment (detection Unclear risk bias)

All outcomes All outcomes

Selective reporting (reporting bias) High risk

Other bias $\quad$ Low risk
Incomplete outcome data (attrition bias) Low risk

"double-blind". "...injections were provided by two non blinded nurses who scrupulously concealed the information on dose assignment from patients and the treating clinical research team. Blinding details not reported

Drop-outs have been reported per group of intervention. Overall losses to follow-up data balanced across intervention groups: $14(42 \%)$ drop-outs at standard dose and $16(43 \%)$ drop-outs at minimal dose, with similar reasons for missing data. Dropouts for clinical reasons (relapse) also balanced

Outcome data (BPRS, MRQ, SCL-90, social performance) incompletely reported

Allocation: randomised.

Blindness: not described.

Duration: 6 months.

Design: parallel group.

Country: not reported.

Participants

Diagnosis: schizophrenia (ICD-10).

$\mathrm{N}=41$.

Age: $21-55$. mean -41 years.

Sex: 17M, 24F.

History: not stated.

Setting: not stated.

Interventions

1 Fluphenazine decanoate: dose $99.3 \mathrm{mg} / \mathrm{IM} /$ month. $\mathrm{N}=21$.

2. Haloperidol decanoate: dose $47.3 \mathrm{mg} / \mathrm{month} . \mathrm{N}=20$.

Outcomes

Behaviour: leaving the study early.

Unable to use -

Global state: CGI (data unusable).

Mental state: PANSS (data unusable). 
Hranov 1998 (Continued)

Adverse effects: UKU (data unusable).

\begin{tabular}{|c|c|c|}
\hline Notes & & \\
\hline \multicolumn{3}{|l|}{ Risk of bias } \\
\hline Bias & Authors' judgement & Support for judgement \\
\hline $\begin{array}{l}\text { Random sequence generation (selection } \\
\text { bias) }\end{array}$ & Unclear risk & “ randomised”. Method not reported. \\
\hline Allocation concealment (selection bias) & Unclear risk & $\begin{array}{l}\text { Details of allocation concealment not re- } \\
\text { ported. }\end{array}$ \\
\hline $\begin{array}{l}\text { Blinding of participants and personnel } \\
\text { (performance bias) } \\
\text { All outcomes }\end{array}$ & Unclear risk & Blinding not reported. \\
\hline $\begin{array}{l}\text { Blinding of outcome assessment (detection } \\
\text { bias) } \\
\text { All outcomes }\end{array}$ & Unclear risk & Blinding not reported. \\
\hline $\begin{array}{l}\text { Incomplete outcome data (attrition bias) } \\
\text { All outcomes }\end{array}$ & High risk & $\begin{array}{l}6(30 \%) \text { participants in the fluphenazine } \\
\text { group and } 4(19 \%) \text { in the haloperidol } \\
\text { group left the study early. Reasons for leav- } \\
\text { ing early not reported }\end{array}$ \\
\hline Selective reporting (reporting bias) & High risk & Outcome results reported incompletely. \\
\hline Other bias & Unclear risk & Source of funding not reported. \\
\hline
\end{tabular}

Jain 1975

\begin{tabular}{ll}
\hline Methods & Allocation: randomised. \\
& Blindness: double. \\
& Duration: 20 weeks, preceded by 2-week washout. \\
Design: parallel group. \\
Country: Canada. \\
\hline
\end{tabular}

\begin{tabular}{ll}
\hline Participants & Diagnosis: schizophrenia. \\
& N $=30$. \\
Age: $24-61$ years, mean -49 years. \\
Sex: 14F, 16M. \\
History: hospitalised for under 1 year. \\
Setting: hospital.
\end{tabular}

Interventions

1. Fluphenazine enanthate: dose $125 \mathrm{mg} / \mathrm{IM}$ biweekly. $\mathrm{N}=15$.

2. Pipothiazine palmitate: dose $250 \mathrm{mg} / \mathrm{IM}$ biweekly. $\mathrm{N}=15$. 
Jain 1975 (Continued)

\begin{tabular}{ll}
\hline Outcomes & Global state: CGI. \\
& Behaviour: leaving the study early. \\
& Adverse effects: TESS. \\
& Unable to use - \\
& Mental state: BPRS (no data). \\
\hline Notes & $73 \%$ drop-out rate in the PP group, data not usable. \\
\hline
\end{tabular}

Risk of bias

\begin{tabular}{lll}
\hline Bias & Authors' judgement & Support for judgement \\
\hline $\begin{array}{l}\text { Random sequence generation (selection } \\
\text { bias) }\end{array}$ & Unclear risk & “randomised”. Method not reported. \\
\hline Allocation concealment (selection bias) & Unclear risk & $\begin{array}{l}\text { Details of allocation concealment not re- } \\
\text { ported. }\end{array}$ \\
\hline
\end{tabular}

Blinding of participants and personnel Unclear risk (performance bias)

“double-blind". Blinding details not reAll outcomes ported.

Blinding of outcome assessment (detection Unclear risk bias)

"double-blind". Blinding details not reported.

All outcomes

Incomplete outcome data (attrition bias) High risk All outcomes

Losses to follow-up unbalanced between groups: 4 (27\%) participants left the study early in the pipothiazine group and $1(5 \%)$ in the fluphenazine group

Selective reporting (reporting bias) High risk

Outcome data incompletely reported for BPRS.

Other bias

Low risk

Partially supported by Public Health Service Grant from the Department of Health, Education and Welfare, Washington D.C

\section{Javed 1991}

Methods

Allocation: randomised.

Blindness: double.

Duration: 12 weeks.

Design: parallel group.

Country: Pakistan. 
Javed 1991 (Continued)

\begin{tabular}{ll}
\hline Participants & Diagnosis: schizophrenia (DSM III). \\
& $\mathrm{N}=45$. \\
& Age: $\mathrm{mean}-50$ years. \\
& Sex: $33 \mathrm{M}, 5 \mathrm{~F}$. \\
& History: stabilised for 6 months on neuroleptics, involved in rehabilitation, duration \\
& illness 13 years. \\
& Setting: not stated. \\
\hline Interventions & 1. Fluphenazine decanoate: dose $25 \mathrm{mg} / \mathrm{IM}$ biweekly. $\mathrm{N}=20$. \\
\hline 2. Flupenthixol decanoate: dose $40 \mathrm{mg} / \mathrm{IM}$ biweekly. $\mathrm{N}=18$. \\
\hline \multirow{2}{*}{ Outcomes } & Behaviour: leaving the study early. \\
& Mental state: HRSD. \\
Adverse effects: $\mathrm{EPSE}, \mathrm{SE}$ checklist. \\
Unable to use - \\
Global state: CGI (no SD). \\
Mental state: BPRS (no SD). \\
\hline Authors contacted. \\
\hline Notes
\end{tabular}

Risk of bias

\begin{tabular}{|c|c|c|}
\hline Bias & Authors' judgement & Support for judgement \\
\hline $\begin{array}{l}\text { Random sequence generation (selection } \\
\text { bias) }\end{array}$ & Unclear risk & "randomised". Method not reported. \\
\hline Allocation concealment (selection bias) & Unclear risk & $\begin{array}{l}\text { Details of allocation concealment not re- } \\
\text { ported. }\end{array}$ \\
\hline $\begin{array}{l}\text { Blinding of participants and personnel } \\
\text { (performance bias) } \\
\text { All outcomes }\end{array}$ & Unclear risk & $\begin{array}{l}\text { "double-blind". Blinding details not re- } \\
\text { ported. }\end{array}$ \\
\hline $\begin{array}{l}\text { Blinding of outcome assessment (detection } \\
\text { bias) } \\
\text { All outcomes }\end{array}$ & Unclear risk & $\begin{array}{l}\text { "double-blind". Blinding details not re- } \\
\text { ported. }\end{array}$ \\
\hline $\begin{array}{l}\text { Incomplete outcome data (attrition bias) } \\
\text { All outcomes }\end{array}$ & Unclear risk & $\begin{array}{l}7 / 45 \text { patients dropped out of the study. } 1 / 7 \\
\text { died and } 6 / 7 \text { "dropped out for reasons not } \\
\text { related to the treatment...did not keep up } \\
\text { their appointments." The number of drop- } \\
\text { outs not reported across the intervention } \\
\text { group }\end{array}$ \\
\hline
\end{tabular}

Selective reporting (reporting bias) High risk

All outcomes reported as mean scores (without SD): CGI, BPRS, Hamilton, SAS, Side effects checklist (side effects not 
Javed 1991 (Continued)

described). Serious adverse events not reported

\begin{tabular}{l|l} 
Other bias $\quad$ Unclear risk $\quad$ Source of funding not reported.
\end{tabular}

Jolley 1990

\begin{tabular}{l|l} 
Methods & Allocation: randomised. \\
& Blindness: double. \\
& Duration: 2 years. \\
Design: 2 year follow-up. \\
Country: United KIngdom.
\end{tabular}

Participants

Diagnosis: schizophrenia (DSM III).

$\mathrm{N}=54$.

Age: not stated.

Sex: not stated.

History: stable patients in remission, who has been free of florid symptoms (delusions, hallucinations, bizarre behaviour and thought disorders) for at least 6 months.

Setting: community.

Interventions

1. Fluphenazine decanoate: (dosage not reported). $\mathrm{N}=27$.

2. Placebo. (dosage not reported). $\mathrm{N}=27$.

Outcomes

Death.

Behaviour: leaving the study early.

Adverse effects: AIMS.

Unable to use -

Adverse effects: SAS (data unusable).

Social ability: SAS (non clinical outcomes, data unusable).

Notes

Risk of bias

\begin{tabular}{l|ll}
\hline Bias & Authors' judgement & Support for judgement \\
\hline $\begin{array}{l}\text { Random sequence generation (selection } \\
\text { bias) }\end{array}$ & Unclear risk & “randomised”. Method not reported. \\
\hline Allocation concealment (selection bias) & Unclear risk & $\begin{array}{l}\text { Details of allocation concealment not re- } \\
\text { ported. }\end{array}$ \\
\hline
\end{tabular}

Blinding of participants and personnel Unclear risk (performance bias)

"double-blind". Blinding details not reAll outcomes ported. 
Jolley 1990 (Continued)

\begin{tabular}{|c|c|c|}
\hline $\begin{array}{l}\text { Blinding of outcome assessment (detection } \\
\text { bias) } \\
\text { All outcomes }\end{array}$ & Unclear risk & $\begin{array}{l}\text { "double-blind". Blinding details not re- } \\
\text { ported. }\end{array}$ \\
\hline $\begin{array}{l}\text { Incomplete outcome data (attrition bias) } \\
\text { All outcomes }\end{array}$ & Low risk & $\begin{array}{l}\text { Losses to follow up balanced across the } \\
\text { groups: } 5(19 \%) \text { in the placebo group and } \\
6(22 \%) \text { in the treatment group }\end{array}$ \\
\hline Selective reporting (reporting bias) & High risk & Not all outcomes have been fully reported. \\
\hline Other bias & Low risk & $\begin{array}{l}\text { Supported by grants from The Department } \\
\text { for Health and Social Security, North West } \\
\text { Thames Regional Research Fund and the } \\
\text { Priory hospital }\end{array}$ \\
\hline
\end{tabular}

Ju 2000

Methods

Allocation: Randomly assigned.

Blindness: not mentioned.

Duration: 12 weeks.

Design: parallel.

Country: China.

\begin{tabular}{ll}
\hline Participants & Diagnosis: schizophrenia (CCMD-2-R). \\
& $\begin{array}{l}\mathrm{N}=152 . \\
\text { Age: } \mathrm{mean}-31 \text { years, range } 18-45 . \\
\text { Sex: } 93 \mathrm{M}, 59 \mathrm{~F} \\
\text { History: course of disease 2-6 years. } \\
\text { Setting: hospital. }\end{array}$ \\
\hline Interventions & $\begin{array}{l}\text { 1. Fluphenazine decanoate: start from } 25 \mathrm{mg} \text {, controlled dose at range of } 25-125 \mathrm{mg}, \\
\text { given once for every } 2 \text { weeks. } \mathrm{N}=49 . \\
\text { 2. Pipothiazine palmitate: start from } 25 \mathrm{mg}, \text { controlled dose at range of } 25-125 \mathrm{mg}, \\
\text { given once for every } 4 \text { weeks. } \mathrm{N}=103\end{array}$ \\
\hline Outcomes & $\begin{array}{l}\text { Global state: clinically important global change. } \\
\text { Behaviour: leaving the study early. } \\
\text { Mental state: BPRS, SANS, SAPS. } \\
\text { Adverse effects: TESS, RSESE. }\end{array}$ \\
\hline
\end{tabular}

Notes

Risk of bias

Bias

Authors' judgement

Support for judgement 


\begin{tabular}{l|l|l}
$\begin{array}{l}\text { Random sequence generation (selection } \\
\text { bias) }\end{array}$ & Unclear risk & Randomised, no further details reported. \\
\hline Allocation concealment (selection bias) & Unclear risk & $\begin{array}{l}\text { Details of allocation concealment not re- } \\
\text { ported. }\end{array}$ \\
\hline
\end{tabular}

Blinding of participants and personnel Unclear risk

Not mentioned.

(performance bias)

All outcomes

\begin{tabular}{lll}
\hline $\begin{array}{l}\text { Blinding of outcome assessment (detection } \\
\text { bias) } \\
\text { All outcomes }\end{array}$ & Unclear risk & Not mentioned. \\
\hline $\begin{array}{l}\text { Incomplete outcome data (attrition bias) } \\
\text { All outcomes }\end{array}$ & Low risk & $\begin{array}{l}\text { All the patients complete the 12-week treat- } \\
\text { ment. }\end{array}$ \\
\hline $\begin{array}{l}\text { Selective reporting (reporting bias) } \\
\text { Other bias }\end{array}$ & Low risk & All the outcomes were reported. \\
\hline
\end{tabular}

Kane 1978

\begin{tabular}{|c|c|}
\hline Methods & $\begin{array}{l}\text { Allocation: randomised. } \\
\text { Blindness: unclear. } \\
\text { Duration: } 2 \text { weeks. } \\
\text { Design: parallel. } \\
\text { Country: United States. }\end{array}$ \\
\hline Participants & $\begin{array}{l}\text { Diagnosis: schizophrenia (criteria not reported). } \\
\mathrm{N}=49 . \\
\text { Age: } 13-37 \text { years. } \\
\text { Sex: } 29 \mathrm{M}, 20 \mathrm{~F} \text {. } \\
\text { History: Recently discharged schizophrenic patients stabilized on oral antipsychotic med- } \\
\text { ication and procyclidine. } \\
\text { Setting: community. }\end{array}$ \\
\hline Interventions & $\begin{array}{l}\text { 1. Fluphenazine enanthate, } 12.5 \mathrm{mg} \text { and } 2 \mathrm{mcg} \text { benztropine mesylate; IM. } \mathrm{N}=12 \\
\text { 2. Fluphenazine decanoate, } 12.5 \mathrm{mg} \text { and } 2 \mathrm{mcg} \text { benztropine mesylate; IM. } \mathrm{N}=13 \\
\text { 3. Fluphenazine enanthate, } 18.75 \mathrm{mg} \mathrm{IM} . \mathrm{N}=14 \\
\text { 4. Fluphenazine decanoate, } 18.75 \mathrm{mg} \text {; } \mathrm{IM} . \mathrm{N}=10\end{array}$ \\
\hline Outcomes & Adverse effects: SAS \\
\hline Notes & $\begin{array}{l}11 / 23 \text { patients receiving fluphenazine decanoate and } 13 / 26 \text { patients receiving } \\
\text { fluphenazine enanthate also took oral antipsychotics. } 3 \text { patients in the fluphenazine enan- } \\
\text { thate and } 2 \text { in the fluphenazine decanoate group received imipramine and } 1 \text { patient in } \\
\text { each group received lithium }\end{array}$ \\
\hline
\end{tabular}


The two fluphenazine decanoate and two fluphenazine enanthate groups were combined in the analysis as the doses are standard doses in each group

\section{Risk of bias}

\begin{tabular}{l|l|l} 
Bias & Authors' judgement & Support for judgement \\
\hline $\begin{array}{l}\text { Random sequence generation (selection } \\
\text { bias) }\end{array}$ & Unclear risk & $\begin{array}{l}\text { "randomly assigned”. Method not re- } \\
\text { ported. }\end{array}$ \\
\hline Allocation concealment (selection bias) & Unclear risk & $\begin{array}{l}\text { Details of allocation concealment not re- } \\
\text { ported. }\end{array}$ \\
\hline
\end{tabular}

Blinding of participants and personnel Unclear risk (performance bias)

Blinding details of personnel and patients All outcomes not reported.

Blinding of outcome assessment (detection Low risk bias)

All outcomes

Incomplete outcome data (attrition bias) Low risk All outcomes

Selective reporting (reporting bias)

High risk

Other bias
"An examination of extrapyramidal side effects was carried out ...by a physician blind to the type of injection received"

Losses to follow-up or missing data balanced across intervention groups, with similar reasons for missing data. "The data was incomplete in 11 patients (22\%) due to noncompliance. 10 of these 11 patients missed the 2-week examination. There was no significant difference in compliance between the fluphenazine enanthate and fluphenazine decanoate groups"

The study reports only the primary outcome i.e. extrapyramidal symptoms. Other expected outcomes (e.g. relapse, hospital admission, non-EPS adverse events) have not been reported

Source of funding not reported. 
Allocation: randomised.

Blindness: double.

Duration: 1 year.

Design: dosage study.

Country: United States.

\begin{tabular}{ll}
\hline Participants & Diagnosis: schizophrenia (RDC). \\
& $\mathrm{N}=126$. \\
& Age: $17-60$ years, mean -29 years. \\
& Sex: $63 \mathrm{M}, 37 \mathrm{~F}$. \\
& History: in state of remission, able to give informed consent. \\
& Setting: community. \\
\hline Interventions & 1. Fluphenazine decanoate (low dose): dose $1.25-5.0 \mathrm{mg} / \mathrm{IM}$ biweekly. $\mathrm{N}=62$. \\
& 2. Fluphenazine decanoate (standard dose): dose $12.5-50 \mathrm{mg} / \mathrm{IM}$ biweekly. $\mathrm{N}=64$ \\
\hline \multirow{2}{*}{ Outcomes } & Behaviour: leaving the study early. \\
& Adverse effects: SDS, SAS. \\
& $\begin{array}{l}\text { Unable to use - } \\
\text { Global State: CGI (no data). } \\
\text { Mental State: BPRS (no data). } \\
\text { Behaviour: SAS-R (data unusable). }\end{array}$ \\
\hline
\end{tabular}

Notes

Risk of bias

\begin{tabular}{|c|c|c|}
\hline Bias & Authors' judgement & Support for judgement \\
\hline $\begin{array}{l}\text { Random sequence generation (selection } \\
\text { bias) }\end{array}$ & Unclear risk & $\begin{array}{l}\text { "randomly assigned". Method not re- } \\
\text { ported. }\end{array}$ \\
\hline Allocation concealment (selection bias) & Unclear risk & $\begin{array}{l}\text { Details of allocation concealment not re- } \\
\text { ported. }\end{array}$ \\
\hline $\begin{array}{l}\text { Blinding of participants and personnel } \\
\text { (performance bias) } \\
\text { All outcomes }\end{array}$ & Unclear risk & $\begin{array}{l}\text { "double-blind". Blinding details not re- } \\
\text { ported. }\end{array}$ \\
\hline
\end{tabular}

\begin{tabular}{|c|c|c|}
\hline $\begin{array}{l}\text { Blinding of outcome assessment (detection } \\
\text { bias) } \\
\text { All outcomes }\end{array}$ & Unclear risk & Blinding details not reported. \\
\hline $\begin{array}{l}\text { Incomplete outcome data (attrition bias) } \\
\text { All outcomes }\end{array}$ & Unclear risk & Losses to follow-up not reported. \\
\hline Selective reporting (reporting bias) & High risk & $\begin{array}{l}\text { Outcome data for BPRS and CGI not re- } \\
\text { ported. }\end{array}$ \\
\hline
\end{tabular}


Kane 1983 (Continued)

\begin{tabular}{|c|c|c|}
\hline Other bias & Low risk & $\begin{array}{l}\text { "...supported, in part, by grants...from the } \\
\text { National Institute of Mental Health" }\end{array}$ \\
\hline
\end{tabular}

Kaneno 1991

Methods

Allocation: randomised.

Blindness: double.

Duration: 6 months.

Design: parallel group.

County: Japan.

\begin{tabular}{ll} 
Participants & Diagnosis: schizophrenia. \\
& N $=259$. \\
Age: $20-65$ years. & Sex: $168 \mathrm{M}, 91 \mathrm{~F}$. \\
& $\begin{array}{l}\text { History: not stated. } \\
\text { Setting: hospital and community. }\end{array}$ \\
\hline Interventions & $\begin{array}{l}\text { 1. Fluphenazine decanoate: dose } 12-50 \mathrm{mg} / \mathrm{ml} / \mathrm{IM} \text { administered } 6 \text { times at } 4 \text {-week in- } \\
\text { tervals. } \mathrm{N}=127 .\end{array}$ \\
& $\begin{array}{l}\text { 2. Haloperidol: dose } 3.0-12.1 \mathrm{mg} \text { administered } 6 \text { times. } \mathrm{N}=132 \\
\text { Outcomes }\end{array}$ \\
& $\begin{array}{l}\text { Suicide. } \\
\text { Behaviour: leaving the study early. } \\
\text { Unable to use - } \\
\text { Mental state: BPRS, KORS (no SD). } \\
\text { Adverse effects: OSR (no SD). }\end{array}$ \\
\hline Notes & Article mostly written in Japanese, risk of bias assessed from the English section only
\end{tabular}

Risk of bias

\begin{tabular}{|c|c|c|}
\hline Bias & Authors' judgement & Support for judgement \\
\hline $\begin{array}{l}\text { Random sequence generation (selection } \\
\text { bias) }\end{array}$ & Unclear risk & Randomisation details not reported. \\
\hline
\end{tabular}

bias)

Allocation concealment (selection bias) Unclear risk

Details of allocation concealment not reported.

Blinding of participants and personnel Unclear risk

"double blind".

(performance bias)

All outcomes

Blinding of outcome assessment (detection Unclear risk

bias)

All outcomes

Blinding details not reported. 
Kaneno 1991 (Continued)

\begin{tabular}{l|l|l}
$\begin{array}{l}\text { Incomplete outcome data (attrition bias) } \\
\text { All outcomes }\end{array}$ & Unclear risk & $\begin{array}{l}\text { All participants (completed and termi- } \\
\text { nated) were entered to the analysis. No } \\
\text { missing data }\end{array}$ \\
\hline Selective reporting (reporting bias) & High risk & $\begin{array}{l}\text { Data not reported fully for mental state and } \\
\text { adverse effects }\end{array}$ \\
\hline Other bias & Unclear risk & The paper is in Japanese. \\
\hline
\end{tabular}

Kelly 1977

Methods

Allocation: randomised.

Blindness: single.

Duration: 9 months.

Design: parallel group.

Country: UK.

\begin{tabular}{ll} 
Participants & Diagnosis: schizophrenia (Schneider 1st Rank). \\
& $\mathrm{N}=60$. \\
& Age: $18-65$ years, mean -42 years. \\
& Sex: $18 \mathrm{M}, 35 \mathrm{~F}$. \\
& History: not stated. \\
& Setting: community. \\
& Excluded: epilepsy, ECT, brain damage, pregnancy, marked metal retardation or parkin- \\
& sonism \\
\hline Interventions & 1. Fluphenazine decanoate: dose $1 \mathrm{ml} / \mathrm{IM}$ every 3 weeks. $\mathrm{N}=30$. \\
& 2. Flupenthixol decanoate: dose $1 \mathrm{ml} / \mathrm{IM}$ every 3 weeks. $\mathrm{N}=30$ \\
& Medication adjusted weeks $1-9$, stable thereafter. \\
\hline \multirow{2}{*}{ Outcomes } & Leaving the study early. \\
& Global state: relapse. \\
& Unable to use - \\
& Mental state: BPRS (no SD). \\
Adverse effects: EPS (no data).
\end{tabular}

Notes

Risk of bias

\begin{tabular}{|c|c|c|}
\hline Bias & Authors' judgement & Support for judgement \\
\hline $\begin{array}{l}\text { Random sequence generation (selection } \\
\text { bias) }\end{array}$ & Unclear risk & $\begin{array}{l}\text { "randomly allocated". Method not re- } \\
\text { ported. }\end{array}$ \\
\hline Allocation concealment (selection bias) & Low risk & $\begin{array}{l}\text { "the key to the allocation [was] known only } \\
\text { to the hospital pharmacist and to the nurse } \\
\text { administering the injections" }\end{array}$ \\
\hline
\end{tabular}


Kelly 1977 (Continued)

Blinding of participants and personnel Unclear risk (performance bias)

All outcomes

Blinding of outcome assessment (detection Unclear risk

bias)

All outcomes

Incomplete outcome data (attrition bias) Low risk

All outcomes

Selective reporting (reporting bias)

High risk

Unclear risk

Other bias
Insufficient information: patients may have blinded, nurses were unblinded. “...the key to the allocation [was] known only to the nurse administering the injections."

Blinding details not reported. reported for BPRS.

Number and reasons for missing data balanced. 60 randomised, 53 completed patients. 6 patients relapsed ( 3 in each group and 1 became pregnant

Source of funding not reported.

Keskiner 1971

Methods

Allocation: randomised.

Blindness: double.

Duration: 8 weeks ( 4 weeks before cross-over).

Design: cross-over.

Country: United States.

Darticipants
N $=12$.
Age: $25-51$ years, mean -38 years.
Sex: $3 \mathrm{M}, 9 \mathrm{~F}$.
History: duration of illness $5-25$ years (mean 14 years).
Setting: hospital.

Interventions

1. Fluphenazine enanthate: dose $1 \mathrm{mg} / \mathrm{kg}$ body weight $/ \mathrm{IM}$ single dose. $\mathrm{N}=6$.

2. Fluphenazine decanoate: dose $1 \mathrm{mg} / \mathrm{kg}$ body weight $\mathrm{IM}$ single dose. $\mathrm{N}=6$

Outcomes

Behaviour: leaving the study early.

Unable to use -

Global state: GES (data unusable).

Mental state: BPRS (data unusable).

Adverse effects: TESS (data unusable).

Notes

Risk of bias 


\begin{tabular}{|c|c|c|}
\hline Bias & Authors' judgement & Support for judgement \\
\hline $\begin{array}{l}\text { Random sequence generation (selection } \\
\text { bias) }\end{array}$ & Unclear risk & $\begin{array}{l}\text { "randomly separated". Method not re- } \\
\text { ported. }\end{array}$ \\
\hline Allocation concealment (selection bias) & Unclear risk & $\begin{array}{l}\text { Details of allocation concealment not re- } \\
\text { ported. }\end{array}$ \\
\hline $\begin{array}{l}\text { Blinding of participants and personnel } \\
\text { (performance bias) } \\
\text { All outcomes }\end{array}$ & Unclear risk & $\begin{array}{l}\text { "double-blind". Blinding details not re- } \\
\text { ported. }\end{array}$ \\
\hline $\begin{array}{l}\text { Blinding of outcome assessment (detection } \\
\text { bias) } \\
\text { All outcomes }\end{array}$ & Unclear risk & $\begin{array}{l}\text { "double-blind". Blinding details not re- } \\
\text { ported. }\end{array}$ \\
\hline $\begin{array}{l}\text { Incomplete outcome data (attrition bias) } \\
\text { All outcomes }\end{array}$ & Low risk & All participants completed the study. \\
\hline Selective reporting (reporting bias) & High risk & Reports reported incompletely. \\
\hline Other bias & Low risk & $\begin{array}{l}\text { "Supported, in part, by Psychiatric Re- } \\
\text { search Foundation of Missouri and The } \\
\text { Squibb Institute for Medical Research, new } \\
\text { brunswick, NJ" }\end{array}$ \\
\hline
\end{tabular}

\section{Khazaie 2005}

\begin{tabular}{|c|c|}
\hline Methods & $\begin{array}{l}\text { Allocation: randomised. } \\
\text { Blindness: double blind. } \\
\text { Duration: } 54 \text { weeks. } \\
\text { Design: parallel. } \\
\text { Country: Iran. }\end{array}$ \\
\hline Participants & $\begin{array}{l}\text { Diagnosis: schizophrenia or schizoaffective disorder (DSM IV). } \\
\mathrm{N}=50 \text {. } \\
\text { Age: mean }-34 \text { years. } \\
\text { Sex: } 36 \mathrm{M}, 14 \mathrm{~F} \text {. } \\
\text { History: duration of illness mean } 12.0 \text { years }(\mathrm{SD}=6.6) \\
\text { Setting: community. }\end{array}$ \\
\hline Interventions & $\begin{array}{l}\text { 1. Fluphenazine decanoate: } 25 \mathrm{mg} \text { every } 2 \text { weeks. } \mathrm{N}=25 \text {. } \\
\text { 2. Fluphenazine decanoate: } 25 \mathrm{mg} \text { every } 6 \text { weeks. } \mathrm{N}=25 \text {. } \\
\text { All patients, in both groups, received injections every two weeks; group } 1 \text { received } \\
\text { fluphenazine at each time i.e. every two weeks and group } 2 \text { received placebo injections } \\
\text { between each fluphenazine injection }\end{array}$ \\
\hline
\end{tabular}




\begin{tabular}{|c|c|c|}
\hline Outcomes & \multicolumn{2}{|c|}{$\begin{array}{l}\text { Mental state: BPRS. } \\
\text { Clinical impression: CGI. } \\
\text { Quality of life: Quality of life Scale. } \\
\text { Extrapyramidal adverse effects: Maryland Psychiatric Research Center Involuntary } \\
\text { Movement Scale } \\
\text { Not used in review - } \\
\text { General functioning: Level of Functioning Scale. }\end{array}$} \\
\hline Notes & & \\
\hline \multicolumn{3}{|l|}{ Risk of bias } \\
\hline Bias & Authors' judgement & Support for judgement \\
\hline $\begin{array}{l}\text { Random sequence generation (selection } \\
\text { bias) }\end{array}$ & Unclear risk & $\begin{array}{l}\text { "randomly assigned". Method not re- } \\
\text { ported. }\end{array}$ \\
\hline Allocation concealment (selection bias) & Unclear risk & $\begin{array}{l}\text { Details of allocation concealment not re- } \\
\text { ported. }\end{array}$ \\
\hline $\begin{array}{l}\text { Blinding of participants and personnel } \\
\text { (performance bias) } \\
\text { All outcomes }\end{array}$ & Unclear risk & $\begin{array}{l}\text { "double-blind". Blinding details not re- } \\
\text { ported. }\end{array}$ \\
\hline $\begin{array}{l}\text { Blinding of outcome assessment (detection } \\
\text { bias) } \\
\text { All outcomes }\end{array}$ & Unclear risk & $\begin{array}{l}\text { "double-blind". Blinding details not re- } \\
\text { ported. }\end{array}$ \\
\hline $\begin{array}{l}\text { Incomplete outcome data (attrition bias) } \\
\text { All outcomes }\end{array}$ & Low risk & $\begin{array}{l}\text { Losses to follow-up balanced in numbers } \\
\text { across allocation groups: } 7 \text { participants } \\
\text { from the } 2 \text {-week group and } 6 \text { from the } 6 \text { - } \\
\text { week group. Reasons for drop-outs not re- } \\
\text { ported per allocation group }\end{array}$ \\
\hline Selective reporting (reporting bias) & Low risk & All outcome results have been reported. \\
\hline Other bias & Unclear risk & Source of funding not reported. \\
\hline
\end{tabular}

\section{Kissling 1985}

Methods

Allocation: randomised (coin throwing).

Blindness: double.

Duration: 6 months.

Design: parallel group.

Country: Germany. 


\section{Kissling 1985 (Continued)}

\begin{tabular}{ll}
\hline Participants & Diagnosis: schizophrenia, schizoaffective psychosis (DSM III). \\
& N 54. \\
Age: FD - mean age 28 years, HD - mean age 35 years. \\
Sex: $24 \mathrm{M}, 7 \mathrm{~F}$. \\
History: on oral medication, required depot treatment for $>6$ months, able to give \\
informed consent. \\
Setting: not stated.
\end{tabular}

Risk of bias

\begin{tabular}{|c|c|c|}
\hline Bias & Authors' judgement & Support for judgement \\
\hline $\begin{array}{l}\text { Random sequence generation (selection } \\
\text { bias) }\end{array}$ & Low risk & "random allocation (coin throwing)". \\
\hline Allocation concealment (selection bias) & Unclear risk & $\begin{array}{l}\text { Details of allocation concealment not re- } \\
\text { ported. }\end{array}$ \\
\hline $\begin{array}{l}\text { Blinding of participants and personnel } \\
\text { (performance bias) } \\
\text { All outcomes }\end{array}$ & Unclear risk & $\begin{array}{l}\text { "double-blind". Blinding details not re- } \\
\text { ported. }\end{array}$ \\
\hline $\begin{array}{l}\text { Blinding of outcome assessment (detection } \\
\text { bias) } \\
\text { All outcomes }\end{array}$ & Unclear risk & $\begin{array}{l}\text { "double-blind". Blinding details not re- } \\
\text { ported. }\end{array}$ \\
\hline $\begin{array}{l}\text { Incomplete outcome data (attrition bias) } \\
\text { All outcomes }\end{array}$ & High risk & $\begin{array}{l}\text { Losses to follow-up/missing data imbal- } \\
\text { anced in numbers or reasons across inter- } \\
\text { vention groups. } 10(30 \%) \text { of participants } \\
\text { in the haloperidol group left the study early } \\
\text { and } 13(60 \%) \text { in the fluphenazine group }\end{array}$ \\
\hline Selective reporting (reporting bias) & High risk & $\begin{array}{l}\text { All outcomes have been reported, however } \\
\text { data presented is not usable }\end{array}$ \\
\hline
\end{tabular}




\section{Kissling 1985 (Continued)}

$\begin{array}{lll}\text { Other bias } & \text { Unclear risk } \quad \text { Source of funding not reported. }\end{array}$

Kreisman 1988

\begin{tabular}{|c|c|}
\hline Methods & $\begin{array}{l}\text { Allocation: randomised. } \\
\text { Blindness: double. } \\
\text { Duration: } 1 \text { year. } \\
\text { Design: dosage study. } \\
\text { Country: United States. }\end{array}$ \\
\hline Participants & $\begin{array}{l}\text { Diagnosis: schizophrenia (Research Diagnostic Criteria - Spitzer 1977). } \\
\text { N = } 132 . \\
\text { Age: } 17-60 \text { years. } \\
\text { Sex: } 91 \mathrm{M}, 41 \mathrm{~F} \text {. } \\
\text { History: 'were in remission, at a stable clinical plateau'. } \\
\text { Setting: community. } \\
\text { Excluded: presumptive tardive dyskinesia, neurological disorders, serious substance } \\
\text { abuse, mental retardation, physical illnesses, or requiring adjunctive medication except } \\
\text { for antiparkinsonian agents and minor tranquillisers }\end{array}$ \\
\hline Interventions & $\begin{array}{l}\text { 1. Fluphenazine decanoate (low dose): dose } 1.25-5 \mathrm{mg} / \mathrm{cc} \text { biweekly. } \mathrm{N}=66 \text {. } \\
\text { 2. Fluphenazine decanoate (high dose): dose } 12.5-50 \mathrm{mg} / \mathrm{cc} \text { biweekly. } \mathrm{N}=66\end{array}$ \\
\hline Outcomes & $\begin{array}{l}\text { Global state: relapse. } \\
\text { Unable to use - } \\
\text { Global state: GAS (data unusable). } \\
\text { Mental state: BPRS (no SD). } \\
\text { Behaviour: SAS II, PRS (no usable data). }\end{array}$ \\
\hline
\end{tabular}

Notes

Risk of bias

\begin{tabular}{|c|c|c|}
\hline Bias & Authors' judgement & Support for judgement \\
\hline $\begin{array}{l}\text { Random sequence generation (selection } \\
\text { bias) }\end{array}$ & Unclear risk & $\begin{array}{l}\text { "randomly assigned". Method not re- } \\
\text { ported. }\end{array}$ \\
\hline
\end{tabular}

Allocation concealment (selection bias) Unclear risk

Details of allocation concealment not reported.

Blinding of participants and personnel Unclear risk (performance bias)

“double-blind". Blinding details not reported.

All outcomes

Blinding of outcome assessment (detection Unclear risk

bias)

"double-blind". Blinding details not reported.

All outcomes

Fluphenazine decanoate (depot) and enanthate for schizophrenia (Review)

Copyright @ 2016 The Cochrane Collaboration. Published by John Wiley \& Sons, Ltd. 
Kreisman 1988 (Continued)

\begin{tabular}{l|l|l}
$\begin{array}{l}\text { Incomplete outcome data (attrition bias) } \\
\text { All outcomes }\end{array}$ & High risk & $\begin{array}{l}132 \text { participants entered the study, the final } \\
\text { sample was 51. Reasons for losses to follow- } \\
\text { up not reported }\end{array}$ \\
\hline Selective reporting (reporting bias) & High risk & Outcomes reported as P values. \\
\hline Other bias & Unclear risk & Source of funding not reported \\
\hline
\end{tabular}

Kurland 1966

Methods

Allocation: randomised.

Blindness: double.

Duration: 24 weeks (first arm 12 weeks).

Design: cross-over.

Country: United States.

\begin{tabular}{ll}
\hline Participants & Diagnosis: schizophrenia. \\
& $\mathrm{N}=19$. \\
Age: $23-53$ years. & $\begin{array}{l}\text { Sex: all male. } \\
\text { History: chronically ill. } \\
\text { Setting: hospital. }\end{array}$ \\
\hline Interventions & 1. Fluphenazine decanoate: dose mean $25 \mathrm{mg} / \mathrm{IM}$ monthly. $\mathrm{N}=9$. \\
& 2. Fluphenazine enanthate: dose mean $22.8 \mathrm{mg} / \mathrm{IM}$ monthly. $\mathrm{N}=1$
\end{tabular}

\begin{tabular}{ll} 
Outcomes & $\begin{array}{l}\text { Adverse effects. } \\
\text { Unable to use - } \\
\text { Physiological: weight measures, BP (non-clinical outcomes). }\end{array}$ \\
\hline Notes & $\begin{array}{l}\text { No continuous outcomes measured. } \\
\text { Data put in depot vs depot category in both FE \& FD treatment groups }\end{array}$ \\
\hline
\end{tabular}

Risk of bias

\begin{tabular}{|c|c|c|}
\hline Bias & Authors' judgement & Support for judgement \\
\hline $\begin{array}{l}\text { Random sequence generation (selection } \\
\text { bias) }\end{array}$ & Unclear risk & $\begin{array}{l}\text { "randomly assigned". Method not re- } \\
\text { ported. }\end{array}$ \\
\hline Allocation concealment (selection bias) & Low risk & $\begin{array}{l}\text { "Medication was administered under a } \\
\text { code number known only to the pharma- } \\
\text { cist." }\end{array}$ \\
\hline $\begin{array}{l}\text { Blinding of participants and personnel } \\
\text { (performance bias) } \\
\text { All outcomes }\end{array}$ & Unclear risk & $\begin{array}{l}\text { "double-blind". Blinding details not re- } \\
\text { ported. }\end{array}$ \\
\hline
\end{tabular}

Fluphenazine decanoate (depot) and enanthate for schizophrenia (Review)

Copyright @ 2016 The Cochrane Collaboration. Published by John Wiley \& Sons, Ltd. 
Kurland 1966 (Continued)

\begin{tabular}{|c|c|c|}
\hline $\begin{array}{l}\text { Blinding of outcome assessment (detection } \\
\text { bias) } \\
\text { All outcomes }\end{array}$ & Unclear risk & $\begin{array}{l}\text { "double-blind". Blinding details not re- } \\
\text { ported. }\end{array}$ \\
\hline $\begin{array}{l}\text { Incomplete outcome data (attrition bias) } \\
\text { All outcomes }\end{array}$ & Low risk & All patients completed the study. \\
\hline Selective reporting (reporting bias) & Low risk & All outcomes reported. \\
\hline Other bias & Low risk & $\begin{array}{l}\text { "grant-in aid from the Squibb Institute for } \\
\text { Medical Research" }\end{array}$ \\
\hline
\end{tabular}

Lehmann 1980

\begin{tabular}{|c|c|c|}
\hline Methods & \multicolumn{2}{|c|}{$\begin{array}{l}\text { Allocation: randomised. } \\
\text { Blindness: double. } \\
\text { Duration: } 24 \text { weeks. } \\
\text { Desgin: dosage study. } \\
\text { Country: Germany. }\end{array}$} \\
\hline Participants & \multicolumn{2}{|c|}{$\begin{array}{l}\text { Diagnosis: schizophrenia (ICD 2951). } \\
\mathrm{N}=40 \text {. } \\
\text { Age: } 35-38 \text { years. } \\
\text { Sex: } 27 \mathrm{M}, 13 \mathrm{~F} \text {. } \\
\text { History: all patients chronically ill and resistant to standard doses of neuroleptics. } \\
\text { Setting: not stated. }\end{array}$} \\
\hline Interventions & \multicolumn{2}{|c|}{$\begin{array}{l}\text { 1. Fluphenazine decanoate: dose } 225 \mathrm{mg} / \text { day. } \mathrm{N}=20 \text {. } \\
\text { 2. Fluphenazine decanoate: dose } 25 \mathrm{mg} / \text { day. } \mathrm{N}=20 \text {. }\end{array}$} \\
\hline Outcomes & \multicolumn{2}{|c|}{$\begin{array}{l}\text { Global state: GRS. } \\
\text { Behaviour: leaving the study early. } \\
\text { Unable to use - } \\
\text { Mental state: EWL-K (no usable data). }\end{array}$} \\
\hline Notes & \multicolumn{2}{|l|}{ Article in German. } \\
\hline \multicolumn{3}{|l|}{ Risk of bias } \\
\hline Bias & Authors' judgement & Support for judgement \\
\hline $\begin{array}{l}\text { Random sequence generation (selection } \\
\text { bias) }\end{array}$ & Unclear risk & Randomised, details not reported. \\
\hline Allocation concealment (selection bias) & Unclear risk & Not reported. \\
\hline
\end{tabular}




\section{Lehmann 1980 (Continued)}

Blinding of participants and personnel Unclear risk (performance bias)

All outcomes

Blinding of outcome assessment (detection Unclear risk bias)

All outcomes

Incomplete outcome data (attrition bias) Low risk All outcomes

Selective reporting (reporting bias) Unclear risk

Other bias

Unclear risk
To assure the double-blindness, the patients whose doses were reduced were given placebo injections

Double-blind, details not reported.

tients were analysed after 24 weeks. There was one drop-out from the high-dosage group

\section{Leong 1989}

\section{Methods}

Allocation: randomised.

Blindness: partial.

Duration: 28 weeks.

Design: parallel group.

Country: Singapore.

\begin{tabular}{ll} 
Participants & Diagnosis: schizophrenia (ICD-295). \\
& $\mathrm{N}=60$. \\
& Age: $18-65$ years, mean -38 years. \\
& Sex: $27 \mathrm{M}, 33 \mathrm{~F}$. \\
& History: able to give informed consent, patients in remission. \\
& Setting: community. \\
\hline Interventions & 1. Fluphenazine decanoate: dose $12.5-50 \mathrm{mg} / \mathrm{IM}$ monthly. $\mathrm{N}=30$. \\
& $\begin{array}{l}\text { 2. Pipothiazine palmitate: dose } 25-50 \mathrm{mg} / \mathrm{IM} \text { monthly. } \mathrm{N}=30 . \\
\text { Flexible dose. }\end{array}$ \\
\hline
\end{tabular}

Outcomes

Global state: CGI, need for additional medication.

Mental state: BPRS.

Behaviour: leaving the study early.

Adverse effects: various measures, EPS.

Notes

Risk of bias 


\section{Leong 1989 (Continued)}

\begin{tabular}{|c|c|c|}
\hline $\begin{array}{l}\text { Random sequence generation (selection } \\
\text { bias) }\end{array}$ & High risk & $\begin{array}{l}\text { "randomised", "On admission, the patients } \\
\text { were assigned the next available study num- } \\
\text { ber in numerical sequence and allocated } \\
\text { to receive either pipothiazine palmitate or } \\
\text { fluphenazine decanoate" }\end{array}$ \\
\hline Allocation concealment (selection bias) & Unclear risk & $\begin{array}{l}\text { Details of allocation concealment not re- } \\
\text { ported. }\end{array}$ \\
\hline $\begin{array}{l}\text { Blinding of participants and personnel } \\
\text { (performance bias) } \\
\text { All outcomes }\end{array}$ & High risk & $\begin{array}{l}\text { "The administration of the study medica- } \\
\text { tions was open to the person giving the in- } \\
\text { jections..." }\end{array}$ \\
\hline $\begin{array}{l}\text { Blinding of outcome assessment (detection } \\
\text { bias) } \\
\text { All outcomes }\end{array}$ & Low risk & $\begin{array}{l}\text { “...the patients' symptoms and side effects. } \\
\text {..were assessed by one of the investigators } \\
\text { who had no knowledge of which study } \\
\text { medication had been prescribed." }\end{array}$ \\
\hline $\begin{array}{l}\text { Incomplete outcome data (attrition bias) } \\
\text { All outcomes }\end{array}$ & Low risk & All participants completed the trial. \\
\hline Selective reporting (reporting bias) & Low risk & All outcomes reported. \\
\hline Other bias & Unclear risk & Source of funding not reported. \\
\hline
\end{tabular}

Levenson 1976

Methods

Allocation: randomised.

Blindness: double.

Duration: 21 days.

Design: 3 treatment groups.

Country: United States.

Participants

Diagnosis: schizophrenia (Spitzerian criteria).

$\mathrm{N}=12$.

Age: $18-53$ years, mean -30 years.

Sex: $4 \mathrm{M}, 8 \mathrm{~F}$.

History: able to give informed consent.

Setting: hospital.

Interventions

1. Fluphenazine decanoate: dose $2.5-7.5 \mathrm{mg} /$ day. $\mathrm{N} 5$.

2. Thiothixine: dose $5-15 \mathrm{mg} /$ day. $\mathrm{N}=3$.

3. Haloperidol: dose $2.5-7.5 \mathrm{mg} /$ day. $\mathrm{N}=4$.

Outcomes

Behaviour: leaving the study early.

Adverse effects.

Unable to use - 
Levenson 1976 (Continued)

Mental state: BPRS (no usable data).

\begin{tabular}{|c|c|c|}
\hline Notes & & \\
\hline \multicolumn{3}{|l|}{ Risk of bias } \\
\hline Bias & Authors' judgement & Support for judgement \\
\hline $\begin{array}{l}\text { Random sequence generation (selection } \\
\text { bias) }\end{array}$ & Unclear risk & $\begin{array}{l}\text { "randomly assigned". Method not re- } \\
\text { ported. }\end{array}$ \\
\hline Allocation concealment (selection bias) & Unclear risk & $\begin{array}{l}\text { Details of allocation concealment not re- } \\
\text { ported. }\end{array}$ \\
\hline $\begin{array}{l}\text { Blinding of participants and personnel } \\
\text { (performance bias) } \\
\text { All outcomes }\end{array}$ & Unclear risk & $\begin{array}{l}\text { "double-blind". Blinding details not re- } \\
\text { ported. }\end{array}$ \\
\hline $\begin{array}{l}\text { Blinding of outcome assessment (detection } \\
\text { bias) } \\
\text { All outcomes }\end{array}$ & Unclear risk & $\begin{array}{l}\text { "double-blind". Blinding details not re- } \\
\text { ported. }\end{array}$ \\
\hline $\begin{array}{l}\text { Incomplete outcome data (attrition bias) } \\
\text { All outcomes }\end{array}$ & Low risk & $\begin{array}{l}\text { "Five patients received an administrative } \\
\text { discharge from the study prior ton remis- } \\
\text { sion for reasons unrelated to their illness, } \\
\text { to the medication they were receiving, or } \\
\text { to any other pertinent study variable" }\end{array}$ \\
\hline Selective reporting (reporting bias) & High risk & $\begin{array}{l}\text { Clinical outcome (BPRS) reported incom- } \\
\text { pletely. }\end{array}$ \\
\hline Other bias & Unclear risk & Source of funding not reported. \\
\hline
\end{tabular}

Lundin 1990

Methods

Allocation: randomised.

Blindness: double.

Duration: 1 year (preceded by 6 month 'run-in' period).

Design: parallel group.

Country: Norway.

Participants

Diagnosis: schizophrenia (NIMH Collaborative Study/ DSM III).

$\mathrm{N}=58$.

Age: $18-65$ years.

Sex: 46M, 12F.

History: $>3$ months satisfactory response on depot, duration illness $6-<24$ months, able to give informed consent.

Setting: community. 


\section{Lundin 1990 (Continued)}

\begin{tabular}{|c|c|c|}
\hline Interventions & \multicolumn{2}{|c|}{$\begin{array}{l}\text { 1. Fluphenazine decanoate: dose mean } 34.8 \mathrm{mg} / \mathrm{IM} \text { monthly. } \mathrm{N}=30 \text {. } \\
\text { 2. Flupenthixol decanoate: dose mean } 54.7 \mathrm{mg} / \mathrm{IM} \text { monthly. } \mathrm{N}=28\end{array}$} \\
\hline Outcomes & \multicolumn{2}{|c|}{$\begin{array}{l}\text { Behaviour: leaving the study early. } \\
\text { Unable to use - } \\
\text { Global state: TES (no data). } \\
\text { Mental state: BPRS, CPRS (no data). } \\
\text { Adverse effects: EPS, HRSD, CSE (no data). } \\
\text { Social ability: KAS (non clinical outcome, data unusable). }\end{array}$} \\
\hline Notes & \multicolumn{2}{|l|}{ Authors contacted. } \\
\hline \multicolumn{3}{|l|}{ Risk of bias } \\
\hline Bias & Authors' judgement & Support for judgement \\
\hline $\begin{array}{l}\text { Random sequence generation (selection } \\
\text { bias) }\end{array}$ & Unclear risk & $\begin{array}{l}\text { "randomly allocated". Method not re- } \\
\text { ported. }\end{array}$ \\
\hline Allocation concealment (selection bias) & Unclear risk & $\begin{array}{l}\text { Details of allocation concealment not re- } \\
\text { ported. }\end{array}$ \\
\hline $\begin{array}{l}\text { Blinding of participants and personnel } \\
\text { (performance bias) } \\
\text { All outcomes }\end{array}$ & Unclear risk & $\begin{array}{l}\text { "double-blind". Blinding details not re- } \\
\text { ported. }\end{array}$ \\
\hline $\begin{array}{l}\text { Blinding of outcome assessment (detection } \\
\text { bias) } \\
\text { All outcomes }\end{array}$ & Unclear risk & $\begin{array}{l}\text { "double-blind". Blinding details not re- } \\
\text { ported. }\end{array}$ \\
\hline $\begin{array}{l}\text { Incomplete outcome data (attrition bias) } \\
\text { All outcomes }\end{array}$ & Low risk & $\begin{array}{l}\text { Losses to follow-up / missing data bal- } \\
\text { anced across intervention groups, with sim- } \\
\text { ilar reasons for missing data }\end{array}$ \\
\hline Selective reporting (reporting bias) & High risk & $\begin{array}{l}\text { Results reported incompletely: BPRS, } \\
\text { CPRS, CSE, SAS, HRSD, KAS }\end{array}$ \\
\hline Other bias & Unclear risk & Source of funding not reported. \\
\hline
\end{tabular}


Methods

Blindness: double.
Duration: 1 year.
Design: parallel group.
Country: Canada.

Diagnosis: schizophrenia.

$\mathrm{N}=49$.

Age: $28-54$ years, mean -40 years.

Sex: 16M, 23F.

History: duration illness 1-21 years, mean -12 years.

Setting: community.

1. Fluphenazine decanoate: dose 25-37.5/IM every 28 days. $\mathrm{N}=24$.

2. Fluphenazine enanthate: dose $25-37.5 \mathrm{mg} / \mathrm{IM}$ every 25 days. $\mathrm{N}=25$

Global state: need for additional medication.

Mental state: BPRS.

Behaviour: leaving the study early.

Side effects: Bordeleau Scale.

Notes

Risk of bias

\section{Bias}

Random sequence generation (selection Unclear risk bias)

Allocation concealment (selection bias) Unclear risk

\section{Support for judgement}

"randomly assigned". Method not reported.

Details of allocation concealment not reported.

"double-blind". Blinding details not reported.
Blinding of participa
(performance bias)

All outcomes

Blinding of outcome assessment (detection Unclear risk bias)

All outcomes

Incomplete outcome data (attrition bias) High risk

All outcomes

High risk

"double-blind". Blinding details not reported.
Selective reporting (reporting bias) Low risk
Losses to follow-up/missing data imbalanced in numbers and reasons across intervention groups. Fluphenazine enanthate $3(12 \%)$ and fluphenazine decaonate 7 $(29 \%)$

Outcomes have been reported: BPRS, extrapyramidal symptoms, other adverse 
events and serious adverse events

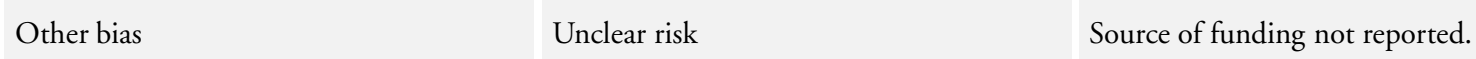

Magnus 1979

Methods

Blindness: open.
Duration: 6 months.
Design: parallel group.
Country: UK.

Participants

Interventions

1. Fluphenazine decanoate: every $2-3$ weeks, dose range $50-100 \mathrm{mg} / \mathrm{IM} . \mathrm{N}=26$.

2. Fluspirilene: weekly, dose range 6-12 $\mathrm{mg} / \mathrm{IM} . \mathrm{N}=24$.

Indiviually adjusted doses.

Diagnosis: schizophrenia.

$\mathrm{N}=50$.

Age: 'approximately equal in both groups'.

Sex: male and female 'approximately equal in both groups'

History: newly admitted to hospital (either first episode or relapse).

Setting: community and hospital.
Global state: need for additional medication.

Behaviour: leaving the study early.

Adverse effects.

Unable to use -

Mental state: BPRS (no SD) self and nurse's assessment (no data).

Social ability: WWBRS (non-clinical outcomes, data unusable)

\section{Notes}

Authors contacted.

Unable to complete risk of bias table - PDF missing.

\begin{tabular}{|c|c|c|}
\hline Bias & Authors' judgement & Support for judgement \\
\hline $\begin{array}{l}\text { Random sequence generation (selection } \\
\text { bias) }\end{array}$ & Unclear risk & $\begin{array}{l}\text { "Patients were allocated...according to a } \\
\text { pre-arranged randomised prescribing list" }\end{array}$ \\
\hline Allocation concealment (selection bias) & Unclear risk & $\begin{array}{l}\text { Details of allocation concealment not re- } \\
\text { ported. }\end{array}$ \\
\hline
\end{tabular}

Blinding of participants and personnel High risk (performance bias)

Open trial.

All outcomes 
Magnus 1979 (Continued)

\begin{tabular}{l|l|l}
\hline $\begin{array}{l}\text { Blinding of outcome assessment (detection } \\
\text { bias) } \\
\text { All outcomes }\end{array}$ & High risk & Open trial. \\
\hline $\begin{array}{l}\text { Incomplete outcome data (attrition bias) } \\
\text { All outcomes }\end{array}$ & Low risk & $\begin{array}{l}\text { "All but two (one in each group) completed } \\
\text { six months treatment" }\end{array}$ \\
\hline Selective reporting (reporting bias) & High risk & $\begin{array}{l}\text { Not all outcomes reported: no SD reported } \\
\text { for BPRS and no data for nurse's assessment }\end{array}$ \\
\hline Other bias & Unclear risk & $\begin{array}{l}\text { Smith Kline \& French Laboratories pro- } \\
\text { vided statistical help. }\end{array}$ \\
\hline
\end{tabular}

Malm 1974

\begin{tabular}{|c|c|}
\hline Methods & $\begin{array}{l}\text { Allocation: randomised. } \\
\text { Blindness: double. } \\
\text { Duration: } 8 \text { weeks. } \\
\text { Design: parallel group. } \\
\text { Country: Sweden. }\end{array}$ \\
\hline Participants & $\begin{array}{l}\text { Diagnosis: schizophrenia. } \\
\mathrm{N}=62 . \\
\text { Age: } 18-65 \text { years. } \\
\text { Sex: } 21 \mathrm{M}, 36 \mathrm{~F} \text {. } \\
\text { History: duration illness } 2-39 \text { years, mean } \sim 15 \text { years. } \\
\text { Setting: hospital. }\end{array}$ \\
\hline Interventions & $\begin{array}{l}\text { 1. Fluphenazine enanthate: dose } 7.5-50 \mathrm{mg} / \mathrm{IM} \text {, mean } 28.5 \mathrm{mg} / \mathrm{IM} \text { biweekly. } \mathrm{N}=26 \text {. } \\
\text { 2. Fluspirilene: dose } 1-14 \mathrm{mg} / \mathrm{IM} \text {, mean } 5.7 \mathrm{mg} / \mathrm{IM} \text { weekly. } \mathrm{N}=31\end{array}$ \\
\hline Outcomes & $\begin{array}{l}\text { Global state: need for additional medication. } \\
\text { Behaviour: leaving the study early. } \\
\text { Unable to use - } \\
\text { Mental state: S-Scale (no data). } \\
\text { Behaviour: ADL (no data). } \\
\text { Adverse effects: SE scale (no SD). } \\
\text { Physiological: various measures (non-clinical outcomes, data unusable) }\end{array}$ \\
\hline
\end{tabular}

Notes

Risk of bias

\begin{tabular}{lll}
\hline Bias & Authors' judgement & Support for judgement \\
\hline $\begin{array}{l}\text { Random sequence generation (selection } \\
\text { bias) }\end{array}$ & Unclear risk & $\begin{array}{l}\text { "allocated randomly". Method not re- } \\
\text { ported. }\end{array}$
\end{tabular}




\begin{tabular}{|c|c|c|}
\hline Allocation concealment (selection bias) & Unclear risk & $\begin{array}{l}\text { Details of allocation concealment not re- } \\
\text { ported. }\end{array}$ \\
\hline $\begin{array}{l}\text { Blinding of participants and personnel } \\
\text { (performance bias) } \\
\text { All outcomes }\end{array}$ & Unclear risk & $\begin{array}{l}\text { "double-blind". Patients blinded. Nurses } \\
\text { administering the injections: not blinded } \\
\text { to the study medications }\end{array}$ \\
\hline
\end{tabular}

Blinding of outcome assessment (detection Unclear risk

Blinding details not reported. bias)

All outcomes

Incomplete outcome data (attrition bias) Unclear risk All outcomes
62 participants were enrolled but number randomised to each group not reported. 5 participants were withdrawn during the first two months, intervention groups not reported

Outcomes incompletely reported: S-Scale, Side effects, Nurses' ratings (ADL)

"Supported, in part, by a grant from LEO Research Foundation, Helsingborg."

Marder 1987

Methods

Participants

Interventions

Outcomes
1. Fluphenazine decanoate (low dose): dose mean $5 \mathrm{mg} / \mathrm{IM}$ biweekly. $\mathrm{N}=35$.

2. Fluphenzazine decanoate (standard): dose mean $25 \mathrm{mg} / \mathrm{IM}$ biweekly. $\mathrm{N}=31$

Allocation: randomised.

Blindness: double.

Duration: 2 years.

Design: dosage study.

Country: United States.

Diagnosis: schizophrenia (DSM III).

$\mathrm{N}=66$.

Age: mean -35 years.

Sex: all male.

History: drug free for a month, duration illness mean 24 months $(5 \mathrm{mg}), 170$ months (25 mg).

Setting: community and hospital.

Global state: relapse.

Behaviour: leaving the study early.

Adverse effects: need for additional medication.

Unable to use -

Mental state: BPRS (no data).

Adverse effects: Hopkins SCL-90R, side-effects scale (no data) 
Marder 1987 (Continued)

\begin{tabular}{|c|c|c|}
\hline Notes & & \\
\hline \multicolumn{3}{|l|}{ Risk of bias } \\
\hline Bias & Authors' judgement & Support for judgement \\
\hline $\begin{array}{l}\text { Random sequence generation (selection } \\
\text { bias) }\end{array}$ & Unclear risk & "randomised“. Method not reported. \\
\hline Allocation concealment (selection bias) & Unclear risk & $\begin{array}{l}\text { Details of allocation concealment not re- } \\
\text { ported. }\end{array}$ \\
\hline $\begin{array}{l}\text { Blinding of participants and personnel } \\
\text { (performance bias) } \\
\text { All outcomes }\end{array}$ & Unclear risk & $\begin{array}{l}\text { Double-blind. Blinding details not re- } \\
\text { ported. }\end{array}$ \\
\hline $\begin{array}{l}\text { Blinding of outcome assessment (detection } \\
\text { bias) } \\
\text { All outcomes }\end{array}$ & Unclear risk & $\begin{array}{l}\text { Double-blind. Blinding details not re- } \\
\text { ported. }\end{array}$ \\
\hline $\begin{array}{l}\text { Incomplete outcome data (attrition bias) } \\
\text { All outcomes }\end{array}$ & Unclear risk & $\begin{array}{l}2 / 35 \text { and } 6 / 31 \text { participants left the study } \\
\text { early during the first six months", reasons } \\
\text { not reported }\end{array}$ \\
\hline Selective reporting (reporting bias) & Unclear risk & $\begin{array}{l}\text { Outcomes incompletely reported: SCL-90, } \\
\text { SE Scale, IMEPS, Subjective EPS Rating } \\
\text { Scale. P values for most outcomes and data } \\
\text { at } 1 \text { month (SCL-90) and three months } \\
\text { (side effects) }\end{array}$ \\
\hline Other bias & Low risk & $\begin{array}{l}\text { "...supported by Veterans Administration } \\
\text { Medical Research and the UCLA Mental } \\
\text { Mental Health Clinical Research Center } \\
\text { for the Study of schizophrenia; and the na- } \\
\text { tional Institute of mental Health grant...E. } \\
\text { R. Squibb and Sons...provided the low dose } \\
\text { formulation of fluphenazine decanoate." }\end{array}$ \\
\hline
\end{tabular}


Methods
Allocation: randomised.

Blindness: double.

Duration: 6 months (preceded by pretrial of 6 weeks).

Design: dosage study.

Country: UK.

Diagnosis: schizophrenia (Kraepelinian).
Participants
Age: $18-60$ years.
Sex: $22 \mathrm{M}, 28 \mathrm{~F}$.
History: disabled, able to give informed consent, minimum hospital stay > 12 months.
Setting: hospital.

Interventions

1. Fluphenazine decanoate (VHD): dose mean $250 \mathrm{mg} / \mathrm{IM}$ weekly. $\mathrm{N}=25$.

2. Fluphenazine decanoate (standard): dose mean $12.5 \mathrm{mg} / \mathrm{IM}$ weekly. $\mathrm{N}=25$

$\begin{array}{ll}\text { Outcomes } & \text { Global state: need for additional medication. } \\ \text { Mental state: BPRS. } \\ \text { Behaviour: leaving the study early. } \\ \text { Adverse effects: EPS Scale. } \\ \text { Unable to use - } \\ \text { Behaviour: WWBRS (no data). } \\ \text { Physiological measures: weight (non-clinical outcomes, data unusable) }\end{array}$

Risk of bias

\begin{tabular}{|c|c|c|}
\hline Bias & Authors' judgement & Support for judgement \\
\hline $\begin{array}{l}\text { Random sequence generation (selection } \\
\text { bias) }\end{array}$ & Unclear risk & $\begin{array}{l}\text { "Random allocation", no further details re- } \\
\text { ported. }\end{array}$ \\
\hline
\end{tabular}

Allocation concealment (selection bias) Low risk

Blinding of participants and personnel Low risk (performance bias)

All outcomes
"Allocation to the experimental or control group was carried out by the hospital pharmacist"

"Double-blind design and entailed the 'double-dummy' technique." "The manufacturer prepared 10-ml ampules of fluphenazine decanoate, $25 \mathrm{mg} / \mathrm{ml}$ and inactive preparation containing only sesame oil, as well as $0.5 \mathrm{ml}$ ampules with $25 \mathrm{mg} /$ $\mathrm{ml}$ of the drug and a sesame oil placebo". "All hospital personnel were 'blind".

"Double-blind", blinding of outcome assessors not reported.

bias)

All outcomes

Fluphenazine decanoate (depot) and enanthate for schizophrenia (Review) 
McClelland 1976 (Continued)

\begin{tabular}{l|ll}
\hline $\begin{array}{l}\text { Incomplete outcome data (attrition bias) } \\
\text { All outcomes }\end{array}$ & Low risk & $\begin{array}{l}\text { Two participants in the VHD group left the } \\
\text { study early and three in the standard dose } \\
\text { group }\end{array}$ \\
\hline Selective reporting (reporting bias) & High risk & No data were reported for the WWBRS. \\
\hline Other bias & Unclear risk & Source of funding not reported. \\
\hline
\end{tabular}

\section{McCreadie 1980}

Methods

Allocation: randomised.

Blindness: double.

Duration: 9 months.

Design: parallel group.

Country: United Kingdom (Scotland).

\begin{tabular}{ll}
\hline Participants & Diagnosis: schizophrenia (Feighner's Criteria). \\
& $\mathrm{N}=35$. \\
& Age: $19-70$ years, mean $47-55$ years. \\
& Sex: all male. \\
& History: on antipsychotics for mean 4 years, duration illness $18-26$ years, able to give \\
& informed consent. \\
& Setting: hospital and community. \\
\hline
\end{tabular}

Interventions

1. Fluphenazine decanoate: dose mean $12.5 \mathrm{mg} / \mathrm{IM}$, maximum $50 \mathrm{mg} / \mathrm{IM}$ weekly. $\mathrm{N}=$ 18.

2. Pimozide: dose mean $8 \mathrm{mg}$, maximum $32 \mathrm{mg}$ every 4 days/week. $\mathrm{N}=16$

\begin{tabular}{lll} 
Outcomes & $\begin{array}{l}\text { Global state: relapse, need for additional medication. } \\
\text { Adverse effects: Kraweicka scale. }\end{array}$ & $\begin{array}{l}\text { Unable to use - } \\
\text { Mental state: Hamilton-Lorr scale (no data). } \\
\text { Behaviour: Wing Ward Behaviour Scale (no data). }\end{array}$ \\
\hline Notes & N differs in the paper and abstract. & \\
\hline Risk of bias & & Support for judgement \\
\hline Bias & Authors' judgement & "randomly allocated". Method not re- \\
\hline $\begin{array}{l}\text { Random sequence generation (selection } \\
\text { bias) }\end{array}$ & Unclear risk & ported. \\
\hline Allocation concealment (selection bias) & Unclear risk & $\begin{array}{l}\text { Details of allocation concealment not re- } \\
\text { ported. }\end{array}$
\end{tabular}


McCreadie 1980 (Continued)

\begin{tabular}{|c|c|c|}
\hline $\begin{array}{l}\text { Blinding of participants and personnel } \\
\text { (performance bias) } \\
\text { All outcomes }\end{array}$ & Unclear risk & $\begin{array}{l}\text { "double-blind". "To ensure } \\
\text { double-blind conditions patients received } \\
\text { active fluphenazine injections and placebo } \\
\text { pimozide tablets, or placebo injections and } \\
\text { active tablets." Participants blinded. Blind- } \\
\text { ing details of personnel unclear }\end{array}$ \\
\hline $\begin{array}{l}\text { Blinding of outcome assessment (detection } \\
\text { bias) } \\
\text { All outcomes }\end{array}$ & Unclear risk & $\begin{array}{l}\text { "double-blind". Blinding details of out- } \\
\text { come assessors unclear }\end{array}$ \\
\hline $\begin{array}{l}\text { Incomplete outcome data (attrition bias) } \\
\text { All outcomes }\end{array}$ & Low risk & $\begin{array}{l}\text { Losses to follow-up and missing data bal- } \\
\text { anced across intervention groups ( } 4 / 16 \text { vs } \\
3 / 18 \text { ), with similar reasons for missing } \\
\text { data (relapse: } 3 / 16 \text { vs } 3 / 18 ; 1 \text { participant } \\
\text { dropped out due to non-compliance with } \\
\text { medication) }\end{array}$ \\
\hline Selective reporting (reporting bias) & High risk & $\begin{array}{l}\text { Hamiiton-Lorr, Wing and Griffiths scales' } \\
\text { results not reported }\end{array}$ \\
\hline Other bias & Unclear risk & $\begin{array}{l}\text { Funding source not reported. Medication } \\
\text { supplied by Janssen Pharmaceutical and } \\
\text { Squibb }\end{array}$ \\
\hline
\end{tabular}

McCreadie 1982

\begin{tabular}{|c|c|}
\hline Methods & $\begin{array}{l}\text { Allocation: randomised. } \\
\text { Blindness: double. } \\
\text { Duration: } 9 \text { months. } \\
\text { Design: parallel group. } \\
\text { Country: United Kingdom (Scotland). }\end{array}$ \\
\hline Participants & $\begin{array}{l}\text { Diagnosis: schizophrenia (Feighner's criteria). } \\
\mathrm{N}=28 \text {. } \\
\text { Age: } 27-70 \text { years, mean }-55 \text { years. } \\
\text { Sex: all male. } \\
\text { History: duration illness }>27 \text { yrs. } \\
\text { Setting: hospital. }\end{array}$ \\
\hline Interventions & $\begin{array}{l}\text { 1. Fluphenazine decanoate: dose range } 2-25 \mathrm{mg} / \mathrm{IM} \text {, mean } 14 \mathrm{mg} / \mathrm{IM} \text { biweekly. } \mathrm{N}=15 \text {. } \\
\text { 2. Pimozide: dose range } 10-60 \mathrm{mg} \text {, mean } 40 \mathrm{mg} \text {, weekly. } \mathrm{N}=13\end{array}$ \\
\hline Outcomes & $\begin{array}{l}\text { Mental state: Krawiecka sub-scales. } \\
\text { Behaviour: leaving the study early. } \\
\text { Adverse effects: parkinsonism, tardive dyskinesia. } \\
\text { Unable to use - } \\
\text { Mental state: HLS (no data). }\end{array}$ \\
\hline
\end{tabular}


McCreadie 1982 (Continued)

Behaviour: WWBRS (no data).

\begin{tabular}{|c|c|c|}
\hline Notes & \multicolumn{2}{|l|}{ Authors contacted. } \\
\hline \multicolumn{3}{|l|}{ Risk of bias } \\
\hline Bias & Authors' judgement & Support for judgement \\
\hline $\begin{array}{l}\text { Random sequence generation (selection } \\
\text { bias) }\end{array}$ & Unclear risk & $\begin{array}{l}\text { "randomly allocated". Method not re- } \\
\text { ported }\end{array}$ \\
\hline Allocation concealment (selection bias) & Unclear risk & $\begin{array}{l}\text { Details of allocation concealment not re- } \\
\text { ported. }\end{array}$ \\
\hline $\begin{array}{l}\text { Blinding of participants and personnel } \\
\text { (performance bias) } \\
\text { All outcomes }\end{array}$ & Unclear risk & $\begin{array}{l}\text { "double-blind". "To ensure double blind } \\
\text { conditions, patients received either active } \\
\text { pimozide tablets and dummy fluphenazine } \\
\text { injections or dummy tablets and active in- } \\
\text { jections." Blinding details of personnel, un- } \\
\text { clear }\end{array}$ \\
\hline $\begin{array}{l}\text { Blinding of outcome assessment (detection } \\
\text { bias) } \\
\text { All outcomes }\end{array}$ & Unclear risk & $\begin{array}{l}\text { "double-blind". Blinding details of out- } \\
\text { come assessors, unclear }\end{array}$ \\
\hline $\begin{array}{l}\text { Incomplete outcome data (attrition bias) } \\
\text { All outcomes }\end{array}$ & Low risk & $\begin{array}{l}\text { Losses to follow-up balanced across inter- } \\
\text { vention groups ( } 38 \% \text { vs } 40 \%) \text {, with sim- } \\
\text { ilar reasons (exacerbation, adverse events) } \\
\text { for missing data }\end{array}$ \\
\hline Selective reporting (reporting bias) & High risk & $\begin{array}{l}\text { Hamilton-Lorr scale, Wing Ward Be- } \\
\text { haviour Scale results not reported }\end{array}$ \\
\hline Other bias & Low risk & $\begin{array}{l}\text { "...supported by a research grant from } \\
\text { Dumfries and Galloway Health Board". } \\
\text { Medication and other materials provided } \\
\text { by Janssen Pharmaceutical and Squibb } \\
\text { Limited }\end{array}$ \\
\hline
\end{tabular}


Methods
Allocation: randomised.

Blindness: double.

Duration: 48 weeks. (preceded by 12 weeks 'run in' period where additional medication allowed).

Design: parallel group.

Country: United Kingdom (Scotland).
Diagnosis: schizophrenia (Feighner (1972).

$\mathrm{N}=38$.

Age: $31-71$ years, mean -56 years.

Sex: 22M, 16F.

History: previously on antipsychotics, consent given by next of kin.

Setting: hospital.
Interventions

Outcomes
1. Fluphenazine decanoate: dose mean $106 \mathrm{mg} / \mathrm{IM} /$ week, week 12 dose mean 105/IM monthly. $\mathrm{N}=19$.

2. Haloperidol decanoate: dose mean $127 \mathrm{mg} / \mathrm{IM}$, week 12 dose mean $120 \mathrm{mg} / \mathrm{IM}$ monthly. $\mathrm{N}=19$

Global state: Global 5-point scale, need for additional medication.

Behaviour: leaving the study early.

Adverse effects: AIMS, SAS, Parkinsonism.

5 people unaccounted for in th FD group.

Risk of bias

Bias

Authors' judgement

Support for judgement

Random sequence generation (selection Unclear risk bias)

"randomly allocated". Method not reported

Allocation concealment (selection bias) Unclear risk

Details of allocation concealment not reported.

Blinding of participants and personnel Unclear risk (performance bias)

"double-blind". Blinding details not reported.

All outcomes

Blinding of outcome assessment (detection Unclear risk bias)

"double-blind". Blinding details not reported.

All outcomes

Incomplete outcome data (attrition bias) High risk

All outcomes
Five patients left the study during the runin period and 17 patients on haloperidol and 16 on fluphenazine entered the study proper. Losses to follow-up/missing data balanced in numbers $6 / 17$ vs $4 / 16$ but imbalanced in reasons (relapse $6 / 17$ vs $1 /$ 
McKane 1987 (Continued)

16; non-compliance: $0 / 17$ vs $2 / 16$; adverse events: $0 / 17$ vs $1 / 16$ ) across intervention groups

\begin{tabular}{|c|c|c|}
\hline Selective reporting (reporting bias) & Low risk & $\begin{array}{l}\text { Outcome results reported: Krawieka, } \\
\text { Wing, SAS, AIMS. }\end{array}$ \\
\hline Other bias & High risk & $\begin{array}{l}\text { "Janssen Pharmaceutical...financial and } \\
\text { other assistance". }\end{array}$ \\
\hline
\end{tabular}

\section{McLaren 1992}

\begin{tabular}{|c|c|}
\hline Methods & $\begin{array}{l}\text { Allocation: randomised. } \\
\text { Blindness: double. } \\
\text { Duration: } 1 \text { year. } \\
\text { Design: parallel group. } \\
\text { Country: United Kingdom. }\end{array}$ \\
\hline Participants & $\begin{array}{l}\text { Diagnosis: schizophrenia (ICD-9). } \\
\mathrm{N}=47 \text {. } \\
\text { Age: } 20-65 \text { yrs. } \\
\text { Sex: } 27 \mathrm{M}, 20 \mathrm{~F} \text {. } \\
\text { History: good physical health, received antipsychotics for at least } 1 \text { year previously, } \\
\text { duration illness } 18 \text { years, able to give informed consent. } \\
\text { Setting: community. }\end{array}$ \\
\hline Interventions & $\begin{array}{l}\text { 1. Fluphenazine decanoate: dose } 16-300 \mathrm{mg} / \mathrm{IM} / \text { month, mean } 103 \mathrm{mg} / \mathrm{IM} / \mathrm{month} . \mathrm{N} \\
=24 \text {. } \\
\text { 2. Bromperidol decanoate: dose } 67-400 \mathrm{mg} / \mathrm{IM} / \text { month, mean } 242 \mathrm{mg} / \mathrm{IM} / \mathrm{month} . \mathrm{N}= \\
23\end{array}$ \\
\hline Outcomes & $\begin{array}{l}\text { Global state: relapse, need for additional medication. } \\
\text { Behaviour: leaving the study early. } \\
\text { Symptoms: NSRS. } \\
\text { Unable to use - } \\
\text { Mental state: KWS, MARDRS (no data). } \\
\text { Social ability: MRSS (non clinical outcome, data unusable). } \\
\text { Adverse effects: AIMS (data unusable), SAS (no data). } \\
\text { Physiological measures: weight, blood samples (non-clinical outcomes, data unusable) }\end{array}$ \\
\hline
\end{tabular}

Notes

Risk of bias

\begin{tabular}{lll}
\hline Bias & Authors' judgement & Support for judgement \\
\hline $\begin{array}{l}\text { Random sequence generation (selection } \\
\text { bias) }\end{array}$ & Unclear risk & $\begin{array}{l}\text { "randomly allocated". Method not re- } \\
\text { ported. }\end{array}$
\end{tabular}


McLaren 1992 (Continued)

\begin{tabular}{|c|c|c|}
\hline Allocation concealment (selection bias) & Unclear risk & $\begin{array}{l}\text { Details of allocation concealment not re- } \\
\text { ported. }\end{array}$ \\
\hline $\begin{array}{l}\text { Blinding of participants and personnel } \\
\text { (performance bias) } \\
\text { All outcomes }\end{array}$ & Low risk & "double-blind" "identical ampoules". \\
\hline $\begin{array}{l}\text { Blinding of outcome assessment (detection } \\
\text { bias) } \\
\text { All outcomes }\end{array}$ & Unclear risk & $\begin{array}{l}\text { "double-blind". Blinding details of out- } \\
\text { come assessment not reported }\end{array}$ \\
\hline $\begin{array}{l}\text { Incomplete outcome data (attrition bias) } \\
\text { All outcomes }\end{array}$ & Unclear risk & $\begin{array}{l}\text { "Two patients, both on fluphenazine de- } \\
\text { canoate withdrew consent". "Five patients, } \\
\text { all in the bromperidol decanoate group } \\
\text { were withdrawn from the study...following } \\
\text { relapse", "One other patient, on bromperi- } \\
\text { dol decanoate, was lost to follow-up during } \\
\text { the sixth week of the study having deterio- } \\
\text { rated before contact was lost" }\end{array}$ \\
\hline Selective reporting (reporting bias) & High risk & $\begin{array}{l}\text { Data not reported or incomplete for } \\
\text { the NSRS, Krawiecka-Goldberg scale, } \\
\text { MARDRS, MRSS, SAS, AIMS }\end{array}$ \\
\hline Other bias & High risk & financial support: Jannsen Pharmaceutical. \\
\hline
\end{tabular}

\section{Odejide 1982}

\begin{tabular}{ll} 
Methods & $\begin{array}{l}\text { Allocation: randomised. } \\
\text { Blindness: double. } \\
\text { Duration: } 12 \text { months. } \\
\text { Design: parallel group. } \\
\text { Country: Nigeria. }\end{array}$ \\
\hline Participants & $\begin{array}{l}\text { Diagnosis: schizophrenia (ICD-9). } \\
\text { N }=70 .\end{array}$ \\
Age: not stated. \\
Sex: not stated. \\
History: treated with FD $<2$ years, $<2$ acute \\
Setting: community. \\
\hline Interventions & $\begin{array}{l}\text { 1. Fluphenazine decanoate: dose } 25 \text { mg/IM ever } \\
\text { 2. Placebo. } \mathrm{N}=35 .\end{array}$ \\
\hline Outcomes & $\begin{array}{l}\text { Global state: need for additional medication. } \\
\text { Behaviour: leaving the study early. } \\
\text { Adverse effects: AIMS. } \\
\text { Unable to use - }\end{array}$ \\
\hline
\end{tabular}


Odejide 1982 (Continued)

Mental state: BPRS, PSE (no data).

\begin{tabular}{|c|c|c|}
\hline Notes & \multicolumn{2}{|c|}{2 drop-outs unaccounted for in th FD group. } \\
\hline \multicolumn{3}{|l|}{ Risk of bias } \\
\hline Bias & Authors' judgement & Support for judgement \\
\hline $\begin{array}{l}\text { Random sequence generation (selection } \\
\text { bias) }\end{array}$ & Unclear risk & "randomised”. Method not reported. \\
\hline Allocation concealment (selection bias) & Unclear risk & $\begin{array}{l}\text { Details of allocation concealment not re- } \\
\text { ported. }\end{array}$ \\
\hline $\begin{array}{l}\text { Blinding of participants and personnel } \\
\text { (performance bias) } \\
\text { All outcomes }\end{array}$ & Unclear risk & $\begin{array}{l}\text { "double-blind". "patients were unaware of } \\
\text { the contents of their injections". Blinding } \\
\text { details of other personnel (e.g. nurses who } \\
\text { administered the injections), not reported }\end{array}$ \\
\hline $\begin{array}{l}\text { Blinding of outcome assessment (detection } \\
\text { bias) } \\
\text { All outcomes }\end{array}$ & Low risk & $\begin{array}{l}\text { "The psychiatrist who evaluated follow-up } \\
\text { status, was blind to treatment status..." }\end{array}$ \\
\hline $\begin{array}{l}\text { Incomplete outcome data (attrition bias) } \\
\text { All outcomes }\end{array}$ & Low risk & $\begin{array}{l}53 / 70 \text { participants completed the study. } \\
\text { Losses to follow-up or missing data bal- } \\
\text { anced across intervention groups }\end{array}$ \\
\hline Selective reporting (reporting bias) & High risk & $\begin{array}{l}\text { Outcomes not reported (PSE) or incom- } \\
\text { pletely reported (BPRS). }\end{array}$ \\
\hline Other bias & Unclear risk & Source of funding not reported. \\
\hline
\end{tabular}

Pinto 1979

\begin{tabular}{ll} 
Methods & Allocation: randomised. \\
& Blindness: double. \\
& Duration: 18 months (preceded by 3 months 'run-in' period - medication unchanged). \\
& Design: parallel group. \\
& Country: United Kingdom. \\
\hline Participants & Diagnosis: schizophrenia. \\
& N = 64. \\
Age: not stated. & Sex: not stated. \\
& History: receiving depot for at least 6 months, stable - no hospital admission for at least \\
& 3 months prior to trial. \\
& Setting: community.
\end{tabular}


Pinto 1979 (Continued)

\begin{tabular}{|c|c|c|}
\hline Interventions & \multicolumn{2}{|c|}{$\begin{array}{l}\text { 1. Fluphenazine decanoate: dose mean } 25 \mathrm{mg} / \mathrm{IM} \text { every } 3 \text { weeks (initial dose } 12.5 \mathrm{mg} \text { ). } \\
\mathrm{N}=33 \text {. } \\
2 \text { Flupenthixol decanoate: dose mean } 36.6 \mathrm{mg} / \mathrm{IM} \text { every } 3 \text { weeks (initial dose } 20 \mathrm{mg} \text { ). } \\
\mathrm{N}=31\end{array}$} \\
\hline Outcomes & \multicolumn{2}{|c|}{$\begin{array}{l}\text { Global state: need for additional medication. } \\
\text { Adverse effects: EPSE. } \\
\text { Leaving the study early. } \\
\text { Unable to use - } \\
\text { Mental state: BPRS (no SD). }\end{array}$} \\
\hline Notes & \multicolumn{2}{|l|}{ Authors contacted. } \\
\hline \multicolumn{3}{|l|}{ Risk of bias } \\
\hline Bias & Authors' judgement & Support for judgement \\
\hline $\begin{array}{l}\text { Random sequence generation (selection } \\
\text { bias) }\end{array}$ & Unclear risk & $\begin{array}{l}\text { "randomly allocated“. Method not re- } \\
\text { ported. }\end{array}$ \\
\hline Allocation concealment (selection bias) & Unclear risk & $\begin{array}{l}\text { Details of allocation concealment not re- } \\
\text { ported. }\end{array}$ \\
\hline $\begin{array}{l}\text { Blinding of participants and personnel } \\
\text { (performance bias) } \\
\text { All outcomes }\end{array}$ & Unclear risk & $\begin{array}{l}\text { "double-blind“. Patients blinded, nurses } \\
\text { unblinded. "injections were prepared and } \\
\text { administered by nursing staff...neither the } \\
\text { clinician nor the patients were aware of the } \\
\text { treatment allocation }\end{array}$ \\
\hline $\begin{array}{l}\text { Blinding of outcome assessment (detection } \\
\text { bias) } \\
\text { All outcomes }\end{array}$ & Low risk & $\begin{array}{l}\text { "...rating clinicians in ignorance of the al- } \\
\text { location of patients to treatment groups" }\end{array}$ \\
\hline $\begin{array}{l}\text { Incomplete outcome data (attrition bias) } \\
\text { All outcomes }\end{array}$ & High risk & $\begin{array}{l}\text { Losses to follow-up/missing data imbal- } \\
\text { anced in numbers across intervention } \\
\text { groups. "a total of eight patients all in the } \\
\text { fluphenazine group, dropped-out of the } \\
\text { trial..." }\end{array}$ \\
\hline Selective reporting (reporting bias) & High risk & $\begin{array}{l}\text { Outcome data incompletely reported } \\
\text { (BPRS). }\end{array}$ \\
\hline Other bias & Unclear risk & Source of funding not reported. \\
\hline
\end{tabular}


Methods

(1)

Blindness: double.

Duration: 1 year (six weeks prior to study entry, participants were stabilised on fluphenazine decanoate $0.5-2 \mathrm{~mL} / 2$ weeks.

Design: parallel group.

Country: United States.
Diagnosis: schizophrenia (RDC).

$\mathrm{N}=60$.

Age: $17-49$ years.

Sex: 41M,19F.

History: $<2$ psychotic episodes, able to give informed consent.

Setting: community.
1. Fluphenazine decanoate: dose $0.5-4 \mathrm{IM} / \mathrm{mL}$ biweekly. $\mathrm{N}=29$.

2. Penfluridol (oral): dose 20-160 mg, weekly. $\mathrm{N}=27$.

Outcomes

Global state: need for additional medication.

Behaviour: leaving the study early.

Unable to use -

Global state: CGI (no data).

Mental state: BPRS (no data).

Adverse effects: KAS (no data).

Social ability: SAS (non clinical outcome, data unusable).

Notes Authors contacted.

Risk of bias

\begin{tabular}{|c|c|c|}
\hline Bias & Authors' judgement & Support for judgement \\
\hline $\begin{array}{l}\text { Random sequence generation (selection } \\
\text { bias) }\end{array}$ & Unclear risk & "random". Method not reported. \\
\hline Allocation concealment (selection bias) & Unclear risk & $\begin{array}{l}\text { Details of allocation concealment not re- } \\
\text { ported. }\end{array}$ \\
\hline $\begin{array}{l}\text { Blinding of participants and personnel } \\
\text { (performance bias) } \\
\text { All outcomes }\end{array}$ & Unclear risk & $\begin{array}{l}\text { "double-blind". Blinding details not re- } \\
\text { ported. }\end{array}$ \\
\hline $\begin{array}{l}\text { Blinding of outcome assessment (detection } \\
\text { bias) } \\
\text { All outcomes }\end{array}$ & Unclear risk & $\begin{array}{l}\text { "double-blind". Blinding details not re- } \\
\text { ported. }\end{array}$ \\
\hline $\begin{array}{l}\text { Incomplete outcome data (attrition bias) } \\
\text { All outcomes }\end{array}$ & High risk & $\begin{array}{l}\text { "After } 56 \text { patients completed the study, the } \\
\text { Food and Drug Administration suspended } \\
\text { the use penfluridol", "these four patients } \\
\text { whose failure to complete the study is un- }\end{array}$ \\
\hline
\end{tabular}


Quitkin 1978 (Continued)

\begin{tabular}{|c|c|c|}
\hline & & $\begin{array}{l}\text { related to the clinical efficacy... will not be } \\
\text { considered in further analysis" } \\
35 / 56 \text { participants completed the study; } \\
4 \text { from the penfluridol and } 8 \text { from the } \\
\text { fluphenazine group left the study early }\end{array}$ \\
\hline Selective reporting (reporting bias) & High risk & $\begin{array}{l}\text { Outcome data not reported: BPRS, CGI, } \\
\text { KASP, KASR. }\end{array}$ \\
\hline Other bias & Unclear risk & Source of funding not reported. \\
\hline
\end{tabular}

Rifkin 1977

\begin{tabular}{|c|c|}
\hline Methods & $\begin{array}{l}\text { Allocation: randomised. } \\
\text { Blindness: double. } \\
\text { Duration: } 1 \text { year (psychotherapy given every } 2 \text { weeks for first } 6 \text { months, monthly there- } \\
\text { after). } \\
\text { Design: } 3 \text { treatment groups. } \\
\text { Country: United States. }\end{array}$ \\
\hline Participants & $\begin{array}{l}\text { Diagnosis: schizophrenia (Kraepelinian). } \\
\mathrm{N}=73 \text {. } \\
\text { Age: } 17-38 \text { years, mean } \\
\text { Sex: } 50 \mathrm{M}, 23 \mathrm{~F} \text {. } \\
\text { History: } 16 \text { participants acutely ill, stable while receiving FD/F HCL for } 4 \text { weeks, able } \\
\text { to give informed consent. } \\
\text { Setting: community. }\end{array}$ \\
\hline Interventions & $\begin{array}{l}\text { 1.Fluphenazine decanoate: dose range } 0.5-2.0 \mathrm{IM} / \mathrm{mL} \text {, mean } 0.5 \mathrm{~mL} / \mathrm{IM} \text { biweekly. } \mathrm{N}= \\
\text { 23. } \\
\text { 2. Fluphenazine hydrochloride (oral): dose range } 5-20 \mathrm{mg} \text {, mean } 5 \mathrm{mg} / \text { daily. } \mathrm{N}=28 \text {. } \\
\text { 3. Placebo. } \mathrm{N}=22 \text {. }\end{array}$ \\
\hline Outcomes & $\begin{array}{l}\text { Behaviour: leaving the study early. } \\
\text { Mental state: relapse. } \\
\text { Adverse effects: toxicity. } \\
\text { Unable to use - } \\
\text { Global State: CGI (no data). } \\
\text { Mental state: BPRS (patient evaluation, no data). } \\
\text { Adverse effects: KAS (no data). }\end{array}$ \\
\hline Notes & $\begin{array}{l}\mathrm{N} \text { differs in paper I for chronic patients compared to paper II. } \\
\text { Continuous data reported in paper II but not usable- not separated into separate groups }\end{array}$ \\
\hline
\end{tabular}

Risk of bias

Risk of bias

Bias

Authors' judgement

Support for judgement

Fluphenazine decanoate (depot) and enanthate for schizophrenia (Review)

Copyright $\odot 2016$ The Cochrane Collaboration. Published by John Wiley \& Sons, Ltd. 
Rifkin 1977 (Continued)

\begin{tabular}{|c|c|c|}
\hline $\begin{array}{l}\text { Random sequence generation (selection } \\
\text { bias) }\end{array}$ & Unclear risk & "random". Method not reported. \\
\hline Allocation concealment (selection bias) & Unclear risk & $\begin{array}{l}\text { Details of allocation concealment not re- } \\
\text { ported. }\end{array}$ \\
\hline $\begin{array}{l}\text { Blinding of participants and personnel } \\
\text { (performance bias) } \\
\text { All outcomes }\end{array}$ & Unclear risk & $\begin{array}{l}\text { "double-blind". Patients blinded: "All pa- } \\
\text { tients received biweekly injections and } \\
\text { daily pills-some being placebo." Blinding } \\
\text { details of personnel not reported }\end{array}$ \\
\hline $\begin{array}{l}\text { Blinding of outcome assessment (detection } \\
\text { bias) } \\
\text { All outcomes }\end{array}$ & Unclear risk & $\begin{array}{l}\text { "double-blind". Blinding details not re- } \\
\text { ported. }\end{array}$ \\
\hline $\begin{array}{l}\text { Incomplete outcome data (attrition bias) } \\
\text { All outcomes }\end{array}$ & Unclear risk & $\begin{array}{l}\text { Number of drop-outs are balanced across } \\
\text { the intervention groups but reasons have } \\
\text { not been reported }\end{array}$ \\
\hline Selective reporting (reporting bias) & Unclear risk & Outcomes not fully reported. \\
\hline Other bias & Low risk & $\begin{array}{l}\text { “...supported by national Institute of Men- } \\
\text { tal Health grant..." }\end{array}$ \\
\hline
\end{tabular}

Rossi 1990

Methods

Allocation: randomised.

Blindness: double.

Duration: 6 months.

Design: parallel group.

Country:

\begin{tabular}{|c|c|}
\hline Participants & $\begin{array}{l}\text { Diagnosis: schizophrenia (DSM III-R). } \\
\mathrm{N}=30 \text {. } \\
\text { Age: } 19-42 \text { years, mean }-29 \text { years. } \\
\text { Sex: } 18 \mathrm{M}, 13 \mathrm{~F} \text {. } \\
\text { History: duration of illness }(<1 \text { year } \mathrm{n}=6),(1-6 \text { year } \\
\text { Setting: community. }\end{array}$ \\
\hline Interventions & $\begin{array}{l}\text { 1. Fluphenazine decanoate: dose } 25-50 \mathrm{mg} / \mathrm{IM} \text {, mear } \\
\text { 2. Bromperidol decanoate: dose } 50-100 \mathrm{mg} / \mathrm{IM} \text {, mea }\end{array}$ \\
\hline Outcomes & $\begin{array}{l}\text { Behaviour: leaving the study early. } \\
\text { Unable to use - } \\
\text { Global state: CGI (no SD). } \\
\text { Mental state: BPRS (no SD). } \\
\text { Behaviour: CBS (no SD). } \\
\text { Side effects: DOTES, TESS, EPSE (data unusable). }\end{array}$ \\
\hline
\end{tabular}


Rossi 1990 (Continued)

\begin{tabular}{lll}
\hline Notes & Unable to complete 'Risk of bias' table - article in Italian \\
\hline Risk of bias & & \\
\hline Bias & Authors' judgement & Support for judgement \\
\hline Allocation concealment (selection bias) & Unclear risk & B - Unclear \\
\hline
\end{tabular}

Russell 1982

\begin{tabular}{|c|c|c|}
\hline Methods & \multicolumn{2}{|c|}{$\begin{array}{l}\text { Allocation: randomised. } \\
\text { Blindness: double. } \\
\text { Duration: } 6 \text { months. } \\
\text { Design: parallel group. } \\
\text { Country: United Kingdom. }\end{array}$} \\
\hline Participants & \multicolumn{2}{|c|}{$\begin{array}{l}\text { Diagnosis: schizophrenia (ICD-9). } \\
\mathrm{N}=33 \text {. } \\
\text { Age: mean }-36 \text { years. } \\
\text { Sex: } 12 \mathrm{M}, 16 \mathrm{~F} \text {. } \\
\text { History: duration illness } 9 \text { years, able to give informed consent. } \\
\text { Setting: unclear. }\end{array}$} \\
\hline Interventions & \multicolumn{2}{|c|}{$\begin{array}{l}\text { 1. Fluphenazine decanoate: dose mean } 12.5 \mathrm{mg} / \mathrm{IM} \text {, maximum dose } 25.5 \mathrm{mg} / \mathrm{IM} \text { every } \\
2-3 \text { weeks. } \mathrm{N}=13 \text {. } \\
\text { 2. Fluspiriline decanoate: dose mean } 3 \mathrm{mg} / \mathrm{IM} \text {, maximum dose } 10.94 \mathrm{mg} / \mathrm{IM} \text { weekly. } \mathrm{N} \\
=20\end{array}$} \\
\hline Outcomes & \multicolumn{2}{|c|}{$\begin{array}{l}\text { Global state: need for additional medication. } \\
\text { Behaviour: leaving the study early. } \\
\text { Adverse effects: EPRS. } \\
\text { Unable to use - } \\
\text { Global state: CGI (no SD). } \\
\text { Mental state: BPRS (no SD). } \\
\text { Adverse effects: SAS (no data). } \\
\text { Behaviour: MACC-BAS (no data). }\end{array}$} \\
\hline Notes & \multicolumn{2}{|l|}{ Authors contacted. } \\
\hline \multicolumn{3}{|l|}{ Risk of bias } \\
\hline Bias & Authors' judgement & Support for judgement \\
\hline $\begin{array}{l}\text { Random sequence generation (selection } \\
\text { bias) }\end{array}$ & Unclear risk & "random allocation". Method not reported. \\
\hline
\end{tabular}




\section{Russell 1982 (Continued)}

\begin{tabular}{|c|c|c|}
\hline Allocation concealment (selection bias) & Unclear risk & $\begin{array}{l}\text { Details of allocation concealment not re- } \\
\text { ported. }\end{array}$ \\
\hline $\begin{array}{l}\text { Blinding of participants and personnel } \\
\text { (performance bias) } \\
\text { All outcomes }\end{array}$ & Unclear risk & $\begin{array}{l}\text { "double-blind". Subjects } \\
\text { blinded "...placebo injections being given. } \\
\text {..in the intervening weeks...". Blinding de- } \\
\text { tails of personnel not reported }\end{array}$ \\
\hline $\begin{array}{l}\text { Blinding of outcome assessment (detection } \\
\text { bias) } \\
\text { All outcomes }\end{array}$ & Low risk & $\begin{array}{l}\text { "double-blind". "...a second psychiatrist } \\
\text { rated each patient blind..." }\end{array}$ \\
\hline $\begin{array}{l}\text { Incomplete outcome data (attrition bias) } \\
\text { All outcomes }\end{array}$ & Unclear risk & $\begin{array}{l}\text { Two fluspirilene and three fluphenazine pa- } \\
\text { tients dropped out after one week", reasons } \\
\text { not reported }\end{array}$ \\
\hline Selective reporting (reporting bias) & High risk & $\begin{array}{l}\text { BPRS and CGI incompletely reported (P } \\
\text { values). SAS and MACC Behavior Adjust- } \\
\text { ment Scale results not reported }\end{array}$ \\
\hline Other bias & Unclear risk & $\begin{array}{l}\text { "...supported by McNeil (Canada) labora- } \\
\text { tories. }\end{array}$ \\
\hline
\end{tabular}

\section{Schlosberg 1978}

Methods
Allocation: randomised.

Blindness: double.

Duration: 9 months (depot), 3 months (placebo)*.

Design: parallel group.

Country: Israel.
Participants

Diagnosis: schizophrenia.

$\mathrm{N}=75$ (12 in placebo trial).

Age: mean 42 years.

Sex: not stated.

History: duration illness mean -17 years.

Setting: not stated.

Interventions

1. Fluphenazine decanoate: dose $6.25-50 \mathrm{mg} / \mathrm{IM}$ monthly. $\mathrm{N}=30$.

2. Pipothiazine palmitate: dose $6.25-50 \mathrm{mg} / \mathrm{IM}$ monthly. $\mathrm{N}=30$

Outcomes

Leaving the study.

Global Impression.

Side effects.

Unable to use -

Mental state: BPRS (no SD).

Behaviour: NOSIE (no SD).

Fluphenazine decanoate (depot) and enanthate for schizophrenia (Review)

Copyright @ 2016 The Cochrane Collaboration. Published by John Wiley \& Sons, Ltd. 
Schlosberg 1978 (Continued)

\begin{tabular}{|c|c|c|}
\hline Notes & * Wash-out period 14 days. & \\
\hline \multicolumn{3}{|l|}{ Risk of bias } \\
\hline Bias & Authors' judgement & Support for judgement \\
\hline $\begin{array}{l}\text { Random sequence generation (selection } \\
\text { bias) }\end{array}$ & Unclear risk & $\begin{array}{l}\text { "randomly assigned". Method not re- } \\
\text { ported. }\end{array}$ \\
\hline Allocation concealment (selection bias) & Unclear risk & $\begin{array}{l}\text { Details of allocation concealment not re- } \\
\text { ported. }\end{array}$ \\
\hline $\begin{array}{l}\text { Blinding of participants and personnel } \\
\text { (performance bias) } \\
\text { All outcomes }\end{array}$ & Unclear risk & $\begin{array}{l}\text { "double-blind". Patients blinded: "The } \\
\text { drugs injected were pipotiazine palmitate, } \\
\text { fluphenazine decanoate, and placebo...". } \\
\text { Blinding details of personnel not reported }\end{array}$ \\
\hline $\begin{array}{l}\text { Blinding of outcome assessment (detection } \\
\text { bias) } \\
\text { All outcomes }\end{array}$ & Unclear risk & $\begin{array}{l}\text { "double-blind". Blinding details not re- } \\
\text { ported. }\end{array}$ \\
\hline $\begin{array}{l}\text { Incomplete outcome data (attrition bias) } \\
\text { All outcomes }\end{array}$ & Unclear risk & Number lost to follow-up not reported. \\
\hline Selective reporting (reporting bias) & High risk & $\begin{array}{l}\text { BPSD and NOISE results incompletely re- } \\
\text { ported. }\end{array}$ \\
\hline Other bias & Unclear risk & Source of funding not reported. \\
\hline
\end{tabular}

Schneider 1981

Methods

Allocation: randomised.

Blindness: double.

Duration: 1 year (preceded by 2 weeks washout).

Design: parallel group.

Country: United States.

\begin{tabular}{|c|c|}
\hline Participants & $\begin{array}{l}\text { Diagnosis: schizophrenia (DSM II). } \\
\mathrm{N}=59 \text {. } \\
\text { Age: } 21-65 \text { years, mean }-45 \text { years. } \\
\text { Sex: } 51 \mathrm{M}, 8 \mathrm{~F} \text {. } \\
\text { History: duration illness mean }-21 \text { years, able to give informed consent. } \\
\text { Setting: hospital. }\end{array}$ \\
\hline Interventions & $\begin{array}{l}\text { 1. Fluphenazine decanoate: dose } 12.5-400 \mathrm{mg} / \mathrm{IM} \text { every } 2-5 \text { weeks. } \mathrm{N}=27 \text {. } \\
\text { 2. Pipothiazine palmitate: dose } 50-400 \mathrm{mg} / \mathrm{IM} \text { every } 2-5 \text { weeks. } \mathrm{N}=32\end{array}$ \\
\hline
\end{tabular}


Schneider 1981 (Continued)

\begin{tabular}{|c|c|c|}
\hline Outcomes & \multicolumn{2}{|c|}{$\begin{array}{l}\text { Leaving the study early. } \\
\text { Unable to use - } \\
\text { Global state: CGI (no data). } \\
\text { Physiological measures: blood samples (non-clinical outcome, data unusable) }\end{array}$} \\
\hline Notes & \multicolumn{2}{|c|}{$67 \%$ attrition rate in the treatment group, therefore the data are not usable } \\
\hline \multicolumn{3}{|l|}{ Risk of bias } \\
\hline Bias & Authors' judgement & Support for judgement \\
\hline $\begin{array}{l}\text { Random sequence generation (selection } \\
\text { bias) }\end{array}$ & Unclear risk & $\begin{array}{l}\text { "randomly assigned". Method not re- } \\
\text { ported. }\end{array}$ \\
\hline Allocation concealment (selection bias) & Unclear risk & $\begin{array}{l}\text { Details of allocation concealment not re- } \\
\text { ported. }\end{array}$ \\
\hline $\begin{array}{l}\text { Blinding of participants and personnel } \\
\text { (performance bias) } \\
\text { All outcomes }\end{array}$ & Unclear risk & $\begin{array}{l}\text { "double-blind". Blinding details not re- } \\
\text { ported. }\end{array}$ \\
\hline $\begin{array}{l}\text { Blinding of outcome assessment (detection } \\
\text { bias) } \\
\text { All outcomes }\end{array}$ & Unclear risk & $\begin{array}{l}\text { "double-blind". Blinding details not re- } \\
\text { ported. }\end{array}$ \\
\hline $\begin{array}{l}\text { Incomplete outcome data (attrition bias) } \\
\text { All outcomes }\end{array}$ & Unclear risk & $\begin{array}{l}\text { Number of drop-outs not reported per allo- } \\
\text { cation group. } 59 \text { patients were randomised, } \\
\text { "a total of } 34 \text { patients remained in the study } \\
\text { long enough to have at least } 10 \text { blood anal- } \\
\text { yses" }\end{array}$ \\
\hline Selective reporting (reporting bias) & High risk & Data not reported for CGI. \\
\hline Other bias & Unclear risk & Source of funding not reported. \\
\hline
\end{tabular}

\section{Schooler 1976}

Methods

Allocation: randomised.

Blindness: double.

Duration: 1 year.

Design: parallel group.

Country: United States.

Participants

Diagnosis: schizophrenia (Schneiderian 1st rank).

$\mathrm{N}=197$.

Age: $18-55$ years, mean -30 years.

Sex: 58M, 42F. 
Schooler 1976 (Continued)

History: newly admitted from the community.

Setting: community.

\begin{tabular}{ll} 
Interventions & $\begin{array}{l}\text { 1. Fluphenazine decanoate: dose } 12.5-100 \mathrm{mg} / \mathrm{IM} \text {, mean } 34.7 \mathrm{mg} / \mathrm{IM} \text { every } 3 \text { weeks. } \mathrm{N} \\
=102 .\end{array}$ \\
& 2. Fluphenazine (orally): dose max $60 \mathrm{mg}$, mean $25.2 \mathrm{mg} / \mathrm{IM}$ daily. $\mathrm{N}=95$ \\
\hline \multirow{2}{*}{ Outcomes } & $\begin{array}{l}\text { Leaving the study early. } \\
\text { Additional medication. } \\
\text { Side effects: TESS. }\end{array}$ \\
\hline Notes & No continuous outcomes measured.
\end{tabular}

Risk of bias

\begin{tabular}{|c|c|c|}
\hline Bias & Authors' judgement & Support for judgement \\
\hline $\begin{array}{l}\text { Random sequence generation (selection } \\
\text { bias) }\end{array}$ & Unclear risk & $\begin{array}{l}\text { "randomly assigned". Method not re- } \\
\text { ported. }\end{array}$ \\
\hline Allocation concealment (selection bias) & Unclear risk & $\begin{array}{l}\text { Details of allocation concealment not re- } \\
\text { ported. }\end{array}$ \\
\hline $\begin{array}{l}\text { Blinding of participants and personnel } \\
\text { (performance bias) } \\
\text { All outcomes }\end{array}$ & Unclear risk & $\begin{array}{l}\text { Patients blinded; blinding details of per- } \\
\text { sonnel not reported."...an injection of de- } \\
\text { pot fluphenazine plus oral placebo or depot } \\
\text { placebo plus oral fluphenazine. Both injec- } \\
\text { tions and pills...administered under dou- } \\
\text { ble-blind conditions.” }\end{array}$ \\
\hline $\begin{array}{l}\text { Blinding of outcome assessment (detection } \\
\text { bias) } \\
\text { All outcomes }\end{array}$ & Unclear risk & $\begin{array}{l}\text { "double-blind". Blinding details not re- } \\
\text { ported. }\end{array}$ \\
\hline $\begin{array}{l}\text { Incomplete outcome data (attrition bias) } \\
\text { All outcomes }\end{array}$ & Unclear risk & $\begin{array}{l}\text { Insufficient information. Losses to follow- } \\
\text { up not reported. }\end{array}$ \\
\hline Selective reporting (reporting bias) & High risk & $\begin{array}{l}\text { Incomplete data. "Treatment emergent } \\
\text { symptoms were evaluated... by both a } \\
\text { physician and a nurse... The data in this pa- } \\
\text { per are the nurse's ratings" }\end{array}$ \\
\hline Other bias & Low risk & $\begin{array}{l}\text { "...supported by the National Institute of } \\
\text { Mental Health grants..." }\end{array}$ \\
\hline
\end{tabular}




\begin{tabular}{|c|c|}
\hline Methods & $\begin{array}{l}\text { Allocation: randomised. } \\
\text { Blindness: double. } \\
\text { Duration: } 1 \text { year. } \\
\text { Design: parallel group. } \\
\text { Country: USA. }\end{array}$ \\
\hline Participants & $\begin{array}{l}\text { Diagnosis: schizophrenia. } \\
\mathrm{N}=214^{*} \text {. } \\
\text { Age: mean }-29 \text { years. } \\
\text { Sex: not stated. } \\
\text { History: not stated. } \\
\text { Setting: community. }\end{array}$ \\
\hline Interventions & $\begin{array}{l}\text { 1. Fluphenazine decanoate: (dose and frequency not stated). } \mathrm{N}=107 \text {. } \\
\text { 2. Fluphenazine hydrochloride: (dose and frequency not stated). } \mathrm{N}=107\end{array}$ \\
\hline Outcomes & $\begin{array}{l}\text { Relapse. } \\
\text { Unable to use - } \\
\text { Mental state: BPRS (no SD). } \\
\text { Side effects: SCL-9 (no SD). }\end{array}$ \\
\hline Notes & *Maintenance phase \\
\hline
\end{tabular}

Risk of bias

\begin{tabular}{|c|c|c|}
\hline Bias & Authors' judgement & Support for judgement \\
\hline $\begin{array}{l}\text { Random sequence generation (selection } \\
\text { bias) }\end{array}$ & Unclear risk & $\begin{array}{l}\text { "randomly assigned". Method not re- } \\
\text { ported. }\end{array}$ \\
\hline Allocation concealment (selection bias) & Unclear risk & $\begin{array}{l}\text { Details of allocation concealment not re- } \\
\text { ported. }\end{array}$ \\
\hline $\begin{array}{l}\text { Blinding of participants and personnel } \\
\text { (performance bias) } \\
\text { All outcomes }\end{array}$ & Unclear risk & $\begin{array}{l}\text { "double blind". Blinding details not re- } \\
\text { ported. }\end{array}$ \\
\hline $\begin{array}{l}\text { Blinding of outcome assessment (detection } \\
\text { bias) } \\
\text { All outcomes }\end{array}$ & Unclear risk & $\begin{array}{l}\text { "double blind". Blinding details not re- } \\
\text { ported. }\end{array}$ \\
\hline $\begin{array}{l}\text { Incomplete outcome data (attrition bias) } \\
\text { All outcomes }\end{array}$ & Unclear risk & $\begin{array}{l}\text { Insufficient information. Losses to follow- } \\
\text { up not reported. }\end{array}$ \\
\hline Selective reporting (reporting bias) & High risk & $\begin{array}{l}\text { BPRS and SCL- } 90 \text { results incompletely re- } \\
\text { ported. }\end{array}$ \\
\hline Other bias & Unclear risk & Source of funding not reported. \\
\hline
\end{tabular}


Methods

\begin{tabular}{|l} 
Blindness: double. \\
Duration: 1 year. \\
Design: parallel group. \\
Country: United States.
\end{tabular}

Diagnosis: schizophrenia.

$\mathrm{N}=290^{*}$.

Age: $18-55$ years, mean -29 years.

Sex: 170M, 120F.

History: able to give informed consent.

Setting: initially in hospital for 7-9 weeks intensive treatment, followed by community

Interventions

1. Fluphenazine decanoate: dose $12.5-100 \mathrm{mg} / \mathrm{IM}$, mean $34.2 \mathrm{mg} / \mathrm{IM}$ every 3 weeks. $\mathrm{N}$

$=143$.

2. Fluphenazine hydrochloride (oral): dose $2.5-60 \mathrm{mg}$, mean $24.8 \mathrm{mg}$ daily. $\mathrm{N}=147$

\begin{tabular}{l|l} 
Outcomes & Leaving the study early. \\
& Side effects: DOTES, SCL-90. \\
& Unable to use - \\
& Global state: CGI, Community Nursing Assessmen \\
& Mental state: BPRS, HRSD (no data). \\
& Social ability: SAS (non clinical outcomes, data \\
\hline Notes & Results for both FD \& FHCL groups together. \\
& $\begin{array}{l}\text { Authors contacted } \\
* 214 \text { entered maintenance phase. }\end{array}$ \\
\hline
\end{tabular}

Risk of bias

Bias

Random sequence generation (selection Unclear risk bias)

Allocation concealment (selection bias)

Unclear risk

\section{Support for judgement}

"stratified by sex, randomly assigned". Method not reported.

Details of allocation concealment not reported.

"double-blind". "...either fluphenazine decanoate and oral placebo or fluphenazine hydrochloride and placebo injection." $\mathrm{Pa}$ tients blinded. Blinding details of personnel not reported

"double blind". Blinding details not reported.
Blinding of outcome assessment (detection Unclear risk bias)

All outcomes 
Schooler 1980 (Continued)

\begin{tabular}{|l|l}
$\begin{array}{l}\text { Incomplete outcome data (attrition bias) } \\
\text { All outcomes }\end{array}$ Low risk & $\begin{array}{l}290 \text { participants were randomised to the } \\
\text { "intensive phase" of the study lasting } 7 \text { to } 9 \\
\text { weeks. "76 participants were removed from } \\
\text { treatment during the intensive treatment } \\
\text { phase. There were no significant differences } \\
\text { between the two treatments in reasons for } \\
\text { or number of these terminations" } \\
\text { "214 (107 from each of the two treat- } \\
\text { ment regimes) entered the one-year"main- } \\
\text { tenance phase" of the study". } \\
\text { "Patients continued to receive study treat- } \\
\text { ment for a maximum of one year or un- } \\
\text { til termination for either treatment-related } \\
\text { or administrative reasons". Survival analy- } \\
\text { sis was performed in the study }\end{array}$
\end{tabular}

Selective reporting (reporting bias) High risk

Incomplete report of outcome data (BPRS, Hopkins Symptom Checklist-90, CGI), no data Hamilton Depression Scale. Full BPRS data (mean SD) not reported per allocation group

\begin{tabular}{|c|c|c|}
\hline Other bias & Low risk & $\begin{array}{l}\text { "...supported by the Nations Institute of } \\
\text { Mental Health grants..." }\end{array}$ \\
\hline
\end{tabular}

\section{Schooler 1997}

\begin{tabular}{|c|c|}
\hline Methods & $\begin{array}{l}\text { Allocation: randomised. } \\
\text { Blindness: double. } \\
\text { Duration: } 16-24 \text { weeks. } \\
\text { Design: dosage study. } \\
\text { Country: United States. }\end{array}$ \\
\hline Participants & $\begin{array}{l}\text { Diagnosis: schizophrenia (DSM III). } \\
\mathrm{N}=313 \text {. } \\
\text { Age: mean } 29.6 \text { years. } \\
\text { Sex: } 207 \mathrm{M} 106 \mathrm{~F} \text {. } \\
\text { History: acutely ill. } \\
\text { Setting: community and /or hospital. }\end{array}$ \\
\hline Interventions & $\begin{array}{l}\text { 1. Fluphenazine decanoate (low dose): dose } 2.5-10 \mathrm{mg} \text { biweekly. } \mathrm{N}=106 \text {. } \\
\text { 2. Fluphenazine decanoate (standard): dose } 12.5-50 \mathrm{mg} \text { biweekly. } \mathrm{N}=107\end{array}$ \\
\hline Outcomes & $\begin{array}{l}\text { Rehospitalised. } \\
\text { Unable to use - } \\
\text { Global impression: CGI (no data). } \\
\text { Mental state: BPRS, SANS (no data). } \\
\text { Side effects: AIMS, EPS, Early Signs Questionnaire (no data). }\end{array}$ \\
\hline
\end{tabular}

Fluphenazine decanoate (depot) and enanthate for schizophrenia (Review) 
Schooler 1997 (Continued)

Family therapy strategies: (non-clinical outcomes, data unusable)

\begin{tabular}{|c|c|c|}
\hline Notes & \multicolumn{2}{|l|}{ Authors contacted. } \\
\hline \multicolumn{3}{|l|}{ Risk of bias } \\
\hline Bias & Authors' judgement & Support for judgement \\
\hline $\begin{array}{l}\text { Random sequence generation (selection } \\
\text { bias) }\end{array}$ & Unclear risk & $\begin{array}{l}\text { "randomly assigned". Method not re- } \\
\text { ported. }\end{array}$ \\
\hline Allocation concealment (selection bias) & Unclear risk & $\begin{array}{l}\text { Details of allocation concealment not re- } \\
\text { ported. }\end{array}$ \\
\hline $\begin{array}{l}\text { Blinding of participants and personnel } \\
\text { (performance bias) } \\
\text { All outcomes }\end{array}$ & Unclear risk & $\begin{array}{l}\text { "double-blind". Blinding details not re- } \\
\text { ported. }\end{array}$ \\
\hline $\begin{array}{l}\text { Blinding of outcome assessment (detection } \\
\text { bias) } \\
\text { All outcomes }\end{array}$ & Unclear risk & $\begin{array}{l}\text { "double-blind". Blinding details not re- } \\
\text { ported. }\end{array}$ \\
\hline $\begin{array}{l}\text { Incomplete outcome data (attrition bias) } \\
\text { All outcomes }\end{array}$ & Unclear risk & $\begin{array}{l}\text { Information on losses to follow-up not re- } \\
\text { ported. }\end{array}$ \\
\hline Selective reporting (reporting bias) & High risk & $\begin{array}{l}\text { Outcomes not reported: BPRS, SANS, } \\
\text { CGI, Neurological Rating Scale (extrapyra- } \\
\text { midal symptoms), Early Signs Question- } \\
\text { naire. Family treatment strategies outcomes } \\
\text { incompletely reported }\end{array}$ \\
\hline Other bias & Unclear risk & $\begin{array}{l}\text { Source of funding unclear. "All dou- } \\
\text { ble-blind medication supplies as well as } \\
\text { open label fluphenazine decanoate and } \\
\text { fluphenazine hydrochloride were provided } \\
\text { courtesy of Bristol-Myers Squibb Com- } \\
\text { pany...". "multicentre clinical trial...in col- } \\
\text { laboration with the Division of Treatment } \\
\text { and Clinical Research of the national Insti- } \\
\text { tute of Mental Health" }\end{array}$ \\
\hline
\end{tabular}


Methods
Allocation: randomised.

Blindness: double.

Duration: 48 weeks.

Design: parallel group.

Country: UK.

\begin{tabular}{ll}
\hline Participants & Diagnosis: schizophrenia (DSM III). \\
& N $=59$. \\
Age: $30-81$ years, mean -52 years. \\
Sex: $34 \mathrm{M}, 25 \mathrm{~F}$. \\
History: duration illness 22 years, able to give informed consent. \\
Setting: not stated. \\
\hline
\end{tabular}

Interventions

1. Fluphenazine decanoate: dose $100 \mathrm{mg} / \mathrm{IM} /$ monthly. $\mathrm{N}=29$.

2. Haloperidol decanoate: dose $100 \mathrm{mg} / \mathrm{IM} /$ monthly. $\mathrm{N}=30$.

\begin{tabular}{ll} 
Outcomes & Leaving the study early. \\
& Additional medication. \\
& Side effects: EPS Rating Scale, AIMS. \\
& Unable to use - \\
& Mental state: CPRS (data unusable). \\
& Physiological measures: weight (non-clinical outcomes, data unusable) \\
\hline Notes & N and drop-out numbers for each group changes.
\end{tabular}

Risk of bias

\begin{tabular}{l|l|l}
\hline Bias & Authors' judgement & Support for judgement \\
\hline $\begin{array}{l}\text { Random sequence generation (selection } \\
\text { bias) }\end{array}$ & Unclear risk & $\begin{array}{l}\text { "randomly allocated". Method not re- } \\
\text { ported. }\end{array}$ \\
\hline $\begin{array}{l}\text { Allocation concealment (selection bias) } \\
\text { Alinding of participants and personnel }\end{array}$ & Unclear risk & $\begin{array}{l}\text { Details of allocation concealment not re- } \\
\text { ported. }\end{array}$ \\
\hline $\begin{array}{l}\text { All outcomes } \\
\text { Blinding of outcome assessment (detection } \\
\text { bias) } \\
\text { All outcomes }\end{array}$ & Unclear risk & $\begin{array}{l}\text { "double-blind". Blinding details not re- } \\
\text { ported. }\end{array}$ \\
\hline $\begin{array}{l}\text { Incomplete outcome data (attrition bias) } \\
\text { All outcomes }\end{array}$ & High risk & $\begin{array}{l}\text { "double-blind". Blinding details not re- } \\
\text { ported. }\end{array}$ \\
\hline
\end{tabular}


Sharma 1991 (Continued)

\begin{tabular}{|c|c|c|}
\hline Selective reporting (reporting bias) & High risk & CPRS results reported incompletely. \\
\hline Other bias & Unclear risk & Source of funding not reported. \\
\hline
\end{tabular}

\section{Shenoy 1981}

\begin{tabular}{|c|c|c|}
\hline Methods & \multicolumn{2}{|c|}{$\begin{array}{l}\text { Allocation: randomised. } \\
\text { Blindness: double. } \\
\text { Duration: } 6 \text { weeks. } \\
\text { Design: parallel group. } \\
\text { Country: USA. }\end{array}$} \\
\hline Participants & \multicolumn{2}{|c|}{$\begin{array}{l}\text { Diagnosis: schizophrenia (DSM III criteria). } \\
\mathrm{N}=31 . \\
\text { Age: mean } 37.4 \text { (11.33) years, range 23-59. } \\
\text { Sex: not reported. } \\
\text { History: chronic ambulatory schizophrenia patients treated with fluphenazine decanoate } \\
\text { for two years. } \\
\text { Setting: outpatients. }\end{array}$} \\
\hline Interventions & \multicolumn{2}{|c|}{$\begin{array}{l}\text { 1. Placebo, every } 3 \text { weeks. } N=17 \\
\text { 2. Fluphenazine decanoate (dose not reported), every } 3 \text { weeks. } N=14\end{array}$} \\
\hline Outcomes & \multicolumn{2}{|c|}{$\begin{array}{l}\text { Global state: relapse, GAS. } \\
\text { Mental State: SADS. } \\
\text { Unable to use - } \\
\text { Adverse effects: AIMS (data not reported per treatment group) }\end{array}$} \\
\hline Notes & \multicolumn{2}{|c|}{$\begin{array}{l}\text { Participants in group } 1 \text { were given placebo injections as a "drug holiday". At the end of } \\
\text { the } 6 \text {-week study they were returned to their routine active medication }\end{array}$} \\
\hline \multicolumn{3}{|l|}{ Risk of bias } \\
\hline Bias & Authors' judgement & Support for judgement \\
\hline $\begin{array}{l}\text { Random sequence generation (selection } \\
\text { bias) }\end{array}$ & Unclear risk & $\begin{array}{l}\text { "patients were randomly assigned". } \\
\text { Method not reported. }\end{array}$ \\
\hline Allocation concealment (selection bias) & Unclear risk & $\begin{array}{l}\text { Details of allocation concealment not re- } \\
\text { ported. }\end{array}$ \\
\hline $\begin{array}{l}\text { Blinding of participants and personnel } \\
\text { (performance bias) } \\
\text { All outcomes }\end{array}$ & Unclear risk & $\begin{array}{l}\text { "double-blind". Blinding details not re- } \\
\text { ported. }\end{array}$ \\
\hline
\end{tabular}




\section{Shenoy 1981 (Continued)}

\begin{tabular}{lll}
$\begin{array}{l}\text { Blinding of outcome assessment (detection } \\
\text { bias) } \\
\text { All outcomes }\end{array}$ & Unclear risk & $\begin{array}{l}\text { "double-blind". Blinding details not re- } \\
\text { ported. }\end{array}$ \\
\hline $\begin{array}{l}\text { Incomplete outcome data (attrition bias) } \\
\text { All outcomes }\end{array}$ & Low risk & $\begin{array}{l}\text { None of the patients relapsed. } 3 \text { partici- } \\
\text { pants did not complete the trial: } 2 \text { in the } \\
\text { active and } 1 \text { in the placebo group. All drop- } \\
\text { out were due to "failure to meet appoint- } \\
\text { ments" }\end{array}$ \\
\hline Selective reporting (reporting bias) & Low risk & All outcomes have been reported. \\
\hline Other bias & Unclear risk & Source of funding has not been reported. \\
\hline
\end{tabular}

\section{Shu 1983}

Methods

Allocation: randomised.

Blindness: double.

Duration: 6 weeks.

Design: parallel group.

Country: China.

\begin{tabular}{|c|c|c|}
\hline Participants & \multicolumn{2}{|c|}{$\begin{array}{l}\text { Diagnosis: schizophrenia. } \\
\mathrm{N}=34 . \\
\text { Age: } 15-48 \text { years. } \\
\text { Sex: all male. } \\
\text { History: not stated. } \\
\text { Setting: hospital. }\end{array}$} \\
\hline Interventions & \multicolumn{2}{|c|}{$\begin{array}{l}\text { 1. Fluphenazine decanoate: (dose and frequency not stated). } \mathrm{N}=16 \text {. } \\
\text { 2. Penflurdidol + placebo: (dose and frequency not stated). } \mathrm{N}=18\end{array}$} \\
\hline Outcomes & \multicolumn{2}{|l|}{$\begin{array}{l}\text { Global state: CGI. } \\
\text { Leaving the study early. } \\
\text { Side effects: SAS. }\end{array}$} \\
\hline \multicolumn{3}{|l|}{ Notes } \\
\hline \multicolumn{3}{|l|}{ Risk of bias } \\
\hline Bias & Authors' judgement & Support for judgement \\
\hline $\begin{array}{l}\text { Random sequence generation (selection } \\
\text { bias) }\end{array}$ & Unclear risk & "randomised". Method not reported. \\
\hline Allocation concealment (selection bias) & Unclear risk & $\begin{array}{l}\text { Details of allocation concealment nc } \\
\text { ported. }\end{array}$ \\
\hline
\end{tabular}




\section{Shu 1983 (Continued)}

\begin{tabular}{l|l|l}
$\begin{array}{l}\text { Blinding of participants and personnel } \\
\text { (performance bias) } \\
\text { All outcomes }\end{array}$ & Unclear risk & $\begin{array}{l}\text { "double-blind". Blinding details not re- } \\
\text { ported. }\end{array}$ \\
\hline $\begin{array}{l}\text { Blinding of outcome assessment (detection } \\
\text { bias) } \\
\text { All outcomes }\end{array}$ & Unclear risk & $\begin{array}{l}\text { "double-blind". Blinding details not re- } \\
\text { ported. }\end{array}$ \\
\hline $\begin{array}{l}\text { Incomplete outcome data (attrition bias) } \\
\text { All outcomes }\end{array}$ & Low risk & $\begin{array}{l}\text { Losses to follow-up balanced across in- } \\
\text { tervention groups: } 3 \text { (18\%) lost in the } \\
\text { fluphenazine group and 2 (11\%) in the } \\
\text { penflurdidol group, with similar reasons for } \\
\text { missing data (exacerbation) }\end{array}$ \\
\hline Selective reporting (reporting bias) & Low risk & $\begin{array}{l}\text { Outcome data have been reported: CGI, A } \\
\text { standardized psychiatric Assessment Scale } \\
\text { for Chronic psychiatric Patients, rating } \\
\text { Scale for Extramidal Side Effects (Simpson } \\
\text { G M) }\end{array}$ \\
\hline
\end{tabular}

\section{Simon 1978}

\begin{tabular}{|c|c|}
\hline Methods & $\begin{array}{l}\text { Allocation: randomised. } \\
\text { Blindness: open. } \\
\text { Duration: } 18 \text { months. } \\
\text { Design: } 3 \text { treatment groups. } \\
\text { Country: France. }\end{array}$ \\
\hline Participants & $\begin{array}{l}\text { Diagnosis: schizophrenia (French classification of mental illness). } \\
\mathrm{N}=181 . \\
\text { Age: } 21-45 \text { years. } \\
\text { Sex: } 117 \mathrm{M}, 64 \mathrm{~F} \text {. } \\
\text { History: duration illness 3-10 years. } \\
\text { Setting: community and/or hospital. }\end{array}$ \\
\hline Interventions & $\begin{array}{l}\text { 1. Fluphenazine decanoate: dose mean } 88 \mathrm{mg} / \mathrm{IM} \text { every } 22 \text { days. } \mathrm{N}=57 \text {. } \\
\text { 2. Pipothiazine decanoate: dose mean } 90 \mathrm{mg} / \mathrm{IM} \text { every } 25 \text { days. } \mathrm{N}=61 \text {. } \\
\text { 3. Standard oral neuroleptics: no further details. } \mathrm{N}=63 \text {. }\end{array}$ \\
\hline Outcomes & $\begin{array}{l}\text { Leaving the study early. } \\
\text { Global state: CGI. } \\
\text { Mental state: BPRS, NOSIE. } \\
\text { Additional medication. } \\
\text { Side effects. }\end{array}$ \\
\hline
\end{tabular}

Notes

Fluphenazine decanoate (depot) and enanthate for schizophrenia (Review)

Copyright @ 2016 The Cochrane Collaboration. Published by John Wiley \& Sons, Ltd. 
Simon 1978 (Continued)

Risk of bias

\begin{tabular}{|c|c|c|}
\hline Bias & Authors' judgement & Support for judgement \\
\hline $\begin{array}{l}\text { Random sequence generation (selection } \\
\text { bias) }\end{array}$ & Unclear risk & $\begin{array}{l}\text { "balanced randomization". Method not re- } \\
\text { ported. }\end{array}$ \\
\hline Allocation concealment (selection bias) & Unclear risk & $\begin{array}{l}\text { Details of allocation concealment not re- } \\
\text { ported. }\end{array}$ \\
\hline $\begin{array}{l}\text { Blinding of participants and personnel } \\
\text { (performance bias) } \\
\text { All outcomes }\end{array}$ & High risk & “open study" \\
\hline $\begin{array}{l}\text { Blinding of outcome assessment (detection } \\
\text { bias) } \\
\text { All outcomes }\end{array}$ & High risk & “open study" \\
\hline $\begin{array}{l}\text { Incomplete outcome data (attrition bias) } \\
\text { All outcomes }\end{array}$ & High risk & $\begin{array}{l}39 \text { participants left the study early, } 14 \text { in the } \\
\text { fluphenazine group, } 15 \text { in the pipothiazine } \\
\text { group and } 10 \text { in the standard neuroleptics } \\
\text { group. Reasons were similar across groups. } \\
\text { "The } 18 \text { dropouts due to independent } \\
\text { causes and those lost to follow up were not } \\
\text { considered in the analysis" }\end{array}$ \\
\hline Selective reporting (reporting bias) & Low risk & All outcome data has been reported. \\
\hline Other bias & Unclear risk & $\begin{array}{l}\text { "...supported by a grant from the Fonda- } \\
\text { tion de L'Industrie pharmaceutique pour la } \\
\text { recherche..." }\end{array}$ \\
\hline
\end{tabular}

Singh 1979

Methods

Allocation: randomised.

Blindness: double.

Duration: 44 weeks.

Design: parallel group.

Country: Canada.

Participants

Diagnosis: schizophrenia (DSM-II).

$\mathrm{N}=30$.

Age: $29-59$ years, mean -44 years.

Sex: 24M, 6F.

History: duration illness 3-32 years.

Setting: community. 
Singh 1979 (Continued)

\begin{tabular}{ll} 
Interventions & $\begin{array}{l}\text { 1. Fluphenazine enanthate: dose } 25-75 \mathrm{mg} / \mathrm{IM} \text {, mean } 44.2 \mathrm{mg} / \mathrm{IM} / \mathrm{monthly} . \mathrm{N}=15 . \\
\text { 2. Pipothiazine palmitate: dose } 100-150 \mathrm{mg} / \mathrm{IM}, \text { mean } 125 \mathrm{mg} / \mathrm{IM} / \mathrm{mon} \text { thly. } \mathrm{N}=15\end{array}$ \\
\hline \multirow{2}{*}{ Outcomes } & $\begin{array}{l}\text { Mental state: BPRS. } \\
\text { Side effects. } \\
\text { Unable to use - } \\
\text { Physicological measures: (non-clinical outcomes, data unusable) }\end{array}$ \\
\hline &
\end{tabular}

Notes

Risk of bias

Risk of bias

\begin{tabular}{|c|c|c|}
\hline Bias & Authors' judgement & Support for judgement \\
\hline $\begin{array}{l}\text { Random sequence generation (selection } \\
\text { bias) }\end{array}$ & Unclear risk & $\begin{array}{l}\text { "randomly assigned". Method not re- } \\
\text { ported. }\end{array}$ \\
\hline Allocation concealment (selection bias) & Unclear risk & $\begin{array}{l}\text { Details of allocation concealment not re- } \\
\text { ported. }\end{array}$ \\
\hline $\begin{array}{l}\text { Blinding of participants and personnel } \\
\text { (performance bias) } \\
\text { All outcomes }\end{array}$ & Unclear risk & $\begin{array}{l}\text { "double blind". Patients blinded; " ...test } \\
\text { medications were administered blind...on } \\
\text { alternate two weeks, patients in the pipoti- } \\
\text { azine group received an injection of sesame } \\
\text { oil...". Blinding details of personnel not re- } \\
\text { ported }\end{array}$ \\
\hline $\begin{array}{l}\text { Blinding of outcome assessment (detection } \\
\text { bias) } \\
\text { All outcomes }\end{array}$ & Unclear risk & $\begin{array}{l}\text { "double blind". Blinding details not re- } \\
\text { ported. }\end{array}$ \\
\hline $\begin{array}{l}\text { Incomplete outcome data (attrition bias) } \\
\text { All outcomes }\end{array}$ & Low risk & All patients completed the study. \\
\hline Selective reporting (reporting bias) & Low risk & $\begin{array}{l}\text { Outcome data reported for BPRS and side } \\
\text { effects. }\end{array}$ \\
\hline Other bias & Unclear risk & Source of funding not reported. \\
\hline
\end{tabular}




\begin{tabular}{ll} 
Methods & $\begin{array}{l}\text { Allocation: randomised. } \\
\text { Blindness: double. } \\
\text { Duration: } 6 \text { months. } \\
\text { Design: } 3 \text { treatment groups. } \\
\text { Country: not reported. }\end{array}$ \\
\hline Participants & $\begin{array}{l}\text { Diagnosis: schizophrenia. } \\
\text { N }=154 .\end{array}$ \\
& $\begin{array}{l}\text { Age: not stated. } \\
\text { Sex: not stated. } \\
\text { History: chronic. } \\
\text { Setting: hospital. }\end{array}$ \\
\hline Interventions & \begin{tabular}{l} 
1. Fluphenazine decanoate: (dose and frequency not stated). $\mathrm{N}=50$. \\
2. Pipothiazine palmitate (oral): (dose and frequency not stated). $\mathrm{N}=52$. \\
3. Pipothiazine palmate (oral, non-blinded): (dose and frequency not stated). $\mathrm{N}=52$ \\
\hline Outcomes
\end{tabular} \\
\hline Leaving the study early. \\
Mental state: BPRS* \\
Unable to use - \\
Side effects: TESS (data unusable). \\
\hline
\end{tabular}

Notes

Risk of bias

\section{Bias}

Random sequence generation (selection Unclear risk bias)

Allocation concealment (selection bias) Unclear risk

\section{Support for judgement}

“randomised”. Method not reported.

Details of allocation concealment not reported.

"double blindness in pipotiazine palmitate group and Fluphenazine Decanoate group, and one non-blindness pipotiazine palmitate group". Details not reported

"double blindness in pipotiazine palmitate group and Fluphenazine Decanoate group, and one non-blindness pipotiazine palmitate group". Details not reported

Losses to follow-up not reported.

Incomplete outcome data (attrition bias) Unclear risk All outcomes

Selective reporting (reporting bias)

High risk

Outcome data for TESS not reported fully. 
Song 1993 (Continued)

\begin{tabular}{|c|c|c|}
\hline Other bias & Unclear risk & Source of funding not reported. \\
\hline
\end{tabular}

Van Praag 1970

$\begin{array}{ll}\text { Methods } & \text { Allocation: randomised. } \\ \text { Blindness: double. } \\ \text { Duration: } 8 \text { weeks, follow-up } 4 \text { weeks. } \\ \text { Design: parallel group. } \\ \text { Country: The Netherlands. }\end{array}$

\begin{tabular}{ll}
\hline Participants & Diagnosis: psychotic*. \\
& $\mathrm{N}=25$. \\
& Age: not stated. \\
Sex: not stated \\
History: chronic and acute. \\
Setting: hospital. \\
\hline
\end{tabular}

Interventions

1. Fluphenazine enanthate: dose mean $25 \mathrm{mg} / \mathrm{IM}+$ oral placebo every 3 weeks. $\mathrm{N}=13$.

2. Fluphenazine oral + depot placebo: dose and frequency not reported. $\mathrm{N}=12$

All received concomitant orphenadrine (Disipal) $50 \mathrm{mg}$ tds.

\begin{tabular}{|c|c|c|}
\hline Outcomes & \multicolumn{2}{|c|}{$\begin{array}{l}\text { Additional medication. } \\
\text { Unable to use - } \\
\text { Side effects: EPS checklist (no data). } \\
\text { Behaviour: Wing Scale - Scale A (no data), Scale B: (authors own scale }{ }^{* *} \text { ). } \\
\text { Physiological measures: (non-clinical outcomes, data unusable) }\end{array}$} \\
\hline Notes & \multicolumn{2}{|c|}{$\begin{array}{l}\text { * Group } 1 \text { were acutely ill. } \\
\text { Group } 2 . \text { were chronically ill. } \\
{ }^{* *} \text { Marshall } 1998 .\end{array}$} \\
\hline \multicolumn{3}{|l|}{ Risk of bias } \\
\hline Bias & Authors' judgement & Support for judgement \\
\hline $\begin{array}{l}\text { Random sequence generation (selection } \\
\text { bias) }\end{array}$ & Unclear risk & "divided at random". Method not reported. \\
\hline Allocation concealment (selection bias) & Unclear risk & $\begin{array}{l}\text { Details of allocation concealment not re- } \\
\text { ported. }\end{array}$ \\
\hline
\end{tabular}

Blinding of participants and personnel Low risk (performance bias)

All outcomes
"One category received active fluphenazine orally and placebo injections; the other received oral placebos and injections of fluphenazine enanthate...The test drug and placebo were contained in identical capsules...Active fluid and placebo 
Van Praag 1970 (Continued)

fluid were indistinguishable...Strict double-blind conditions prevailed throughout the experiment."

Blinding of outcome assessment (detection Low risk bias)

"Strict double-blind conditions prevailed throughout the experiment."

All outcomes

Incomplete outcome data (attrition bias) High risk

All outcomes

Missing data imbalanced across intervention groups $8 \%$ (oral group) versus 25\% (depot group): 2 participants in the depo category left the study early due to deterioration of symptoms, a further patient in the depot category left due to encephalitis. 1 participant in the oral category due to severe oligophrenia

\begin{tabular}{lll}
\hline Selective reporting (reporting bias) & High risk & Outcome data not fully reported. \\
\hline Other bias & Unclear risk & Source of funding not reported. \\
\hline
\end{tabular}

Van Praag 1973

\begin{tabular}{|c|c|}
\hline Methods & $\begin{array}{l}\text { Allocation: randomised. } \\
\text { Blindness: double. } \\
\text { Duration: } 4 \text { weeks. } \\
\text { Design: parallel group. } \\
\text { Country: The Netherlands. }\end{array}$ \\
\hline Participants & $\begin{array}{l}\text { Diagnosis: acutely psychotic. } \\
\mathrm{N}=33 \text {. } \\
\text { Age: } 19-70 \text { years, mean }-42 \text { years. } \\
\text { Sex: } 19 \mathrm{~F}, 11 \mathrm{M} \text {. } \\
\text { History: not stated. } \\
\text { Setting: hospital. }\end{array}$ \\
\hline Interventions & $\begin{array}{l}\text { 1. Fluphenazine decanoate: dose } 25 \mathrm{mg} / \mathrm{IM} \text { every } 3 \text { weeks. } \mathrm{N}=15 \text {. } \\
\text { 2. Fluphenazine enanthate: dose } 25 \mathrm{mg} / \mathrm{IM} \text { every } 3 \text { weeks. } \mathrm{N}=18\end{array}$ \\
\hline Outcomes & $\begin{array}{l}\text { Leaving the study early. } \\
\text { Additional medication. } \\
\text { Unable to use - } \\
\text { Behaviour: Wing Scale - A \& B (no data). }\end{array}$ \\
\hline Notes & Data put in depot vs depot category in both FE \& FD treatment $g$ \\
\hline
\end{tabular}

Risk of bias 
Van Praag 1973 (Continued)

\begin{tabular}{|c|c|c|}
\hline Bias & Authors' judgement & Support for judgement \\
\hline $\begin{array}{l}\text { Random sequence generation (selection } \\
\text { bias) }\end{array}$ & Unclear risk & "randomly divided". Method not reported. \\
\hline Allocation concealment (selection bias) & Unclear risk & $\begin{array}{l}\text { Details of allocation concealment not re- } \\
\text { ported. }\end{array}$ \\
\hline $\begin{array}{l}\text { Blinding of participants and personnel } \\
\text { (performance bias) } \\
\text { All outcomes }\end{array}$ & Unclear risk & $\begin{array}{l}\text { "The conditions of a double-blind test were } \\
\text { ensured". Blinding details not reported }\end{array}$ \\
\hline $\begin{array}{l}\text { Blinding of outcome assessment (detection } \\
\text { bias) } \\
\text { All outcomes }\end{array}$ & Low risk & $\begin{array}{l}\text { "The evaluators- physicians and nurses- } \\
\text { were blind to the distribution of enanthate } \\
\text { and decanoate..." }\end{array}$ \\
\hline $\begin{array}{l}\text { Incomplete outcome data (attrition bias) } \\
\text { All outcomes }\end{array}$ & Low risk & $\begin{array}{l}\text { Missing data balanced across intervention } \\
\text { groups: } 2(14 \%) \text { participants from the } \\
\text { enanthate group and } 1(6 \%) \text { from the de- } \\
\text { canoate group, with similar reasons for } \\
\text { missing data }\end{array}$ \\
\hline Selective reporting (reporting bias) & High risk & $\begin{array}{l}\text { Outcome data not reported (Wing Scale A } \\
\text { and Scale B) }\end{array}$ \\
\hline Other bias & Unclear risk & Source of funding not reported \\
\hline
\end{tabular}

\section{Walker 1983}

\begin{tabular}{ll}
\hline Methods & Allocation: randomised. \\
& Blindness: double. \\
& Duration: 24 weeks (preceded by 12 week open trial). \\
& Design: parallel group. \\
& Country: United Kingdom. \\
\hline Participants & Diagnosis: schizophrenia. \\
& $\mathrm{N}=39$. \\
& Age: $23-67$ years, mean -45 years. \\
& Sex: male and female. \\
& History: currently maintained on depot neuroleptics, at least one hospitalisation, dura- \\
tion illness $1-20$ years. & Setting: community. \\
\hline Interventions & $\begin{array}{l}\text { 1.Fluphenazine decanoate: dose } 12.5 \mathrm{mg} / \text { weeks-37.5 mg/4 weeks, mean } 24.8 \mathrm{mg} / \mathrm{IM} \\
\text { every } 3-4 \text { weeks. } \mathrm{N}=20 .\end{array}$ \\
\hline $\begin{array}{l}\text { 2. Clopenthixol decanoate: dose } 200 \mathrm{mg} / 4 \text { weeks }-600 \mathrm{mg} / 2 \text { weeks, mean } 220 \mathrm{mg} / \mathrm{IM} \\
\text { every } 3-4 \text { weeks. } \mathrm{N}=19\end{array}$ \\
\hline
\end{tabular}


Walker 1983

\begin{tabular}{ll}
\hline Outcomes & Side effects: Side Effects Inventory. \\
& Unable to use - \\
& Global state: CGI, Krawiecka, Goldberg \& Vaughan Rating Scale (no SD). \\
& Mental state: BPRS (no SD). \\
& Physiological measures: blood/liver tests, weight, BP ( non-clinical outcomes, data un- \\
& usable) \\
\hline \multirow{2}{*}{ Notes } & Authors contacted. \\
& Analysis: last observation carried forward. \\
\hline
\end{tabular}

Risk of bias

\begin{tabular}{|c|c|c|}
\hline Bias & Authors' judgement & Support for judgement \\
\hline $\begin{array}{l}\text { Random sequence generation (selection } \\
\text { bias) }\end{array}$ & Unclear risk & $\begin{array}{l}\text { "randomly allocated". Method not re- } \\
\text { ported. }\end{array}$ \\
\hline Allocation concealment (selection bias) & Unclear risk & $\begin{array}{l}\text { Details of allocation concealment not re- } \\
\text { ported. }\end{array}$ \\
\hline $\begin{array}{l}\text { Blinding of participants and personnel } \\
\text { (performance bias) } \\
\text { All outcomes }\end{array}$ & Unclear risk & $\begin{array}{l}\text { "double-blind". Blinding details not re- } \\
\text { ported. }\end{array}$ \\
\hline $\begin{array}{l}\text { Blinding of outcome assessment (detection } \\
\text { bias) } \\
\text { All outcomes }\end{array}$ & Unclear risk & $\begin{array}{l}\text { "double-blind". Blinding details not re- } \\
\text { ported. }\end{array}$ \\
\hline $\begin{array}{l}\text { Incomplete outcome data (attrition bias) } \\
\text { All outcomes }\end{array}$ & Unclear risk & $\begin{array}{l}\text { Missed data per allocation group unclear. } \\
\text { "Forty five patients entered the trial. Six pa- } \\
\text { tients failed to return following the first in- } \\
\text { terview and so were discounted. One pa- } \\
\text { tient returned to her home country, and so } \\
\text { failed to attend for the final assessment" }\end{array}$ \\
\hline Selective reporting (reporting bias) & High risk & $\begin{array}{l}\text { Outcome data incompletely reported (SD } \\
\text { not reported): CGI, BPRS, Krawiecka, } \\
\text { Goldberg \& Vaughan Rating Scale }\end{array}$ \\
\hline Other bias & Unclear risk & Source of funding not reported. \\
\hline
\end{tabular}


Methods
Allocation: randomised.

Blindness: double.

Duration: 2 years.

Design: parallel group.

Country: Sweden.

\begin{tabular}{ll} 
Participants & Diagnosis: schizophrenia (Bleuler's criteria). \\
& $\mathrm{N}=32$. \\
& Age: $26-67$ years, mean -41 years. \\
& Sex: $15 \mathrm{M}, 17 \mathrm{~F}$. \\
& History: stabilised on depots, relapse in connection with withdrawal; duration illness \\
mean -14 years. & Setting: not stated. \\
\hline Interventions & 1. Fluphenazine decanoate: dose mean $27 \mathrm{mg} / \mathrm{IM}$ every 3 weeks. $\mathrm{N}=15$. \\
& 2. Flupenthixol decanoate: dose mean $31 \mathrm{mg} / \mathrm{IM}$ every 3 weeks. $\mathrm{N}=17$ \\
\hline Outcomes & $\begin{array}{l}\text { Leaving the study early. } \\
\text { Side effects: SRSE, AIMS. } \\
\text { Unable to use - } \\
\text { Global state: CGI (no data). } \\
\text { Mental state: CPRS (no data). }\end{array}$ \\
\hline Notes & Authors contacted.
\end{tabular}

Risk of bias

\section{Bias}

Random sequence generation (selection Unclear risk bias)

Allocation concealment (selection bias) Unclear risk

Blinding of participants and personnel Unclear risk (performance bias)

All outcomes

Blinding of outcome assessment (detection Low risk bias)

All outcomes

Incomplete outcome data (attrition bias) Unclear risk All outcomes

Selective reporting (reporting bias)

\section{Support for judgement}

"allocated randomly". Method not reported.

Details of allocation concealment not reported.

"double-blind". Blinding details not reported.

"The injections were given by a nurse who did not participate in the assessment."

Missing data balanced across intervention groups (27\% fluphenazine group vs $40 \%$ flupenthixol group). Reasons for losses to follow-up not reported

Incomplete outcome data: CGI, CPRS. 
Wistedt 1983 (Continued)

\begin{tabular}{l|l} 
Other bias $\quad$ Unclear risk $\quad$ Source of funding not reported.
\end{tabular}

\section{Wistedt 1984}

\begin{tabular}{|c|c|}
\hline Methods & $\begin{array}{l}\text { Allocation: randomised. } \\
\text { Blindness: double. } \\
\text { Duration: } 20 \text { weeks. } \\
\text { Design: parallel group. } \\
\text { Country: Sweden. }\end{array}$ \\
\hline Participants & $\begin{array}{l}\text { Diagnosis: schizophrenia (RDC). } \\
\mathrm{N}=51 . \\
\text { Age range: } 21-63 \text { years. } \\
\text { Sex: } 33 \mathrm{M}, 18 \mathrm{~F} \text {. } \\
\text { History: } 6 \text { months treatment forseen, duration illness }<12 \text { years, able to give informed } \\
\text { consent. } \\
\text { Setting: } 4 \text { weeks in hospital, thereafter in the community. }\end{array}$ \\
\hline Interventions & $\begin{array}{l}\text { 1. Fluphenazine decanoate: dose mean } 84 \mathrm{mg} / \mathrm{IM} / \text { monthly. } \mathrm{N}=26 \text {. } \\
\text { 2. Haloperidol decanoate: dose mean } 122 \mathrm{mg} / \mathrm{IM} / \text { monthly. } \mathrm{N}=25 \\
\text { Depot }(\mathrm{FD} / \mathrm{HD}) \text { dose range: } 25-100 \mathrm{mg} / \text { injection, initially adjusted at } 2 \mathrm{nd} \text { injection } \\
(\max .300 \mathrm{mg})\end{array}$ \\
\hline
\end{tabular}

Outcomes

Global State: CGI.

Mental state: CPRS.

Leaving the study early.

Additional medication.

Side effects: EPS, AIMS.

Unable to use -

Physiological measures: drug plasma levels, weight changes (non clinical outcomes, data unusable)

Notes

Risk of bias

\begin{tabular}{|c|c|c|}
\hline Bias & Authors' judgement & Support for judgement \\
\hline $\begin{array}{l}\text { Random sequence generation (selection } \\
\text { bias) }\end{array}$ & Unclear risk & $\begin{array}{l}\text { "allocated to the two groups according to a } \\
\text { randomisation list...Patients were balanced } \\
\text { in groups of six" Details not reported }\end{array}$ \\
\hline Allocation concealment (selection bias) & Unclear risk & $\begin{array}{l}\text { Details of allocation concealment not re- } \\
\text { ported. }\end{array}$ \\
\hline
\end{tabular}

Blinding of participants and personnel Unclear risk (performance bias)

"Injections were give double-blind". BlindAll outcomes

Fluphenazine decanoate (depot) and enanthate for schizophrenia (Review)

Copyright @ 2016 The Cochrane Collaboration. Published by John Wiley \& Sons, Ltd. 


\section{Wistedt 1984 (Continued)}

\begin{tabular}{l|l|l}
$\begin{array}{l}\text { Blinding of outcome assessment (detection } \\
\text { bias) } \\
\text { All outcomes }\end{array}$ & Unclear risk & Blinding details not reported. \\
\hline $\begin{array}{l}\text { Incomplete outcome data (attrition bias) } \\
\text { All outcomes }\end{array}$ & Low risk & $\begin{array}{l}\text { Missing data balanced across interven- } \\
\text { tion groups (15\% fluphenazine vs } 15 \% \\
\text { haloperidol), with similar reasons for miss- } \\
\text { ing data }\end{array}$ \\
\hline Selective reporting (reporting bias) & Low risk & All primary outcomes reported: CPRS, \\
\hline CGI, EPS.
\end{tabular}

Woggon 1977

\begin{tabular}{|c|c|c|}
\hline Methods & \multicolumn{2}{|l|}{$\begin{array}{l}\text { Allocation: randomised. } \\
\text { Blindness: double. } \\
\text { Duration: } 6 \text { months. } \\
\text { Design: parallel group. } \\
\text { Country: Germany. }\end{array}$} \\
\hline Participants & \multicolumn{2}{|l|}{$\begin{array}{l}\text { Diagnosis: schizophrenia (ICD Nr). } \\
\mathrm{N}=61 . \\
\text { Age: } 21-79 \text { years. } \\
\text { Sex: } 36 \mathrm{M}, 25 \mathrm{~F} \text {. } \\
\text { History: } 6 \text { months treatment. } \\
\text { Setting: community. }\end{array}$} \\
\hline Interventions & \multicolumn{2}{|c|}{$\begin{array}{l}\text { 1. Fluphenazine decanoate: dose } 25-37.5 \mathrm{mg} / \mathrm{IM} \text { every } 3 \text { weeks. } \mathrm{N}=30 \text {. } \\
\text { 2. Pipothiazine palmitate: dose } 100 \mathrm{mg} / \mathrm{IM} \text { every } 4 \text { weeks. } \mathrm{N}=31\end{array}$} \\
\hline Outcomes & \multicolumn{2}{|l|}{$\begin{array}{l}\text { Leaving the study early. } \\
\text { Unable to use - } \\
\text { Side effects: (data unusable). }\end{array}$} \\
\hline Notes & \multicolumn{2}{|l|}{ Article in German. } \\
\hline \multicolumn{3}{|l|}{ Risk of bias } \\
\hline Bias & Authors' judgement & Support for judgement \\
\hline $\begin{array}{l}\text { Random sequence generation (selection } \\
\text { bias) }\end{array}$ & Unclear risk & "Randomised", no further details reported. \\
\hline Allocation concealment (selection bias) & Unclear risk & Not reported. \\
\hline
\end{tabular}




\begin{tabular}{l|l|l}
$\begin{array}{l}\text { Blinding of participants and personnel } \\
\text { (performance bias) } \\
\text { All outcomes }\end{array}$ & Unclear risk & $\begin{array}{l}\text { The tests were performed double-blind. } \\
\text { The dosage of both drugs and the addi- } \\
\text { tional medications were prescribed by an } \\
\text { investigator who himself did not take part } \\
\text { in the ratings }\end{array}$ \\
\hline $\begin{array}{l}\text { Blinding of outcome assessment (detection } \\
\text { bias) } \\
\text { All outcomes }\end{array}$ & Unclear risk & Double-blind, details not reported. \\
\hline $\begin{array}{l}\text { Incomplete outcome data (attrition bias) } \\
\text { All outcomes }\end{array}$ & Low risk & No losses to follow-up. \\
\hline $\begin{array}{l}\text { Selective reporting (reporting bias) } \\
\text { Other bias }\end{array}$ & Unclear risk & Data for side effects not usable, other out- \\
\hline
\end{tabular}

\section{Diagnostic tools:}

CCMD-2-R: Chinese Classification of Mental Disorders, Second Edition, Revised

DSM III - Diagnostic Statistical Manual, version 3

ICD-9 - International Classification of Diseases, version 9

RDC - Research Diagnostic Criteria

Rating scales

Global state:

CGI - Clinical Global Impression

GAS - Global Assessment Scale

GRS - Global Rating Scale

GES - Global Evaluation Scale

KWS - Krawiecka-Goldberg Scale

PRS - Patient Rejection Scale

TES - Therapeutic Effects Scale

Mental state:

BPRS - Brief Psychiatric Rating Scale

CPRS - Comprehensive Psychopathological Rating Scale

EWL-K - List of Attributes self rating scale.

HLS - Hamilton-Lorr Scale

HRSD - Hamilton Psychiatric Rating Scale for Depression

KGS -

KORS - Keio University's Simplified Rating Scale for Psychiatric Symptoms

KWS -

MIE - Mental Illness Evaluation

PANSS - Positive and Negative Syndrome Scale

PSE - Wing Ward Present State Examination

SANS - Scale for Assessment of Negative Symptoms

SAPS - Scale for Assessment of Positive Symptoms

S-Scale - The Symptom Scale 


\section{Behaviour:}

CBS - Current Behaviour Schedule

MACC-BAS - MACC Behaviour Adjustment Scale

PRS -

WWBRS - Wing Ward Behaviour Rating Scale

Symptom scales:

HSC - Hopkins Symptom Checklist

MRSS - Morningside Rehabilitation Rating Scale

NSRS - Negative Symptom Rating Scale

SSI - Springfield Symptom Index

SCL-90 - Symptom Checklist -90

Social behaviour:

ADL - Activities of Daily Living

KAS - Katz Adjustment Scale

SAS - Social Adjustment Scale

SRE - Schedule of Recent Events

SBAS - Social Behaviour Assement Schedule

SPS - Social Performance Schedule

Side effects

AIMS - Abnormal Involuntary Movement Side effects

Bordeleau Scale

CSE - Clinical Side Effects Scale

DOTES - Dosage Record \& Treatment Emergent Symptom Scale

EPMS - Extrapyramidal Motor Side-effects

EPSS - Extrapyramidal Side-effects Symptoms

EPS -Extrapyramidal symptom scale

IMEPS - Involuntary Movement and EPS Scale

MARDRS- Montgomery-Asberg Depression Rating Scale

MRQ - Medication Response Questionnaire

NOSIE - Nurses Observation Scale for Inpatient Evaluation

OSR - Overall Safety Rating

RSESE - Rating Scale for Extrapyramidal Side Effects

SAS - Simpson and Angus Scale

SDS - Simpson Dyskinesia Scale

SRSE - Simpson Rating Scale for EPS

SEC -Side Effects Checklist

SCL-9 Side effects Check List 9

STESS - Total Score of Side Effects Self Rating

TESF - Treatment Emergent Symptom Form

TESS - Treatment Emergent Symptoms Scale

UKU - Side Effects Rating Scale

\section{Miscellaneous:}

bid - twice daily

BMI - body mass index

BP - Blood Pressure

EE - Expressed Emotion

IM - intramuscular

NIMH - National Institute of Mental Health

SD - standard deviation

tds - three times daily

VHD - Very High Dose

Fluphenazine decanoate (depot) and enanthate for schizophrenia (Review)

Copyright $\Subset 2016$ The Cochrane Collaboration. Published by John Wiley \& Sons, Ltd. 
Characteristics of excluded studies [ordered by study ID]

\begin{tabular}{|c|c|}
\hline Study & Reason for exclusion \\
\hline Abuzzahab 1976a & Allocation: not randomised. \\
\hline Abuzzahab 1976b & $\begin{array}{l}\text { Allocation: double blind. } \\
\text { Participants: people with schizophrenia. } \\
\text { Interventions: oral fluphenazine versus pimozide. }\end{array}$ \\
\hline Abuzzahab 1977 & Allocation: not randomised. \\
\hline Abuzzahab 1980 & $\begin{array}{l}\text { Allocation: double blind. } \\
\text { Participants: people with psychopathology. } \\
\text { Interventions: fluphenazine } \mathrm{HCl} \text { versus pimozide. }\end{array}$ \\
\hline Ahlfors 1971 & $\begin{array}{l}\text { Allocation: randomly selected. } \\
\text { Participants: people with schizophrenia. } \\
\text { Interventions: fluphenazine enanthate versus pipotiazine undecylenic ester. } \\
\text { Outcomes: no data presented. }\end{array}$ \\
\hline Ahlfors 1973 & $\begin{array}{l}\text { Allocation: randomised. } \\
\text { Particpants: people with schizophrenia. } \\
\text { Interventions: fluphenazine enanthate versus pipotiazine undecylenate. } \\
\text { Outcomes: no usable data, authors contacted. }\end{array}$ \\
\hline Altamura 1987 & Allocation: not randomised. \\
\hline Angst 1975 & $\begin{array}{l}\text { Allocation: double blind. } \\
\text { Participants: people with schizophrenia. } \\
\text { Interventions: fluphenazine decanoate versus fluspirilen versus penfluridol versus perphenazino enanthate } \\
\text { versus pipothiazine palmitate. } \\
\text { Outcomes: no usable data. }\end{array}$ \\
\hline Arato 1979 & Allocation: not randomised (retrospective study). \\
\hline Astrup 1974 & Allocation: not randomised. \\
\hline Balon 1982 & $\begin{array}{l}\text { Allocation: double blind - cross-over study. } \\
\text { Participants: people with schizophrenia. } \\
\text { Interventions: depot fluphenazine decanoate versus hydroxyprotepine decanoate. } \\
\text { Outcomes: no usable data. }\end{array}$ \\
\hline Bankier 1968 & $\begin{array}{l}\text { Allocation: randomised. } \\
\text { Participants: people with schizophrenia. } \\
\text { Interventions: trifluoperazine versus placebo. }\end{array}$ \\
\hline
\end{tabular}


(Continued)

\begin{tabular}{ll} 
Bao 1991 & $\begin{array}{l}\text { Allocation: randomised. } \\
\text { Participants: people with schizophrenia. } \\
\text { Interventions: flupenthixol decanoate versus chlorpromazine. }\end{array}$ \\
\hline Barnes 2010 & $\begin{array}{l}\text { Allocation: randomised. } \\
\text { Participants: people with schizophrenia. } \\
\text { Interventions: first generation antipsychotics versus second generation (non-clozapine) antipsychotics }\end{array}$ \\
\hline Barsa 1965 & $\begin{array}{l}\text { Allocation: double blind. } \\
\text { Participants: not specified. }\end{array}$ \\
\hline Bastie 1974 & Allocation: not randomised. \\
\hline Benassi 1968 & Allocation: not randomised. \\
\hline Berliner 1974 & Allocation: not randomised. \\
\hline Bilone 1988 & Allocation: not randomised. \\
\hline Bloch 2004 & $\begin{array}{l}\text { Allocation: randomised. } \\
\text { Participants: people with schizophrenia. } \\
\text { Interventions: lidocaine-prilocaine cream versus placebo to reduce pain injection site pain of depot antipsy- } \\
\text { chotics }\end{array}$ \\
\hline
\end{tabular}

Boyer $1987 \quad$ Allocation: randomised.

Participants: people with schizophrenia.

Interventions: amisulpride versus fluphenazine.

Outcomes: no usable data (no SDs).

Brankovic $1998 \quad$ Allocation: not randomised.

Breier 1987 Allocation: double blind.

Participants: people with schizophrenia.

Interventions: fluphenazine - withdrawal study.

Caranza $1973 \quad$ Allocation: not randomised.

$\begin{array}{ll}\text { Carpenter } 1992 & \text { Allocation: randomised. } \\ & \text { Participants: people with schizophrenia. } \\ & \text { Interventions: fluphenazine versus placebo versus diazepam. } \\ & \text { Outcomes: withdrawal study. }\end{array}$

Carpenter 1993 Allocation: randomised.

Participants: people with schizophrenia.

Interventions: Fluphenazine decanoate $1 \mathrm{cc}$, every 2 weeks versus fluphenazine decanoate $1 \mathrm{cc}$, every 6 weeks.

Outcome data: no usable data reported (conference proceeding) 
(Continued)

\begin{tabular}{|c|c|}
\hline Carpenter 1999 & $\begin{array}{l}\text { Allocation: randomised. } \\
\text { Participants: people with schizophrenia. } \\
\text { Interventions: fluphenazine decanoate injection } 2 / 52 \text { versus } 6 / 52 \text { with oral fluphenazine prescribed as re- } \\
\text { quired }\end{array}$ \\
\hline Casacchia 1989 & $\begin{array}{l}\text { Allocation: randomised. } \\
\text { Participants: people with schizophrenia. } \\
\text { Interventions: bromperidol decanoate versus fluphenazine decanoate. } \\
\text { Outcomes: no usable data. }\end{array}$ \\
\hline Castellini & Allocation: open - cross-over study. \\
\hline Chacon 1972 & Allocation: double blind - cross-over study. \\
\hline Chacon 1973 & $\begin{array}{l}\text { Allocation: double blind. } \\
\text { Participants: people with schizophrenia. } \\
\text { Interventions: fluphenazine decanoate versus chloropromazine. } \\
\text { Outcomes: no usable data, authors contacted. }\end{array}$ \\
\hline Charalampous 1977 & $\begin{array}{l}\text { Allocation: random double-blind fashion. } \\
\text { Participants: people with schizophrenia. } \\
\text { Interventions: oral fluphenazine versus pentofluridol. }\end{array}$ \\
\hline Chien 1974 & $\begin{array}{l}\text { Allocation: randomised. } \\
\text { Participants: people with psychotic illnesses including schizophrenia. } \\
\text { Interventions: fluphenazine enanthate versus different dosages of antiparkinson drugs (not antipsychotics) }\end{array}$ \\
\hline Childers 1964 & $\begin{array}{l}\text { Allocation: randomised. } \\
\text { Participants: people with schizophrenia. } \\
\text { Interventions: electro convulsive therapy (ECT) versus oral fluphenazine versus chlorpromazine versus chlor- } \\
\text { promazine with ECT }\end{array}$ \\
\hline Chouinard 1970 & $\begin{array}{l}\text { Allocation: randomised. } \\
\text { Participants: people with schizophrenia. } \\
\text { Interventions: oral fluphenazine versus pimozide. }\end{array}$ \\
\hline Chowdhury 1980 & $\begin{array}{l}\text { Allocation: randomised. } \\
\text { Participants: people with schizophrenia. } \\
\text { Interventions: fluphenazine decanoate versus flupenthixol decanoate. } \\
\text { Outcomes: no usable data, authors contacted. }\end{array}$ \\
\hline Clark 1971 & $\begin{array}{l}\text { Allocation: randomised. } \\
\text { Participants: people with schizophrenia. } \\
\text { Interventions: oral fluphenazine versus chlorpromazine versus thioridazine versus placebo }\end{array}$ \\
\hline Cohen 1985 & Allocation: not randomised. \\
\hline
\end{tabular}

Fluphenazine decanoate (depot) and enanthate for schizophrenia (Review)

Copyright () 2016 The Cochrane Collaboration. Published by John Wiley \& Sons, Ltd. 
(Continued)

\begin{tabular}{|c|c|}
\hline Cole 1967 & $\begin{array}{l}\text { Allocation: randomised. } \\
\text { Participants: people with schizophrenia. } \\
\text { Interventions: oral fluphenazine versus chlorpromazine versus acetophenazine }\end{array}$ \\
\hline Cookson 1991 & $\begin{array}{l}\text { Allocation: double blind. } \\
\text { Participants: people with schizophrenia. } \\
\text { Interventions: haloperidol decanoate versus fluphenazine decanoate. } \\
\text { Outcomes: no usable data. }\end{array}$ \\
\hline Coufal 1981 & Allocation: not randomised. \\
\hline Curry 1979 & Allocation: double blind - cross-over study. \\
\hline Curson 1985 & $\begin{array}{l}\text { Allocation: randomised. } \\
\text { Participants: people with schizophrenia. } \\
\text { Interventions: fluphenazine decanoate and flupenthixol decanoate versus placebo, the data for the two } \\
\text { antipsychotics (depot and oral) were analysed as one group }\end{array}$ \\
\hline Curson 1986 & Allocation: not randomised. \\
\hline De Alarcon 1969 & Allocation: not randomised - case reports. \\
\hline De Buck 1973 & $\begin{array}{l}\text { Allocation: randomised. } \\
\text { Participants: people with schizophrenia. } \\
\text { Interventions: oral fluphenazine (dosage study). }\end{array}$ \\
\hline
\end{tabular}

Del Giudice 1975 Allocation: randomised.

Participants: people with schizophrenia.

Interventions: fluphenazine enanthate versus fluphenazine hydrochloride (orally).

Outcomes: no usable data, no continuous outcomes measured.

Dencker $1978 \quad$ Allocation: randomised.

Participants: people with schizophrenia.

Intervention: high - low doses of fluphenazine enanthate.

Outcomes: no usable data.

Dencker $1981 \quad$ Allocation: not randomised.

Dengler $1969 \quad$ Allocation: not randomised.

DeWolfe $1971 \quad$ Allocation: randomised

Participants: people with schizophrenia.

Interventions: fluphenazine enanthate versus thorazine-stelazine (orally).

Outcomes: data not usable, drop-out rate $60 \%$ in 6-week trial

Donlon $19761 \quad$ Allocation: randomised.

Participants: people with schizophrenia.

Interventions: fluphenazine decanoate versus fluphenazine enanthate.

Outcomes: no usable data. 
(Continued)

\begin{tabular}{|c|c|}
\hline Donlon 1977 & $\begin{array}{l}\text { Allocation: randomised. } \\
\text { Participants: people with schizophrenia. } \\
\text { Interventions: oral fluphenazine versus pimozide. }\end{array}$ \\
\hline Donlon 1978 & Allocation: quasi-randomised. \\
\hline Doongaji 1988 & $\begin{array}{l}\text { Allocation: randomised. } \\
\text { Participants: people with schizophrenia. } \\
\text { Interventions: fluphenazine decanoate versus penfluridol. } \\
\text { Outcomes: no usable data, authors contacted. }\end{array}$ \\
\hline Dossenbach 1997 & $\begin{array}{l}\text { Allocation: randomised. } \\
\text { Participants: people with schizophrenia. } \\
\text { Interventions: oral fluphenazine versus olanzapine. }\end{array}$ \\
\hline Downing 1963 & $\begin{array}{l}\text { Allocation: randomised. } \\
\text { Participants: people with schizophrenia. } \\
\text { Interventions: chlorpromazine versus fluphenazine versus thioridazine versus placebo. } \\
\text { Outcomes: no usable data. }\end{array}$ \\
\hline Emsley 1999 & $\begin{array}{l}\text { Allocation: randomised. } \\
\text { Participants: people with schizophrenia. } \\
\text { Interventions: quetiapine versus haloperidol with fluphenazine prescribed (4-week run-in phase) }\end{array}$ \\
\hline Engelhardt 1973 & $\begin{array}{l}\text { Allocation: randomised. } \\
\text { Participants: people with schizophrenia. } \\
\text { Interventions: oral fluphenazine versus haloperidol versus placebo }\end{array}$ \\
\hline Engstrand 1969 & Allocation: not randomised. \\
\hline Faltus 1974 & Allocation: not randomised. \\
\hline Faretra 1970 & $\begin{array}{l}\text { Allocation: randomised. } \\
\text { Participants: people with schizophrenia. } \\
\text { Interventions: oral fluphenazine versus haloperidol. }\end{array}$ \\
\hline Ferenc 2000 & $\begin{array}{l}\text { Allocation: double blind. } \\
\text { Participants: people with schizophrenia. } \\
\text { Interventions: oral fluphenazine versus olanzapine. }\end{array}$ \\
\hline
\end{tabular}

Filip $1985 \quad$ Allocation: randomised.

Participants: people with schizophrenia.

Interventions: fluphenazine decanoate versus oxyprothepin decanoate with cross over at 6 months.

Outcomes: no usable data - results provided at 12 months without separating the treatments

Floru $1974 \quad$ Allocation: not randomised.

Floru $1975 \quad$ Allocation: not randomised.

Fluphenazine decanoate (depot) and enanthate for schizophrenia (Review)

Copyright @ 2016 The Cochrane Collaboration. Published by John Wiley \& Sons, Ltd. 
(Continued)

\begin{tabular}{|c|c|}
\hline Giannelli 1990 & Allocation: not randomised. \\
\hline Gillis 1981 & $\begin{array}{l}\text { Allocation: randomised. } \\
\text { Participants: people with schizophrenia. } \\
\text { Interventions: no usable data. }\end{array}$ \\
\hline Gitlin 1988 & $\begin{array}{l}\text { Allocation: randomised. } \\
\text { Participants: people with schizophrenia. } \\
\text { Interventions: fluphenazine decanoate versus placebo. } \\
\text { Outcomes: no usable data (plasma study). }\end{array}$ \\
\hline Gitlin 2001 & $\begin{array}{l}\text { Allocation: randomised cross-over trial. } \\
\text { Participants: people with schizophrenia. } \\
\text { Interventions: fluphenazine decanoate versus placebo. } \\
\text { Outcomes: no usable data, no results reported for first phase of the study }\end{array}$ \\
\hline Goff 2005 & $\begin{array}{l}\text { Allocation: randomised. } \\
\text { Participants: people with schizophrenia. } \\
\text { Interventions: d-cycloserine as add-on to conventional antipsychotics versus placebo add-on }\end{array}$ \\
\hline Goldberg 1967 & $\begin{array}{l}\text { Allocation: randomised. } \\
\text { Participants: people with schizophrenia. } \\
\text { Interventions: chlorpromazine versus fluphenazine versus thioridazine. } \\
\text { Outcomes: no usable data. }\end{array}$ \\
\hline Goldberg 1968 & $\begin{array}{l}\text { Allocation: randomised. } \\
\text { Participants: people with schizophrenia. } \\
\text { Interventions: placebo versus thioridazine versus chlorpromazine versus fluphenazine. } \\
\text { Outcomes: no usable data. }\end{array}$ \\
\hline Goldberg 1970 & $\begin{array}{l}\text { Allocation: randomised. } \\
\text { Participants: people with schizophrenia. } \\
\text { Interventions: prolixin enanthate versus oral phenothiazines }\end{array}$ \\
\hline Goldberg 1981 & Allocation: randomised - withdrawal study. \\
\hline Gopalakrishnan 2006 & $\begin{array}{l}\text { Allocation: randomised. } \\
\text { Participants: people with schizophrenia. } \\
\text { Interventions: sildenafil versus placebo. }\end{array}$ \\
\hline Grosser 1970 & $\begin{array}{l}\text { Allocation: randomised. } \\
\text { Participants: people with schizophrenia. } \\
\text { Interventions: fluphenazine decanoate versus fluphenazine enanthate. } \\
\text { Outcomes: no usable data. }\end{array}$ \\
\hline Haider 1968 & $\begin{array}{l}\text { Allocation: randomised. } \\
\text { Participants: people with schizophrenia. } \\
\text { Interventions: fluphenazine enanthate versus fluphenazine (oral). } \\
\text { Outcomes: no usable data. }\end{array}$ \\
\hline
\end{tabular}

Fluphenazine decanoate (depot) and enanthate for schizophrenia (Review)

Copyright () 2016 The Cochrane Collaboration. Published by John Wiley \& Sons, Ltd. 
(Continued)

\begin{tabular}{|c|c|}
\hline Hall 1968 & $\begin{array}{l}\text { Allocation: randomised. } \\
\text { Participants: people with schizophrenia. } \\
\text { Interventions: oral fluphenazine versus haloperidol. }\end{array}$ \\
\hline
\end{tabular}

Hamilton $1979 \quad$ Allocation: randomised.

Participants: people with schizophrenia.

Interventions: fluphenazine decanoate versus flupenthixol decanoate.

Outcomes: no usable data, no outcomes measured.

Hanlon $1965 \quad$ Allocation: randomised.

Participants: people with schizophrenia.

Interventions: oral fluphenazine versus chlorpromazine, thioridazine, trifluoperazine, prochlorpromazine, perphenazine, thiopropazate and trifluperazine

Harper 1976 Allocation: double blind - cross-over study.

Participants: people with schizophrenia.

Interventions: chlorpromazine depot preparations versus fluphenazine.

Outcomes: no usable data.

Haslam 1975 Allocation: randomised.

Participants: people with schizophrenia.

Interventions: fluphenazine decanoate versus flupenthixol decanoate.

Outcomes: no usable data, data difficult to interpret.

Held $1970 \quad$ Allocation: randomised.

Participants: people with schizophrenia.

Interventions: phenothiazines and placebo.

Outcomes: no usable data.

Hirsch 1973 Allocation: randomised.

Participants: people with schizophrenia.

Interventions: fluphenazine decanoate and placebo, withdrawal study

Hirsch 1978 Allocation: double blind.

Participants: people with schizophrenia.

Interventions: fluphenazine versus flupenthixol.

Outcomes: no usable data.

Hirsch $1989 \quad$ Allocation: randomised.

Participants: people with schizophrenia.

Interventions: fluphenazine decanoate versus active injections with haloperidol prescribed as required

Hogarty $1995 \quad$ Allocation: randomised.

Participants: people with schizophrenia.

Interventions: fluphenazine decanoate - low dose versus standard dose.

Outcomes: fluphenazine decanoate measured against anxiolytics or antidepressants not antipsychotics

Holden 1970

Allocation: double blind - cross-over study.

Fluphenazine decanoate (depot) and enanthate for schizophrenia (Review)

Copyright @ 2016 The Cochrane Collaboration. Published by John Wiley \& Sons, Ltd. 
(Continued)

\begin{tabular}{|c|c|}
\hline Holt 1984 & Allocation: not randomised. \\
\hline Hsu 1967 & $\begin{array}{l}\text { Allocation: randomised. } \\
\text { Participants: people suffering from psychotic disorders, including schizophrenia. } \\
\text { Interventions: fluphenazine enanthate versus placebo. } \\
\text { Outcomes: no usable data. }\end{array}$ \\
\hline Inderbitzen 1994 & Allocation: not randomised. \\
\hline Inderbitzin 1993 & $\begin{array}{l}\text { Allocation: randomised. } \\
\text { Participants: people with schizophrenia. } \\
\text { Interventions: reduced fluphenazine decanoate dose by } 50 \%(10 \% \text { per month for } 5 \text { months) versus at least } \\
20 \text { mg fluphenazine decanoate every four weeks. } \\
\text { Outcome data: no usable data reported (conference proceeding) }\end{array}$ \\
\hline Ionescu 1983 & Allocation: not randomised. \\
\hline Iqbal 1978 & Allocation: not randomised. \\
\hline Irwin 1986 & $\begin{array}{l}\text { Allocation: double blind. } \\
\text { Participants: people with schizophrenia. } \\
\text { Interventions: } 5 \text {-HT versus placebo. }\end{array}$ \\
\hline Itil 1970a & Allocation: not randomised. \\
\hline Itil $1970 \mathrm{~b}$ & Allocation: double blind - cross-over study. \\
\hline Itil 1971 & $\begin{array}{l}\text { Allocation: double blind. } \\
\text { Participants: people with schizophrenia. } \\
\text { Interventions: fluphenazine hydrochloride. } \\
\text { Outcomes: no usable data. }\end{array}$ \\
\hline Itil 1978 & Allocation: not randomised. \\
\hline Jakovljevic 1999 & $\begin{array}{l}\text { Allocation: randomised. } \\
\text { Participants: people with schizophrenia. } \\
\text { Interventions: oral fluphenazine versus olanzapine. }\end{array}$ \\
\hline James 1977 & $\begin{array}{l}\text { Allocation: randomised. } \\
\text { Participants: people with schizophrenia. } \\
\text { Interventions: fluphenazine decanoate versus penfluridol. } \\
\text { Outcomes: no usable data (no SD). }\end{array}$ \\
\hline Johnson 1975 & Allocation: not randomised. \\
\hline Jones 2006 & $\begin{array}{l}\text { Allocation: randomised. } \\
\text { Participants: people with schizophrenia. } \\
\text { Interventions: first generation antipsychotics versus second generation antipsychotics }\end{array}$ \\
\hline
\end{tabular}

Fluphenazine decanoate (depot) and enanthate for schizophrenia (Review)

Copyright () 2016 The Cochrane Collaboration. Published by John Wiley \& Sons, Ltd. 
(Continued)

\begin{tabular}{ll} 
Kabes 1980a & $\begin{array}{l}\text { Allocation: “divided randomly into } 2 \text { groups" - cross-over study. } \\
\text { Participants: people with schizophrenia. } \\
\text { Interventions: depot preparations plus fluphenazine, oxyprothepine/oxyprotepin. } \\
\text { Outcomes: no usable data. }\end{array}$ \\
\hline Kabes 1980b & $\begin{array}{l}\text { Allocation: randomised. } \\
\text { Participants: people with schizophrenia. } \\
\text { Interventions: oxyprothepin decanoate versus fluphenazine decanoate - medication crossed over at } 6 \text { months. } \\
\text { Outcomes: no usable data - results presented at } 12 \text { months without differentiating each treatment arm }\end{array}$ \\
\hline Kabes 1981 & $\begin{array}{l}\text { Allocation: double blind - cross-over study. } \\
\text { Participants: people with schizophrenia. } \\
\text { Interventions: oxyprothepin decanoate versus fluphenazine decanoate. } \\
\text { Outcomes: no usable data. }\end{array}$ \\
\hline
\end{tabular}

Kane 1979

Allocation: randomised.

Participants: people with schizophrenia.

Interventions: fluphenazine decanoate and placebo.

Outcomes: withdrawal study.

Kane 1982

Allocation: randomised.

Participants: people with acute first episode schizophrenia.

Interventions: fluphenazine decanoate versus placebo.

Outcomes: no usable data, authors contacted.

Kane 1983 b Allocation: not randomised - review article.

Keith $2002 \quad$ Allocation: randomised.

Participants: people with schizophrenia.

Interventions: Continuous low dose $(2.5-10 \mathrm{mg})$ fluphenazine decanoate versus standard dose (12.5-50 mg) versus targeted dose (vehicle), delivered by injection every two weeks for two years.

Outcome data: no usable data reported (conference proceeding)

Kelly $1999 \quad$ Allocation: not randomised.

Kenway $1971 \quad$ Allocation: randomised - cross-over study.

Keskiner 1968a Allocation: not randomised.

Keskiner 1968b Allocation: randomised.

Participants: people with schizophrenia.

Interventions: fluphenazine decanoate versus placebo.

Outcomes: withdrawal study.

King $1979 \quad$ Allocation: randomised.

Participants: people with schizophrenia.

Interventions: family therapy in conjunction with high- and low-dose phenothiazines

Fluphenazine decanoate (depot) and enanthate for schizophrenia (Review) 
(Continued)

Kinon 1993

Allocation: randomised.

Participants: people with schizophrenia.

Interventions: fluphenazine high dose versus fluphenazine low dose versus haloperidol.

Outcomes: no usable data.

Kinross-Wright 1963 Allocation: not randomised.

Knights $1979 \quad$ Allocation: not randomised.

Kong $1989 \quad$ Allocation: not randomised.

Landmark $1994 \quad$ Allocation: randomised.

Participants: people with schizophrenia.

Interventions: fluphenazine decanoate versus fluphenazine hydrochloride (oral).

Outcomes: no usable data, no clinical outcomes reported.

Lapierre $1975 \quad$ Allocation: randomised.

Participants: people with schizophrenia.

Interventions: fluphenazine versus pimozide.

Outcomes: no usable data.

Lapierre $1976 \quad$ Allocation: randomised.

Participants: people with schizophrenia.

Interventions: fluphenazine versus pimozide + half of each group received psychotherapy.

Outcomes: no usable data.

Lapierre $1978 \quad$ Allocation: randomised.

Participants: people with schizophrenia.

Interventions: oral fluphenazine versus penfluridol.

Lapierre $1983 \quad$ Allocation: randomised.

Participants: people with schizophrenia.

Interventions: pipothiazine palmitate versus fluphenazine decanoate.

Outcomes: no usable data.

Lasky $1962 \quad$ Allocation: randomised.

Participants: people with schizophrenia.

Interventions: chlorpromazine versus thioridazine versus chlorprothixene versus triflupromazine.

Outcomes: no usable data - drop outs $>50 \%$.

Leff $1971 \quad$ Allocation: randomised.

Particpants: people with schizophrenia.

Interventions: trifluperazine versus chlorpromazine.

Leff 1973 Allocation: randomised.

Participants: people with schizophrenia.

Interventions: maintenance therapy and life events. 
(Continued)

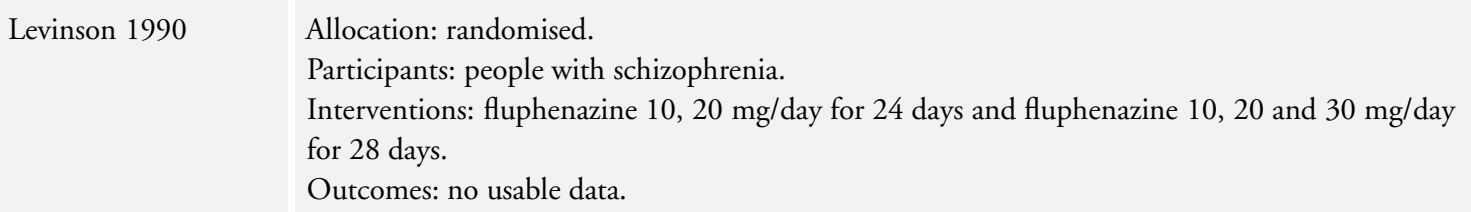

Lewis 2003

Allocation: randomised.

Participants: people with schizophrenia.

Interventions: first generation antipsychotics versus second generation antipsychotics

Litman $1994 \quad$ Allocation: double blind.

Participants: people with schizophrenia.

Interventions: fluphenazine versus benztropine (1st phase) and fluphenazine versus clozapine 92nd phase).

Outcomes: no usable data.

Ljubin 2000

Allocation: randomised.

Participants: people with schizophrenia.

Interventions: oral fluphenazine versus olanzapine.

Mahmoud $2004 \quad$ Allocation: randomised.

Participants: people with schizophrenia.

Interventions: risperidone versus conventional antipsychotics

Marder $1986 \quad$ Allocation: randomised.

Participants: people with schizophrenia.

Interventions: fluphenazine decanoate (dosage study).

Outcomes: no usable data.

Marder $1989 \quad$ Allocation: randomised.

Participants: people with schizophrenia.

Interventions: fluphenazine decanoate versus fluphenazine (oral).

Outcomes: no usable data, drug metabolism study - no clinical outcomes measured

$\begin{array}{ll}\text { Marder 1990a } & \text { Allocation: randomised. } \\ & \text { Participants: people with schizophrenia. } \\ & \text { Interventions: fluphenazine decanoate (dosage study). } \\ & \text { Outcomes: no usable data, authors contacted. }\end{array}$

Marder 1991a Allocation: randomised.

Participants: people with schizophrenia.

Interventions: fluphenazine decanoate (dosage study).

Outcomes: no usable data, pharmacological study - no clinical outcomes reported

Marder 1991b Allocattion: randomised.

Participants: people with schizophrenia.

Interventions: fluphenazine decanoate versus placebo.

Outcomes: no usable data, trial of different measuring procedures 
(Continued)

\begin{tabular}{|c|c|}
\hline Marder 1996 & $\begin{array}{l}\text { Allocation: randomised. } \\
\text { Particpants: people with schizophrenia. } \\
\text { Interventions: fluphenazine decanoate versus placebo. } \\
\text { Outcomes: no usable data. }\end{array}$ \\
\hline Marder 2002 & $\begin{array}{l}\text { Allocation: randomised. } \\
\text { Particpants: people with schizophrenia. } \\
\text { Interventions: low-dose fluphenazine decanoate versus medium dose fluphenazine decanoate versus high- } \\
\text { dose fluphenazine decanoate. } \\
\text { Outcomes: no usable data. }\end{array}$ \\
\hline Martenyi 2000 & $\begin{array}{l}\text { Allocation: double blind. } \\
\text { Participants: people with schizophrenia. } \\
\text { Interventions: olanzapine versus fluphenazine. } \\
\text { Outcomes: no usable data. }\end{array}$ \\
\hline Martin 1972 & Allocation: not randomised. \\
\hline Mattes 1984 & $\begin{array}{l}\text { Allocation: double blind. } \\
\text { Participants: people with schizophrenia. } \\
\text { Interventions: lithium versus fluphenazine (oral and decanoate) versus placebo. } \\
\text { Outcomes: no usable data. }\end{array}$ \\
\hline McCreadie 1983 & Allocation: not randomised. \\
\hline McCreadie 1986 & $\begin{array}{l}\text { Allocation: double blind. } \\
\text { Participants: people with schizophrenia. } \\
\text { Interventions: haloperidol versus fluphenazine. } \\
\text { Outcomes: no usable data. }\end{array}$ \\
\hline
\end{tabular}

Meco 1987

Allocation: not randomised but double blinded.

Participants: people with schizophrenia.

Interventions: fluphenazine decanoate versus haloperidol decanoate.

Outcomes: no usable data, authors contacted.

Mimica $1998 \quad$ Allocation: randomised.

Participants: people with schizophrenia.

Interventions: oral fluphenazine versus olanzapine.

Montejo $2010 \quad$ Allocation: not randomised.

Morris $1970 \quad$ Allocation: randomised - cross-over study.

National $1964 \quad$ Allocation: "randomly assigned".

Participants: people with schizophrenia.

Interventions: oral fluphenazine versus chlorpromazine versus thioridazine versus placebo 
(Continued)

\begin{tabular}{|c|c|}
\hline Nestoros 1978 & $\begin{array}{l}\text { Allocation: "randomly assigned" } \\
\text { Participants: people with schizophrenia. } \\
\text { Interventions: oral fluphenazine versus butaclamol. }\end{array}$ \\
\hline Owen 1993 & Allocation: admitted sequentially - cross-over study. \\
\hline Palma 1997 & $\begin{array}{l}\text { Allocation: randomised. } \\
\text { Participants: people with schizophrenia. } \\
\text { Interventions: flupenthixol decanoate versus other neuroleptics including fluphenazine decanoate. } \\
\text { Outcomes: fluphenazine decanoate results not presented separately from the other neuroleptics }\end{array}$ \\
\hline Pichot 1988 & $\begin{array}{l}\text { Allocation: randomised. } \\
\text { Participants: people with schizophrenia. } \\
\text { Interventions: oral fluphenazine versus amisulpride. }\end{array}$ \\
\hline Pickar 1987 & Allocation: review of studies. \\
\hline Pickar 1992 & Allocation: double blind - cross-over study. \\
\hline Pickar 1994 & Allocation: double blind - cross-over study. \\
\hline Pollack 1964 & $\begin{array}{l}\text { Allocation: randomised. } \\
\text { Partcipants: people with schizophrenia. } \\
\text { Interventions: fluphenazine decanoate versus placebo. } \\
\text { Outcomes: no usable data. }\end{array}$ \\
\hline Preussler 1995 & $\begin{array}{l}\text { Allocation: randomised. } \\
\text { Participants: people with schizophrenia. } \\
\text { Interventions: clozapine versus fluphenazine. } \\
\text { Outcomes: no usable data. }\end{array}$ \\
\hline Preussler 1997 & $\begin{array}{l}\text { Allocation: randomised. } \\
\text { Participants: people with schizophrenia. } \\
\text { Interventions: clozapine versus fluphenazine. } \\
\text { Outcomes: no usable data. }\end{array}$ \\
\hline Quitkin 1975 & $\begin{array}{l}\text { Allocation: "randomly assigned". } \\
\text { Participants: people with schizophrenia. } \\
\text { Interventions: oral fluphenazine (dosage study). }\end{array}$ \\
\hline Quitkin 1977 & $\begin{array}{l}\text { Allocation: randomised. } \\
\text { Participants: people with schizophrenia. } \\
\text { Interventions: fluphenazine decanoate versus penfluridol. } \\
\text { Outcomes: no usable data, preliminary report. }\end{array}$ \\
\hline
\end{tabular}

Ravaris $1965 \quad$ Allocation: not randomised. 
(Continued)

\begin{tabular}{|c|c|}
\hline Ravaris 1967 & $\begin{array}{l}\text { Allocation: randomised. } \\
\text { Participants: people with schizophrenia. } \\
\text { Interventions: fluphenazine enanthate versus fluphenazine (oral). } \\
\text { Outcomes: no usable data. }\end{array}$ \\
\hline Rifkin 1976 & $\begin{array}{l}\text { Allocation: randomised. } \\
\text { Participants: people with schizophrenia. } \\
\text { Interventions: fluphenazine decanoate versus fluphenazine (oral) versus placebo. } \\
\text { Outcomes: no usable data. }\end{array}$ \\
\hline Roose 1982 & Allocation: not randomised. \\
\hline Rossger 1997 & $\begin{array}{l}\text { Allocation: randomised. } \\
\text { Participants: people with schizophrenia. } \\
\text { Interventions: clozapine versus fluphenazine. } \\
\text { Outcomes: no usable data. }\end{array}$ \\
\hline Saxena 1996 & Allocation: non-specific - authors contacted (conference abstract) \\
\hline Schausberger 1999 & $\begin{array}{l}\text { Allocation: randomised. } \\
\text { Participants: people with schizophrenia. } \\
\text { Interventions: oral fluphenazine versus olanzapine. }\end{array}$ \\
\hline Schipper 1971 & Allocation: not randomised. \\
\hline Schooler 1971 & $\begin{array}{l}\text { Allocation: randomised. } \\
\text { Participants: people with schizophrenia. } \\
\text { Interventions: acetophenazine maleate versus chlorpromazine versus fluphenazine hydrochloride. } \\
\text { Outcomes: no usable data. }\end{array}$ \\
\hline Schooler 1977 & Allocation: not randomised - double blinded. \\
\hline Schubert 1988 & $\begin{array}{l}\text { Allocation: randomised. } \\
\text { Participants: people with schizophrenia. } \\
\text { Interventions: fluphenazine versus haloperidol. } \\
\text { Outcomes: no usable data. }\end{array}$ \\
\hline Shafti 2009 & $\begin{array}{l}\text { Allocation: randomised. } \\
\text { Participants: people with schizophrenia. } \\
\text { Interventions: fluphenazine versus placebo as add-on to olanzapine }\end{array}$ \\
\hline Simpson 1970 & $\begin{array}{l}\text { Allocation: randomised. } \\
\text { Participants: people with schizophrenia. } \\
\text { Interventions: fluphenazine. } \\
\text { Outcomes: no usable data. }\end{array}$ \\
\hline Siris 1990 & $\begin{array}{l}\text { Allocation: randomised. } \\
\text { Participants: people with schizophrenia. } \\
\text { Interventions: imipramine versus placebo as add-on to fluphenazine decanoate and benztropine }\end{array}$ \\
\hline
\end{tabular}

Fluphenazine decanoate (depot) and enanthate for schizophrenia (Review)

Copyright () 2016 The Cochrane Collaboration. Published by John Wiley \& Sons, Ltd. 
(Continued)

\begin{tabular}{ll} 
Siris 1991 & $\begin{array}{l}\text { Allocation: randomised. } \\
\text { Participants: people with schizophrenia. } \\
\text { Interventions: imipramine versus placebo as add-on to fluphenazine decanoate and benztropine }\end{array}$ \\
\hline Steingard 1994 & $\begin{array}{l}\text { Allocation: randomised. } \\
\text { Participants: people with schizophrenia. } \\
\text { Interventions: fluphenazine versus placebo. } \\
\text { Outcomes: no usable data. }\end{array}$ \\
\hline Stevens 1973 & $\begin{array}{l}\text { Allocation: randomised. } \\
\text { Participants: people with schizophrenia. } \\
\text { Interventions: fluphenazine decanoate versus placebo. } \\
\text { Outcomes: no usable data. }\end{array}$ \\
\hline
\end{tabular}

Tegeler $1985 \quad$ Allocation: randomised.

Participants: people with schizophrenia.

Interventions: fluphenazine decanoate versus clopenthixol decanoate.

Outcomes: no usable data, authors contacted.

Tetreault $1969 \quad$ Allocation: randomised.

Participants: people with schizophrenia.

Interventions: fluphenazine enanthate versus oral fluphenazine bichloralhydrate

Tran $1998 \quad$ Allocation: randomised.

Participants: people with schizophrenia.

Interventions: olanzapine versus fluphenazine.

Outcomes: no usable data.

Tsai $2004 \quad$ Allocation: randomised.

Participants: people with schizophrenia.

Interventions: sarcosine versus placebo as add-on to antipsychotics

Tsai $2006 \quad$ Allocation: randomised.

Participants: people with schizophrenia.

Interventions: D-alanine versus placebo as add-on to antipsychotics as add-on to antipsychotics

Turner $1966 \quad$ Allocation: randomised.

Participants: not described.

Turner $2004 \quad$ Allocation: not randomised.

Ushakov $1990 \quad$ Allocation: not randomised, case series.

van Putten $1986 \quad$ Allocation: randomised.

Participants: people with schizophrenia.

Interventions: 1st report - haloperidol (dosage study), 2nd report - fluphenazine (dosage study).

Outcomes: no usable data. 
(Continued)

\begin{tabular}{ll}
\hline van Putten 1991 & $\begin{array}{l}\text { Allocation: randomised. } \\
\text { Participants: people with schizophrenia. } \\
\text { Interventions: fluphenazine (dosage study). } \\
\text { Outcomes: no usable data. }\end{array}$ \\
\hline Verster 1998 & $\begin{array}{l}\text { Allocation: randomised. } \\
\text { Participants: people with schizophrenia. } \\
\text { Interventions: fluphenazine decanoate versus generic substitute }\end{array}$ \\
\hline Vestre 1962 & $\begin{array}{l}\text { Allocation: randomised. } \\
\text { Participants: people with schizophrenia. } \\
\text { Interventions: oral fluphenazine versus triflupromazine versus phenobarbital }\end{array}$ \\
\hline Viala 1988 & Allocation: not randomised. \\
\hline Villeneuve 1970 & Allocation: not randomised. \\
\hline Vinar 1970 & $\begin{array}{l}\text { Allocation: double blind. } \\
\text { Participants: people with schizophrenia. } \\
\text { Interventions: fluphenazine versus fluphenazine long-acting form. } \\
\text { Outcomes: no usable data. }\end{array}$ \\
\hline
\end{tabular}

Weiden $1993 \quad$ Allocation: randomised.

Participants: people with schizophrenia.

Interventions: fluphenazine decanoate (dosage study).

Outcomes: no usable data, prescribing patterns study.

Wiles $1990 \quad$ Allocation: double blind.

Participants: people with schizophrenia.

Interventions: haloperidol decanoate versus fluphenazine decanoate.

Outcomes: no usable data, authors contacted.
Winter $1973 \quad$ Allocation: randomised.
Participants: people with schizophrenia.
Interventions: fluphenazine decanoate versus fluspirilene decanoate.
Outcomes: no usable data.

Wistedt $1981 \quad$ Allocation: randomised.

Participants: people with schizophrenia.

Interventions: fluphenazine decanoate and flupenthixol decanoate versus placebo.

Outcomes: no usable data, the two drug treatments are grouped as one group

Wistedt 1983a Allocation: randomised.

Participants: people with schizophrenia.

Interventions: fluphenazine decanoate and flupenthixol decanoate versus placebo.

Outcomes: no usable data - both drugs placed in one group. 
(Continued)

Wistedt 1983b Allocation: randomised.

Participants: people with schizophrenia.

Interventions: discontinuation study.

Zapletalek $1981 \quad$ Allocation: not randomised.

Characteristics of studies awaiting assessment [ordered by study ID]

\section{Angst 1973}

Methods

Participants

Interventions

Outcomes

Notes Article in German

\section{del Giudice 1970}

Methods

Participants

Interventions

Outcomes

Notes Missing PDF

Jue 1996

Methods

Participants

Interventions

Outcomes

Notes Missing PDF 
Kabes 1984

Methods

Participants

Interventions

Outcomes

Notes Missing PDF

Ravanic 1996

Methods

Participants

Interventions

Outcomes

Notes Missing PDF

Ushakov 1990a

\begin{tabular}{ll}
\hline Methods & Unclear \\
\hline Participants & People with schizophrenia \\
\hline Interventions & $\begin{array}{l}\text { 1. Fluphenazine decanoate (moditen-depot) } \\
\text { 2. haloperidol decanoate }\end{array}$ \\
\hline Outcomes & Efficacy \\
\hline Notes & Article in Russian \\
\hline
\end{tabular}


DATA ANDANALYSES

\section{Comparison 1. FLUPHENAZINE DECANOATE vs PLACEBO}

\begin{tabular}{|c|c|c|c|c|}
\hline Outcome or subgroup title & $\begin{array}{l}\text { No. of } \\
\text { studies }\end{array}$ & $\begin{array}{c}\text { No. of } \\
\text { participants }\end{array}$ & Statistical method & Effect size \\
\hline 1 Death & 1 & 54 & Risk Ratio (M-H, Fixed, 95\% CI) & $5.0[0.25,99.51]$ \\
\hline 2 Global state: 1 . Relapse & 5 & & Risk Ratio (M-H, Random, 95\% CI) & Subtotals only \\
\hline $\begin{array}{l}2.1 \text { short term ( } 6 \text { weeks to } 5 \\
\text { months) }\end{array}$ & 1 & 31 & Risk Ratio (M-H, Random, 95\% CI) & $0.0[0.0,0.0]$ \\
\hline $\begin{array}{l}2.2 \text { medium term ( } 6 \text { months } \\
\text { to } 1 \text { year) }\end{array}$ & 3 & 196 & Risk Ratio (M-H, Random, 95\% CI) & $0.62[0.24,1.60]$ \\
\hline $\begin{array}{l}2.3 \text { longer term (more than } 1 \\
\text { year) }\end{array}$ & 1 & 54 & Risk Ratio (M-H, Random, 95\% CI) & $0.35[0.19,0.64]$ \\
\hline $\begin{array}{l}3 \text { Global state: } 2 \text {. GAS (short term } \\
-6 \text { weeks to } 5 \text { months) (high } \\
\text { score }=\text { worse) }\end{array}$ & 1 & 28 & Mean Difference (IV, Fixed, 95\% CI) & $3.61[-4.41,11.63]$ \\
\hline 4 Leaving the study early & 6 & & Risk Ratio (M-H, Fixed, 95\% CI) & Subtotals only \\
\hline $\begin{array}{l}4.1 \text { short term ( } 6 \text { weeks to } 5 \\
\text { months) }\end{array}$ & 1 & 31 & Risk Ratio (M-H, Fixed, 95\% CI) & $2.43[0.24,24.07]$ \\
\hline $\begin{array}{l}4.2 \text { medium term ( } 6 \text { months } \\
\text { to } 1 \text { year) }\end{array}$ & 4 & 216 & Risk Ratio (M-H, Fixed, 95\% CI) & $1.30[0.77,2.19]$ \\
\hline $\begin{array}{l}4.3 \text { longer term (more than } 1 \\
\text { year) }\end{array}$ & 1 & 54 & Risk Ratio (M-H, Fixed, 95\% CI) & $0.47[0.23,0.96]$ \\
\hline $\begin{array}{l}5 \text { Mental state: } 1 . \text { BPRS (endpoint } \\
\text { scores }- \text { high score }=\text { worse) }\end{array}$ & 1 & 16 & Mean Difference (IV, Fixed, 95\% CI) & $-2.03[-4.51,0.45]$ \\
\hline $\begin{array}{l}6 \text { Mental state: } 2 \text {. Depression } \\
\text { (medium term - } 6 \text { months to } 1 \\
\text { year) }\end{array}$ & 1 & 70 & Risk Ratio (M-H, Fixed, 95\% CI) & $1.0[0.45,2.22]$ \\
\hline $\begin{array}{l}7 \text { Adverse effects: } 1 \text {. Movement } \\
\text { disorders - tardive dyskinesia } \\
\text { (longer term - more than } 1 \\
\text { year) }\end{array}$ & 1 & 54 & Risk Ratio (M-H, Fixed, 95\% CI) & $0.83[0.62,1.11]$ \\
\hline 8 Adverse effects: 2 . Toxicity & 1 & 45 & Risk Ratio (M-H, Fixed, 95\% CI) & $7.65[1.04,56.26]$ \\
\hline
\end{tabular}

\section{Comparison 2. FLUPHENAZINE DECANOATE vs ORAL NEUROLEPTICS}

\begin{tabular}{lcclc} 
Outcome or subgroup title & $\begin{array}{c}\text { No. of } \\
\text { studies }\end{array}$ & $\begin{array}{c}\text { No. of } \\
\text { participants }\end{array}$ & Statistical method & Effect size \\
\hline $\begin{array}{c}\text { 1 Global state: } 1 \text {. No clinically } \\
\text { important global change }\end{array}$ & 3 & & Risk Ratio (M-H, Fixed, 95\% CI) & Subtotals only \\
$\begin{array}{l}1.1 \text { immediate (0 to 5 weeks) } \\
1.2 \text { medium term (6 months }\end{array}$ & 2 & 74 & Risk Ratio (M-H, Fixed, 95\% CI) & $0.61[0.46,0.81]$ \\
$\quad \begin{array}{l}1 \\
\text { to } 1 \text { year) }\end{array}$ & 9 & 102 & Risk Ratio (M-H, Fixed, 95\% CI) & $0.85[0.56,1.27]$ \\
2 Global state: 2. Relapse & & Risk Ratio (M-H, Random, 95\% CI) & Subtotals only \\
\hline
\end{tabular}

Fluphenazine decanoate (depot) and enanthate for schizophrenia (Review)

Copyright @ 2016 The Cochrane Collaboration. Published by John Wiley \& Sons, Ltd. 
2.1 medium term (6 months to 1 year)

2.2 longer term (more than 1 year)

3 Global state: 3. Clinical Global Impression (short term - 6

weeks to 5 months) (high score = worse)

4 Leaving the study early 4.1 immediate (0-5 weeks)

4.2 short term (6 weeks to 5 months)

4.3 medium term (6 months to 1 year)

4.4 longer term (more than 1 year)

5 Behaviour: 1. NOSIE-30 endpoint scores (high score $=$ poor)

6 Behaviour: 2. skewed data (endpoint scores)

7 Mental state: 1. BPRS - endpoint scores (longer term - more than 1 year) (high score $=$ poor $)$

8 Mental state: 2 . Depression 8.1 medium term (6 months to 1 year)

8.2 longer term (more than 1 year)

9 Adverse effects: 1a. Movement disorders - general

9.1 medium term (6 months to 1 year)

9.2 longer term (more than 1 year)

10 Adverse effects: 1b. Movement disorders - akathisia

11 Adverse effects: 1c.

Movement disorders - needing anticholinergic drugs

11.1 immediate (0 to 5 weeks) 11.2 medium term (6 months to 1 year)

11.3 longer term (more than 1 year)

12 Adverse effects: 1d. Movement disorders - tardive dyskinesia

12.1 medium term (6 months to 1 year)

12.2 longer term (more than 1 year)
6

Other data

Risk Ratio (M-H, Fixed, 95\% CI) Risk Ratio (M-H, Fixed, 95\% CI)

Risk Ratio (M-H, Fixed, 95\% CI)

Risk Ratio (M-H, Fixed, 95\% CI)

Risk Ratio (M-H, Fixed, 95\% CI)

Risk Ratio (M-H, Fixed, 95\% CI)

Risk Ratio (M-H, Fixed, 95\% CI)

Risk Ratio (M-H, Fixed, 95\% CI)

Risk Ratio (M-H, Fixed, 95\% CI)

Risk Ratio (M-H, Random, 95\% CI)

Risk Ratio (M-H, Random, 95\% CI)

Risk Ratio (M-H, Random, 95\% CI)

Risk Ratio (M-H, Random, 95\% CI)

Risk Ratio (M-H, Fixed, 95\% CI)

Risk Ratio (M-H, Fixed, 95\% CI)

120
$1.46[0.75,2.83]$

$1.25[0.81,1.95]$

$-0.10[-2.79,2.59]$

Subtotals only $0.32[0.01,7.30]$

$1.69[0.32,8.85]$

$0.96[0.73,1.25]$

$1.20[0.61,2.36]$

$-0.56[-6.92,5.80]$

No numeric data

$-0.75[-5.75,4.25]$

Subtotals only $0.89[0.60,1.32]$

$1.53[0.91,2.57]$

Subtotals only

$0.47[0.24,0.91]$

$0.4[0.12,1.28]$

$20.54[1.25,337.94]$

Subtotals only

$0.0[0.0,0.0]$

$0.86[0.21,3.45]$

$1.04[0.86,1.25]$

Subtotals only

$0.62[0.41,0.93]$

$0.16[0.01,2.99]$

Fluphenazine decanoate (depot) and enanthate for schizophrenia (Review)

Copyright $\Subset 2016$ The Cochrane Collaboration. Published by John Wiley \& Sons, Ltd. 
13 Adverse effects: 1e. Movement disorders - tremor (longer term - more than 1 year)

14 Adverse effects: 1f. Movement disorders - average score

(Simpson \& Angus, 0 to 5 weeks, high = poor)

15 Adverse effects: 2. Blurred vision - medium term (6 months to 1 year)

16 Adverse effects: 3. Toxicity medium term ( 6 months to 1 year)

17 Adverse effects: 4. General adverse effects

17.1 immediate ( 0 to 5 weeks)

17.2 medium term (6 months to 1 year)

18 SENSITIVITY ANALYSIS

Global state: 2. Relapse
Subtotals only

\section{Comparison 3. FLUPHENAZINE DECANOATE vs OTHER DEPOT NEUROLEPTICS}

\begin{tabular}{|c|c|c|c|c|}
\hline Outcome or subgroup title & $\begin{array}{l}\text { No. of } \\
\text { studies }\end{array}$ & $\begin{array}{c}\text { No. of } \\
\text { participants }\end{array}$ & Statistical method & Effect size \\
\hline 1 Death & 1 & 38 & Risk Ratio (M-H, Fixed, 95\% CI) & $3.0[0.13,69.31]$ \\
\hline $\begin{array}{l}2 \text { Global state: } 1 \text {. No clinically } \\
\text { important global change }\end{array}$ & 4 & 339 & Risk Ratio (M-H, Fixed, 95\% CI) & $1.07[0.97,1.18]$ \\
\hline $\begin{array}{l}2.1 \text { short term (6 weeks to } 5 \\
\text { months) }\end{array}$ & 1 & 152 & Risk Ratio (M-H, Fixed, 95\% CI) & $1.16[0.89,1.51]$ \\
\hline $\begin{array}{l}2.2 \text { medium term ( } 6 \text { months } \\
\text { to } 1 \text { year) }\end{array}$ & 3 & 187 & Risk Ratio (M-H, Fixed, 95\% CI) & $1.04[0.96,1.12]$ \\
\hline 3 Global state: 2. Relapse & 16 & & Risk Ratio (M-H, Fixed, 95\% CI) & Subtotals only \\
\hline $\begin{array}{l}3.1 \text { short term ( } 6 \text { weeks to } 5 \\
\text { months) }\end{array}$ & 1 & 51 & Risk Ratio (M-H, Fixed, 95\% CI) & $0.96[0.27,3.43]$ \\
\hline $\begin{array}{l}3.2 \text { medium term ( } 6 \text { months } \\
\text { to } 1 \text { year) }\end{array}$ & 11 & 581 & Risk Ratio (M-H, Fixed, 95\% CI) & $0.82[0.56,1.18]$ \\
\hline $\begin{array}{l}3.3 \text { longer term (more than } 1 \\
\text { year) }\end{array}$ & 4 & 252 & Risk Ratio (M-H, Fixed, 95\% CI) & $1.22[0.77,1.92]$ \\
\hline $\begin{array}{l}\text { 4 Global state: } 3 \text {. Severly ill } \\
\text { (medium term } 6 \text { months to } 1 \\
\text { year) }\end{array}$ & 1 & 60 & Risk Ratio (M-H, Fixed, 95\% CI) & $1.07[0.94,1.23]$ \\
\hline $\begin{array}{l}5 \text { Global state: } 4 \text {. Needing } \\
\text { additional antipsychotic } \\
\text { treatment ( } 6 \text { months to } 1 \text { year) }\end{array}$ & 2 & 91 & Risk Ratio (M-H, Fixed, 95\% CI) & $0.53[0.14,1.96]$ \\
\hline
\end{tabular}

Fluphenazine decanoate (depot) and enanthate for schizophrenia (Review) 
6 Global state: 5 . Clinical Global Impression. (short term - 6 weeks to 5 months) (skewed data)

7 Global state: 6. Clinical Global Impression. (medium term - 6 months to 1 year)

8 Global state: 7. Clinical Global Impression - not improved $($ high score $=$ poor $)$

8.1 short term (6 weeks to 5 months)

8.2 medium term (6 months to 1 year)

9 Leaving the study early

9.1 immediate ( 0 to 5 weeks)

9.2 short term (6 weeks to 5 months)

9.3 medium term (6 months to 1 year)

9.4 By more than 1 year

10 Behaviour: 1. NOSIE-30 endpoint scores (high score $=$ poor)

11 Mental state: 1. BPRS

(endpoint scores - high score $=$ poor)

11.1 short term (6 weeks to 5 months)

11.2 medium term (6 months to 1 year)

11.3 longer term (more than one year)

12 Mental state: 2. BPRS (endpoint scores 6 months to 1 year - dichotomous data)

13 Mental state: 3. Depression (6 months to 1 year)

14 Mental state: 4. SAPS and

SANS (endpoint scores - high score $=$ poor $)($ skewed data $)$

14.1 SAPS

14.2 SANS

15 Adverse effects: 1a. Movement disorders - general

15.1 immediate term ( 0 to 5 weeks)

15.2 short term (6 weeks to 5 months)

15.3 medium term (6 months to 1 year)
Other data

No numeric data

Mean Difference (IV, Fixed, 95\% CI)

$-0.10[-0.41,0.21]$

Risk Ratio (M-H, Fixed, 95\% CI)

Subtotals only

$2.5[0.53,11.70]$

$0.75[0.18,3.07]$

Subtotals only

$0.0[0.0,0.0]$

$0.76[0.32,1.84]$

$1.13[0.89,1.44]$

$0.89[0.64,1.23]$

$-5.21[-10.85,0.43]$

Subtotals only

Mean Difference (IV, Fixed, 95\% CI)

$1.11[0.86,1.36]$

$1.20[1.10,1.30]$

$0.85[-2.32,4.03]$

$0.91[0.59,1.43]$

$1.02[0.81,1.28]$

No numeric data

Other data

Other data

No numeric data No numeric data

$1.11[0.91,1.35]$

Risk Ratio (M-H, Fixed, 95\% CI)

12 Risk Ratio (M-H, Fixed, 95\% CI)

$0.93[0.24,3.68]$

$2.0[0.43,9.32]$

$1.08[0.86,1.34]$

Fluphenazine decanoate (depot) and enanthate for schizophrenia (Review)

Copyright @ 2016 The Cochrane Collaboration. Published by John Wiley \& Sons, Ltd. 
15.4 longer term (more than 1 year)

16 Adverse effects: $1 \mathrm{~b}$.

Movement disorders - needing anticholinergic drugs

16.1 short term (6 weeks to 5 months)

16.2 medium term (6 months to 1 year)

16.3 longer term (more than 1 year)

17 Adverse effects: 1c. Movement disorders - parkinsonism 17.1 immediate (0 to 5 weeks) 17.2 medium term (6 months to 1 year)

17.3 longer term (more than 1 year)

18 Adverse effects: 1d. Movement disorders - tardive dyskinesia: longer term (more than 1 year)

19 Adverse effects: 1e. Movement disorders - tremor

19.1 short term (6 weeks to 5 months)

19.2 medium term (6 months to 1 year)

20 Adverse effects: 1f. Movement disorders - endpoint scores (short term - 6 weeks to 5 months)

20.1 TESS $($ high $=$ poor $)$ 20.2 RSESE (high = poor)

21 Adverse effects: 2. Blurred vision

21.1 medium term (6 months to 1 year)

21.2 longer term (more than 1 year)

22 Adverse effects: 3. Dry mouth: longer term (more than 1 year)

23 Adverse effects: 4. General adverse effects

23.1 short term (6 weeks to 5 months)

23.2 medium term (6 months to 1 year)

24 SENSITIVITY ANALYSIS

Global state: 2. Relapse
Subtotals only

$1.48[0.96,2.28]$

$1.24[0.93,1.64]$

$1.26[0.86,1.83]$

$1.12[0.47,2.69]$

$0.93[0.24,3.68]$

$1.25[0.37,4.21]$

$1.07[0.07,16.71]$

$0.62[0.32,1.23]$

150 Risk Ratio (M-H, Fixed, 95\% CI)

Risk Ratio (M-H, Fixed, 95\% CI)

Subtotals only

Risk Ratio (M-H, Fixed, 95\% CI)

$1.36[0.76,2.46]$

$1.14[0.73,1.78]$

152 Risk Ratio (M-H, Fixed, 95\% CI)

Other data

No numeric data

Other data

Other data

Risk Ratio (M-H, Fixed, 95\% CI)

32

Risk Ratio (M-H, Fixed, 95\% CI)

64 Risk Ratio (M-H, Fixed, 95\% CI)

32 Risk Ratio (M-H, Fixed, 95\% CI)

Risk Ratio (M-H, Fixed, 95\% CI)

88

Risk Ratio (M-H, Fixed, 95\% CI)

249

Risk Ratio (M-H, Fixed, 95\% CI)

Risk Ratio (M-H, Fixed, 95\% CI)
No numeric data No numeric data Subtotals only

$0.88[0.44,1.78]$

$17.88[1.08,294.82]$

$0.72[0.38,1.37]$

Subtotals only

$1.36[1.07,1.74]$

$1.04[0.83,1.32]$

Subtotals only

Fluphenazine decanoate (depot) and enanthate for schizophrenia (Review)

Copyright $\odot 2016$ The Cochrane Collaboration. Published by John Wiley \& Sons, Ltd. 


\section{Comparison 4. FLUPHENAZINE DECANOATE - DOSAGE STUDIES - HIGH DOSE vs STANDARD}

\begin{tabular}{|c|c|c|c|c|}
\hline Outcome or subgroup title & $\begin{array}{l}\text { No. of } \\
\text { studies }\end{array}$ & $\begin{array}{c}\text { No. of } \\
\text { participants }\end{array}$ & Statistical method & Effect size \\
\hline $\begin{array}{l}1 \text { Global state: } 1 \text {. Relapse (medium } \\
\text { term - } 6 \text { months to } 1 \text { year) }\end{array}$ & 2 & 182 & Risk Ratio (M-H, Random, 95\% CI) & $2.11[0.30,14.91]$ \\
\hline $\begin{array}{l}2 \text { Global state: } 2 \text {. Needing } \\
\text { additional antispsychotic } \\
\text { treatment (medium term - } 6 \\
\text { months to } 1 \text { year) }\end{array}$ & 1 & 50 & Risk Ratio (M-H, Fixed, 95\% CI) & $1.67[0.45,6.24]$ \\
\hline $\begin{array}{l}3 \text { Global state: } 3 \text {. Not improved } \\
\text { (medium term - } 6 \text { months to } 1 \\
\text { year) }\end{array}$ & 1 & & Risk Ratio (M-H, Fixed, 95\% CI) & Subtotals only \\
\hline 3.1 nurse rated & 1 & 40 & Risk Ratio (M-H, Fixed, 95\% CI) & $1.58[1.09,2.30]$ \\
\hline 3.2 psychiatrist rated & 1 & 40 & Risk Ratio (M-H, Fixed, 95\% CI) & $1.15[0.77,1.74]$ \\
\hline $\begin{array}{l}4 \text { Leaving the study early (medium } \\
\text { term - } 6 \text { months to } 1 \text { year) }\end{array}$ & 2 & 90 & Risk Ratio (M-H, Fixed, 95\% CI) & $0.6[0.15,2.36]$ \\
\hline $\begin{array}{l}5 \text { Mental state: BPRS endpoint } \\
\text { scores (medium term }-6 \\
\text { months to } 1 \text { year, high score }= \\
\text { poor) }\end{array}$ & 1 & 50 & Mean Difference (IV, Fixed, 95\% CI) & $-0.03[-5.79,5.73]$ \\
\hline $\begin{array}{l}6 \text { Adverse effects: Movement } \\
\text { disorders - needing } \\
\text { anticholinergic drugs (medium } \\
\text { term - } 6 \text { months to } 1 \text { year) }\end{array}$ & 1 & 50 & Risk Ratio (M-H, Fixed, 95\% CI) & $1.67[0.45,6.24]$ \\
\hline
\end{tabular}

\section{Comparison 5. FLUPHENAZINE DECANOATE - DOSAGE STUDIES - LOW DOSE vs STANDARD}

\begin{tabular}{|c|c|c|c|c|}
\hline Outcome or subgroup title & $\begin{array}{l}\text { No. of } \\
\text { studies }\end{array}$ & $\begin{array}{c}\text { No. of } \\
\text { participants }\end{array}$ & Statistical method & Effect size \\
\hline 1 Global state: Relapse & 6 & & Risk Ratio (M-H, Random, 95\% CI) & Subtotals only \\
\hline $\begin{array}{l}1.1 \text { medium term (6 months } \\
\text { to } 1 \text { year) }\end{array}$ & 3 & 471 & Risk Ratio (M-H, Random, 95\% CI) & $3.33[0.77,14.51]$ \\
\hline $\begin{array}{l}1.2 \text { longer term (more than } 1 \\
\text { year) }\end{array}$ & 3 & 172 & Risk Ratio (M-H, Random, 95\% CI) & $0.84[0.38,1.89]$ \\
\hline 2 Leaving the study early & 3 & & Risk Ratio (M-H, Fixed, 95\% CI) & Subtotals only \\
\hline $\begin{array}{l}2.1 \text { longer term (more than } 1 \\
\text { year) }\end{array}$ & 3 & 172 & Risk Ratio (M-H, Fixed, 95\% CI) & $0.67[0.33,1.36]$ \\
\hline $\begin{array}{l}3 \text { Adverse effects: } 1 \text {. Movement } \\
\text { disorders (medium term - } 6 \\
\text { months to } 1 \text { year) }\end{array}$ & 2 & & Risk Ratio (M-H, Fixed, 95\% CI) & Subtotals only \\
\hline 3.1 Tardive dyskinesia & 1 & 126 & Risk Ratio (M-H, Fixed, 95\% CI) & $0.52[0.10,2.72]$ \\
\hline
\end{tabular}




\section{Comparison 6. FLUPHENAZINE ENANTHATE vs PLACEBO}

\begin{tabular}{|c|c|c|c|c|}
\hline Outcome or subgroup title & $\begin{array}{l}\text { No. of } \\
\text { studies }\end{array}$ & $\begin{array}{c}\text { No. of } \\
\text { participants }\end{array}$ & Statistical method & Effect size \\
\hline $\begin{array}{l}1 \text { Adverse effects: Movement } \\
\text { disorders - general }\end{array}$ & 1 & & Risk Ratio (M-H, Fixed, 95\% CI) & Subtotals only \\
\hline $\begin{array}{l}\text { 1.1 Needing anticholinergic } \\
\text { drugs (short term - } 6 \text { weeks to } \\
5 \text { months) }\end{array}$ & 1 & 25 & Risk Ratio (M-H, Fixed, 95\% CI) & $9.69[0.58,163.02]$ \\
\hline
\end{tabular}

\section{Comparison 7. FLUPHENAZINE ENANTHATE vs ORAL NEUROLEPTICS}

\begin{tabular}{|c|c|c|c|c|}
\hline Outcome or subgroup title & $\begin{array}{l}\text { No. of } \\
\text { studies }\end{array}$ & $\begin{array}{c}\text { No. of } \\
\text { participants }\end{array}$ & Statistical method & Effect size \\
\hline $\begin{array}{l}1 \text { Global state: No clinically } \\
\text { important global change } \\
\text { (immediate - } 0 \text { to } 5 \text { weeks) }\end{array}$ & 1 & 31 & Risk Ratio (M-H, Fixed, 95\% CI) & $0.67[0.27,1.66]$ \\
\hline $\begin{array}{l}2 \text { Adverse effects: Movement } \\
\text { disorders - general }\end{array}$ & 1 & & Risk Ratio (M-H, Fixed, 95\% CI) & Subtotals only \\
\hline $\begin{array}{l}2.1 \text { movement disorders: } \\
\text { immediate ( } 0 \text { to } 5 \text { weeks })\end{array}$ & 1 & 31 & Risk Ratio (M-H, Fixed, 95\% CI) & $2.34[0.53,10.30]$ \\
\hline $\begin{array}{l}2.2 \text { side effects: immediate }(0 \\
\text { to } 5 \text { weeks) }\end{array}$ & 1 & 31 & Risk Ratio (M-H, Fixed, 95\% CI) & $2.81[0.94,8.45]$ \\
\hline $\begin{array}{l}2.3 \text { parkinsonism: immediate } \\
\text { ( } 0 \text { to } 5 \text { weeks })\end{array}$ & 1 & 31 & Risk Ratio (M-H, Fixed, 95\% CI) & $6.56[0.91,47.21]$ \\
\hline
\end{tabular}

\section{Comparison 8. FLUPHENAZINE ENANTHATE vs OTHER DEPOT NEUROLEPTICS}

\begin{tabular}{|c|c|c|c|c|}
\hline Outcome or subgroup title & $\begin{array}{l}\text { No. of } \\
\text { studies }\end{array}$ & $\begin{array}{c}\text { No. of } \\
\text { participants }\end{array}$ & Statistical method & Effect size \\
\hline $\begin{array}{l}1 \text { Global state: } 1 \text {. Needing } \\
\text { additional antipsychotic } \\
\text { treatment ( } 6 \text { months to } 1 \text { year) }\end{array}$ & 2 & 65 & Risk Ratio (M-H, Fixed, 95\% CI) & $0.5[0.24,1.05]$ \\
\hline 2 Global state: 2. Relapse & 2 & & Risk Ratio (M-H, Fixed, 95\% CI) & Subtotals only \\
\hline
\end{tabular}


2.1 short term (6 weeks to 5 months)

2.2 medium term (6 months to 1 year)

3 Leaving the study early

3.1 immediate ( 0 to 5 weeks)

3.2 short term (6 weeks to 5 months)

3.3 medium term (6 months to 1 year)

4 Mental state: 1 . BPRS - endpoint scores (medium term - 6

months to 1 year) (high score $=$ poor)

5 Mental state: 2. Depression (medium term - 6 months to 1 year)

6 Adverse effects: 1a. Movement disorders - general (medium term - 6 months to 1 year)

7 Adverse effects: 1b. Movement anticholinergic drugs

7.1 short term (6 weeks to 5 months)

7.2 medium term (6 months to 1 year)

8 Adverse effects: 1c. Movement disorders - tardive dyskinesia: medium term (6 months to 1 year)

9 Adverse effects: $1 \mathrm{~d}$. Movement disorders - tremor (medium term - 6 months to 1 year)

10 Adverse effects: 2. Blurred vision (medium term - 6 months to 1 year)

11 Adverse effects: 3. Dry mouth (medium term - 6 months to 1 disorders - needing additional

Risk Ratio (M-H, Fixed, 95\% CI)

Risk Ratio (M-H, Fixed, 95\% CI)

Mean Difference (IV, Fixed, 95\% CI)

Risk Ratio (M-H, Fixed, 95\% CI)

$2.38[0.66,8.61]$

$0.33[0.04,2.87]$

Subtotals only 0.09 [0.01, 0.62]

2.38 [0.66, 8.61]

$0.33[0.04,2.87]$

$0.40[0.34,0.46]$

$7.0[0.39,124.83]$

$1.52[0.75,3.07]$

Subtotals only

$2.86[1.16,7.06]$

$1.02[0.76,1.35]$

$0.89[0.46,1.71]$ year) 


\section{Comparison 9. FLUPHENAZINE ENANTHATE - DOSAGE STUDIES - LOW DOSE vs INTERMEDI- ATE/HIGH DOSE}

\begin{tabular}{lcccc} 
Outcome or subgroup title & $\begin{array}{c}\text { No. of } \\
\text { studies }\end{array}$ & $\begin{array}{c}\text { No. of } \\
\text { participants }\end{array}$ & Statistical method & Effect size \\
\hline $\begin{array}{c}1 \text { Global state: Relapse (short term } \\
-6 \text { weeks to 5 months) }\end{array}$ & 1 & 104 & Risk Ratio (M-H, Fixed, 95\% CI) & $9.35[2.28,38.29]$ \\
$\begin{array}{c}2 \text { Leaving the study early (short } \\
\text { term - 6 weeks to 5 months) }\end{array}$ & 1 & 104 & Risk Ratio (M-H, Fixed, 95\% CI) & $3.12[0.66,14.74]$ \\
\hline
\end{tabular}

Comparison 10. FLUPHENAZINE DECANAOTE vs FLUPHENAZINE ENANTHATE

\begin{tabular}{|c|c|c|c|c|}
\hline Outcome or subgroup title & $\begin{array}{l}\text { No. of } \\
\text { studies }\end{array}$ & $\begin{array}{c}\text { No. of } \\
\text { participants }\end{array}$ & Statistical method & Effect size \\
\hline $\begin{array}{l}1 \text { Global state: } 1 . \text { Needing } \\
\text { additional antipsychotic } \\
\text { treatment }\end{array}$ & 2 & & Risk Ratio (M-H, Fixed, 95\% CI) & Subtotals only \\
\hline 1.1 immediate (0 to 5 weeks) & 1 & 33 & Risk Ratio (M-H, Fixed, 95\% CI) & $0.39[0.18,0.86]$ \\
\hline $\begin{array}{l}1.2 \text { mediium term ( } 6 \text { months } \\
\text { to } 1 \text { year) }\end{array}$ & 1 & 48 & Risk Ratio (M-H, Fixed, 95\% CI) & $1.0[0.15,6.53]$ \\
\hline 2 Global state: 2. Relapse & 4 & & Risk Ratio (M-H, Fixed, 95\% CI) & Subtotals only \\
\hline 2.1 immediate ( 0 to 5 weeks) & 2 & 44 & Risk Ratio (M-H, Fixed, 95\% CI) & $0.66[0.18,2.43]$ \\
\hline $\begin{array}{l}2.2 \text { short term ( } 6 \text { weeks to } 5 \\
\text { months) }\end{array}$ & 1 & 30 & Risk Ratio (M-H, Fixed, 95\% CI) & $2.29[0.70,7.48]$ \\
\hline $\begin{array}{l}2.3 \text { medium term ( } 6 \text { months } \\
\text { to } 1 \text { year) }\end{array}$ & 1 & 49 & Risk Ratio (M-H, Fixed, 95\% CI) & $2.43[0.71,8.32]$ \\
\hline $\begin{array}{l}3 \text { Behavioiur: Leaving the study } \\
\text { early }\end{array}$ & 5 & & Risk Ratio (M-H, Fixed, 95\% CI) & Subtotals only \\
\hline 3.1 immediate ( 0 to 5 weeks) & 2 & 44 & Risk Ratio (M-H, Fixed, 95\% CI) & $0.66[0.18,2.43]$ \\
\hline $\begin{array}{l}3.2 \text { short term ( } 6 \text { weeks to } 5 \\
\text { months) }\end{array}$ & 2 & 42 & Risk Ratio (M-H, Fixed, 95\% CI) & $2.29[0.70,7.48]$ \\
\hline $\begin{array}{l}3.3 \text { medium term ( } 6 \text { months } \\
\text { to } 1 \text { year) }\end{array}$ & 1 & 49 & Risk Ratio (M-H, Fixed, 95\% CI) & $2.43[0.71,8.32]$ \\
\hline $\begin{array}{l}4 \text { Mental State: BPRS medium } \\
\text { term }(6 \text { months to } 1 \text { year }- \text { high } \\
\text { score }=\text { poor })\end{array}$ & 1 & 39 & Mean Difference (IV, Fixed, 95\% CI) & $0.0[-3.93,3.93]$ \\
\hline $\begin{array}{l}5 \text { Adverse effects: 1a. Movement } \\
\text { disorders - general }\end{array}$ & 3 & & Risk Ratio (M-H, Fixed, 95\% CI) & Subtotals only \\
\hline 5.1 Immediate (0 to 5 weeks) & 1 & 49 & Risk Ratio (M-H, Fixed, 95\% CI) & $2.65[0.82,8.64]$ \\
\hline $\begin{array}{l}5.2 \text { short term ( } 6 \text { weeks to } 5 \\
\text { months) }\end{array}$ & 2 & 49 & Risk Ratio (M-H, Fixed, 95\% CI) & $1.14[0.79,1.64]$ \\
\hline $\begin{array}{l}6 \text { Adverse effects: } 1 \mathrm{~b} \text {. Movement } \\
\text { disorders - needing } \\
\text { anticholinergic drugs }\end{array}$ & 4 & & Risk Ratio (M-H, Fixed, 95\% CI) & Subtotals only \\
\hline 6.1 immediate ( 0 to 5 weeks) & 1 & 33 & Risk Ratio (M-H, Fixed, 95\% CI) & $0.29[0.12,0.70]$ \\
\hline
\end{tabular}

Fluphenazine decanoate (depot) and enanthate for schizophrenia (Review)

Copyright @ 2016 The Cochrane Collaboration. Published by John Wiley \& Sons, Ltd. 
6.2 short term (6 weeks to 5 months)

6.3 medium term (6 months to 1 year)

7 Adverse effects: 1c. Movement disorders - parkinsonism (short term - 6 weeks to 5 months)

8 Adverse effects: $1 \mathrm{~d}$. Movement disorders - akathisia (Immediate - 0 to 5 weeks)

9 Adverse effects: 2. General adverse effects (immediate - 0 to 5 weeks)

\section{Comparison 11. FLUPHENAZINE DECANOATE - DOSAGE STUDIES - 2 WEEKS vs 6 WEEKS}

\begin{tabular}{|c|c|c|c|c|}
\hline Outcome or subgroup title & $\begin{array}{l}\text { No. of } \\
\text { studies }\end{array}$ & $\begin{array}{c}\text { No. of } \\
\text { participants }\end{array}$ & Statistical method & Effect size \\
\hline 1 Global state: 1. Relapse (1 year) & 1 & 37 & Risk Ratio (M-H, Random, 95\% CI) & $0.89[0.55,1.44]$ \\
\hline 2 Leaving the study early (1 year) & 1 & 50 & Risk Ratio (M-H, Fixed, 95\% CI) & $1.17[0.46,2.98]$ \\
\hline $\begin{array}{l}3 \text { Mental state: } 1 . \text { BPRS - endpoint } \\
\text { scores (1 year) (high score }= \\
\text { poor) }\end{array}$ & 1 & & Mean Difference (Fixed, 95\% CI) & Subtotals only \\
\hline 3.1 Total & 1 & 37 & Mean Difference (Fixed, 95\% CI) & $2.72[-1.16,6.60]$ \\
\hline 3.2 Thought disorder & 1 & 37 & Mean Difference (Fixed, 95\% CI) & $-0.39[-1.51,0.73]$ \\
\hline $\begin{array}{l}4 \text { Adverse effects: } 1 . \text { Movement } \\
\text { disorders - MPRC (1 year, high } \\
\text { = poor) }\end{array}$ & 1 & & Mean Difference (Fixed, 95\% CI) & Subtotals only \\
\hline 4.1 Parkinsonian symptoms & 1 & 37 & Mean Difference (Fixed, 95\% CI) & $1.3[-0.03,2.63]$ \\
\hline 4.2 Dyskinesia & 1 & 37 & Mean Difference (Fixed, 95\% CI) & $2.4[-1.77,6.57]$ \\
\hline $\begin{array}{l}5 \text { Quality of life: Quality of life } \\
\text { scale (1 year) (high score = } \\
\text { good) }\end{array}$ & 1 & 37 & Mean Difference (Fixed, 95\% CI) & $1.42[-9.68,12.52]$ \\
\hline
\end{tabular}




\section{Analysis I.I. Comparison I fLUPHENAZINE DECANOATE vs PLACEBO, Outcome I Death.}

Review: Fluphenazine decanoate (depot) and enanthate for schizophrenia

Comparison: I FLUPHENAZINE DECANOATE vs PLACEBO

Outcome: I Death

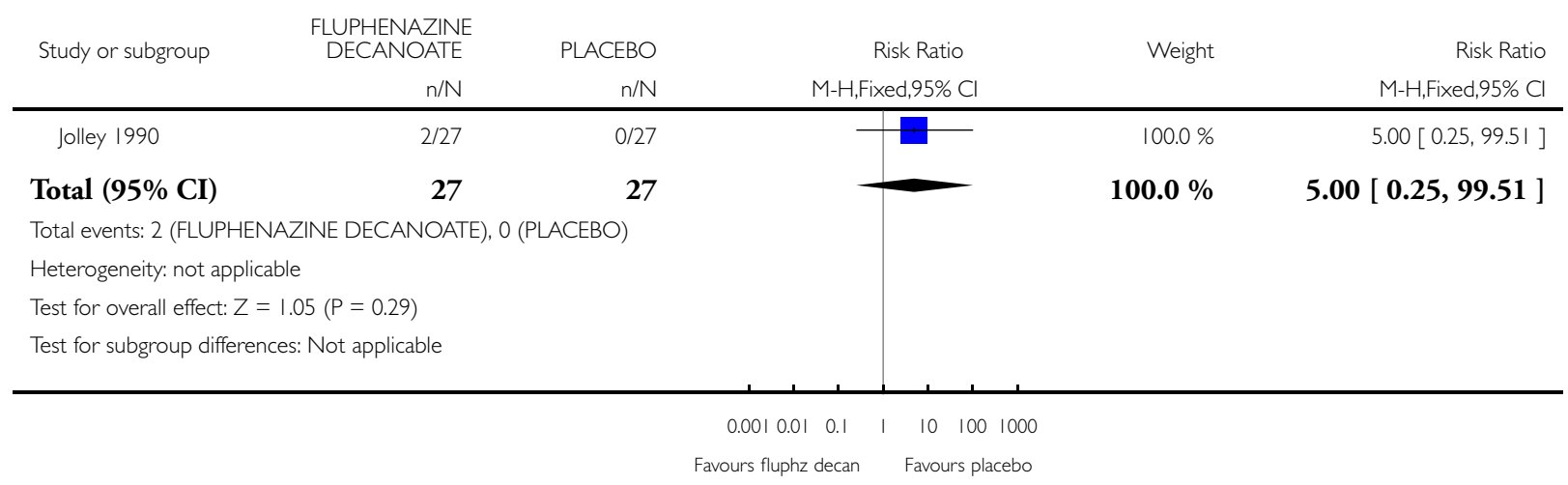




\section{Analysis I.2. Comparison I FLUPHENAZINE DECANOATE vs PLACEBO, Outcome 2 Global state: I. Relapse.}

\begin{tabular}{|c|c|c|c|c|c|}
\hline \multicolumn{6}{|c|}{ Comparison: I FLUPHENAZINE DECANOATE vs PLACEBO } \\
\hline \multicolumn{6}{|c|}{ Outcome: 2 Global state: I. Relapse } \\
\hline Study or subgroup & $\begin{array}{l}\text { FLUPHENAZINE } \\
\text { DECANOATE }\end{array}$ & $\begin{array}{r}\text { PLACEBO } \\
n / N \\
\end{array}$ & $\begin{array}{c}\text { Risk Ratio } \\
\text { M- } \\
\text { H,Random,95\% } \\
\text { Cl }\end{array}$ & Weight & $\begin{array}{c}\text { Risk Ratio } \\
\text { M- } \\
\text { H,Random,95\% } \\
\text { Cl }\end{array}$ \\
\hline \multicolumn{6}{|c|}{ I short term ( 6 weeks to 5 months) } \\
\hline Shenoy |981 & $0 / 14$ & $0 / 17$ & & & Not estimable \\
\hline Subtotal $(95 \% \mathrm{CI})$ & 14 & 17 & & & Not estimable \\
\hline \multicolumn{6}{|c|}{ Total events: 0 (FLUPHENAZINE DECANOATE), 0 (PLACEBO) } \\
\hline \multicolumn{6}{|c|}{ Heterogeneity: not applicable } \\
\hline \multicolumn{6}{|c|}{ Test for overall effect: not applicable } \\
\hline \multicolumn{6}{|c|}{2 medium term ( 6 months to I year) } \\
\hline Hirsch 1975 & $7 / 40$ & $28 / 41$ & $\longrightarrow$ & $30.6 \%$ & $0.26[0.13,0.52]$ \\
\hline Odejide 1982 & $15 / 35$ & $23 / 35$ & $\rightarrow$ & $34.0 \%$ & $0.65[0.42,1.02]$ \\
\hline Rifkin 1977 & $20 / 23$ & $15 / 22$ & + & $35.3 \%$ & $1.28[0.92,1.77]$ \\
\hline Subtotal $(95 \% \mathrm{CI})$ & 98 & 98 & - & $100.0 \%$ & $0.62[0.24,1.60]$ \\
\hline \multicolumn{6}{|c|}{ Total events: 42 (FLUPHENAZINE DECANOATE), 66 (PLACEBO) } \\
\hline \multicolumn{6}{|c|}{ Heterogeneity: $\mathrm{Tau}^{2}=0.63 ; \mathrm{Chi}^{2}=24.16, \mathrm{df}=2(\mathrm{P}<0.0000 \mathrm{I}) ; \mathrm{I}^{2}=92 \%$} \\
\hline \multicolumn{6}{|c|}{ Test for overall effect: $Z=0.99(P=0.32)$} \\
\hline \multicolumn{6}{|c|}{3 longer term (more than I year) } \\
\hline Jolley 1990 & $8 / 27$ & 23/27 & - & $100.0 \%$ & $0.35[0.19,0.64]$ \\
\hline Subtotal $(95 \% \mathrm{CI})$ & 27 & 27 & & $100.0 \%$ & $0.35[0.19,0.64]$ \\
\hline \multicolumn{6}{|c|}{ Total events: 8 (FLUPHENAZINE DECANOATE), 23 (PLACEBO) } \\
\hline \multicolumn{6}{|c|}{ Heterogeneity: not applicable } \\
\hline Test for overall effect: $Z=$ & $=0.00059)$ & & & & \\
\hline
\end{tabular}


Analysis I.3. Comparison I FLUPHENAZINE DECANOATE vs PLACEBO, Outcome 3 Global state: 2. GAS (short term - 6 weeks to 5 months) (high score $=$ worse).

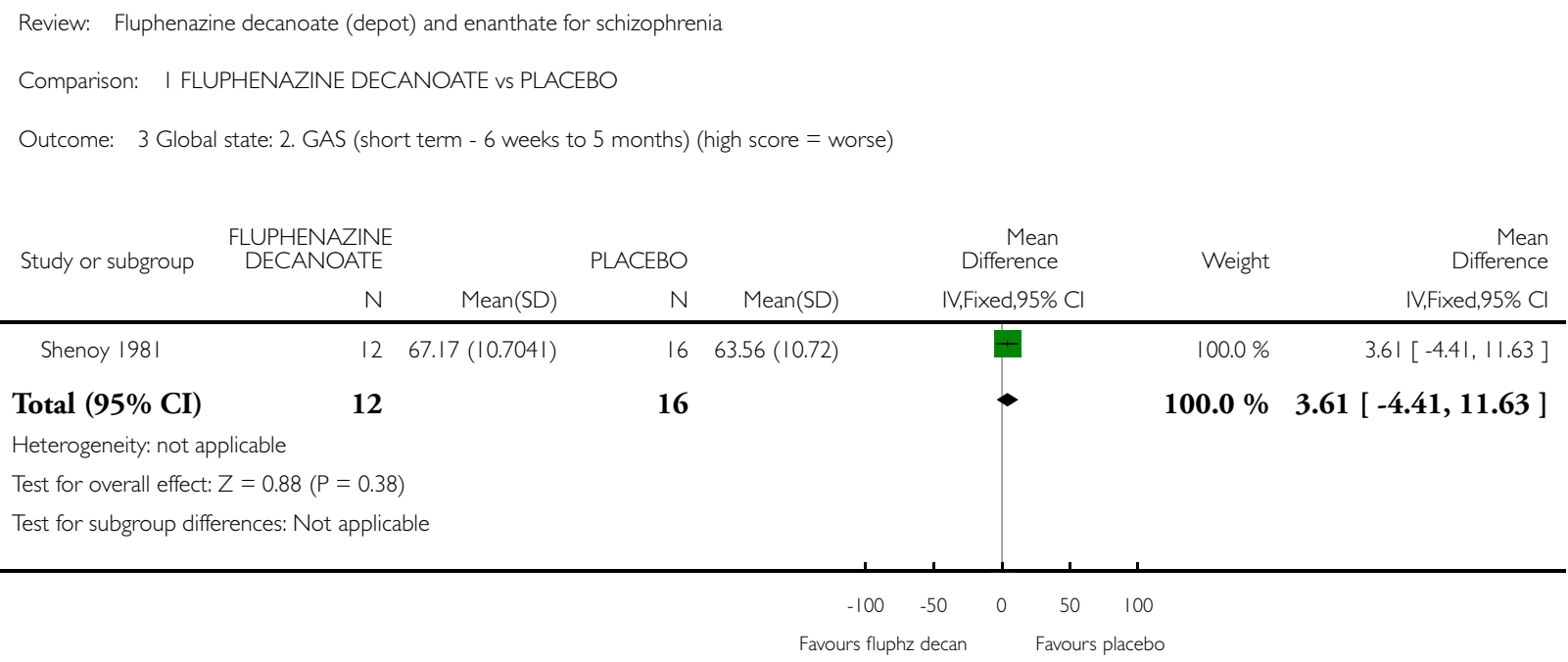


Analysis I.4. Comparison I FLUPHENAZINE DECANOATE vs PLACEBO, Outcome 4 Leaving the study early.

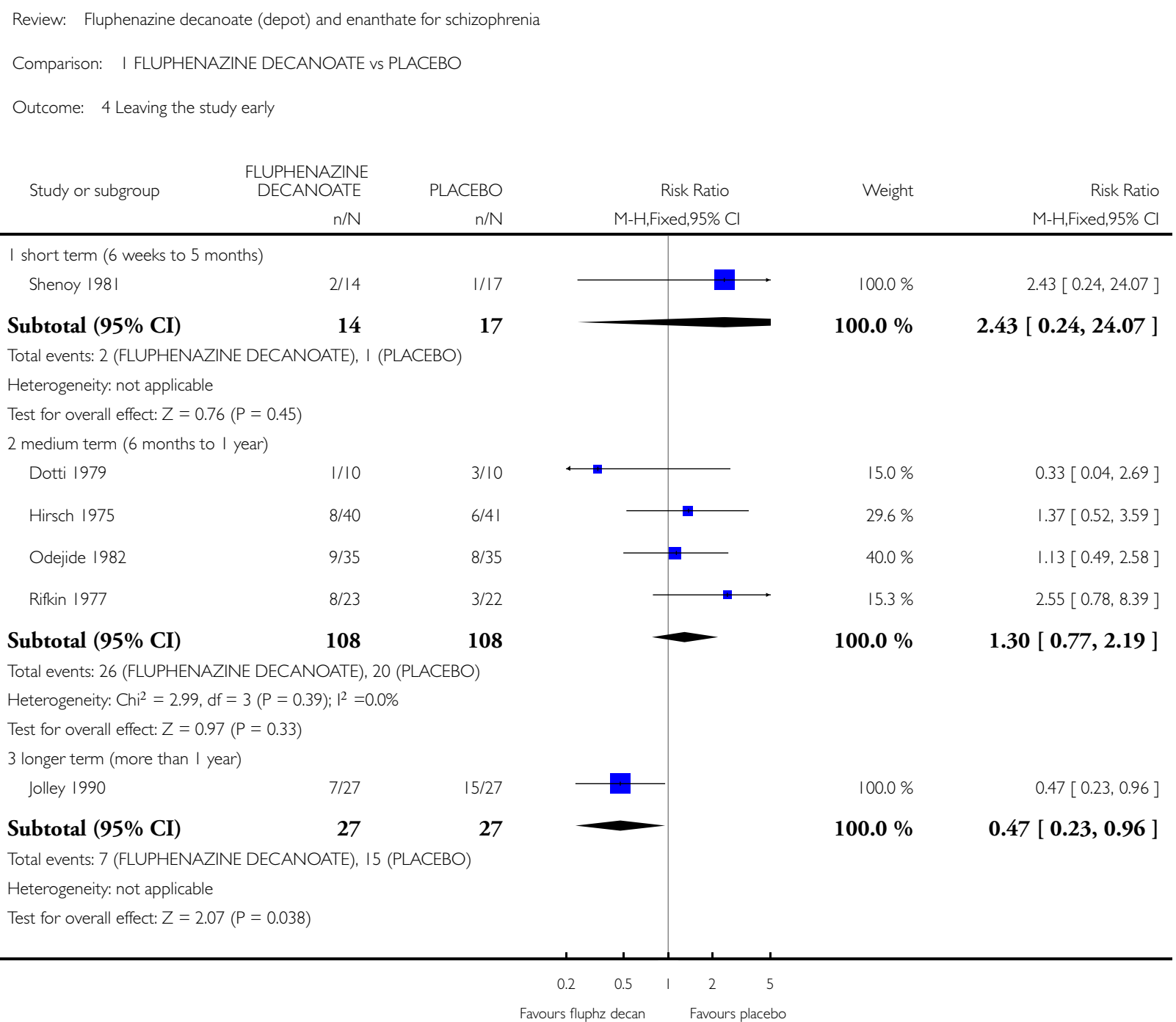


Analysis I.5. Comparison I FLUPHENAZINE DECANOATE vs PLACEBO, Outcome 5 Mental state: I. BPRS (endpoint scores - high score $=$ worse).

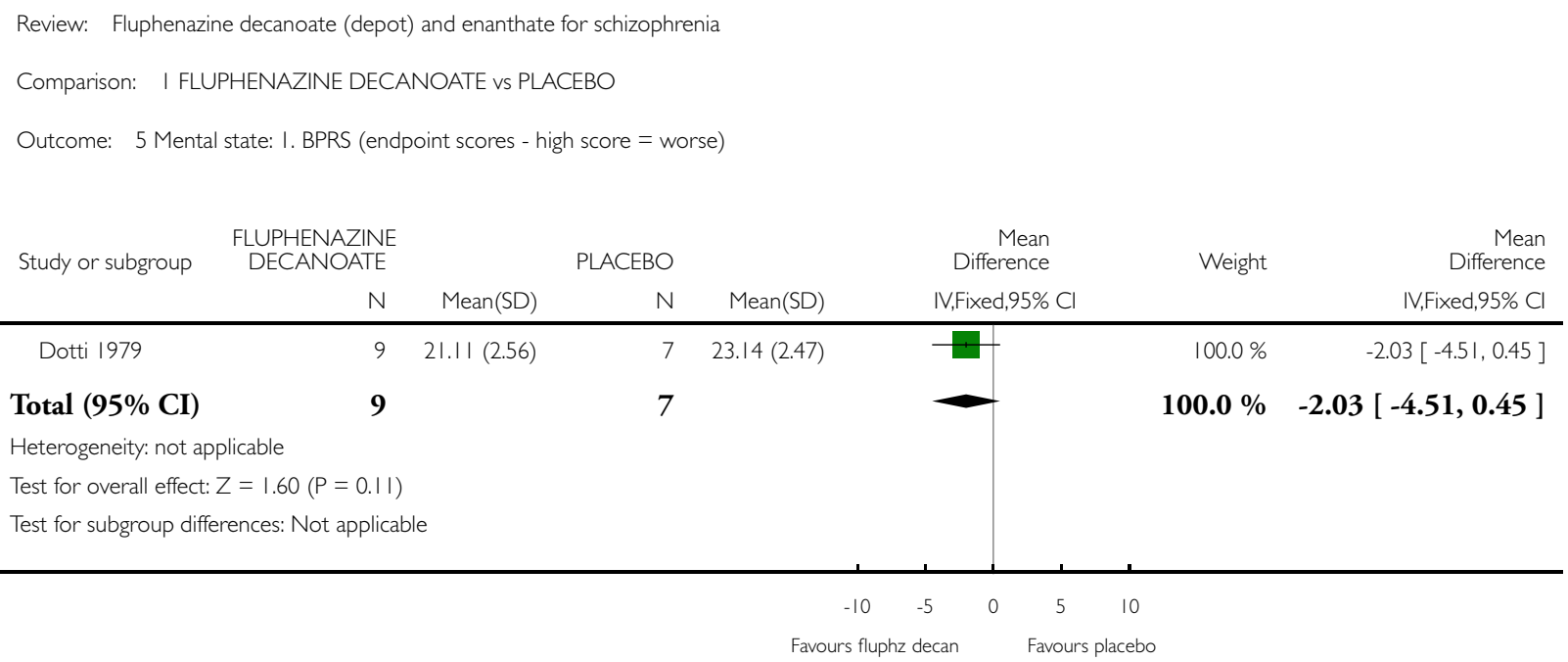

\section{Analysis I.6. Comparison I fLUPHENAZINE DECANOATE vs PLACEBO, Outcome 6 Mental state: 2. Depression (medium term - 6 months to I year).}

Review: Fluphenazine decanoate (depot) and enanthate for schizophrenia
Comparison: I FLUPHENAZINE DECANOATE vs PLACEBO
Outcome: 6 Mental state: 2. Depression (medium term - 6 months to I year)

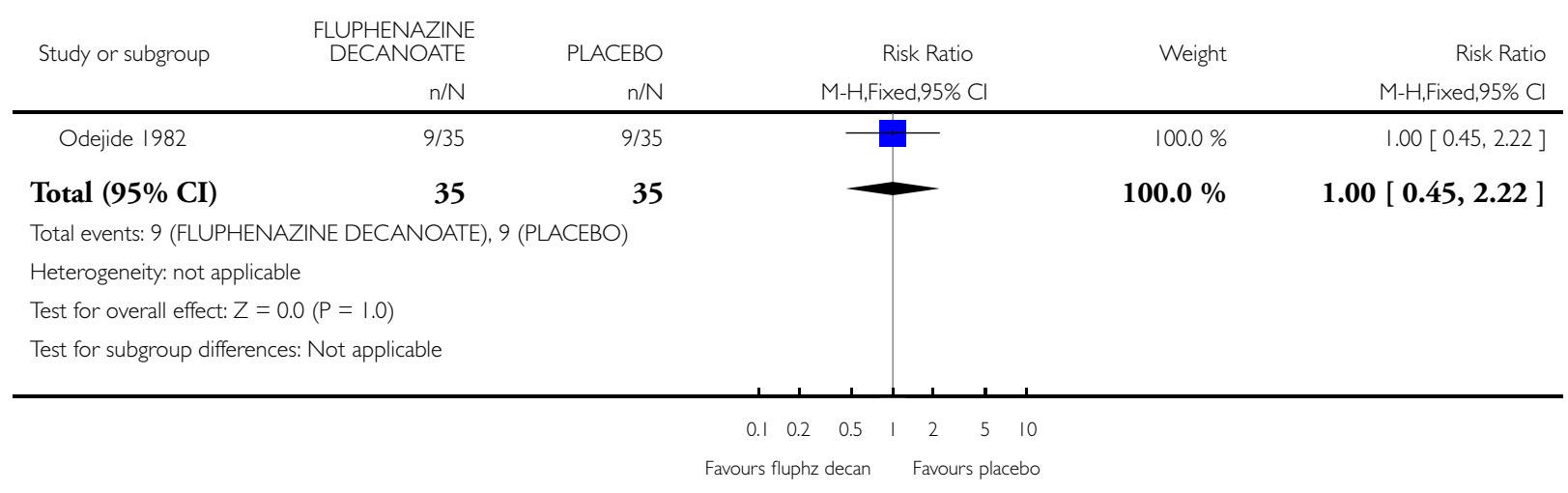


Analysis I.7. Comparison I FLUPHENAZINE DECANOATE vs PLACEBO, Outcome 7 Adverse effects: I. Movement disorders - tardive dyskinesia (longer term - more than I year).

Review: Fluphenazine decanoate (depot) and enanthate for schizophrenia

Comparison: I FLUPHENAZINE DECANOATE vs PLACEBO

Outcome: 7 Adverse effects: I. Movement disorders - tardive dyskinesia (longer term - more than I year)

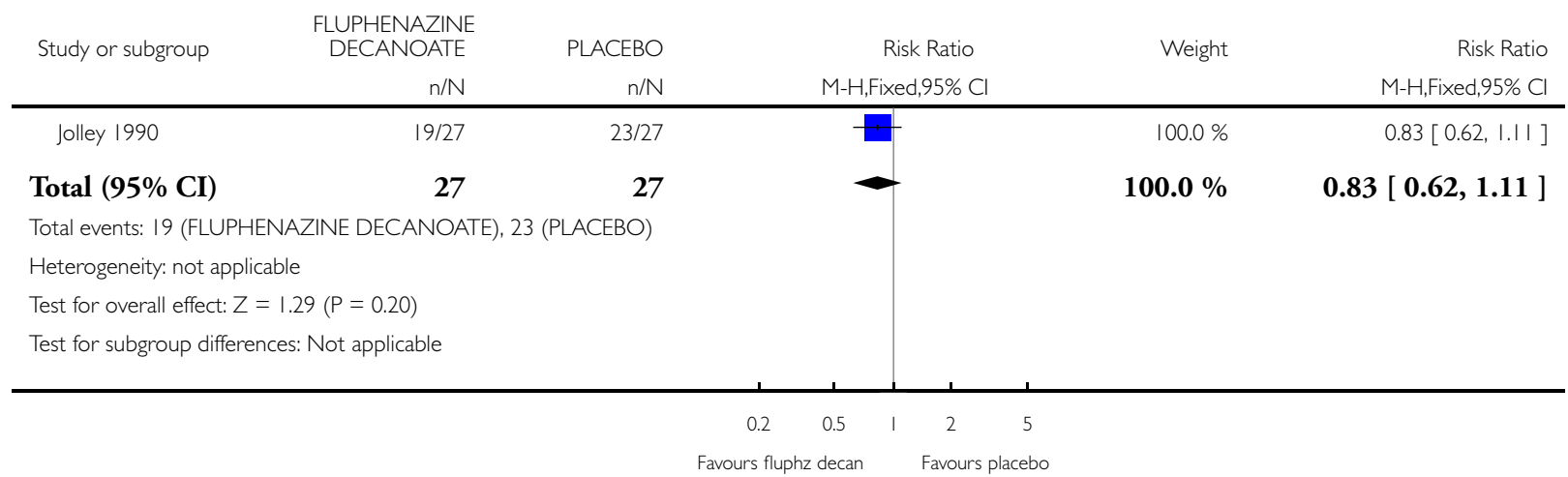

\section{Analysis I.8. Comparison I FLUPHENAZINE DECANOATE vs PLACEBO, Outcome 8 Adverse effects: 2.} Toxicity.

Review: Fluphenazine decanoate (depot) and enanthate for schizophrenia

Comparison: I FLUPHENAZINE DECANOATE vs PLACEBO

Outcome: 8 Adverse effects: 2. Toxicity

\begin{tabular}{|c|c|c|c|c|c|}
\hline Study or subgroup & $\begin{array}{l}\text { FLUPHENAZINE } \\
\text { DECANOATE }\end{array}$ & PLACEBO & Risk Ratio & Weight & Risk Ratio \\
\hline & $n / N$ & $\mathrm{n} / \mathrm{N}$ & M-H,Fixed,95\% Cl & & M-H,Fixed,95\% Cl \\
\hline Rifkin 1977 & $8 / 23$ & $1 / 22$ & W & $100.0 \%$ & $7.65[1.04,56.26]$ \\
\hline Total (95\% CI) & 23 & 22 & $\longrightarrow$ & $100.0 \%$ & {$[1.04,56.26]$} \\
\hline
\end{tabular}

Total events: 8 (FLUPHENAZINE DECANOATE), I (PLACEBO)

Heterogeneity: not applicable

Test for overall effect: $Z=2.00(P=0.046)$

Test for subgroup differences: Not applicable 
Analysis 2.I. Comparison 2 fLUPHENAZINE DECANOATE vs ORAL NEUROLEPTICS, Outcome I Global state: I. No clinically important global change.

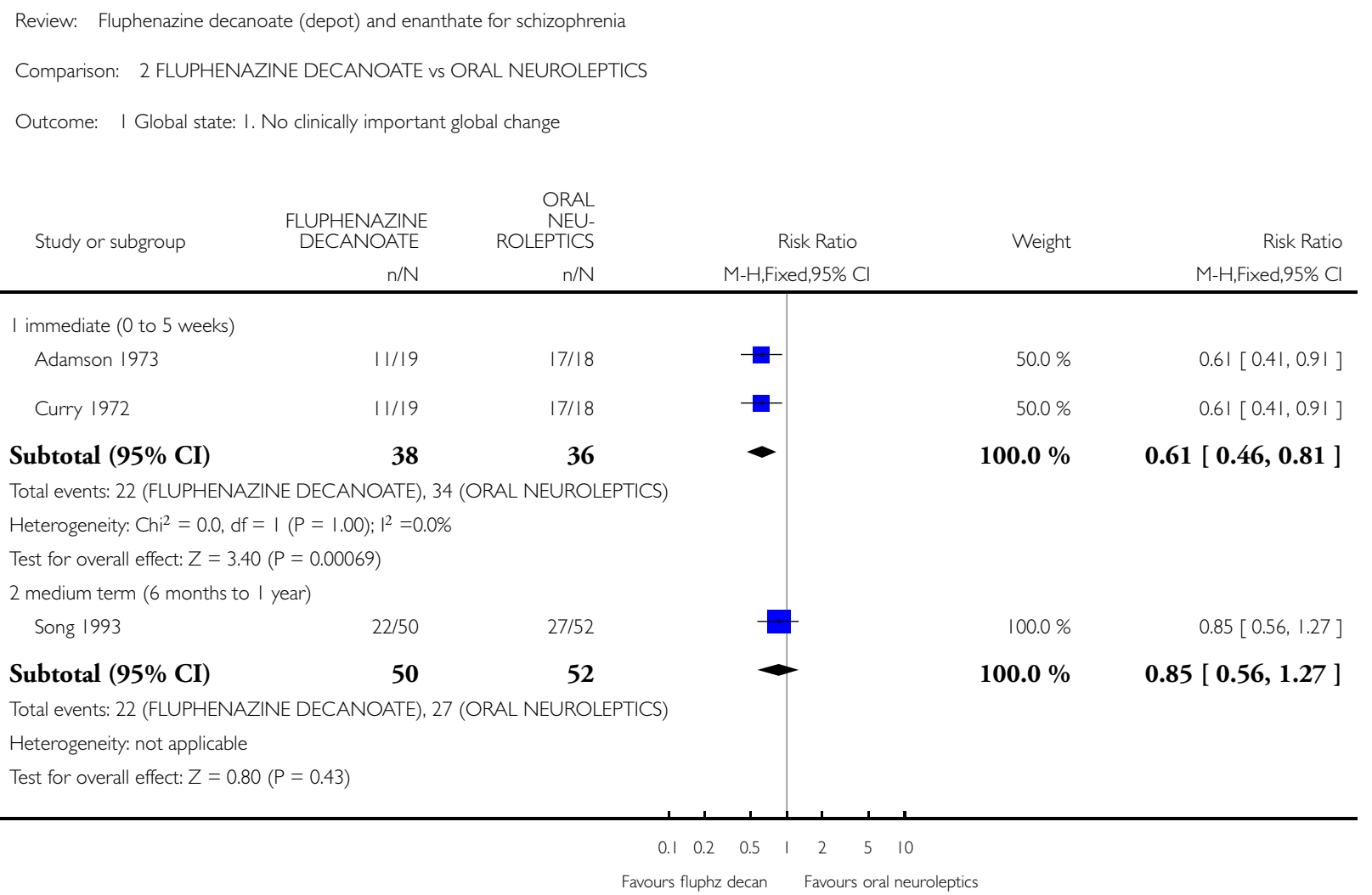


Analysis 2.2. Comparison 2 FLUPHENAZINE DECANOATE vs ORAL NEUROLEPTICS, Outcome 2 Global state: 2. Relapse.

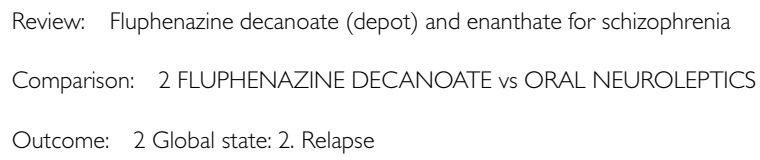

$211 \quad 208$

208

Total events: 103 (FLUPHENAZINE DECANOATE), 88 (ORAL NEUROLEPTICS)

Heterogeneity: Tau $^{2}=0.46 ; \mathrm{Chi}^{2}=20.42, \mathrm{df}=5(\mathrm{P}=0.00 \mathrm{I}) ; \mathrm{I}^{2}=76 \%$

Test for overall effect: $Z=1.12(P=0.26)$

2 longer term (more than I year)

$\begin{array}{lcc}\text { Falloon } 1978 & 8 / 20 & 5 / 24 \\ \text { Hogarty } 1979 & 13 / 27 & 13 / 25 \\ \text { Simon } 1978 & 14 / 57 & 10 / 63 \\ \text { M } & \mathbf{1 0 4} & \mathbf{1 1 2}\end{array}$

Subtotal (95\% CI)

104

112

$\begin{array}{ll}19.6 \% & 1.92[0.74,4.95] \\ 49.4 \% & 0.93[0.54,1.59] \\ 31.0 \% & 1.55[0.75,3.20]\end{array}$

Total events: 35 (FLUPHENAZINE DECANOATE), 28 (ORAL NEUROLEPTICS)

Heterogeneity: $\mathrm{Tau}^{2}=0.03 ; \mathrm{Chi}^{2}=2.38, \mathrm{df}=2(\mathrm{P}=0.30) ; \mathrm{I}^{2}=16 \%$

Test for overall effect: $Z=1.00(P=0.32)$

Test for subgroup differences: $C^{2} i^{2}=0.14, d f=I(P=0.7 I), I^{2}=0.0 \%$

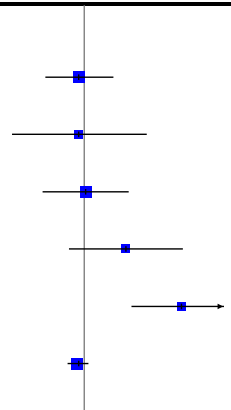

$\begin{array}{ll}19.2 \% & 0.89[0.43,1.86] \\ 11.3 \% & 0.89[0.21,3.80] \\ 16.8 \% & 1.04[0.41,2.62] \\ 13.5 \% & 2.48[0.73,8.40] \\ 15.0 \% & 8.12[2.75,23.92] \\ 24.2 \% & 0.88[0.70,1.11]\end{array}$

$100.0 \%$

$1.46[0.75,2.83]$

$100.0 \%$ 
Analysis 2.3. Comparison 2 FLUPHENAZINE DECANOATE vs ORAL NEUROLEPTICS, Outcome 3 Global state: 3. Clinical Global Impression (short term - 6 weeks to 5 months) (high score = worse).

Review: Fluphenazine decanoate (depot) and enanthate for schizophrenia

Comparison: 2 FLUPHENAZINE DECANOATE vs ORAL NEUROLEPTICS

Outcome: 3 Global state: 3 . Clinical Global Impression (short term - 6 weeks to 5 months) (high score $=$ worse)

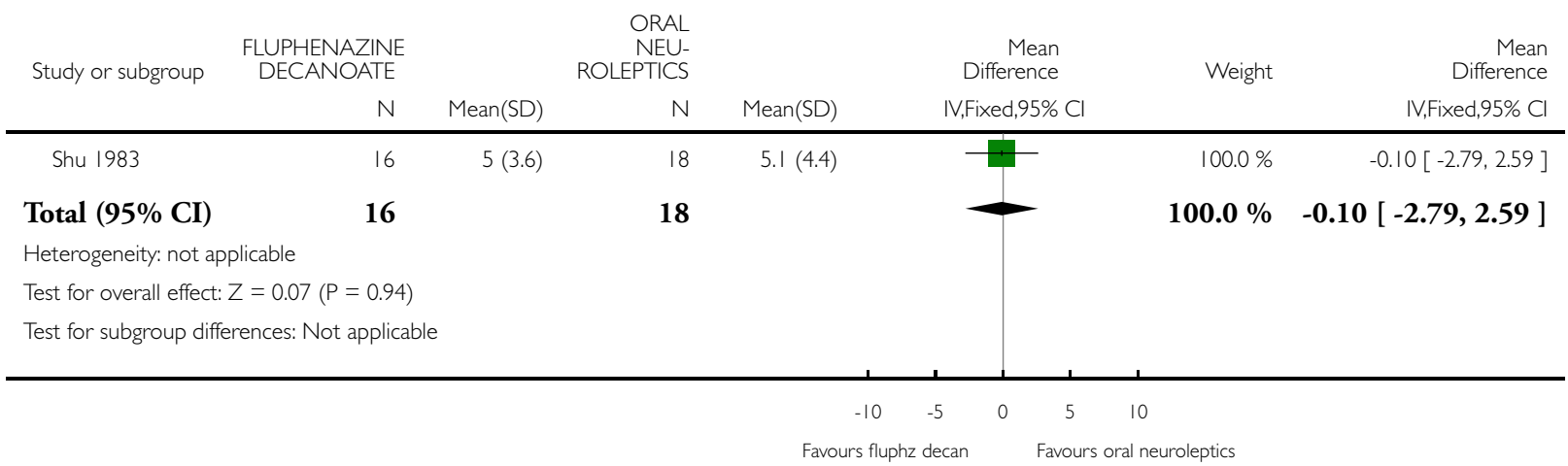




\section{Analysis 2.4. Comparison 2 FLUPHENAZINE DECANOATE vs ORAL NEUROLEPTICS, Outcome 4 Leaving the study early.}

Review: Fluphenazine decanoate (depot) and enanthate for schizophrenia

Comparison: 2 FLUPHENAZINE DECANOATE vs ORAL NEUROLEPTICS

Outcome: 4 Leaving the study early

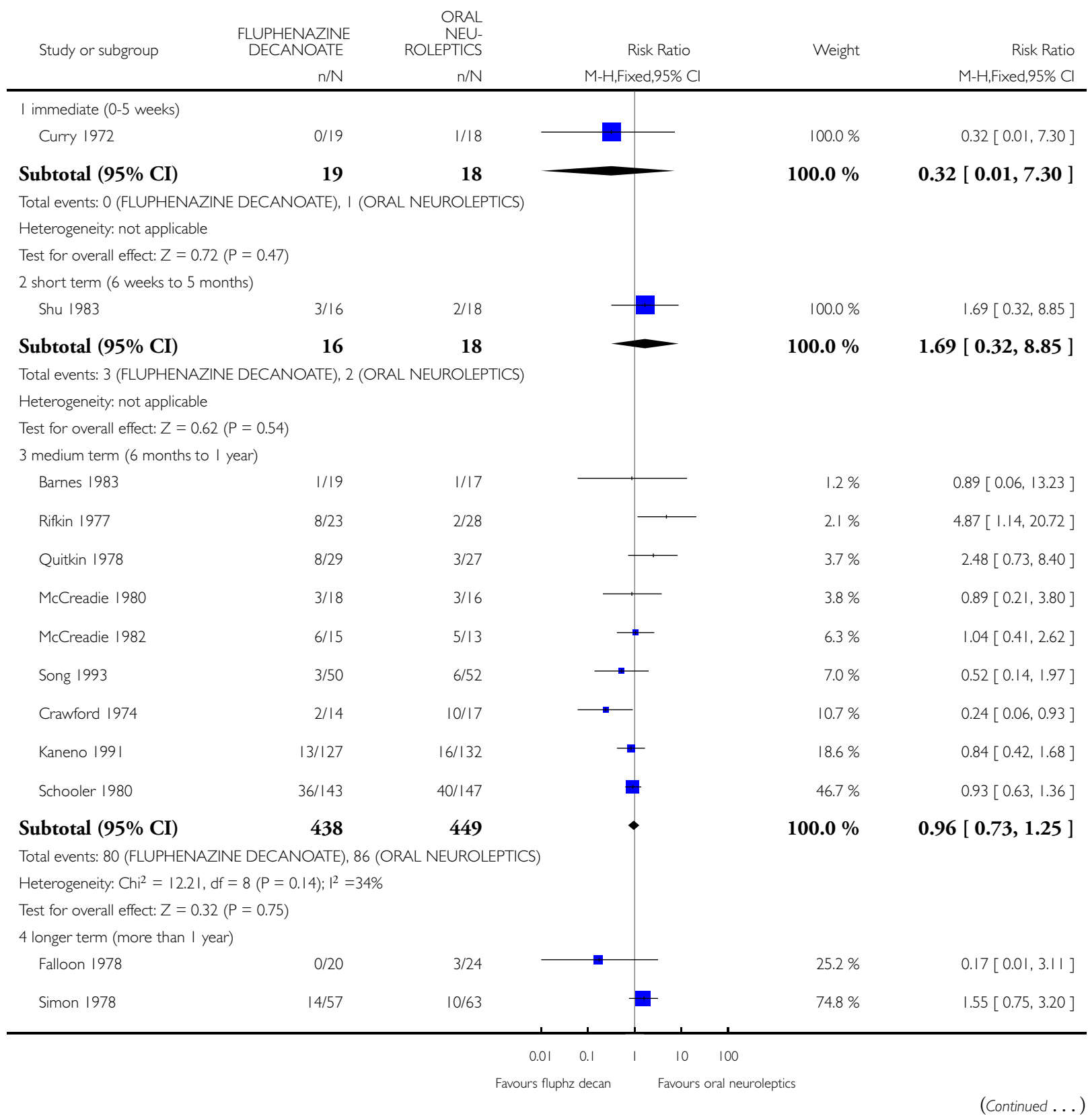

Fluphenazine decanoate (depot) and enanthate for schizophrenia (Review)

Copyright (c) 2016 The Cochrane Collaboration. Published by John Wiley \& Sons, Ltd. 


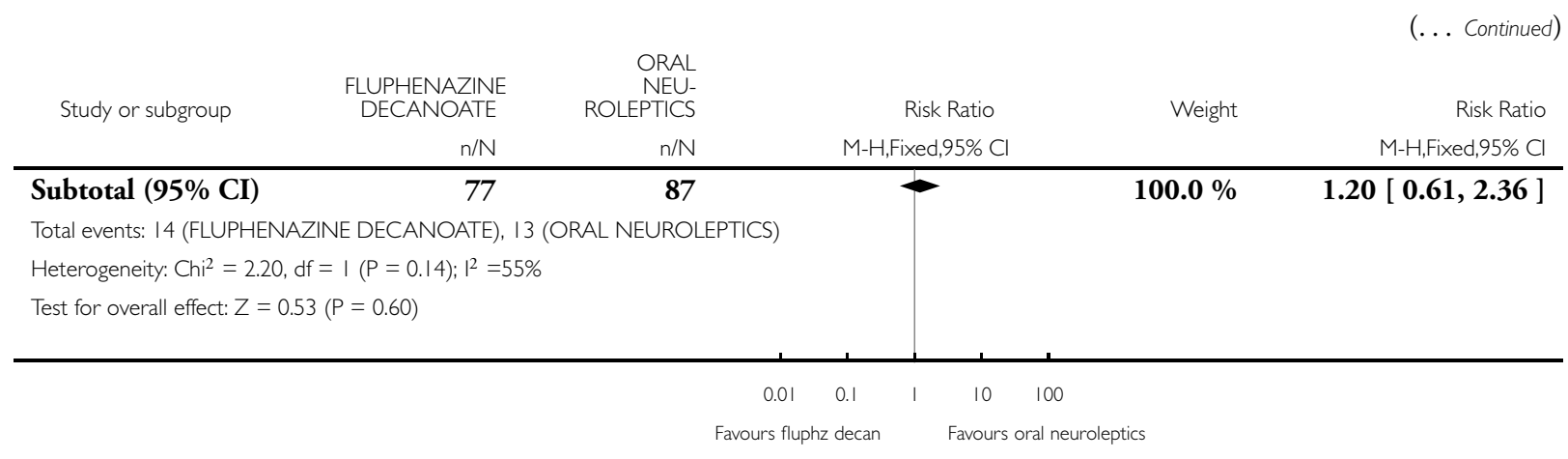

\section{Analysis 2.5. Comparison 2 FLUPHENAZINE DECANOATE vs ORAL NEUROLEPTICS, Outcome 5 Behaviour: I. NOSIE-30 - endpoint scores (high score = poor).}

Review: Fluphenazine decanoate (depot) and enanthate for schizophrenia

Comparison: 2 FLUPHENAZINE DECANOATE vs ORAL NEUROLEPTICS

Outcome: 5 Behaviour: I. NOSIE-30 - endpoint scores (high score = poor)

\begin{tabular}{|c|c|c|c|c|c|c|c|}
\hline \multirow[t]{2}{*}{ Study or subgroup } & $\begin{array}{l}\text { FLUPHENAZINE } \\
\text { DECANOATE }\end{array}$ & & $\begin{array}{r}\text { ORAL } \\
\text { NEU- } \\
\text { ROLEPTICS }\end{array}$ & & \multirow{2}{*}{$\begin{array}{c}\text { Mean } \\
\text { Difference } \\
\text { IV,Fixed,95\% Cl }\end{array}$} & \multirow[t]{2}{*}{ Weight } & \multirow{2}{*}{$\begin{array}{r}\text { Mean } \\
\text { Difference } \\
\text { IV,Fixed,95\% C }\end{array}$} \\
\hline & $N$ & Mean(SD) & $N$ & Mean(SD) & & & \\
\hline Simon 1978 & 57 & $152.25(\mid 6.97)$ & 63 & $|52.8|(\mid 8.55)$ & & $100.0 \%$ & $-0.56[-6.92,5.80]$ \\
\hline Total (95\% CI) & 57 & & 63 & & $\varphi$ & $100.0 \%$ & $-0.56[-6.92,5.80]$ \\
\hline \multicolumn{8}{|c|}{ Heterogeneity: not applicable } \\
\hline \multicolumn{8}{|c|}{ Test for overall effect: $Z=0.17(P=0.86)$} \\
\hline \multicolumn{8}{|c|}{ Test for subgroup differences: Not applicable } \\
\hline & & & & -100 & -50 & 100 & \\
\hline \multicolumn{8}{|c|}{ Favours fluphz decan $\quad$ Favours oral neuroleptics } \\
\hline
\end{tabular}


Analysis 2.6. Comparison 2 FLUPHENAZINE DECANOATE vs ORAL NEUROLEPTICS, Outcome 6 Behaviour: 2. skewed data (endpoint scores).

Behaviour: 2. skewed data (endpoint scores)

\begin{tabular}{llllll}
\hline Study & Intervention & mean & SD & N \\
\hline Barnes 1983 & Fluphenazine decanoate & 5.7 & 4.1 & 19 \\
\hline Barnes 1983 & Pimozide & 4.2 & 5.5 & 17 \\
\hline
\end{tabular}

Analysis 2.7. Comparison 2 FLUPHENAZINE DECANOATE vs ORAL NEUROLEPTICS, Outcome 7 Mental state: I. BPRS - endpoint scores (longer term - more than I year) (high score = poor).

Review: Fluphenazine decanoate (depot) and enanthate for schizophrenia

Comparison: 2 FLUPHENAZINE DECANOATE vs ORAL NEUROLEPTICS

Outcome: 7 Mental state: I. BPRS - endpoint scores (longer term - more than I year) (high score = poor)

\begin{tabular}{|c|c|c|c|c|c|c|c|}
\hline \multirow[t]{2}{*}{ Study or subgroup } & $\begin{array}{c}\text { FLUPHENAZINE } \\
\text { DECANOATE }\end{array}$ & & $\begin{array}{r}\text { ORAL } \\
\text { NEU- } \\
\text { ROLEPTICS }\end{array}$ & & $\begin{array}{r}\text { Mean } \\
\text { Difference }\end{array}$ & \multirow[t]{2}{*}{ Weight } & \multirow{2}{*}{$\begin{array}{r}\text { Mean } \\
\text { Difference } \\
\text { IV,Fixed, } 95 \% \mathrm{Cl}\end{array}$} \\
\hline & $\mathrm{N}$ & Mean(SD) & $\mathrm{N}$ & Mean(SD) & IV,Fixed,95\% Cl & & \\
\hline Simon 1978 & 57 & $60.33(14.8)$ & 63 & $61.08(12.98)$ & & $100.0 \%$ & $-0.75[-5.75,4.25]$ \\
\hline Total (95\% CI) & 57 & & 63 & & & $100.0 \%$ & $-0.75[-5.75,4.25]$ \\
\hline \multicolumn{8}{|c|}{ Heterogeneity: not applicable } \\
\hline \multicolumn{8}{|c|}{ Test for overall effect: $Z=0.29(P=0.77)$} \\
\hline \multicolumn{8}{|c|}{ Test for subgroup differences: Not applicable } \\
\hline & & & & -10 & -5 & 10 & \\
\hline & & & & Favours flup & Favours & al neuroleptics & \\
\hline
\end{tabular}




\section{Analysis 2.8. Comparison 2 FLUPHENAZINE DECANOATE vs ORAL NEUROLEPTICS, Outcome 8}

Mental state: 2. Depression.

Review: Fluphenazine decanoate (depot) and enanthate for schizophrenia

Comparison: 2 FLUPHENAZINE DECANOATE vs ORAL NEUROLEPTICS

Outcome: 8 Mental state: 2. Depression

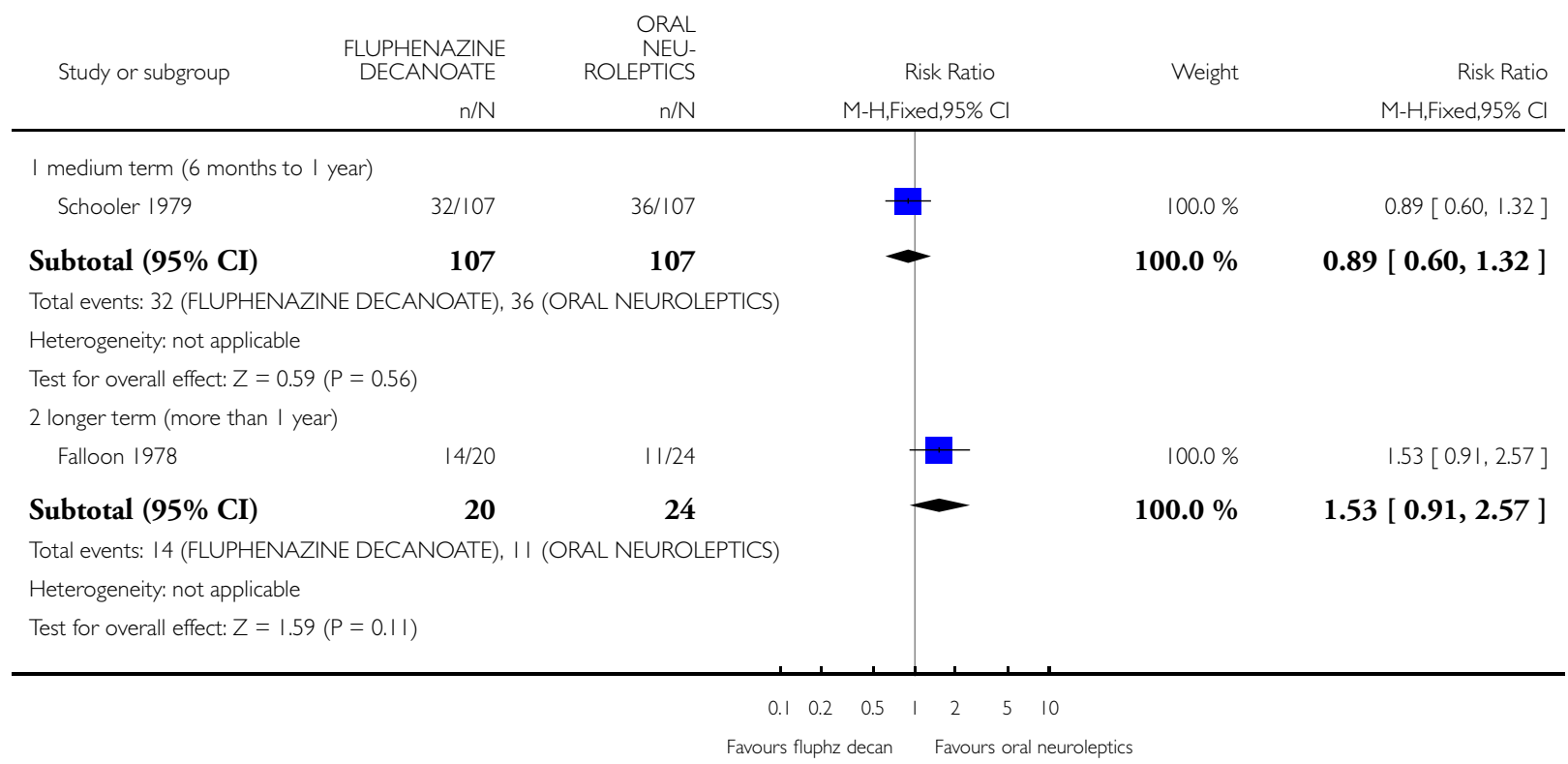


Analysis 2.9. Comparison 2 fLUPHENAZINE DECANOATE vs ORAL NEUROLEPTICS, Outcome 9 Adverse effects: Ia. Movement disorders - general.

Review: Fluphenazine decanoate (depot) and enanthate for schizophrenia

Comparison: 2 FLUPHENAZINE DECANOATE vs ORAL NEUROLEPTICS

Outcome: 9 Adverse effects: Ia. Movement disorders - general

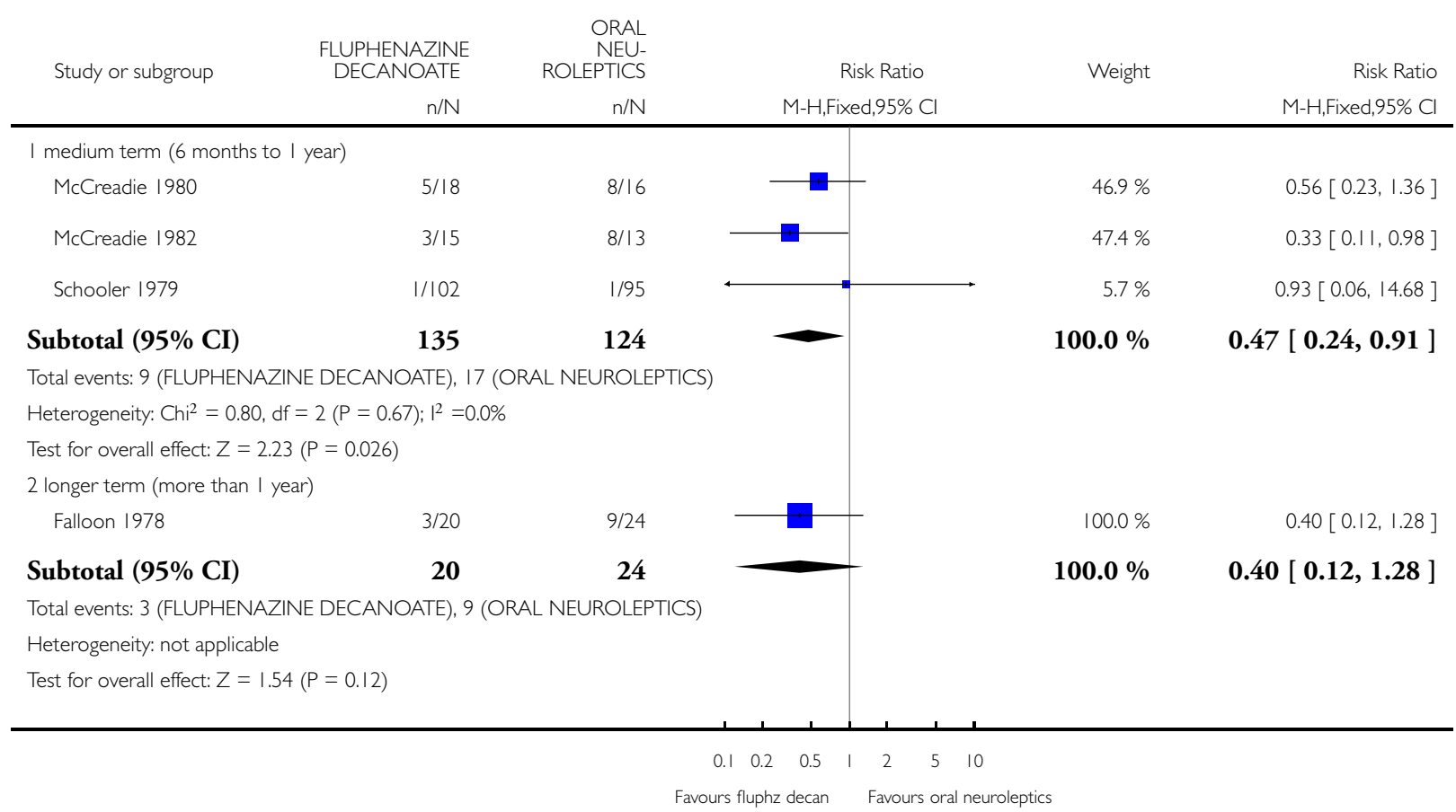




\section{Analysis 2.10. Comparison 2 FLUPHENAZINE DECANOATE vs ORAL NEUROLEPTICS, Outcome I0}

\section{Adverse effects: Ib. Movement disorders - akathisia.}

Review: Fluphenazine decanoate (depot) and enanthate for schizophrenia

Comparison: 2 FLUPHENAZINE DECANOATE vs ORAL NEUROLEPTICS

Outcome: 10 Adverse effects: Ib. Movement disorders - akathisia

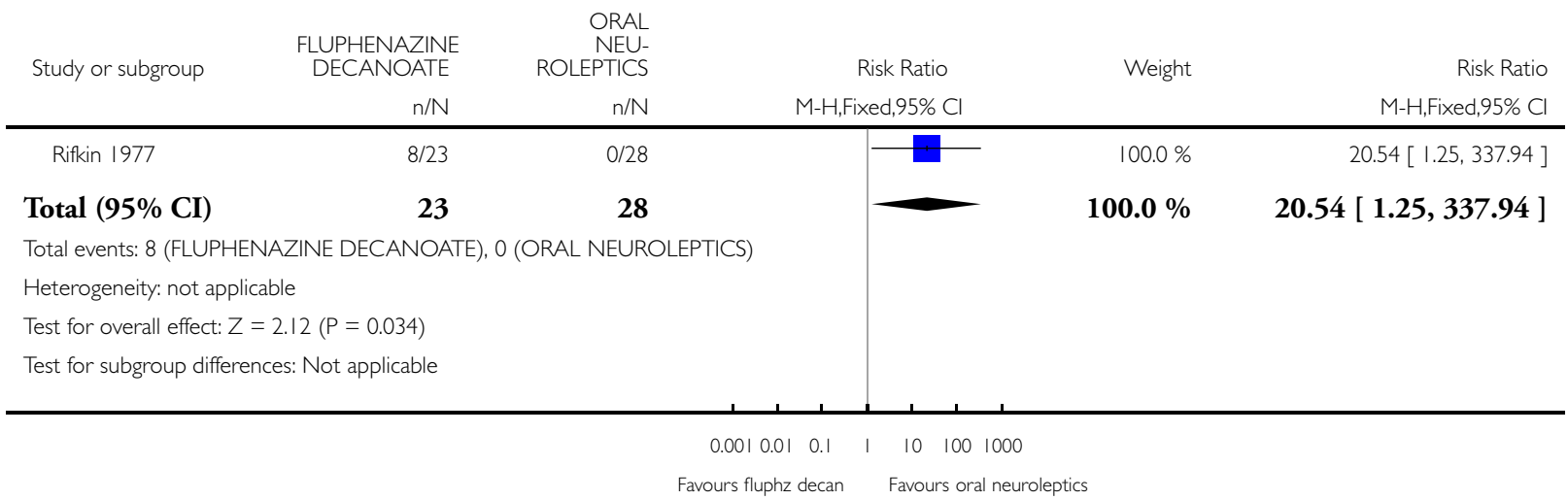


Analysis 2.1 I. Comparison 2 fLUPHENAZINE DECANOATE vs ORAL NEUROLEPTICS, Outcome I I Adverse effects: Ic. Movement disorders - needing anticholinergic drugs.

Review: Fluphenazine decanoate (depot) and enanthate for schizophrenia

Comparison: 2 FLUPHENAZINE DECANOATE vs ORAL NEUROLEPTICS

Outcome: II Adverse effects: Ic. Movement disorders - needing anticholinergic drugs

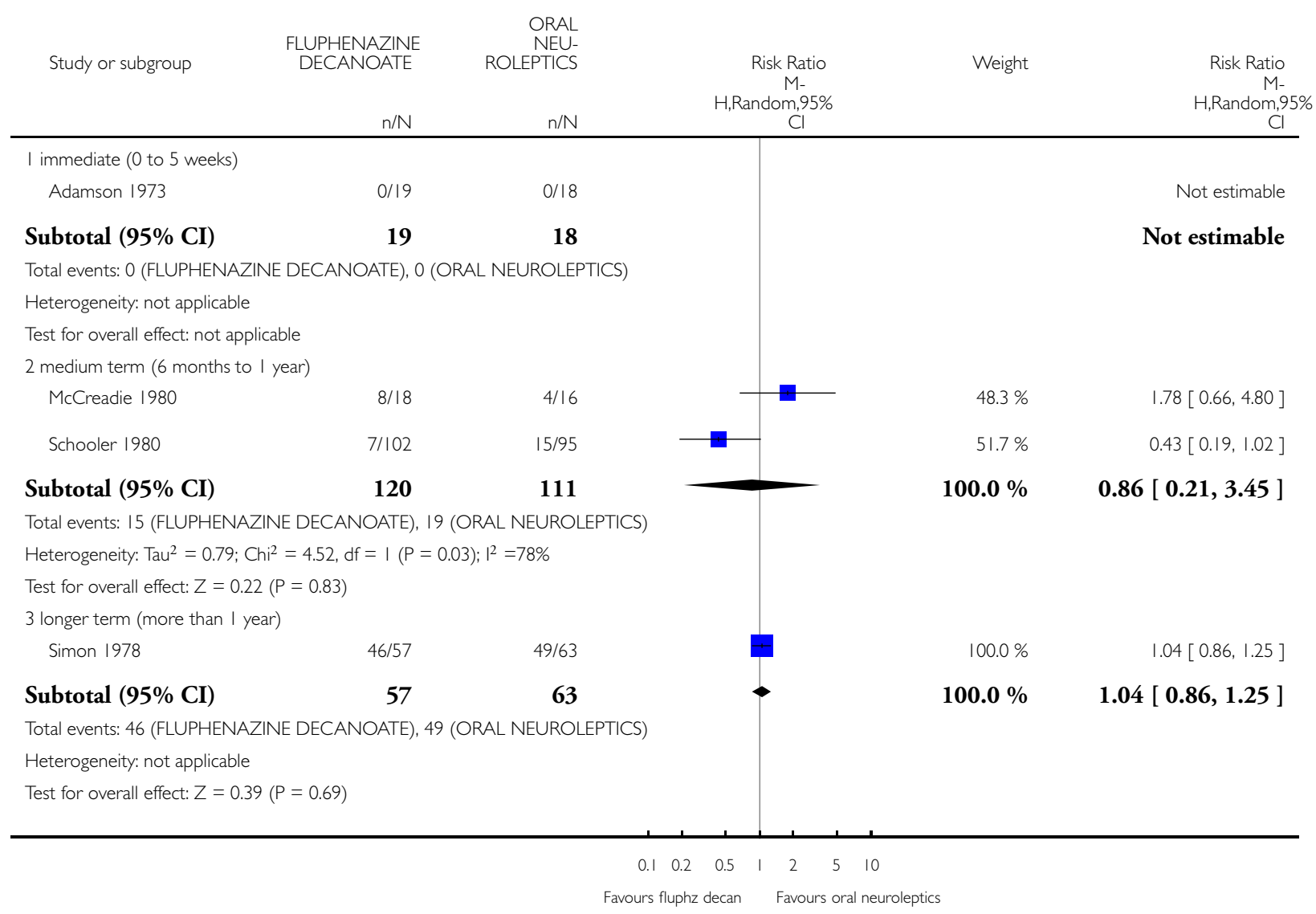


Analysis 2.12. Comparison 2 fLUPHENAZINE DECANOATE vs ORAL NEUROLEPTICS, Outcome I 2 Adverse effects: Id. Movement disorders - tardive dyskinesia.

Review: Fluphenazine decanoate (depot) and enanthate for schizophrenia

Comparison: 2 FLUPHENAZINE DECANOATE vs ORAL NEUROLEPTICS

Outcome: 12 Adverse effects: Id. Movement disorders - tardive dyskinesia

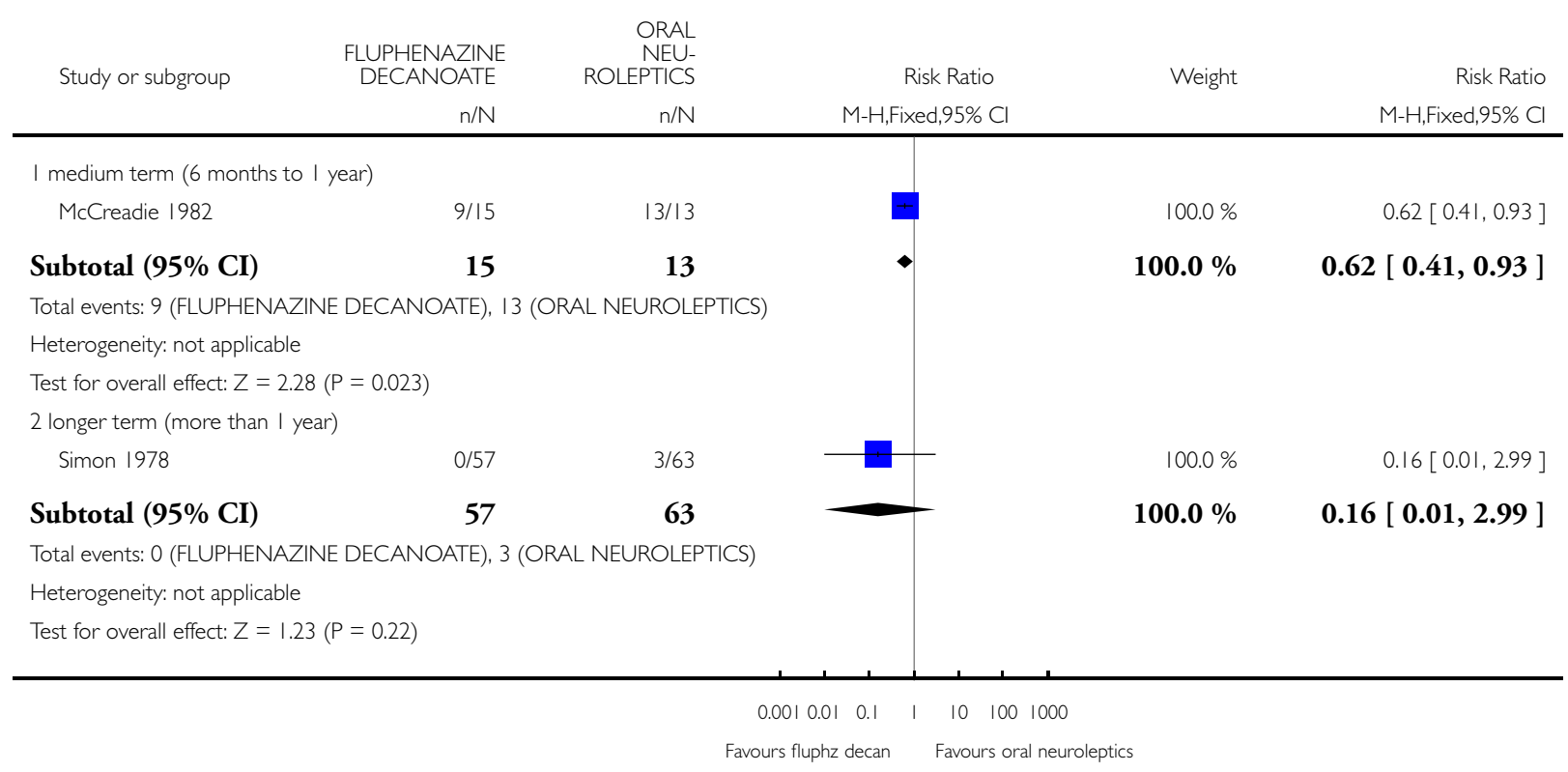


Analysis 2.13. Comparison 2 FLUPHENAZINE DECANOATE vs ORAL NEUROLEPTICS, Outcome 13 Adverse effects: Ie. Movement disorders - tremor (longer term - more than I year).

Review: Fluphenazine decanoate (depot) and enanthate for schizophrenia

Comparison: 2 FLUPHENAZINE DECANOATE vs ORAL NEUROLEPTICS

Outcome: 13 Adverse effects: Ie. Movement disorders - tremor (longer term - more than I year)

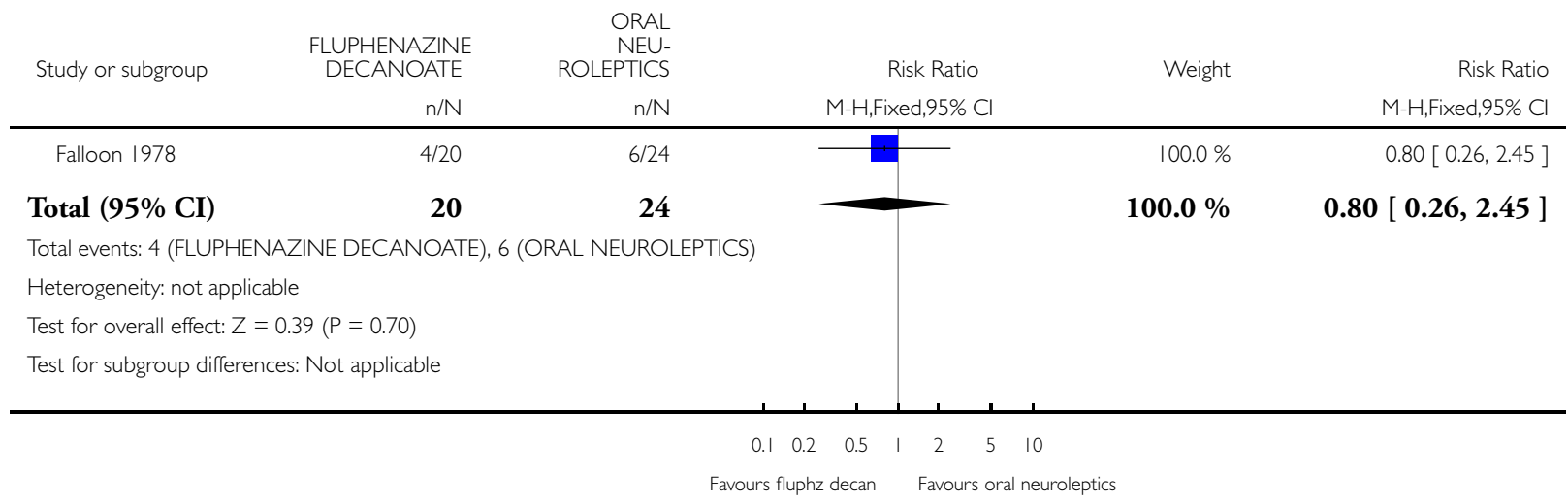

Analysis 2.14. Comparison 2 FLUPHENAZINE DECANOATE vs ORAL NEUROLEPTICS, Outcome I4 Adverse effects: If. Movement disorders - average score (Simpson \& Angus, 0 to 5 weeks, high = poor).

Review: Fluphenazine decanoate (depot) and enanthate for schizophrenia

Comparison: 2 FLUPHENAZINE DECANOATE vs ORAL NEUROLEPTICS

Outcome: 14 Adverse effects: If. Movement disorders - average score (Simpson \% Angus, 0 to 5 weeks, high = poor)

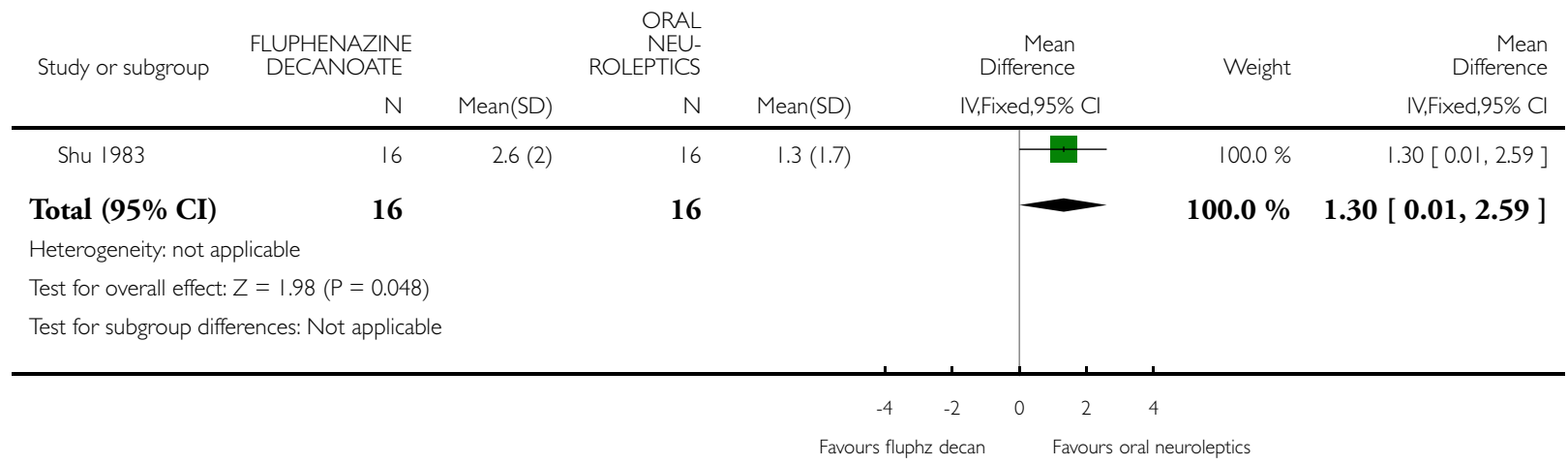


Analysis 2.15. Comparison 2 FLUPHENAZINE DECANOATE vs ORAL NEUROLEPTICS, Outcome I5 Adverse effects: 2 . Blurred vision - medium term (6 months to I year).

Review: Fluphenazine decanoate (depot) and enanthate for schizophrenia

Comparison: 2 FLUPHENAZINE DECANOATE vs ORAL NEUROLEPTICS

Outcome: 15 Adverse effects: 2. Blurred vision - medium term (6 months to I year)

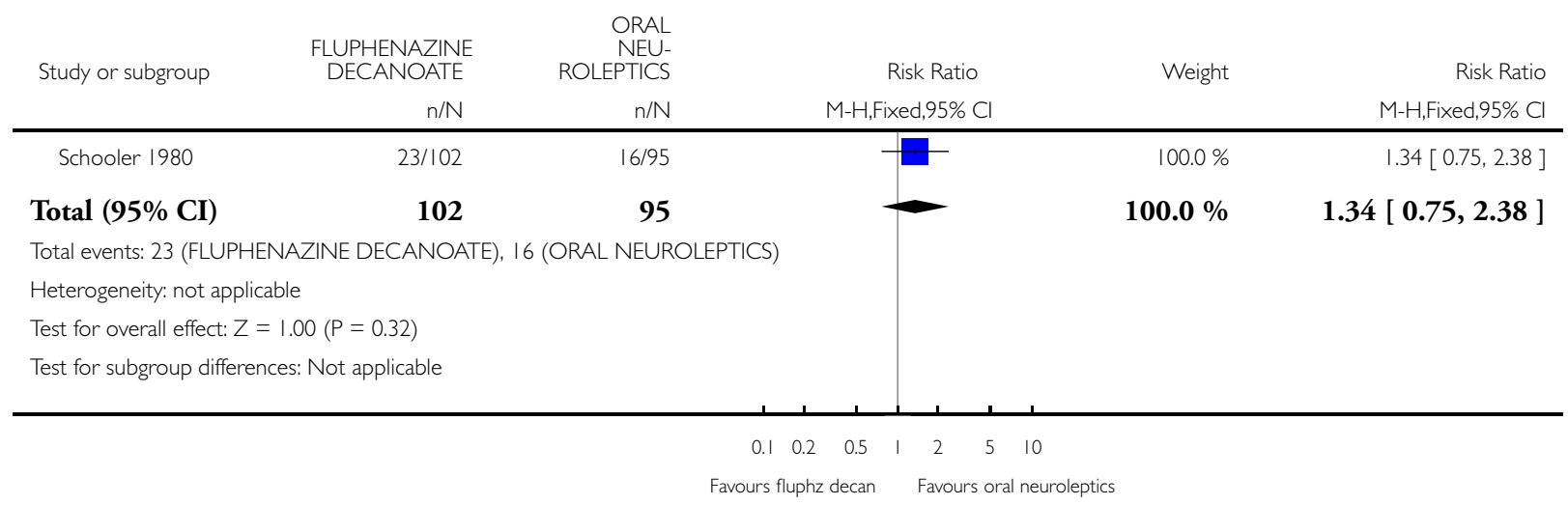

Analysis 2.16. Comparison 2 FLUPHENAZINE DECANOATE vs ORAL NEUROLEPTICS, Outcome I6 Adverse effects: 3 . Toxicity - medium term (6 months to I year).

Review: Fluphenazine decanoate (depot) and enanthate for schizophrenia

Comparison: 2 FLUPHENAZINE DECANOATE vs ORAL NEUROLEPTICS

Outcome: 16 Adverse effects: 3. Toxicity - medium term (6 months to I year)

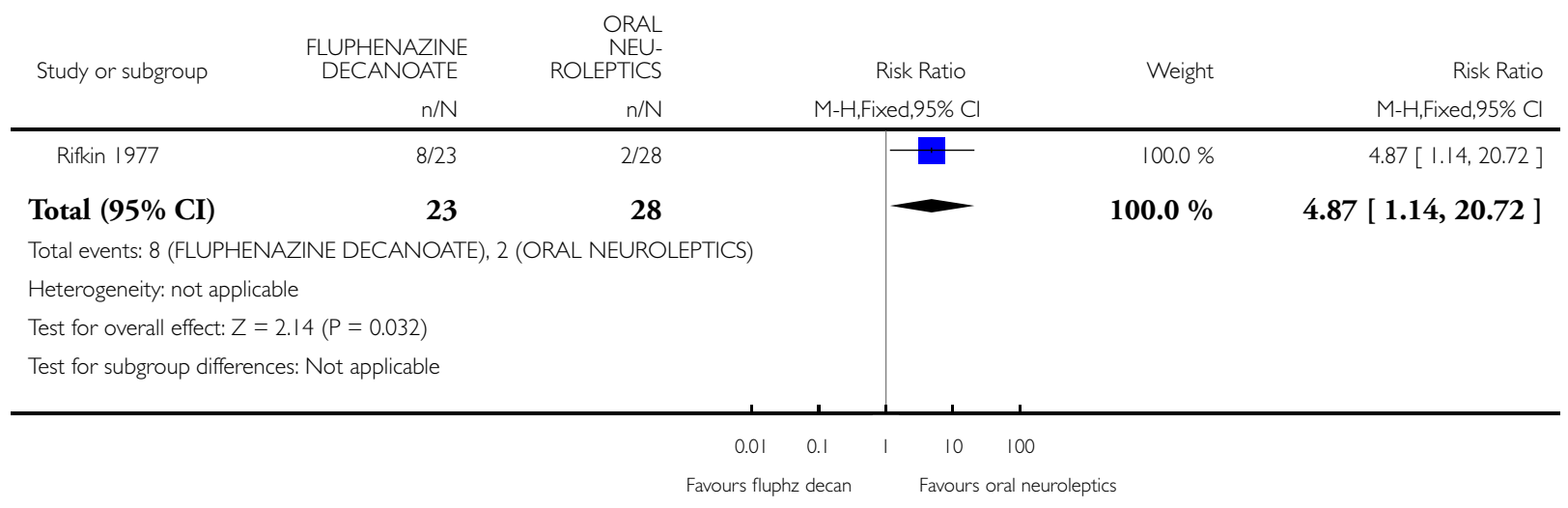


Analysis 2.17. Comparison 2 FLUPHENAZINE DECANOATE vs ORAL NEUROLEPTICS, Outcome I7 Adverse effects: 4. General adverse effects.

Review: Fluphenazine decanoate (depot) and enanthate for schizophrenia

Comparison: 2 FLUPHENAZINE DECANOATE vs ORAL NEUROLEPTICS

Outcome: 17 Adverse effects: 4. General adverse effects

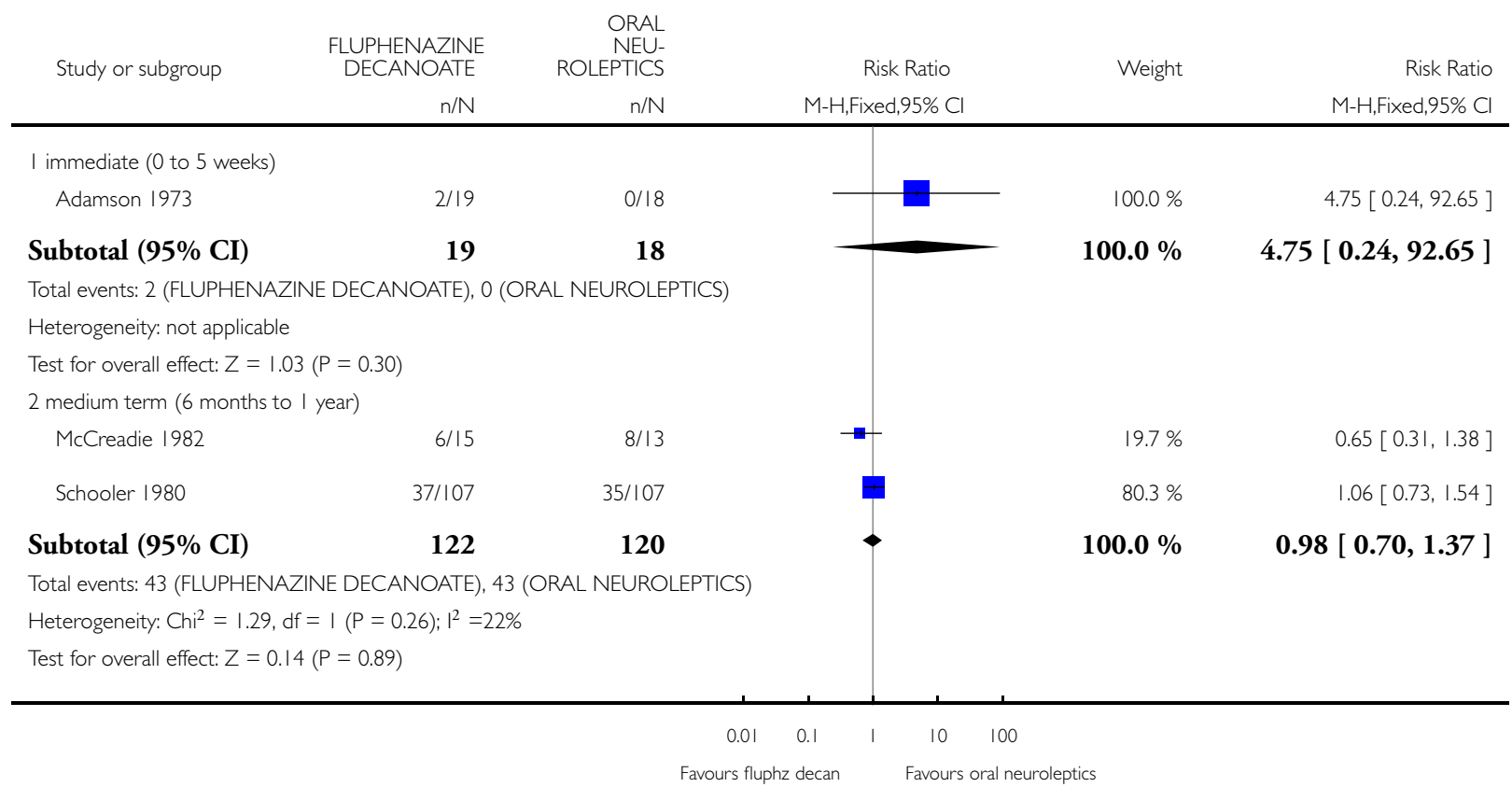


Analysis 2.18. Comparison 2 FLUPHENAZINE DECANOATE vs ORAL NEUROLEPTICS, Outcome 18 SENSITIVITY ANALYSIS Global state: 2. Relapse.

Review: Fluphenazine decanoate (depot) and enanthate for schizophrenia

Comparison: 2 FLUPHENAZINE DECANOATE vs ORAL NEUROLEPTICS

Outcome: 18 SENSITIVITY ANALYSIS Global state: 2. Relapse

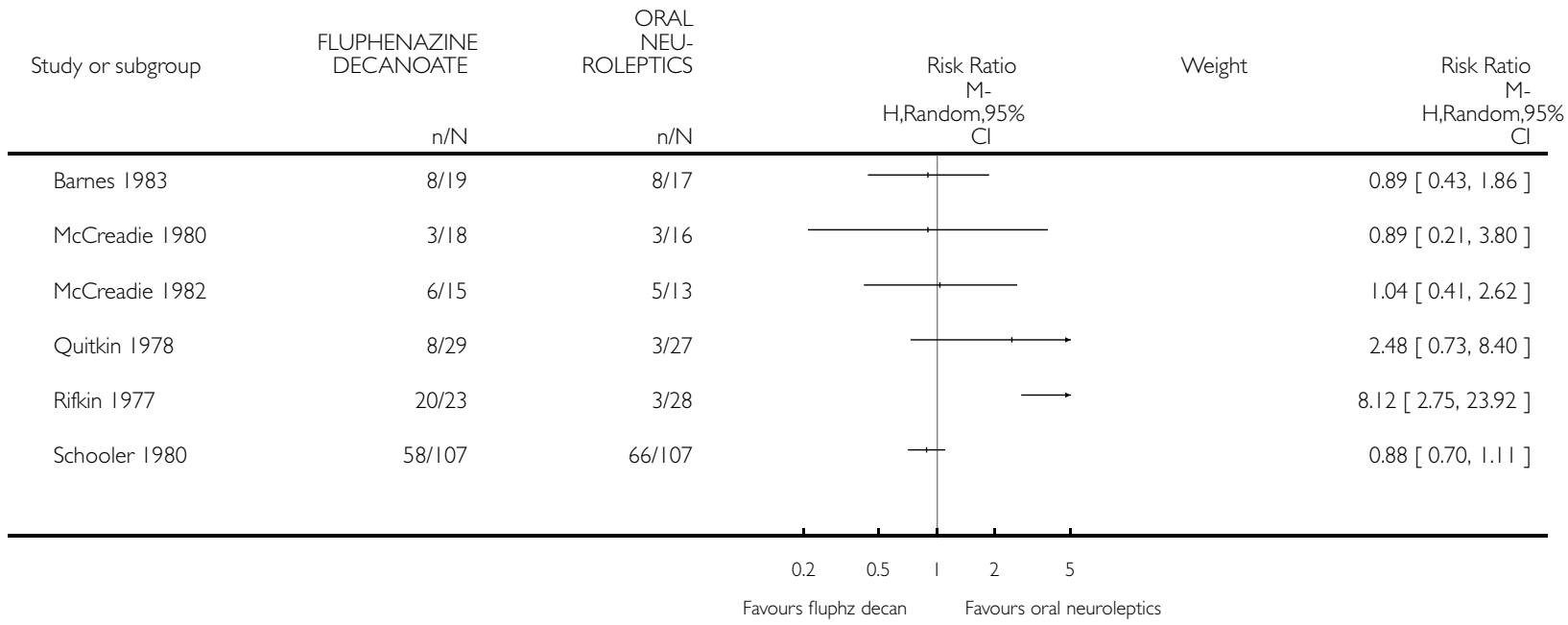

Analysis 3.I. Comparison 3 FLUPHENAZINE DECANOATE vs OTHER DEPOT NEUROLEPTICS, Outcome I Death.

Review: Fluphenazine decanoate (depot) and enanthate for schizophrenia

Comparison: 3 FLUPHENAZINE DECANOATE vs OTHER DEPOT NEUROLEPTICS

Outcome: I Death

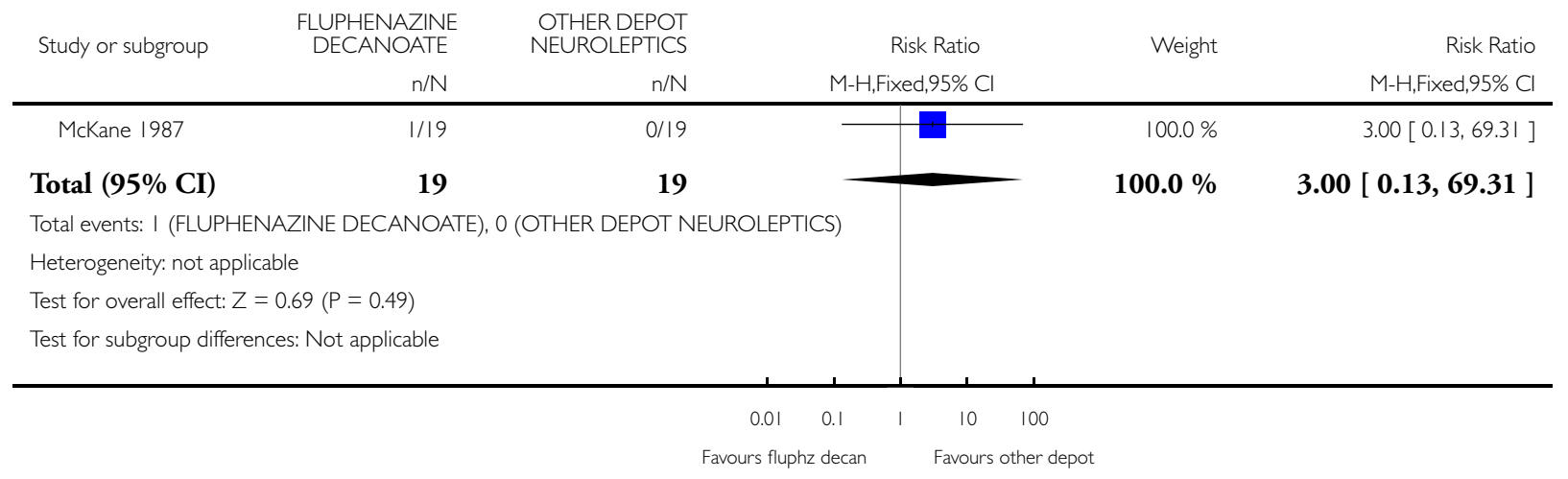

Fluphenazine decanoate (depot) and enanthate for schizophrenia (Review)

Copyright @ 2016 The Cochrane Collaboration. Published by John Wiley \& Sons, Ltd. 


\section{Analysis 3.2. Comparison 3 FLUPHENAZINE DECANOATE vs OTHER DEPOT NEUROLEPTICS,}

Outcome 2 Global state: I. No clinically important global change.

Review: Fluphenazine decanoate (depot) and enanthate for schizophrenia

Comparison: 3 FLUPHENAZINE DECANOATE vs OTHER DEPOT NEUROLEPTICS

Outcome: 2 Global state: I. No clinically important global change

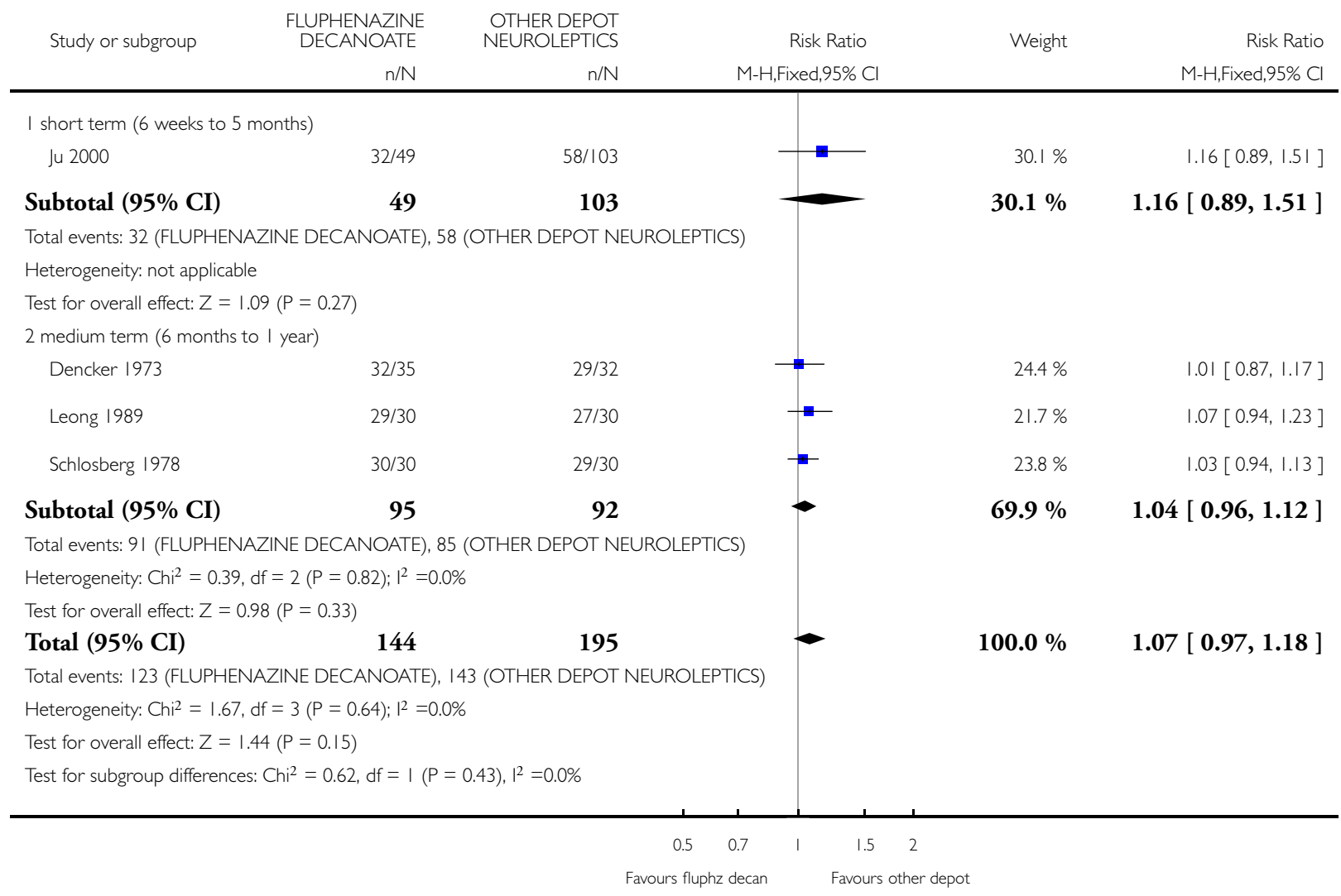




\section{Analysis 3.3. Comparison 3 FLUPHENAZINE DECANOATE vs OTHER DEPOT NEUROLEPTICS, Outcome 3 Global state: 2. Relapse.}

Review: Fluphenazine decanoate (depot) and enanthate for schizophrenia

Comparison: 3 FLUPHENAZINE DECANOATE vs OTHER DEPOT NEUROLEPTICS

Outcome: 3 Global state: 2. Relapse

$\begin{array}{lcc}\text { Study or subgroup } & \text { FLUPHENAZINE } & \text { OTHER DEPOT } \\ & \text { DECANOATE } & \text { NEUROLEPTICS }\end{array}$

$$
\mathrm{n} / \mathrm{N} \quad \mathrm{n} / \mathrm{N}
$$

$\mathrm{n} / \mathrm{N}$

Risk Ratio

Weight

Risk Ratio

M-H,Fixed,95\% Cl

M-H,Fixed,95\% Cl

I short term (6 weeks to 5 months)

Wistedt 1984

$4 / 26 \quad 4 / 25$

$4 / 25$

$100.0 \%$

$0.96[0.27,3.43]$

Subtotal (95\% CI)

26

25

$100.0 \%$

Total events: 4 (FLUPHENAZINE DECANOATE), 4 (OTHER DEPOT NEUROLEPTICS)

Heterogeneity: not applicable

Test for overall effect: $Z=0.06(P=0.95)$

2 medium term (6 months to I year)

$$
\text { Chouinard } 1984
$$

$1 / 36$

Cookson 1986

Cookson 1986

Dencker 1973

Kelly 1977

Leong 1989

Lundin 1990

Magnus 1979

McLaren 1992

Russell 1982

Schlosberg 1978

Sharma 199|

Subtotal (95\% CI)

290

$0 / 9$

$6 / 35$

$3 / 28$

0/30

$9 / 30$

1/26

$0 / 24$

$3 / 13$

$9 / 30$

$6 / 29$

Total events: 38 (FLUPHENAZINE DECANOATE), 47 (OTHER DEPOT NEUROLEPTICS)

Heterogeneity: $\mathrm{Chi}^{2}=8.59, \mathrm{df}=10(\mathrm{P}=0.57) ; \mathrm{I}^{2}=0.0 \%$

Test for overall effect: $Z=1.07(P=0.28)$

3 longer term (more than I year)

$\begin{array}{ll}\text { McKane } 1987 & \text { 2/ } \\ \text { Pinto } 1979 & \text { 14/53 } \\ \text { Simon } 1978 & 6 / 15\end{array}$

Subtotal $(95 \% \mathrm{CI})$

124

$3 / 19$

$8 / 33$

$4 / 57$

$6 / 15$

Total events: 30 (FLUPHENAZINE DECANOATE), 26 (OTHER DEPOT NEUROLEPTICS)

Heterogeneity: $\mathrm{Chi}^{2}=4.89, \mathrm{df}=3(\mathrm{P}=0.18) ; \mathrm{I}^{2}=39 \%$

Test for overall effect: $Z=0.85(P=0.39)$

$8 / 17$

128
$0 / 36$

$2 / 10$

$3 / 32$

$3 / 26$

$1 / 30$

| |/28

$1 / 26$

$6 / 23$

$2 / 20$

$8 / 30$

10/30

291

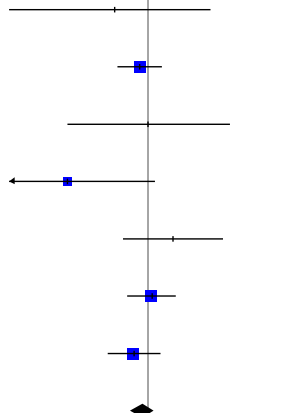

$1.0 \%$

$4.9 \%$

$6.4 \%$

$6.3 \%$

$3.1 \%$

$23.2 \%$

$2.0 \%$

$13.5 \%$

$3.2 \%$

$16.3 \%$

$20.0 \%$

$100.0 \%$

$11.8 \%$

$2.0 \%$

$56.8 \%$

$29.4 \%$

$100.0 \%$

$0.96[0.27,3.43$ ]

$$
\begin{array}{r}
3.00[0.13,71.28] \\
0.22[0.01,4.05] \\
1.83[0.50,6.71] \\
0.93[0.21,4.20] \\
0.33[0.01,7.87] \\
0.76[0.37,1.56] \\
1.00[0.07,15.15] \\
0.07[0.00,1.24] \\
2.31[0.44,11.98] \\
1.13[0.50,2.52] \\
0.62[0.26,1.49] \\
\mathbf{0 . 8 2}[\mathbf{0 . 5 6 , 1 . 1 8}]
\end{array}
$$

$$
0.67[0.13,3.55]
$$

$16.00[0.96,266.03]$

$1.00[0.53,1.88]$

$0.85[0.38,1.89]$

$1.22[0.77,1.92]$

Fluphenazine decanoate (depot) and enanthate for schizophrenia (Review)

Copyright @ 2016 The Cochrane Collaboration. Published by John Wiley \& Sons, Ltd. 


\section{Analysis 3.4. Comparison 3 FLUPHENAZINE DECANOATE vs OTHER DEPOT NEUROLEPTICS,}

Outcome 4 Global state: 3. Severly ill (medium term 6 months to I year).

Review: Fluphenazine decanoate (depot) and enanthate for schizophrenia

Comparison: 3 FLUPHENAZINE DECANOATE vs OTHER DEPOT NEUROLEPTICS

Outcome: 4 Global state: 3 . Severly ill (medium term 6 months to I year)

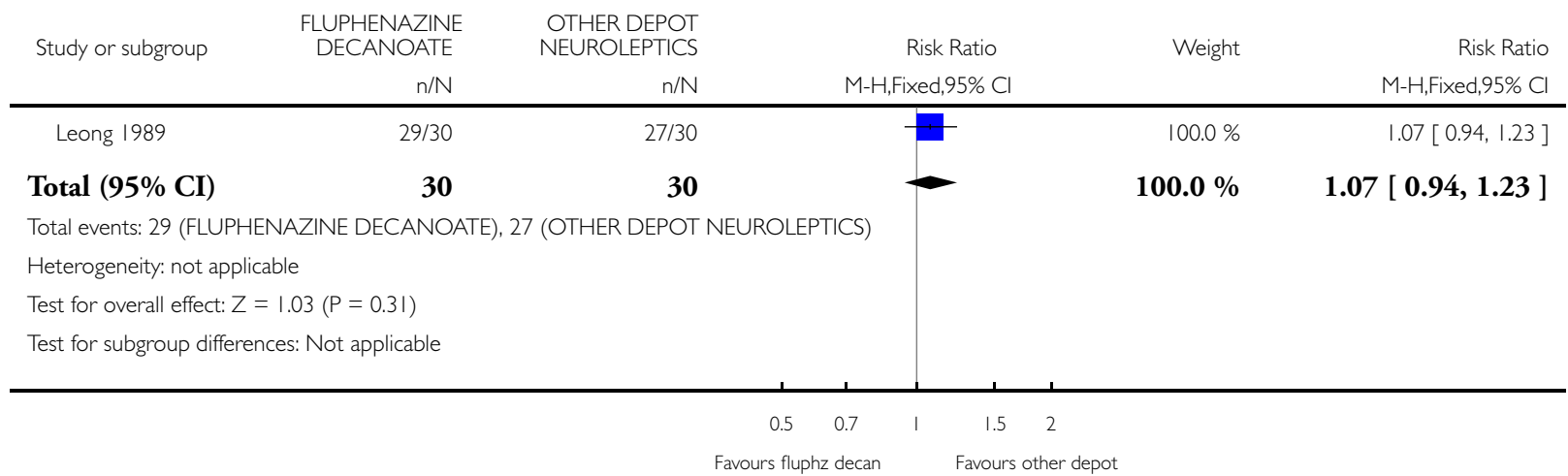


Analysis 3.5. Comparison 3 FLUPHENAZINE DECANOATE vs OTHER DEPOT NEUROLEPTICS, Outcome 5 Global state: 4. Needing additional antipsychotic treatment (6 months to I year).

Review: Fluphenazine decanoate (depot) and enanthate for schizophrenia

Comparison: 3 FLUPHENAZINE DECANOATE vs OTHER DEPOT NEUROLEPTICS

Outcome: 5 Global state: 4 . Needing additional antipsychotic treatment (6 months to I year)

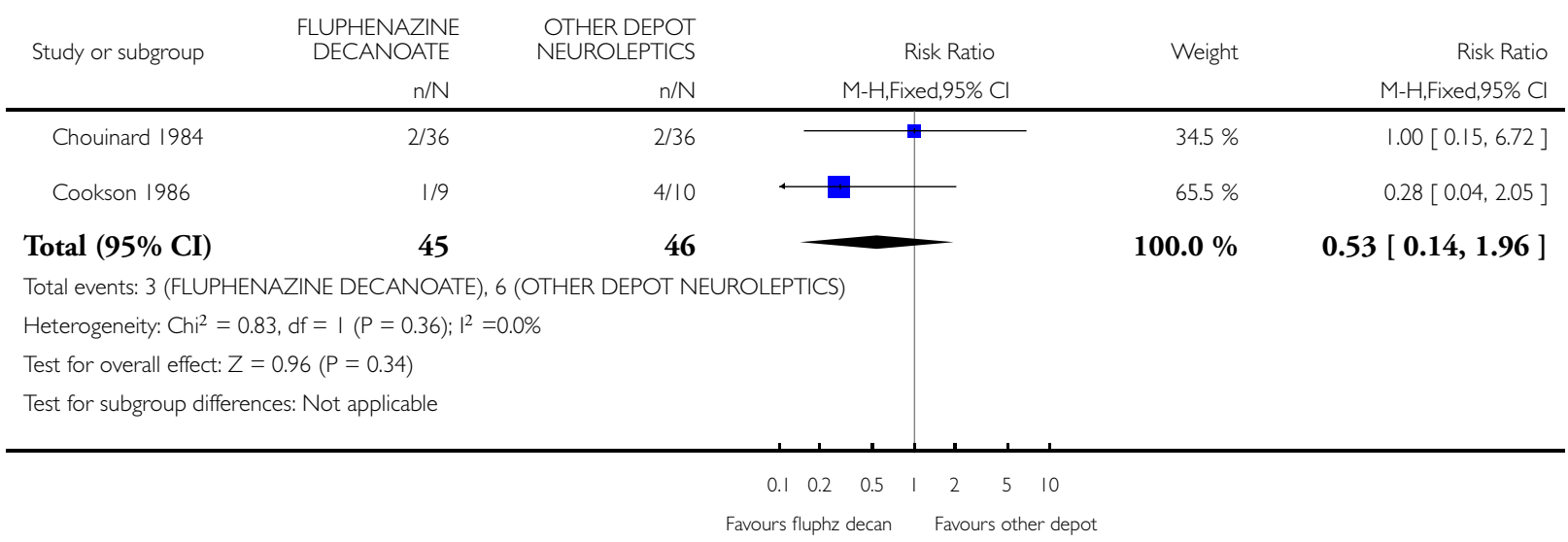

Analysis 3.6. Comparison 3 FLUPHENAZINE DECANOATE vs OTHER DEPOT NEUROLEPTICS, Outcome 6 Global state: 5. Clinical Global Impression. (short term - 6 weeks to 5 months) (skewed data).

Global state: 5. Clinical Global Impression. (short term - 6 weeks to 5 months) (skewed data)

\begin{tabular}{llllll}
\hline Study & Intervention & mean & SD & N \\
\hline Wistedt 1984 & Fluphenazine decanoate & 2.9 & 2 & 26 \\
\hline Wistedt 1984 & Pipothiazine & 2.9 & 1.5 & 25 \\
\hline
\end{tabular}


Analysis 3.7. Comparison 3 FLUPHENAZINE DECANOATE vs OTHER DEPOT NEUROLEPTICS, Outcome 7 Global state: 6. Clinical Global Impression. (medium term - 6 months to I year).

Review: Fluphenazine decanoate (depot) and enanthate for schizophrenia

Comparison: 3 FLUPHENAZINE DECANOATE vs OTHER DEPOT NEUROLEPTICS

Outcome: 7 Global state: 6 . Clinical Global Impression. (medium term - 6 months to I year)

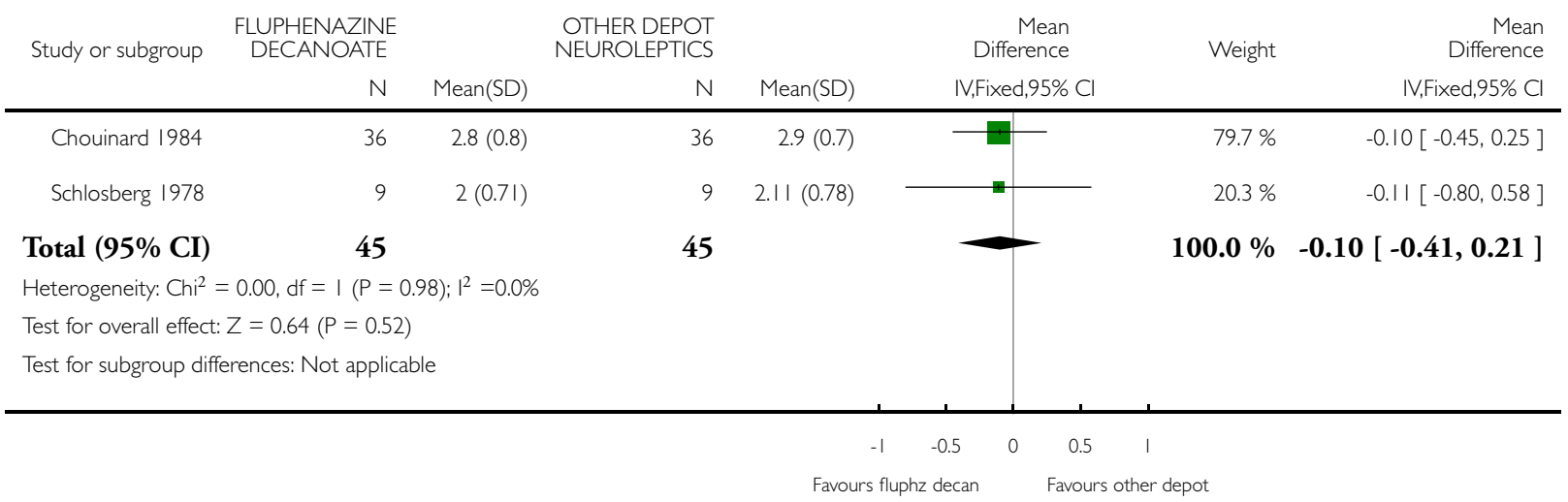


Analysis 3.8. Comparison 3 FLUPHENAZINE DECANOATE vs OTHER DEPOT NEUROLEPTICS, Outcome 8 Global state: 7. Clinical Global Impression - not improved (high score = poor).

Review: Fluphenazine decanoate (depot) and enanthate for schizophrenia

Comparison: 3 FLUPHENAZINE DECANOATE vs OTHER DEPOT NEUROLEPTICS

Outcome: 8 Global state: 7. Clinical Global Impression - not improved (high score = poor)

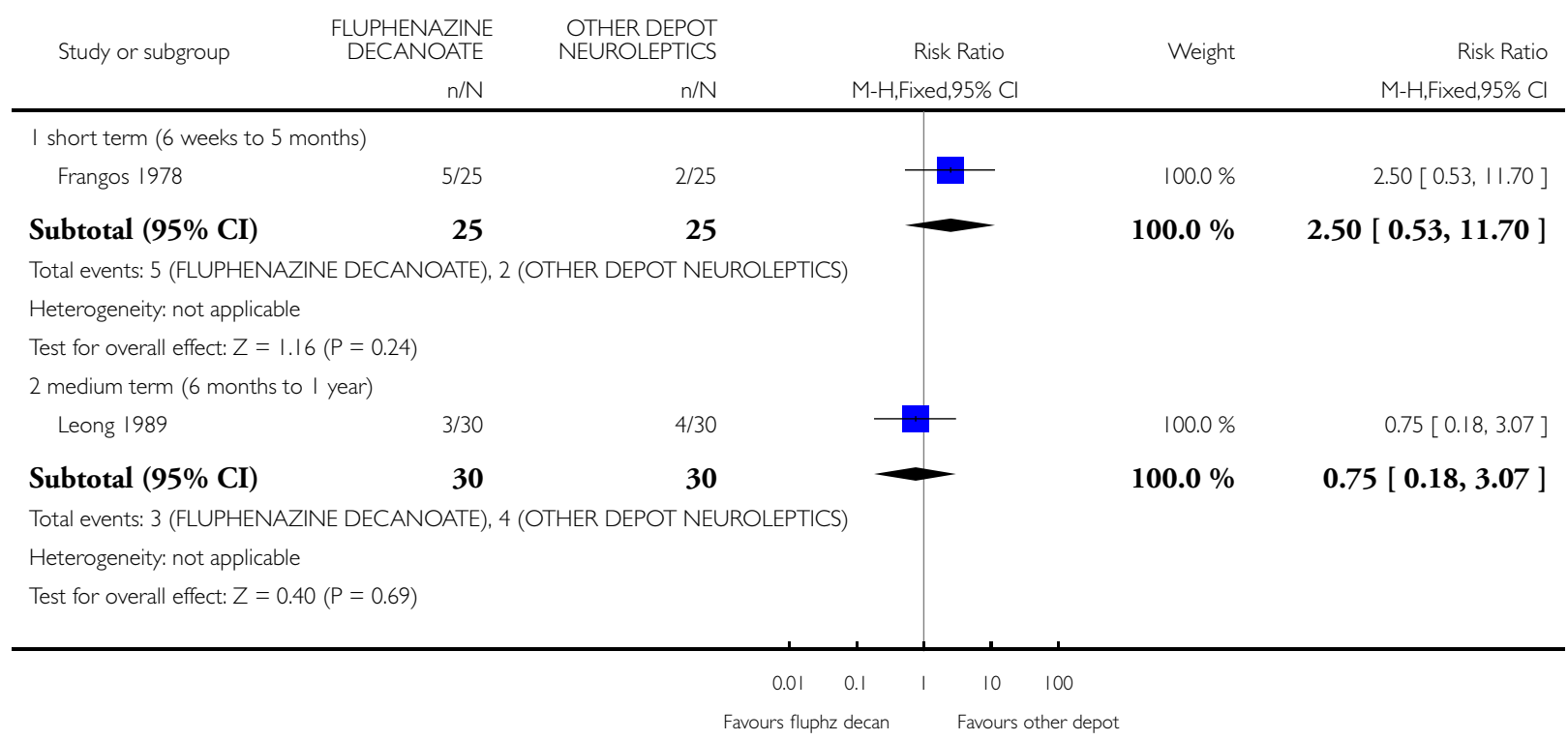




\section{Analysis 3.9. Comparison 3 FLUPHENAZINE DECANOATE vs OTHER DEPOT NEUROLEPTICS, Outcome 9 Leaving the study early.}

Review: Fluphenazine decanoate (depot) and enanthate for schizophrenia

Comparison: 3 FLUPHENAZINE DECANOATE vs OTHER DEPOT NEUROLEPTICS

Outcome: 9 Leaving the study early

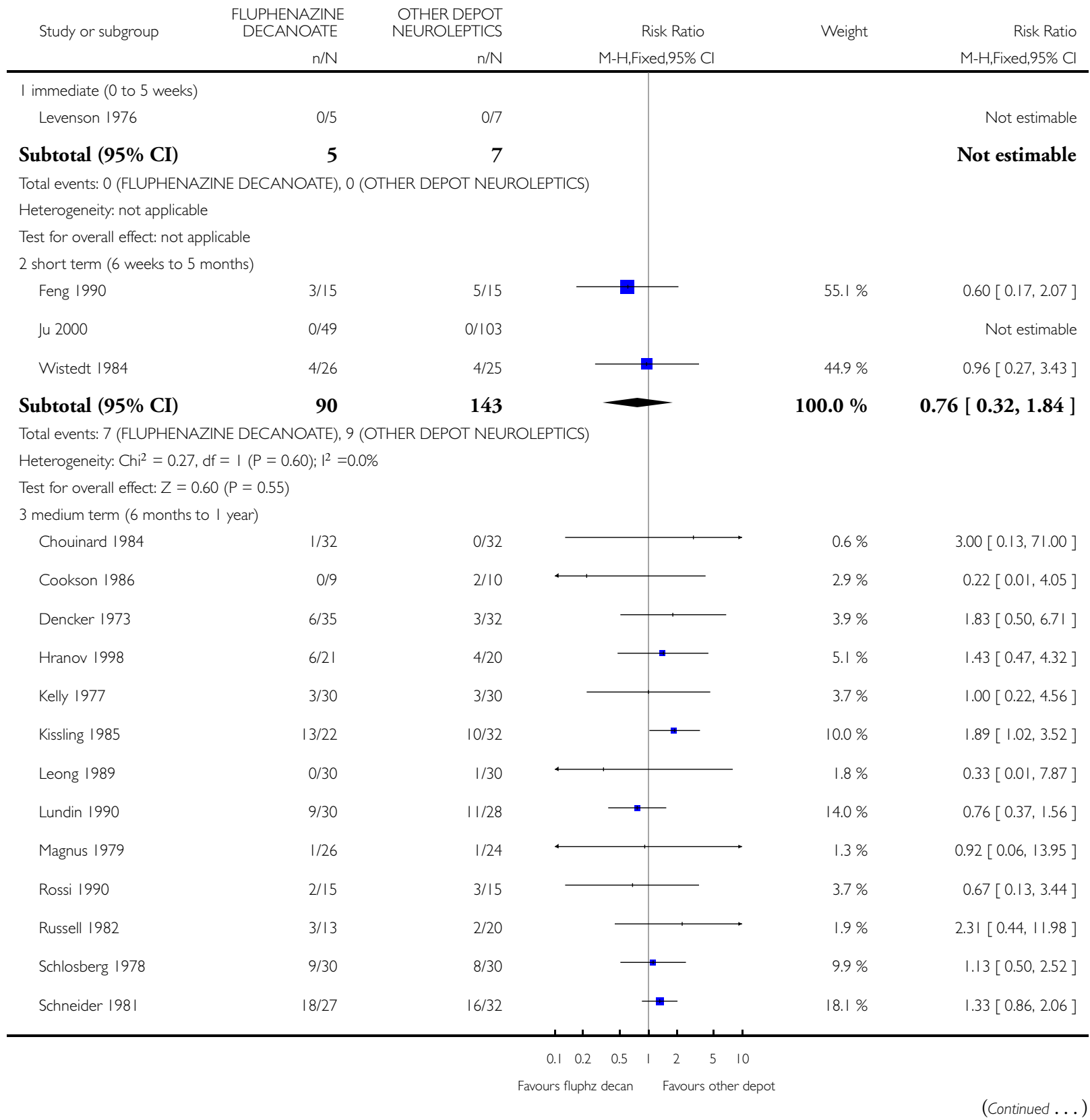

Fluphenazine decanoate (depot) and enanthate for schizophrenia (Review) 


\begin{tabular}{|c|c|c|c|c|c|}
\hline \multirow[t]{2}{*}{ Study or subgroup } & $\begin{array}{l}\text { FLUPHENAZINE } \\
\text { DECANOATE }\end{array}$ & $\begin{array}{r}\text { OTHER DEPOT } \\
\text { NEUROLEPTICS }\end{array}$ & Risk Ratio & \multicolumn{2}{|r|}{$\begin{array}{c}\text { (... Continued) } \\
\text { Risk Ratio }\end{array}$} \\
\hline & $\mathrm{n} / \mathrm{N}$ & $\mathrm{n} / \mathrm{N}$ & M-H,Fixed,95\% Cl & & M-H,Fixed,95\% Cl \\
\hline Sharma 1991 & $6 / 29$ & $10 / 30$ & $\longrightarrow$ & $12.1 \%$ & $0.62[0.26,1.49]$ \\
\hline Woggon 1977 & 9/30 & $9 / 31$ & 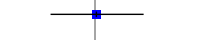 & $10.9 \%$ & $1.03[0.48,2.24]$ \\
\hline Subtotal $(95 \% \mathrm{CI})$ & 379 & 396 & - & $100.0 \%$ & $1.13[0.89,1.44]$ \\
\hline \multicolumn{6}{|c|}{ Total events: 86 (FLUPHENAZINE DECANOATE), 83 (OTHER DEPOT NEUROLEPTICS) } \\
\hline \multicolumn{6}{|c|}{ Heterogeneity: $\mathrm{Ch}^{2}=10.22, \mathrm{df}=14(\mathrm{P}=0.75) ;\left.\right|^{2}=0.0 \%$} \\
\hline \multicolumn{6}{|c|}{ Test for overall effect: $Z=0.98(P=0.33)$} \\
\hline \multicolumn{6}{|l|}{4 By more than I year } \\
\hline Dencker 1973 & $10 / 34$ & $21 / 33$ & - & $41.1 \%$ & $0.46[0.26,0.83]$ \\
\hline McKane 1987 & $7 / 19$ & $8 / 19$ & & $15.4 \%$ & $0.88[0.40,1.93]$ \\
\hline Pinto 1979 & $8 / 33$ & $0 / 31$ & & $1.0 \%$ & $16.00[0.96,266.03]$ \\
\hline Simon 1978 & $14 / 57$ & $15 / 61$ & & $28.0 \%$ & $1.00[0.53,1.88]$ \\
\hline Wistedt 1983 & $6 / 15$ & $8 / 17$ & -1 & $14.5 \%$ & $0.85[0.38,1.89]$ \\
\hline Subtotal $(95 \% \mathrm{CI})$ & 158 & 161 & 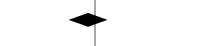 & $100.0 \%$ & $0.89[0.64,1.23]$ \\
\hline \multicolumn{6}{|c|}{ Total events: 45 (FLUPHENAZINE DECANOATE), 52 (OTHER DEPOT NEUROLEPTICS) } \\
\hline \multicolumn{6}{|c|}{ Heterogeneity: $\mathrm{Chi}^{2}=9.04, \mathrm{df}=4(\mathrm{P}=0.06) ; \mathrm{I}^{2}=56 \%$} \\
\hline \multicolumn{6}{|c|}{ Test for overall effect: $Z=0.72(P=0.47)$} \\
\hline
\end{tabular}


Analysis 3.10. Comparison 3 FLUPHENAZINE DECANOATE vs OTHER DEPOT NEUROLEPTICS, Outcome 10 Behaviour: I. NOSIE-30 - endpoint scores (high score = poor).

Review: Fluphenazine decanoate (depot) and enanthate for schizophrenia

Comparison: 3 FLUPHENAZINE DECANOATE vs OTHER DEPOT NEUROLEPTICS

Outcome: 10 Behaviour: I. NOSIE-30 - endpoint scores (high score = poor)

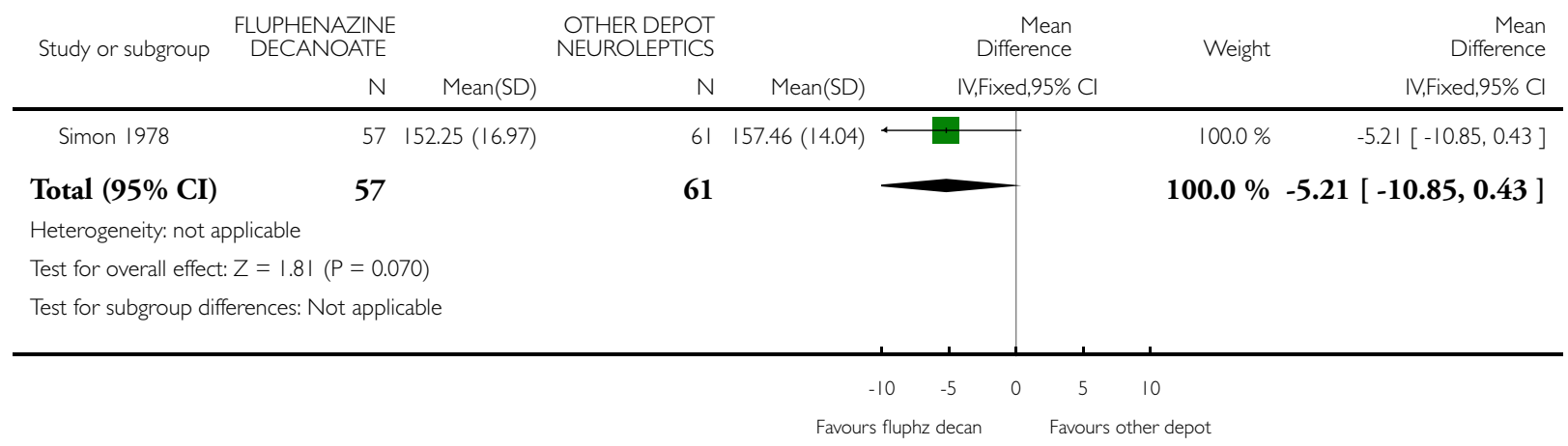

Analysis 3.1 I. Comparison 3 FLUPHENAZINE DECANOATE vs OTHER DEPOT NEUROLEPTICS, Outcome I I Mental state: I. BPRS (endpoint scores - high score = poor).

Review: Fluphenazine decanoate (depot) and enanthate for schizophrenia

Comparison: 3 FLUPHENAZINE DECANOATE vs OTHER DEPOT NEUROLEPTICS

Outcome: II Mental state: I. BPRS (endpoint scores - high score = poor)

\begin{tabular}{ccrrrr} 
& FLUPHENAZINE & OTHER DEPOT & & Mean & Mean \\
Study or subgroup & DECANOATE & NEUROLEPTICS & Difference & Weight \\
N & Mean(SD) & N & Mean(SD) & IV,Fixed,95\% Cl & IV,Fixed,95\% Cl \\
\hline
\end{tabular}

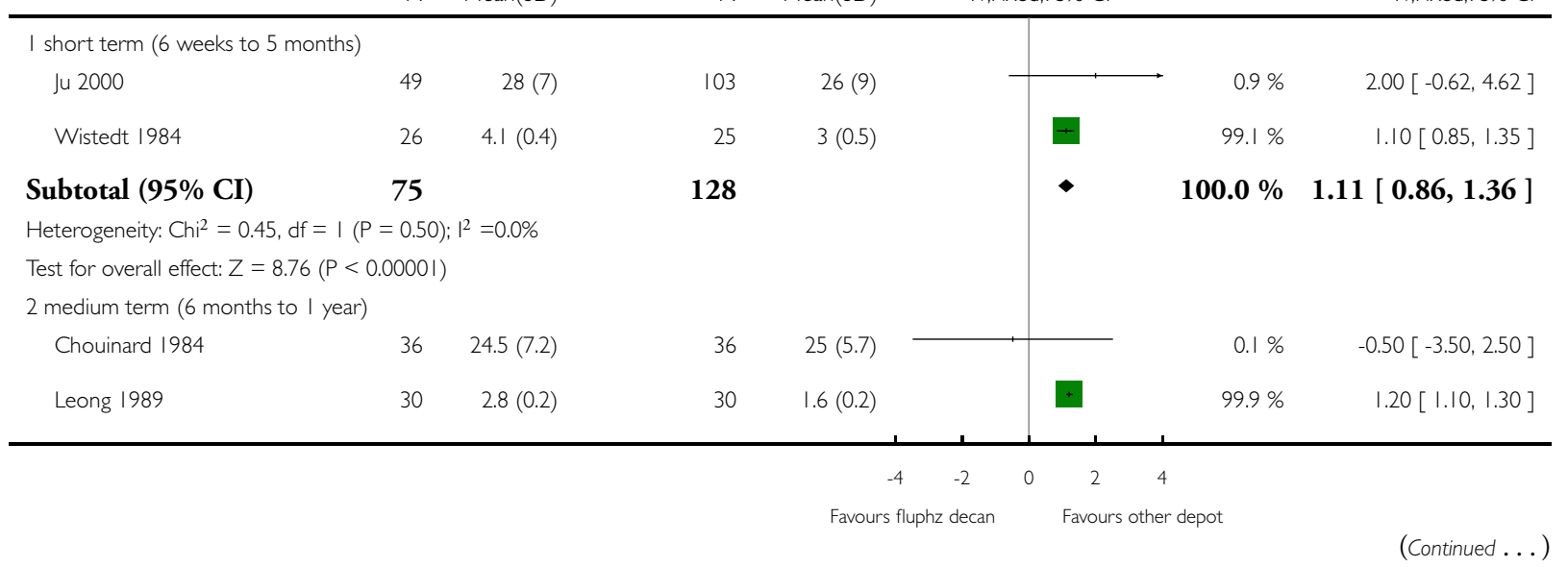




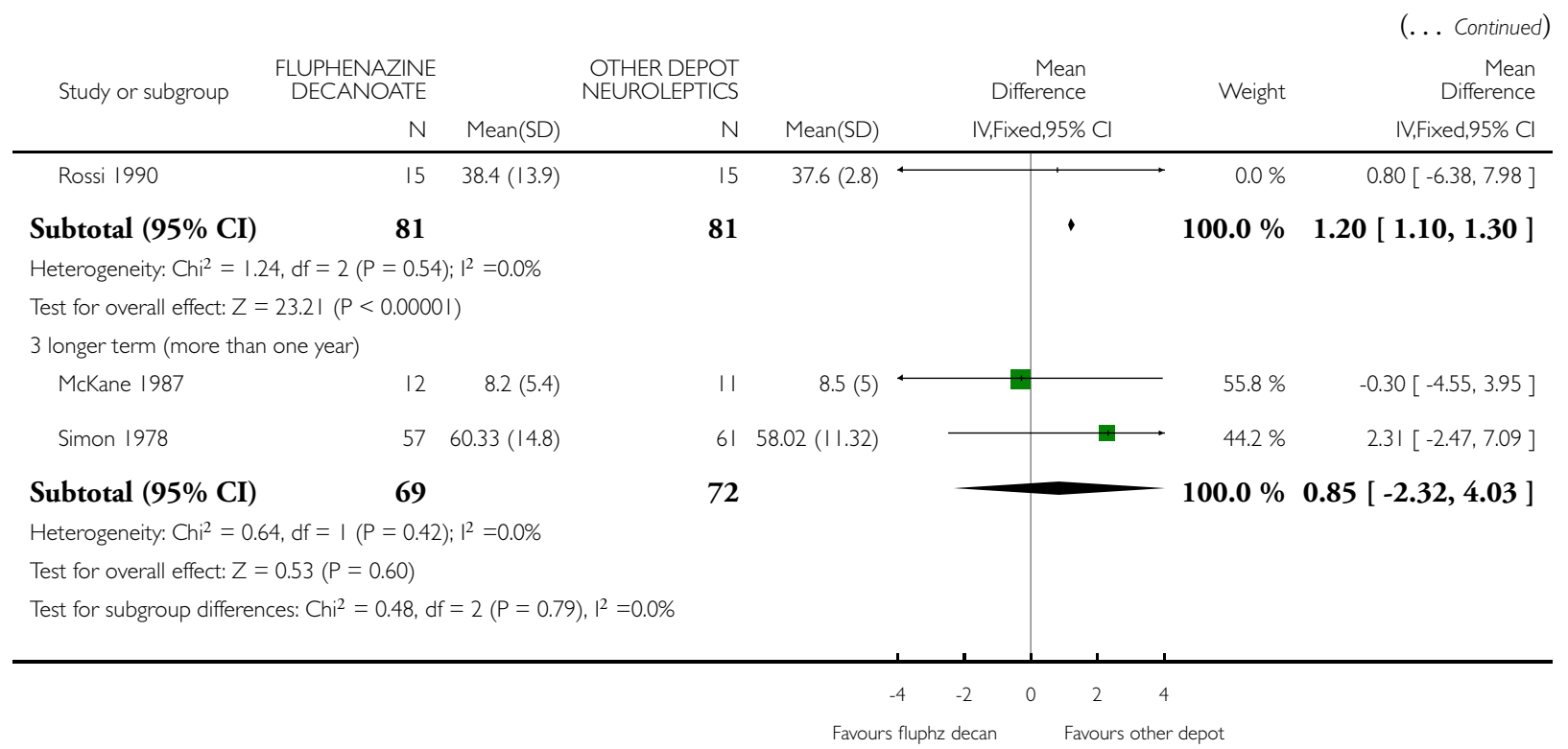

\section{Analysis 3.12. Comparison 3 FLUPHENAZINE DECANOATE vs OTHER DEPOT NEUROLEPTICS,} Outcome I 2 Mental state: 2. BPRS (endpoint scores 6 months to I year - dichotomous data).

Review: Fluphenazine decanoate (depot) and enanthate for schizophrenia

Comparison: 3 FLUPHENAZINE DECANOATE vs OTHER DEPOT NEUROLEPTICS

Outcome: 12 Mental state: 2. BPRS (endpoint scores 6 months to I year - dichotomous data)

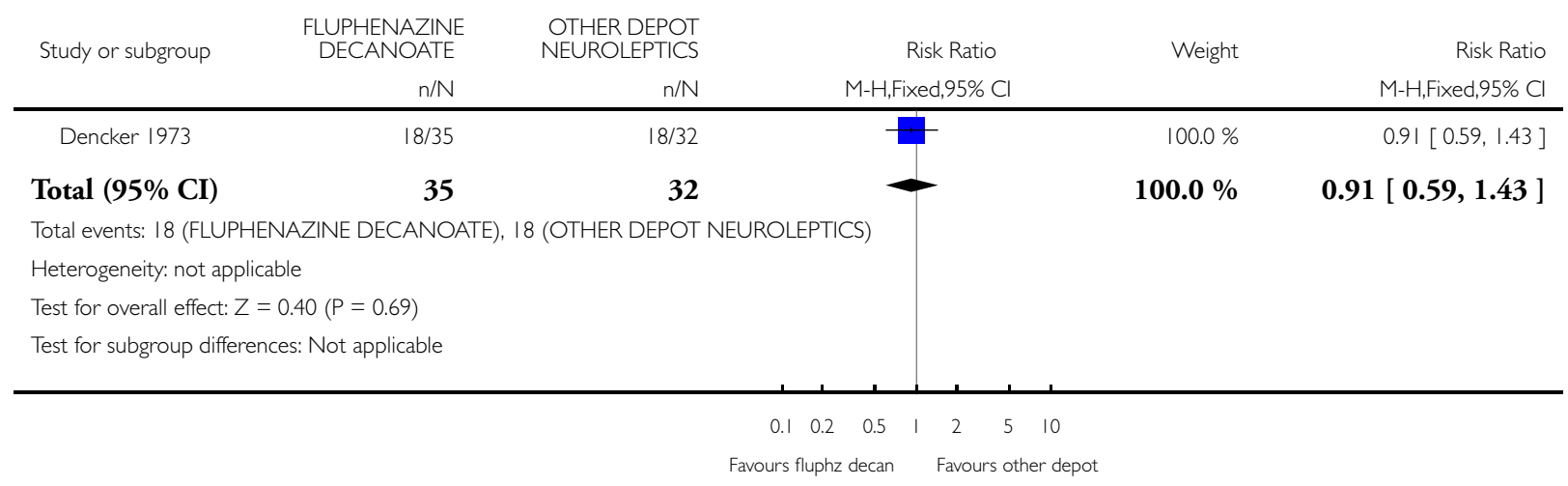




\section{Analysis 3.13. Comparison 3 FLUPHENAZINE DECANOATE vs OTHER DEPOT NEUROLEPTICS,}

Outcome 13 Mental state: 3. Depression (6 months to I year).

Review: Fluphenazine decanoate (depot) and enanthate for schizophrenia

Comparison: 3 FLUPHENAZINE DECANOATE vs OTHER DEPOT NEUROLEPTICS

Outcome: 13 Mental state: 3. Depression (6 months to I year)

\begin{tabular}{|c|c|c|c|c|c|}
\hline \multirow[t]{2}{*}{ Study or subgroup } & $\begin{array}{l}\text { FLUPHENAZINE } \\
\text { DECANOATE }\end{array}$ & $\begin{array}{l}\text { OTHER DEPOT } \\
\text { NEUROLEPTICS }\end{array}$ & Risk Ratio & Weight & Risk Ratio \\
\hline & $n / N$ & $n / N$ & M-H,Fixed,95\% Cl & & M-H,Fixed,95\% Cl \\
\hline Dencker 1973 & $29 / 35$ & $26 / 32$ & & $100.0 \%$ & $1.02[0.81,1.28]$ \\
\hline Total (95\% CI) & 35 & 32 & & $100.0 \%$ & $1.02[0.81,1.28]$ \\
\hline
\end{tabular}

Total events: 29 (FLUPHENAZINE DECANOATE), 26 (OTHER DEPOT NEUROLEPTICS)

Heterogeneity: not applicable

Test for overall effect: $Z=0.17(P=0.86)$

Test for subgroup differences: Not applicable

Analysis 3.14. Comparison 3 FLUPHENAZINE DECANOATE vs OTHER DEPOT NEUROLEPTICS, Outcome 14 Mental state: 4. SAPS and SANS (endpoint scores - high score $=$ poor) (skewed data).

Mental state: 4. SAPS and SANS (endpoint scores - high score = poor) $($ skewed data)

\begin{tabular}{|c|c|c|c|c|}
\hline Study & Intervention & mean & SD & $\mathbf{N}$ \\
\hline \multicolumn{5}{|l|}{ SAPS } \\
\hline Ju 2000 & Fluphenazine decanoate & 31.4 & 21.1 & 49 \\
\hline Ju 2000 & Pipothiazine palmitate & 20.1 & 23.1 & 103 \\
\hline \multicolumn{5}{|l|}{ SANS } \\
\hline Ju 2000 & Fluphenazine decanoate & 14.6 & 11.4 & 49 \\
\hline Ju 2000 & Pipothiazine palmitate & 9.4 & 14.7 & 103 \\
\hline
\end{tabular}




\section{Analysis 3.15. Comparison 3 FLUPHENAZINE DECANOATE vs OTHER DEPOT NEUROLEPTICS, Outcome 15 Adverse effects: Ia. Movement disorders - general.}

Review: Fluphenazine decanoate (depot) and enanthate for schizophrenia

Comparison: 3 FLUPHENAZINE DECANOATE vs OTHER DEPOT NEUROLEPTICS

Outcome: 15 Adverse effects: Ia. Movement disorders - general

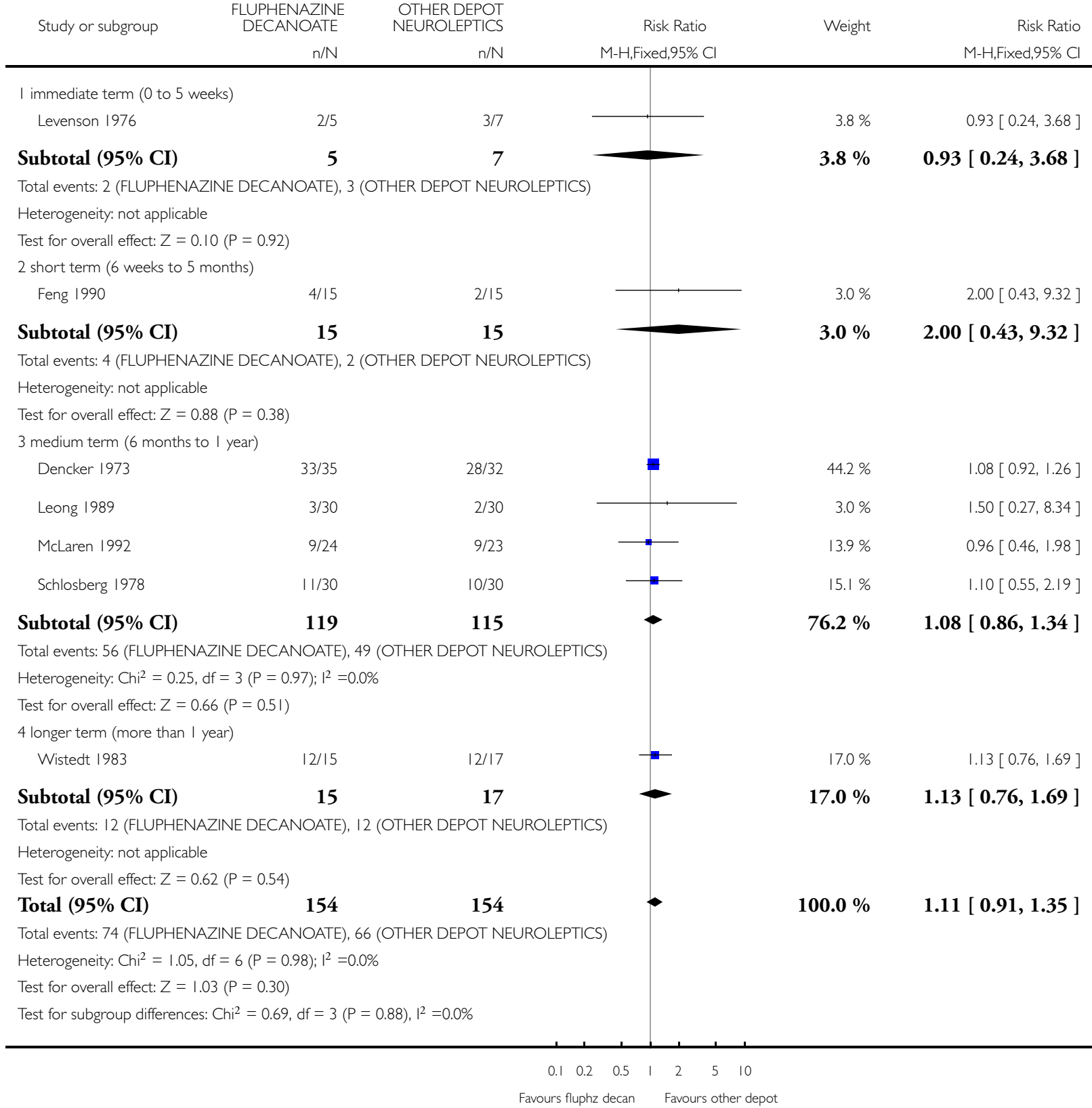

Fluphenazine decanoate (depot) and enanthate for schizophrenia (Review)

Copyright @ 2016 The Cochrane Collaboration. Published by John Wiley \& Sons, Ltd. 


\section{Analysis 3.16. Comparison 3 FLUPHENAZINE DECANOATE vs OTHER DEPOT NEUROLEPTICS,}

Outcome 16 Adverse effects: Ib. Movement disorders - needing anticholinergic drugs.

Review: Fluphenazine decanoate (depot) and enanthate for schizophrenia

Comparison: 3 FLUPHENAZINE DECANOATE vs OTHER DEPOT NEUROLEPTICS

Outcome: 16 Adverse effects: Ib. Movement disorders - needing anticholinergic drugs

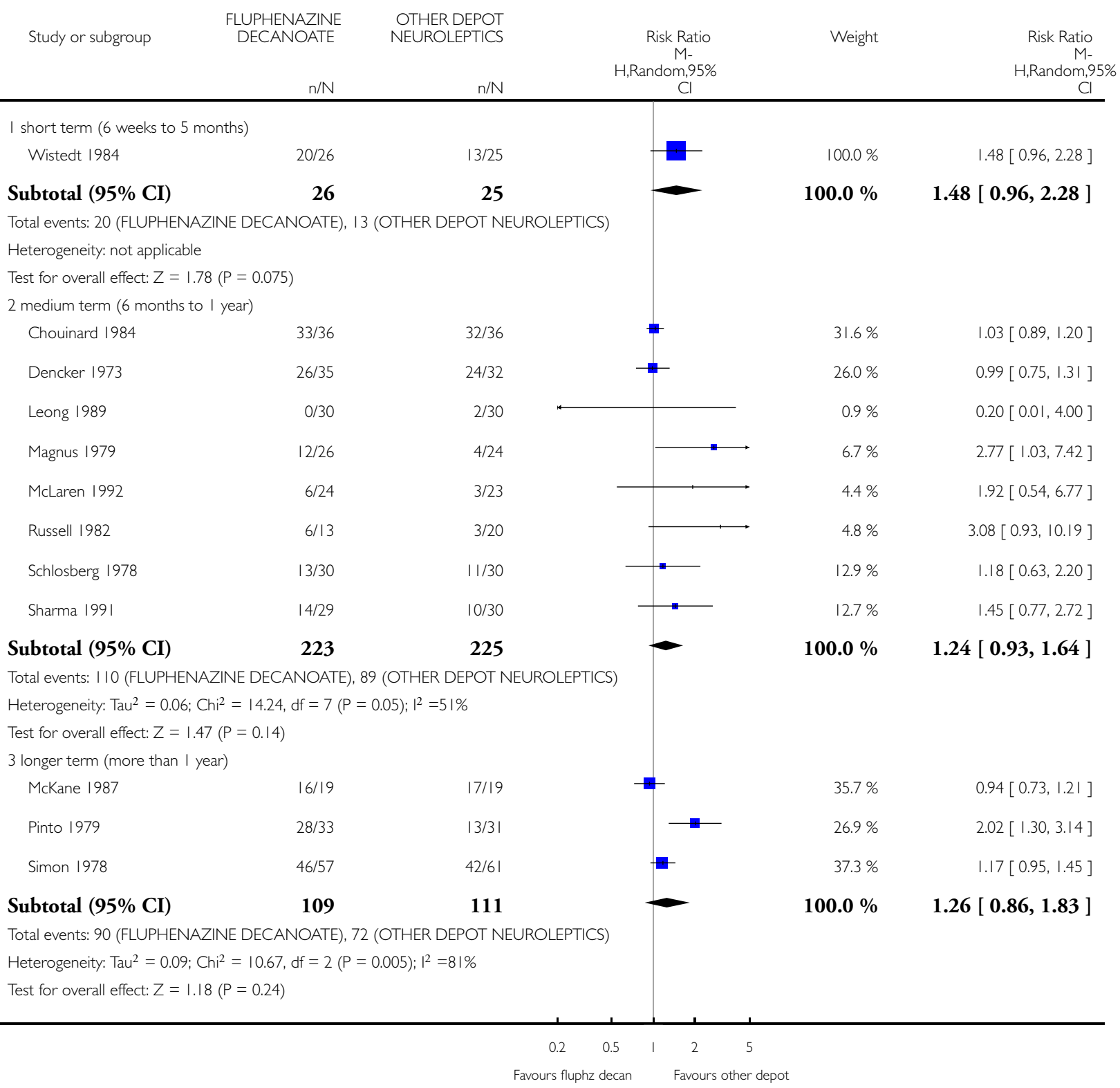




\section{Analysis 3.17. Comparison 3 FLUPHENAZINE DECANOATE vs OTHER DEPOT NEUROLEPTICS,}

Outcome 17 Adverse effects: Ic. Movement disorders - parkinsonism.

Review: Fluphenazine decanoate (depot) and enanthate for schizophrenia

Comparison: 3 FLUPHENAZINE DECANOATE vs OTHER DEPOT NEUROLEPTICS

Outcome: 17 Adverse effects: Ic. Movement disorders - parkinsonism

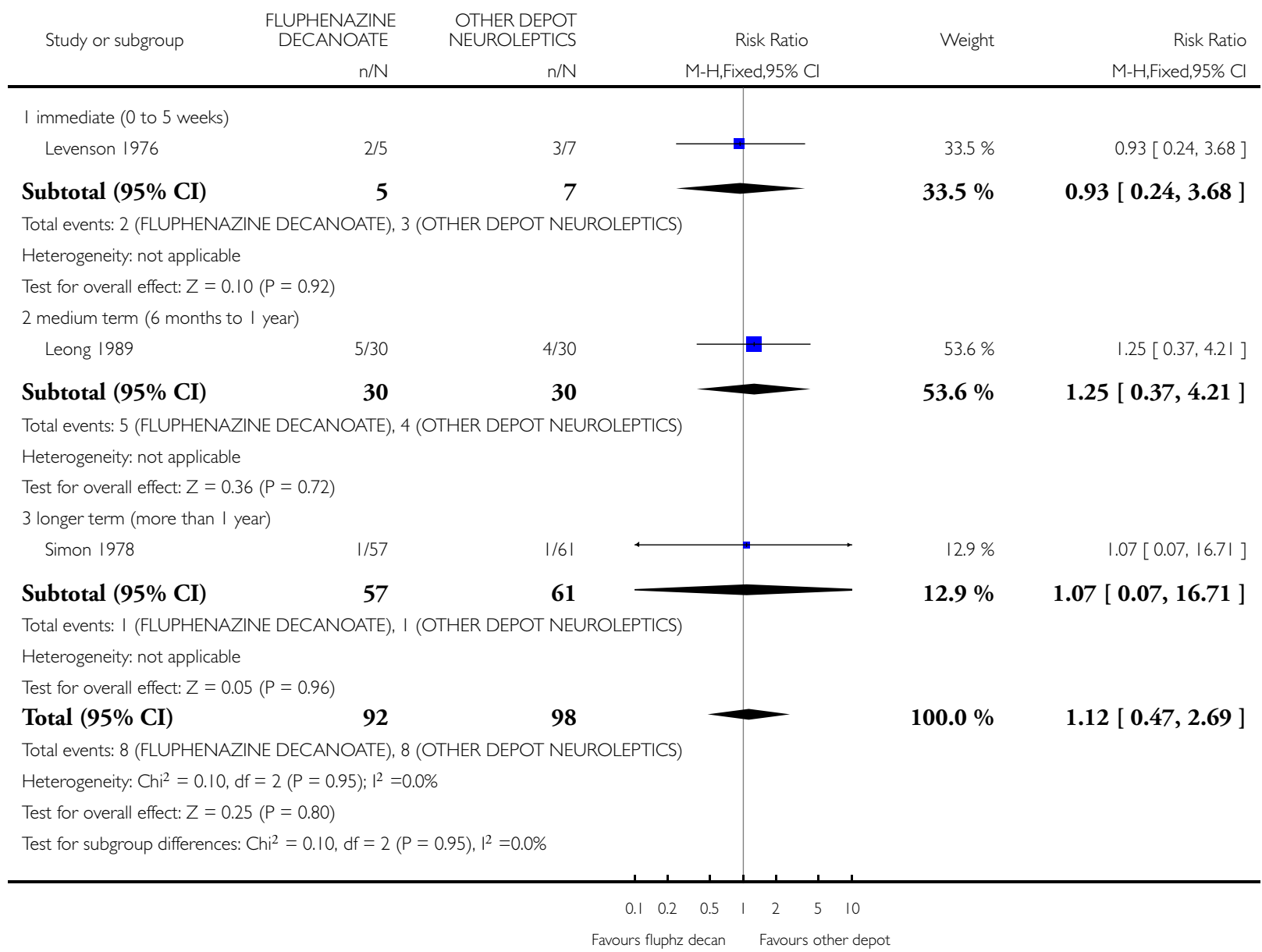


Analysis 3.18. Comparison 3 FLUPHENAZINE DECANOATE vs OTHER DEPOT NEUROLEPTICS, Outcome 18 Adverse effects: Id. Movement disorders - tardive dyskinesia: longer term (more than I year).

Review: Fluphenazine decanoate (depot) and enanthate for schizophrenia

Comparison: 3 FLUPHENAZINE DECANOATE vs OTHER DEPOT NEUROLEPTICS

Outcome: 18 Adverse effects: Id. Movement disorders - tardive dyskinesia: longer term (more than I year)

\begin{tabular}{|c|c|c|c|c|c|}
\hline \multirow[t]{2}{*}{ Study or subgroup } & $\begin{array}{l}\text { FLUPHENAZINE } \\
\text { DECANOATE }\end{array}$ & $\begin{array}{l}\text { OTHER DEPOT } \\
\text { NEUROLEPTICS }\end{array}$ & Risk Ratio & \multirow[t]{2}{*}{ Weight } & \multirow{2}{*}{$\begin{array}{r}\text { Risk Ratio } \\
\text { M-H,Fixed,95\% Cl }\end{array}$} \\
\hline & $\mathrm{n} / \mathrm{N}$ & $\mathrm{n} / \mathrm{N}$ & M-H,Fixed,95\% Cl & & \\
\hline Simon 1978 & $0 / 57$ & $3 / 61$ & 4 & $26.5 \%$ & $0.15[0.01,2.89]$ \\
\hline Wistedt 1983 & $7 / 15$ & $10 / 17$ & & $73.5 \%$ & $0.79[0.41,1.55]$ \\
\hline Total $(95 \% \mathrm{CI})$ & 72 & 78 & & $100.0 \%$ & $0.62[0.32,1.23]$ \\
\hline \multicolumn{6}{|c|}{ Total events: 7 (FLUPHENAZINE DECANOATE), I3 (OTHER DEPOT NEUROLEPTICS) } \\
\hline \multicolumn{6}{|c|}{ Heterogeneity: Chi $^{2}=1.37, \mathrm{df}=1(P=0.24) ; I^{2}=27 \%$} \\
\hline \multicolumn{6}{|c|}{ Test for overall effect: $Z=1.37(P=0.17)$} \\
\hline Test for subgroup diff & s: Not applicable & & & & \\
\hline
\end{tabular}

$\begin{array}{lllllll}0.1 & 0.2 & 0.5 & 1 & 2 & 5 & 10\end{array}$

Favours fluphz decan Favours other depot 


\section{Analysis 3.19. Comparison 3 FLUPHENAZINE DECANOATE vs OTHER DEPOT NEUROLEPTICS,}

Outcome 19 Adverse effects: le. Movement disorders - tremor.

Review: Fluphenazine decanoate (depot) and enanthate for schizophrenia

Comparison: 3 FLUPHENAZINE DECANOATE vs OTHER DEPOT NEUROLEPTICS

Outcome: 19 Adverse effects: le. Movement disorders - tremor

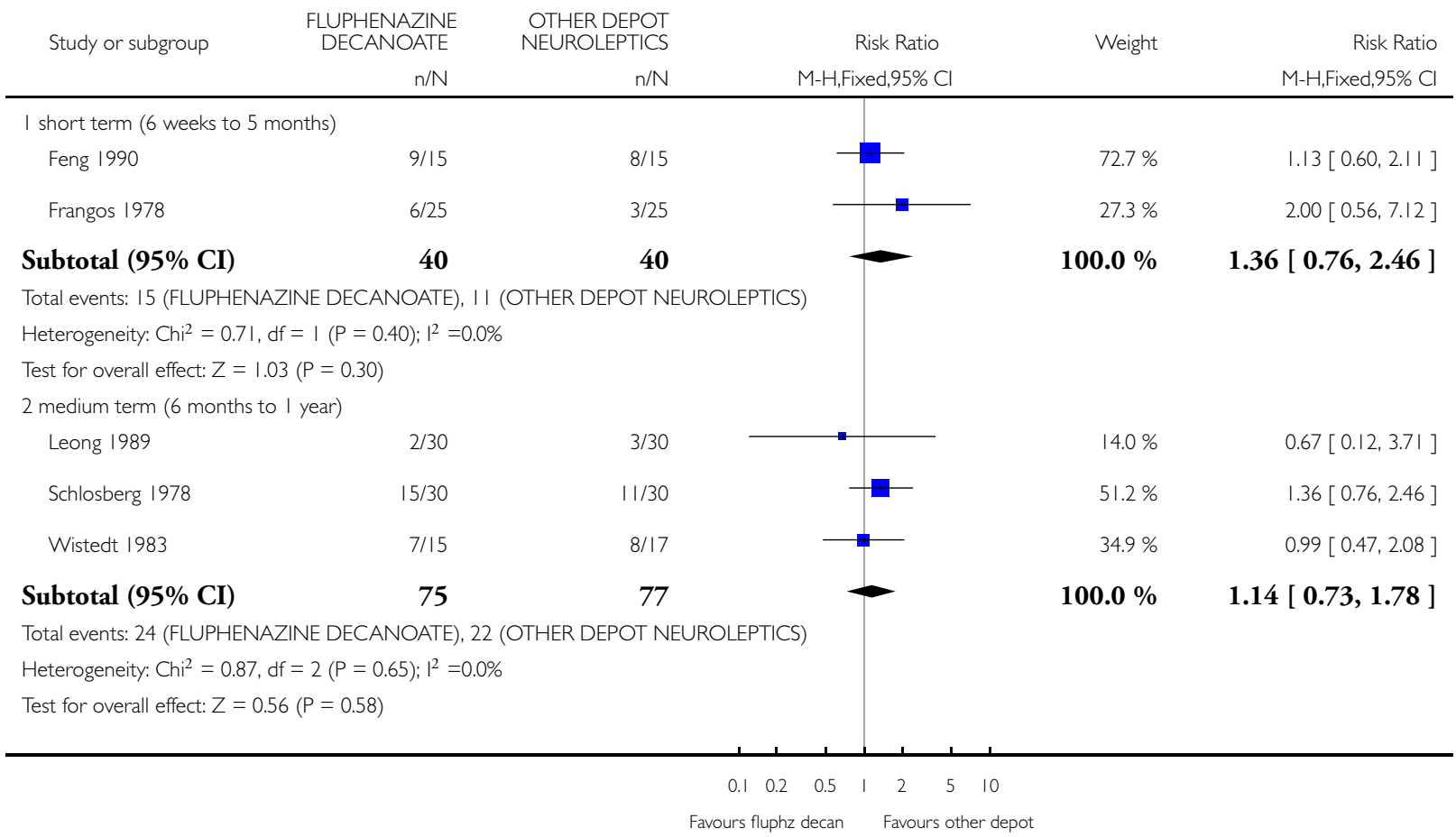

Analysis 3.20. Comparison 3 FLUPHENAZINE DECANOATE vs OTHER DEPOT NEUROLEPTICS, Outcome 20 Adverse effects: If. Movement disorders - endpoint scores (short term - 6 weeks to 5 months).

Adverse effects: 1f. Movement disorders - endpoint scores (short term - 6 weeks to 5 months)

\begin{tabular}{lllll}
\hline Study & Intervention & mean & SD & N \\
\hline TESS (high = poor) & & & & TESS (high = p \\
\hline Ju 2000 & Fluphenazine decanoate & 10.2 & 8.1 & 49 \\
\hline Ju $2000 \quad$ Pipothiazine palmitate & 2.6 & 3.9 & 103 \\
\hline RSESE (high = poor) & & & \\
\hline Ju $2000 \quad$ Fluphenazine decanoate & 7.5 & 7 & 49
\end{tabular}


Adverse effects: 1f. Movement disorders - endpoint scores (short term - 6 weeks to 5 months) (Continued)

$\begin{array}{lllll}\text { Ju } 2000 & \text { Pipothiazine palmitate } & 1.0 & 2.1 & 103\end{array}$

\section{Analysis 3.21. Comparison 3 FLUPHENAZINE DECANOATE vs OTHER DEPOT NEUROLEPTICS,} Outcome 2 I Adverse effects: 2. Blurred vision.

Review: Fluphenazine decanoate (depot) and enanthate for schizophrenia

Comparison: 3 FLUPHENAZINE DECANOATE vs OTHER DEPOT NEUROLEPTICS

Outcome: 21 Adverse effects: 2. Blurred vision

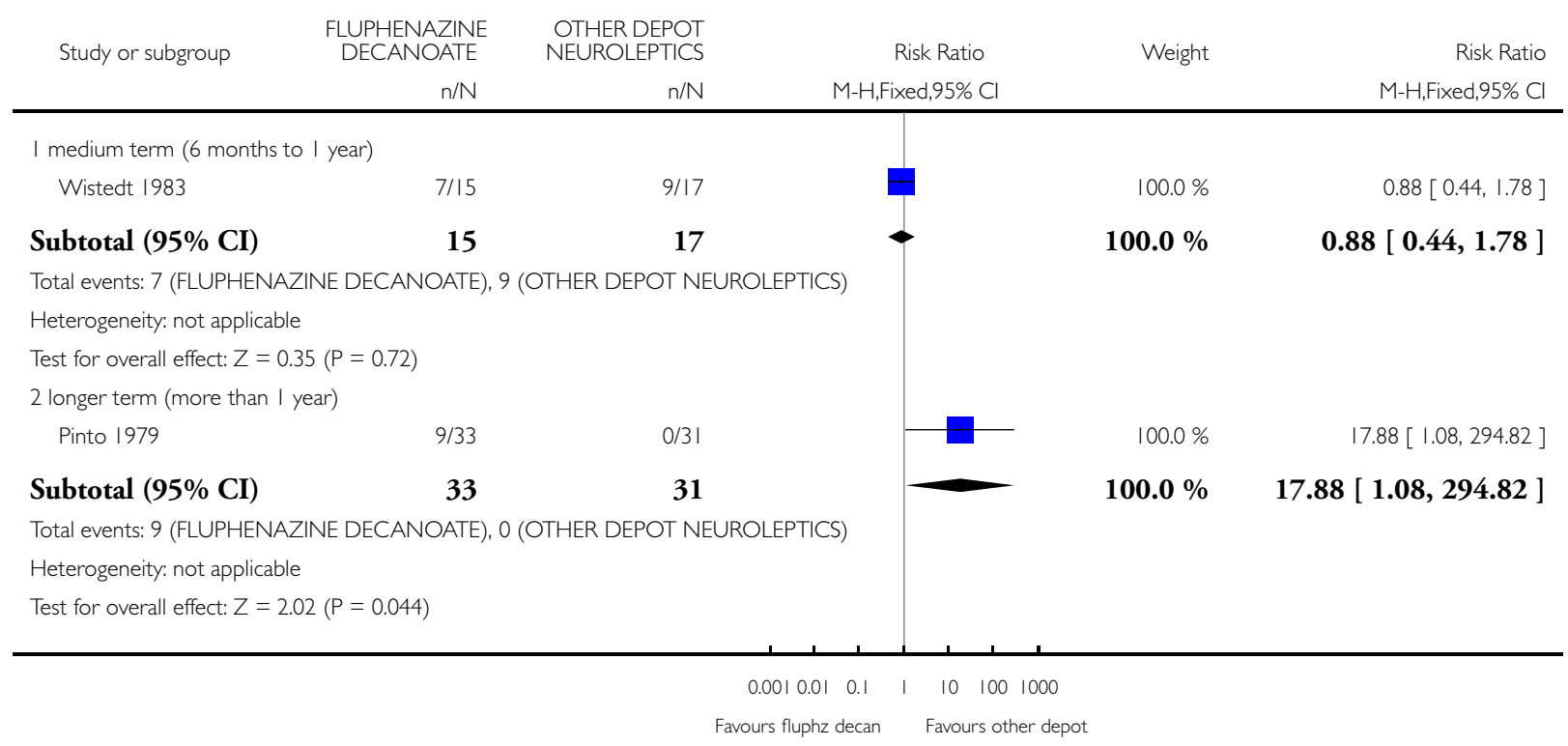




\section{Analysis 3.22. Comparison 3 FLUPHENAZINE DECANOATE vs OTHER DEPOT NEUROLEPTICS,}

Outcome 22 Adverse effects: 3. Dry mouth: longer term (more than I year).

Review: Fluphenazine decanoate (depot) and enanthate for schizophrenia

Comparison: 3 FLUPHENAZINE DECANOATE vs OTHER DEPOT NEUROLEPTICS

Outcome: 22 Adverse effects: 3. Dry mouth: longer term (more than I year)

\begin{tabular}{|c|c|c|c|c|c|}
\hline \multirow[t]{2}{*}{ Study or subgroup } & $\begin{array}{c}\text { FLUPHENAZINE } \\
\text { DECANOATE }\end{array}$ & $\begin{array}{l}\text { OTHER DEPOT } \\
\text { NEUROLEPTICS }\end{array}$ & Risk Ratio & \multirow[t]{2}{*}{ Weight } & \multirow{2}{*}{$\begin{array}{r}\text { Risk Ratio } \\
\text { M-H,Fixed,95\% Cl }\end{array}$} \\
\hline & $\mathrm{n} / \mathrm{N}$ & $\mathrm{n} / \mathrm{N}$ & M-H,Fixed,95\% Cl & & \\
\hline Wistedt 1983 & $7 / 15$ & $11 / 17$ & & $100.0 \%$ & $0.72[0.38,1.37]$ \\
\hline Total (95\% CI) & 15 & 17 & & $100.0 \%$ & $0.72[0.38,1.37]$ \\
\hline \multicolumn{6}{|c|}{ Total events: 7 (FLUPHENAZINE DECANOATE), II (OTHER DEPOT NEUROLEPTICS) } \\
\hline \multicolumn{6}{|c|}{ Heterogeneity: not applicable } \\
\hline \multicolumn{6}{|c|}{ Test for overall effect: $Z=0.99(P=0.32)$} \\
\hline \multicolumn{6}{|c|}{ Test for subgroup differences: Not applicable } \\
\hline
\end{tabular}

\section{Analysis 3.23. Comparison 3 FLUPHENAZINE DECANOATE vs OTHER DEPOT NEUROLEPTICS,} Outcome 23 Adverse effects: 4. General adverse effects.

Review: Fluphenazine decanoate (depot) and enanthate for schizophrenia

Comparison: 3 FLUPHENAZINE DECANOATE vs OTHER DEPOT NEUROLEPTICS

Outcome: 23 Adverse effects: 4. General adverse effects

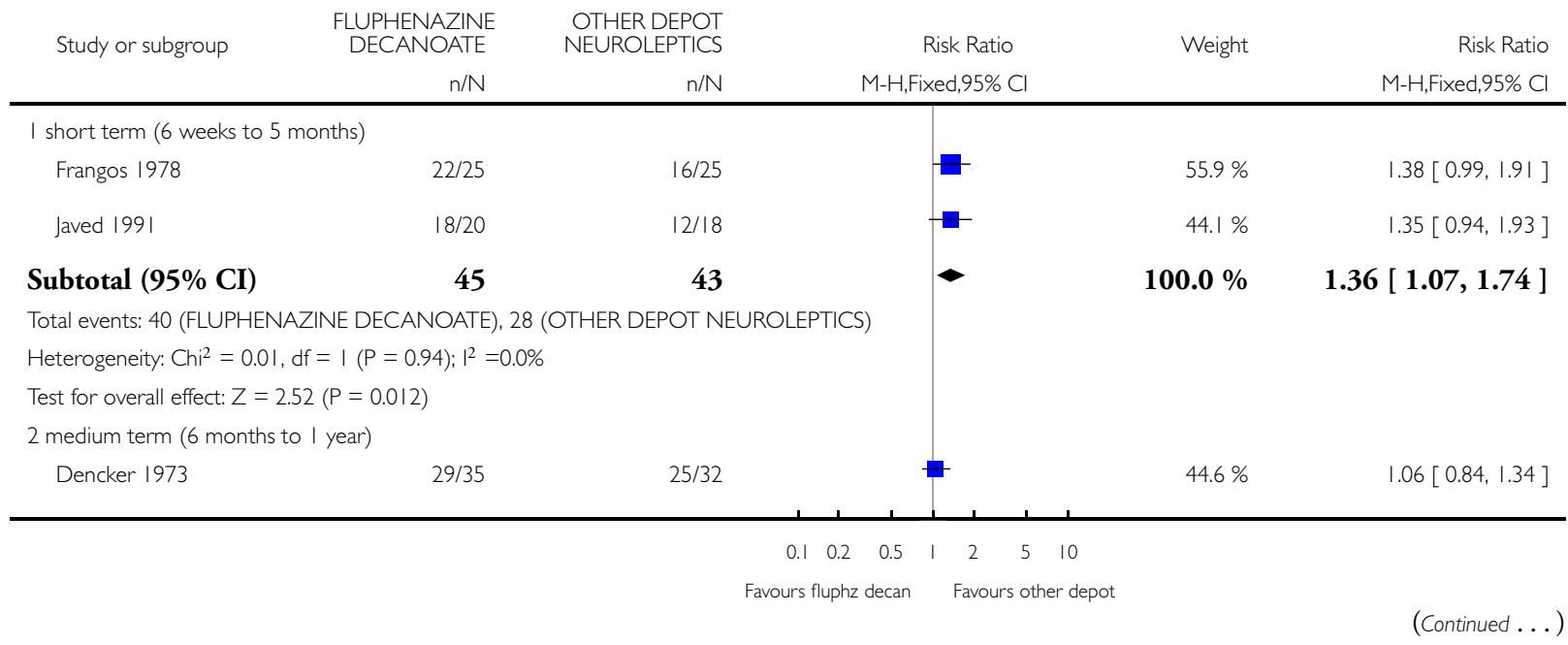




\begin{tabular}{|c|c|c|c|c|c|}
\hline \multirow[t]{2}{*}{ Study or subgroup } & $\begin{array}{l}\text { FLUPHENAZINE } \\
\text { DECANOATE }\end{array}$ & $\begin{array}{l}\text { OTHER DEPOT } \\
\text { NEUROLEPTICS }\end{array}$ & Risk Ratio & \multirow[t]{2}{*}{ Weight } & $\begin{array}{r}\text { (... Continued) } \\
\text { Risk Ratio }\end{array}$ \\
\hline & $\mathrm{n} / \mathrm{N}$ & $\mathrm{n} / \mathrm{N}$ & M-H,Fixed,95\% Cl & & M-H,Fixed,95\% Cl \\
\hline Leong 1989 & $6 / 30$ & $2 / 30$ & 7 & $3.4 \%$ & $3.00[0.66,13.69]$ \\
\hline Magnus 1979 & $15 / 26$ & $4 / 24$ & $\longrightarrow$ & $7.1 \%$ & $3.46[1.33,8.98]$ \\
\hline Russell 1982 & $3 / 13$ & $8 / 20$ & 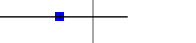 & $10.8 \%$ & $0.58[0.19,1.78]$ \\
\hline Walker 1983 & $9 / 20$ & $19 / 19$ & - & $34.1 \%$ & $0.46[0.29,0.75]$ \\
\hline Subtotal $(95 \%$ CI) & 124 & 125 & - & $100.0 \%$ & $1.04[0.83,1.32]$ \\
\hline \multicolumn{6}{|c|}{ Total events: 62 (FLUPHENAZINE DECANOATE), 58 (OTHER DEPOT NEUROLEPTICS) } \\
\hline \multicolumn{6}{|c|}{ Heterogeneity: $\mathrm{Chi}^{2}=20.14, \mathrm{df}=4(P=0.00047) ; 1^{2}=80 \%$} \\
\hline \multicolumn{6}{|c|}{ Test for overall effect: $Z=0.34(P=0.73)$} \\
\hline
\end{tabular}

\section{Analysis 3.24. Comparison 3 FLUPHENAZINE DECANOATE vs OTHER DEPOT NEUROLEPTICS, Outcome 24 SENSITIVITY ANALYSIS Global state: 2. Relapse.}

Review: Fluphenazine decanoate (depot) and enanthate for schizophrenia

Comparison: 3 FLUPHENAZINE DECANOATE vs OTHER DEPOT NEUROLEPTICS

Outcome: 24 SENSITIVITY ANALYSIS Global state: 2. Relapse

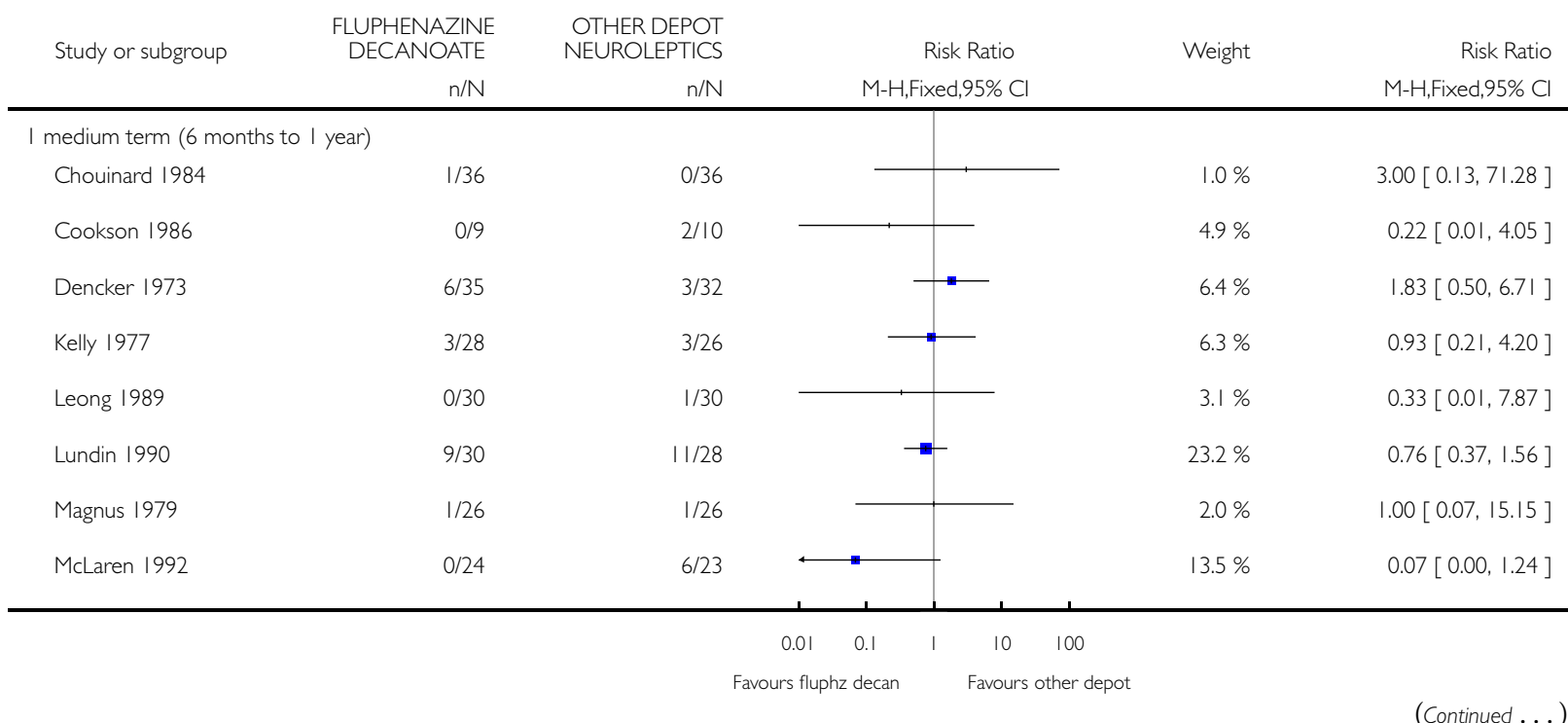




\begin{tabular}{|c|c|c|c|c|c|}
\hline \multirow[t]{2}{*}{ Study or subgroup } & $\begin{array}{l}\text { FLUPHENAZINE } \\
\text { DECANOATE }\end{array}$ & $\begin{array}{l}\text { OTHER DEPOT } \\
\text { NEUROLEPTICS }\end{array}$ & Risk Ratio & \multirow[t]{2}{*}{ Weight } & $\begin{array}{r}\text { (... Continued) } \\
\text { Risk Ratio }\end{array}$ \\
\hline & $\mathrm{n} / \mathrm{N}$ & $\mathrm{n} / \mathrm{N}$ & M-H,Fixed, $95 \% \mathrm{Cl}$ & & M-H,Fixed,95\% Cl \\
\hline Russell 1982 & $3 / 13$ & $2 / 20$ & $\longrightarrow$ & $3.2 \%$ & $2.31[0.44,11.98]$ \\
\hline Schlosberg 1978 & $9 / 30$ & $8 / 30$ & $\rightarrow$ & $16.3 \%$ & $1.13[0.50,2.52]$ \\
\hline Sharma 1991 & $6 / 29$ & $10 / 30$ & $\rightarrow$ & $20.0 \%$ & $0.62[0.26,1.49]$ \\
\hline Subtotal $(95 \% \mathrm{CI})$ & 290 & 291 & 4 & $100.0 \%$ & $0.82[0.56,1.18]$ \\
\hline \multicolumn{6}{|c|}{ Total events: 38 (FLUPHENAZINE DECANOATE), 47 (OTHER DEPOT NEUROLEPTICS) } \\
\hline \multicolumn{6}{|c|}{ Heterogeneity: $\mathrm{Ch}^{2}=8.59, \mathrm{df}=10(\mathrm{P}=0.57) ; \mathrm{I}^{2}=0.0 \%$} \\
\hline \multicolumn{6}{|c|}{ Test for overall effect: $Z=1.07(P=0.28)$} \\
\hline & & & 10 & 100 & \\
\hline & & & Favour: & ther depot & \\
\hline
\end{tabular}

\section{Analysis 4.I. Comparison 4 FLUPHENAZINE DECANOATE - DOSAGE STUDIES - HIGH DOSE vS} STANDARD, Outcome I Global state: I. Relapse (medium term - 6 months to I year).

Review: Fluphenazine decanoate (depot) and enanthate for schizophrenia

Comparison: 4 FLUPHENAZINE DECANOATE - DOSAGE STUDIES - HIGH DOSE vS STANDARD

Outcome: I Global state: I. Relapse (medium term - 6 months to I year)

\begin{tabular}{|c|c|c|c|c|c|}
\hline Study or subgroup & $\begin{array}{r}\text { FLUPHZ } \\
\text { DECAN HIGH } \\
\text { DOSE } \\
n / N\end{array}$ & $\begin{array}{r}\text { FLUPHZ } \\
\text { DECAN } \\
\text { STANRD DOSE } \\
\mathrm{n} / \mathrm{N}\end{array}$ & $\begin{array}{c}\text { Risk Ratio } \\
\text { M- } \\
\text { H,Random,95\% } \\
\mathrm{Cl}\end{array}$ & Weight & $\begin{array}{c}\text { Risk Ratio } \\
\text { M- } \\
\text { H,Random,95\% } \\
\text { Cl }\end{array}$ \\
\hline Kreisman 1988 & $30 / 66$ & $6 / 66$ & + & $57.2 \%$ & $5.00[2.23,11.21]$ \\
\hline McClelland 1976 & $2 / 25$ & $3 / 25$ & - & $42.8 \%$ & $0.67[0.12,3.65]$ \\
\hline Total (95\% CI) & 91 & 91 & 0 & $100.0 \%$ & $2.11[0.30,14.91]$ \\
\hline \multicolumn{6}{|c|}{ Total events: 32 (FLUPHZ DECAN HIGH DOSE), 9 (FLUPHZ DECAN STANRD DOSE) } \\
\hline \multicolumn{6}{|c|}{ Heterogeneity: $\operatorname{Tau}^{2}=1.57 ; \mathrm{Chi}^{2}=4.40, \mathrm{df}=\mathrm{I}(P=0.04) ; \mathrm{I}^{2}=77 \%$} \\
\hline \multicolumn{6}{|c|}{ Test for overall effect: $Z=0.75(P=0.45)$} \\
\hline Test for subgroup diff & : Not applicable & & & & \\
\hline
\end{tabular}

$0.0010 .01 \quad 0.1 \quad 1 \quad 10 \quad 100 \quad 1000$

Favours high dose Favours standard dose 
Analysis 4.2. Comparison 4 FLUPHENAZINE DECANOATE - DOSAGE STUDIES - HIGH DOSE vS STANDARD, Outcome 2 Global state: 2. Needing additional antispsychotic treatment (medium term - 6 months to I year).

Review: Fluphenazine decanoate (depot) and enanthate for schizophrenia

Comparison: 4 FLUPHENAZINE DECANOATE - DOSAGE STUDIES - HIGH DOSE vs STANDARD

Outcome: 2 Global state: 2. Needing additional antispsychotic treatment (medium term - 6 months to I year)

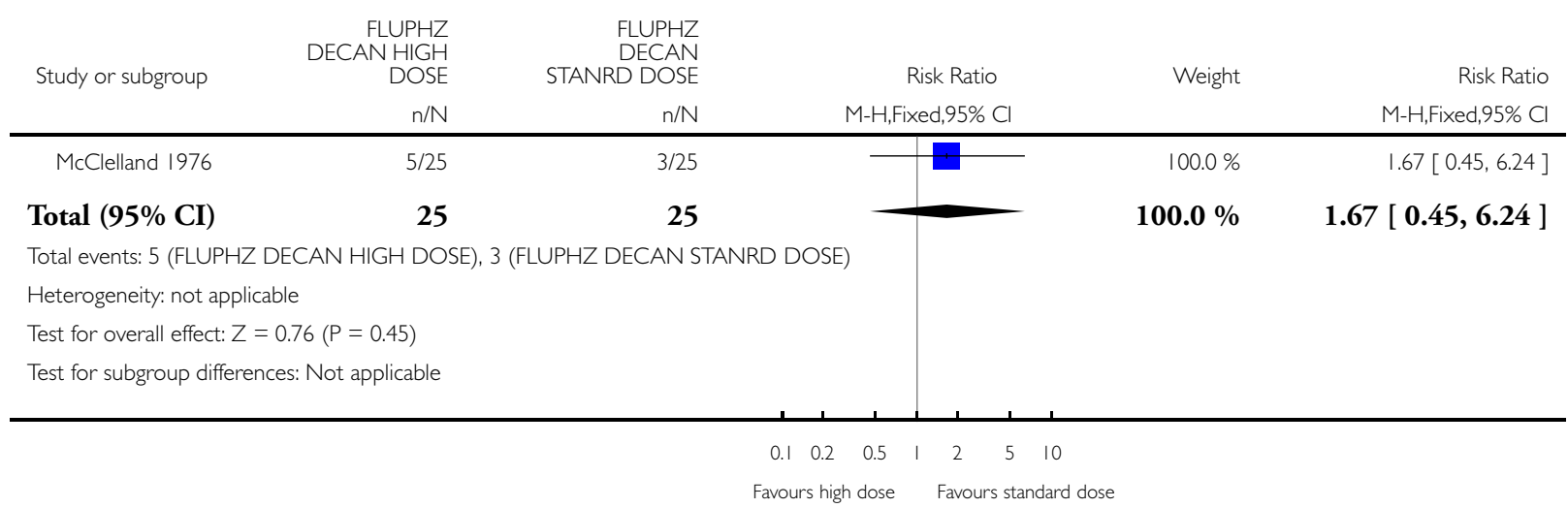


Analysis 4.3. Comparison 4 FLUPHENAZINE DECANOATE - DOSAGE STUDIES - HIGH DOSE vS STANDARD, Outcome 3 Global state: 3. Not improved (medium term - 6 months to I year).

Review: Fluphenazine decanoate (depot) and enanthate for schizophrenia

Comparison: 4 FLUPHENAZINE DECANOATE - DOSAGE STUDIES - HIGH DOSE vS STANDARD

Outcome: 3 Global state: 3. Not improved (medium term - 6 months to I year)

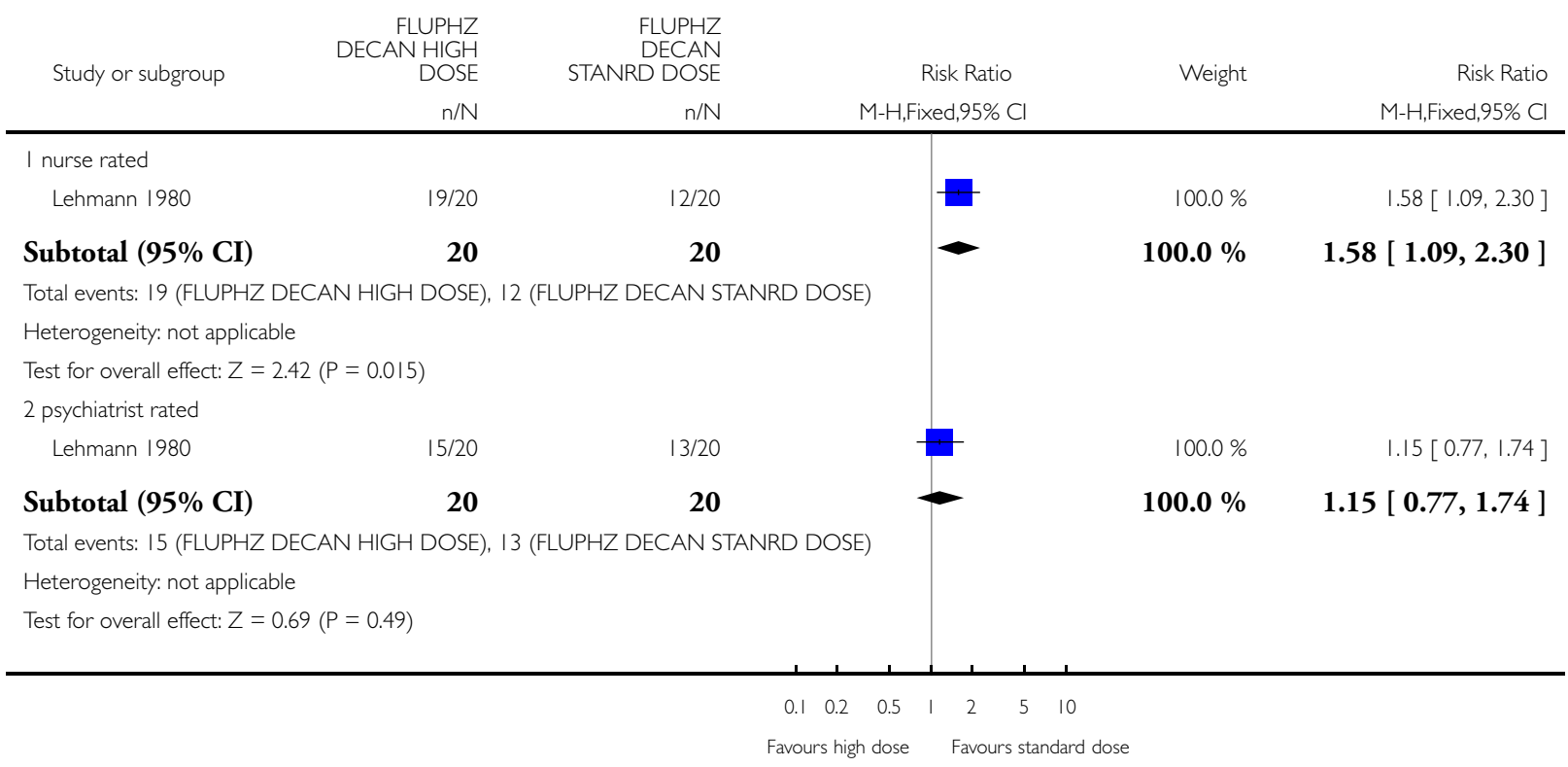


Analysis 4.4. Comparison 4 FLUPHENAZINE DECANOATE - DOSAGE STUDIES - HIGH DOSE vS STANDARD, Outcome 4 Leaving the study early (medium term - 6 months to I year).

Review: Fluphenazine decanoate (depot) and enanthate for schizophrenia

Comparison: 4 FLUPHENAZINE DECANOATE - DOSAGE STUDIES - HIGH DOSE vs STANDARD

Outcome: 4 Leaving the study early (medium term - 6 months to I year)

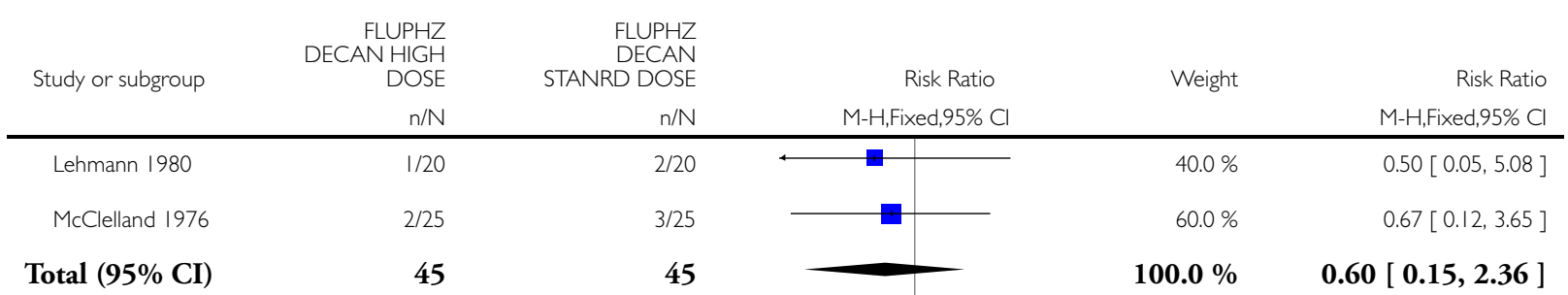

Total events: 3 (FLUPHZ DECAN HIGH DOSE), 5 (FLUPHZ DECAN STANRD DOSE)

Heterogeneity: Chi $^{2}=0.04, \mathrm{df}=\mathrm{I}(\mathrm{P}=0.84) ; \mathrm{I}^{2}=0.0 \%$

Test for overall effect: $Z=0.73(P=0.46)$

Test for subgroup differences: Not applicable

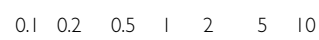

Favours high dose Favours standard dose

Analysis 4.5. Comparison 4 FLUPHENAZINE DECANOATE - DOSAGE STUDIES - HIGH DOSE vs STANDARD, Outcome 5 Mental state: BPRS endpoint scores (medium term - 6 months to $I$ year, high score = poor).

Review: Fluphenazine decanoate (depot) and enanthate for schizophrenia

Comparison: 4 FLUPHENAZINE DECANOATE - DOSAGE STUDIES - HIGH DOSE vs STANDARD

Outcome: 5 Mental state: BPRS endpoint scores (medium term - 6 months to I year, high score $=$ poor)

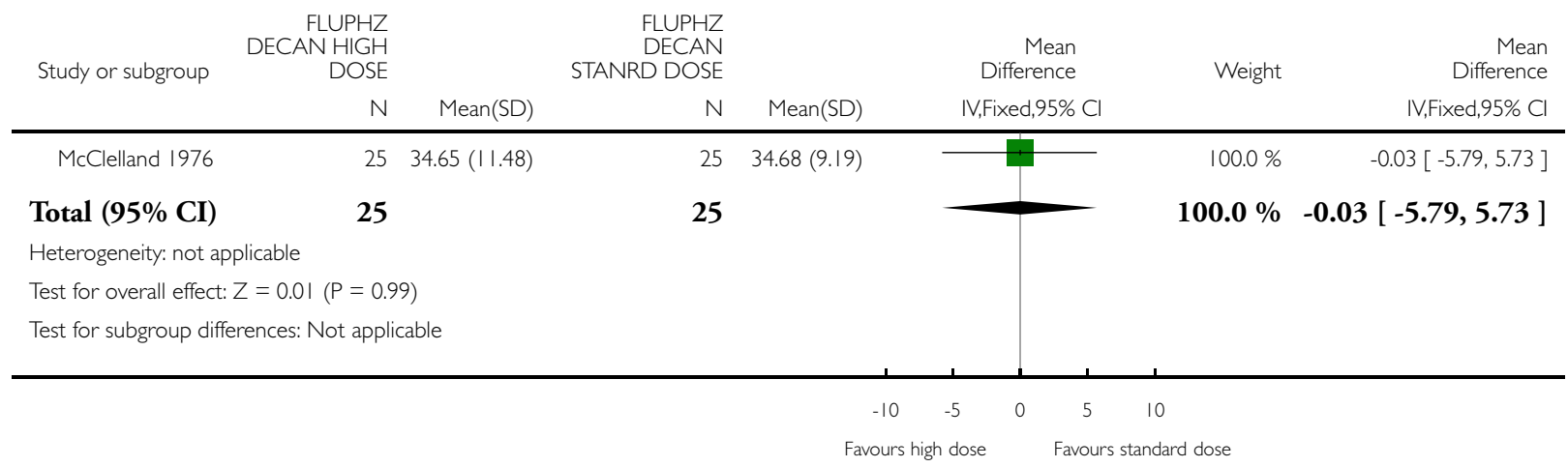


Analysis 4.6. Comparison 4 FLUPHENAZINE DECANOATE - DOSAGE STUDIES - HIGH DOSE vS STANDARD, Outcome 6 Adverse effects: Movement disorders - needing anticholinergic drugs (medium term 6 months to I year).

Review: Fluphenazine decanoate (depot) and enanthate for schizophrenia

Comparison: 4 FLUPHENAZINE DECANOATE - DOSAGE STUDIES - HIGH DOSE vs STANDARD

Outcome: 6 Adverse effects: Movement disorders - needing anticholinergic drugs (medium term - 6 months to I year)

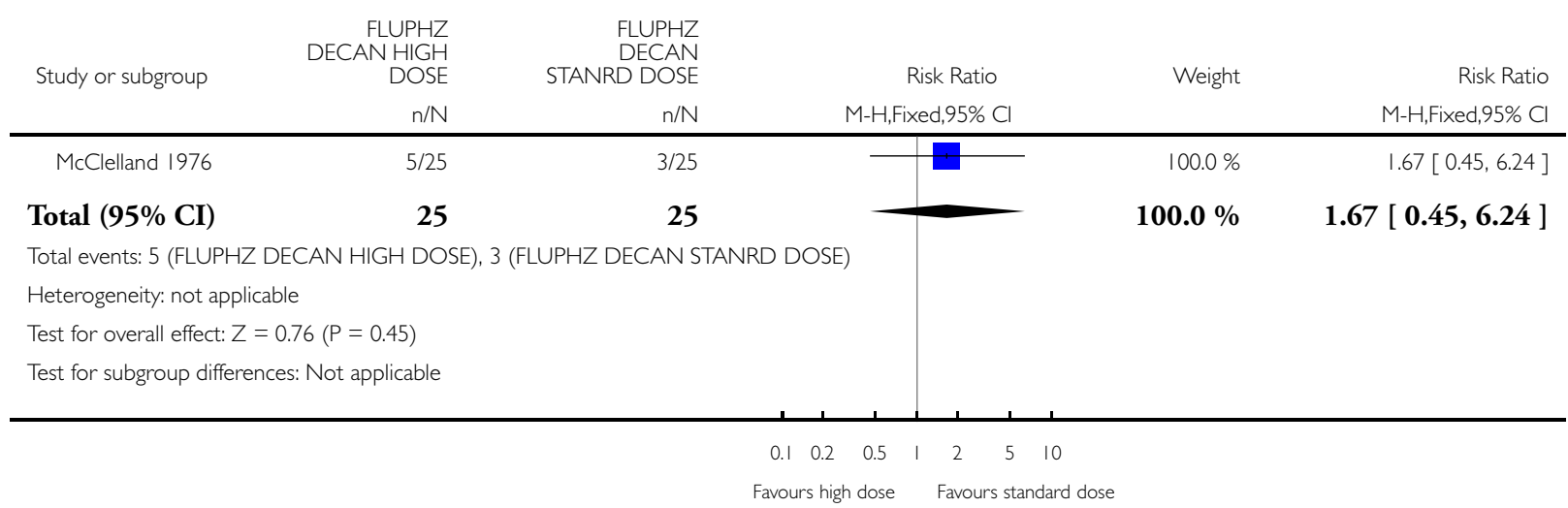




\section{Analysis 5.I. Comparison 5 FLUPHENAZINE DECANOATE - DOSAGE STUDIES - LOW DOSE vS STANDARD, Outcome I Global state: Relapse.}

Review: Fluphenazine decanoate (depot) and enanthate for schizophrenia

Comparison: 5 FLUPHENAZINE DECANOATE - DOSAGE STUDIES - LOW DOSE vs STANDARD

Outcome: I Global state: Relapse

\begin{tabular}{|c|c|c|c|c|c|}
\hline Study or subgroup & $\begin{array}{r}\text { FLUPHZ } \\
\text { DECAN LOW } \\
\text { DOSE } \\
\mathrm{n} / \mathrm{N} \\
\end{array}$ & $\begin{array}{r}\text { FLUPHZ } \\
\text { DECAN } \\
\text { STANRD DOSE }\end{array}$ & $\begin{array}{c}\text { Risk Ratio } \\
\text { M- } \\
\text { H,Random,95\% } \\
\text { Cl } \\
\end{array}$ & Weight & $\begin{array}{c}\text { Risk Ratio } \\
\text { M- } \\
\text { H,Random,95\% } \\
\text { Cl } \\
\end{array}$ \\
\hline \multicolumn{6}{|c|}{ I medium term (6 months to I year) } \\
\hline Kane 1983 & $26 / 62$ & $3 / 64$ & $\longrightarrow$ & $30.5 \%$ & $8.95[2.85,28.05]$ \\
\hline Kreisman 1988 & $30 / 66$ & $6 / 66$ & $\longrightarrow$ & $33.5 \%$ & $5.00[2.23,11.21]$ \\
\hline Schooler 1997 & $27 / 107$ & $27 / 106$ & & $36.0 \%$ & $0.99[0.62,1.57]$ \\
\hline Subtotal (95\% CI) & 235 & 236 & & $100.0 \%$ & $3.33[0.77,14.51]$ \\
\hline \multicolumn{6}{|c|}{ Total events: 83 (FLUPHZ DECAN LOW DOSE), 36 (FLUPHZ DECAN STANRD DOSE) } \\
\hline \multicolumn{6}{|c|}{ Heterogeneity: Tau $^{2}=1.51 ; \mathrm{Chi}^{2}=21.86, \mathrm{df}=2(\mathrm{P}=0.00002) ; \mathrm{I}^{2}=91 \%$} \\
\hline \multicolumn{6}{|c|}{ Test for overall effect: $Z=1.60(P=0.11)$} \\
\hline \multicolumn{6}{|c|}{2 longer term (more than I year) } \\
\hline Asarnow 1988 & $3 / 22$ & $1 / 14$ & $\longrightarrow$ & $12.2 \%$ & $1.91[0.22,16.58]$ \\
\hline Hogarty 1988 & $16 / 37$ & $14 / 33$ & & $66.2 \%$ & $1.02[0.59,1.75]$ \\
\hline Marder 1987 & $2 / 35$ & $6 / 31$ & & $21.6 \%$ & $0.30[0.06,1.36]$ \\
\hline Subtotal (95\% CI) & 94 & 78 & & $100.0 \%$ & $0.84[0.38,1.89]$ \\
\hline \multicolumn{6}{|c|}{ Total events: 2 I (FLUPHZ DECAN LOW DOSE), 2 I (FLUPHZ DECAN STANRD DOSE) } \\
\hline \multicolumn{6}{|c|}{ Heterogeneity: $\operatorname{Tau}^{2}=0.18 ; \mathrm{Chi}^{2}=2.78, \mathrm{df}=2(\mathrm{P}=0.25) ; \mathrm{I}^{2}=28 \%$} \\
\hline \multicolumn{6}{|c|}{ Test for overall effect: $Z=0.42(P=0.68)$} \\
\hline
\end{tabular}


Analysis 5.2. Comparison 5 FLUPHENAZINE DECANOATE - DOSAGE STUDIES - LOW DOSE vS STANDARD, Outcome 2 Leaving the study early.

Review: Fluphenazine decanoate (depot) and enanthate for schizophrenia

Comparison: 5 FLUPHENAZINE DECANOATE - DOSAGE STUDIES - LOW DOSE vs STANDARD

Outcome: 2 Leaving the study early

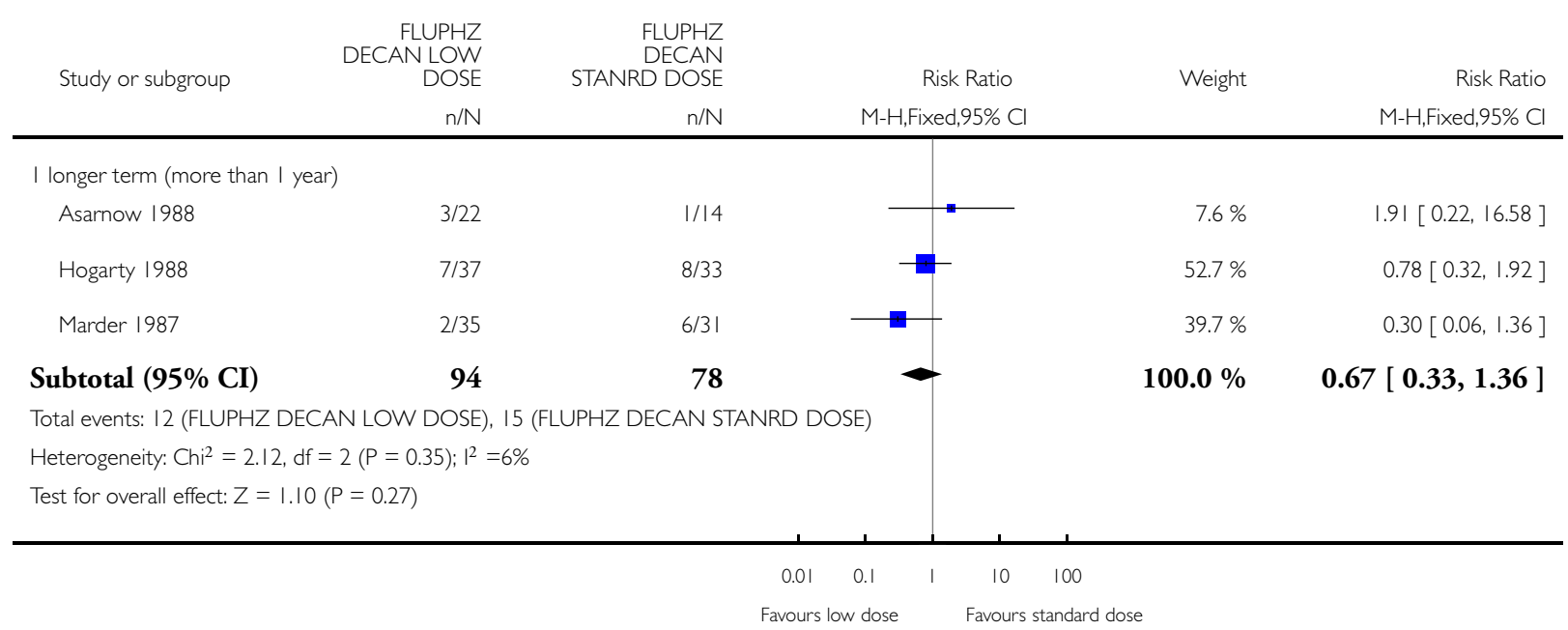


Analysis 5.3. Comparison 5 FLUPHENAZINE DECANOATE - DOSAGE STUDIES - LOW DOSE vS STANDARD, Outcome 3 Adverse effects: I. Movement disorders (medium term - 6 months to I year).

Review: Fluphenazine decanoate (depot) and enanthate for schizophrenia

Comparison: 5 FLUPHENAZINE DECANOATE - DOSAGE STUDIES - LOW DOSE vs STANDARD

Outcome: 3 Adverse effects: I. Movement disorders (medium term - 6 months to I year)

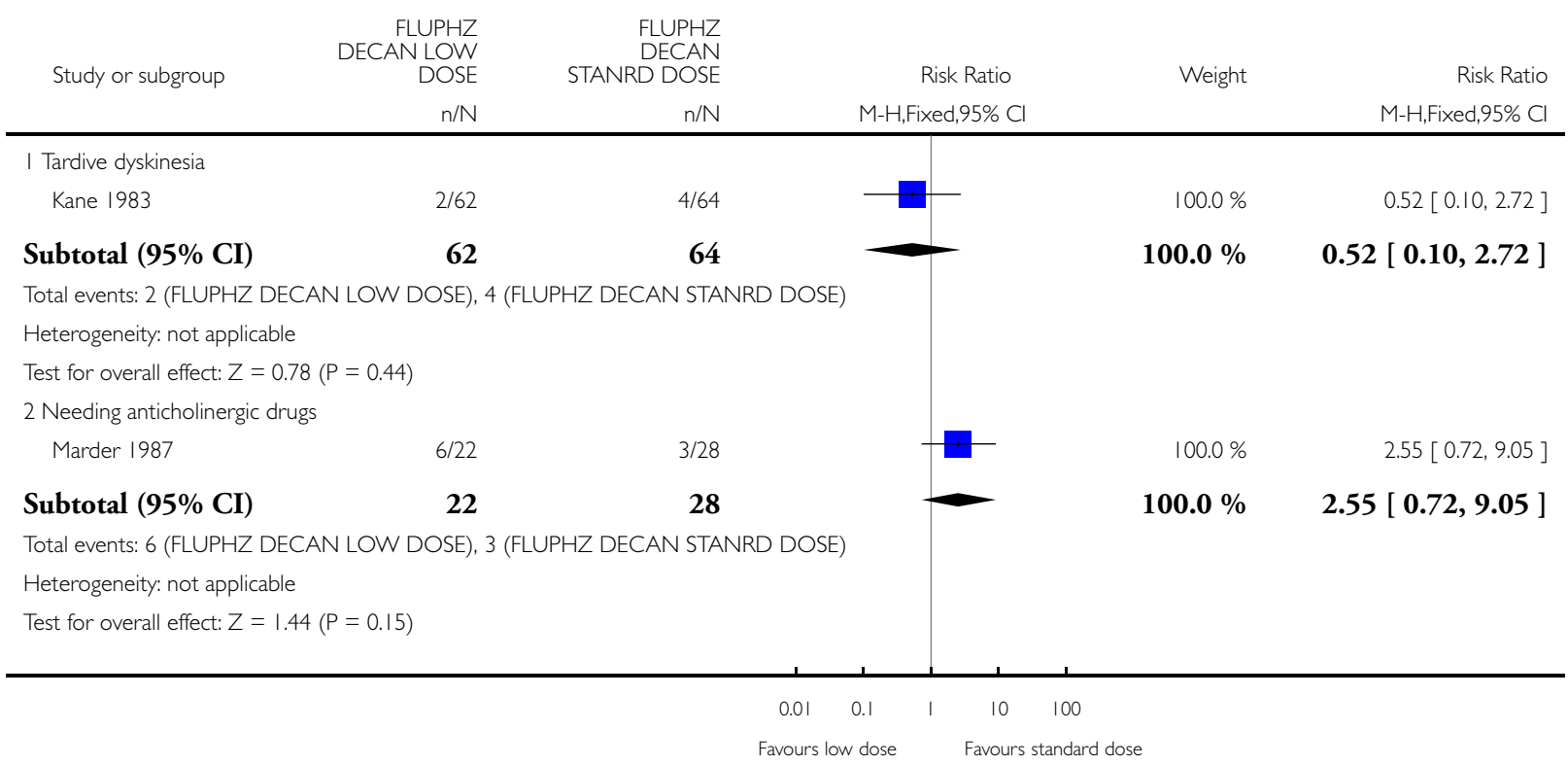

Analysis 5.4. Comparison 5 FLUPHENAZINE DECANOATE - DOSAGE STUDIES - LOW DOSE vS STANDARD, Outcome 4 Adverse effects: 2. Continuous data - skewed data (endpoint scores, high = poor).

Adverse effects: 2. Continuous data - skewed data (endpoint scores, high = poor)

\begin{tabular}{|c|c|c|c|c|c|}
\hline Study & Intervention & & mean & SD & $\mathbf{N}$ \\
\hline Kane 1983 & $\begin{array}{l}\text { Fluphenazine } \\
\text { (low dose) }\end{array}$ & decanoate & 0.52 & 1.00 & 62 \\
\hline Kane 1983 & $\begin{array}{l}\text { Fluphenazine } \\
\text { (standard dose) }\end{array}$ & decanoate & 1.04 & 2.42 & 64 \\
\hline
\end{tabular}


Analysis 6.I. Comparison 6 FLUPHENAZINE ENANTHATE vs PLACEBO, Outcome I Adverse effects: Movement disorders - general.

\begin{tabular}{|c|c|c|c|c|c|}
\hline \multicolumn{6}{|c|}{ Comparison: 6 FLUPHENAZINE ENANTHATE vs PLACEBO } \\
\hline \multirow[t]{2}{*}{ Study or subgroup } & $\begin{array}{l}\text { FLUPHENAZINE } \\
\text { ENANTHATE }\end{array}$ & PLACEBO & \multirow{2}{*}{$\begin{array}{c}\text { Risk Ratio } \\
\text { M-H,Fixed,95\% Cl }\end{array}$} & \multirow[t]{2}{*}{ Weight } & Risk Ratic \\
\hline & $\mathrm{n} / \mathrm{N}$ & $\mathrm{n} / \mathrm{N}$ & & & M-H,Fixed,95\% C \\
\hline \multicolumn{6}{|c|}{ I Needing anticholinergic drugs (short term - 6 weeks to 5 months) } \\
\hline Van Praag 1970 & $4 / 12$ & $0 / 13$ & 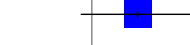 & $100.0 \%$ & $9.69[0.58,163.02]$ \\
\hline Subtotal $(95 \%$ CI $)$ & 12 & 13 & & $100.0 \%$ & $9.69[0.58,163.02]$ \\
\hline \multicolumn{6}{|c|}{ Total events: 4 (FLUPHENAZINE ENANTHATE), 0 (PLACEBO) } \\
\hline \multicolumn{6}{|c|}{ Heterogeneity: not applicable } \\
\hline \multicolumn{6}{|c|}{ Test for overall effect: $Z=1.58(P=0.11)$} \\
\hline
\end{tabular}

Analysis 7.I. Comparison 7 FLUPHENAZINE ENANTHATE vs ORAL NEUROLEPTICS, Outcome I Global state: No clinically important global change (immediate - 0 to 5 weeks).

Review: Fluphenazine decanoate (depot) and enanthate for schizophrenia

Comparison: 7 FLUPHENAZINE ENANTHATE vs ORAL NEUROLEPTICS

Outcome: I Global state: No clinically important global change (immediate - 0 to 5 weeks)

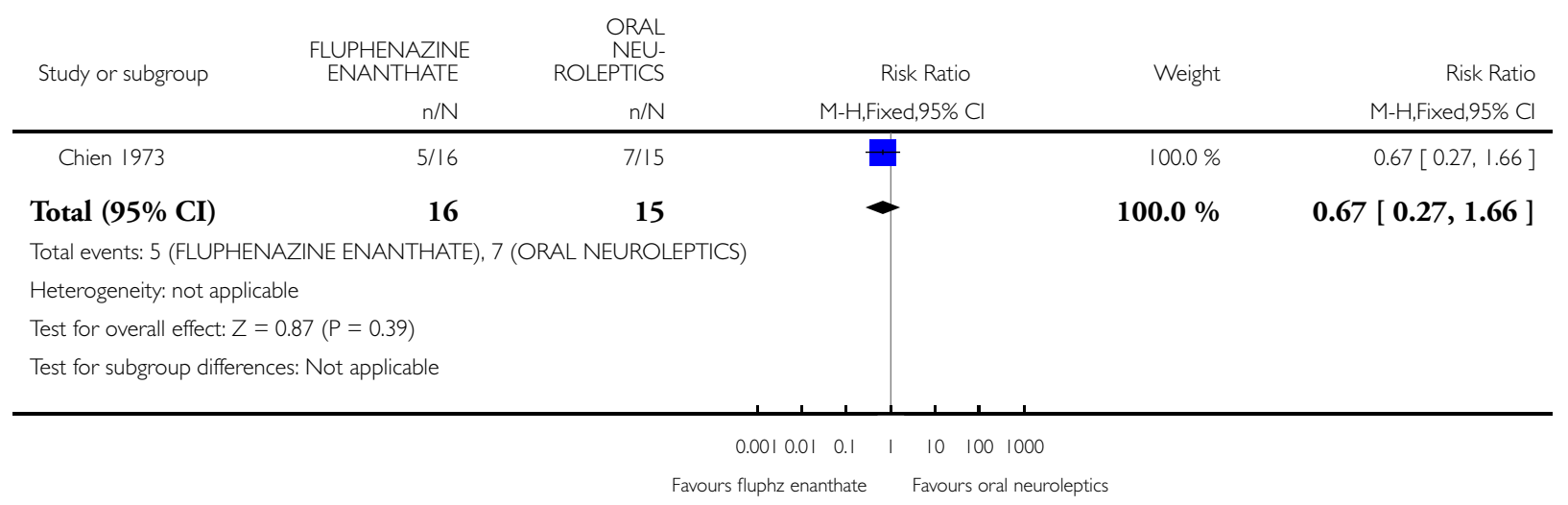


Analysis 7.2. Comparison 7 FLUPHENAZINE ENANTHATE vs ORAL NEUROLEPTICS, Outcome 2 Adverse effects: Movement disorders - general.

Review: Fluphenazine decanoate (depot) and enanthate for schizophrenia

Comparison: 7 FLUPHENAZINE ENANTHATE vs ORAL NEUROLEPTICS

Outcome: 2 Adverse effects: Movement disorders - general

\begin{tabular}{|c|c|c|c|c|c|}
\hline \multirow[t]{2}{*}{ Study or subgroup } & $\begin{array}{l}\text { FLUPHENAZINE } \\
\text { ENANTHATE }\end{array}$ & $\begin{array}{r}\text { ORAL } \\
\text { NEU- } \\
\text { ROLEPTICS }\end{array}$ & \multirow{2}{*}{$\begin{array}{c}\text { Risk Ratio } \\
\text { M-H,Fixed,95\% Cl }\end{array}$} & \multirow[t]{2}{*}{ Weight } & \multirow{2}{*}{$\begin{array}{r}\text { Risk Ratio } \\
\text { M-H,Fixed,95\% C }\end{array}$} \\
\hline & $\mathrm{n} / \mathrm{N}$ & $\mathrm{n} / \mathrm{N}$ & & & \\
\hline \multicolumn{6}{|c|}{ I movement disorders: immediate ( 0 to 5 weeks) } \\
\hline Chien 1973 & $5 / 16$ & $2 / 15$ & & $100.0 \%$ & $2.34[0.53,10.30]$ \\
\hline Subtotal (95\% CI) & 16 & 15 & & $100.0 \%$ & $2.34[0.53,10.30]$ \\
\hline \multicolumn{6}{|c|}{ Total events: 5 (FLUPHENAZINE ENANTHATE), 2 (ORAL NEUROLEPTICS) } \\
\hline \multicolumn{6}{|c|}{ Heterogeneity: not applicable } \\
\hline \multicolumn{6}{|c|}{ Test for overall effect: $Z=1.13(P=0.26)$} \\
\hline \multicolumn{6}{|c|}{2 side effects: immediate ( 0 to 5 weeks) } \\
\hline Chien 1973 & $9 / 16$ & $3 / 15$ & & $100.0 \%$ & $2.81[0.94,8.45]$ \\
\hline Subtotal (95\% CI) & 16 & 15 & & $100.0 \%$ & $2.81[0.94,8.45]$ \\
\hline \multicolumn{6}{|c|}{ Total events: 9 (FLUPHENAZINE ENANTHATE), 3 (ORAL NEUROLEPTICS) } \\
\hline \multicolumn{6}{|c|}{ Heterogeneity: not applicable } \\
\hline \multicolumn{6}{|c|}{ Test for overall effect: $Z=1.84(P=0.066)$} \\
\hline \multicolumn{6}{|c|}{3 parkinsonism: immediate ( 0 to 5 weeks) } \\
\hline Chien 1973 & $7 / 16$ & $1 / 15$ & & $100.0 \%$ & $6.56[0.91,47.21]$ \\
\hline Subtotal (95\% CI) & 16 & 15 & & $100.0 \%$ & $6.56[0.91,47.21]$ \\
\hline \multicolumn{6}{|c|}{ Total events: 7 (FLUPHENAZINE ENANTHATE), I (ORAL NEUROLEPTICS) } \\
\hline \multicolumn{6}{|c|}{ Heterogeneity: not applicable } \\
\hline \multicolumn{6}{|c|}{ Test for overall effect: $Z=1.87(P=0.062)$} \\
\hline
\end{tabular}


Analysis 8.I. Comparison 8 FLUPHENAZINE ENANTHATE vs OTHER DEPOT NEUROLEPTICS, Outcome I Global state: I. Needing additional antipsychotic treatment (6 months to I year).

Review: Fluphenazine decanoate (depot) and enanthate for schizophrenia

Comparison: 8 FLUPHENAZINE ENANTHATE vs OTHER DEPOT NEUROLEPTICS

Outcome: I Global state: I. Needing additional antipsychotic treatment (6 months to I year)

\begin{tabular}{|c|c|c|c|c|c|}
\hline \multirow[t]{2}{*}{ Study or subgroup } & $\begin{array}{l}\text { FLUPHENAZINE } \\
\text { ENANTHATE }\end{array}$ & $\begin{array}{l}\text { OTHER DEPOT } \\
\text { NEUROLEPTICS }\end{array}$ & Risk Ratio & \multirow[t]{2}{*}{ Weight } & \multirow{2}{*}{$\begin{array}{r}\text { Risk Ratio } \\
\text { M-H,Fixed,95\% Cl }\end{array}$} \\
\hline & $n / N$ & $n / N$ & M-H,Fixed,95\% Cl & & \\
\hline Albert 1980 & $4 / 11$ & $13 / 22$ & $+1-10$ & $59.1 \%$ & $0.62[0.26,1.45]$ \\
\hline Chouinard 1978 & $2 / 16$ & $6 / 16$ & - & $40.9 \%$ & $0.33[0.08,1.41]$ \\
\hline Total $(95 \%$ CI $)$ & 27 & 38 & & $100.0 \%$ & $0.50[0.24,1.05]$ \\
\hline \multicolumn{6}{|c|}{ Total events: 6 (FLUPHENAZINE ENANTHATE), 19 (OTHER DEPOT NEUROLEPTICS) } \\
\hline \multicolumn{6}{|c|}{ Heterogeneity: $\mathrm{Chi}^{2}=0.53, \mathrm{df}=\mathrm{I}(\mathrm{P}=0.47) ; \mathrm{I}^{2}=0.0 \%$} \\
\hline \multicolumn{6}{|c|}{ Test for overall effect: $Z=1.84(P=0.066)$} \\
\hline Test for subgroup diff & s: Not applicable & & & & \\
\hline
\end{tabular}

\section{$\begin{array}{lllllll}0.1 & 0.2 & 0.5 & \mid & 2 & 5 & 10\end{array}$}

Favours fluphz enanthate Favours other depot neuro 


\section{Analysis 8.2. Comparison 8 FLUPHENAZINE ENANTHATE vs OTHER DEPOT NEUROLEPTICS,}

\section{Outcome 2 Global state: 2. Relapse.}

Review: Fluphenazine decanoate (depot) and enanthate for schizophrenia

Comparison: 8 FLUPHENAZINE ENANTHATE vs OTHER DEPOT NEUROLEPTICS

Outcome: 2 Global state: 2. Relapse

\begin{tabular}{|c|c|c|c|c|c|}
\hline \multirow[t]{2}{*}{ Study or subgroup } & $\begin{array}{l}\text { FLUPHENAZINE } \\
\text { ENANTHATE }\end{array}$ & \multirow{2}{*}{$\begin{array}{r}\text { OTHER DEPOT } \\
\text { NEUROLEPTICS } \\
\mathrm{n} / \mathrm{N}\end{array}$} & Risk Ratio & \multirow[t]{2}{*}{ Weight } & \multirow{2}{*}{$\begin{array}{r}\text { Risk Ratio } \\
\mathrm{M}-\mathrm{H}, \text { Fixed,95\% Cl }\end{array}$} \\
\hline & $\mathrm{n} / \mathrm{N}$ & & M-H,Fixed,95\% Cl & & \\
\hline \multicolumn{6}{|c|}{ I short term (6 weeks to 5 months) } \\
\hline Malm 1974 & $6 / 26$ & $3 / 31$ & & $100.0 \%$ & $2.38[0.66,8.61]$ \\
\hline Subtotal (95\% CI) & 26 & 31 & & $100.0 \%$ & $2.38[0.66,8.61]$ \\
\hline \multicolumn{6}{|c|}{ Total events: 6 (FLUPHENAZINE ENANTHATE), 3 (OTHER DEPOT NEUROLEPTICS) } \\
\hline \multicolumn{6}{|c|}{ Heterogeneity: not applicable } \\
\hline \multicolumn{6}{|c|}{ Test for overall effect: $Z=1.33(P=0.18)$} \\
\hline \multicolumn{6}{|c|}{2 medium term ( 6 months to I year) } \\
\hline Chouinard 1978 & $1 / 16$ & $3 / 16$ & & $100.0 \%$ & $0.33[0.04,2.87]$ \\
\hline Subtotal (95\% CI) & 16 & 16 & & $100.0 \%$ & $0.33[0.04,2.87]$ \\
\hline \multicolumn{6}{|c|}{ Total events: I (FLUPHENAZINE ENANTHATE), 3 (OTHER DEPOT NEUROLEPTICS) } \\
\hline \multicolumn{6}{|c|}{ Heterogeneity: not applicable } \\
\hline Test for overall effect: $Z=$ & $(P=0.32)$ & & & & \\
\hline
\end{tabular}




\section{Analysis 8.3. Comparison 8 FLUPHENAZINE ENANTHATE vs OTHER DEPOT NEUROLEPTICS, Outcome 3 Leaving the study early.}

Review: Fluphenazine decanoate (depot) and enanthate for schizophrenia

Comparison: 8 FLUPHENAZINE ENANTHATE vs OTHER DEPOT NEUROLEPTICS

Outcome: 3 Leaving the study early

\begin{tabular}{|c|c|c|c|c|c|}
\hline \multirow[t]{2}{*}{ Study or subgroup } & $\begin{array}{c}\text { FLUPHENAZINE } \\
\text { ENANTHATE }\end{array}$ & $\begin{array}{l}\text { OTHER DEPOT } \\
\text { NEUROLEPTICS }\end{array}$ & Risk Ratio & \multirow[t]{2}{*}{ Weight } & \multirow{2}{*}{$\begin{array}{r}\text { Risk Ratio } \\
\text { M-H,Fixed,95\% Cl }\end{array}$} \\
\hline & $n / N$ & $\mathrm{n} / \mathrm{N}$ & M-H,Fixed,95\% Cl & & \\
\hline \multicolumn{6}{|c|}{ I immediate (0 to 5 weeks) } \\
\hline Jain 1975 & $1 / 15$ & $11 / 15$ & & $100.0 \%$ & $0.09[0.01,0.62]$ \\
\hline Subtotal (95\% CI) & 15 & 15 & & $100.0 \%$ & $0.09[0.01,0.62]$ \\
\hline \multicolumn{6}{|c|}{ Total events: I (FLUPHENAZINE ENANTHATE), I I (OTHER DEPOT NEUROLEPTICS) } \\
\hline \multicolumn{6}{|c|}{ Heterogeneity: not applicable } \\
\hline \multicolumn{6}{|c|}{ Test for overall effect: $Z=2.45(P=0.014)$} \\
\hline \multicolumn{6}{|c|}{2 short term (6 weeks to 5 months) } \\
\hline Malm 1974 & $6 / 26$ & $3 / 31$ & & $100.0 \%$ & $2.38[0.66,8.61]$ \\
\hline Subtotal (95\% CI) & 26 & 31 & & $100.0 \%$ & $2.38[0.66,8.61]$ \\
\hline \multicolumn{6}{|c|}{ Total events: 6 (FLUPHENAZINE ENANTHATE), 3 (OTHER DEPOT NEUROLEPTICS) } \\
\hline \multicolumn{6}{|c|}{ Heterogeneity: not applicable } \\
\hline \multicolumn{6}{|c|}{ Test for overall effect: $Z=1.33(P=0.18)$} \\
\hline \multicolumn{6}{|c|}{3 medium term (6 months to I year) } \\
\hline Chouinard 1978 & $1 / 16$ & $3 / 16$ & & $100.0 \%$ & $0.33[0.04,2.87]$ \\
\hline Subtotal (95\% CI) & 16 & 16 & & $100.0 \%$ & $0.33[0.04,2.87]$ \\
\hline \multicolumn{6}{|c|}{ Total events: I (FLUPHENAZINE ENANTHATE), 3 (OTHER DEPOT NEUROLEPTICS) } \\
\hline \multicolumn{6}{|c|}{ Heterogeneity: not applicable } \\
\hline \multicolumn{6}{|c|}{ Test for overall effect: $Z=1.00(P=0.32)$} \\
\hline
\end{tabular}


Analysis 8.4. Comparison 8 FLUPHENAZINE ENANTHATE vs OTHER DEPOT NEUROLEPTICS, Outcome 4 Mental state: I. BPRS - endpoint scores (medium term - 6 months to I year) (high score = poor).

Review: Fluphenazine decanoate (depot) and enanthate for schizophrenia

Comparison: 8 FLUPHENAZINE ENANTHATE vs OTHER DEPOT NEUROLEPTICS

Outcome: 4 Mental state: I. BPRS - endpoint scores (medium term - 6 months to I year) (high score = poor)

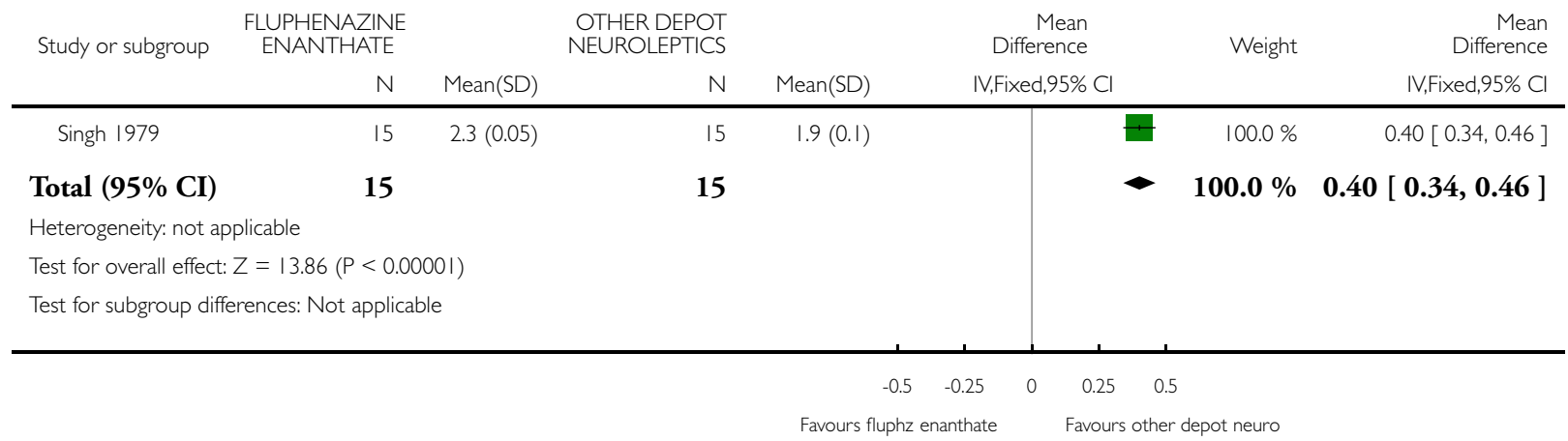

Analysis 8.5. Comparison 8 FLUPHENAZINE ENANTHATE vs OTHER DEPOT NEUROLEPTICS, Outcome 5 Mental state: 2. Depression (medium term - 6 months to I year).

Review: Fluphenazine decanoate (depot) and enanthate for schizophrenia

Comparison: 8 FLUPHENAZINE ENANTHATE vs OTHER DEPOT NEUROLEPTICS

Outcome: 5 Mental state: 2. Depression (medium term - 6 months to I year)

\begin{tabular}{|c|c|c|c|c|c|}
\hline \multirow[t]{2}{*}{ Study or subgroup } & $\begin{array}{l}\text { FLUPHENAZINE } \\
\text { ENANTHATE }\end{array}$ & $\begin{array}{l}\text { OTHER DEPOT } \\
\text { NEUROLEPTICS }\end{array}$ & Risk Ratio & \multirow[t]{2}{*}{ Weight } & \multirow{2}{*}{$\begin{array}{r}\text { Risk Ratio } \\
\text { M-H,Fixed,95\% Cl }\end{array}$} \\
\hline & $\mathrm{n} / \mathrm{N}$ & $\mathrm{n} / \mathrm{N}$ & M-H,Fixed,95\% Cl & & \\
\hline Singh 1979 & $3 / 15$ & $0 / 15$ & r & $100.0 \%$ & $7.00[0.39,124.83]$ \\
\hline Total (95\% CI) & 15 & 15 & & $100.0 \%$ & $7.00[0.39,124.83]$ \\
\hline \multicolumn{6}{|c|}{ Total events: 3 (FLUPHENAZINE ENANTHATE), 0 (OTHER DEPOT NEUROLEPTICS) } \\
\hline \multicolumn{6}{|c|}{ Heterogeneity: not applicable } \\
\hline \multicolumn{6}{|c|}{ Test for overall effect: $Z=1.32(P=0.19)$} \\
\hline Test for subgroup diff & es: Not applicable & & & & \\
\hline
\end{tabular}

$$
\begin{array}{cccc}
0.0010 .01 \quad 0.1 \quad 1 \quad 10 \quad 1001000 \\
\text { Favours fluphz enanthate } \quad \text { Favours other depot neuro }
\end{array}
$$


Analysis 8.6. Comparison 8 FLUPHENAZINE ENANTHATE vs OTHER DEPOT NEUROLEPTICS, Outcome 6 Adverse effects: Ia. Movement disorders - general (medium term - 6 months to I year).

Review: Fluphenazine decanoate (depot) and enanthate for schizophrenia

Comparison: 8 FLUPHENAZINE ENANTHATE vs OTHER DEPOT NEUROLEPTICS

Outcome: 6 Adverse effects: Ia. Movement disorders - general (medium term - 6 months to I year)

\begin{tabular}{|c|c|c|c|c|c|}
\hline \multirow[t]{2}{*}{ Study or subgroup } & $\begin{array}{c}\text { FLUPHENAZINE } \\
\text { ENANTHATE }\end{array}$ & $\begin{array}{l}\text { OTHER DEPOT } \\
\text { NEUROLEPTICS }\end{array}$ & Risk Ratio & \multirow[t]{2}{*}{ Weight } & \multirow{2}{*}{$\begin{array}{r}\text { Risk Ratio } \\
\text { M-H,Fixed,95\% Cl}\end{array}$} \\
\hline & $\mathrm{n} / \mathrm{N}$ & $\mathrm{n} / \mathrm{N}$ & M-H,Fixed,95\% Cl & & \\
\hline Albert 1980 & $0 / 11$ & $1 / 22$ & $\longleftrightarrow$ & $14.6 \%$ & $0.64[0.03,14.52]$ \\
\hline Singh 1979 & $10 / 15$ & $6 / 15$ & & $85.4 \%$ & $1.67[0.81,3.41]$ \\
\hline Total $(95 \%$ CI $)$ & 26 & 37 & & $100.0 \%$ & $1.52[0.75,3.07]$ \\
\hline \multicolumn{6}{|c|}{ Total events: IO (FLUPHENAZINE ENANTHATE), 7 (OTHER DEPOT NEUROLEPTICS) } \\
\hline \multicolumn{6}{|c|}{ Heterogeneity: $\mathrm{Chi}^{2}=0.36, \mathrm{df}=\mathrm{I}(\mathrm{P}=0.55) ; \mathrm{I}^{2}=0.0 \%$} \\
\hline \multicolumn{6}{|c|}{ Test for overall effect: $Z=1.16(P=0.25)$} \\
\hline Test for subgroup diff & s: Not applicable & & & & \\
\hline
\end{tabular}

\section{$\begin{array}{lllllll}0.1 & 0.2 & 0.5 & 1 & 2 & 5 & 10\end{array}$}

Favours fluphz enanthate Favours other depot neuro 
Analysis 8.7. Comparison 8 FLUPHENAZINE ENANTHATE vs OTHER DEPOT NEUROLEPTICS, Outcome 7 Adverse effects: Ib. Movement disorders - needing additional anticholinergic drugs.

Review: Fluphenazine decanoate (depot) and enanthate for schizophrenia

Comparison: 8 FLUPHENAZINE ENANTHATE vs OTHER DEPOT NEUROLEPTICS

Outcome: 7 Adverse effects: Ib. Movement disorders - needing additional anticholinergic drugs

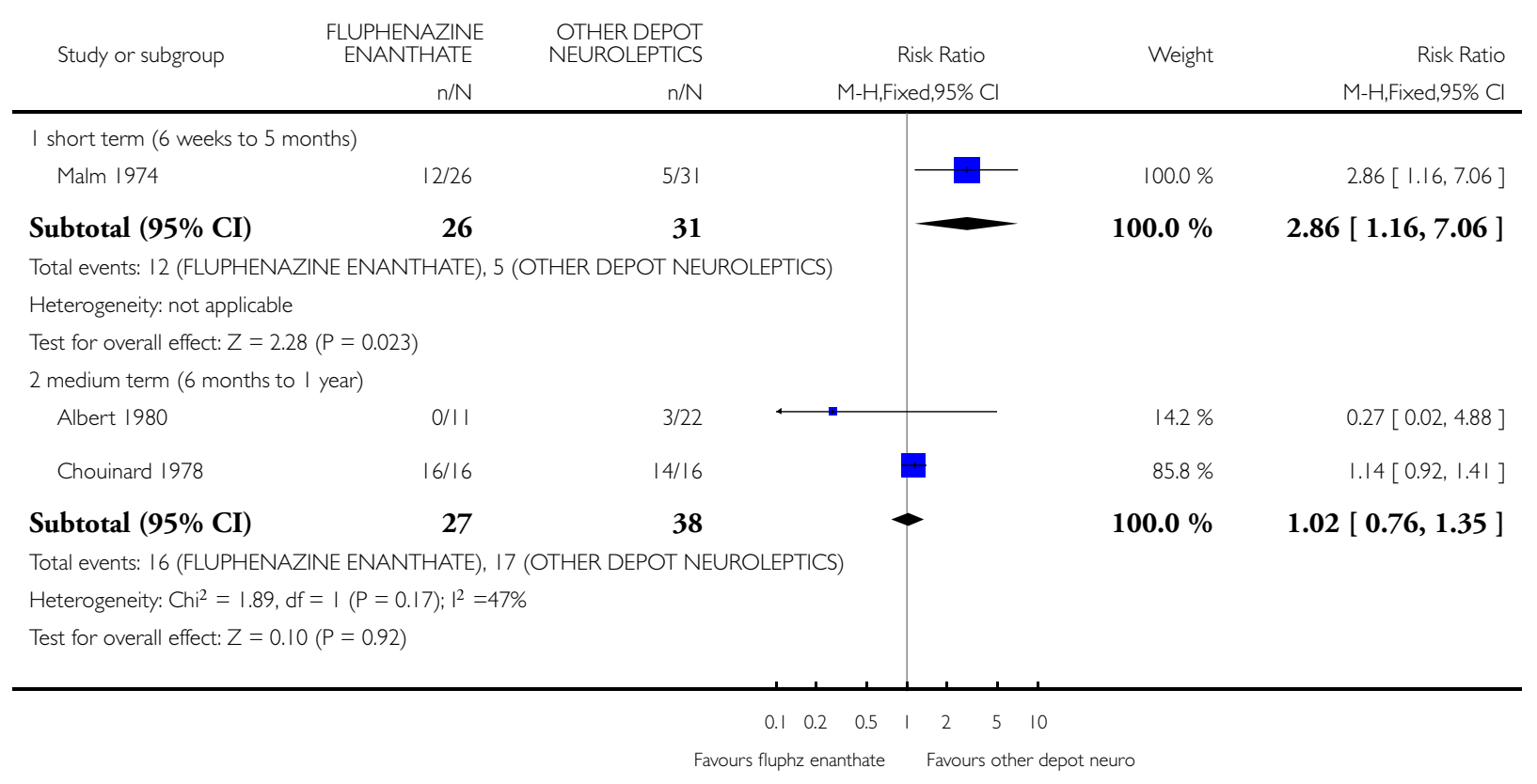


Analysis 8.8. Comparison 8 FLUPHENAZINE ENANTHATE vs OTHER DEPOT NEUROLEPTICS, Outcome 8 Adverse effects: Ic. Movement disorders - tardive dyskinesia: medium term (6 months to I year).

Review: Fluphenazine decanoate (depot) and enanthate for schizophrenia

Comparison: 8 FLUPHENAZINE ENANTHATE vs OTHER DEPOT NEUROLEPTICS

Outcome: 8 Adverse effects: Ic. Movement disorders - tardive dyskinesia: medium term (6 months to I year)

\begin{tabular}{|c|c|c|c|c|c|}
\hline \multirow[t]{2}{*}{ Study or subgroup } & $\begin{array}{l}\text { FLUPHENAZINE } \\
\text { ENANTHATE }\end{array}$ & $\begin{array}{l}\text { OTHER DEPOT } \\
\text { NEUROLEPTICS }\end{array}$ & Risk Ratio & Weight & Risk Ratio \\
\hline & $\mathrm{n} / \mathrm{N}$ & $\mathrm{n} / \mathrm{N}$ & M-H,Fixed,95\% Cl & & M-H,Fixed,95\% Cl \\
\hline Chouinard 1978 & $8 / 16$ & $9 / 16$ & -15 & $100.0 \%$ & $0.89[0.46,1.71]$ \\
\hline Total (95\% CI) & 16 & 16 & & $100.0 \%$ & $0.89[0.46,1.71]$ \\
\hline
\end{tabular}

Total events: 8 (FLUPHENAZINE ENANTHATE), 9 (OTHER DEPOT NEUROLEPTICS)

Heterogeneity: not applicable

Test for overall effect: $Z=0.35(P=0.72)$

Test for subgroup differences: Not applicable

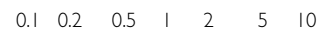

Favours fluphz enanthate Favours other depot neuro

Analysis 8.9. Comparison 8 FLUPHENAZINE ENANTHATE vs OTHER DEPOT NEUROLEPTICS, Outcome 9 Adverse effects: Id. Movement disorders - tremor (medium term - 6 months to I year).

Review: Fluphenazine decanoate (depot) and enanthate for schizophrenia

Comparison: 8 FLUPHENAZINE ENANTHATE vs OTHER DEPOT NEUROLEPTICS

Outcome: 9 Adverse effects: Id. Movement disorders - tremor (medium term - 6 months to I year)

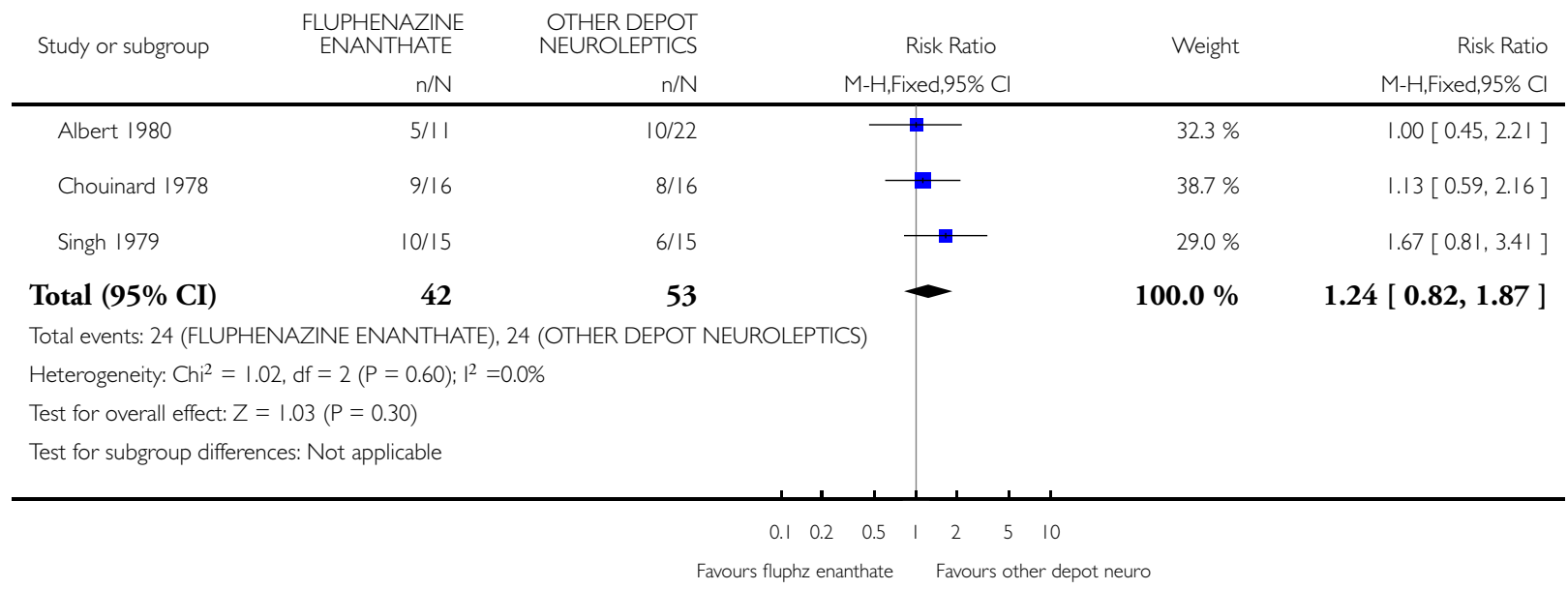

Fluphenazine decanoate (depot) and enanthate for schizophrenia (Review)

Copyright @ 2016 The Cochrane Collaboration. Published by John Wiley \& Sons, Ltd. 


\section{Analysis 8.10. Comparison 8 FLUPHENAZINE ENANTHATE vs OTHER DEPOT NEUROLEPTICS,}

Outcome 10 Adverse effects: 2. Blurred vision (medium term - 6 months to I year).

Review: Fluphenazine decanoate (depot) and enanthate for schizophrenia

Comparison: 8 FLUPHENAZINE ENANTHATE vs OTHER DEPOT NEUROLEPTICS

Outcome: 10 Adverse effects: 2. Blurred vision (medium term - 6 months to I year)

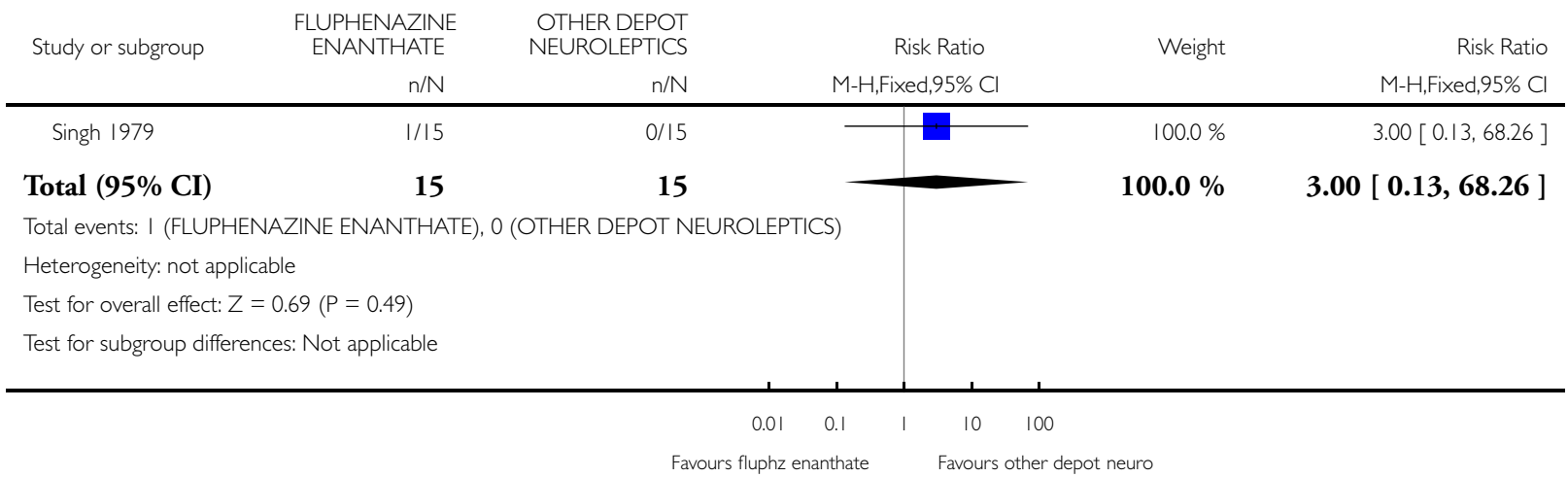

Analysis 8.I I. Comparison 8 FLUPHENAZINE ENANTHATE vs OTHER DEPOT NEUROLEPTICS, Outcome II Adverse effects: 3. Dry mouth (medium term - 6 months to I year).

Review: Fluphenazine decanoate (depot) and enanthate for schizophrenia

Comparison: 8 FLUPHENAZINE ENANTHATE vs OTHER DEPOT NEUROLEPTICS

Outcome: II Adverse effects: 3. Dry mouth (medium term - 6 months to I year)

\begin{tabular}{|c|c|c|c|c|c|}
\hline \multirow[t]{2}{*}{ Study or subgroup } & $\begin{array}{l}\text { FLUPHENAZINE } \\
\text { ENANTHATE }\end{array}$ & $\begin{array}{l}\text { OTHER DEPOT } \\
\text { NEUROLEPTICS }\end{array}$ & Risk Ratio & \multirow[t]{2}{*}{ Weight } & \multirow{2}{*}{$\begin{array}{r}\text { Risk Ratio } \\
\text { M-H,Fixed,95\% Cl }\end{array}$} \\
\hline & $\mathrm{n} / \mathrm{N}$ & $\mathrm{n} / \mathrm{N}$ & M-H,Fixed,95\% Cl & & \\
\hline Chouinard 1978 & $4 / 16$ & $9 / 16$ & $\square$ & $90.0 \%$ & $0.44[0.17,1.15]$ \\
\hline Singh 1979 & $4 / 15$ & $1 / 15$ & & $10.0 \%$ & $4.00[0.50,31.74]$ \\
\hline Total $(95 \% \mathrm{CI})$ & 31 & 31 & & $100.0 \%$ & $0.80[0.36,1.76]$ \\
\hline \multicolumn{6}{|c|}{ Total events: 8 (FLUPHENAZINE ENANTHATE), I0 (OTHER DEPOT NEUROLEPTICS) } \\
\hline \multicolumn{6}{|c|}{ Heterogeneity: Chi $^{2}=3.78, d f=I(P=0.05) ;\left.\right|^{2}=74 \%$} \\
\hline \multicolumn{6}{|c|}{ Test for overall effect: $Z=0.55(P=0.58)$} \\
\hline \multicolumn{6}{|c|}{ Test for subgroup differences: Not applicable } \\
\hline
\end{tabular}


Analysis 9.I. Comparison 9 FLUPHENAZINE ENANTHATE - DOSAGE STUDIES - LOW DOSE vS INTERMEDIATE/HIGH DOSE, Outcome I Global state: Relapse (short term - 6 weeks to 5 months).

Review: Fluphenazine decanoate (depot) and enanthate for schizophrenia

Comparison: 9 FLUPHENAZINE ENANTHATE - DOSAGE STUDIES - LOW DOSE vs INTERMEDIATE/HIGH DOSE

Outcome: I Global state: Relapse (short term - 6 weeks to 5 months)

\begin{tabular}{|c|c|c|c|c|c|}
\hline \multirow[t]{2}{*}{ Study or subgroup } & $\begin{array}{r}\text { FLUPHZINE } \\
\text { ENANTH LOW } \\
\text { DOSE }\end{array}$ & $\begin{array}{r}\text { FLUPHZ ENAN } \\
\text { INT/HIGH DOSE }\end{array}$ & Risk Ratio & \multirow[t]{2}{*}{ Weight } & \multirow{2}{*}{$\begin{array}{r}\text { Risk Ratio } \\
\text { M-H,Fixed,95\% Cl }\end{array}$} \\
\hline & $\mathrm{n} / \mathrm{N}$ & $\mathrm{n} / \mathrm{N}$ & M-H,Fixed,95\% Cl & & \\
\hline Goldstein 1978 & $|8 / 5|$ & $2 / 53$ & 7 & $100.0 \%$ & $9.35[2.28,38.29]$ \\
\hline Total (95\% CI) & 51 & 53 & $\longrightarrow$ & $100.0 \%$ & $9.35[2.28,38.29]$ \\
\hline
\end{tabular}

Total events: 18 (FLUPHZINE ENANTH LOW DOSE), 2 (FLUPHZ ENAN INT/HIGH DOSE)

Heterogeneity: not applicable

Test for overall effect: $Z=3.11(P=0.0019)$

Test for subgroup differences: Not applicable

Analysis 9.2. Comparison 9 FLUPHENAZINE ENANTHATE - DOSAGE STUDIES - LOW DOSE vS INTERMEDIATE/HIGH DOSE, Outcome 2 Leaving the study early (short term - 6 weeks to 5 months).

Review: Fluphenazine decanoate (depot) and enanthate for schizophrenia

Comparison: 9 FLUPHENAZINE ENANTHATE - DOSAGE STUDIES - LOW DOSE vs INTERMEDIATE/HIGH DOSE

Outcome: 2 Leaving the study early (short term - 6 weeks to 5 months)

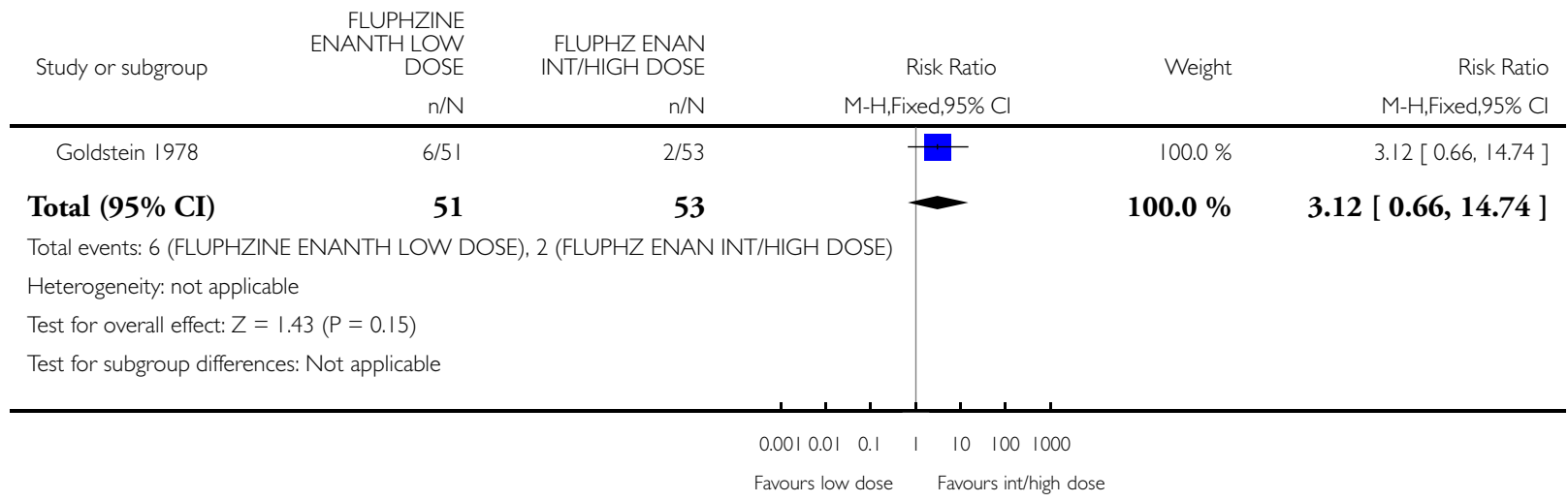




\section{Analysis 10.1. Comparison 10 FLUPHENAZINE DECANAOTE vs FLUPHENAZINE ENANTHATE,}

Outcome I Global state: I. Needing additional antipsychotic treatment.

Review: Fluphenazine decanoate (depot) and enanthate for schizophrenia

Comparison: 10 FLUPHENAZINE DECANAOTE vs FLUPHENAZINE ENANTHATE

Outcome: I Global state: I. Needing additional antipsychotic treatment

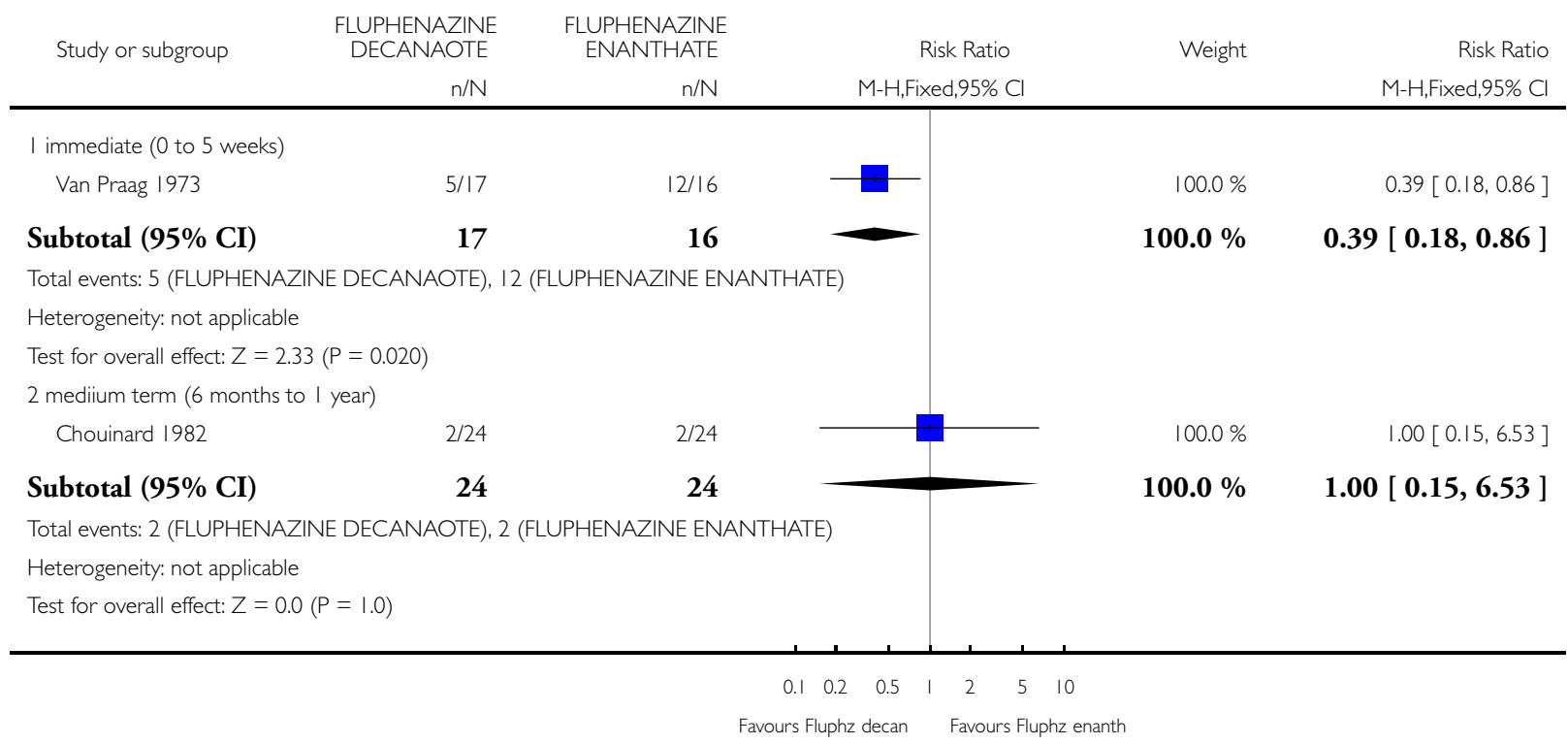




\section{Analysis 10.2. Comparison 10 FLUPHENAZINE DECANAOTE vs FLUPHENAZINE ENANTHATE,}

Outcome 2 Global state: 2. Relapse.

Review: Fluphenazine decanoate (depot) and enanthate for schizophrenia

Comparison: 10 FLUPHENAZINE DECANAOTE vs FLUPHENAZINE ENANTHATE

Outcome: 2 Global state: 2. Relapse

\begin{tabular}{|c|c|c|c|c|c|}
\hline \multirow[t]{2}{*}{ Study or subgroup } & $\begin{array}{l}\text { FLUPHENAZINE } \\
\text { DECANAOTE }\end{array}$ & $\begin{array}{c}\text { FLUPHENAZINE } \\
\text { ENANTHATE }\end{array}$ & Risk Ratio & \multirow[t]{2}{*}{ Weight } & \multirow{2}{*}{$\begin{array}{r}\text { Risk Ratio } \\
\text { M-H,Fixed,95\% Cl}\end{array}$} \\
\hline & $\mathrm{n} / \mathrm{N}$ & $\mathrm{n} / \mathrm{N}$ & M-H,Fixed,95\% Cl & & \\
\hline \multicolumn{6}{|l|}{ I immediate (0 to 5 weeks) } \\
\hline Altamura 1985 & $2 / 6$ & $2 / 5$ & & $51.4 \%$ & $0.83[0.18,3.96]$ \\
\hline Van Praag 1973 & $1 / 17$ & $2 / 16$ & & $48.6 \%$ & $0.47[0.05,4.70]$ \\
\hline Subtotal (95\% CI) & 23 & 21 & & $100.0 \%$ & $0.66[0.18,2.43]$ \\
\hline \multicolumn{6}{|c|}{ Total events: 3 (FLUPHENAZINE DECANAOTE), 4 (FLUPHENAZINE ENANTHATE) } \\
\hline \multicolumn{6}{|c|}{ Heterogeneity: Chi $^{2}=0.17, \mathrm{df}=1(P=0.68) ;\left.\right|^{2}=0.0 \%$} \\
\hline \multicolumn{6}{|c|}{ Test for overall effect: $Z=0.63(P=0.53)$} \\
\hline \multicolumn{6}{|c|}{2 short term ( 6 weeks to 5 months) } \\
\hline Donlon 1976 & $6 / 14$ & $3 / 16$ & & $100.0 \%$ & $2.29[0.70,7.48]$ \\
\hline Subtotal (95\% CI) & 14 & 16 & & $100.0 \%$ & $2.29[0.70,7.48]$ \\
\hline \multicolumn{6}{|c|}{ Total events: 6 (FLUPHENAZINE DECANAOTE), 3 (FLUPHENAZINE ENANTHATE) } \\
\hline \multicolumn{6}{|c|}{ Heterogeneity: not applicable } \\
\hline \multicolumn{6}{|c|}{ Test for overall effect: $Z=1.37(P=0.17)$} \\
\hline \multicolumn{6}{|c|}{3 medium term (6 months to I year) } \\
\hline MacCrimmon 1978 & $7 / 24$ & $3 / 25$ & & $100.0 \%$ & $2.43[0.71,8.32]$ \\
\hline Subtotal (95\% CI) & 24 & 25 & & $100.0 \%$ & $2.43[0.71,8.32]$ \\
\hline \multicolumn{6}{|c|}{ Total events: 7 (FLUPHENAZINE DECANAOTE), 3 (FLUPHENAZINE ENANTHATE) } \\
\hline \multicolumn{6}{|c|}{ Heterogeneity: not applicable } \\
\hline Test for overall effect: $Z=$ & $(P=0.16)$ & & & & \\
\hline
\end{tabular}




\section{Analysis 10.3. Comparison I0 FLUPHENAZINE DECANAOTE vs FLUPHENAZINE ENANTHATE,}

Outcome 3 Behavioiur: Leaving the study early.

Review: Fluphenazine decanoate (depot) and enanthate for schizophrenia

Comparison: 10 FLUPHENAZINE DECANAOTE vs FLUPHENAZINE ENANTHATE

Outcome: 3 Behavioiur: Leaving the study early

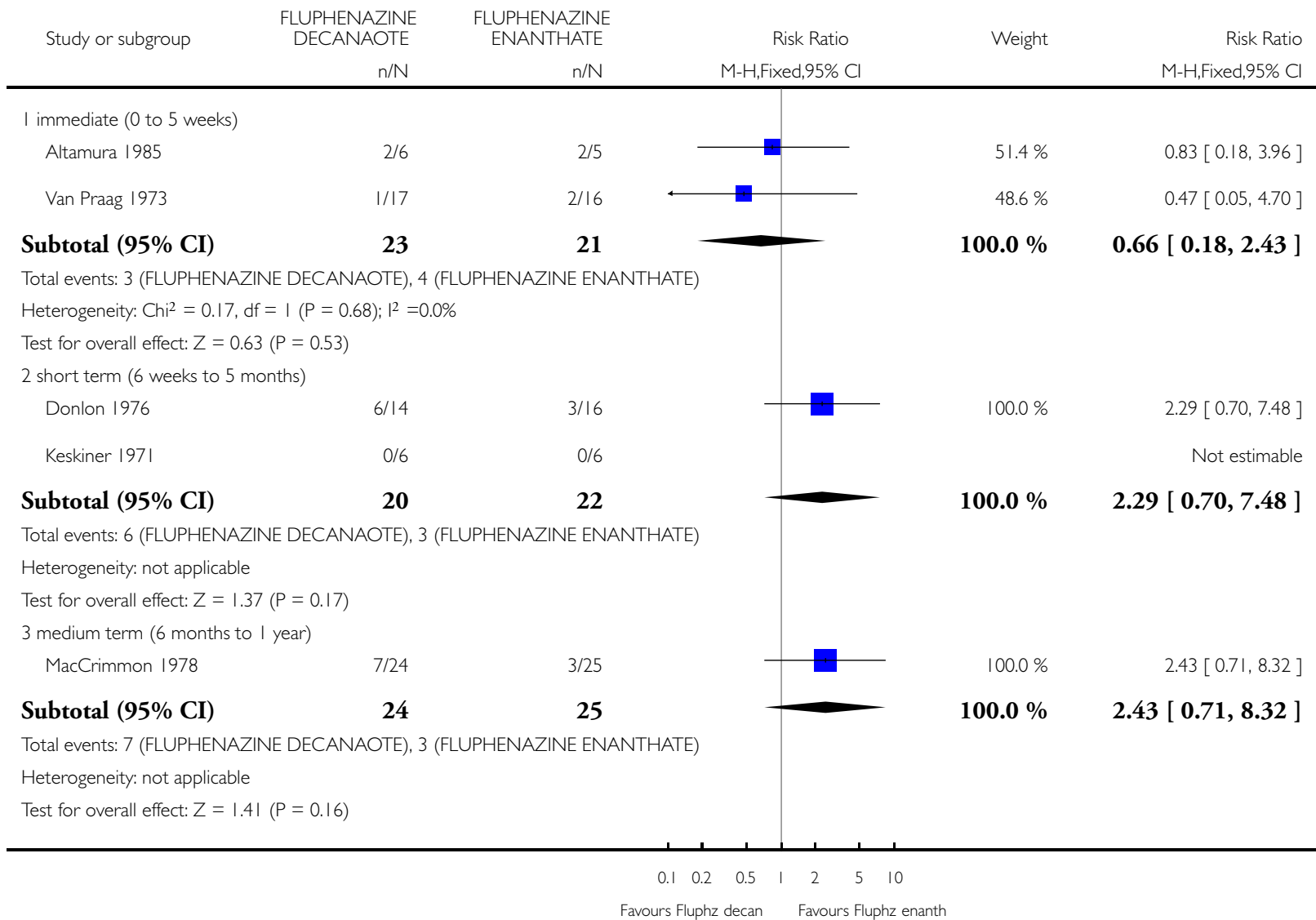


Analysis 10.4. Comparison 10 fLUPHENAZINE DECANAOTE vs FLUPHENAZINE ENANTHATE, Outcome 4 Mental State: BPRS medium term $(6$ months to I year - high score $=$ poor $)$.

Review: Fluphenazine decanoate (depot) and enanthate for schizophrenia

Comparison: I0 FLUPHENAZINE DECANAOTE vs FLUPHENAZINE ENANTHATE

Outcome: 4 Mental State: BPRS medium term (6 months to I year - high score = poor)

\begin{tabular}{|c|c|c|c|c|c|c|c|}
\hline \multirow[t]{2}{*}{ Study or subgroup } & \multirow{2}{*}{$\begin{array}{r}\text { FLUPHENAZINE } \\
\text { DECANAOTE } \\
\text { N }\end{array}$} & \multicolumn{3}{|c|}{$\begin{array}{c}\text { FLUPHENAZINE } \\
\text { ENANTHATE }\end{array}$} & $\begin{array}{r}\text { Mean } \\
\text { Difference }\end{array}$ & \multirow[t]{2}{*}{ Weight } & \multirow{2}{*}{$\begin{array}{r}\text { Mean } \\
\text { Difference } \\
\text { IV,Fixed,95\% Cl }\end{array}$} \\
\hline & & Mean(SD) & $N$ & Mean(SD) & IV,Fixed,95\% Cl & & \\
\hline MacCrimmon 1978 & 17 & $26(7)$ & 22 & $26(5)$ & 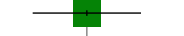 & $100.0 \%$ & $0.0[-3.93,3.93]$ \\
\hline Total (95\% CI) & 17 & & 22 & & & $100.0 \%$ & $0.0[-3.93,3.93]$ \\
\hline \multicolumn{8}{|c|}{ Heterogeneity: not applicable } \\
\hline \multicolumn{8}{|c|}{ Test for overall effect: $Z=0.0(P=1.0)$} \\
\hline \multicolumn{8}{|c|}{ Test for subgroup differences: Not applicable } \\
\hline & & & & -10 & -5 & 10 & \\
\hline \multicolumn{8}{|c|}{ Favours Fluphz decan } \\
\hline
\end{tabular}

\section{Analysis 10.5. Comparison 10 FLUPHENAZINE DECANAOTE vs FLUPHENAZINE ENANTHATE, Outcome 5 Adverse effects: la. Movement disorders - general.}

Review: Fluphenazine decanoate (depot) and enanthate for schizophrenia

Comparison: 10 FLUPHENAZINE DECANAOTE vs FLUPHENAZINE ENANTHATE

Outcome: 5 Adverse effects: Ia. Movement disorders - general

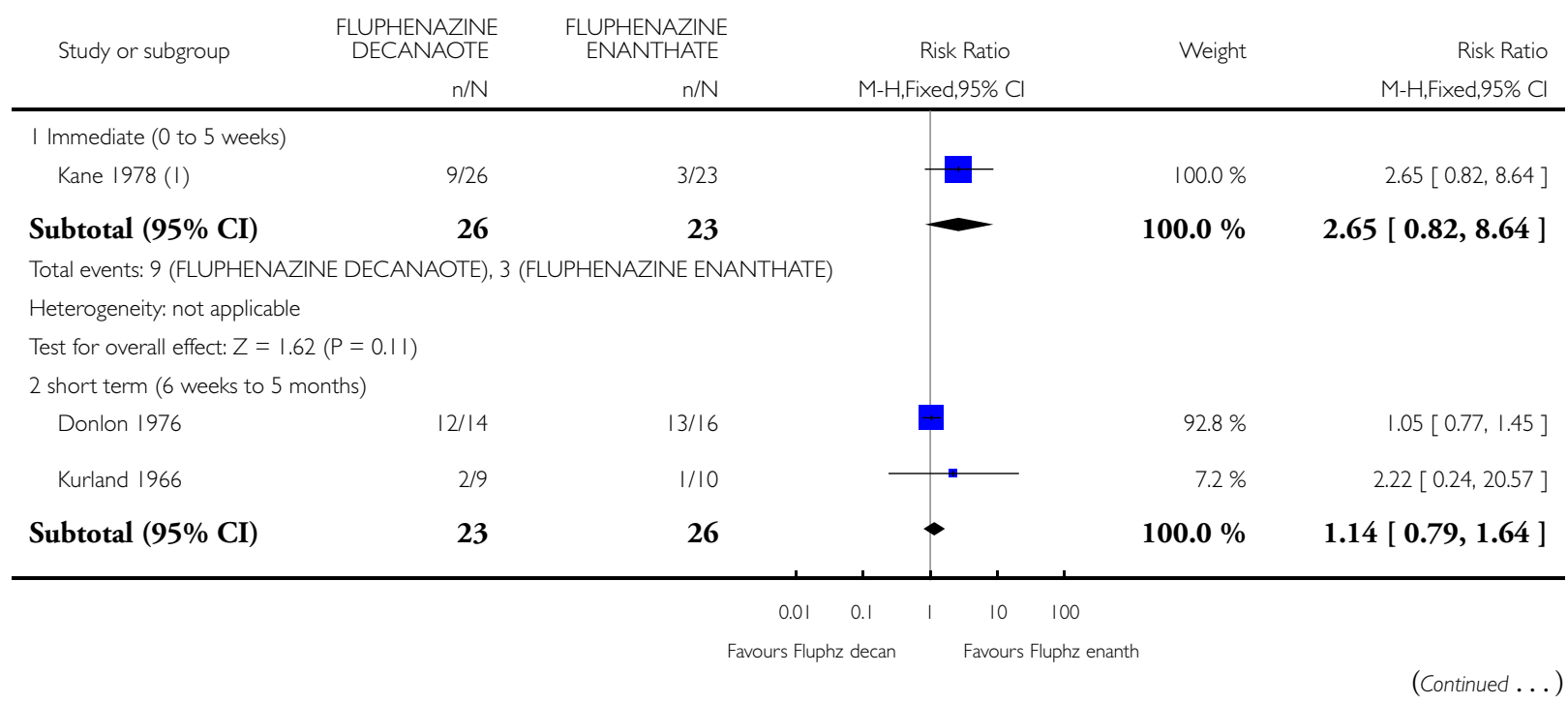

Fluphenazine decanoate (depot) and enanthate for schizophrenia (Review)

Copyright @ 2016 The Cochrane Collaboration. Published by John Wiley \& Sons, Ltd. 
Total events: 14 (FLUPHENAZINE DECANAOTE), 14 (FLUPHENAZINE ENANTHATE)

Heterogeneity: Chi $^{2}=0.57, \mathrm{df}=\mathrm{I}(\mathrm{P}=0.45) ; \mathrm{I}^{2}=0.0 \%$

Test for overall effect: $Z=0.71(P=0.48)$

Test for subgroup differences: $\mathrm{Chi}^{2}=1.80, \mathrm{df}=\mathrm{I}(\mathrm{P}=0.18), \mathrm{I}^{2}=45 \%$

$\begin{array}{cccc}0.01 & 0.1 & 1 & 10 \\ \text { Favours Fluphz decan } & & \text { Favours Fluphz enanth }\end{array}$

(I) 2 point increase on the Simpson Angus Scale

Analysis 10.6. Comparison 10 FLUPHENAZINE DECANAOTE vs FLUPHENAZINE ENANTHATE,

Outcome 6 Adverse effects: Ib. Movement disorders - needing anticholinergic drugs.

Review: Fluphenazine decanoate (depot) and enanthate for schizophrenia

Comparison: 10 FLUPHENAZINE DECANAOTE vs FLUPHENAZINE ENANTHATE

Outcome: 6 Adverse effects: Ib. Movement disorders - needing anticholinergic drugs

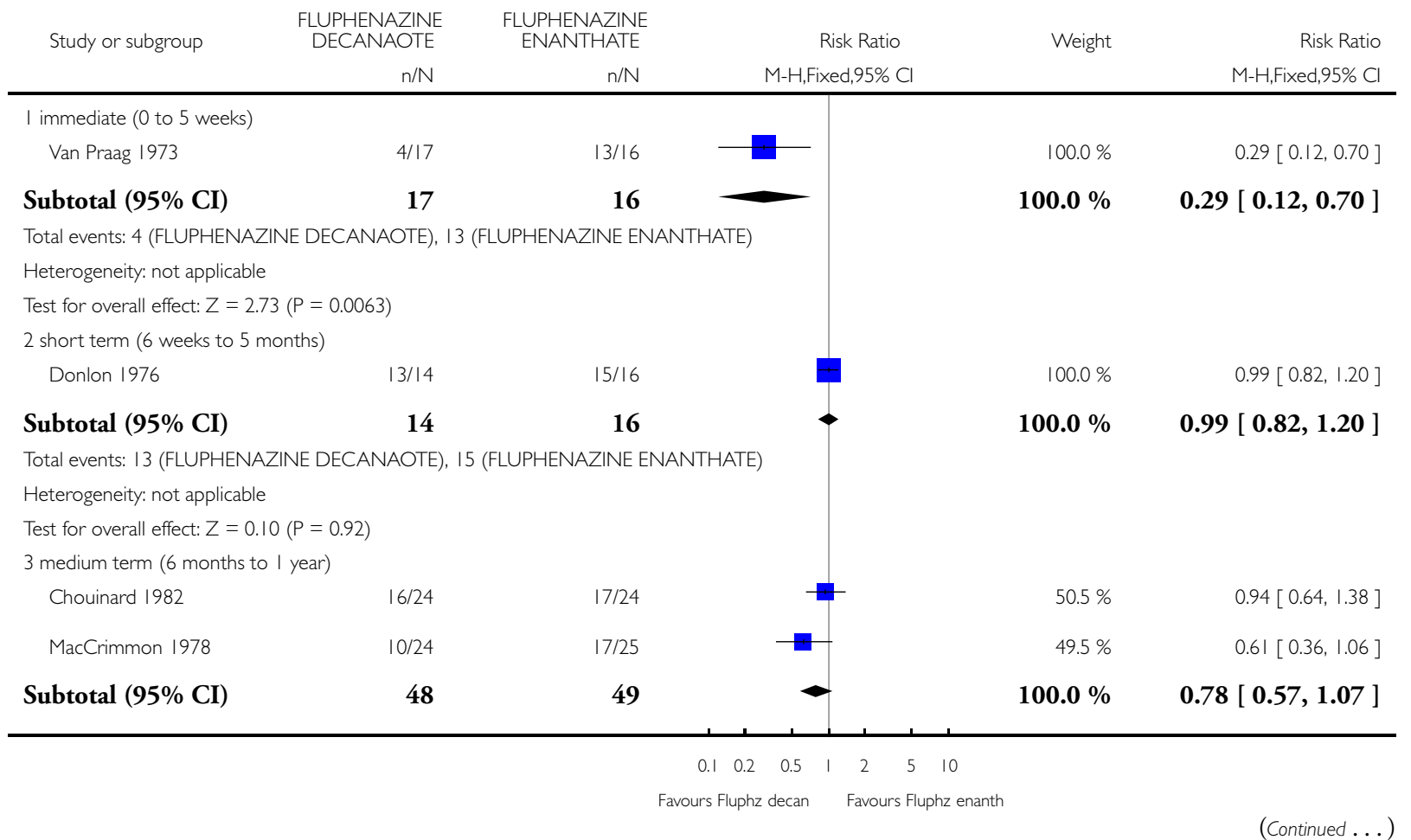

Fluphenazine decanoate (depot) and enanthate for schizophrenia (Review)

Copyright () 2016 The Cochrane Collaboration. Published by John Wiley \& Sons, Ltd. 
Total events: 26 (FLUPHENAZINE DECANAOTE), 34 (FLUPHENAZINE ENANTHATE)

Heterogeneity: $\mathrm{Chi}^{2}=1.69, \mathrm{df}=\mathrm{I}(\mathrm{P}=0.19) ; \mathrm{I}^{2}=41 \%$

Test for overall effect: $Z=1.53(P=0.12)$

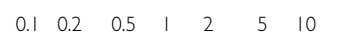

Favours Fluphz decan Favours Fluphz enanth

Analysis 10.7. Comparison 10 FLUPHENAZINE DECANAOTE vs FLUPHENAZINE ENANTHATE, Outcome 7 Adverse effects: Ic. Movement disorders - parkinsonism (short term - 6 weeks to 5 months).

Review: Fluphenazine decanoate (depot) and enanthate for schizophrenia

Comparison: 10 FLUPHENAZINE DECANAOTE vs FLUPHENAZINE ENANTHATE

Outcome: 7 Adverse effects: Ic. Movement disorders - parkinsonism (short term - 6 weeks to 5 months)

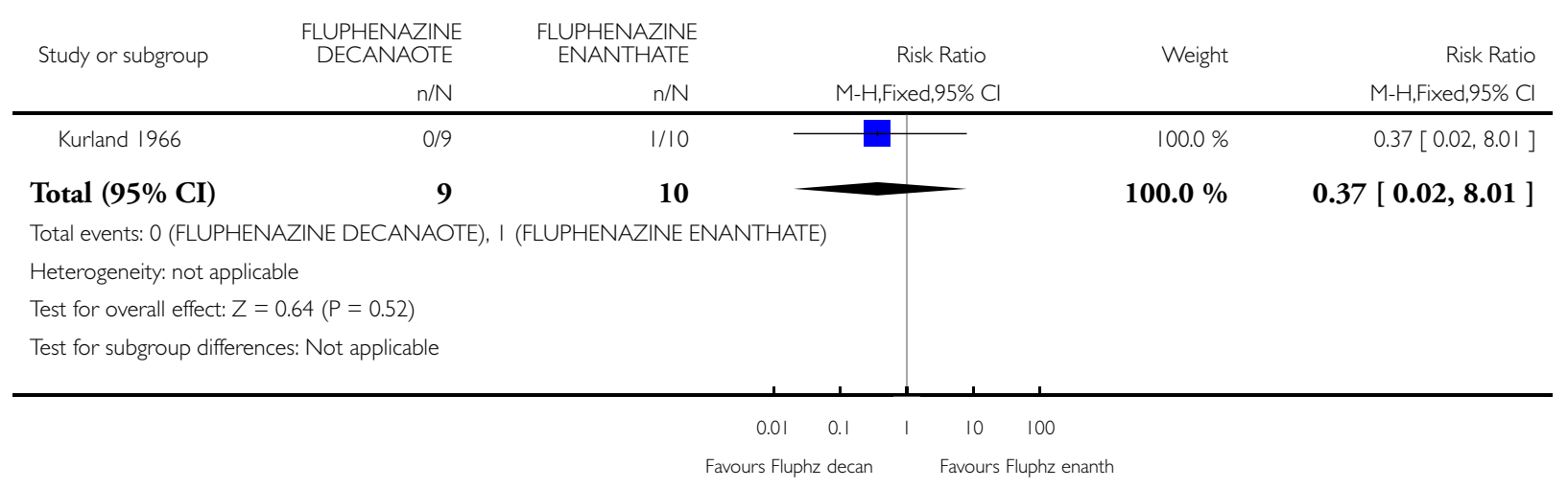


Analysis 10.8. Comparison 10 FLUPHENAZINE DECANAOTE vs FLUPHENAZINE ENANTHATE, Outcome 8 Adverse effects: Id. Movement disorders - akathisia (Immediate - 0 to 5 weeks).

Review: Fluphenazine decanoate (depot) and enanthate for schizophrenia

Comparison: 10 FLUPHENAZINE DECANAOTE vs FLUPHENAZINE ENANTHATE

Outcome: 8 Adverse effects: Id. Movement disorders - akathisia (Immediate - 0 to 5 weeks)

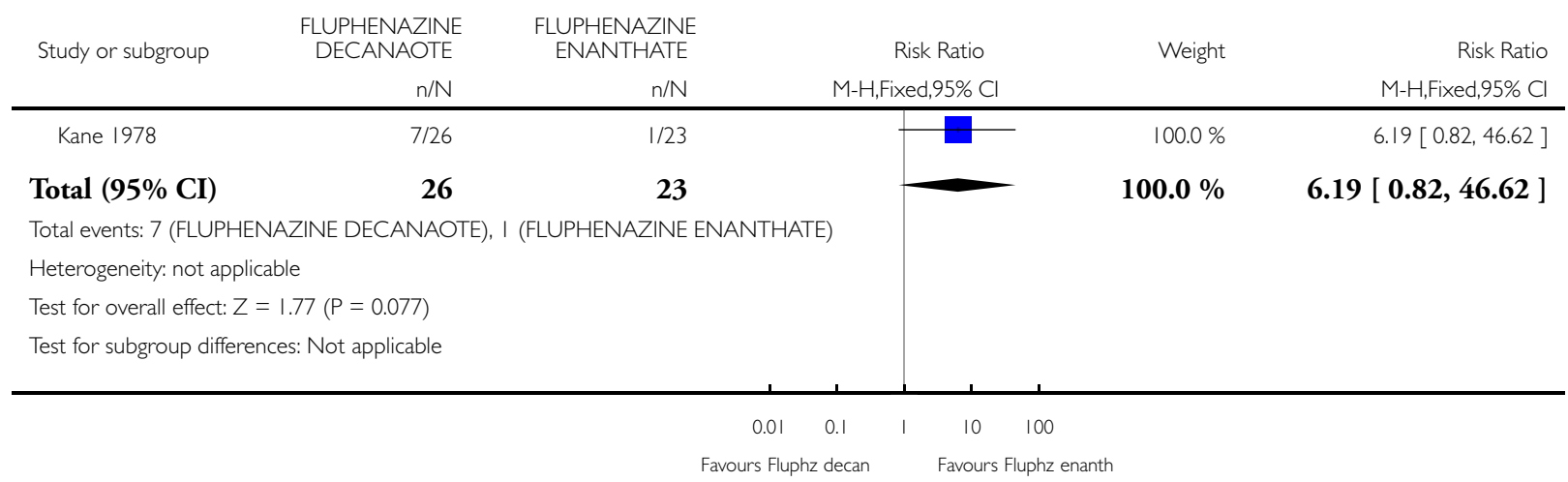

Analysis 10.9. Comparison 10 FLUPHENAZINE DECANAOTE vs FLUPHENAZINE ENANTHATE, Outcome 9 Adverse effects: 2. General adverse effects (immediate - 0 to 5 weeks).

Review: Fluphenazine decanoate (depot) and enanthate for schizophrenia

Comparison: 10 FLUPHENAZINE DECANAOTE vs FLUPHENAZINE ENANTHATE

Outcome: 9 Adverse effects: 2. General adverse effects (immediate - 0 to 5 weeks)

\begin{tabular}{|c|c|c|c|c|c|}
\hline Study or subgroup & $\begin{array}{r}\text { FLUPHENAZINE } \\
\text { DECANAOTE } \\
n / N\end{array}$ & $\begin{array}{r}\text { FLUPHENAZINE } \\
\text { ENANTHATE } \\
n / N\end{array}$ & $\begin{array}{c}\text { Risk Ratio } \\
\text { M-H,Fixed,95\% Cl }\end{array}$ & Weight & $\begin{array}{r}\text { Risk Ratio } \\
\text { M-H,Fixed,95\% Cl }\end{array}$ \\
\hline Altamura 1985 & $0 / 6$ & $5 / 5$ & & $100.0 \%$ & $0.08[0.01,1.14]$ \\
\hline Total $(95 \% \mathrm{CI})$ & 6 & 5 & & $100.0 \%$ & $0.08[0.01,1.14]$ \\
\hline \multicolumn{6}{|c|}{ Total events: 0 (FLUPHENAZINE DECANAOTE), 5 (FLUPHENAZINE ENANTHATE) } \\
\hline \multicolumn{6}{|c|}{ Heterogeneity: not applicable } \\
\hline \multicolumn{6}{|c|}{ Test for overall effect: $Z=1.87(P=0.062)$} \\
\hline \multicolumn{6}{|c|}{ Test for subgroup differences: Not applicable } \\
\hline
\end{tabular}


Analysis I I.I. Comparison I I FLUPHENAZINE DECANOATE - DOSAGE STUDIES - 2 WEEKS vs 6 WEEKS, Outcome I Global state: I. Relapse (I year).

Review: Fluphenazine decanoate (depot) and enanthate for schizophrenia

Comparison: II FLUPHENAZINE DECANOATE - DOSAGE STUDIES - 2 WEEKS vs 6 WEEKS

Outcome: I Global state: I. Relapse (I year)

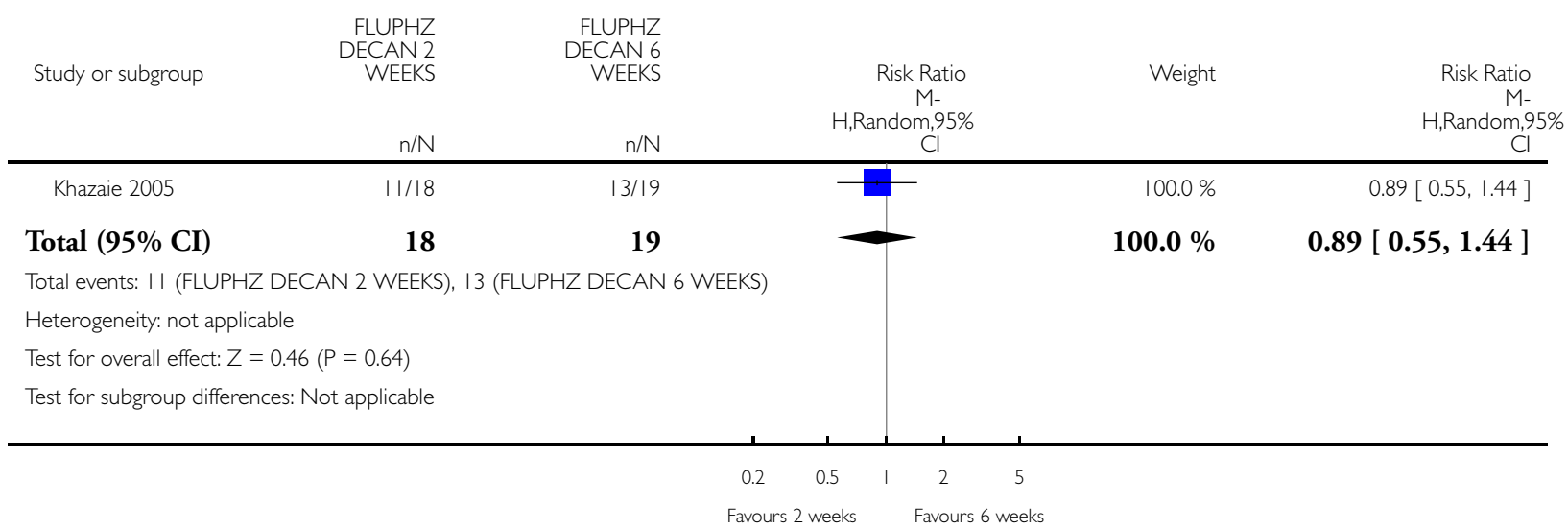

Analysis I I.2. Comparison I I FLUPHENAZINE DECANOATE - DOSAGE STUDIES - 2 WEEKS vs 6 WEEKS, Outcome 2 Leaving the study early (I year).

Review: Fluphenazine decanoate (depot) and enanthate for schizophrenia

Comparison: II FLUPHENAZINE DECANOATE - DOSAGE STUDIES - 2 WEEKS vs 6 WEEKS

Outcome: 2 Leaving the study early (I year)

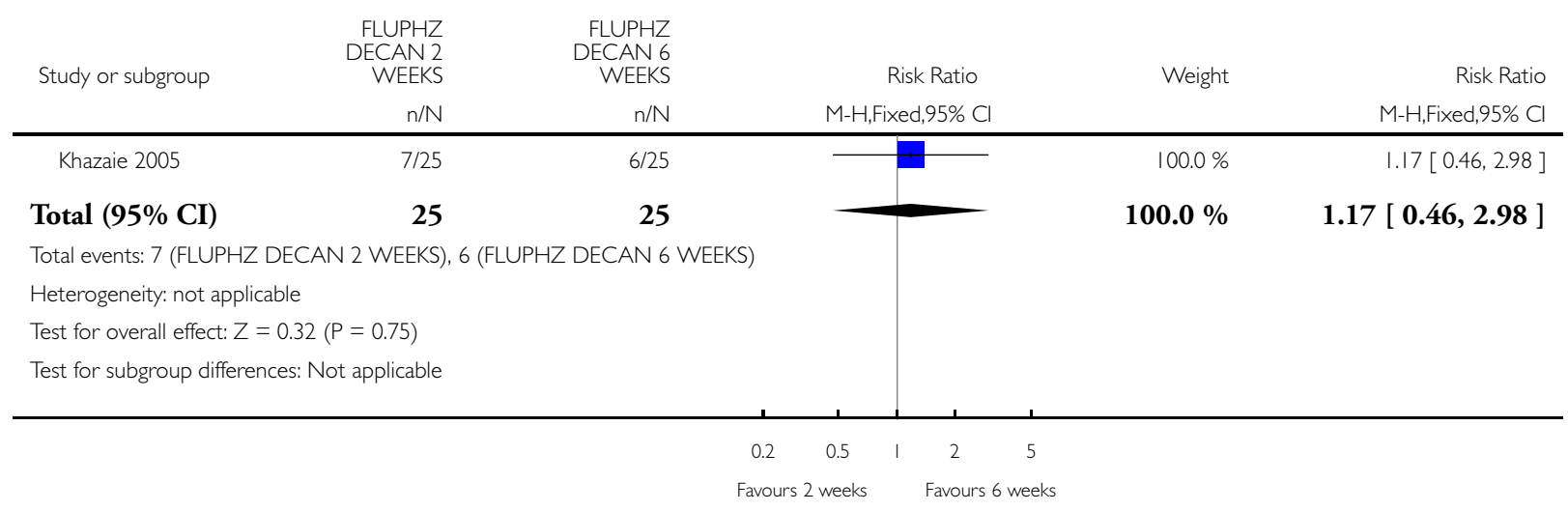


Analysis I I.3. Comparison I I FLUPHENAZINE DECANOATE - DOSAGE STUDIES - 2 WEEKS vs 6 WEEKS, Outcome 3 Mental state: I. BPRS - endpoint scores (I year) (high score = poor).

Review: Fluphenazine decanoate (depot) and enanthate for schizophrenia

Comparison: II FLUPHENAZINE DECANOATE - DOSAGE STUDIES - 2 WEEKS vs 6 WEEKS

Outcome: 3 Mental state: I. BPRS - endpoint scores (I year) (high score = poor)

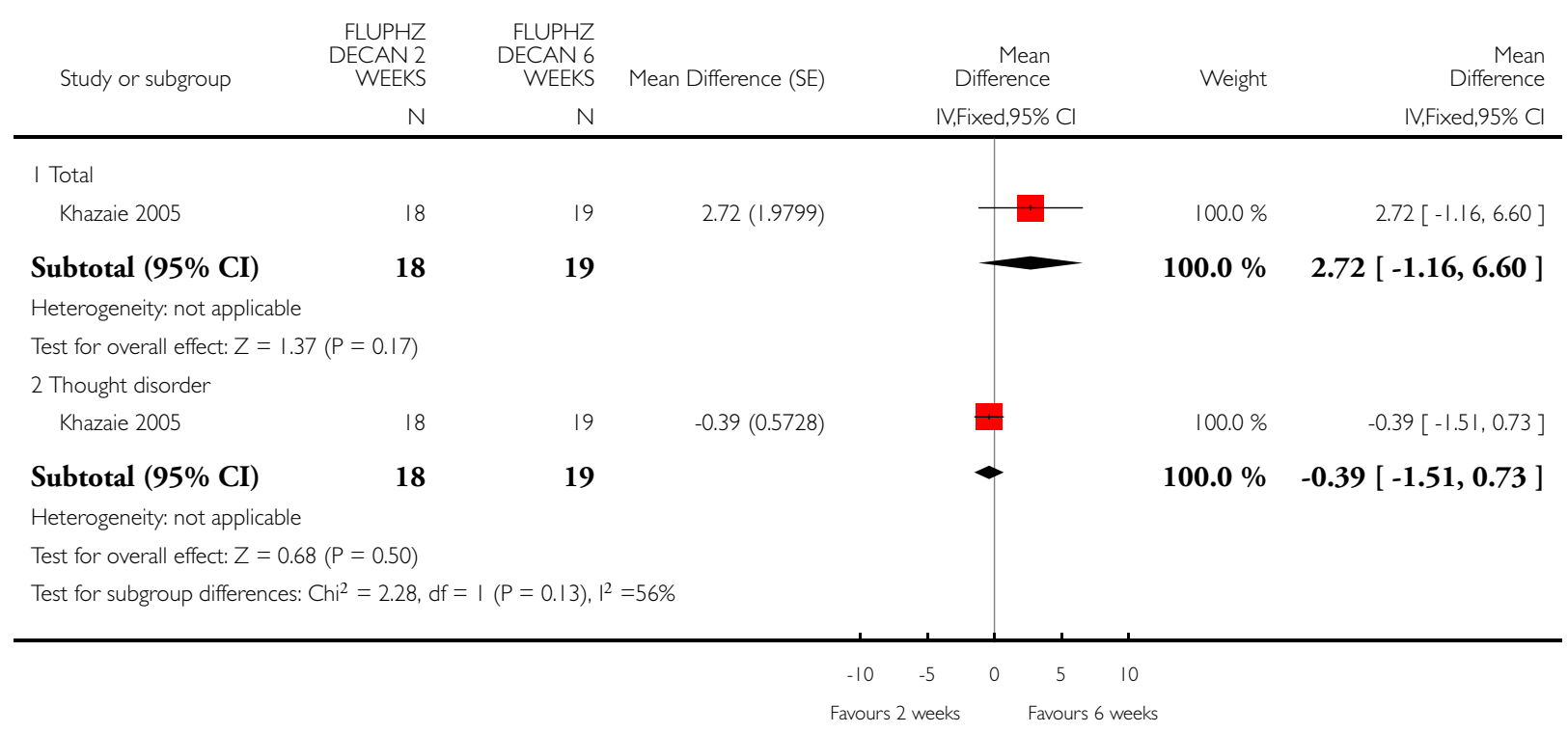


Analysis I I.4. Comparison I I FLUPHENAZINE DECANOATE - DOSAGE STUDIES - 2 WEEKS vs 6 WEEKS, Outcome 4 Adverse effects: I. Movement disorders - MPRC (I year, high = poor).

Review: Fluphenazine decanoate (depot) and enanthate for schizophrenia

Comparison: II FLUPHENAZINE DECANOATE - DOSAGE STUDIES - 2 WEEKS vs 6 WEEKS

Outcome: 4 Adverse effects: I. Movement disorders - MPRC (I year, high = poor $)$

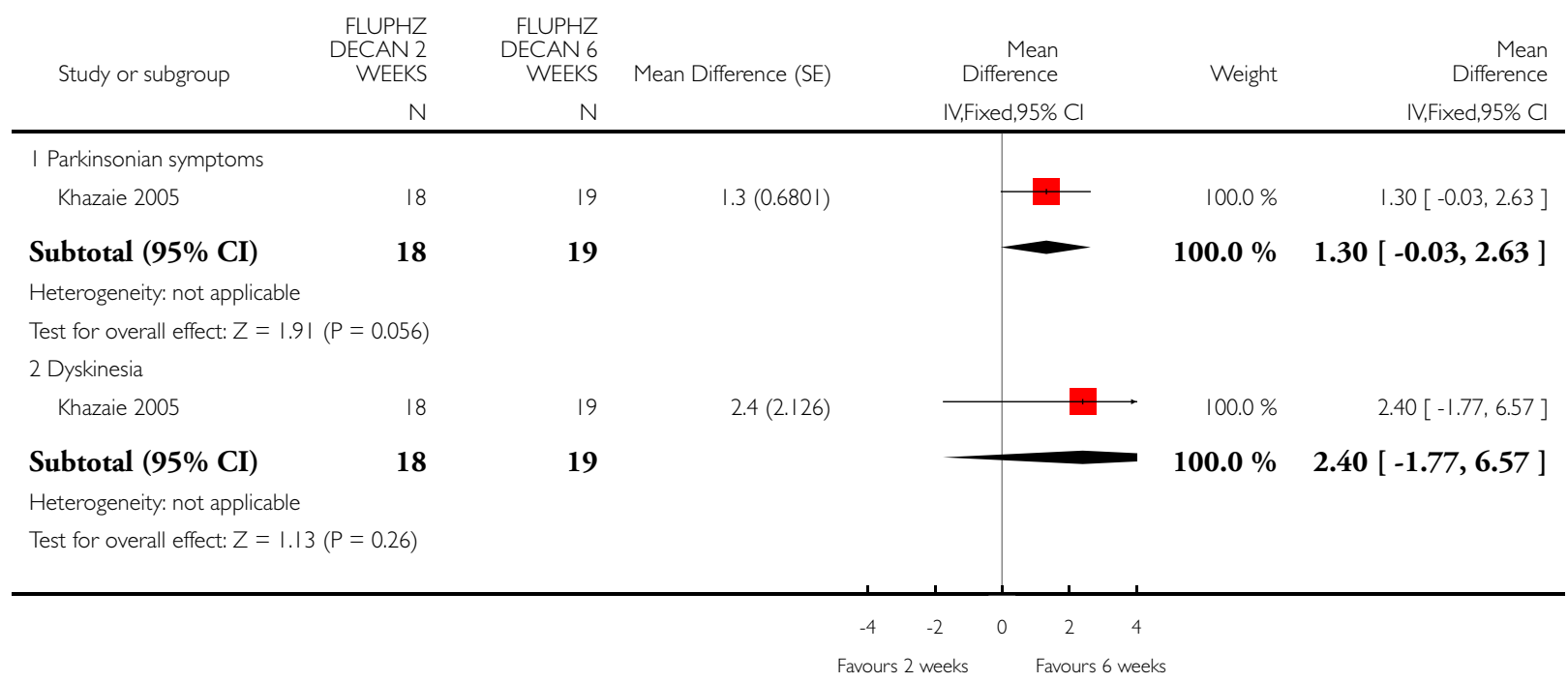

Analysis II.5. Comparison II FLUPHENAZINE DECANOATE - DOSAGE STUDIES - 2 WEEKS vs 6 WEEKS, Outcome 5 Quality of life: Quality of life scale (I year) (high score $=$ good).

Review: Fluphenazine decanoate (depot) and enanthate for schizophrenia

Comparison: II FLUPHENAZINE DECANOATE - DOSAGE STUDIES - 2 WEEKS vs 6 WEEKS

Outcome: 5 Quality of life: Quality of life scale (I year) (high score $=$ good)

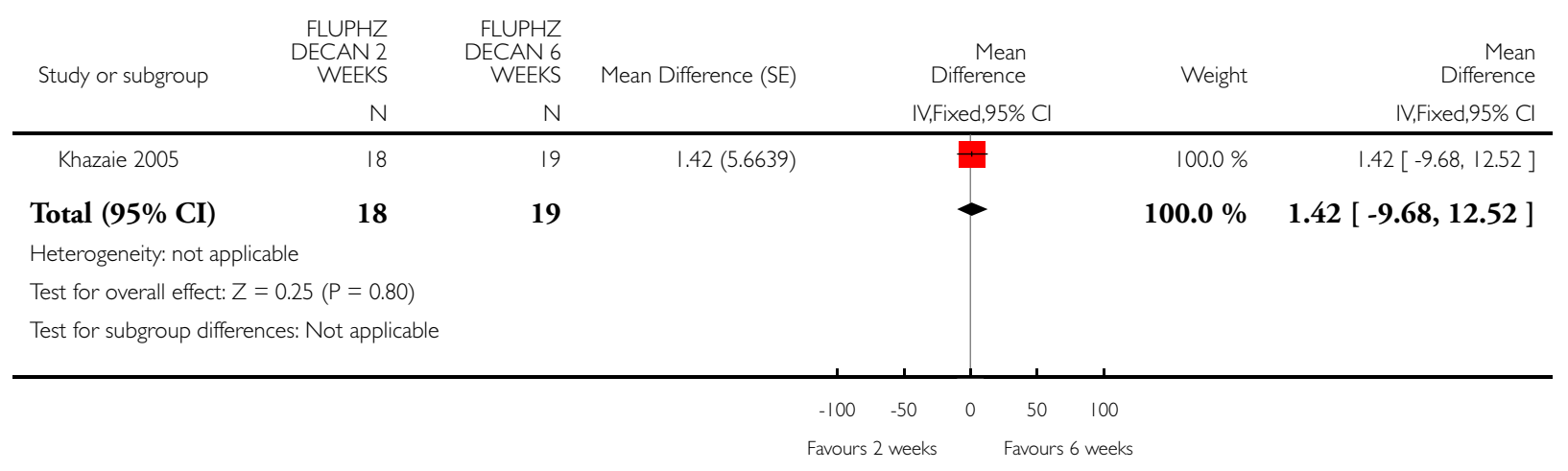

Fluphenazine decanoate (depot) and enanthate for schizophrenia (Review)

Copyright $\odot 2016$ The Cochrane Collaboration. Published by John Wiley \& Sons, Ltd. 


\section{ADDITIONAL TABLES}

\section{Table 1. Suggested design of future trials}

\begin{tabular}{ll}
\hline Methods & $\begin{array}{l}\text { Allocation: randomised - clearly described generation of sequence and concealment of allocation. } \\
\text { Blindness: double - described and tested. } \\
\text { Duration: } 12 \text { months minimum. }\end{array}$ \\
\hline Participants & $\begin{array}{l}\text { Diagnosis: schizophrenia (operational criteria). } \\
\mathrm{N}=300^{*} \\
\text { Age: any. } \\
\text { Gender: both. }\end{array}$ \\
\hline History: any. \\
\hline Interventions & $\begin{array}{l}\text { 1. Fluphenazine decanoate/enanthate: clinically acceptable dose. } \mathrm{N}=150 \\
\text { 2. Oral antipsychotic: clinically acceptable dose. } \mathrm{N}=150 .\end{array}$ \\
\hline Outcomes & $\begin{array}{l}\text { Death and all causes of mortality** } \\
\text { Clinical global state - relapse**; clinically significant change in global state**; leaving the study early** } \\
\text { Service utilisation outcomes - hospital admission**, time in hospital. } \\
\text { Adverse effects: extrapyramidal adverse effects**, other adverse effects. } \\
\text { Economic outcomes. }\end{array}$ \\
\hline Notes & $\begin{array}{l}\text { * The number of participants needed to gain sufficient power to highlight about a } 10 \% \text { difference between groups } \\
\text { for primary outcome depends on the primary outcome used and the prevalence/magnitude of this outcome. } \mathrm{N}= \\
\text { 300 is approximately the size of study to detect a } 10 \% \text { difference in improvement with } 80 \% \text { certainty } \\
\text { ** Primary outcome }\end{array}$ \\
\hline
\end{tabular}

\section{AP PENDICES}

\section{Appendix I. Previous search strategies}

1. We updated previous searches in May 2002 using the Cochrane Schizophrenia Group's Register search phrase:

[ fluphen* or *fluphen* or *modec* or *moditen* or *eutimox* or *flufen* or *prolixin* or *siqualone* or *anaten* or *dapotum* or *decazate* or *lyoridin* in title, abstract, index terms of [REFERENCE] or [(fluphenaz* AND depot*) in interventions of STUDY]

2. Details of previous electronic searches.

2.1 Electronic searching

Relevant randomised trials were identified by searching several electronic databases (the Cochrane Schizophrenia Group's Register of Trials, the Cochrane Library, Biological Abstracts, EMBASE, MEDLINE, PsycLIT and SCISEARCH).

2.2 We searched the Cochrane Schizophrenia Group's Register (1998) using the phrase:

(FLUPHEN* ${ }^{*}$ and DECANOATE or ENANTHATE ) or ((DEPOT* or (LONG and ACTING) or (DELAY* and ACTION)) and (FLUPHEN* or MODEC* or MODITEN* or EUTIMOX* or FLUFEN* or PROLIXIN* or SIQUALONE* or ANATEN* or DAPOTUM* or DECAZATE* or LYORIDIN*) or $(\# 44=2$ and $\# 44=230)$ or $\# 44=549$ ) 
2.3 We searched the COCHRANE LIBRARY (Issue 2, 1998) using the Cochrane Schizophrenia Group's phrase for schizophrenia (see Group search strategy) combined with the phrase:

(FLUPHEN* ${ }^{*}$ and DECANOATE or ENANTHATE) or ((DEPOT* or (LONG and ACTING) or (DELAY* and ACTION)) and (FLUPHEN* ${ }^{*}$ or MODEC* or MODITEN* ${ }^{*}$ or EUTIMOX* or FLUFEN* or PROLIXIN* or SIQUALONE* or ANATEN* or DAPOTUM* ${ }^{*}$ or DECAZATE* or LYORIDIN*)) or (FLUPHEN* ME and DELAYED-ACTION-PREPARATIONS* ME))]

2.4 We searched BIOLOGICAL ABSTRACTS (January 1982 to June 1998 - current disc issue) using the Cochrane Schizophrenia Group's phrase for randomised controlled trials and schizophrenia (see Group search strategy) combined with the phrase:

[and (FLUPHENAZINE near1 DECANOATE or ENANTHATE) or ((DEPOT* or (LONG near4 ACTING) or (DELAY* near2 ACTION)) near (FLUPHENAZINE or MODEC* or MODITEN* or EUTIMOX* or FLUFEN* or PROLIXIN* or SIQUALONE* or ANATEN* or DAPOTUM* or DECAZOTE* or LYONRIDIN*)]

2.5 EMBASE (January 1980 to June 1998 - current disc issue): we searched this database using the Cochrane Schizophrenia Group's phrase for randomised controlled trials and schizophrenia (see Group search strategy) combined with the phrase:

(FLUPHEN* near1 DECANOATE or ENANTHATE) or ((DEPOT* or (LONG near4 ACTING) or (DELAY* near2 ACTION)) near (FLUPHEN* ${ }^{*}$ or MODITEN* ${ }^{*}$ or MODEC* or FLUFEN* or EUTIMOX* or PROLIXIN* or SIQUALONE* or ANATEN* or DAPOTUM* $^{*}$ or DECAZATE* or LYORIDIN*) or "FLUPHENAZINE-DECANOATE"/ all subheadings]

2.6 We searched MEDLINE (January 1966 to June 1998 - current disc issue) using the Cochrane Schizophrenia Group's phrase for randomised controlled trials and schizophrenia (see Group search strategy) combined with the phrase:

(FLUPHEN* near1 DECANOATE or ENANTHATE) or ((DEPOT* or (LONG near4 ACTING) or (DELAY* near2 ACTION)) near (FLUPHEN* ${ }^{*}$ or MODEC* ${ }^{*}$ or MODITEN* ${ }^{*}$ or EUTIMOX* or FLUFEN* or PROLIXIN* or SEQUALONE* or ANATEN* or DAPOTUM* or DECAZATE* or LYORIDIN*) or ("FLUPHENAZINE"/ all subheadings and explode "DELAYED-ACTIONPREPARATIONS”/ all subheadings))]

2.7 We searched PsycLIT (January 1974 to June 1998 - current disc issue) using the Cochrane Schizophrenia Group's phrase for randomised controlled trials and schizophrenia (see Group search strategy) combined with the phrase:

(FLUPHEN* near1 DECANOATE or ENANTHATE) or ((DEPOT* or (LONG near4 ACTING) or (DELAY* near2 ACTION)) near (FLUPHEN* or MODEC* or MODITEN* or EUTIMOX* or FLUFEN* or PROLIXIN* or SIQUALONE* or ANATEN* or DAPOTUM* or DECAZATE* or LYORIDIN)

\section{Appendix 2. Previous methods: data collection and analyses}

\section{Study selection}

In the original review, all the studies we identified were inspected by the principal reviewer (SQ). A randomly selected sample of $10 \%$ of all reports was re-inspected by $\mathrm{AD}$ in order to ensure selection was reliable. Where disagreement occurred, we resolved this by discussion, where there was still doubt, we acquired the full article for further inspection. Once we had obtained the full articles, SQ and AD independently decided whether they met the review criteria. We resolved disagreement by discussion and when this was not possible sought further information. We added these trials to the list of those awaiting assessment pending acquisition of further information. For the updated version of this review, JR inspected and data extracted all studies.

\section{Assessment of methodological quality}

We allocated trials to three quality categories, as described in The Cochrane Collaboration Handbook (Alderson 2004). Again, we resolved disputes by discussion. When this was not possible and further information was necessary to clarify which category to allocate a trial to, we did not enter data and allocated the trial to the list of those awaiting assessment. We included only trials in Category A or $\mathrm{B}$ in the review.

\section{Data collection}

In the first version of this review SQ and AD independently extracted data from selected trials. JR did this for the updated version. Again, we resolved disputes by discussion. When this was not possible and further information was necessary to resolve the dilemma, we did not enter data and added this outcome of the trial to the list of those awaiting assessment.

\section{Data synthesis}

4.1 Incomplete data.

Where more than $30 \%$ of those randomised were lost to follow-up by six months, or $50 \%$ by beyond that time, we felt data to be too prone to bias and did not used these outcomes.

4.2 Dichotomous - yes/no - data.

Fluphenazine decanoate (depot) and enanthate for schizophrenia (Review)

Copyright @ 2016 The Cochrane Collaboration. Published by John Wiley \& Sons, Ltd. 
4.2.1 Statistics: For binary outcomes, for example, 'admitted' or 'not admitted', we estimated a Relative Risk with $95 \%$ confidence interval. Where possible, we calculated the number needed to treat statistic (NNT) taking into account the event rate in the control group.

4.2.2 Intention-to-treat: We present data on a 'once-randomised-always-analyse' basis. Those who were lost to follow-up are all assumed to have the negative outcome, with the exception of death, which was coded separately. For example, for the outcome of relapse, we considered those who were lost to follow-up all to have relapsed.

4.2.3 Data reporting

4.3 Continuous - scale - data

4.3.1 Normal data: Mental health continuous data are often not 'normally' distributed. To avoid the pitfall of applying parametric tests to non-parametric data we applied the following standards to all data before inclusion: i. standard deviations and means had to be reported in the paper or had to be obtainable from the authors; ii. when a continuous outcome started from a finite number (such as 0 ), the standard deviation, when multiplied by 2, had to be less than the mean (as otherwise the mean is unlikely to be an appropriate measure of the centre of the distribution - Altman 1996). We did not enter data not meeting the second standard into the RevMan calculator (which assumes a normal distribution). However, data not meeting these standards can be reported in the 'Other data types' of the results section if they have been analysed with appropriate non-parametric tests. If continuous data were recording change, where the finite parameters of the measure were unclear, the reviewers decided whether the data were usable or not.

4.3.2 Rating scales: A wide range of instruments is available to measure mental health outcomes. These instruments vary in quality and many are not valid, or are ad hoc. For outcome instruments some minimum standards have to be set. They could be that: i. the psychometric properties of the instrument should have been described in a peer-reviewed journal; ii. not written or modified by one of the trialists; iii. the instrument should either be: (a) a self report, or (b) completed by an independent rater or relative (not the therapist); and iv. the instrument should be a global assessment of an area of functioning (Marshall 1998).

4.3.3 Endpoint versus change data: where possible we presented endpoint data and if both endpoint and change data were available for the same outcomes then we only reported the former in this review.

4.3.4 Cluster trials

Studies increasingly employ 'cluster randomisation' (such as randomisation by clinician or practice) but analysis and pooling of clustered data poses problems. Firstly, authors often fail to account for intra class correlation in clustered studies, leading to a 'unit of analysis' error (Divine 1992) whereby P values are spuriously low, confidence intervals unduly narrow and statistical significance overestimated. This causes type I errors (Bland 1997; Gulliford 1999).

Where clustering was not accounted for in primary studies, we presented data in a table, with a $\left(^{*}\right)$ symbol to indicate the presence of a probable unit of analysis error. In subsequent versions of this review we will seek to contact first authors of studies to obtain intra-class correlation co-efficients of their clustered data and to adjust for this using accepted methods (Gulliford 1999). If clustering had been incorporated into the analysis of primary studies, we would have presented these data as if from a non-cluster randomised study, but would have adjusted for the clustering effect.

We have sought statistical advice and have been advised that the binary data as presented in a report should be divided by a 'design effect'. This is calculated using the mean number of participants per cluster $(\mathrm{m})$ and the intraclass correlation coefficient (ICC) [Design effect $\left.=1+(\mathrm{m}-1)^{*} \mathrm{ICC}\right]$ (Donner 2002). If the ICC was not reported it was assumed to be 0.1 (Ukoumunne 1999).

\section{Heterogeneity}

Firstly, we considered all the included studies within any comparison to judge clinical heterogeneity. We then used visual inspection of graphs to investigate the possibility of statistical heterogeneity. This was supplemented using, primarily, the I-squared statistic. This provides an estimate of the percentage of variability due to heterogeneity rather than chance alone. Where the I-squared estimate was greater than or equal to $75 \%$, we interpreted this as indicating the presence of high levels of heterogeneity (Higgins 2003). If inconsistency was high, we did not summate these data, but presented them separately and investigated reasons for heterogeneity. Data were presented using a fixed-effect model for homogeneous data and a random-effects model for heterogeneous data.

\section{Tables and figures}

Where possible we entered data into RevMan in such a way that the area to the left of the line of no effect indicated a favourable outcome for the fluphenazine esters.

Fluphenazine decanoate (depot) and enanthate for schizophrenia (Review) 


\section{WHAT'S NEW}

Last assessed as up-to-date: 1 December 2013.

\begin{tabular}{l|ll}
\hline Date & Event & Description \\
\hline 25 February 2016 & Amended & $\begin{array}{l}\text { The comparison interventions in five studies (Falloon 1978, McCreadie 1980, McCreadie 1982, } \\
\text { Quitkin 1978 \& Rifkin 1977) were incorrectly described as IM for the comparison Fluphenazine } \\
\text { decanoate IM versus oral neuroleptics. All now are correctly described as oral. Leaving the study } \\
\text { early data from Magnus 1979 have been removed for this comparison. These corrections do not } \\
\text { change results }\end{array}$ \\
\hline
\end{tabular}

\section{H I S T O R Y}

Protocol first published: Issue 2, 1996

Review first published: Issue 4, 1997

\begin{tabular}{lll}
\hline Date & Event & Description \\
\hline 1 May 2014 & $\begin{array}{l}\text { New citation required but conclusions have not } \\
\text { changed }\end{array}$ & $\begin{array}{l}\text { Additional data from three new trials have been added } \\
\text { to the review, no change to overall conclusions of review }\end{array}$ \\
\hline 4 December 2013 & New search has been performed & $\begin{array}{l}\text { Results from } 2013 \text { search added to the review. Method } \\
\text { sections amended to include new Cochrane methodol- } \\
\text { ogy }\end{array}$ \\
\hline 25 January 2011 & Amended & byline corrected \\
\hline 30 October 2008 & Amended & Converted to new review format. \\
\hline
\end{tabular}

\section{CONTRIBUTIONSOFAUTHORS}

Seema Quraishi - prepared protocol, undertook searches, selected and acquired studies, extracted data, summated data, produced report (2002, 2011).

Maurice Eisenbruch - prepared the protocol, undertook searches, selected and acquired studies, extracted data, summated data, produced reports $(2002,2011)$.

Anthony David - acquired funding, helped prepare protocol, select studies, extract data, and produce the report (2002, 2011).

Clive Adams - acquired funding, helped prepare protocol, undertook searches, selected and acquired studies, extracted and summated data, produce the reports and prepared the updated review $(2002,2011,2013)$.

John Rathbone - selected and acquired studies, extracted and summated data and prepared the updated review (2002, 2011).

Enhance Reviews - Nicola Maayan and Rosie Asher screened studies, extracted data for two new studies, assessed the risk of bias for all included studies, prepared 'Summary of findings' tables and prepared the updated review (2013). 


\section{DECLARATIONSOF INTEREST}

The authors have declared no conflicts of interest.

Enhance Reviews: is a company that carries out systematic reviews mostly for the public sector, it currently does not provide services for the pharmaceutical industry.

\section{SOURCES OF SUPPORT}

\section{Internal sources}

- No sources of support supplied

\section{External sources}

- NHS-ROCD Health Technology Assessment Programme., UK.

\section{DIFFERENCES BETWEEN PROTOCOLANDREVIEW}

We have updated our methods since publication of the protocol to reflect advances in Cochrane methodology.

\section{INDEX TERMS}

\section{Medical Subject Headings (MeSH)}

Administration, Oral; Antipsychotic Agents [administration \& dosage; *therapeutic use]; Delayed-Action Preparations [administration $\&$ dosage; therapeutic use]; Fluphenazine [administration \& dosage; *analogs \& derivatives; therapeutic use]; Injections, Intramuscular; Randomized Controlled Trials as Topic; Schizophrenia [drug therapy]

\section{MeSH check words}

Humans 